\title{
Hydrostatic Machine Tool Spindles
}

\author{
by \\ Kevin L. Wasson \\ Bachelor of Science in Mechanical Engineering \\ University of Oklahoma \\ May 9, 1992 \\ Master of Science in Mechanical Engineering \\ Massachusetts Institute of Technology \\ February 16, 1994
}
Submitted to the Department of Mechanical Engineering in Partial Fulfillment of the Requirements for the Degree of
Doctor of Philosophy
at the
Massachusetts Institute of Technology

June 4, 1996

C 1996 Massachusetts Institute of Technology

All rights reserved

Signature of Author

Department of Mechanical Engineering

June 41996

Certified by

Professor Alexander H. Slocum

Thesis Supervisor

Certified by

Professor John H. Lienhard V

Thesis Supervisor

Accepted by

Professor Ain A. Sonin

Chairman, Graduate Committee 


\title{
Hydrostatic Machine Tool Spindles
}

\author{
by \\ Kevin L. Wasson \\ Submitted to the Department of Mechanical Engineering \\ on June 4, 1996 in partial fulfillment of the requirements for \\ the Degree of Master of Science in \\ Mechanical Engineering
}

\begin{abstract}
This work investigates the application of hydrostatic bearings, particularly those of the self-compensating type, to precision machine tool spindles. First, relations are developed that can be used to calculate the hydraulic resistances of various bearing land flows in tilted and untilted orientations. These relations are then applied to predicting the loadcarrying characteristics of several different types of hydrostatic bearings. The bearing calculations are then integrated with shaft bending calculations to predict static spindle stiffness. Relations are also presented to calculate the frictional and thermal characteristics of the bearings at high speeds. The relations developed are compared to experimental data collected on three prototype test spindles. These spindles were found to have excellent qualities that represent a significant advance in machine tool spindle technology. Finally, a design case study is presented that illustrates how the methods developed in this work may be applied to practical spindle design.
\end{abstract}

Thesis Supervisor: Dr. Alexander H. Slocum

Title: Professor of Mechanical Engineering

Thesis Supervisor: Dr. John H. Lienhard V

Title: Professor of Mechanical Engineering 


\section{ACKNOWLEDGMENTS}

A substantial portion of the work presented in this thesis resulted from my involvement in a cooperative spindle development project. A competetive award from the NIST Advanced Technology Program, NIST Cooperative Agreement - Spindles 70NANB4H1500, provided $44 \%$ cofunding for the work described here. The remaining funding was provided by the Project Team, consisting of the following organizations and personnel:

Aesop, Inc. (Aesop)

Alex Slocum, Rick Slocum

Ford Motor Company (Ford) Jack Grant, Dave Chesney, Ming Chen, Dave Donavan, Nathaniel Field

General Motors Corporation (GM)

Mike Brink, Ron Schaffa, John Agapiou, Mo Fetouh

Giddings \& Lewis (G\&L)

Bruce Cuppan, Pete Beyer, James Jourden

Manufacturing Laboratories, Inc. (MLI)

Tom Delio, George Tlusty

National Center for Manufacturing Sciences (NCMS)

Jack McCabe, Program Manager; Debbie Howay, Hank Wallace

Olofsson Machine Tools, Inc.

Bob Bair

ORSCO, Inc. (ORSCO)

Cliff Willner, Chris Willner

SETCO Sales Company (Setco-Whitnon)

Hal Wagner, Tom O'Brien, Marty Rejniak, Steve Monahan, John Kirsch

The Torrington Company (Torrington)

Ton DeMarino, Robert Riley, Mark Lamoureaux

National Institute for Standards and Technology (NIST)

Jack Boudreaux, NIST Program Manager 
I would like to thank all of the team members who contributed to the success of the project. Working with them and learning from them has been a pleasure.

While working on the above project, I was also employed by Creare, Inc. of Hanover, NH. I would like to thank Creare, in particular President James Block, for allowing me to work on my doctorate while employed full-time. I would also like to thank Javier Valenzuela for his initiative and thoughtful insights into designing SelfCompensated Bearing \#9 of Chapter 3.

I would like to thank the National Science Foundation for the support in the preliminary stage of this project under grant number DDM - 9201906. I would also like to thank Leblond Makino Machine Tool Company for their early support in developing the HydroSpindle ${ }^{\mathrm{TM}}$.

I would also like to thank Weldon Machine Tool for their continued support in the development of the HydroSpindle ${ }^{\mathrm{TM}}$. In particular, I would like to thank President Jim Flinchbaugh.

I would like to thank my two advisors, Professor Alexander H. Slocum and Professor John H. Lienhard V. During the course my studies, Professor Slocum provided me with constant intellectual challenge and inspired me with the amount of energy and creativity that he puts into his work. I am grateful to Professor Lienhard for the help that he gave me in making sense out of the difficult problems we encountered. Over the course of my studies, I developed great respect for him.

I would like to thank my parents, Marvin and Carolyn wasson, who encouraged me and taught me my work ethic.

This thesis is dedicated to my wife, Sue, who both encouraged me and put up with me over the course of its development. 


\section{TABLE OF CONTENTS}

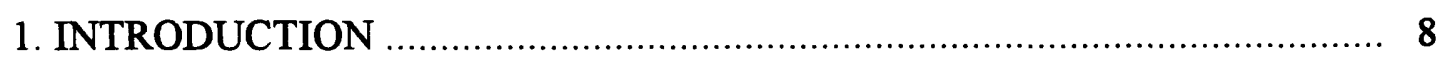

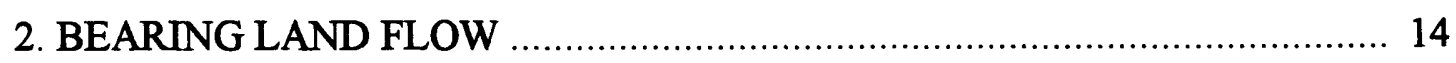

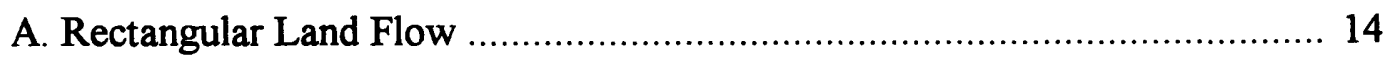

i. Simple Untitled Rectangular Land Flow .......................................... 14

ii. Tilted Rectangular Land Flow ....................................................... 17

iii. Converging Rectangular Land Flow ............................................. 20

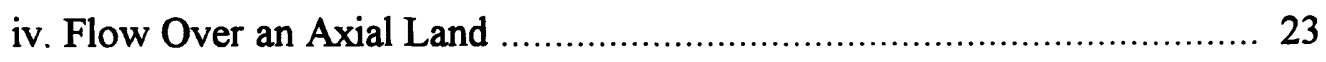

v. Flow Over a Circumferential Land ................................................... 29

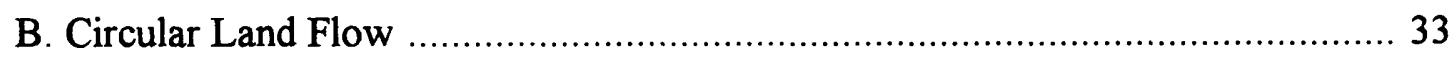

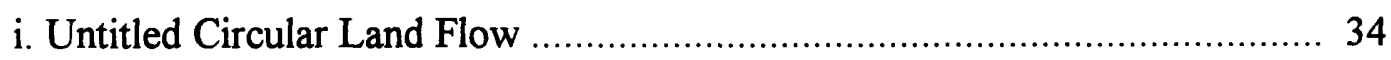

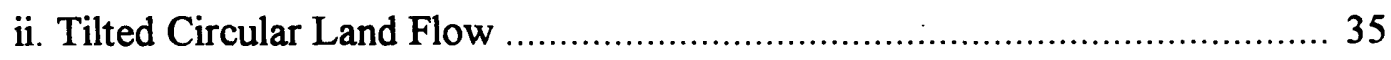

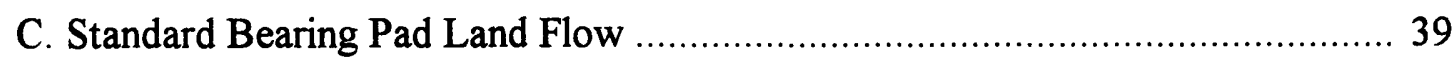

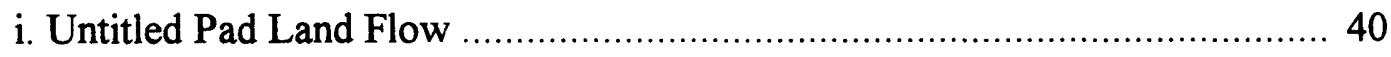

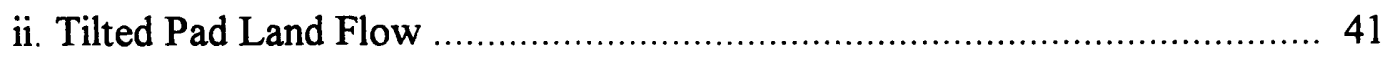

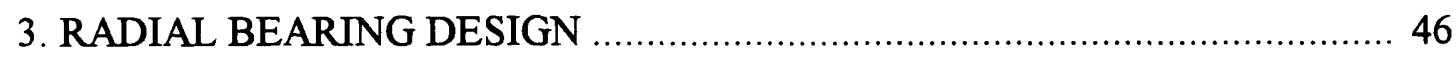

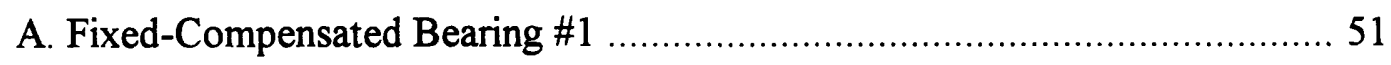

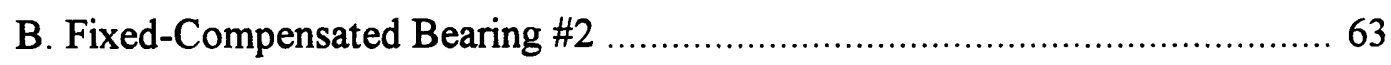

C. Self-Compensated Bearing \#1 ....................................................... 72

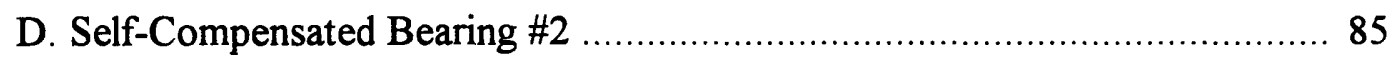

E. Self-Compensated Bearing \#3 ………................................................ 89

F. Self-Compensated Bearing \#4 .......................................................... 94

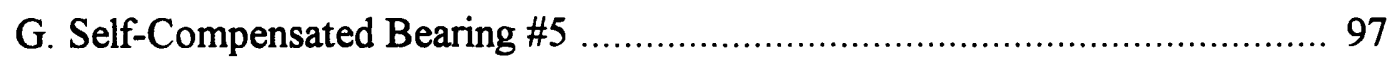

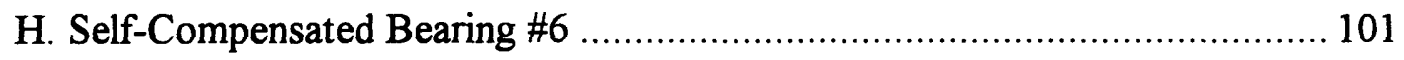

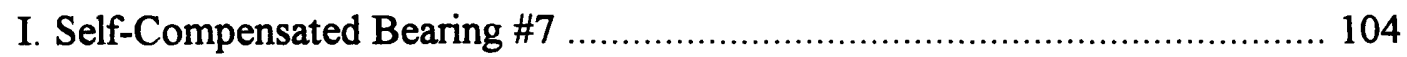

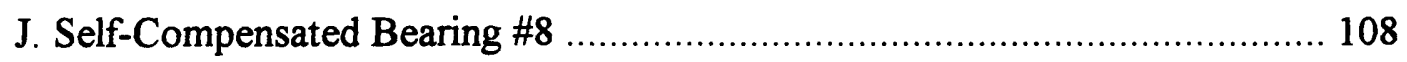

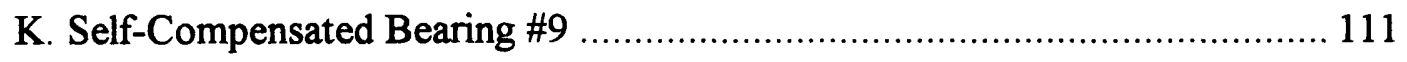

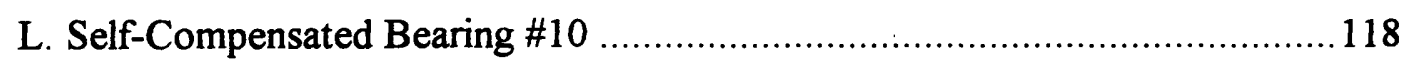

M. Comparison of Twelve Radial Hydrostatic Bearings ............................... 128

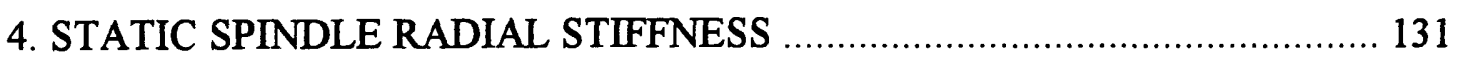

A. Simple Model of a Constant Cross-Section Spindle ................................ 132

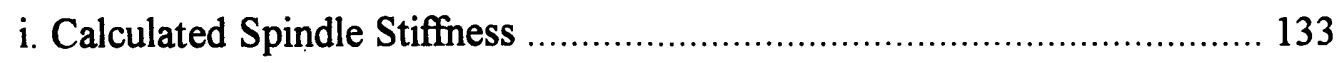

ii. Incorporation of the Effect of Shaft Tilting ......................................... 135

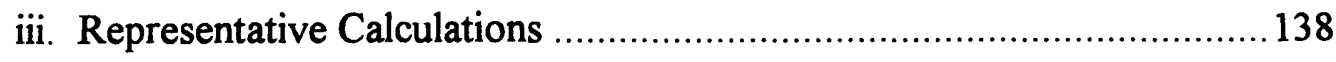


B. Modeling Spindle Stiffness Using Finite Element Methods ........................ 139

i. Benchmark Validation of the Finite Element Model ............................... 141

ii. Shaft Bending with Non-Negligible Shear Deformations .......................143

iii. Development of the Shear Correction Factor ....................................... 144

iv. Formation of the Global Stiffness Matrix ........................................... 148

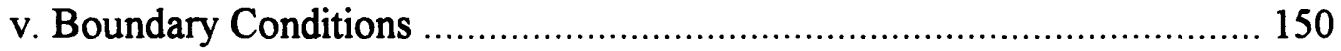

vi. Formation of the Global Load Vector ............................................. 152

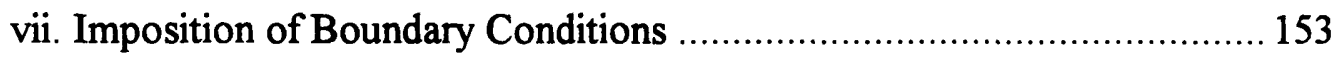

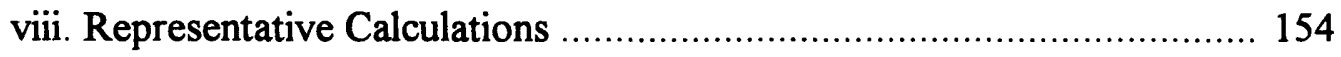

C. Comparison of Spindle Stiffness Using Twelve Bearings ............................ 155

i. Fixed-Compensated Bearing \#1 …................................................. 156

ii. Fixed-Compensated Bearing \#2 ………….................................... 158

iii. Self-Compensated Bearing \#1 .......................................................... 160

iv. Self-Compensated Bearing \#2 …………....................................... 162

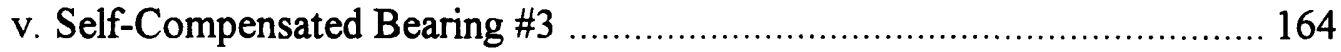

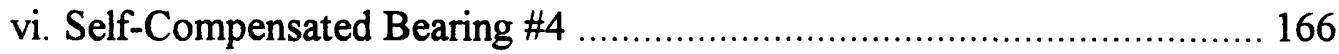

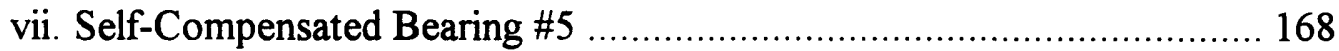

viii. Self-Compensated Bearing \#6 ….................................................. 170

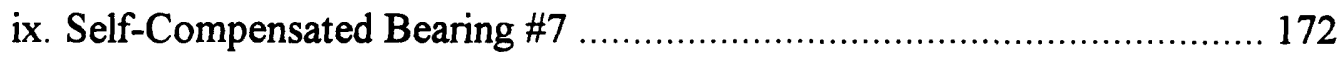

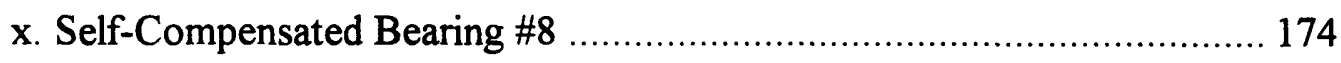

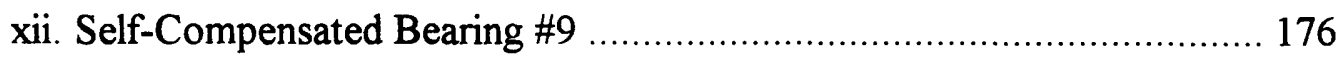

xiii. Self-Compensated Bearing \#10 …............................................... 178

xiv. Comparison of Stiffness with Twelve Different Bearings .................... 180

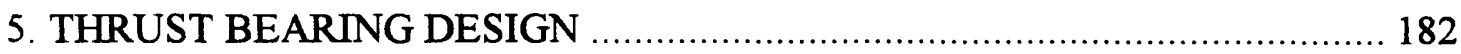

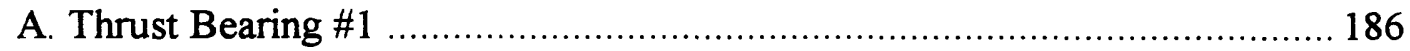

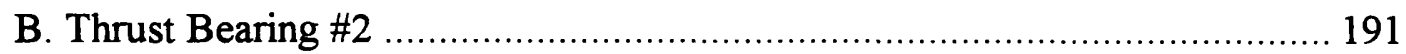

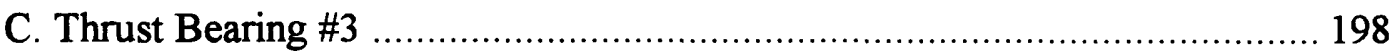

6. FRICTIONAL AND THERMAL CHARACTERISTICS ……......................... 202

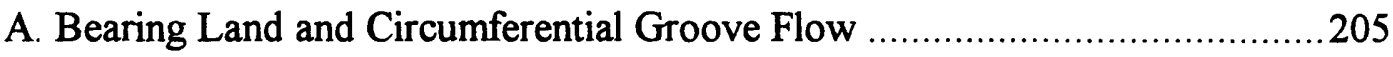

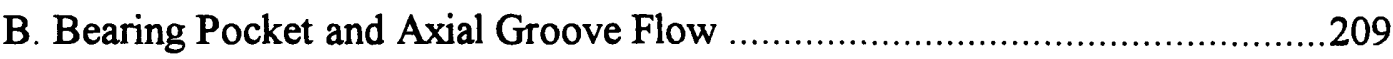

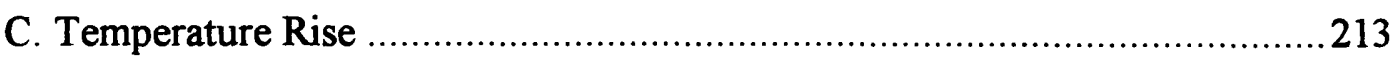

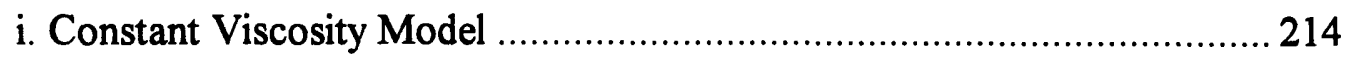

ii. Temperature-Dependent Viscosity Model .......................................... 217

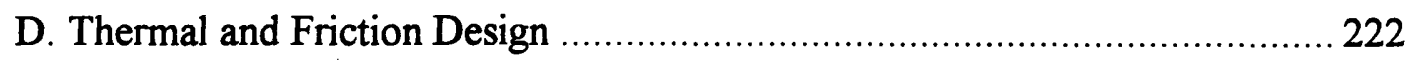

7. EXPERIMENTAL TESTING: THREE PROTOTYPE SPINDLES …...............230

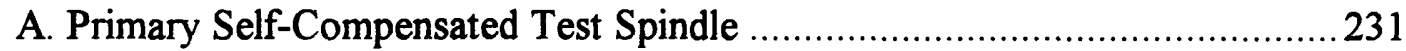




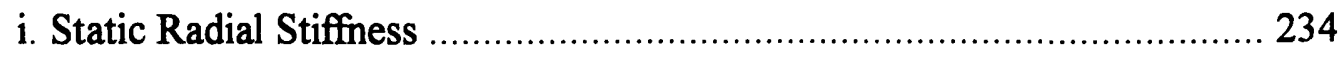

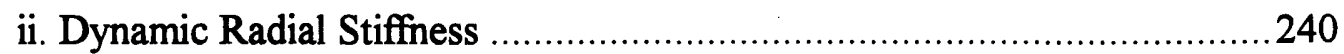

iii. Static Thrust Stiffness and Load Capacity ........................................... 244

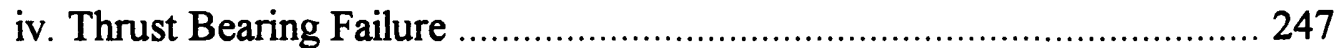

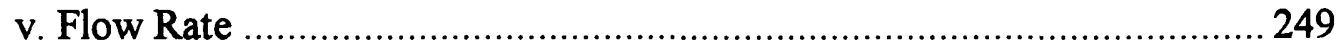

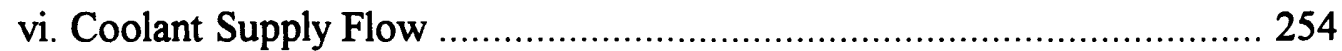

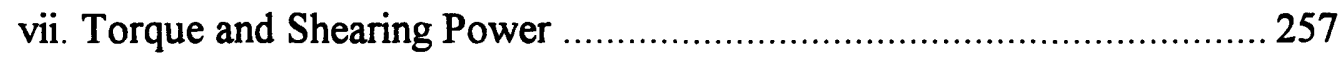

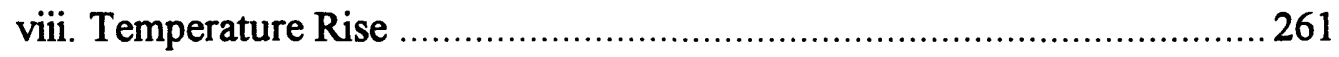

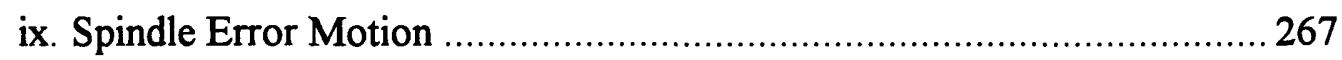

B. Twisted-Groove Spindle \#1: Eight-Pocket Spindle ................................... 270

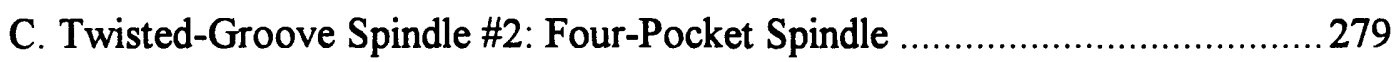

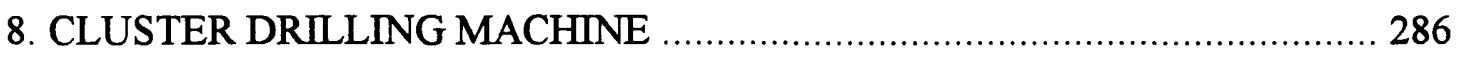

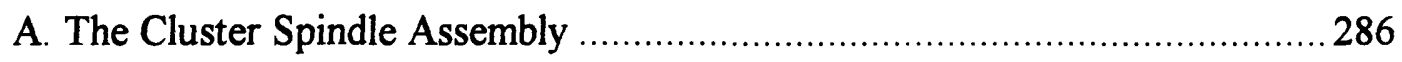

B. Comparison with the Rolling Element Spindle …....................................... 296

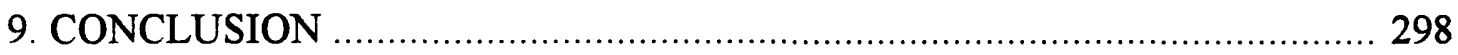

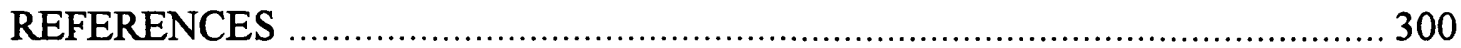

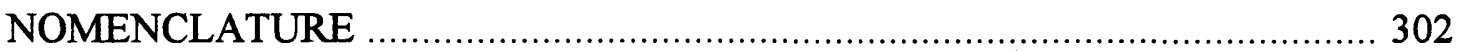

APPENDIX A: BEAM THEORY AND FINITE ELEMENT METHODS ........... 304

APPENDIX B: SOLUTIONS TO FLUID CIRCUITS ……............................. 313

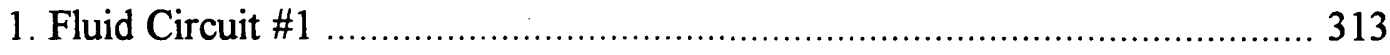

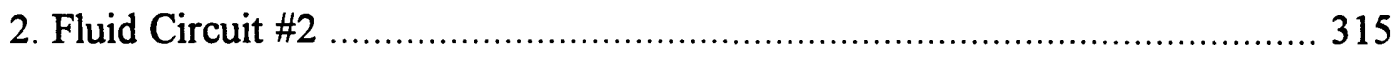

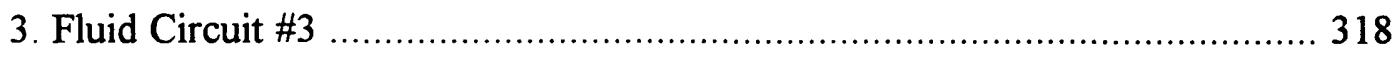

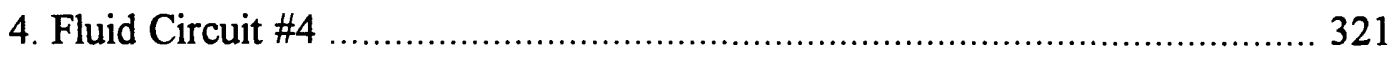

APPENDIX C: CRITERIA FOR LAMINAR, FULLY-DEVELOPED FLOW ..... 327

APPENDIX D: MODEL FOR POCKET PRESSURE GRADIENT ……................337 


\section{INTRODUCTION}

The type of rotary motion bearings principally used in machine tool spindles today are rolling element bearings. Hydrostatic fluid film bearings offer many advantages over rolling element bearings in spindles for milling, drilling, grinding, and other applications. In general, hydrostatic spindles have much higher precision, much higher damping, better crash tolerance, and longer life than rolling element spindles. Higher precision is realized because the fluid film eliminates mechanical contact between the shaft and housing and creates an averaging of the rotational motion that helps to mitigate the effect of manufacturing variations in the bearing geometry and the bearing clearance. High damping is achieved by the squeeze-film effect that occurs in the fluid film. Better crash tolerance is realized with hydrostatic bearings because the higher fluid damping increases the dynamic stiffness of the spindle and enables it to absorb impacts with higher energy. Longer life is achieved because, in the absence of machine crashes and the use of proper filtering, there is no wear.

Other types of bearings that compete with hydrostatic bearings for use in machine tool spindles include hydrodynamic bearings, aerostatic bearings, and magnetic bearings. Hydrodynamic bearings generally cannot generate enough stiffness or load capacity for machine tool applications, particularly when used at low and moderate speeds. The load capacity of aerostatic bearings is also too low for most machine tool applications because air can only be used safely in most industrial environments at pressures less than 200 psi. Electromagnets generate load-supporting pressures no higher than air bearings, and so magnetic bearings cannot generate sufficient load capacity to handle the high cutting loads 
required for most machine tool applications. Magnetic spindles are also usually prohibitively expensive.

Although hydrostatic bearings have many key advantages over other types of bearings for machine tool spindles, they have not seen widespread use by the machine tool industry over the past few decades. This is true primarily because of their high cost and the ergonomic problems associated with their oil supply systems. In addition, hydrostatic spindles have been limited to low speed applications because at high speeds, viscous shearing of the oil creates excessive power consumption, heat generation, and thermal growth errors. To alleviate these problems, new hydrostatic bearings are under development (described herin) that enable water-based coolants to be used as the lubricating fluid and are significantly more easy to manufacture. By pressurizing the bearings with the same coolant that is used to cool the machining process, ergonomic problems are eliminated, sealing the spindle is simplified, and the heat generated by viscous shearing is substantially reduced. In addition, the new bearing methods substantially simplifies the spindle fabrication, thereby reducing manufacturing costs.

The new bearing methods utilize a principle called self-compensation, in which bearing lands integrated into the bearing construction are used to compensate the bearing and enable it to achieve high stiffness without the need for orifices, capillaries, valves, or other compensating devices. Eliminating the need for external compensation substantially simplifies spindle fabrication and reduces spindle manufacturing costs. In addition, since the compensation resistances and the pad resistances both change equally with changes in the nominal clearance between the shaft and housing, the bearing load capacity is 
independent of the bearing clearance. The tolerances on the bearing clearance can therefore be increased, thereby reducing manufacturing costs even further. Selfcompensation also enables the bearing geometry to be readily manufactured on the outer diameter of the shaft rather than on the inner diameter of the housing; this substantially simplifies spindle fabrication since complex bearing geometries can be easily milled and turned onto the surface of the shaft. Also, since all fluid resistances are formed as bearing lands with moving adjacent surfaces, any sludge that forms in the fluid supply system and passes into the bearing will be sheared apart upon entering the bearing. This advantage in particular makes self-compensated bearings attractive because they can be supplied with aqueous fluids such as machine tool coolants in which sludge forms readily with the presence of light. Replacing the oil with coolant makes the bearing ergonomically more attractive and reduces the viscous shearing power consumption of the bearings.

Conventionally, hydrostatic spindle design is accomplished by considering the bearing performance and the overall spindle stiffness separately $[1,2,3]$. The bearings are designed by considering the shaft to move eccentric but parallel to the bearing housing and calculating the restoring force developed by the bearing. The stiffness of the bearings are calculated in this way and optimized to provide maximum stiffness with acceptable pumping power. Next, the shaft is modeled as a beam or series of connected beams supported by springs. The stiffness of the springs used in the spindle calculations are the stiffnesses of the radial bearings previously calculated. The design of the spindle is optimized to provide maximum stiffness at the front face of the spindle or at the toolpoint. This is standard practice for hydrostatic machine tool spindle design. 
The design procedure described above can often provide adequate spindle performance. However, small changes in the bearing geometry can often significantly increase the spindle stiffness. For example, decreasing the width of the bearing lands nearest the front of the spindle can often significantly improve the spindle stiffness. These design improvements are missed when the bearing design is disconnected from the spindle design.

Integration of the bearing design and the spindle design is accomplished in this work with the relations and methods developed in Chapters 2, 3 and 4. In Chapter 2, relations are developed that can be used to calculate the hydraulic resistances of various bearing land flows in tilted orientations. In Chapter 3, these relations are applied to predicting the load-carrying characteristics of several different types of radial hydrostatic bearings. In Chapter 4 , the bearing calculations are integrated with shaft bending calculations to predict static spindle deflections.

The performance of hydrostatic thrust bearings are considered in Chapter 5 . Often the axial stiffness and load capacity of a spindle are just as important as its radial stiffness and load capacity. Relations are developed in Chapter 5 to design and optimize three typical hydrostatic thrust bearings that provide excellent load-carrying performance.

The frictional and thermal characteristics of hydrostatic bearings are considered in Chapter 6. Relations are developed that can be used to predict the viscous shearing power consumption, heat generation, and temperature rise. The design of bearings for optimal thermal performance is considered and several important conclusions are drawn. 
Chapter 7 describes the tests performed at Setco Whitnon on three test spindles. The tests performed on the primary test spindle were very successful. The radial stiffness of the spindle was measured to be over 130,000 pounds per inch at a shaft location 4 " from the front of the air seal. The load capacity of the thrust bearing was tested and found to about 1,100 pounds. The spindle was driven to $18,000 \mathrm{rpm}$ and subjected to impact hits on the end of the shaft exceeding 150 pounds while rotating at $10,000 \mathrm{rpm}$ without failure. Modal plots show that the spindle is very well damped with a dynamic stiffness of about 73,000 pounds per inch at a resonant frequency of $550 \mathrm{~Hz}$. In addition, the temperature rise of the water flowing through the pockets was measured to be $4.2^{\circ} \mathrm{C}$ when the shaft was rotating at $10,000 \mathrm{rpm}, 12.5^{\circ} \mathrm{C}$ when rotating at $18,000 \mathrm{rpm}$. All of these results were predicted by the relations developed in this work within a reasonably close margin, and the behavior of the spindle is believed to be well understood.

The tests performed on the secondary test spindles are also described in Chapter 7. The new spindle configuration has grooves on the surface of the shaft to route the fluid from the compensating lands to the load-supporting pockets. The spindle fabrication is greatly simplified with this scheme. In addition, these spindles require substantially less flow rate and pumping power to achieve the same stiffness. The goals of the tests were to verify the static stiffnesses of the spindles and to verify their stability at high speeds. The two spindles had excellent static stiffnesses that were comparable to that achieved with the primary test spindle. Also, the four-pocket spindle was successfully driven to $20,000 \mathrm{rpm}$ and its stability was verified. These tests verified the basis of the design. 
In Chapter 8, the design of a machine that drills clusters of holes in steel components is presented. Hydrostatic spindles are found to to be clearly superior to rolling element spindles this application. The use of hydrostatic bearings enabled the diameter of the shafts to be increased, thereby greatly increasing the radial stiffness of the spindles. It is expected that the new machine will be able to drill holes with fewer passes than the existing machine, thereby reducing manufacturing costs.

There are three appendices attached to this thesis that are referred to throughout the text. In Appendix A, the matrices of four-node isoparametric beam elements are developed. In Appendix B, four fluid circuits commonly used to model the fluid flow of hydrostatic bearings are described and relations to solve them are presented. In Appendix $\mathrm{C}$, criteria are developed for the flow on the lands of hydrostatic bearing to be laminar and fully-developed. In Appendix D, relations are presented that can be used to calculate the change in flow rate caused by the fluid pumping that occurs within some types of hydrostatic bearings. 


\section{BEARING LAND FLOW}

The load-carrying characteristics of a hydrostatic bearing are predominately determined by the fluid flow across the bearing lands. The lands are used as hydraulic resistances to control the fluid pressure within the bearing pockets such that it has loadcarrying capacity. Clearly, the bearing land flow must be well understood and analytically predictable if a hydrostatic bearing is to be designed properly. In this chapter, the flow of fluid across hydrostatic bearing lands is considered. Relations are developed that can be used to calculate the pressure profiles across rectangular and circular lands. Secondary effects such as tilting of the lands will also be considered. The relations developed in this chapter will be used in Chapters 3 and 4 to predict the load-carrying characteristics of many different types of hydrostatic bearings.

\section{A. Rectangular Land Flow}

\section{A.i. Simple Untilted Rectangular Land Flow}

Consider a land that has a width $\mathrm{L}$ and a uniform gap $\mathrm{h}$. The resistance to flow through the gap can be derived by considering the highly viscous fluid flow shown in Figure 2.1. In the derivation, an assumption of fully-developed laminar flow will be used. These assumptions are valid as long as both the supply pressure is sufficiently low and shaft rotational speed is sufficiently low. Criteria are outlined in Appendix C that can be used to determine whether the laminar, fully-developed assumptions used in this chapter are valid for a operation condition. 


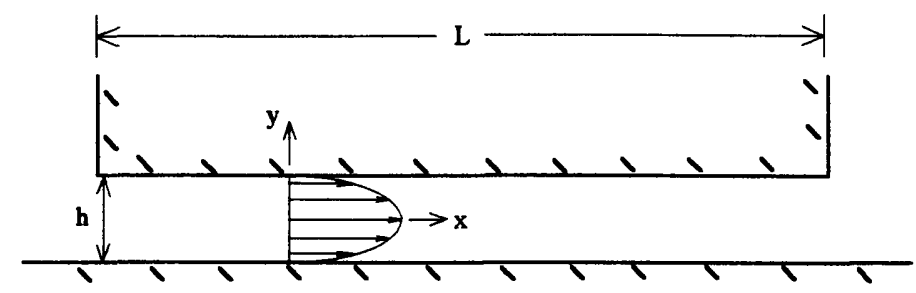

Figure 2.1. Schematic diagram of two-dimensional, fully-developed, highly viscous flow over a flat land of uniform gap.

Consider the Navier-Stokes Equations in which the fluid properties, density and viscosity, are constant. In the $\mathrm{x}$-direction, the equation of motion is:

$$
\frac{\partial u}{\partial t}+u \frac{\partial u}{\partial x}+v \frac{\partial u}{\partial y}+w \frac{\partial u}{\partial z}=-\frac{1}{\rho} \frac{\partial p}{\partial x}+g_{x}+\frac{\mu}{\rho}\left(\frac{\partial^{2} u}{\partial x^{2}}+\frac{\partial^{2} u}{\partial y^{2}}+\frac{\partial^{2} u}{\partial z^{2}}\right)
$$

Since the flow is two-dimensional, $\mathrm{w}=0$ and derivatives with respect to $\mathrm{z}$ are negligible. Since the flow is steady, $\partial u / \partial$ is zero. Body forces are neglected. If the flow is assumed fully-developed, the terms corresponding to the derivatives $\partial u / \partial x$ and $\partial u / \partial x^{2}$ are negligible.

In addition to the equation of motion, consider the equation of mass conservation for this incompressible fluid,

$$
\frac{\partial u}{\partial x}+\frac{\partial}{\partial y}=0
$$

Since $\partial u / \partial x$ is equal to zero, $\partial \nu / \partial y$ must also equal zero. Thus $v$ must be a constant vertically within the pocket. Since $\mathrm{v}$ must equal zero at the bottom of the pocket, it must equal zero at every y within the pocket. If gravity forces are also neglected, Equation (2.1) reduces to: 


$$
0 \approx-\frac{\partial p}{\partial x}+\mu\left(\frac{\partial^{2} u}{\partial y^{2}}\right)
$$

Integrating twice with respect to $y$,

$$
u(y)=C_{1}+C_{2} y+\frac{1}{2 \mu} \frac{\partial p}{\partial x} y^{2}
$$

where $C_{1}$ and $C_{2}$ are integration constants. The two boundary conditions that this equation must satisfy are:

$$
\begin{gathered}
u(y=-h / 2)=0 \\
u(y=h / 2)=0
\end{gathered}
$$

Subject to these constraints, Equation (2.4) becomes:

$$
u=\frac{1}{2 \mu} \frac{\partial p}{\partial x}\left(\frac{h^{2}}{4}-y^{2}\right)
$$

The average velocity can be found by integrating the velocity over the gap of the land:

$$
\bar{u}=\frac{1}{h} \int_{-h / 2}^{h / 2} u d y=-\frac{h^{2}}{12 \mu} \frac{\partial p}{\partial x}
$$

The resistance to flow is simply the pressure difference across the land divided by the flow rate. Since the flow rate is given by $Q=\bar{u} h L_{z}$, where $L_{z}$ is the length of the land in the $z$ direction,

$$
Q=-\frac{h^{3} L_{z}}{12 \mu} \frac{\partial p}{\partial x}
$$

Since the flow is fully developed and unchanging along the $\mathrm{x}$-direction, the pressure gradient is constant and given by the pressure difference across the land divided by the land width, $\mathrm{L}$. The land resistance is then given by: 


$$
\mathrm{R}=\frac{\Delta \mathrm{p}}{\mathrm{Q}}=\frac{12 \mu \mathrm{L}}{\mathrm{h}^{3} \mathrm{~L}_{\mathrm{z}}}
$$

This resistance is a general result that will be used in the derivation of resistances in future sections. It is important to emphasize that this equation is valid only for laminar and fullydeveloped fluid flow across the land, and the criteria developed in Appendix C must be satisfied.

\section{A.ii. Tilted Rectangular Land Flow}

Figure 2.2 below shows a schematic diagram of a rectangular land that is tilted due to pad misalignment. The flow direction is out of the page. The land width in this direction is $L$. The resistance of this land will be derived using the resistance relation derived in previous section. An assumption of highly-viscous, fully-developed fluid flow will again be used.

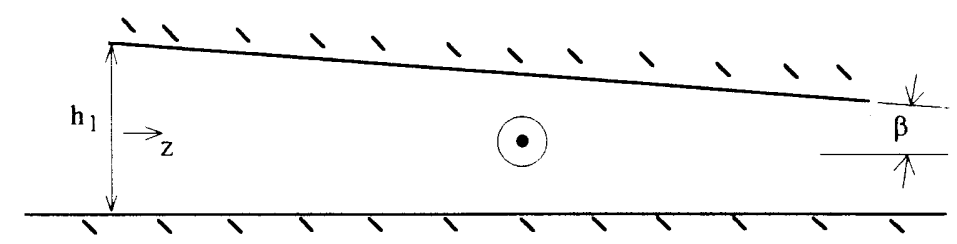

Figure 2.2. Schematic diagram of a flat land that is tiled from pad misalignment. The flow direction is out of the page.

The gap of the land in Figure 2.2 varies linear with $z$, and is given by:

$$
h(x)=h_{1}-z \sin (\beta)
$$


The pressure gradient across the land is constant and given by the pressure difference across the land divided by the land width $\mathrm{L}$. Inserting this pressure gradient and Equation (2.10) into Equation (2.8) and integrating over the length of the land,

$$
Q=\frac{1}{12 \mu} \frac{\Delta p}{L} \int_{0}^{L_{z} \cos (\beta)}\left(h_{1}-z \sin (\beta)\right)^{3} d z
$$

Integrating and rearranging,

$$
Q=\frac{1}{48 \mu} \frac{\Delta p}{L} \frac{h_{1}^{4}}{\sin (\beta)}\left[1-\left(1-\frac{L_{2}}{h_{1}} \cos (\beta) \sin (\beta)\right)^{4}\right]
$$

The resistance is then given by:

$$
R=\frac{48 \mu L \sin (\beta)}{h_{1}^{4}}\left[\frac{1}{1-\left(1-\frac{L_{z}}{h_{1}} \cos (\beta) \sin (\beta)\right)^{4}}\right]
$$

For small angles, this reduces to:

$$
R=\frac{48 \mu L \beta}{h_{1}^{4}}\left[\frac{1}{1-\left(1-\frac{L_{2}}{h_{1}} \beta\right)^{4}}\right]
$$

An alternative approach to using Equation (2.14) for the tilted land resistance may be to use the untilted land resistance, Equation (2.9), with a clearance value that results in an equivalent resistance as that calculated using Equation (2.14). The equivalent clearance to use in Equation (2.9) is given by:

$$
\mathrm{h}_{\mathrm{eq}}=\mathrm{h}_{1}\left[\frac{1}{4} \frac{\mathrm{h}_{1}}{\Delta \mathrm{h}}\left\{1-\left(1-\frac{\Delta \mathrm{h}}{\mathrm{h}_{1}}\right)^{4}\right\}\right]^{1 / 3}
$$


where $\Delta h=h_{1}-h_{2}$. The equivalent $z$-direction location where this clearance occurs is given by:

$$
\frac{\mathrm{z}_{\text {eq }}}{\mathrm{L}_{\mathrm{z}}}=\frac{\mathrm{h}_{1}}{\Delta \mathrm{h}}\left\{1-\left[\frac{1}{4} \frac{\mathrm{h}_{1}}{\Delta \mathrm{h}}\left\{1-\left(1-\frac{\Delta \mathrm{h}}{\mathrm{h}_{1}}\right)^{4}\right\}\right]^{1 / 3}\right\}
$$

This parameter is plotted versus $\Delta \mathrm{h} / \mathrm{h}_{1}$ in Figure 2.3.

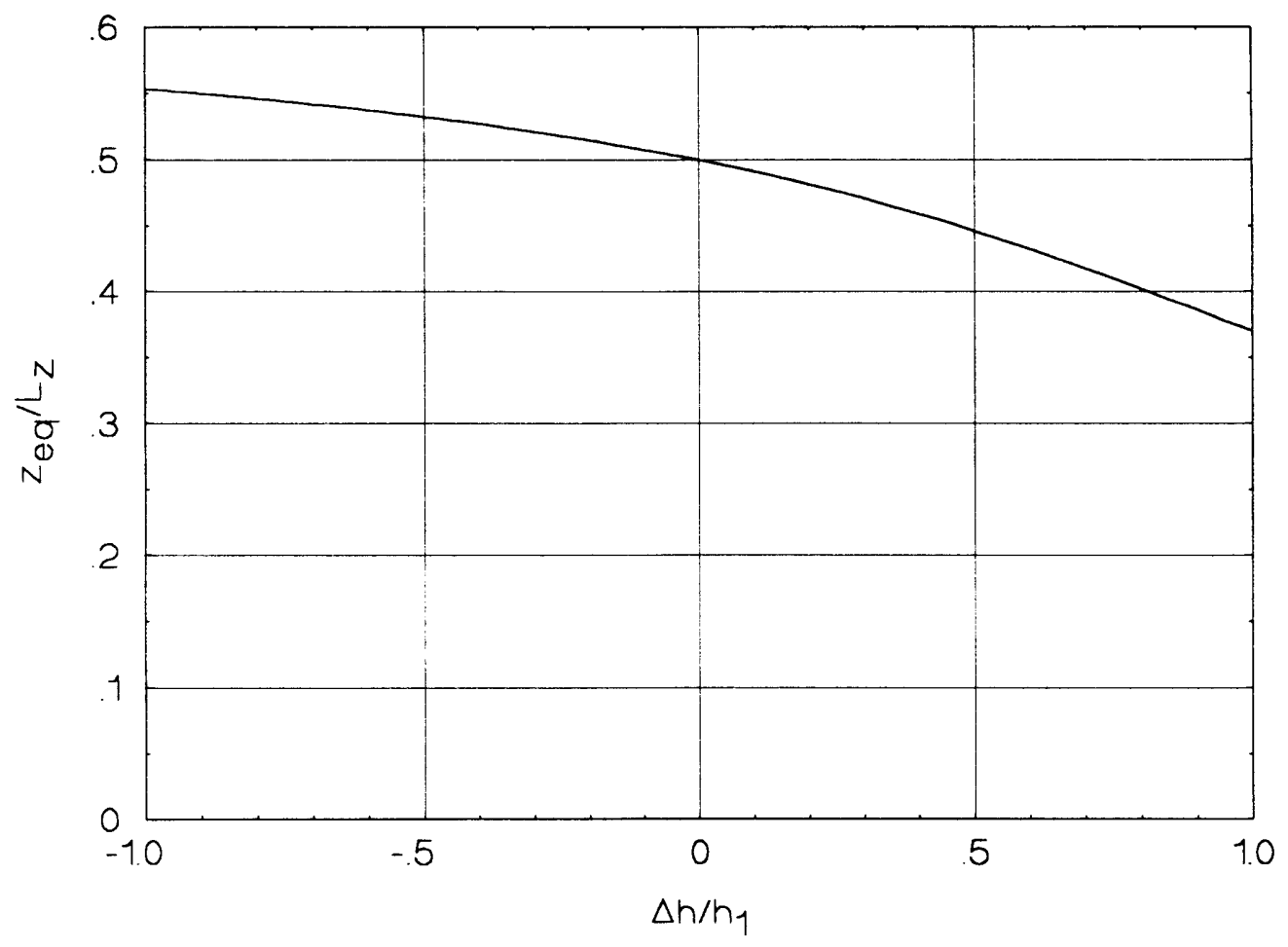

Figure 2.3. Location of the clearance for a tilted land that, when used in the untilted relation of Equation (2.9), will result in the same resistance as that computed using Equation (2.14). 


\section{A.iii. Converging Rectangular Land Flow}

Figure 2.4 below shows a schematic diagram of a rectangular land whose gap is converging due to pad misalignment. The resistance of this land will be derived using the resistance relation derived in section 2.A.i. An assumption of highly-viscous, fullydeveloped fluid flow will again be used.

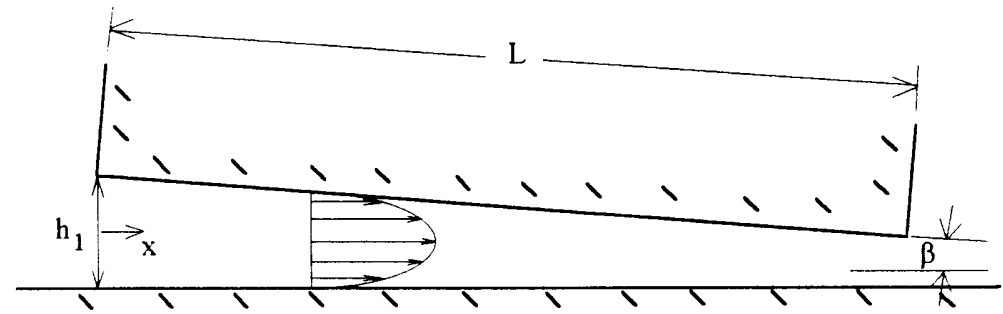

Figure 2.4. Schematic diagram of fully-developed, highly viscous flow over a flat land with a converging gap result from misalignment.

The gap of the land in Figure 2.4 varies linear with $\mathrm{x}$, and is given by:

$$
h(x)=h_{1}-x \sin (\beta)
$$

Inserting this gap relation into equation (2.8) and rearranging,

$$
\frac{\partial p}{\partial x}=-\frac{12 \mu}{\left(h_{1}-x \sin (\beta)\right)^{3}} \frac{Q}{L_{z}}
$$

Integrating with respect to $\mathrm{x}$,

$$
p=-\frac{6 \mu}{\sin (\beta)\left(h_{1}-x \sin (\beta)\right)^{2}} \frac{Q}{L_{z}}+\text { Const }
$$

The pressure is equal to $p_{1}$ at $\mathrm{x}=0$ : 


$$
p-p_{1}=-\frac{6 \mu}{h_{1}^{2} \sin (\beta)} \frac{Q}{L_{2}}\left[\frac{1}{\left(1-\frac{x}{h_{1}} \sin (\beta)\right)^{2}}-1\right]
$$

Evaluating this relation at $\mathrm{x}=\mathrm{L} \cos (\beta)$ gives the pressure difference across the land. The land resistance is the pressure difference divided by the flow rate:

$$
R=\frac{\Delta p}{Q}=\frac{6 \mu}{h_{1}^{2} \sin (\beta) L_{z}}\left[\frac{1}{\left(1-\frac{L}{h_{1}} \cos (\beta) \sin (\beta)\right)^{2}}-1\right]
$$

For small angles, this relation is given approximately by:

$$
R=\frac{\Delta p}{Q}=\frac{6 \mu}{h_{1}^{2} \beta L_{z}}\left[\frac{1}{\left(1-\frac{L}{h_{1}} \beta\right)^{2}}-1\right]
$$

An alternative approach to using Equation (2.22) for the tilted land resistance may be to use the untilted land resistance, Equation (2.9), with a clearance value that results in an equivalent resistance as that calculated using Equation (2.22). The equivalent clearance to use in Equation (2.9) is given by:

$$
\mathrm{h}_{\mathrm{eq}}=\mathrm{h}_{1}\left[\frac{2 \frac{\Delta \mathrm{h}}{\mathrm{h}_{1}}}{\frac{1}{\left(1-\frac{\Delta h}{\mathrm{~h}_{1}}\right)^{2}}-1}\right]^{1 / 3}
$$


where $\Delta h=h_{1}-h_{2}$. The equivalent $x$-direction location where this clearance occurs is given by:

$$
\frac{x_{e q}}{L}=\frac{h_{1}}{\Delta h}\left\{1-\left[\frac{2 \frac{\Delta h}{h_{1}}}{\frac{1}{\left(1-\frac{\Delta h}{h_{1}}\right)^{2}}-1}\right]^{1 / 3}\right\}
$$

This parameter is plotted versus $\Delta \mathrm{h} / \mathrm{h}_{1}$ in Figure 2.5.

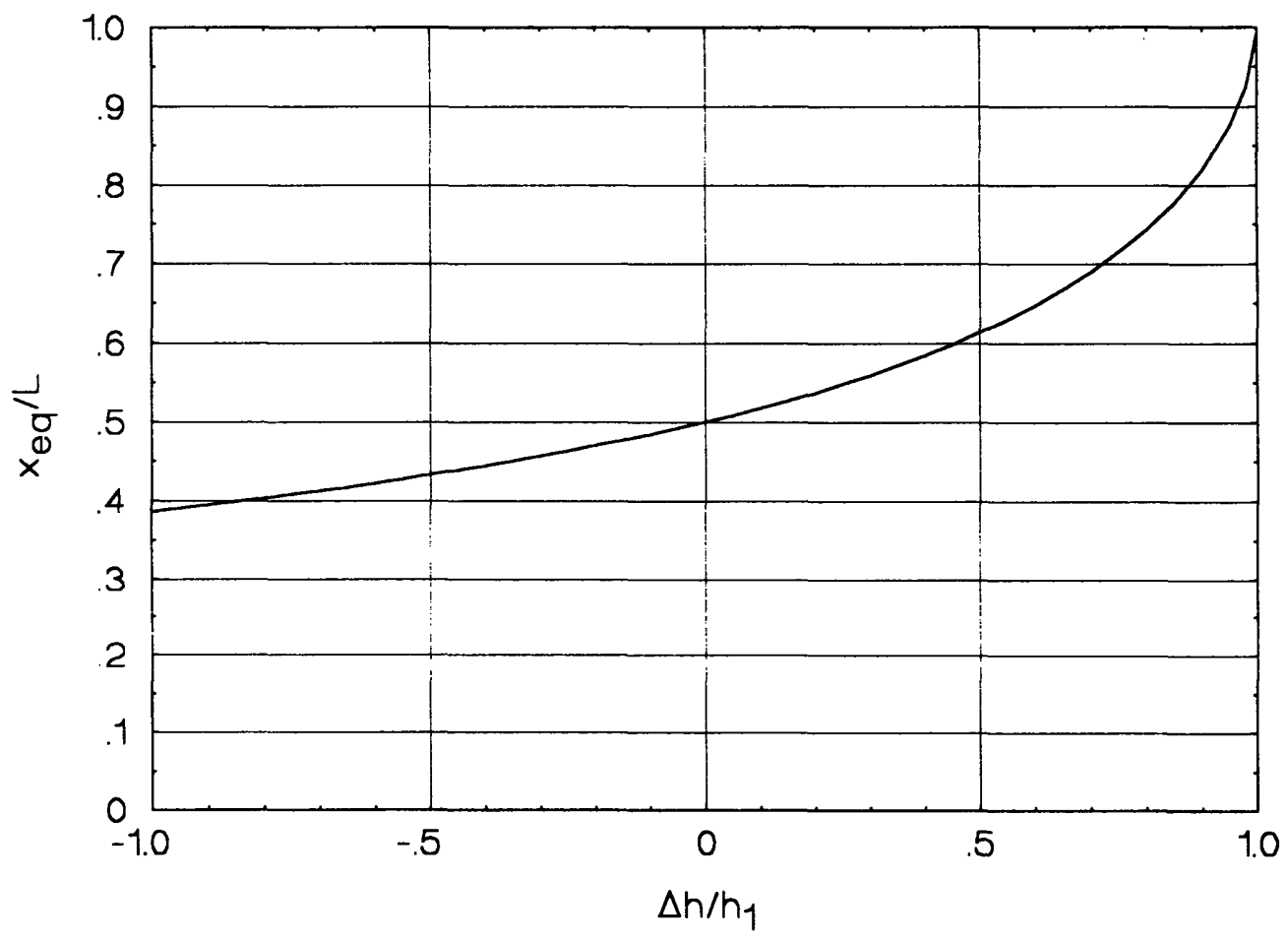

Figure 2.5. Location of the clearance for a tilted land that, when used in the untilted relation of Equation (2.9), will result in the same resistance as that computed using Equation (2.22). 


\section{A.iv. Flow Over an Axial Land}

The fluid flow between a land clearance of a shaft and housing is normally modeled as flow between two flat plates. This approximation is good if the eccentricity of the shaft is small or if the circumferential width of the land is small compared to the circumferential length of the shaft diameter. However, at large eccentricities and large circumferential land widths, the clearance varies significantly across the width of the land. The variation in clearance can effect the resistance and pressure profile across the land. Relations will be developed in this section in which these secondary effects are accounted for.

An axial land on a shaft that is displaced eccentrically is shown schematically in Figure 2.7. The angle $\theta_{c}$ corresponds to the angle between the point of minimum clearance and the center of the axial land. The clearance between a housing and an eccentric shaft is a function of the angle $\theta$, and is given by:

$$
h(x)=h_{o}\left[1-e \cos \left(\theta_{c}+x / D\right)\right]
$$

where $h_{0}$ is the nominal clearance between the shaft and housing. The eccentricity of the shaft, $\mathrm{e}$, is defined as:

$$
e=\frac{\delta}{h_{o}}
$$

where $\delta$ is the distance the shaft is displaced with respect to its concentric position. Inserting the clearance given by Equation (2.25) into Equation (2.8),

$$
\frac{\partial p}{\partial x}=-\frac{12 \mu}{h_{o}^{3}\left[1-e \cos \left(\theta_{c}+x / D\right)\right]^{3}} \frac{Q}{L_{z}}
$$




$$
p-p_{1}=-\frac{12 \mu}{h_{o}^{3}} \frac{Q}{L_{z}} \int_{-L / 2}^{x} \frac{d x^{\prime}}{\left[1-e \cos \left(\theta_{c}+x / D\right)\right]^{3}}
$$

This pressure distribution will be used to find the hydraulic resistance across the land and the force generated by the pressure on the land.

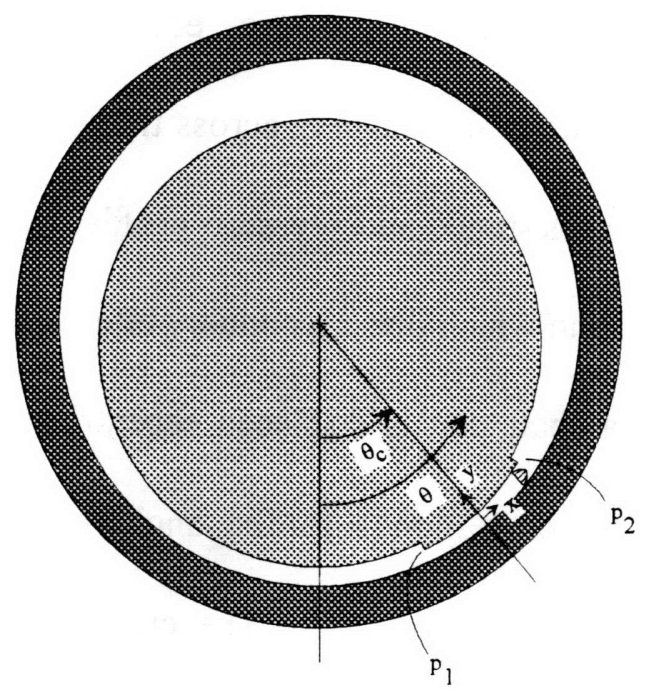

Figure 2.7. Schematic diagram of the fluid flow across an axial land as the shaft is displaced to an eccentric position.

The hydraulic resistance of the axial land is found by considering the total pressure drop across the land:

$$
p_{1}-p_{2}=\frac{12 \mu L}{h_{o}^{3}} \frac{Q}{L_{z}} \int_{-1 / 2}^{1 / 2} \frac{d \xi}{\left[1-e \cos \left(\theta_{c}+\frac{L}{D} \xi\right)\right]^{3}}
$$

where $\xi=x / L$. This makes the hydraulic resistance equal to: 


$$
R_{\text {axial }}=\frac{12 \mu L}{L_{z} h_{o}^{3}} \int_{-1 / 2}^{1 / 2} \frac{d \xi}{\left[1-e \cos \left(\theta_{c}+\frac{L}{D} \xi\right)\right]^{3}}
$$

Figure 2.8 shows plots of the ratio of the resistance of an axial land computed using Equation (2.30) divided by that computed using Equation (2.9) with the clearance corresponding to that of Equation (2.25) with an angle of $\theta_{c}$. The ratio of land width to shaft diameter used for this plot was $\mathrm{L} / \mathrm{D}=0.4$.

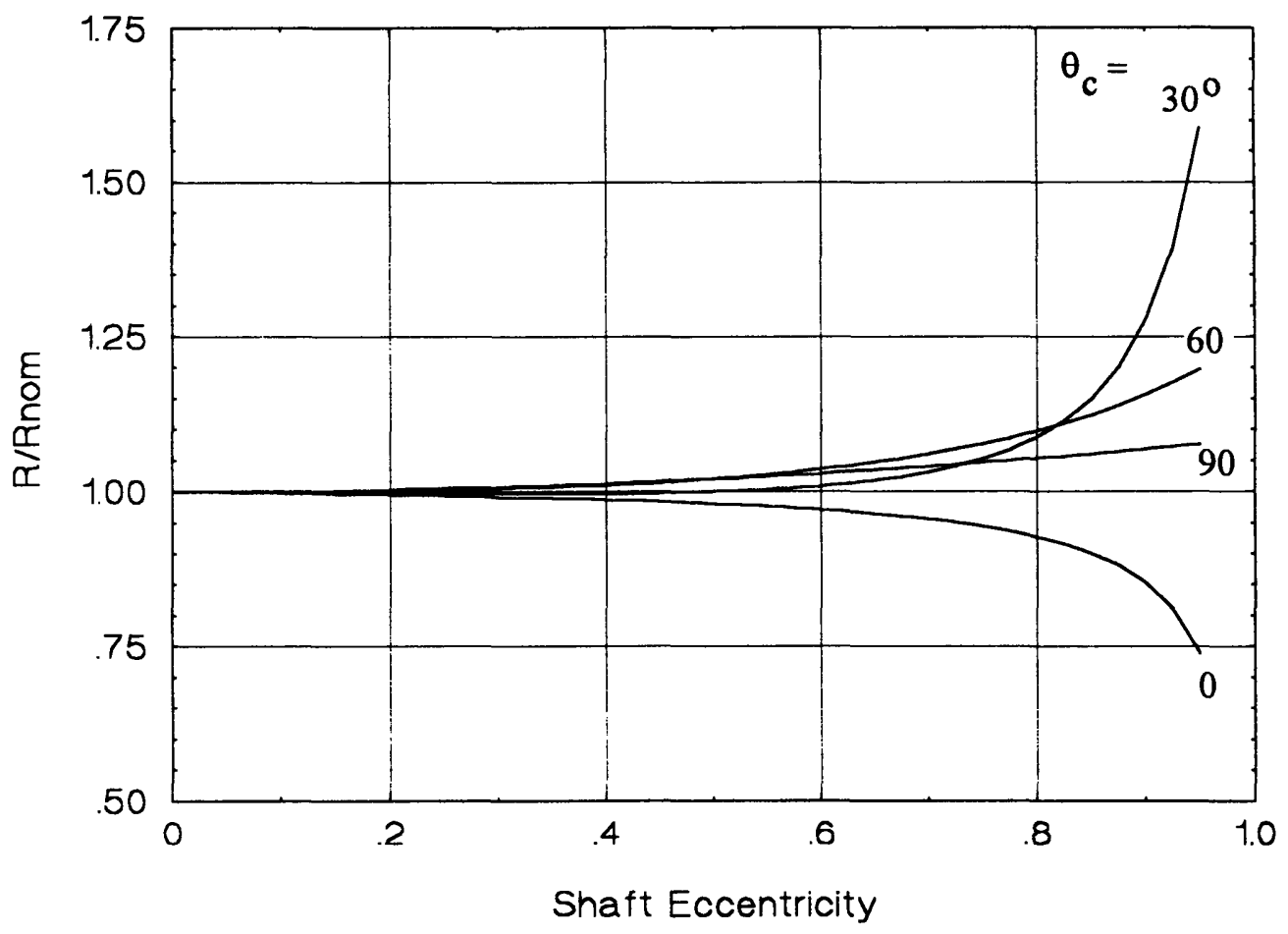

Figure 2.8. Ratio of the resistance of an axial land computed using Equation (2.30) divided by that computed using Equation (2.9) with the clearance corresponding to that of Equation (2.25) with an angle of $\theta_{c}$. The ratio of land width to shaft diameter used for this plot was $\mathrm{L} / \mathrm{D}=0.4$. 
The closed-form solution to the integral of Equation (2.30) is complicated and lengthy. A more compact solution can be obtained by integrating numerically using a 5point Simpson 1/3 Rule numerical integration:

$$
\begin{gathered}
\mathrm{R}_{\text {axial }} \approx \frac{12 \mu \mathrm{L}}{\mathrm{L}_{\mathrm{z}} \mathrm{h}_{\mathrm{o}}^{3}} \mathrm{Z}\left(\mathrm{e}, \mathrm{L} / \mathrm{D}, \theta_{\mathrm{c}}, 1 / 2\right) \\
Z\left(e, L / D, \theta_{c}, a\right)=\frac{a+1 / 2}{12}\left[\frac{1}{g_{1}\left(e, L / D, \theta_{c},-1 / 2\right)}+\frac{4}{g_{1}\left(e, L / D, \theta_{c},-1 / 2+1 / 4(a+1 / 2)\right)}+\right. \\
\frac{2}{g_{1}\left(e, L / D, \theta_{c},-1 / 2+1 / 2(a+1 / 2)\right)}+\frac{4}{g_{1}\left(e, L / D, \theta_{c},-1 / 2+3 / 4(a+1 / 2)\right)}+ \\
\left.\frac{1}{\mathrm{~g}_{1}\left(\mathrm{e}, \mathrm{L} / \mathrm{D}, \theta_{c}, \mathrm{a}\right)}\right]
\end{gathered}
$$

where:

$$
g_{1}\left(e, L / D, \theta_{c}, \xi\right)=\left[1-e \cos \left(\theta_{c}+\frac{L}{D} \xi\right)\right]^{3}
$$

Equations (2.31) through (2.33) may be used to approximately calculate the resistance of Equation (2.30) with a maximum error of approximately $0.5 \%$.

The force generated by the pressure across the axial land will be considered next. Of interest is the net force directed radially toward the center of the shaft, originating from the point corresponding to the angular position $\theta_{c}$. This force is found by integrating the pressure over the land width times the cosine of the angle with respect to the center of the land:

$$
F_{\text {axial }}=L_{z} L \int_{-1 / 2}^{1 / 2} p \cos \left(2 \frac{L}{D} \xi\right) d \xi
$$

Inserting the pressure distribution given by Equation (2.28): 


$$
\begin{gathered}
\frac{F_{\text {axial }}}{L_{z} D}=p_{1} \sin \left(\frac{L}{D}\right)+ \\
\frac{p_{2}-p_{1}}{d \xi} \frac{L}{D} \int_{-1 / 2}^{1 / 2} \cos \left(2 \frac{L}{D} \zeta\right) \int_{-1 / 2}^{\zeta} \frac{d \xi}{\left[1-e \cos \left(\theta_{c}+\frac{L}{D} \xi\right)\right]^{3}} d \zeta
\end{gathered}
$$

For small eccentricities, the change in clearance across the width of the land is very small with respect to the nominal clearance and so the net force developed by the pressure on the land should limit to:

$$
\frac{F_{\text {axial }}}{L_{z} D}=\left(\frac{p_{1}+p_{2}}{2}\right) \sin \left(\frac{L}{D}\right)
$$

Several plots of the ratio of the force calculated using Equation (2.35) to that calculated using Equation (2.36) are shown in Figure 2.9 for a ratio of land width to bearing diameter $\mathrm{L} / \mathrm{D}=0.4$. The pressures considered in this plot are $\mathrm{p}_{2}=0.6 \mathrm{p}_{1}$. 


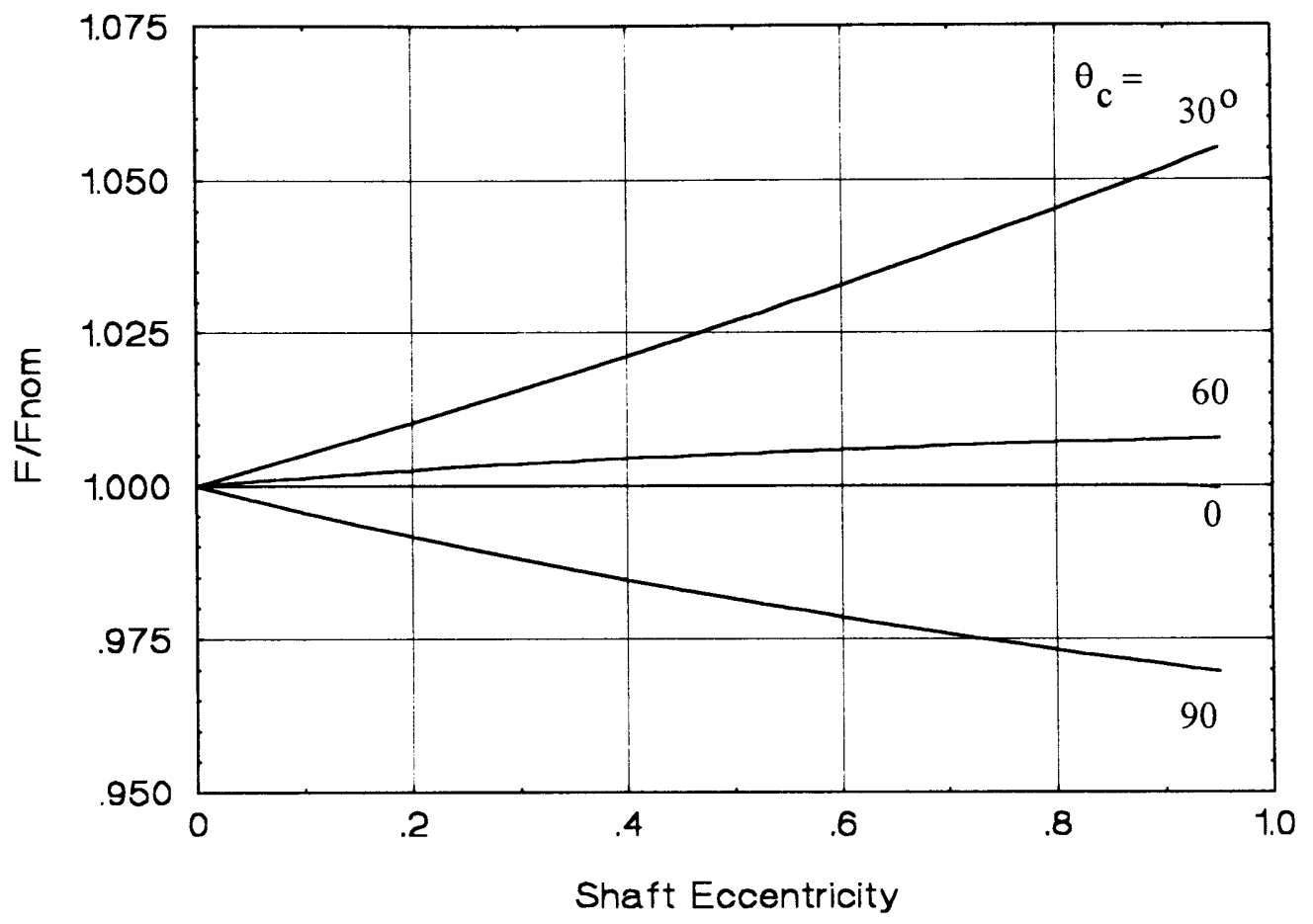

Figure 2.9. Ratio of the force calculated using Equations (2.35) to that calculated using Equation (2.36) are shown for a ratio of land width to bearing diameter $L / D=0.4$. The pressures considered in this plot are $\mathrm{p}_{2}=0.6 \mathrm{p}_{1}$.

The closed-form solution to the integral of Equation (2.35) is complicated and lengthy. A more compact solution can be obtained by integrating numerically using a 5point Simpson 1/3 Rule numerical integration:

$$
\begin{gathered}
\frac{\mathrm{F}_{\text {axial }}}{\mathrm{L}_{\mathrm{z}} \mathrm{D}} \approx \mathrm{p}_{1} \sin \left(\frac{\mathrm{L}}{\mathrm{D}}\right)+ \\
\frac{p_{2}-p_{1}}{Z\left(e, L / D, \theta_{c}, 1 / 2\right)} \frac{L}{D} \frac{1}{12}\left[\cos \left(2 \frac{L}{D}(-1 / 2) Z\left(e, L / D, \theta_{c},-1 / 2\right)\right)+\right. \\
4 \cos \left(2 \frac{L}{D}(-1 / 4) Z\left(e, L / D, \theta_{c},-1 / 4\right)\right)+Z\left(e, L / D, \theta_{c}, 0\right)+
\end{gathered}
$$




$$
\left.4 \cos \left(2 \frac{L}{D}(1 / 4) Z\left(e, L / D, \theta_{c}, 1 / 4\right)\right)+\cos \left(2 \frac{L}{D}(1 / 2) Z\left(e, L / D, \theta_{c}, 1 / 2\right)\right)\right]
$$

where $\mathrm{Z}\left(\mathrm{e}, \mathrm{L} / \mathrm{D}, \theta_{\mathrm{c}}, \mathrm{a}\right)$ and $\mathrm{g} 1\left(\mathrm{e}, \mathrm{L} / \mathrm{D}, \theta_{\mathrm{c}}, \xi\right)$ are given by Equations (2.32) and (2.33). Equation (2.37) may be used to approximately calculate the same value as Equation (2.35) with a maximum error of approximately $2 \%$.

For an axial bearing land that is tilting as a result of shaft bending, the eccentricity varies along the length of the land. The effect of land tilt can be approximately accounted for by combining the solution developed in this section with that developed in Section 2.A.ii. To do this, the equivalent clearance given by Equation (2.15) will be replaced with an equivalent eccentricity. With the assumption that the clearance varies linearly in the axial direction, the equivalent eccentricity of the land is given by:

$$
\mathrm{e}_{\text {axial }}=\frac{1-\left[\frac{\left(1-\mathrm{e}_{1} \cos (\theta)\right)^{4}-\left(1-\mathrm{e}_{2} \cos (\theta)\right)^{4}}{4\left(\mathrm{e}_{2}-\mathrm{e}_{1}\right) \cos (\theta)}\right]^{1 / 3}}{\cos (\theta)}
$$

where $e_{1}$ and $e_{2}$ are the eccentricites at the two ends of the axial land and $\theta$ is the angle from the point of minimum clearance to the circumferential center of the axial land. The general function given by Equation (2.38) will be used in Equations (2.31) and (2.37) to approximately account for the effect of tilting the axial land.

\section{A.v. Flow Over a Circumferential Land}

A circumferential land on a shaft that is displaced eccentrically is shown schematically in Figure 2.10. The fluid flows in the direction into the page across the length width $\mathrm{L}$. The angle $\theta_{\mathrm{c}}$ corresponds to the angle between the point of minimum 
clearance and the center of the circumferential land. Relations will be developed in this section in which the variation in the circumferential direction is accounted for.

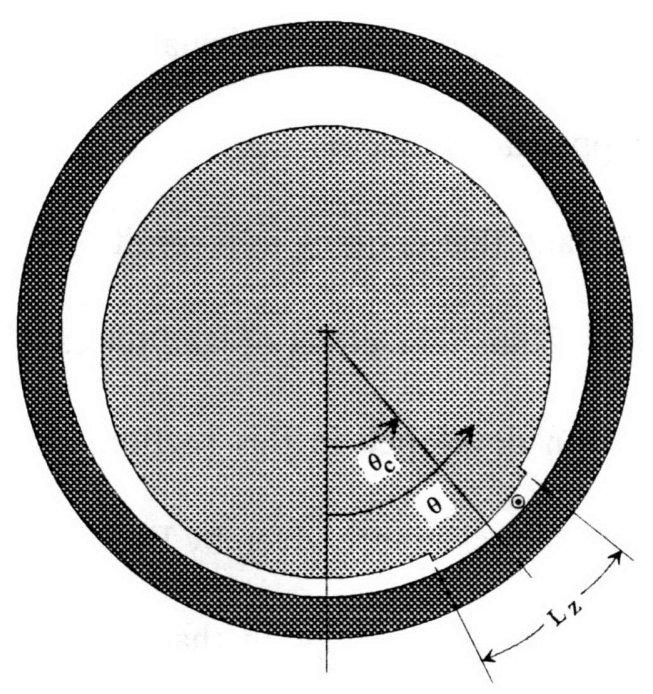

Figure 2.10. Schematic diagram of the fluid flow across an circumferential land as the shaft is displaced to an eccentric position.

The clearance between a housing and an eccentric shaft is a function of the angle $\theta$, and is given by:

$$
h(x)=h_{o}\left[1-e \cos \left(\theta_{c}+x / D\right)\right]
$$

where $h_{0}$ is the nominal clearance between the shaft and housing, and e is the eccentricity of the shaft. The flow rate of fluid across the land is found by inserting the clearance given by Equation (2.39) into Equation (2.8) and noting that the pressure gradient across the land is constant:

$$
\mathrm{Q}=\frac{\mathrm{h}_{\mathrm{o}}^{3} \mathrm{~L}_{\mathrm{z}}}{12 \mu} \frac{\Delta \mathrm{p}}{\mathrm{L}} \int_{-1 / 2}^{1 / 2}\left[1-\mathrm{e} \cos \left(\theta_{\mathrm{c}}+\frac{\mathrm{L}_{\mathrm{z}}}{\mathrm{D}} \xi\right)\right]^{3} \mathrm{~d} \xi
$$


where $\xi=z / L_{z}$. The resistance is therefore given by:

$$
R_{\text {circum }}=\frac{12 \mu L}{h_{o}^{3} L_{z}} \frac{1}{\int_{-1 / 2}^{1 / 2}\left[1-e \cos \left(\theta_{c}+\frac{L_{z}}{D} \xi\right)\right]^{3} d \xi}
$$

Shown in Figure 2.11 are several plots of the ratio of circumferential land resistance calculated using Equation (2.41) divided by that calculated using Equation (2.9) with the clearance corresponding to that of Equation (2.39) with an angle of $\theta_{c}$. The ratio of land length to shaft diameter used for this plot was $L_{z} / D=0.5$.

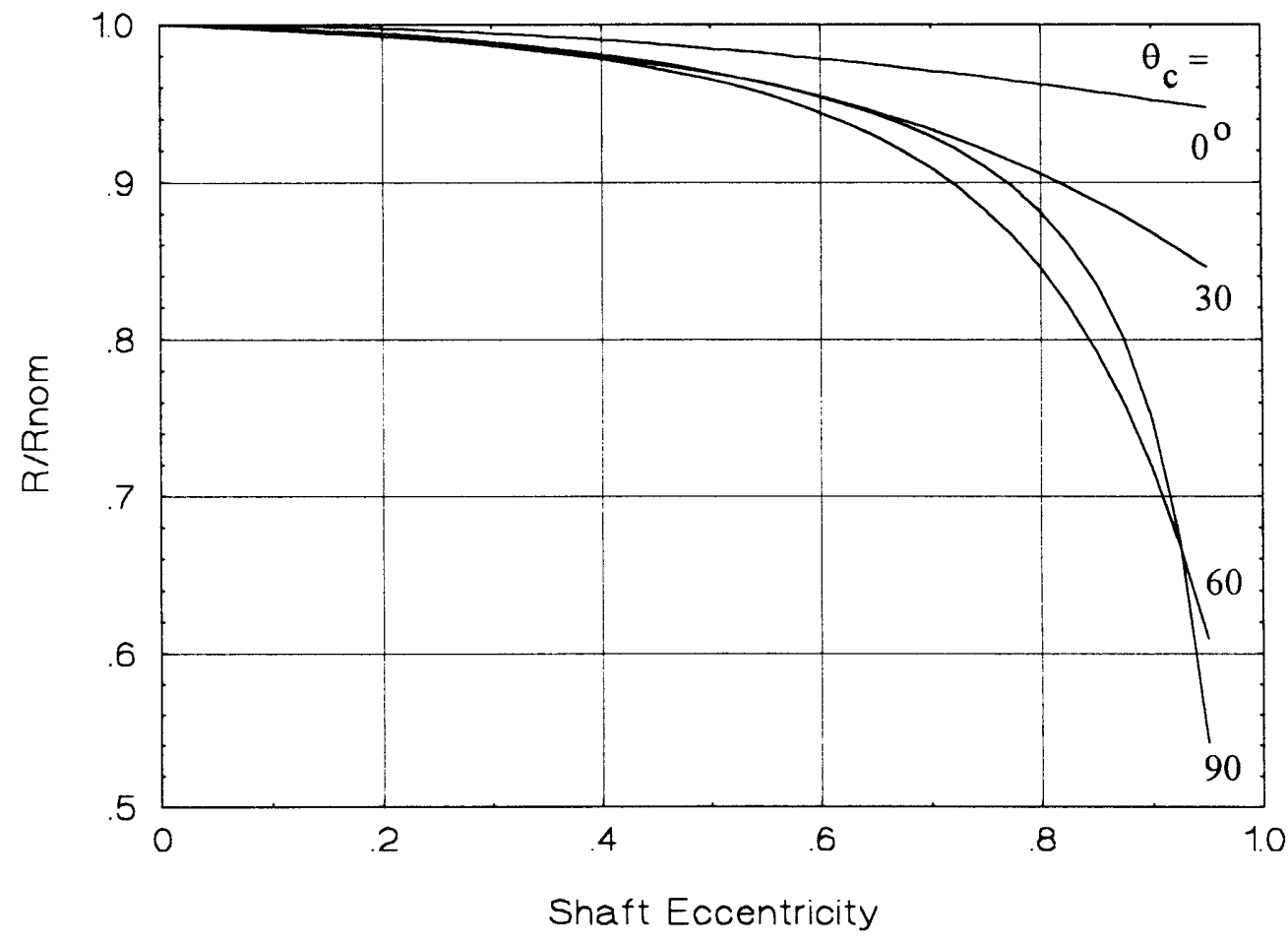

Figure 2.11. Ratio of circumferential land resistance calculated using Equation (2.41) divided by that calculated using Equation (2.9) with the clearance corresponding to that of Equation (2.39) with an angle of $\theta_{c}$. The ratio of land length to shaft diameter used for this plot was $\mathrm{L}_{\mathrm{z}} / \mathrm{D}=0.5$. 
The closed-form solution to the integral of Equation (2.41) is complicated and lengthy. A more compact solution can be obtained by integrating numerically using a 5point Simpson 1/3 Rule numerical integration:

$$
\begin{gathered}
R_{\text {circum }}=\frac{12 \mu \mathrm{L}}{\mathrm{h}_{\mathrm{o}}^{3} \mathrm{~L}_{\mathrm{z}}} \frac{1}{\mathrm{~W}} \\
\mathrm{~W}=\frac{1}{12}\left[\mathrm{~g}_{1}\left(\mathrm{e}, \mathrm{L}_{\mathrm{z}} / \mathrm{D}, \theta_{\mathrm{c}},-1 / 2\right)+4 \mathrm{~g}_{1}\left(\mathrm{e}, \mathrm{L}_{\mathrm{z}} / \mathrm{D}, \theta_{\mathrm{c}},-1 / 4\right)+\right. \\
\left.2 \mathrm{~g}_{1}\left(\mathrm{e}, \mathrm{L}_{\mathrm{z}} / \mathrm{D}, \theta_{\mathrm{c}}, 0\right)+4 \mathrm{~g}_{1}\left(\mathrm{e}, \mathrm{L}_{\mathrm{z}} / \mathrm{D}, \theta_{\mathrm{c}}, 1 / 4\right)+\mathrm{g}_{1}\left(\mathrm{e}, \mathrm{L}_{\mathrm{z}} / \mathrm{D}, \theta_{\mathrm{c}}, 1 / 2\right)\right]
\end{gathered}
$$

where $\mathrm{g}_{1}\left(\mathrm{e}, \mathrm{L} / \mathrm{D}, \theta_{\mathrm{c}}, \xi\right)$ is given by Equation (2.33). Equations (2.42) through (2.43) may be used to approximately calculate the resistance of Equation (2.41) with a maximum error of approximately $0.5 \%$.

For a circumferential bearing land that is tilting as a result of shaft bending, the eccentricity varies across the width of the land. The effect of land tilt can be approximately accounted for by combining the solution developed in this section with that developed in Section 2.A.iii. To do this, the equivalent clearance given by Equation (2.23) will be replaced with an equivalent eccentricity. With the assumption that the clearance varies linearly in the axial direction, the equivalent eccentricity of the land is given by:

$$
\left.\mathrm{e}_{\text {cirum }}=\frac{1-\left[\frac{2\left(1-\mathrm{e}_{1} \cos (\theta)\right)^{2}\left(\mathrm{e}_{2}-\mathrm{e}_{1}\right) \cos (\theta)}{\left(\frac{1-\mathrm{e}_{1} \cos (\theta)}{1-\mathrm{e}_{2} \cos (\theta)}\right)^{2}-1}\right.}{\cos (\theta)}\right]^{1 / 3}
$$


where $e_{1}$ and $e_{2}$ are the eccentricites at the two ends of the land and $\theta$ is the angle from the point of minimum clearance to the circumferential center of the land. The general function given by Equation (2.44) will be used in Equation (2.42) to approximately account for the effect of tilting the axial land.

\section{B. Circular Land Flow}

In this section, the case of fluid flow over a circular land will be considered. Shown in Figure 2.12 is a circular bearing pad in which fluid enters a central pocket and then flows out radially across a land which is at a tight clearance from a supporting member. In all of the derivations that follow, it is assumed that the fluid flow is laminar and fully-developed. The criteria for laminar, fully-developed flow given in Appendix C should be considered before the equations developed in this section are used. Although the criteria given in Appendix $\mathrm{C}$ were developed for rectangular land flow, they may be used for circular land flow by considering the innermost radius, where the fluid velocity is highest. 


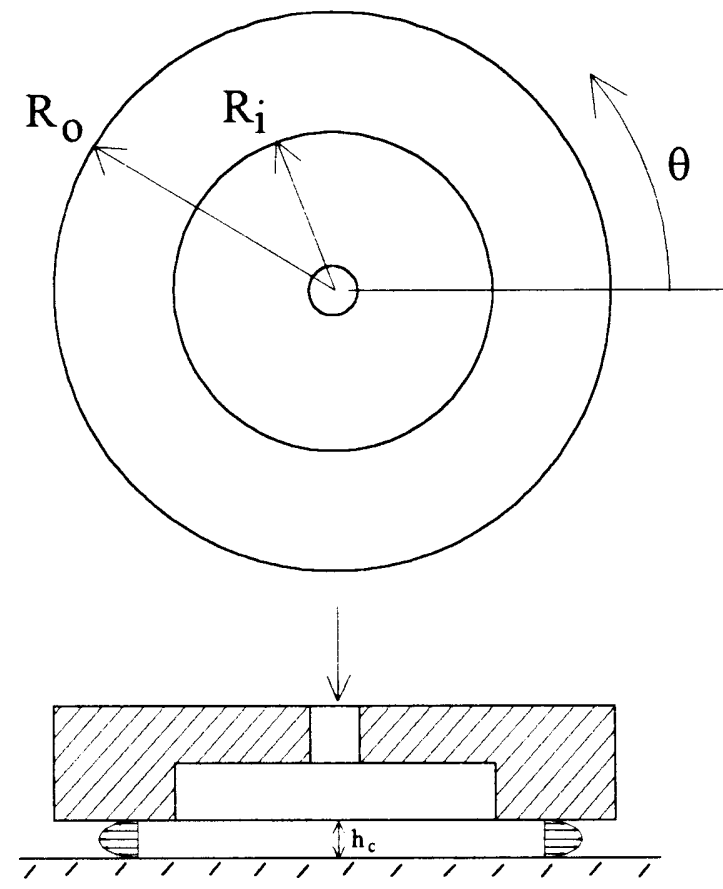

Figure 2.12. Schematic diagram of a circular bearing pad with highly viscous fluid flowing out radially across the circular land.

\section{B.i. Untilted Circular Land Flow}

The nominal, no tilt circular land resistance will be derived first. Modifying Equation (2.8), the flow rate per unit length circumferentially is given by:

$$
\frac{Q}{L_{z}}=-\frac{h^{3}}{12 \mu} \frac{\partial P}{\partial r}
$$

Rearranging,

$$
\frac{d p}{d r}=-\frac{12 \mu}{h^{3}} \frac{Q}{L_{z}}
$$

Integrating around the entire circumference of the pad, 


$$
Q=\int_{0}^{2 \pi}\left(\frac{Q}{L_{z}}\right) r d \theta=-\frac{\pi}{6 \mu} h_{c}^{3} r \frac{\partial p}{\partial r}
$$

Rearranging,

$$
d p=-\frac{6 \mu Q}{\pi h_{c}^{3}} \frac{d r}{r}
$$

Integrating from the inner radius to the outer radius, the pressure difference from the inside of the circular pad to the outside is given by:

$$
\Delta p=\frac{6}{\pi} \frac{\mu Q}{h_{c}^{3}} \ln \left(\frac{R_{o}}{R_{i}}\right)
$$

The nominal circular pad resistance is thus given by:

$$
R_{n o m}=\frac{6}{\pi} \frac{\mu}{h_{c}^{3}} \ln \left(\frac{R_{o}}{R_{i}}\right)
$$

\section{B.ii. Tilted Circular Land Flow}

Figure 2.13 below shows a schematic diagram of a circular pad that is tilted due to pad misalignment. The resistance of this land will be derived similar to the titled rectangular lands of the previous sections of this chapter. An assumption of highlyviscous, fully-developed fluid flow will again be used. 


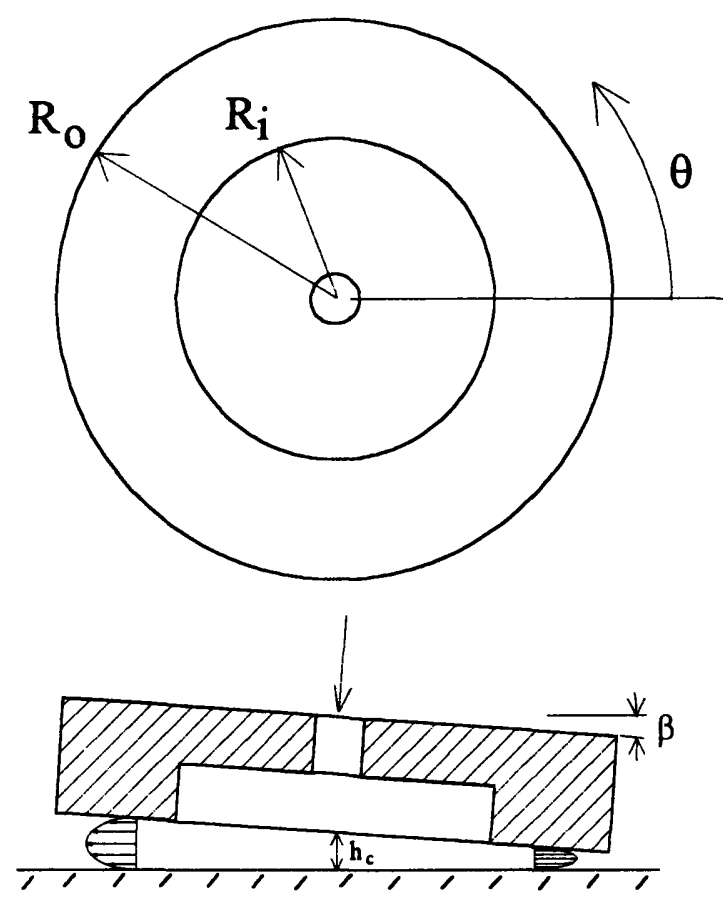

Figure 2.13. Schematic diagram of a tilted circular bearing pad with highly viscous fluid flowing out radially across the circular land.

The gap of the land in Figure $X$ varies linearly with $r$ and $\theta$, and is given by:

$$
h(x)=h_{c}-r \cos (\theta) \sin (\beta)
$$

Modifying Equation (2.8), the flow rate per unit length circumferentially is given by:

$$
\frac{Q}{L_{z}}=-\frac{h_{c}^{3}}{12 \mu} \frac{\partial p}{\partial r}
$$

Rearranging,

$$
\frac{d p}{d r}=-\frac{12 \mu}{h_{c}^{3}} \frac{Q}{L_{z}}
$$

Inserting Equation (2.50),

$$
\frac{d p}{d r}=-12 \mu\left(\frac{Q}{L_{z}}\right) \frac{1}{\left(h_{c}-r \cos (\theta) \sin (\beta)\right)^{3}}
$$


Integrating from the inner radius to the outer radius, the pressure difference from the inside of the circular pad to the outside is given by:

$$
\Delta p=12 \mu\left(\frac{Q}{L_{z}}\right) \int_{R_{i}}^{R_{o}} \frac{d r}{\left(h_{c}-r \cos (\theta) \sin (\beta)\right)^{3}}
$$

Rearranging to give the flow rate per unit length in the circumferential direction,

$$
\frac{Q}{L_{z}}=\frac{\Delta p}{12 \mu} \frac{1}{\int_{R_{t}}^{R_{o}} \frac{d r}{\left(h_{c}-r \cos (\theta) \sin (\beta)\right)^{3}}}
$$

Integrating around the entire circumference of the pad,

$$
Q=\frac{\Delta p}{12 \mu} \int_{0}^{2 \pi} \frac{d \theta}{\int_{R_{i}}^{R_{o}} \frac{d r}{r\left(h_{c}-r \cos (\theta) \sin (\beta)\right)^{3}}}
$$

The tilted circular pad resistance is now given by:

$$
R=\frac{12 \mu}{\int_{0}^{2 \pi} \frac{d \theta}{\int_{R_{i}}^{R_{o}} \frac{d r}{r\left(h_{c}-r \cos (\theta) \sin (\beta)\right)^{3}}}}
$$

When integrated, the analytical relation of Equation (2.57) is very long and cumbersome. Therefore, the lower integral was evaluated analytically and a 5-point Simpson's $1 / 3$ rule numerical integration is used to approximate the upper integral. This results in:

$$
R=\frac{12 \mu}{h_{c}^{3}} \frac{1}{\left(\frac{\pi}{6}\right)\left(\frac{1}{g(0)}+\frac{4}{g(\pi / 4)}+\frac{2}{g(\pi / 2)}+\frac{4}{g(3 \pi / 4)}+\frac{1}{g(\pi)}\right)}
$$

where, using small-angle approximations, 


$$
\begin{gathered}
g(\theta)=\ln \left(\frac{R_{o}}{R_{i}} \frac{\beta \frac{R_{i}}{h_{c}} \cos (\theta)-1}{\left.\beta \frac{R_{o}}{h_{c}} \cos (\theta)-1\right)}+\frac{1 / 2}{\left(\beta \frac{R_{o}}{h_{c}} \cos (\theta)-1\right)^{2}}-\frac{1 / 2}{\left(\beta \frac{R_{i}}{h_{c}} \cos (\theta)-1\right)^{2}}\right. \\
+\frac{1}{\left(\beta \frac{R_{i}}{h_{c}} \cos (\theta)-1\right)}-\frac{1}{\left(\beta \frac{R_{o}}{h_{c}} \cos (\theta)-1\right)}
\end{gathered}
$$

This solution was compared with a very rigorous numerical integration, and agreement was found to be within $1 \times 10^{-3} \%$ over all possible ranges of the variables.

Shown in Figure 2.14 is the reduction in circular pad resistance due to misalignment for several radius ratios. Note that $\beta R_{o} / h_{c}$ is physically constrained to less than a value of 1 or touchdown will occur. As shown, for $R_{0} / R_{i}=1.1$, a reduction in pad resistance of up to $60 \%$ can occur due to misalignment.

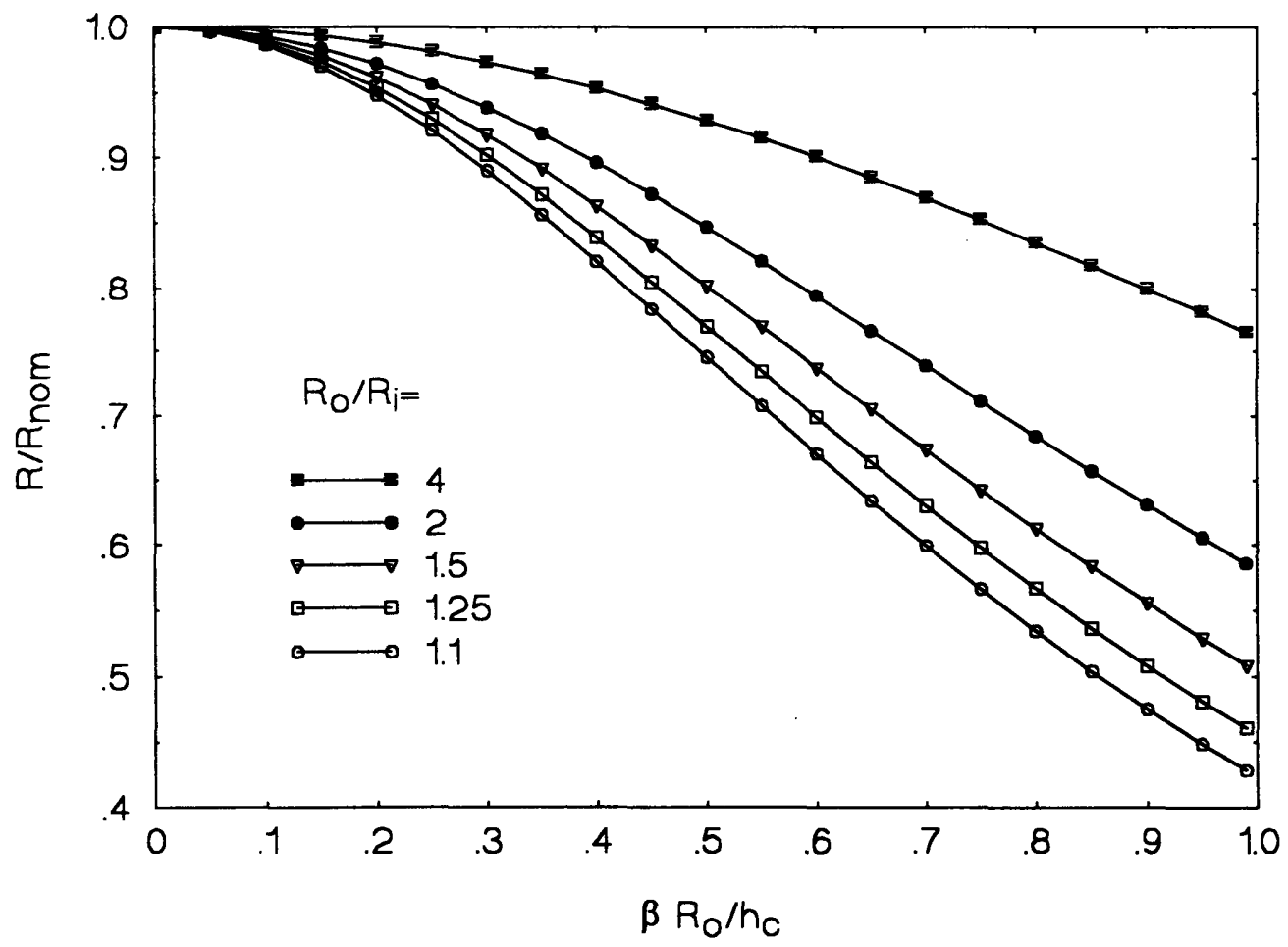

Figure 2.14. Reduction in circular pad resistance resulting from tilt. 
Equations (2.58) through (2.59) are cumbersome for simple calculations, and so an excellent curve fit to the result is given by:

$$
\begin{aligned}
& \frac{\mathrm{R}}{\mathrm{R}_{\text {nom }}}=1-\left\{2.323-0.859\left(\frac{\mathrm{R}_{\mathrm{o}}}{\mathrm{R}_{\mathrm{i}}}\right)+0.0223\left(\frac{\mathrm{R}_{\mathrm{o}}}{\mathrm{R}_{\mathrm{i}}}\right)^{3}\right\}\left[\left(\frac{\beta \mathrm{R}_{\mathrm{o}}}{\mathrm{h}_{\mathrm{c}}}\right)^{2}+\right. \\
& \left.\left\{-0.847+0.231\left(\frac{\mathrm{R}_{\mathrm{o}}}{\mathrm{R}_{\mathrm{i}}}\right)-0.02\left(\frac{\mathrm{R}_{\mathrm{o}}}{\mathrm{R}_{\mathrm{i}}}\right)^{2}\right\}\left(\frac{\beta \mathrm{R}_{\mathrm{o}}}{\mathrm{h}_{\mathrm{c}}}\right)^{3}\right]
\end{aligned}
$$

where $\mathrm{R}_{\mathrm{nom}}$ is the nominal circular pad resistance, given by Equation (2.49). The curve fit given by Equation (2.60) is accurate to within $5 \%$ for $R_{0} / R_{i}<4$. If $\beta R_{0} / h_{c}<0.2$, then Equation (2.60) can be extrapolated up to $R_{0} / R_{i}=6.5$ and will still be accurate to within $5 \%$.

\section{C. Standard Bearing Pad Land Flow}

The flow of fluid across the lands of a standard bearing pad will be considered next. The pad is flat, without any bearing curvature. Although this limits its usefulness for modeling radial hydrostatic bearings, the effect of tilting the pad on its overall hydraulic resistance is instructive. Also, a flat pad may be used as an approximate model of a curved pad in a radial bearing if the pad circumferential width is small compared to the circumferential length of the bearing diameter (i.e., it may be used to good approximation if six or more pads are used). In all of the derivations that follow, it is assumed that the fluid flow is laminar and fully-developed. The criteria for laminar, fullydeveloped flow given in Appendix C should be considered before the equations developed in this section are used. 

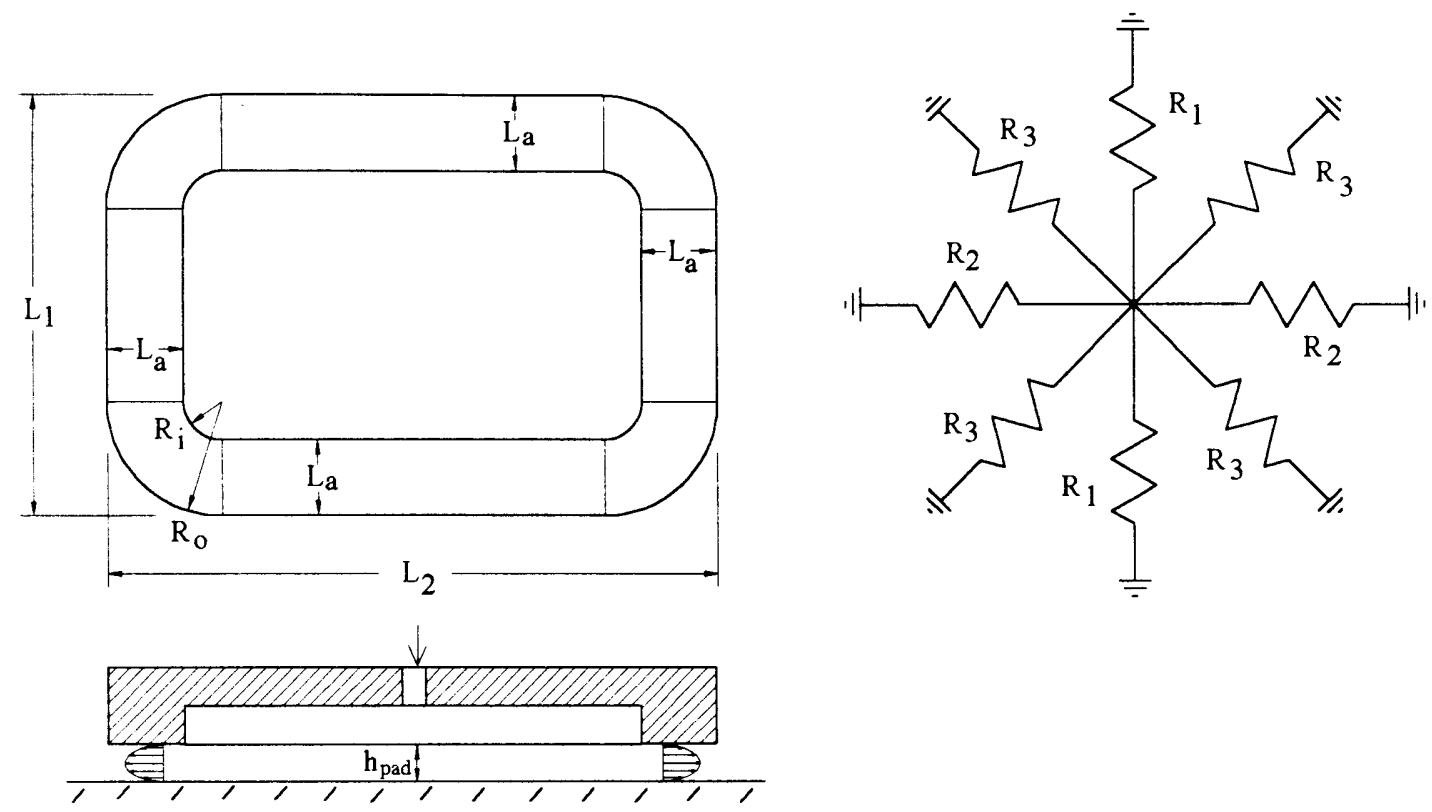

Figure 2.15. Schematic diagram of a tilted bearing pad with highly viscous fluid flowing out of the pocket. The lands form eight resistances in parallel.

\section{C.i. Untilted Pad Land Flow}

The nominal, no tilt total pad resistance will be determined first. The total equivalent pad resistance is found by:

$$
R=\frac{1}{\frac{2}{R_{1}}+\frac{2}{R_{2}}+\frac{4}{R_{3}}}
$$

In this case:

$$
\begin{aligned}
& R_{1}=\frac{12 \mu L_{a}}{h_{p a d}^{3}\left(L_{2}-2 L_{a}-2 R_{i}\right)} \\
& R_{2}=\frac{12 \mu L_{a}}{h_{p a d}^{3}\left(L_{1}-2 L_{a}-2 R_{i}\right)} \\
& R_{3}=\frac{24}{\pi} \frac{\mu}{h_{p a d}^{3}} \ln \left(\frac{R_{o}}{R_{i}}\right)
\end{aligned}
$$


Inserting Equations (2.62) through (2.64) into Equation (2.61), the nominal pad resistance is given by:

$$
R_{n o m}=\frac{6 \mu}{h_{\text {pad }}^{3}} \frac{1}{\frac{\left(L_{2}-2 L_{a}-2 R_{i}\right)}{L_{a}}+\frac{\left(L_{1}-2 L_{a}-2 R_{i}\right)}{L_{a}}+\frac{\pi}{\ln \left(\frac{R_{i}+L_{a}}{R_{i}}\right)}}
$$

\section{2.c.ii. Tilted Pad Land Flow}

A tilted bearing pad is shown schematically in Figure 2.16. The effect of pad tilting on the overall hydraulic resistance of the pad will now be considered. It is again emphasized that, in the derivations that follow, it is assumed that the fluid flowing over the lands is laminar and fully-developed.
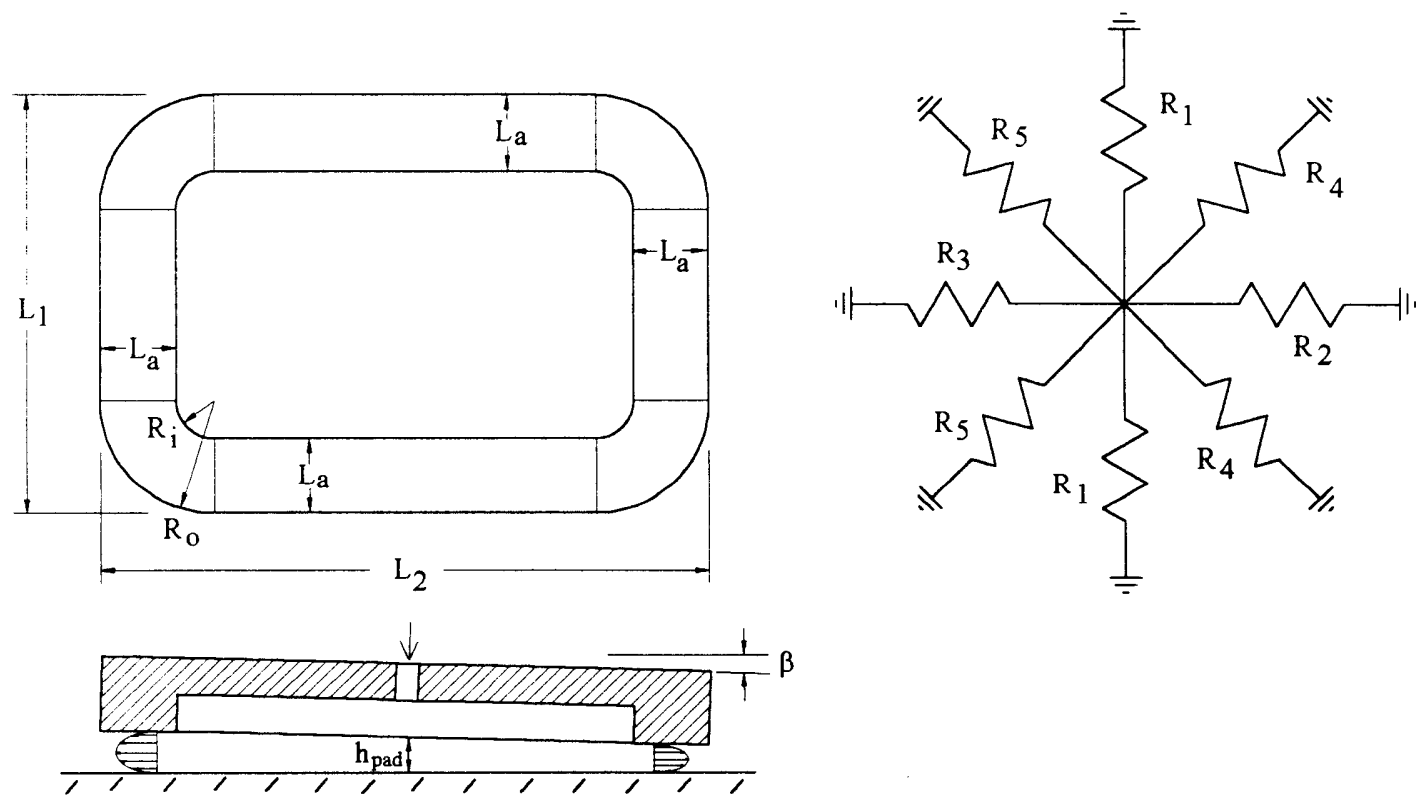

Figure 2.16. Schematic diagram of a tilted bearing pad with highly viscous fluid flowing out of the pocket. The lands form eight resistances in parallel. 
The resistance of a tilted pad will now be found by assembling the individual tilted resistances found in the previous sections. The total equivalent pad resistance is found by:

$$
R=\frac{1}{\frac{2}{R_{1}}+\frac{1}{R_{2}}+\frac{1}{R_{3}}+\frac{2}{R_{4}}+\frac{2}{R_{5}}}
$$

where $R_{1}$ is the resistance of a side rectangular land:

$$
R_{1}=\frac{\frac{48 \mu L_{a} \sin (\beta)}{\left(h_{p a d}+\frac{1}{2}\left(L_{2}-2 L_{a}-2 R_{i}\right) \sin (\beta)\right)^{4}}}{1-\left(1-\frac{L_{2}-2 L_{a}-2 R_{i}}{h_{p a d}+\frac{1}{2}\left(L_{2}-2 L_{a}-2 R_{i}\right) \sin (\beta)} \cos (\beta) \sin (\beta)\right)^{4}}
$$

$\mathbf{R}_{2}$ is the resistance of converging rectangular land:

$$
R_{2}=6 \mu \frac{\frac{1}{\left(1-\frac{L_{a}}{h_{p a d}-\frac{1}{2}\left(L_{2}-2 L_{a}\right) \sin (\beta)} \cos (\beta) \sin (\beta)\right)^{2}}-1}{\left(h_{p a d}-\frac{1}{2}\left(L_{2}-2 L_{a}\right) \sin (\beta)\right)^{2} \sin (\beta)\left(L_{1}-2 L_{a}-2 R_{i}\right)}
$$

$\mathbf{R}_{3}$ is the resistance of the diverging rectangular land:

$$
R_{3}=6 \mu \frac{1-\frac{1}{\left(1+\frac{L_{a}}{h_{p a d}+\frac{1}{2}\left(L_{2}-2 L_{a}\right) \sin (\beta)} \cos (\beta) \sin (\beta)\right)^{2}}}{\left(h_{p a d}+\frac{1}{2}\left(L_{2}-2 L_{a}\right) \sin (\beta)\right)^{2} \sin (\beta)\left(L_{1}-2 L_{a}-2 R_{i}\right)}
$$

$R_{4}$ is the resistance of a quarter of a tilted circular land: 


$$
R_{4}=\frac{\frac{12 \mu}{\left(h_{p a d}-\frac{1}{2}\left(L_{2}-2 L_{a}-2 R_{i}\right) \sin (\beta)\right)^{3}}}{\frac{\pi}{24}\left(\frac{1}{f_{1}(0)}+\frac{4}{f_{1}(\pi / 8)}+\frac{2}{f_{1}(\pi / 4)}+\frac{4}{f_{1}(3 \pi / 8)}+\frac{1}{f_{1}(\pi / 2)}\right)}
$$

where:

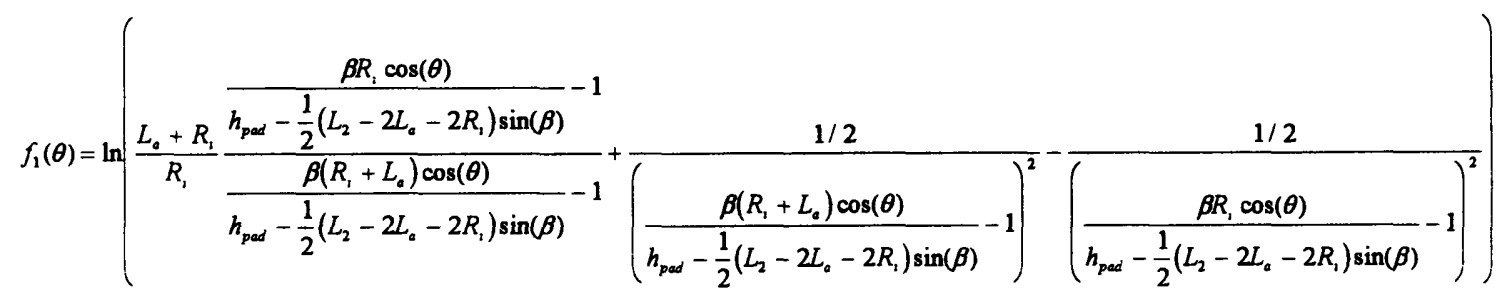

$$
\begin{aligned}
& +\frac{1}{\frac{\beta R_{i} \cos (\theta)}{h_{\text {pad }}-\frac{1}{2}\left(L_{2}-2 L_{a}-2 R_{i}\right) \sin (\beta)}-1}-\frac{1}{\frac{\beta\left(R_{i}+L_{a}\right) \cos (\theta)}{h_{p a d}-\frac{1}{2}\left(L_{2}-2 L_{a}-2 R_{i}\right) \sin (\beta)}-1}
\end{aligned}
$$

and $R_{5}$ is the resistance of a quarter of a tilted circular land:

$$
R_{5}=\frac{\frac{12 \mu}{\left(h_{p a d}+\frac{1}{2}\left(L_{2}-2 L_{a}-2 R_{i}\right) \sin (\beta)\right)^{3}}}{\frac{\pi}{24}\left(\frac{1}{f_{1}(\pi / 2)}+\frac{4}{f_{1}(5 \pi / 8)}+\frac{2}{f_{1}(3 \pi / 4)}+\frac{4}{f_{1}(7 \pi / 8)}+\frac{1}{f_{1}(\pi)}\right)}
$$

where:

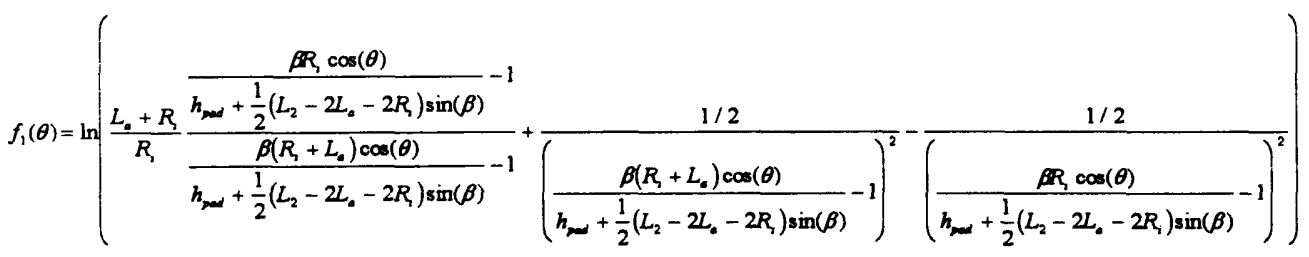

$$
\begin{aligned}
& +\frac{1}{\frac{\beta R_{i} \cos (\theta)}{h_{p a d}+\frac{1}{2}\left(L_{2}-2 L_{a}-2 R_{i}\right) \sin (\beta)}-1}-\frac{1}{\frac{\beta\left(R_{i}+L_{a}\right) \cos (\theta)}{h_{p a d}+\frac{1}{2}\left(L_{2}-2 L_{a}-2 R_{i}\right) \sin (\beta)}-1}
\end{aligned}
$$


Shown in Figure 2.17 is the reduction in pad resistance resulting from tilt for $\mathrm{L}_{\mathbf{a}} / \mathrm{L}_{1}=1 / 6$, $R_{i} / L_{a}=1 / 2$, and several ratios of $L_{2} / L_{1}$. Note that the solution for the tilted hydrostatic bearing pad is subject to the constraints:

$$
\frac{\beta \mathrm{L}_{2} / 2}{\mathrm{~h}_{\mathrm{pad}}}<1, \quad \mathrm{~L}_{2} \geq 2 \mathrm{~L}_{\mathrm{a}}+2 \mathrm{R}_{\mathrm{i}} \quad, \quad \mathrm{L}_{1} \geq 2 \mathrm{~L}_{\mathrm{a}}+2 \mathrm{R}_{\mathrm{i}}
$$

The first constraint insures that touchdown does not occur. The second and third constraints are simply variable definitions stating that the total pad length and width must both exceed the length of the lands and corner radii.

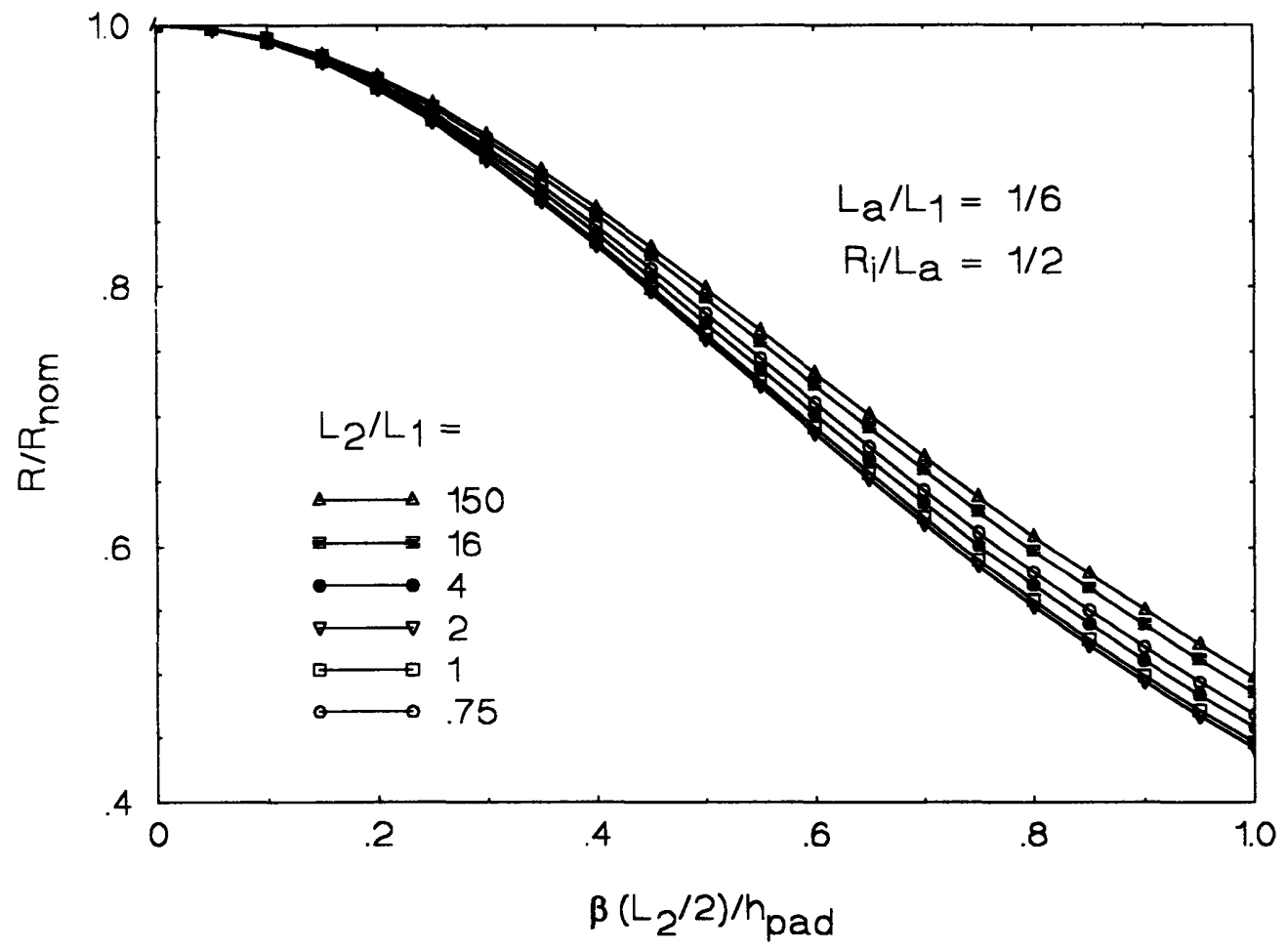

Figure 2.17. Reduction in pad resistance resulting from tilt. 
Equations (2.66) through (2.73) are cumbersome for simple calculations, and so an approximate curve fit to the result is given by:

$$
\frac{R}{R_{\text {nom }}} \approx 1-1.3\left(\frac{\beta L_{2} / 2}{h_{p a d}}\right)^{2}+0.75\left(\frac{\beta L_{2} / 2}{h_{p a d}}\right)^{3}
$$

where $R_{\text {nom }}$ is the nominal no-tilt pad resistance, given by Equation (2.65). The curve fit given by Equation (2.75) is given only for a rough approximation of tilted pad resistance, but is accurate to within about $15 \%$ over the following range of variables:

$$
\begin{aligned}
& 0.05<\frac{L_{a}}{L_{1}}<\frac{1}{2\left(1+\frac{R_{i}}{L_{a}}\right)} \quad, \quad 0.5<\frac{R_{i}}{L_{a}}<1.5 \\
& 0<\frac{\beta L_{2} / 2}{h_{p a d}}<1, \frac{L_{2}}{L_{1}}>3 \frac{L_{a}}{L_{1}}\left(1+\frac{R_{i}}{L_{a}}\right)
\end{aligned}
$$




\section{RADIAL BEARING DESIGN: STIFFNESS, LOAD CAPACITY,}

\section{AND FLOW RATE}

The most important part of designing a hydrostatic machine tool spindle is designing the radial bearings. The primary purpose of the radial bearings are to hold the shaft rigidly in all radial directions while allowing for free angular rotation. This should be accomplished with reasonably low flow rate and pumping power. Often the goals of high stiffness and low pumping power are competing and the most adequate design will depend upon the overall project objective. In some cases, achieving high spindle stiffness is the most important goal and high pumping power is an acceptable cost if it yields higher spindle stiffness. In other cases, the size and cost of the pump must be kept to a minimum, even if that means realizing lower spindle stiffness. In the design of most machine tool spindles, both goals of high stiffness and low pumping power are important and a wellbalanced bearing design is sought. Other important bearing attributes include the bearing damping, shearing power consumption, and temperature rise. These factors will be considered in future chapters. The focus of this chapter will be load-carrying capacity and pumping power.

It should be clearly emphasized that, although it is important to design the radial bearings with high stiffness, the overall goal of a spindle design is not to maximize the bearing stiffness but to maximize the spindle stiffness. Other characteristics of the radial bearings besides their stiffness have an impact on the stiffness of the spindle and must also be considered. First, the effective load center of the bearing is important; how close the effective load center of the front radial bearing is to the spindle nose often has a dramatic 
impact on how stiff the spindle is. Second, how sensitive the front bearing is to shaft displacement at the front of the spindle is very important. The front radial bearing can be designed to have greater sensitivity to shaft deflection near the front of the spindle so that higher spindle stiffness is achieved.

The purpose of this chapter is to appropriately model radial hydrostatic bearings so that their stiffness can be calculated and their design can be integrated into the overall design of the spindle. This is accomplished by calculating the restoring force that the bearing generates when the shaft is moved eccentric and out of parallel with the bearing housing. In other words, the eccentricity of the shaft is a linear function of the axial location within the bearing. By modeling the tilting of the shaft, the effect of shaft bending on the bearing stiffness can be accounted for. Shaft bending particularly affects the stiffness characteristics of the front bearing and must be considered to accurately design and optimize hydrostatic spindles. This will be discussed further in Chapter 4 .

The relations developed in this chapter can be readily implemented into computer programs to quickly design and optimize hydrostatic bearings for machine tool spindles. In the derivations that follow, several important assumptions are made about the bearing characteristics and the operating conditions. It is assumed that:

- The depressed areas of the bearings, including the pockets, supply grooves, and drainage grooves, are at a depth sufficiently great that the pressure drop throughout them is negligible compared to the pressure drop across the bearing land regions. This assumption allows the depressed regions to be modeled as regions of constant pressure and enables fluid circuit theory to be used to solve for the bearing pressures. 
- The fluid is incompressible so that density changes caused by changes in the fluid pressure or temperature are negligible as it passes through the bearing.

- The fluid has a constant dynamic viscosity as it passes through the bearing. This is a good assumption if the temperature rise of the fluid from the bearing inlet to the bearing outlet causes a negligible change in the fluid viscosity.

- The fluid flow on the land areas of the bearing are laminar and fully-developed, as discussed in Chapter 2. In this case, the flow rate across a land is linearly proportional to the pressure difference across it and so the resistances of the fluid circuit are constant and linear fluid circuit theory can be used to solve for the flow rates and pressures.

These assumptions must be considered before the methods and results generated in this chapter are used in hydrostatic bearing design.

Twelve radial hydrostatic bearings are considered in this chapter. In order to compare their relative merits and characteristics, some non-dimensional performance parameters will now be defined. The most important attribute of a bearing is its load carrying efficiency, which is the bearing restoring force divided by the supply pressure times the cross-sectional area:

$$
F_{\text {eff }}=\frac{F_{y}}{P_{s} D L}
$$

The load-carrying efficiency is a strong function of the bearing eccentricity; it is usually fairly linear for deflections up to $50 \%$ of gap closure and then its slope decreases as the shaft comes closer to touch-down. At touch-down, most hydrostatic bearings have loadcarrying efficiencies between 0.4 and 0.6 . The load-carrying efficiency at $75 \%$ of gap 
closure is usually a good indication of the load-capacity of the bearing, and will be used as an indication of such in this chapter.

Perhaps more important than the load-carrying efficiency of a bearing is its stiffness. The specific stiffness quantifies the bearing stiffness:

$$
\mathrm{K}_{\mathrm{spec}}=\frac{\mathrm{F}_{\mathrm{eff}}}{\mathrm{e}}
$$

Hydrostatic bearings behave non-linearly and so the specfic stiffness is a function of the eccentricity. For applications where the cutting loads are low relative to the bearing load capacity, the stiffness of the bearing at small eccentricities is usually the most important consideration. Fortunately, the bearing stiffness is usually highest at small eccentricities. The initial specific stiffness of a bearing generally corresponds to very low eccentricities (e $<0.01$ ), and usually ranges between 0.8 and 1.2 for most hydrostatic bearings, but can be even higher.

The above bearing parameters describe the load-carrying performance of the bearing. Another important consideration is the amount of flow rate that is needed to achieve the load-carrying performance. A parameter that will be used to compare the flow rates for different bearings is the specific flow rate, which is the supply flow rate divided by the flow rate that would occur if the entire length of the bearing were used as a hydraulic resistance:

$$
Q_{\text {spec }}=\frac{Q_{s}}{\left(\frac{P_{s} \pi \mathrm{Dh}_{o}^{3}}{12 \mu \mathrm{L}}\right)}
$$


The specific flow rate of hydrostatic bearings vary from as little as 5 to as much as 50 . The specific flow rate of a bearing is an indication of the pumping power required to support it since the pumping power is the supply pressure times the flow rate.

Relations are developed in the following sections to predict the load-carrying performance of twelve hydrostatic bearings. A representative bearing with a diameter of $80 \mathrm{~mm}$ and a length of $80 \mathrm{~mm}$ is considered in each case and its characteristics are presented. Although the flow rate is considered when the dimensions of the bearing geometries are chosen, the design in each case is primarily optimized to generate the maximum initial specific stiffness. In addition, the dimensions of the bearing geometry are chosen while considering manufacturing limitations. For example, the minimum groove width used is $2 \mathrm{~mm}$; this groove size can be generated fairly easily using conventional machining methods. The minimum land width used is $3 \mathrm{~mm}$; reducing the land width further would make manufacturing variations become significant. Also, the land width should be kept to at least $3 \mathrm{~mm}$ to keep the fluid flow across the lands laminar and fullydeveloped when water is used at supply pressures over 500 psi. Using these limitations and considerations, a representative bearing is designed and optimized for each bearing type. In all cases, the bearing performance presented is that which occurs when the shaft moves parallel to the bearing housing. Although the comparisons generated provide an indication of the relative merits of the various bearings, they do not necessarily indicate which bearings make the best spindles. The performance of spindles that utilize the twelve bearings of this chapter will be demonstrated in Chapter 4. 


\section{A. Fixed-Compensated Bearing \#1}

The pad layout of a conventional, fixed-compensated hydrostatic bearing is shown in Figure 3.1. Fluid enters each pocket through a fixed-resistance device such as a capillary tube. It then flows throughout the pockets, represented by shaded regions, which are at a depth significantly greater than the land clearances such that the fluid pressure throughout each pocket is uniform. The fluid then crosses the lands and out of the bearing to atmospheric pressure. The grooves that run between each successive pocket are used to drain the fluid; they are also at a depth significantly greater than the land clearance such that the fluid pressure throughout them is atmospheric pressure. Note the the lands that run circumferentially on either end of the bearing are modeled as having different widths, $\mathrm{L}_{\text {lmp }}$ and $\mathrm{L}_{\text {lep. }}$. This allows the circumferential land nearest the front of the spindle to be made with a somewhat shorter width than the other bearing lands so that the bearing has higher sensitivity to deflections at the front of the spindle, thereby increasing the spindle stiffness. This will be discussed further in Chapter 4 . 


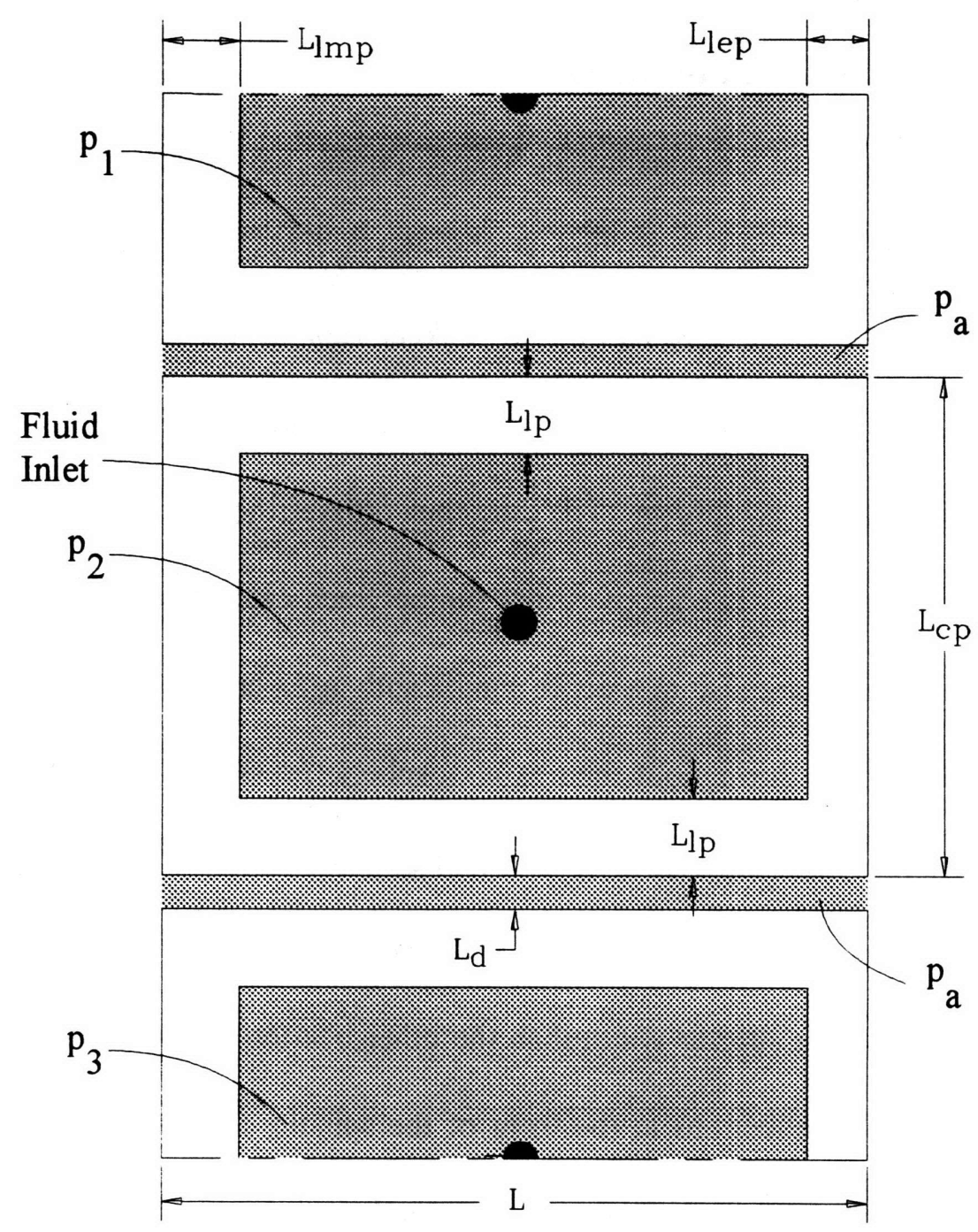

Figure 3.1. Schematic diagram of the pad layout of a conventional, fixed-compensated hydrostatic bearing with drainage grooves between its pockets. The shaded regions represent depressed regions.

\section{A.i. Description of Calculations for Fixed-Compensated Bearing \#1}

Calculating the load-carrying capacity of a bearing is accomplished by moving the shaft to an eccentric position and then calculating the resulting restoring force generated by the bearing. Figure 3.2 shows a schematic diagram of an 8-pocket bearing with its 
shaft displaced to an eccentric position. The shaft is displaced in the y-direction toward pocket \#1, which has a pressure of $p_{1}$. The angular coordinate, $\theta$, begins from the center of pocket \#1.

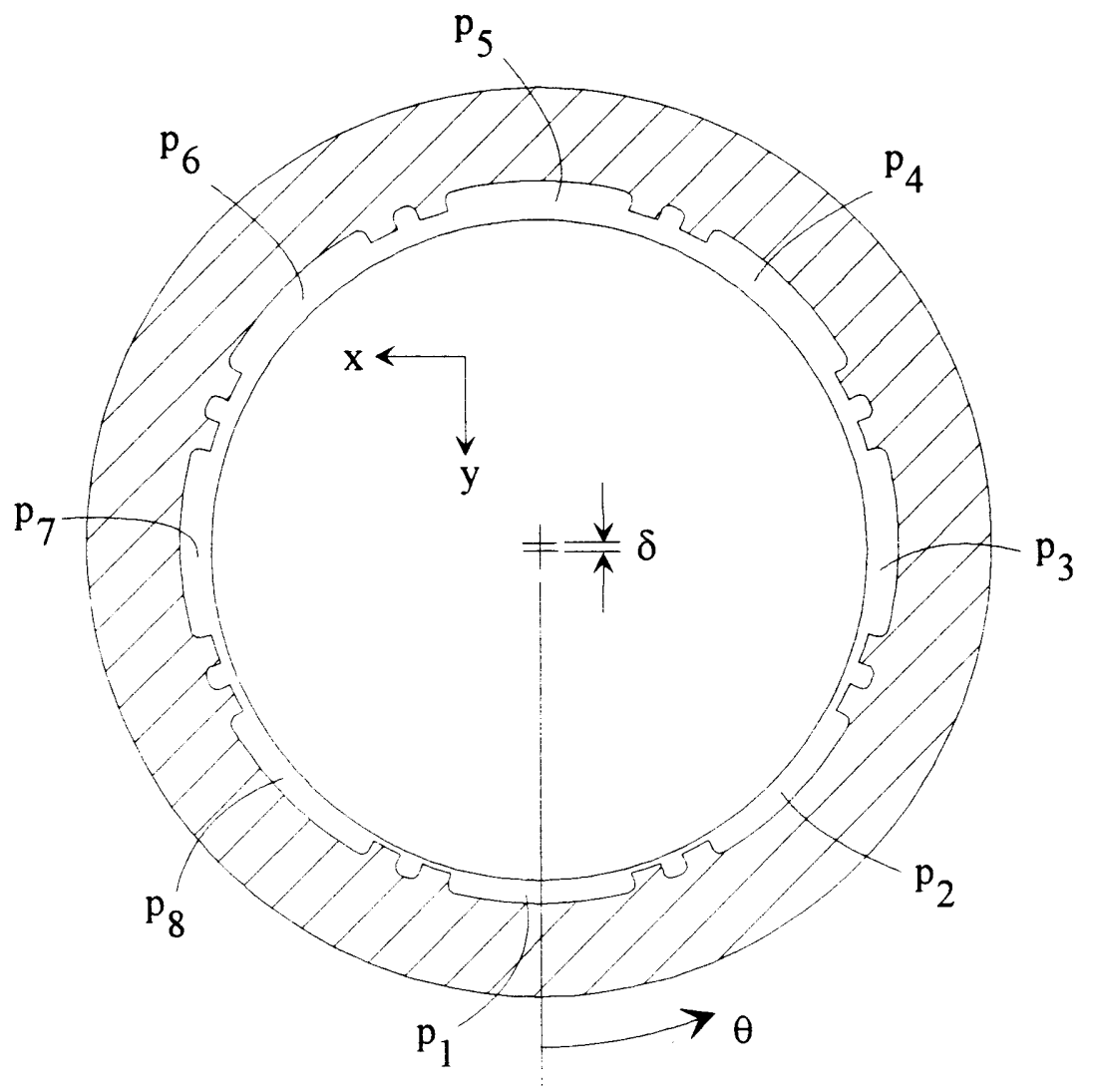

Figure 3.2. Schematic diagram of a shaft displaced to an eccentric position within an 8pocket bearing housing. The clearance is exaggerated for clarity.

Figure 3.3 shows a schematic diagram of a shaft displaced to an eccentric position with respect to the bearing housing and also out of parallel with it. The clearance is exaggerated for clarity. The shaft is modeled as straight with end eccentricities given by:

$$
\mathrm{e}_{\mathrm{m}}=\frac{\delta_{\mathrm{m}}}{\mathrm{h}_{\mathrm{o}}} \quad \mathrm{e}_{\mathrm{e}}=\frac{\delta_{\mathrm{e}}}{\mathrm{h}_{\mathrm{o}}}
$$


where $h_{0}$ is the nominal, radial clearance between the shaft and the housing lands when the shaft is in a concentric position.

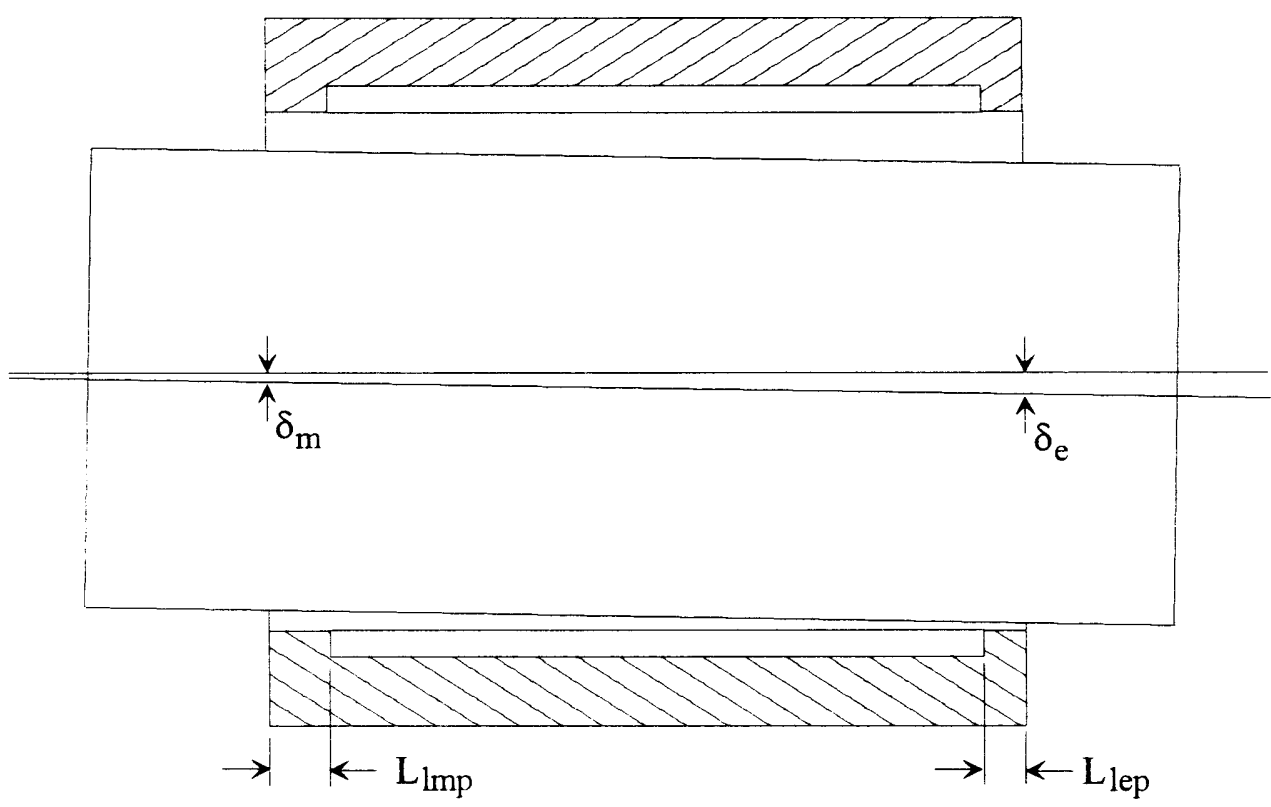

Figure 3.3. Schematic diagram of a shaft displaced to an eccentric position with respect to the bearing housing and also out of parallel with it. The clearance is exaggerated for clarity.

The first step in calculating the restoring force generated by moving the shaft to an eccentric position is to calculate the hydraulic resistance of each bearing land. For each supporting pad, there are four lands over which fluid flows in parallel: the circumferential land with a width $\mathrm{L}_{\text {lep }}$, the circumferential land with a width $\mathrm{L}_{\mathrm{lmp}}$, and the two axial lands with widths of $\mathrm{L}_{\mathrm{lp}}$. The resistance of the two axial lands are given by:

$$
R_{1,1}=R_{\text {axial }}\left(\mu, h_{o}, L_{l p}, L-L_{l m p}-L_{l e p}+L_{m}^{*}+L_{e}^{*}, \theta_{i}+\frac{L_{c p}-L_{l p}}{D}, e_{\text {axal }}\left(e_{1}, e_{2}, \theta_{i}+\frac{L_{c p}-L_{l p}}{D}\right)\right)
$$




$$
R_{2, i}=R_{\text {axial }}\left(\mu, h_{o}, L_{\text {lp }}, L-L_{\text {lmp }}-L_{\text {lep }}++L_{m}^{*}+L_{e}^{*}, \theta_{i}-\frac{L_{c p}-L_{\text {lp }}}{D}, e_{\text {exial }}\left(e_{1}, e_{2}, \theta_{i}-\frac{L_{c p}-L_{l p}}{D}\right)\right)
$$

where $\mathrm{i}$ is the pocket index, $\mathrm{R}_{\mathrm{axial}}$ is the function given by Equation (2.31), $\mathrm{e}_{\text {axial }}$ is the function given by Equation (2.38), and $\theta_{\mathrm{i}}$ is the angle to center of the ith pocket:

$$
\theta_{i}=2 \pi(\mathrm{i}-1) / \mathrm{N} \quad \mathrm{i}=1,2 \cdots, \mathrm{N}
$$

where $\mathrm{N}$ is the number of pockets. The eccentricities $e_{1}$ and $e_{2}$ are those corresponding to the ends of the axial lands:

$$
\begin{aligned}
& e_{1}=e_{m}+\left(e_{e}-e_{m}\right) \frac{L_{l m p}}{L} \\
& e_{2}=e_{e}+\left(e_{m}-e_{e}\right) \frac{L_{l e p}}{L}
\end{aligned}
$$

The variables $\mathrm{L}_{\mathrm{m}}^{*}$ and $\mathrm{L}_{\mathrm{e}}$ are added to the distance perpendicular to the direction of flow to account for the small amount of spreading the fluid experiences near the two ends of the axial land. Recommended relations for these terms are

$$
\begin{aligned}
& \mathrm{L}_{\mathrm{m}}^{*} \approx 0.4\left\{\min \left(\mathrm{L}_{\mathrm{lmp}}, \mathrm{L}_{\mathrm{lp}}\right)\right\} \\
& \mathrm{L}_{\mathrm{e}}^{*} \approx 0.4\left\{\min \left(\mathrm{L}_{\mathrm{lep}}, \mathrm{L}_{\mathrm{lp}}\right)\right\}
\end{aligned}
$$

As long as the land widths are small compared to the pocket axial length, the effect of the fluid spreading is small and the exact magnitudes of these terms do not significantly affect the predicted bearing performance. The resistance of the two circumferential lands are given by:

$$
\begin{array}{r}
\mathbf{R}_{3, \mathrm{i}}=\mathbf{R}_{\text {circum }}\left(\mu, \mathrm{h}_{\mathrm{o}}, \mathrm{L}_{\mathrm{lmp}}, \mathrm{L}_{\mathrm{cp}}-2 \mathrm{~L}_{\mathrm{lp}}+\mathrm{L}_{\mathrm{m}}^{*}, \theta_{\mathrm{i}}, \mathrm{e}_{\text {circum }}\left(\mathrm{e}_{\mathrm{m}}, \mathrm{e}_{1}, \theta_{\mathrm{i}}\right)\right) \\
\mathbf{R}_{4, \mathrm{i}}=\mathbf{R}_{\text {circum }}\left(\mu, \mathrm{h}_{\mathrm{o}}, \mathrm{L}_{\text {lep }}, \mathrm{L}_{\mathrm{cp}}-2 \mathrm{~L}_{\mathrm{lp}}+\mathrm{L}_{\mathrm{e}}^{*}, \theta_{\mathrm{i}}, \mathrm{e}_{\text {circum }}\left(\mathrm{e}_{2}, \mathrm{e}_{\mathrm{e}}, \theta_{\mathrm{i}}\right)\right)
\end{array}
$$


The four resistances given by Equations (3.2), (3.3), (3.9), and (3.10) are evaluated $\mathrm{N}$ times for each pocket of the bearing.

The next step is to calculate the equivalent hydraulic resistance of each pocket corresponding to the resistances $R_{a, i}$ of Circuit \#1 in Appendix B:

$$
\mathbf{R}_{\mathrm{a}, \mathrm{i}}=\frac{1}{\frac{1}{\mathrm{R}_{1, \mathrm{i}}}+\frac{1}{\mathrm{R}_{2, \mathrm{i}}}+\frac{1}{\mathrm{R}_{3, \mathrm{i}}}+\frac{1}{\mathrm{R}_{4, \mathrm{i}}}}
$$

The resistances $R_{c, i}$ of Circuit \#1 correspond to the inlet resistances used to compensate the bearing. The value of these fixed resistances are chosen so that the resistance ratio $\gamma=$ $\mathrm{R}_{\mathrm{c}, \mathrm{i}} / \mathrm{R}_{\mathrm{a}, \mathrm{i}}$ is approximately equal to 1 when the shaft is concentric to the bearing housing. Once the resistances of Circuit \#1 have been calculated, the fluid circuit can be solved for the $\mathrm{N}$ unknown pocket pressures, $\mathrm{p}_{\mathrm{i}}$, and the supply volumetric flow rate, $\mathrm{Q}_{\mathrm{s}}$.

Once the pocket pressures are known, the reaction force of the bearing is calculated by multiplying the pressures times the appropriate areas. The net force generated by pocket $\mathrm{i}$ in the direction of the bearing center is given by:

$$
\mathrm{F}_{\text {net. }}^{1}=\left(\mathrm{L}-\mathrm{L}_{\mathrm{lmp}}-\mathrm{L}_{\text {lep }}\right) \mathrm{D} \sin \left(\frac{\mathrm{L}_{\mathrm{cp}}-2 \mathrm{~L}_{\mathrm{lp}}}{\mathrm{D}}\right) \mathrm{p}_{\mathrm{i}}
$$

The net force generated by pocket $\mathrm{i}$ in the $\mathrm{y}$-direction is given by:

$$
F_{y, i}^{1}=D\left(L-L_{l m p}-L_{l e p}\right) \sin \left(\frac{L_{c p}-2 L_{l p}}{D}\right) p_{i} \cos \left(\theta_{i}\right)
$$

Similarly, the y-direction forces generated by the other bearing areas are given by:

$$
\mathrm{F}_{\mathrm{y}, \mathrm{i}}^{2}=\mathrm{DL}_{\mathrm{lmp}} \sin \left(\frac{\mathrm{L}_{\mathrm{cp}}-2 \mathrm{~L}_{\mathrm{lp}}}{\mathrm{D}}\right)\left(\frac{\mathrm{p}_{\mathrm{i}}}{2}\right) \cos \left(\theta_{\mathrm{i}}\right)
$$




$$
\begin{aligned}
& \mathrm{F}_{\mathrm{y}, \mathrm{i}}^{3}=\mathrm{DL}_{\text {lep }} \sin \left(\frac{\mathrm{L}_{\mathrm{cp}}-2 \mathrm{~L}_{\mathrm{lp}}}{\mathrm{D}}\right)\left(\frac{\mathrm{p}_{\mathrm{i}}}{2}\right) \cos \left(\theta_{\mathrm{i}}\right) \\
& \mathrm{F}_{\mathrm{y}, \mathrm{i}}^{4}=\mathrm{DL}_{\mathrm{lmp}} \sin \left(\frac{\mathrm{L}_{\mathrm{lp}}}{\mathrm{D}}\right)\left(\frac{\mathrm{p}_{\mathrm{i}}}{4}\right) \cos \left(\theta_{\mathrm{i}}+\frac{\mathrm{L}_{\mathrm{cp}}-\mathrm{L}_{\mathrm{lp}}}{\mathrm{D}}\right) \\
& \mathrm{F}_{\mathrm{y}, 1}^{5}=\mathrm{DL}_{\mathrm{lmp}} \sin \left(\frac{\mathrm{L}_{\mathrm{lp}}}{\mathrm{D}}\right)\left(\frac{\mathrm{p}_{\mathrm{i}}}{4}\right) \cos \left(\theta_{1}-\frac{\mathrm{L}_{\mathrm{cp}}-\mathrm{L}_{\mathrm{lp}}}{\mathrm{D}}\right) \\
& \mathrm{F}_{\mathrm{y}, \mathrm{i}}^{6}=\mathrm{DL}_{\mathrm{lep}} \sin \left(\frac{\mathrm{L}_{\mathrm{lp}}}{\mathrm{D}}\right)\left(\frac{\mathrm{p}_{\mathrm{i}}}{4}\right) \cos \left(\theta_{\mathrm{i}}+\frac{\mathrm{L}_{\mathrm{cp}}-\mathrm{L}_{\mathrm{lp}}}{\mathrm{D}}\right) \\
& F_{y, 1}^{7}=D_{l e p} \sin \left(\frac{L_{l p}}{D}\right)\left(\frac{p_{i}}{4}\right) \cos \left(\theta_{i}-\frac{L_{c p}-L_{l p}}{D}\right) \\
& F_{y, i}^{8}=F_{\text {axial }}\left(D, L-L_{\text {lep }}-L_{\text {lmp }}, L_{l p}, p_{i}, 0, e_{\text {axial }}\left(e_{1}, e_{2}, \theta_{i}+\frac{L_{c p}-L_{l p}}{D}\right)\right) \cos \left(\theta_{i}+\frac{L_{c p}-L_{l p}}{D}\right) \\
& F_{y, 1}^{9}=F_{\text {axial }}\left(D, L-L_{\text {lep }}-L_{\text {lmp }}, L_{\text {lp }}, 0, p_{i}, e_{\text {axial }}\left(e_{1}, e_{2}, \theta_{i}-\frac{L_{c p}-L_{l p}}{D}\right)\right) \cos \left(\theta_{i}-\frac{L_{c p}-L_{l p}}{D}\right)
\end{aligned}
$$

where $\mathrm{F}_{\mathrm{axial}}$ is the function given by Equation (2.35). The total y-direction bearing force is calculated by summing the y-direction forces given by Equations (3.13) through (3.21) for all of the $\mathrm{N}$ pockets.

In addition to the calculating the bearing load-carrying capability, the effective load center of the bearing is needed to calculate the stiffness of the spindle. The effective center of the bearing is found by summing the moments about the end of the bearing and dividing by the bearing moment by the bearing force. The bearing moment is computed by multiplying the forces of Equations (3.13) through (3.21) by the axial center of each area:

$$
\mathbf{M}_{\mathrm{y}, \mathrm{i}}^{1}=\left(\mathrm{L}_{\text {lep }}+\frac{\mathrm{L}-\mathrm{L}_{\text {lmp }}-\mathrm{L}_{\text {lep }}}{2}\right) \mathrm{F}_{\mathrm{y}, \mathrm{i}}^{1}
$$




$$
\begin{aligned}
& M_{y, i}^{2}=\left(L-L_{l m p} / 2\right) F_{y, i}^{2} \\
& M_{y, i}^{3}=\left(L_{l e p} / 2\right) F_{y, i}^{3} \\
& M_{y, i}^{4}=\left(L-L_{l m p} / 2\right) F_{y, i}^{4} \\
& M_{y, i}^{5}=\left(L-L_{l m p} / 2\right) F_{y, i}^{5} \\
& M_{y, i}^{6}=\left(L_{l e p} / 2\right) F_{y, i}^{6} \\
& M_{y, i}^{7}=\left(L_{l e p} / 2\right) F_{y, i}^{7} \\
& M_{y, i}^{8}=\left(L_{l e p}+\frac{L-L_{l m p}-L_{l e p}}{2}\right) F_{y, i}^{8} \\
& M_{y, i}^{9}=\left(L_{l e p}+\frac{L-L_{l m p}-L_{l e p}}{2}\right) F_{y, i}^{9}
\end{aligned}
$$

The total $y$-direction bearing moment is calculated by summing the $y$-direction moments given by Equations (3.22) through (3.30) for all of the $\mathrm{N}$ pockets. The effective bearing load center is then given by:

$$
\mathrm{L}_{\mathrm{cen}}=\frac{\mathrm{M}_{\mathrm{y}}}{\mathrm{F}_{\mathrm{y}}}
$$

The parameter $\mathrm{L}_{\text {cen }} / \mathrm{L}$ is important because it gives an indication of how close the effective load center of the front bearing can be placed to the front of the spindle.

\section{A.ii. Representative Calculations for Fixed-Compensated Bearing \#1}

Table 3.1 shows the inputs and outputs for a representative $80 \mathrm{~mm}$ diameter, 80 $\mathrm{mm}$ long bearing. The land widths are $3 \mathrm{~mm}$ and the axial drainage groove width is $2 \mathrm{~mm}$. 
The resistance ratio is 1.5 . As shown, the initial specific stiffness is 0.856 , the loadcarrying efficiency at $75 \%$ of gap closure is 0.542 , and the specific flow rate is 57.4 .

\section{Table 3.1}

\begin{tabular}{|c|c|c|c|c|}
\hline $\begin{array}{l}\text { Operating Conditions } \\
\text { Supply Pressure (Pa,psi,atm) } \\
\text { Eccentricity Ratio em/ee } \\
\text { Eccentricity at End } \\
\text { Eccentricity at Middle End }\end{array}$ & $\begin{array}{c}\text { Ps } \\
\text { lamda } \\
\text { ee } \\
\text { em }\end{array}$ & $\begin{array}{r}4.17 E+06 \\
1 \\
0.01 \\
0.01\end{array}$ & 600 & 40.8 \\
\hline \multicolumn{5}{|l|}{ Fluid Properties } \\
\hline Dynamic Viscosity (N s/m) & mu & 0.0013 & & \\
\hline \multicolumn{5}{|l|}{ Bearing Geometry } \\
\hline Diameter $(m, m m, i n)$ & D & 0.08 & 80 & 3.15 \\
\hline Axial Length $(m, m m, i n)$ & $\mathrm{L}$ & 0.08 & 80 & 3.15 \\
\hline Number of Pockets & $\mathbf{N}$ & 6 & & \\
\hline Nominal Gap $(m, \mu m, i n)$ & ho & 0.000015 & 15 & 0.00059 \\
\hline Nominal Resistance Ratio & gamma & 1.5 & & \\
\hline Pocket Circumferential Land Width ( $\mathrm{m}, \mathrm{mm}$,in) & Llp & 0.003 & 3 & 0.118 \\
\hline Pocket End Axial Land Width $(\mathrm{m}, \mathrm{mm}, \mathrm{in})$ & LImp & 0.003 & 3 & 0.118 \\
\hline Pocket Middle Axial Land Width (m, $\mathrm{mm}$,in) & Llep & 0.003 & 3 & 0.118 \\
\hline Width of Drain Grooves $(m, m m$, in) & Ld & 0.00200 & 2 & 0.079 \\
\hline Pocket Circumferential Length $(\mathrm{m}, \mathrm{mm}$,in) & Lcp & 0.03989 & 39.9 & 1.570 \\
\hline \multicolumn{5}{|l|}{ Bearing Stiffness and Load Capacity } \\
\hline Location of Effective Bearing Force & Lcen_L & 0.500 & & \\
\hline Pressure Difference / Pressure @ ec,ep above & DPP & 0.013 & & \\
\hline $\begin{array}{l}\text { Load Capacity (N,kN,lbf) @ ec,ep given above } \\
\text { Load Carrying Efficiency @ ec,ep given above }\end{array}$ & $\begin{array}{l}\text { Fy } \\
\text { Feff }\end{array}$ & $\begin{array}{r}228 \\
0.009\end{array}$ & 0.23 & 51 \\
\hline 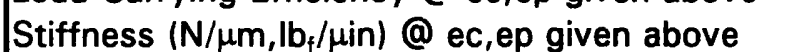 & Ky & 1522 & 8.690 & \\
\hline Specific Stiffness @ ec,ep given above & Kspec & 0.856 & & \\
\hline Initial Specific Stiffness & Kspec,o & 0.856 & & \\
\hline Load Carrying Efficiency @ 75\% of gap closure & Feff, 0.75 & 0.542 & & \\
\hline \multirow{3}{*}{$\begin{array}{l}\text { Flow Rate and Pumping Power } \\
\text { Total Flow Rate }\left(\mathrm{m}^{\wedge} 3 / \mathrm{s}, \mathrm{lpm}, \mathrm{gpm}\right) \\
\text { Pumping Power }(\mathrm{W}, \mathrm{kW}, \mathrm{hp}) \\
\text { Specific Flow Rate }\end{array}$} & & & & \\
\hline & Osupply & $1.62 \mathrm{E}-04$ & & 2.58 \\
\hline & $\begin{array}{l}\text { Ppump } \\
\text { Ospec }\end{array}$ & $\begin{array}{r}677 \\
57.4\end{array}$ & 0.677 & 0.908 \\
\hline $\begin{array}{l}\text { Ratio of Specific Initial Stiffness to Specific } \\
\text { Flow Rate }\end{array}$ & $\mathrm{K} /$ Ospec & 0.0149 & & \\
\hline
\end{tabular}


The load-carrying efficiency of the bearing of Table 3.1 is shown in Figure 3.4 for eccentricities ranging from 0 to 1 . As shown, the response is nearly linear up to an eccentricity of about 0.5 . For higher eccentricities, however, the plots turns off to a touch-down load-carrying efficiency of approximately 0.6 . This response is typical of hydrostatic bearings.

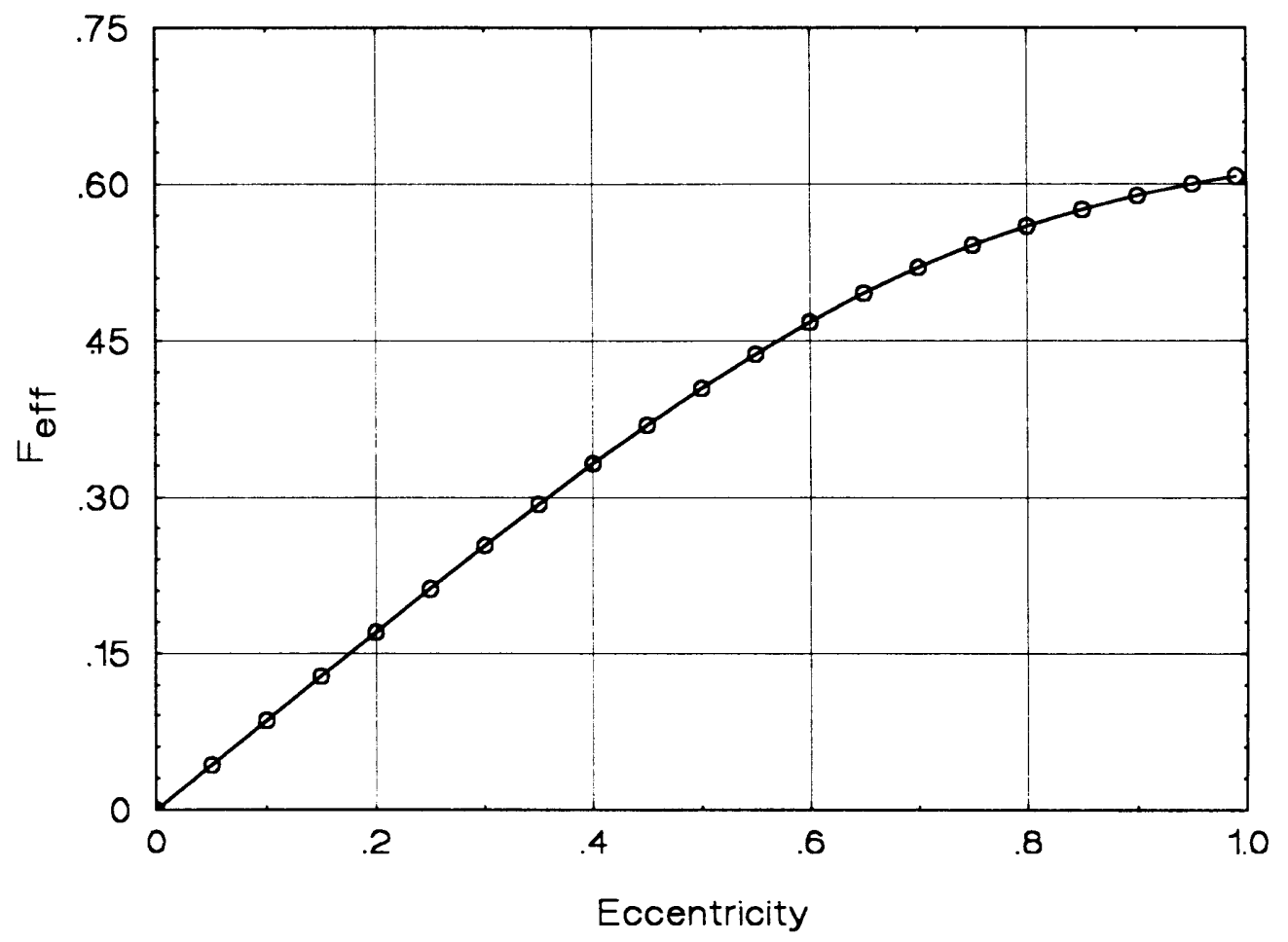

Figure 3.4. Load-carrying efficiency of the 6-pocket, $80 \mathrm{~mm}$ diameter FC Bearing \#1 of Table 3.1.

The effect of the resistance ratio on the performance of the bearing of Table 3.1 is shown in Figure 3.5. As shown, the optimimum resistance ratio that maximizes the initial specific stiffness is 1 ; the stiffness falls off significantly for resistance ratios greater than 
about 1.5 . The load-carrying efficiency at $75 \%$ of gap closure has an optimum for a resistance ratio of approximately 1.4 and remains relatively flat for higher resistance ratios. The specific flow rate decreases dramatically for higher resistance ratios. It is for this reason that bearings are often operated with a resistance ratio between 1.5 and 2.5 .

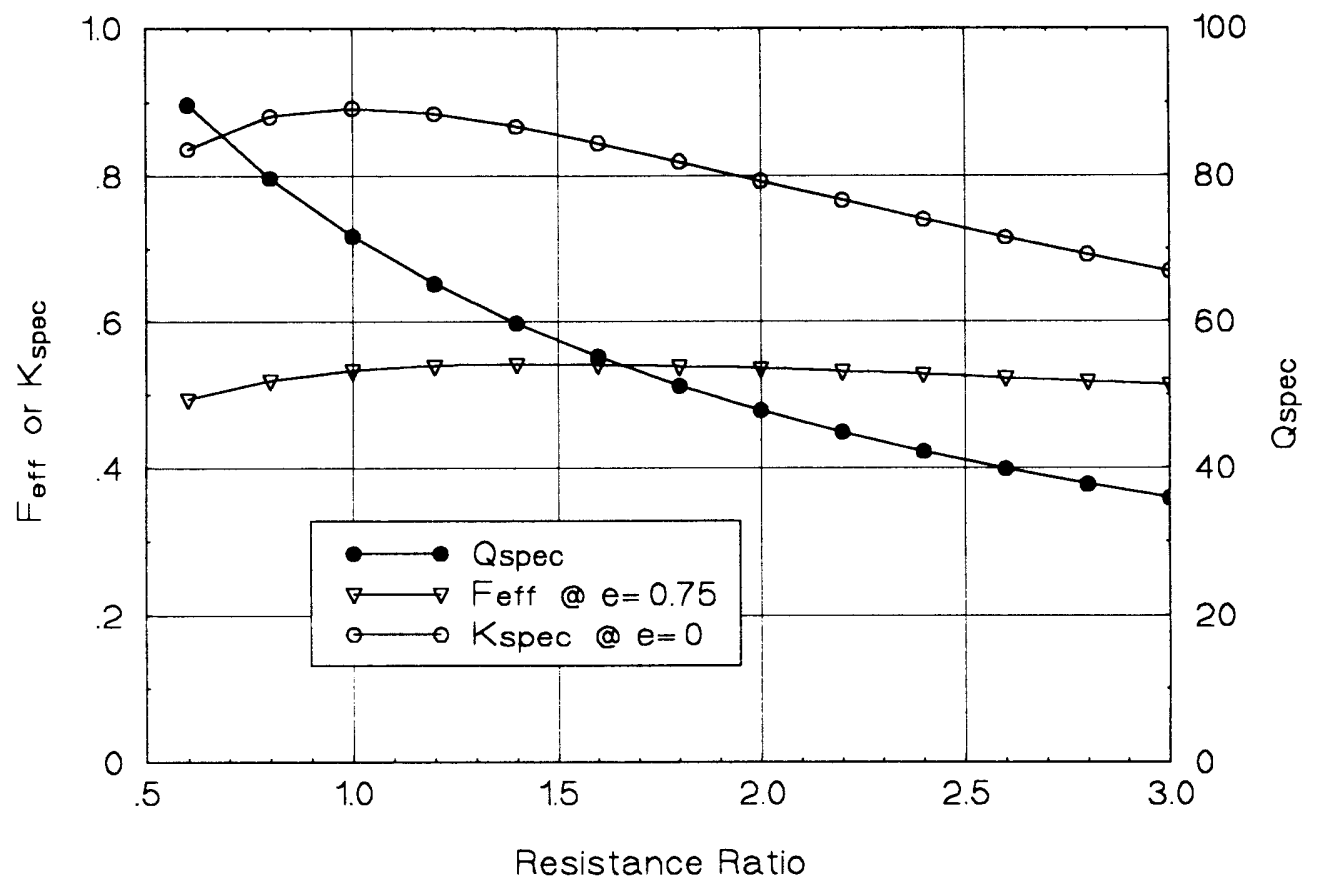

Figure 3.5. Effect of resistance ratio on the 6-pocket, $80 \mathrm{~mm}$ diameter FC Bearing \#1 of Table 3.1.

The effect of land width on the performance of the bearing of Table 3.1 is shown in Figure 3.6. All three land widths, $\mathrm{L}_{\mathrm{lp}}, \mathrm{L}_{\mathrm{lep}}$, and $\mathrm{L}_{\mathrm{lmp}}$, were changed equally. The resistance ratio was kept constant at a value of 1.5. As shown, increasing the land width reduces the specific stiffness and load-carrying efficiency somewhat because the pressure is distrubuted over a lesser percentage of the total bearing area. The specific flow rate is 
also dramatically reduced and wider land widths are sometimes warranted in order to obtain lower pumping power.

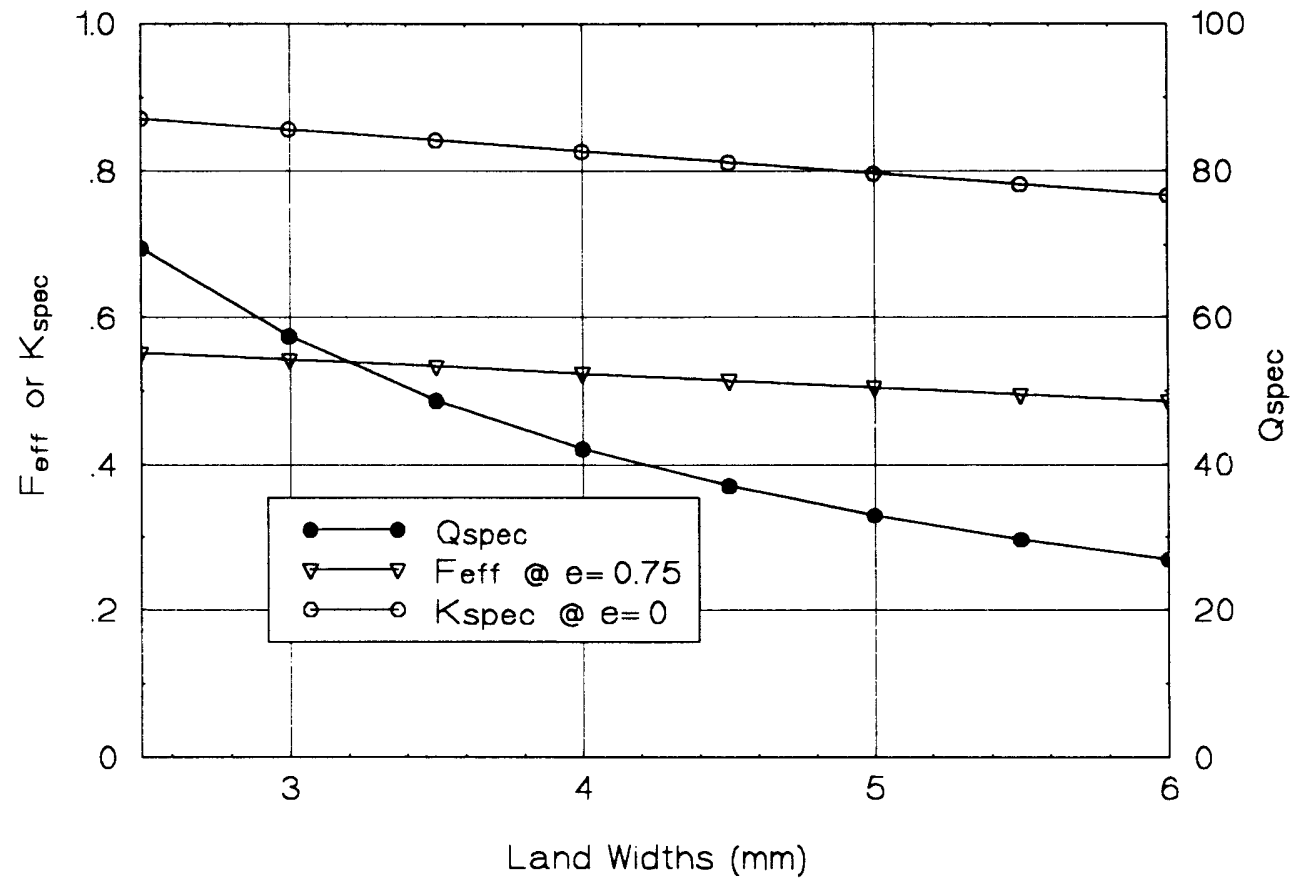

Figure 3.6. Effect of land width on the 6-pocket, $80 \mathrm{~mm}$ diameter FC Bearing \#1 of Table 3.1.

The effect of the number of pockets on the performance of the bearing of Table 3.1 is shown in Figure 3.7. All three land widths, $\mathrm{L}_{\mathrm{lp}}, \mathrm{L}_{\mathrm{lep}}$, and $\mathrm{L}_{\mathrm{lmp}}$, were kept constant at $3 \mathrm{~mm}$ as the number of pockets was changed. The width of the drainage grooves, $L_{d}$, were held constant at $2 \mathrm{~mm}$. As shown, the initial specific stiffness was increased somewhat with more pockets until approximately 6 pockets; the addition of pockets allows the bearing to more effectively utilize the the available area. For more than 8 pockets, however, the pocket area was reduced significantly with the addition of more 
pockets and more drainage grooves. The number of pockets had a lesser effect on the load-carrying efficiency of the bearing. The specific flow rate increased steadily with more pockets as the drainage flow area was increased.

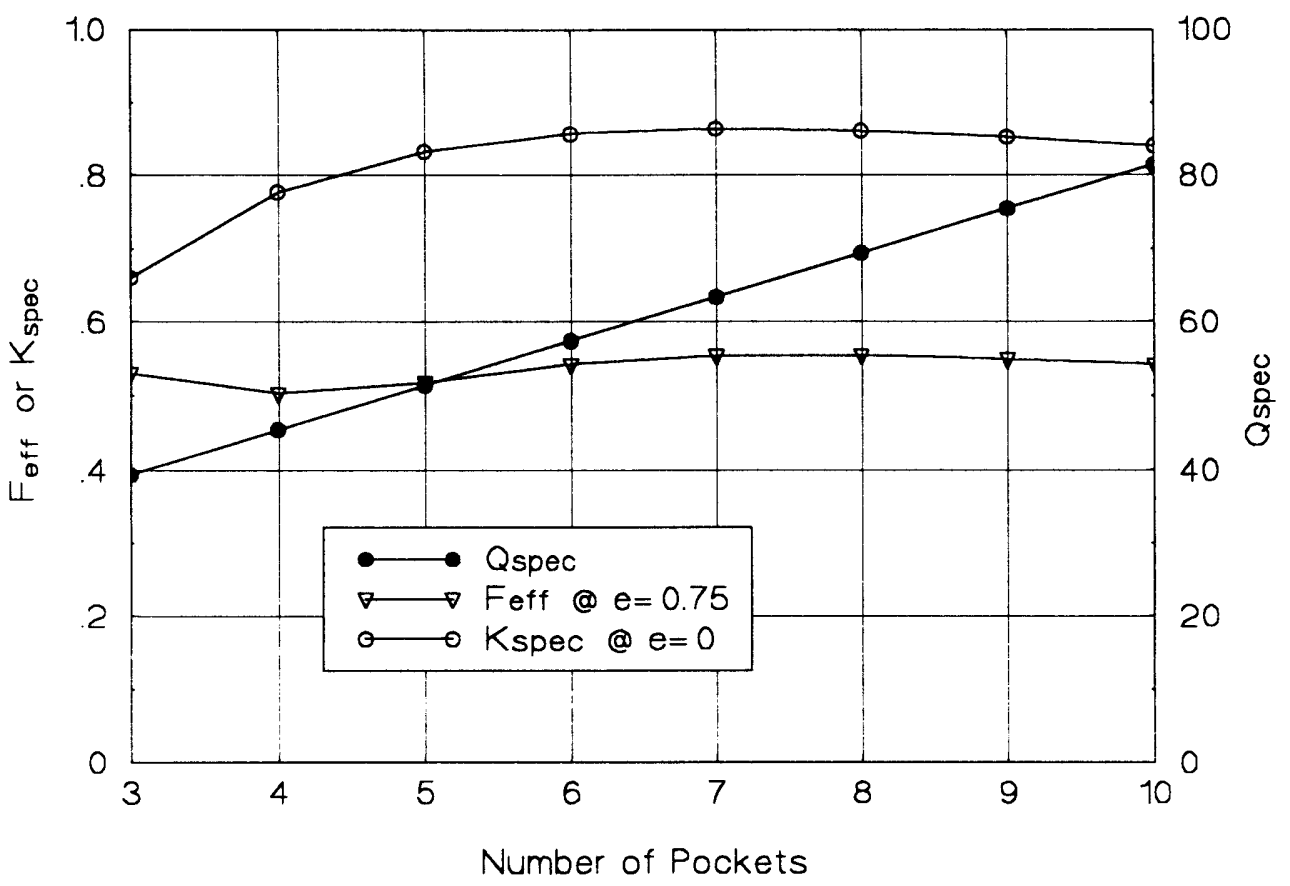

Figure 3.7. Effect of the number of pockets on the $80 \mathrm{~mm}$ diameter FC Bearing \#1 of Table 3.1. The width of each land was $3 \mathrm{~mm}$ and the width of each drainage groove was 2 $\mathrm{mm}$.

\section{B. Fixed-Compensated Bearing \#2}

The pad layout of a second conventional, fixed-compensated hydrostatic bearing is shown in Figure 3.8. Fluid enters each pocket through a fixed-resistance device such as a capillary tube. It then flows throughout the pockets, represented by shaded regions, which are at a depth significantly greater than the land clearances such that the fluid pressure 
throughout each pocket is uniform. The fluid then crosses the circumferential lands and out of the bearing to atmospheric pressure. The axial lands that run between the pockets serve to isolate the pocket pressures and enable a pressure difference to exist between them.

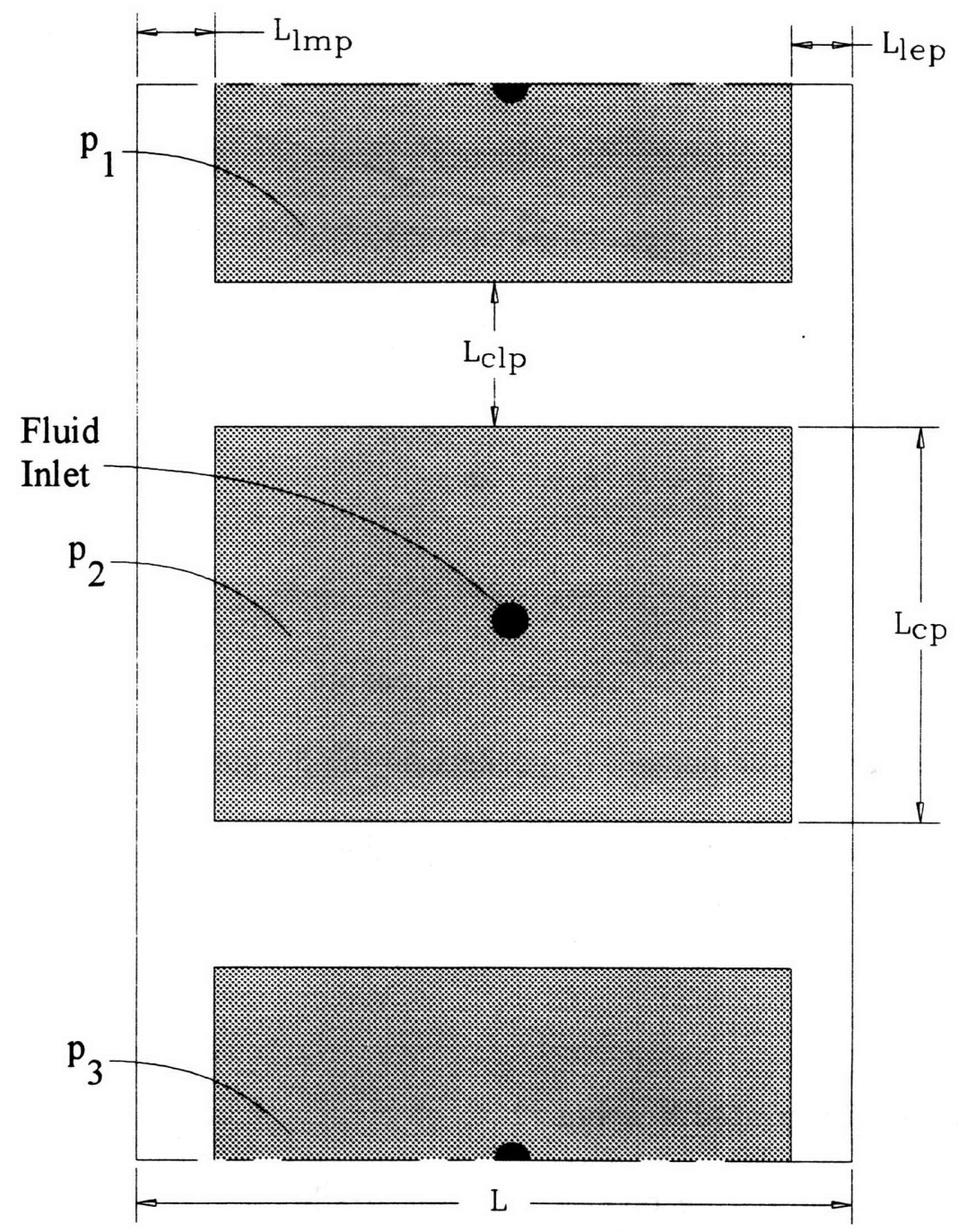

Figure 3.8. Schematic diagram of the pad layout of a conventional, fixed-compensated hydrostatic bearing without drainage grooves between its pockets. The shaded regions represent depressed regions. 


\section{B.i. Description of Calculations for Fixed-Compensated Bearing \#2}

Calculating the load-carrying capacity of a bearing is accomplished in the same manner as the previous bearing; the shaft is moved to an eccentric position and the resulting restoring force generated by the bearing is computed. The first step in calculating the restoring force is to calculate the hydraulic resistance of each bearing land. There are two pocket lands over which fluid flows in parallel: the circumferential land with a width $\mathrm{L}_{\text {lep, }}$, and the circumferential land with a width $\mathrm{L}_{\mathrm{lmp}}$. The resistance of the two circumferential lands are given by:

$$
\begin{aligned}
& \mathbf{R}_{1, i}=\mathbf{R}_{\text {circum }}\left(\mu, h_{o}, L_{\text {lmp }}, L_{c p}+L_{m}^{*}, \theta_{i}, e_{\text {circum }}\left(e_{m}, e_{1}, \theta_{i}\right)\right) \\
& \mathbf{R}_{2, i}=\mathbf{R}_{\text {circum }}\left(\mu, h_{o}, L_{\text {lep }}, L_{c p}+L_{e}^{*}, \theta_{i}, e_{\text {circum }}\left(e_{2}, e_{e}, \theta_{i}\right)\right)
\end{aligned}
$$

where $\mathbf{R}_{\text {circum }}$ is the function given by Equation (2.42), $i$ is the pocket index, and $\theta_{i}$ is the angle to center of the ith pocket:

$$
\theta_{\mathrm{i}}=2 \pi(\mathrm{i}-1) / \mathrm{N} \quad \mathrm{i}=1,2 \cdots, \mathrm{N}
$$

where $\mathrm{N}$ is the number of pockets. Note that the eccentricities $\mathrm{e}_{\mathrm{m}}$ and $\mathrm{e}_{\mathrm{e}}$ are the same bearing end eccentricities as those of FC Bearing $\# 1$, as illustrated in Figure 3.3. The eccentricities $e_{1}$ and $e_{2}$ are those cooresponding to the ends of the axial lands:

$$
\begin{aligned}
& e_{1}=e_{m}+\left(e_{e}-e_{m}\right) \frac{L_{l m p}}{L} \\
& e_{2}=e_{e}+\left(e_{m}-e_{e}\right) \frac{L_{\text {lep }}}{L}
\end{aligned}
$$


The variables $\mathrm{L}_{\mathrm{m}}{ }^{*}$ and $\mathrm{Le}_{\mathrm{e}}{ }^{*}$ are added to the distance perpendicular to the direction of flow to account for the small amount of spreading the fluid experiences near the two ends of the axial land. A recommended relation for these terms are

$$
\begin{aligned}
& \mathrm{L}_{\mathrm{m}}^{*} \approx 0.8\left\{\min \left(\mathrm{L}_{\mathrm{lmp}}, \mathrm{L}_{\mathrm{clp}}\right)\right\} \\
& \mathrm{L}_{\mathrm{e}}^{*} \approx 0.8\left\{\min \left(\mathrm{L}_{\text {lep }}, \mathrm{L}_{\mathrm{clp}}\right)\right\}
\end{aligned}
$$

As long as the land widths are small compared to the pocket axial length, the effect of the fluid spreading is small and the exact magnitudes of these terms do not significantly affect the predicted bearing performance.

The next step is to calculate the equivalent hydraulic resistance of each pocket corresponding to the resistances $R_{a, i}$ of Circuit $\# 2$ in Appendix B:

$$
\mathbf{R}_{\mathrm{a}, \mathrm{i}}=\frac{1}{\frac{1}{\mathbf{R}_{1, \mathrm{i}}}+\frac{1}{\mathbf{R}_{2, \mathrm{i}}}}
$$

The resistances $R_{c, i}$ of Circuit \#2 correspond to the inlet resistances used to compensate the bearing. The value of these fixed resistances are chosen so that the resistance ratio $\gamma=$ $R_{c, i} / R_{a, i}$ is approximately equal to 1 when the shaft is concentric to the bearing housing.

The remaining resistances of Circuit $\# 2$ are the leakage resistances, $R_{b, i}$. The resistance of each axial land is given by:

$$
R_{b, i}=R_{\text {axial }}\left(\mu, h_{o}, L_{c p}, L-L_{l m p}-L_{l e p}+L_{m}^{*}+L_{e}^{*}, \theta_{i}+\frac{L_{c p}+L_{c p p}}{D}, e_{\text {axiel }}\left(e_{1}, e_{2}, \theta_{i}+\frac{L_{c p}+L_{c p p}}{D}\right)\right)
$$

where $R_{\text {axial }}$ is the function given by Equation (2.31), and $e_{\text {axial }}$ is the function given by Equation (2.38). 
Once the resistances of Circuit \#1 have been calculated, the fluid circuit can be solved for the $\mathrm{N}$ unknown pocket pressures, $\mathrm{p}_{\mathrm{i}}$, and the supply volumetric flow rate, $\mathrm{Q}_{s}$. Once the pocket pressures are known, the reaction force of the bearing is calculated by multiplying the pressures times the appropriate areas. The net force generated by pocket $\mathrm{i}$ in the $y$-direction is given by:

$$
\mathrm{F}_{\mathrm{y}, \mathrm{i}}^{1}=\mathrm{D}\left(\mathrm{L}-\mathrm{L}_{\text {lmp }}-\mathrm{L}_{\text {lep }}\right) \sin \left(\frac{\mathrm{L}_{\mathrm{cp}}}{\mathrm{D}}\right) \mathrm{p}_{\mathrm{i}} \cos \left(\theta_{\mathrm{i}}\right)
$$

Similarly, the $y$-direction forces generated by the other bearing areas are given by:

$$
\begin{gathered}
\mathrm{F}_{\mathrm{y}, \mathrm{i}}^{2}=\mathrm{DL}_{\mathrm{lmp}} \sin \left(\frac{\mathrm{L}_{\mathrm{cp}}}{\mathrm{D}}\right)\left(\frac{\mathrm{p}_{\mathrm{i}}}{2}\right) \cos \left(\theta_{\mathrm{i}}\right) \\
\mathrm{F}_{\mathrm{y}, \mathrm{i}}^{3}=\mathrm{DL}_{\text {lep }} \sin \left(\frac{\mathrm{L}_{\mathrm{cp}}}{\mathrm{D}}\right)\left(\frac{\mathrm{p}_{\mathrm{i}}}{2}\right) \cos \left(\theta_{\mathrm{i}}\right) \\
\mathrm{F}_{\mathrm{y}, \mathrm{i}}^{4}=\mathrm{DL}_{\mathrm{lmp}} \sin \left(\frac{\mathrm{L}_{\mathrm{clp}}}{\mathrm{D}}\right)\left(\frac{\mathrm{p}_{\mathrm{i}}+\mathrm{p}_{\mathrm{i}+1}}{4}\right) \cos \left(\theta_{\mathrm{i}}+\frac{\mathrm{L}_{\mathrm{cp}}+\mathrm{L}_{\mathrm{clp}}}{\mathrm{D}}\right) \\
\mathrm{F}_{\mathrm{y}, \mathrm{i}}^{5}=\mathrm{DL}_{\mathrm{lep}} \sin \left(\frac{\mathrm{L}_{\mathrm{clp}}}{\mathrm{D}}\right)\left(\frac{\mathrm{p}_{\mathrm{i}}+\mathrm{p}_{\mathrm{i}+1}}{4}\right) \cos \left(\theta_{\mathrm{i}}+\frac{\mathrm{L}_{\mathrm{cp}}+\mathrm{L}_{\mathrm{clp}}}{\mathrm{D}}\right) \\
\mathrm{F}_{\mathrm{y}, \mathrm{i}}^{6}=\mathrm{F}_{\mathrm{axial}}\left(\mathrm{D}, \mathrm{L}-\mathrm{L}_{\text {lep }}-\mathrm{L}_{\mathrm{lmp}}, \mathrm{L}_{\mathrm{dp}}, \mathrm{p}_{\mathrm{i}}, \mathrm{p}_{\mathrm{i}+1}, \mathrm{e}_{\mathrm{axial}}\left(\mathrm{e}_{1}, \mathrm{e}_{2}, \theta_{\mathrm{i}}+\frac{\mathrm{L}_{\mathrm{cp}}+\mathrm{L}_{\mathrm{lp}}}{\mathrm{D}}\right)\right) \cos \left(\theta_{\mathrm{i}}+\frac{\mathrm{L}_{\mathrm{cp}}+\mathrm{L}_{\mathrm{lp}}}{\mathrm{D}}\right)
\end{gathered}
$$

where $F_{\text {axial }}$ is the function given by Equation (2.35). The total $y$-direction bearing force is calculated by summing the $\mathrm{y}$-direction forces for all of the $\mathrm{N}$ pockets.

In addition to the calculating the bearing load-carrying capability, the effective load center of the bearing is needed to calculate the stiffness of the spindle. The effective center of the bearing is found by summing the moments about the end of the bearing and 
dividing the bearing moment by the bearing force. The bearing moment is computed by multiplying the forces of Equations (3.41) through (3.46) by the axial center of each area:

$$
\begin{array}{r}
M_{y, i}^{1}=\left(L_{\text {lep }}+\frac{L-L_{l m p}-L_{l e p}}{2}\right) F_{y, i}^{1} \\
M_{y, i}^{2}=\left(L-L_{l m p} / 2\right) F_{y, i}^{2} \\
M_{y, i}^{3}=\left(L_{l e p} / 2\right) F_{y, i}^{3} \\
M_{y, i}^{4}=\left(L-L_{l m p} / 2\right) F_{y, i}^{4} \\
M_{y, i}^{5}=\left(L-L_{l m p} / 2\right) F_{y, i}^{5} \\
M_{y, i}^{6}=\left(L_{l e p}+\frac{L-L_{l m p}-L_{l e p}}{2}\right) F_{y, i}^{6}
\end{array}
$$

The total $y$-direction bearing moment is calculated by summing the $y$-direction moments for all of the $\mathrm{N}$ pockets. The effective bearing load center is then computed using Equation (3.31).

\section{B.ii. Representative Calculations for Fixed-Compensated Bearing \#2}

Table 3.2 shows representative calculation results for a $80 \mathrm{~mm}$ diameter, $80 \mathrm{~mm}$ long bearing. The circumferential land widths are $3 \mathrm{~mm}$. The resistance ratio is 1.1 . There are 6 pockets. The axial land width is 0.58 times $\pi \mathrm{D} / \mathrm{N}$, which is $24.3 \mathrm{~mm}$. This land width was found to be result in approximately the maximum specific stiffness for this bearing. As shown, the initial specific stiffness is 0.915 , which is somewhat higher than that of Fixed-Compensated Bearing \#1. The load-carrying efficiency at $75 \%$ of gap closure is 0.562 , which is also higher than that of FC Bearing \#1. Moreover, the specific 
flow rate is 12.1 , nearly 5 times less than that of FC Bearing \#1. Clearly this bearing is superior to that of FC Bearing \#1 since the load-carrying capacity is higher and the pumping power is lower. However, FC Bearing \#1 might be more desirable than FC Bearing \#2 if a higher flow rate is desired; high-speed applications require more flow to maintain low bearing temperature rise.

\section{Table 3.2}

\begin{tabular}{|c|c|c|c|c|}
\hline $\begin{array}{l}\text { Operating Conditions } \\
\text { Supply Pressure (Pa,psi,atm) } \\
\text { Eccentricity Ratio em/ee } \\
\text { Eccentricity at End } \\
\text { Eccentricity at Middle End }\end{array}$ & $\begin{array}{l}\text { Ps } \\
\text { lamda } \\
\text { ee } \\
\text { em }\end{array}$ & $\begin{array}{r}4.17 E+06 \\
1 \\
0.01 \\
0.01\end{array}$ & 600 & 40.8 \\
\hline \multirow{2}{*}{\multicolumn{5}{|c|}{$\begin{array}{l}\text { Fiuid Properties } \\
\text { Dynamic Viscosity ( } \mathrm{s} / \mathrm{m})\end{array}$}} \\
\hline & & & & \\
\hline Diameter $(\mathrm{m}, \mathrm{mm}, \mathrm{in})$ & D & 0.08 & 80 & 3.15 \\
\hline Axial Length $(\mathrm{m}, \mathrm{mm}$,in) & $\mathrm{L}$ & 0.08 & 80 & 3.15 \\
\hline Number of Pockets & $\mathbf{N}$ & 6 & & \\
\hline Nominal Gap $(m, \mu m, i n)$ & ho & 0.000015 & 15 & 0.00059 \\
\hline Nominal Resistance Ratio & gamma & 1.1 & & \\
\hline Axial Land Width (m, mm,in) & Lclp & 0.024 & 24.3 & 0.956 \\
\hline Pocket End Land Width (m, mm, in) & Llmp & 0.003 & & 0.118 \\
\hline Pocket Middle Land Width (m, mm,in) & Llep & 0.003 & & 0.118 \\
\hline Pocket Circumferential Length ( $m, m m$, in) & Lcp & 0.01759 & 17.6 & 0.693 \\
\hline \multicolumn{5}{|l|}{ Bearing Stiffness and Load Capacity } \\
\hline $\begin{array}{l}\text { Location of Effective Bearing Force } \\
\text { Pressure Difference / Pressure @ ec,ep above }\end{array}$ & $\begin{array}{l}\text { Lcen_L } \\
\text { DPP }\end{array}$ & $\begin{array}{l}0.500 \\
0.013\end{array}$ & & \\
\hline Load Capacity (N,kN,lbf) @ ec,ep given above & Fy & 244 & 0.24 & 55 \\
\hline Load Carrying Efficiency @ ec,ep given above & Feff & 0.009 & & \\
\hline Stiffness $\left(N / \mu \mathrm{m}, \mathrm{lb} \mathrm{b}_{f} / \mu \mathrm{in}\right) @$ ec,ep given above & Ky & 1626 & 9.287 & \\
\hline Specific Stiffness @ ec,ep given above & Kspec & 0.915 & & \\
\hline Initial Specific Stiffness & Kspec,o & 0.915 & & \\
\hline Load Carrying Efficiency @ 75\% of gap closure & Feff, 0.75 & 0.562 & & \\
\hline $\begin{array}{l}\text { Flow Rate and Pumping Power } \\
\text { Total Flow Rate (m^3/s,lpm,gpm) } \\
\text { Pumping Power (W,kW,hp) } \\
\text { Specific Flow Rate }\end{array}$ & $\begin{array}{l}\text { Qsupply } \\
\text { Ppump } \\
\text { Qspec }\end{array}$ & $\begin{array}{r}3.43 E-05 \\
143 \\
12.1\end{array}$ & $\begin{array}{r}2.06 \\
0.143\end{array}$ & $\begin{array}{r}0.54 \\
0.192\end{array}$ \\
\hline $\begin{array}{l}\text { Ratio of Specific Initial Stiffness to Specific } \\
\text { Flow Rate }\end{array}$ & K/Ospec & 0.0755 & & \\
\hline
\end{tabular}


The effect of changing the width of the axial land is shown in Figure 3.9. The circumferential land widths, $\mathrm{L}_{\text {lep }}$ and $\mathrm{L}_{\mathrm{lmp}}$, were held constant at $3 \mathrm{~mm}$. The resistance ratio was also held constant at 1.1. As shown, the initial specific stiffness increases with increasing axial land width because it reduces the circumferential leakage between the pockets, thereby enabling a greater pressure difference to exist from one pocket to the next. However, at a land width of about $20 \mathrm{~mm}$, the initial specific stiffness levels off and begins to decline. An important parameter that is qualitatively useful to consider to gain an understanding of this effect is the ratio of the circumferential leakage rate between the pockets to the axial flow rate out of the bearing; increasing the axial land width reduces this parameter until the bearing supply flow rate becomes too low and the parameter begins to rise again, thereby causing the behavior shown in Figure 3.9. The load-carrying efficiency at $75 \%$ of gap closure shown in Figure 3.9 exhibits a similar behavior. The specific flow rate is clearly reduced as the axial land width is increased. A land width of $25 \mathrm{~mm}$ is recommended in this case to achieve near-optimal stiffness and load capacity with a low specific flow rate. 


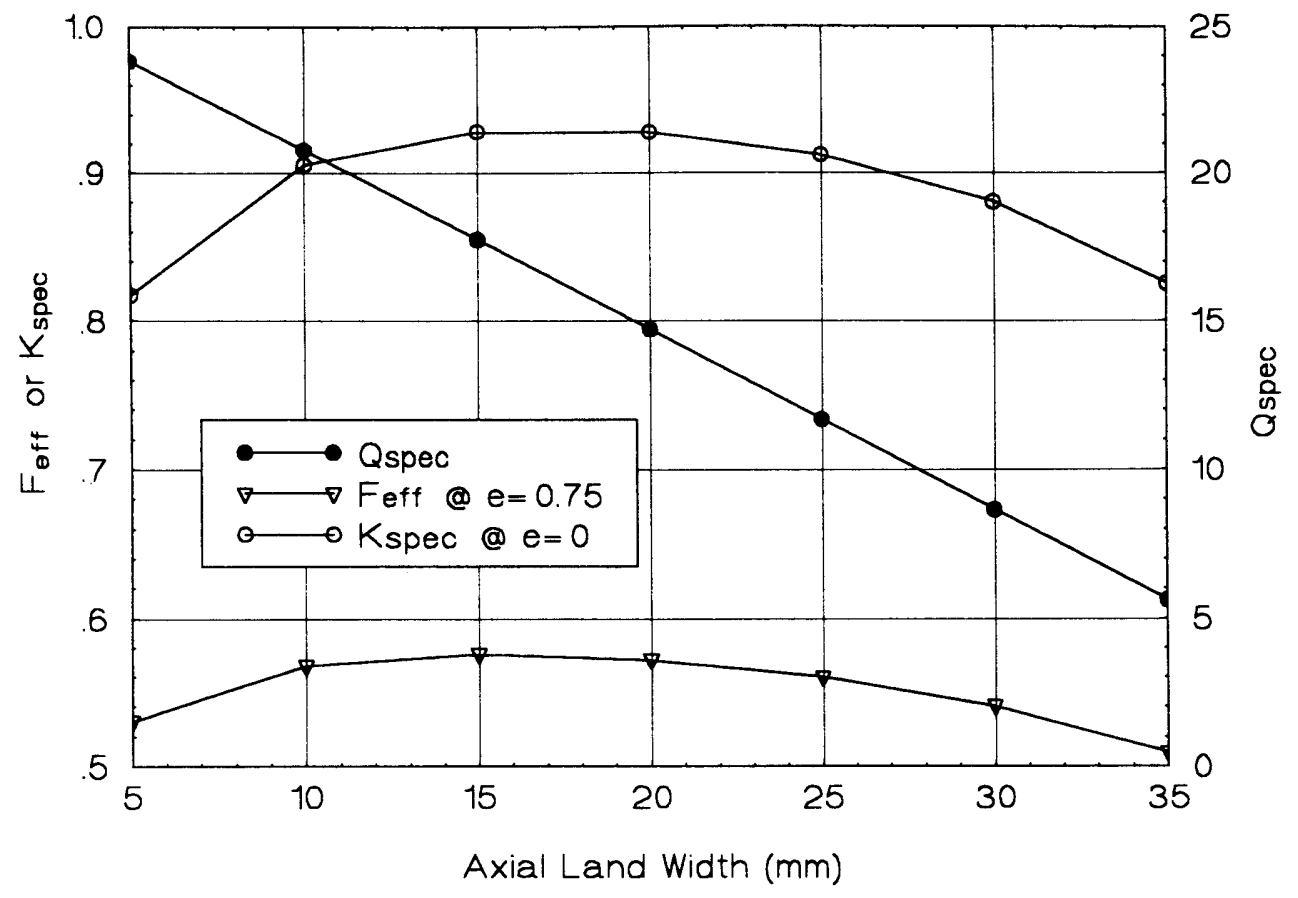

Figure 3.9. Effect of the axial land width on the 6-pocket, $80 \mathrm{~mm}$ diameter FC Bearing \#2 of Table 3.2 .

The effect of increasing the number of pockets of the bearing of Table 3.2 is shown in Figure 3.10. The axial land width was held at a constant percentage of the available length, 0.58 times $\pi \mathrm{D} / \mathrm{N}$. The specific flow rate therefore remained constant. As shown, increasing the number of pockets increases both the initial specific stiffness and the load-carrying efficiency at $75 \%$ of gap closure because the pressure is more effectively spread over the available bearing area. Recall that the stiffness of the Fixed-Compensated Bearing \#1 declined with the addition of too many pockets because the drain area reduced the available bearing support area. FC Bearing \#2, however, does not suffer from that problem, and the stiffness increases asymptotically to an upper-bound value. Note that 6 to 8 pockets are sufficient to obtain the near-optimal bearing performance. 


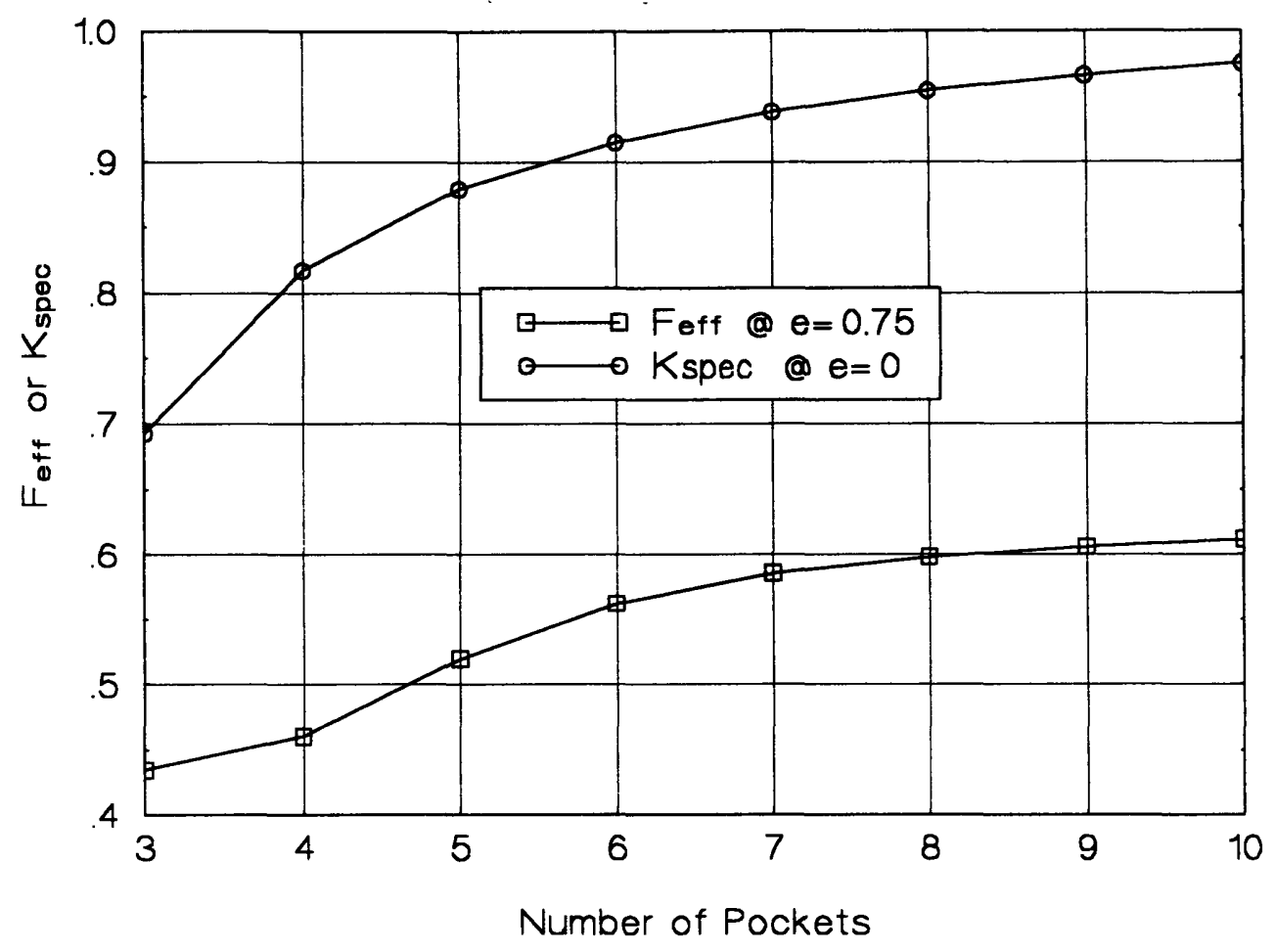

Figure 3.10. Effect of the number of pockets on the load-carrying performance of the 80 mm diameter FC Bearing \#2 of Table 3.2 .

\section{C. Self-Compensated Bearing \#1}

The pad layout of a self-compensated hydrostatic bearing is shown in Figure 3.11. The shaded regions are at a depth significantly greater than the land clearances such that the fluid pressure throughout each area is uniform. This bearing, unlike the previous two bearings considered, does not require any capillaries or orifices for compensation. Instead, small pads on the surface of the bearing are used as the compensation resistances[4]. Fluid enters the fluid supply area, denoted in Figure 3.11 with $\mathbf{P}_{\mathbf{s}}$, through a hole located somewhere in that region. To the right of the supply pressure region is a 
leakage land, of width $\mathrm{L}_{\text {leak}}$, that prevents excessive leakage flow from the supply pressure region to the drain groove which is at atmospheric pressure. Not shown to the left of the bearing is another leakage land which prevents excessive flow from the supply pressure to the exterior of the bearing; alternatively, a thrust bearing or a second radial bearing may be located to the left of this bearing and supplied from the same high-pressure region. After entering the supply pressure region, the fluid flows over the lands of the small compensation pads to a fluid collector groove in the center of the compensators. The fluid is then routed to the opposite side of the bearing to a bearing pocket. The fluid flows throughout the pockets until it crosses the lands and out of the bearing to atmospheric pressure. The axial grooves that run between the pockets serve to isolate the pocket pressures and enable a pressure difference to exist between them. Note that the lands that run circumferentially on either end of the bearing pockets are modeled as having different widths, $\mathrm{L}_{\mathrm{mpp}}$ and $\mathrm{L}_{\text {lep. }}$. This allows the circumferential land nearest the front of the spindle to be made with a somewhat shorter width than the other bearing lands so that the bearing has higher sensitivity to deflections at the front of the spindle, thereby increasing the spindle stiffness. This will be discussed further in Chapter 4. 


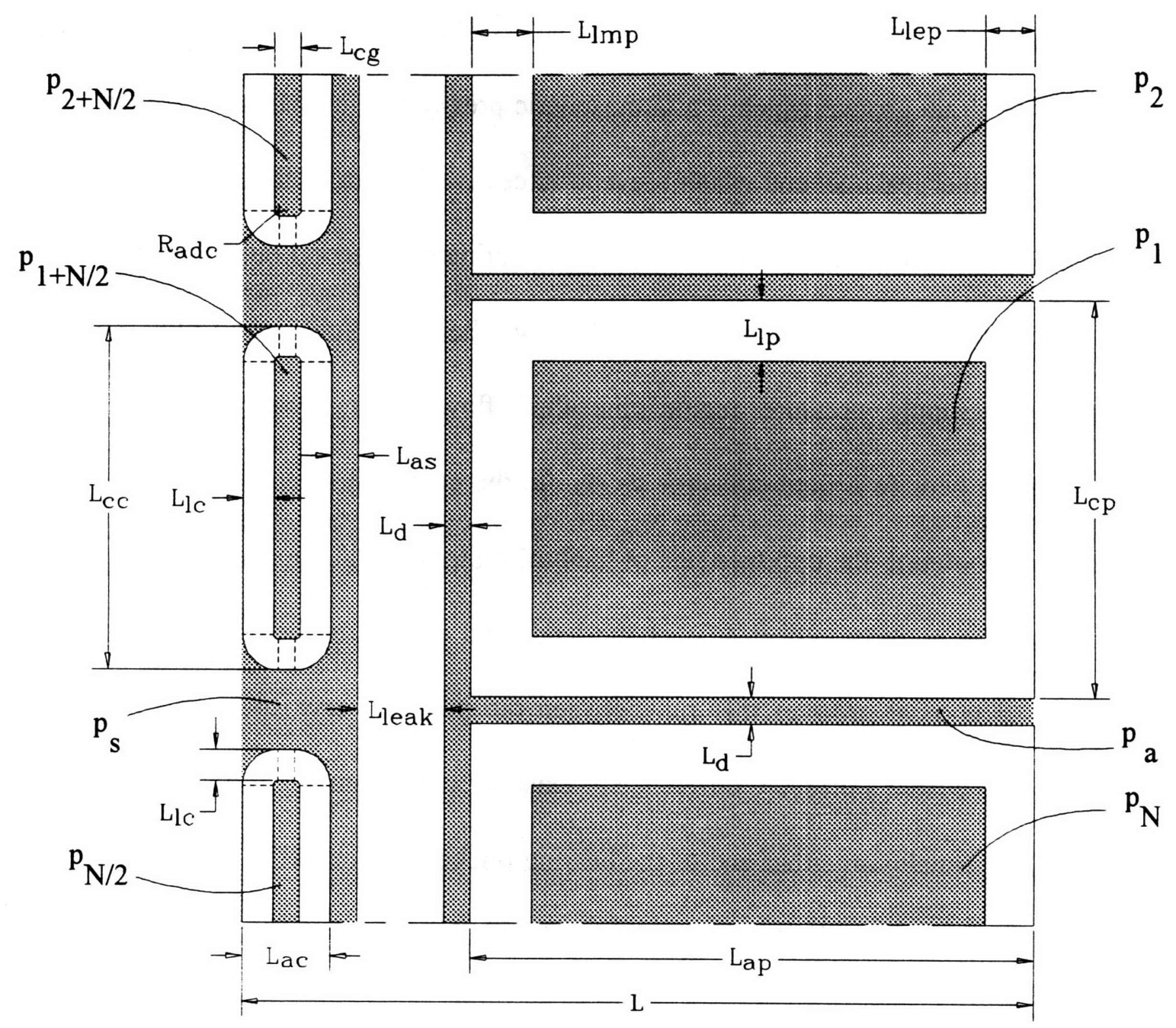

Figure 3.11. Schematic diagram of the pad layout of a self-compensated hydrostatic bearing with drainage grooves between its pockets and a drainage groove between its supply area and its pockets. The shaded regions represent depressed regions.

A schematic diagram of two of the bearing pad/compensator pairs is shown in Figure 3.12 to illustrate how the fluid is routed from the compensators to the supporting pockets. The land geometry may either be located on the inner diameter of a bearing housing, or it may be located on the outer diameter of a shaft located within a housing that has a straight bore and a hole drilled through it leading to the fluid supply region. 


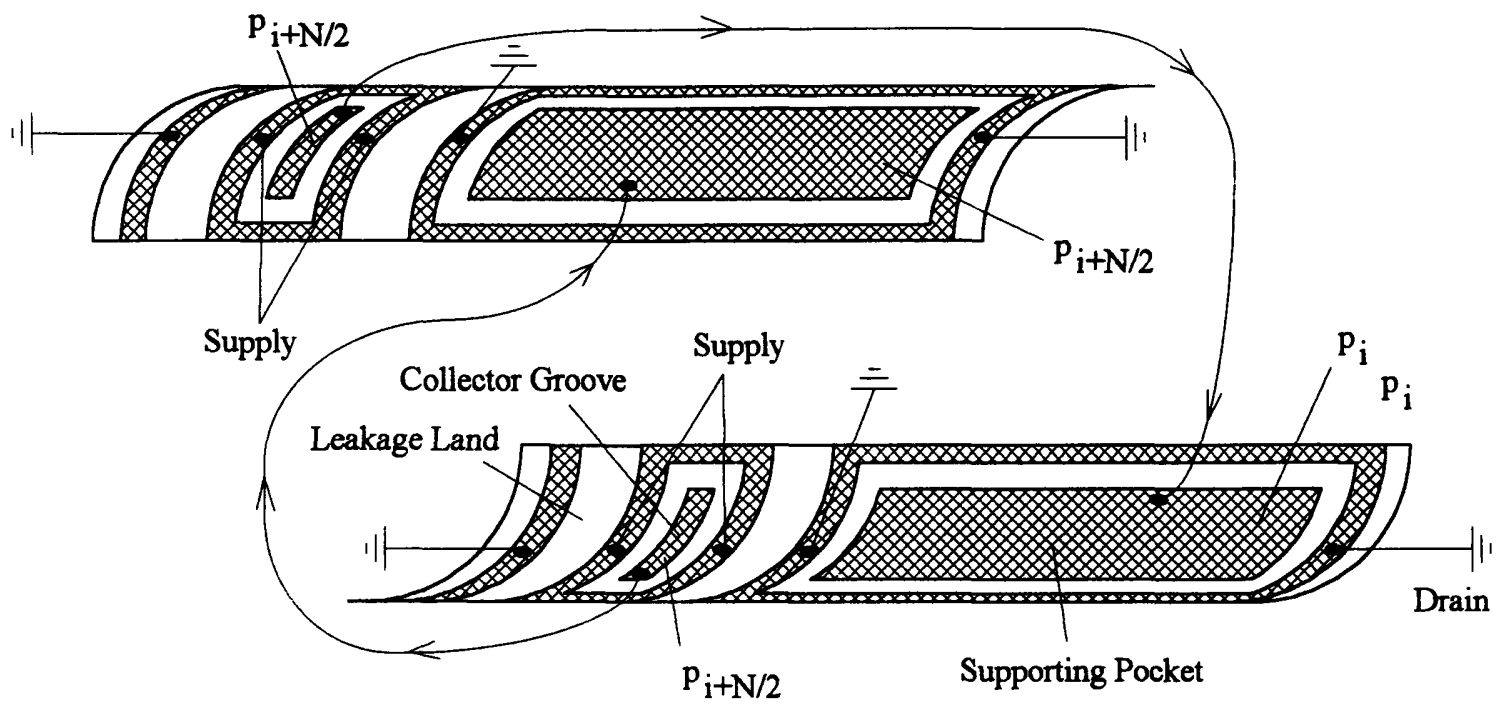

Figure 3.12. Schematic diagram of two pad/compensator pairs of Self-Compensated Bearing \#1.. The hatched regions represent depressed regions.

\section{C.i. Description of Calculations for Bearing \#1}

Calculating the load-carrying capacity of SC Bearing \#1 is the same as that of the previous bearings, except the resistance of the compensators must also be modeled. In the developments that follow, the eccentricity at the edge of the bearing compensators is denoted by $e_{c}$ and the eccentricity at the edge of the furthest pocket lands is denoted by $e_{p}$. The bearing clearance is assumed to vary linearly between these two extreme locations of the bearing. The angular position of the pockets are again denoted by $\theta_{i}$; the first pocket, corresponding to $\theta_{1}=0$, occurs at the location of smallest clearance. The compensators are out of phase with the pockets by $N / 2$; the fluid that enters pocket $i$ is fed from compensator $\mathrm{i}+\mathrm{N} / 2$.

The hydraulic resistances of the supporting-pocket lands will be considered first. There are four pocket lands over which fluid flows in parallel: the circumferential land 
with a width $L_{\text {lep }}$, the circumferential land with a width $L_{\text {lmp }}$, and the two axial lands with widths of $\mathrm{L}_{\mathrm{lp}}$. The resistance of the two axial lands are given by:

$$
\begin{aligned}
& R_{a 1, i}=R_{a x i a l}\left(\mu, h_{o}, L_{\phi}, L_{a p}-L_{b m p}-L_{k p}+L_{m}^{*}+L_{e}^{*}, \theta_{i}+\frac{L_{c p}-L_{\varphi}}{D}, e_{\text {axial }}\left(e_{1}, e_{2}, \theta_{i}+\frac{L_{c p}-L_{p}}{D}\right)\right) \\
& R_{a 2, i}=R_{\text {axial }}\left(\mu, h_{o}, L_{\phi}, L_{a p}-L_{m p}-L_{k p}++L_{m}^{*}+L_{e}^{*}, \theta_{i}-\frac{L_{c p}-L_{\phi}}{D}, e_{a x i a l}\left(e_{1}, e_{2}, \theta_{i}-\frac{L_{c p}-L_{\varphi}}{D}\right)\right)
\end{aligned}
$$

where $\mathrm{i}$ is the pocket index, $\mathbf{R}_{\mathrm{axial}}$ is the function given by Equation (2.31), $\mathbf{e}_{\mathrm{axial}}$ is the function given by Equation (2.38), and $\theta_{\mathrm{i}}$ is the angle to center of the ith pocket:

$$
\theta_{\mathrm{i}}=2 \pi(\mathrm{i}-1) / \mathrm{N} \quad \mathrm{i}=1,2 \cdots, \mathrm{N}
$$

where $\mathrm{N}$ is the number of pockets. The eccentricities $e_{1}$ and $e_{2}$ are those cooresponding to the ends of the axial lands:

$$
\begin{aligned}
& e_{1}=e_{p}+\left(e_{c}-e_{p}\right) \frac{L_{a p}-L_{\text {lmp }}}{L} \\
& e_{2}=e_{p}+\left(e_{c}-e_{p}\right) \frac{L_{\text {lep }}}{L}
\end{aligned}
$$

The variables $L_{m}{ }^{*}$ and $L_{e}{ }^{*}$ are added to the distance perpendicular to the direction of flow to account for the small amount of spreading the fluid experiences near the two ends of the axial land. A recommended relation for these terms are

$$
\begin{aligned}
& \mathrm{L}_{\mathrm{m}}^{*} \approx 0.4\left\{\min \left(\mathrm{L}_{\mathrm{lmp}}, \mathrm{L}_{\mathrm{lp}}\right)\right\} \\
& \mathrm{L}_{\mathrm{e}}^{*} \approx 0.4\left\{\min \left(\mathrm{L}_{\text {lep }}, \mathrm{L}_{\mathrm{lp}}\right)\right\}
\end{aligned}
$$

As long as the land widths are small compared to the pocket axial length, the effect of the fluid spreading is small and the exact magnitudes of these terms do not significantly affect 
the predicted bearing performance. The resistance of the two circumferential lands are given by:

$$
\begin{aligned}
& R_{a 3, i}=R_{\text {circum }}\left(\mu, h_{o}, L_{l m p}, L_{c p}-2 L_{l p}+L_{m}^{*}, \theta_{i}, e_{\text {circum }}\left(e_{m}, e_{1}, \theta_{i}\right)\right) \\
& R_{a 4, i}=R_{\text {circum }}\left(\mu, h_{o}, L_{l e p}, L_{c p}-2 L_{l p}+L_{e}^{*}, \theta_{i}, e_{\text {circum }}\left(e_{2}, e_{p}, \theta_{i}\right)\right)
\end{aligned}
$$

The eccentricities $e_{m}$ corresponds to the end of the bearing pad:

$$
e_{m}=e_{p}+\left(e_{c}-e_{p}\right) \frac{L_{a p}}{L}
$$

The four resistances given by Equations (3.53), (3.54), (3.60), and (3.61) are evaluated N times for each pocket of the bearing. Once all of the pocket land resistances have been calculated, the equivalent hydraulic resistance of each pocket corresponding to the resistances $R_{a, i}$ of Circuit \#1 in Appendix $B$ are given by:

$$
R_{a, i}=\frac{1}{\frac{1}{R_{a 1, i}}+\frac{1}{R_{a 2, i}}+\frac{1}{R_{a 3, i}}+\frac{1}{R_{a 4, i}}}
$$

The resistances $R_{c, i}$ of Circuit \#1 correspond to the inlet resistances used to compensate the bearing. The compensators are designed so that the value of these resistances correspond to a resistance ratio $\gamma=R_{c, i} / R_{a, i}$ that is approximately equal to 1 to 2 when the shaft is concentric to the bearing housing. The resistances of the compensators are calculated similarly to those of the pad resistances. The only difference is the use of the circular land resistances:

$$
\begin{aligned}
& R_{c l, i}=R_{a x i d l}\left(\mu, h_{o}, L_{l c}, L_{a c}-2 L_{l c}-2 R_{a d c}, \theta_{i+N / 2}+\frac{L_{c c}-L_{l c}}{D}, e_{\text {acida }}\left(e_{4}, e_{5}, \theta_{i+N / 2}+\frac{L_{c c}-L_{l c}}{D}\right)\right) \\
& R_{c 2, i}=R_{a x i a l}\left(\mu, h_{o}, L_{l c}, L_{a c}-2 L_{l c}-2 R_{a d c}, \theta_{i+N / 2}-\frac{L_{c c}-L_{l c}}{D}, e_{a x i a l}\left(e_{4}, e_{5}, \theta_{i+N / 2}-\frac{L_{c c}-L_{l c}}{D}\right)\right)
\end{aligned}
$$




$$
\begin{aligned}
& R_{c 3, i}=R_{c i r c u m}\left(\mu, h_{o}, L_{l c}, L_{c c}-2 L_{l c}-2 R_{a d c}, \theta_{i+N / 2}, e_{c i r c u m}\left(e_{c}, e_{3}, \theta_{i+N / 2}\right)\right) \\
& R_{c 4, i}=R_{c i r c u m}\left(\mu, h_{o}, L_{l c}, L_{c c}-2 L_{l c}-2 R_{a d c}, \theta_{i+N / 2}, e_{c i r c u m}\left(e_{6}, e_{7}, \theta_{i+N / 2}\right)\right)
\end{aligned}
$$

Since the circular lands are small compared to the length of the bearing, they may be evaluated using the relation for an untilted land, Equation (2.49), using the mean eccentricities:

$$
\begin{aligned}
& R_{c 5, i}=\frac{24 \mu}{\pi h_{o}^{3}} \ln \left(\frac{L_{l c}+R_{a d c}}{R_{a d c}}\right) \frac{1}{\left(1-e_{3} \cos \left(\theta_{i+N / 2}+\frac{L_{c c}-L_{l c}}{D}\right)\right)^{3}} \\
& R_{c 6, i}=\frac{24 \mu}{\pi h_{o}^{3}} \ln \left(\frac{L_{l c}+R_{a d c}}{R_{a d c}}\right) \frac{1}{\left(1-e_{6} \cos \left(\theta_{i+N / 2}+\frac{L_{c c}-L_{l c}}{D}\right)\right)^{3}} \\
& R_{c 5, i}=\frac{24 \mu}{\pi h_{o}^{3}} \ln \left(\frac{L_{l c}+R_{a d c}}{R_{a d c}}\right) \frac{1}{\left(1-e_{3} \cos \left(\theta_{i+N / 2}-\frac{L_{c c}-L_{l c}}{D}\right)\right)^{3}} \\
& R_{c 6, i}=\frac{24 \mu}{\pi h_{o}^{3}} \ln \left(\frac{L_{l c}+R_{a d c}}{R_{a d c}}\right) \frac{1}{\left(1-e_{6} \cos \left(\theta_{i+N / 2}-\frac{L_{c c}-L_{l c}}{D}\right)\right)^{3}}
\end{aligned}
$$

where:

$$
\begin{gathered}
e_{3}=e_{c}+\left(e_{p}-e_{c}\right) \frac{L_{l c}}{L} \\
e_{4}=e_{c}+\left(e_{p}-e_{c}\right) \frac{L_{l c}+R_{a d c}}{L} \\
e_{5}=e_{c}+\left(e_{p}-e_{c}\right) \frac{L_{a c}-L_{l c}-R_{a d c}}{L} \\
e_{6}=e_{c}+\left(e_{p}-e_{c}\right) \frac{L_{a c}-L_{l c}}{L}
\end{gathered}
$$




$$
e_{7}=e_{c}+\left(e_{p}-e_{c}\right) \frac{L_{a c}}{L}
$$

All eight compensation land resistances act in parallel to yield an overall compensation resistance:

$$
R_{c, i}=\frac{1}{\frac{1}{R_{c 1, i}}+\frac{1}{R_{c 2, i}}+\frac{1}{R_{c 3, i}}+\frac{1}{R_{c 4, i}}+\frac{1}{R_{c 5, i}}+\frac{1}{R_{c 6, i}}+\frac{1}{R_{c 7, i}}+\frac{1}{R_{c 8, i}}}
$$

Once the resistances of Circuit \#1 have been calculated, the fluid circuit can be solved for the $\mathrm{N}$ unknown pocket pressures, $\mathrm{p}_{\mathrm{i}}$, and the primary supply flow rate, $\mathrm{Q}_{\mathrm{s} 1}$. In addition to the primary flow rate, the flow rate across the leakage land must be added to get the total flow bearing supply flow rate:

$$
\begin{aligned}
& Q_{s}=Q_{s 1}+\frac{P_{s}}{R_{\text {leakage }}} \\
& R_{\text {leak }}=\frac{12 \mu L_{\text {leak }}}{\pi D h_{o}^{3}} \frac{1}{1+\frac{3}{2} e_{8}^{2}}
\end{aligned}
$$

where $e_{8}$ is the eccentricity of the shaft at the leakage land:

$$
e_{8}=e_{c}+\left(e_{p}-e_{c}\right) \frac{L_{a c}+L_{a s}+L_{l e a k} / 2}{L}
$$

Once the pocket pressures are known, the reaction force of the bearing is calculated by multiplying the pressures times the appropriate areas. The net force generated by pocket $\mathrm{i}$ in the $\mathrm{y}$-direction is given by:

$$
\mathrm{F}_{\mathrm{y}, \mathrm{i}}^{1}=\mathrm{D}\left(\mathrm{L}_{\mathrm{ap}}-\mathrm{L}_{\mathrm{lmp}}-\mathrm{L}_{\mathrm{lep}}\right) \sin \left(\frac{\mathrm{L}_{\mathrm{cp}}-2 \mathrm{~L}_{\mathrm{lp}}}{\mathrm{D}}\right) \mathrm{p}_{\mathrm{i}} \cos \left(\theta_{\mathrm{i}}\right)
$$

Similarly, the y-direction forces generated by the other bearing pad areas are given by: 


$$
\begin{aligned}
& F_{y, i}^{2}=D L_{l m p} \sin \left(\frac{L_{c p}-2 L_{l p}}{D}\right)\left(\frac{p_{i}}{2}\right) \cos \left(\theta_{i}\right) \\
& F_{y, 1}^{3}=D L_{\text {lep }} \sin \left(\frac{L_{c p}-2 L_{\text {lp }}}{D}\right)\left(\frac{p_{i}}{2}\right) \cos \left(\theta_{i}\right) \\
& \mathrm{F}_{\mathrm{y}, \mathrm{i}}^{4}=\mathrm{DL}_{\mathrm{lmp}} \sin \left(\frac{\mathrm{L}_{\mathrm{lp}}}{\mathrm{D}}\right)\left(\frac{\mathrm{p}_{\mathrm{i}}}{4}\right) \cos \left(\theta_{\mathrm{i}}+\frac{\mathrm{L}_{\mathrm{cp}}-\mathrm{L}_{\mathrm{lp}}}{\mathrm{D}}\right) \\
& \mathrm{F}_{\mathrm{y}, \mathrm{i}}^{\mathrm{s}}=\mathrm{DL}_{\mathrm{lmp}} \sin \left(\frac{\mathrm{L}_{\mathrm{lp}}}{\mathrm{D}}\right)\left(\frac{\mathrm{p}_{\mathrm{i}}}{4}\right) \cos \left(\theta_{\mathrm{i}}-\frac{\mathrm{L}_{\mathrm{cp}}-\mathrm{L}_{\mathrm{lp}}}{\mathrm{D}}\right) \\
& \mathrm{F}_{\mathrm{y}, \mathrm{i}}^{6}=\mathrm{DL}_{\mathrm{lep}} \sin \left(\frac{\mathrm{L}_{\mathrm{lp}}}{\mathrm{D}}\right)\left(\frac{\mathrm{p}_{\mathrm{i}}}{4}\right) \cos \left(\theta_{\mathrm{i}}+\frac{\mathrm{L}_{\mathrm{cp}}-\mathrm{L}_{\mathrm{lp}}}{\mathrm{D}}\right) \\
& \mathrm{F}_{\mathrm{y}, \mathrm{i}}^{7}=\mathrm{DL}_{\mathrm{lep}} \sin \left(\frac{\mathrm{L}_{\mathrm{lp}}}{\mathrm{D}}\right)\left(\frac{\mathrm{p}_{\mathrm{i}}}{4}\right) \cos \left(\theta_{\mathrm{i}}-\frac{\mathrm{L}_{\mathrm{cp}}-\mathrm{L}_{\mathrm{lp}}}{\mathrm{D}}\right) \\
& F_{y, 1}^{8}=F_{\text {axial }}\left(D, L_{\text {ap }}-L_{\text {lep }}-L_{\text {lmp }}, L_{l p}, p_{i}, 0, e_{\text {axial }}\left(e_{1}, e_{2}, \theta_{i}+\frac{L_{\text {cp }}-L_{l p}}{D}\right)\right) \cos \left(\theta_{i}+\frac{L_{c p}-L_{l p}}{D}\right) \\
& F_{y, 1}^{9}=F_{\text {axial }}\left(D, L_{\text {ap }}-L_{\text {lep }}-L_{\text {lmp }}, L_{\text {lp }}, 0, p_{i}, e_{\text {axial }}\left(e_{1}, e_{2}, \theta_{i}-\frac{L_{\text {cp }}-L_{\text {lp }}}{D}\right)\right) \cos \left(\theta_{i}-\frac{L_{c p}-L_{l p}}{D}\right)
\end{aligned}
$$

where $F_{\text {axial }}$ is the function given by Equation (2.35). Similarly, the forces generated by the compensators are added.

$$
\begin{gathered}
F_{y, i}^{10}=2 D L_{l c} \sin \left(\frac{L_{c c}-2 L_{l c}}{D}\right)\left(\frac{p_{i+N / 2}}{2}\right) \cos \left(\theta_{i}\right) \\
F_{y, i}^{11}=D\left(L_{a c}-2 L_{l c}\right) \sin \left(\frac{L_{c c}-2 L_{l c}}{D}\right) p_{i+N / 2} \cos \left(\theta_{i}\right) \\
F_{y, i}^{12}=F_{a x i a l}\left(D, L_{a c}-L_{l e p}-2 L_{l c}, L_{l c}, p_{i+N / 2}, 0, e_{a x i a l}\left(e_{4}, e_{5}, \theta_{i}+\frac{L_{c c}-L_{l c}}{D}\right)\right) \cos \left(\theta_{i}+\frac{L_{c c}-L_{l c}}{D}\right)
\end{gathered}
$$




$$
\begin{aligned}
& F_{y, i}^{13}=F_{a x i a l}\left(D, L_{a c}-L_{l e p}-2 L_{l c}, L_{l c}, p_{i+N / 2}, 0, e_{a x i d e}\left(e_{4}, e_{s}, \theta_{i}-\frac{L_{c c}-L_{l c}}{D}\right)\right) \cos \left(\theta_{i}-\frac{L_{c c}-L_{l c}}{D}\right) \\
& F_{y, i}^{14}=\left[\frac{\pi}{2}\left\{\left(R_{a d c}+L_{l c}\right)^{2}-R_{a d c}^{2}\right\}-\right. \\
& \frac{\pi R_{a d c}^{2}}{2 \ln \left(\frac{R_{a d c}+L_{k c}}{R_{a d c}}\right)}\left\{\left(\frac{R_{a d c}+L_{k c}}{R_{a d c}}\right)^{2}\left[2 \ln \left(\frac{R_{a d c}+L_{k c}}{R_{a d c}}\right)-1\right]+1\right\} p_{i+N / 2} \cos \left(\theta_{i}+\frac{L_{c c}-L_{k c}}{D}\right) \\
& F_{y, i}^{15}=\left[\frac{\pi}{2}\left\{\left(R_{a d c}+L_{l c}\right)^{2}-R_{a d c}^{2}\right\}-\right. \\
& \frac{\pi R_{a d c}^{2}}{2 \ln \left(\frac{R_{a d c}+L_{k c}}{R_{a d c}}\right)}\left\{\left(\frac{R_{a d c}+L_{k c}}{R_{a d c}}\right)^{2}\left[2 \ln \left(\frac{R_{a d c}+L_{l c}}{R_{a d c}}\right)-1\right]+1\right\} p_{i+N / 2} \cos \left(\theta_{i}-\frac{L_{c c}-L_{k c}}{D}\right)
\end{aligned}
$$

The total net bearing force in the y-direction is found by adding all of the pocket and compensator forces. Note that the compensator pressures are out of phase with the loadsupporting pocket pressures by $\mathrm{N} / 2$. This means that the net force generated in the $y$ direction by the compensators will be negative. The compensators tend to reduce the load-capacity of the bearing and so their areas should be made as small as possible.

The effective load center of the bearing, the bearing moment divided by the bearing force, is also needed to calculate the stiffness of the spindle. The bearing moment is computed similarly to that of bearings of previous sections, and will not be given here. The effective load center of SC Bearing \#1 differs from that of previous bearings in that the negative force contributed by the compensators tends to push the effective center of 
the bearing further toward the end of the bearing. This is beneficial to the stiffness of the spindle, and will be illustrated in Chapter 4.

\section{C.ii. Representative Calculations for SC Bearing \#1}

Table 3.3 shows a representative $80 \mathrm{~mm}$ diameter, $80 \mathrm{~mm}$ long bearing. The pocket land widths are $3 \mathrm{~mm}$ and the axial drainage groove width is $2 \mathrm{~mm}$. The compensator land widths are $3 \mathrm{~mm}$ and the collector groove width is $2 \mathrm{~mm}$. The compensator length is 0.95 times $\pi \mathrm{D} / \mathrm{N}$ in order to make the resistance ratio as small as possible; the realized resistance ratio is 2.22 . It would be desirable to reduce the resistance ratio further, but that would require reducing the compensator land width or increasing the pocket land width. The land width of the compensator should be at least 3 $\mathrm{mm}$ for manufacturability and to keep the flow over its lands fully-developed. Increasing the pocket land width reduces the effective pocket area and reduces the bearing stiffness. Although the design has a high resistance ratio, it is certainly adequate and the performance is representative of this type of bearing. As shown, the initial specific stiffness is 1.001 , the load-carrying efficiency at $75 \%$ of gap closure is 0.496 , and the specific flow rate is 50.5 .

The leakage land width was set at $10 \mathrm{~mm}$. This created $0.36 \mathrm{gpm}$ of leakage flow rate, which is small compared to $1.91 \mathrm{gpm}$ of total flow rate. Reducing the land width would enable more load-supporting area and therefore higher bearing stiffness. However, the higher stiffness would come with a penalty of higher flow rate and pumping power. 
Table 3.3

\begin{tabular}{|c|c|c|c|c|}
\hline $\begin{array}{l}\text { Operating Conditions } \\
\text { Supply Pressure (Pa,psi,atm) } \\
\text { Eccentricity Ratio ec/ep } \\
\text { Eccentricity at Pocket End } \\
\text { Eccentricity at Compensator End }\end{array}$ & $\begin{array}{c}\text { Ps } \\
\text { lamda } \\
\text { ep } \\
\text { ec }\end{array}$ & $\begin{array}{r}4.17 E+06 \\
1 \\
0.01 \\
0.01\end{array}$ & 600 & 40.8 \\
\hline $\begin{array}{l}\text { Fluid Properties } \\
\text { Dynamic Viscosity ( } \mathrm{s} / \mathrm{m})\end{array}$ & mu & 0.0013 & & \\
\hline \multicolumn{5}{|l|}{ Bearing Geometry } \\
\hline Diameter $(\mathrm{m}, \mathrm{mm}, \mathrm{in})$ & D & 0.08 & 80 & 3.15 \\
\hline Axial Length $(\mathrm{m}, \mathrm{mm}$, in) & $\mathbf{L}$ & 0.08 & 80 & 3.15 \\
\hline Number of Pockets & $\mathbf{N}$ & 6 & & \\
\hline Nominal Gap $(m, \mu m, i n)$ & ho & 0.000015 & \multicolumn{2}{|c|}{150.00059} \\
\hline Compensator Land Width (m, mm,in) & Llc & 0.003 & 3 & 0.118 \\
\hline Max Compensator Circumf. Length (m, mm,in) & $\max$ Lcc & 0.0419 & 41.9 & 1.649 \\
\hline Compensator Circumf. Length (m, mm, in) & Lcc & 0.0398 & 39.8 & 1.567 \\
\hline Compensator Collector Groove Width $(\mathrm{m}, \mathrm{mm}$, in) & Lcg & 0.002 & 2 & 0.079 \\
\hline Compensator Corner Radii (m,mm,in) & Radc & 0.0007 & 0.7 & 0.026 \\
\hline Supply Groove Width (m, mm,in) & Las & 0.002 & 2 & 0.079 \\
\hline Leakage Land Length $(\mathrm{m}, \mathrm{mm}, \mathrm{in})$ & Lleak & 0.01 & 10 & 0.394 \\
\hline Pocket Circumferential Land Width ( $m, m m, i n)$ & Llp & 0.003 & 3 & 0.118 \\
\hline Pocket End Axial Land Width (m, mm, in) & LImp & 0.003 & 3 & 0.118 \\
\hline Pocket Middle Axial Land Width (m, mm,in) & Llep & 0.003 & 3 & 0.118 \\
\hline Width of Drain Grooves $(\mathrm{m}, \mathrm{mm}$, in) & Ld & 0.00200 & 2 & 0.079 \\
\hline Pocket Axial Length (m,mm,in) & Lap & 0.05800 & 58.0 & 2.283 \\
\hline Pocket Circumferential Length (m, mm,in) & Lcp & 0.03989 & 39.9 & 1.570 \\
\hline Nominal Resistance Ratio & gamma & 2.22 & & \\
\hline \multicolumn{5}{|l|}{ Bearing Stiffness and Load Capacity } \\
\hline Location of Effective Bearing Force & Lcen_L & 0.303 & & \\
\hline Pressure Difference / Pressure @ ec,ep above & DPP & 0.024 & & \\
\hline Load Capacity (N,kN,Ibf) @ ec,ep given above & Fy & 267 & 0.27 & 60 \\
\hline Load Carrying Efficiency @ ec,ep given above & Feff & 0.010 & & \\
\hline Stiffness $\left(N / \mu m, l b_{f} / \mu i n\right) @$ ec,ep given above & Ky & 1779 & 10.159 & \\
\hline Specific Stiffness @ ec,ep given above & Kspec & 1.001 & & \\
\hline Initial Specific Stiffness & Kspec,o & 1.001 & & \\
\hline Load Carrying Efficiency @ 75\% of gap closure & Feff, 0.75 & 0.469 & & \\
\hline \multicolumn{5}{|l|}{ Flow Rate and Pumping Power } \\
\hline $\begin{array}{l}\text { Leakage Flow Rate }\left(m^{\wedge} 3 / s, 1 p m, g p m\right) \\
\text { Total Flow Rate }(m ` 3 / s, 1 p m, g p m)\end{array}$ & $\begin{array}{c}\text { Qleak } \\
\text { Osupply }\end{array}$ & $\begin{array}{l}2.27 \mathrm{E}-05 \\
1.20 \mathrm{E}-04\end{array}$ & $\begin{array}{l}1.36 \\
7.22\end{array}$ & $\begin{array}{l}0.36 \\
1.91\end{array}$ \\
\hline $\begin{array}{l}\text { Pumping Power (W,kW,hp) } \\
\text { Specific Flow Rate }\end{array}$ & $\begin{array}{l}\text { Ppump } \\
\text { Ospec }\end{array}$ & $\begin{array}{r}501 \\
50.5\end{array}$ & 0.501 & 0.673 \\
\hline $\begin{array}{l}\text { Ratio of Specific Initial Stiffness to Specific } \\
\text { Flow Rate }\end{array}$ & K/Qspec & 0.0198 & & \\
\hline
\end{tabular}


Also note in Table 3.3 that the effective load center of the bearing, $\mathrm{L}_{\text {cen }} / \mathrm{L}$, is equal to 0.303 . The distance to the effective center is defined with respect to the pocket end of the bearing, and so the effective center is biased toward the pocket end of the bearing. By placing the front bearing pocket near the front of the spindle, the stiffness of the spindle can be increased. This will be discussed further in Chapter 4.

Note that the load-carrying performance of SC Bearing \#1 is superior to that of FC Bearing \#1. The initial specific stiffness of SCB \#1 is 1.00 , while that of FCB \#1 is 0.856 . This is unexpected since the axial length of the load-supporting pockets of SCB \#1 are only $\mathrm{L}_{\text {ap }}=58 \mathrm{~mm}$, compared to $\mathrm{L}=80 \mathrm{~mm}$ for FCB \#1. However, the stiffness of SCB \#1 is higher because of the enhanced feedback of the compensator resistances. As the shaft is displaced toward pocket \#1, the resistance of pocket \#1 increases. The compensator feeding pocket $\# 1$ is on the opposite side of the shaft, so its resistance decreases. This creates a higher pressure in pocket \#1 than if the compensator resistance remained fixed. It is this enhanced feedback mechanism that causes SCB \#1 to have higher stiffness than FCB \#1. This is summarized in Table 3.4. The difference in pressure between the lower and upper pads, $p_{1}-p_{4}$, illustrates the enhanced feedback of SCB \#1. Note that the ratio of initial specific stiffness is 1.17 , not $(1.80)(0.725)=1.31$ expected. Slightly lower stiffness of SCB \#1 is realized because of the small negative stiffness contributed by its compensators.

\section{Table 3.4.}

\begin{tabular}{|c|c|c|c|}
\hline & FC Bearing \#1 & SC Bearing \#1 & Ratio \\
\hline Pad Length, L or $\mathrm{L}_{\text {ap }}$ & $80 \mathrm{~mm}$ & $58 \mathrm{~mm}$ & 0.725 \\
\hline$\left(\mathrm{p}_{1}-\mathrm{p}_{4}\right) / \mathrm{P}_{\mathrm{s}} @ \mathrm{e}, \mathrm{e}=0.01$ & 0.0133 & 0.0240 & 1.80 \\
\hline $\mathrm{K}_{\text {spec }, \mathrm{o}}$ & 0.856 & 1.00 & 1.17 \\
\hline
\end{tabular}




\section{D. Self-Compensated Bearing \#2}

The pad layout of Self-Compensated Bearing $\# 2$ is shown in Figure 3.13. The shaded regions are at a depth significantly greater than the land clearances such that the fluid pressure throughout each area is uniform. This bearing is identical to SC Bearing \#1 except that it does not have drainage grooves between its pockets. Fluid enters the fluid supply area, denoted in Figure 3.13 with $\mathbf{P}_{\mathrm{s}}$, through a hole located somewhere in that region. To the right of the supply pressure region is a leakage land, of width $L_{\text {leak, }}$, that prevents excessive leakage flow from the supply pressure region to the drain groove. After entering the supply pressure region, the fluid flows over the lands of the small compensation pads to a fluid collector groove in the center of the compensators. The fluid is then routed to the opposite side of the bearing to a pocket. The fluid flows throughout the pockets until it crosses the lands and out of the bearing to atmospheric pressure. The axial lands that run between the pockets serve to isolate the pocket pressures and enable a pressure difference to exist between them. 


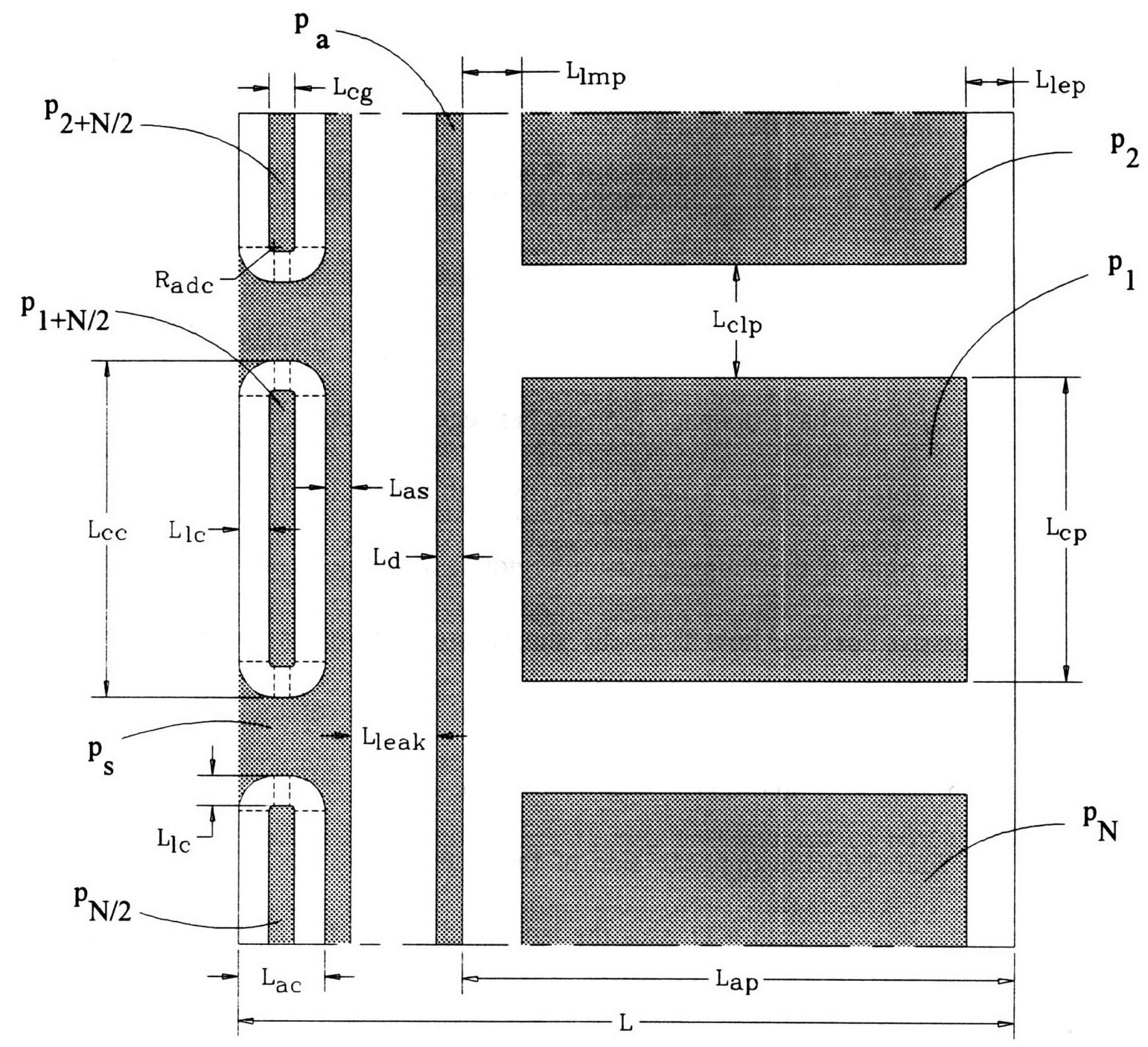

Figure 3.13. Schematic diagram of the pad layout of a self-compensated hydrostatic bearing without drainage grooves between its pockets but with a drainage groove between its supply area and its pockets. The shaded regions represent depressed regions.

The procedure for calculating the load-carrying capacity of SC Bearing \#2 is nearly identical to that of the previous bearing. The only difference is that the circumferential leakage across the axial lands must be accounted for by setting up and 
solving Circuit \#2 of Appendix B. This was explained in detail for FC Bearing \#2, and will not be repeated here.

Table 3.5 shows a representative $80 \mathrm{~mm}$ diameter, $80 \mathrm{~mm}$ long bearing. The pocket land widths are $3 \mathrm{~mm}$. The compensator land widths are $3 \mathrm{~mm}$ and the collector groove width is $2 \mathrm{~mm}$. An axial land width of $25 \mathrm{~mm}$ was found to be approximately optimal, providing maximum initial stiffness. The compensator length is 0.45 times $\pi \mathrm{D} / \mathrm{N}$ in order to make the resistance ratio approximately equal to 1 . The performance shown is representative of this type of bearing. The initial specific stiffness is 1.29 , the loadcarrying efficiency at $75 \%$ of gap closure is 0.540 , and the specific flow rate is 27.7 .

The leakage land width was set at $10 \mathrm{~mm}$. This created $0.36 \mathrm{gpm}$ of leakage flow rate, which is a significant percentage of the $0.88 \mathrm{gpm}$ of total flow rate. Increasing the land width further would reduce the leakage flow rate and the pumping power. However, the lower flow rate would come with a penalty of lower bearing stiffness as less of the available load-supporting area of the bearing is utilized.

The performance of SC Bearing \#2 is clearly superior to that of SC Bearing \#1 because it has higher initial stiffness, higher load capacity, and lower flow rate. These results were expected since the performance of FC Bearing \#2 was superior to that of FC Bearing \#1. SCB \#2 is preferred over SCB \#1 in all cases except where higher flow rate is desired. At high rotational speeds, the heat generated by the bearing can be high and so a greater flow rate is desired to remove the heat being generated and result in lower bearing temperature rise. SCB \#2 may be preferable in this case. 
Table 3.5

\begin{tabular}{|c|c|c|c|c|}
\hline $\begin{array}{l}\text { Operating Conditions } \\
\text { Supply Pressure (Pa,psi,atm) } \\
\text { Eccentricity Ratio ec/ep } \\
\text { Eccentricity at Pocket End } \\
\text { Eccentricity at Compensator End }\end{array}$ & $\begin{array}{l}\text { Ps } \\
\text { lamda } \\
\text { ep } \\
\text { ec }\end{array}$ & $\begin{array}{r}4.17 \mathrm{E}+06 \\
1 \\
0.01 \\
0.01\end{array}$ & 600 & 40.8 \\
\hline \multicolumn{5}{|l|}{ Fluid Properties } \\
\hline \multicolumn{5}{|l|}{ Bearing Geometry } \\
\hline Diameter $(\mathrm{m}, \mathrm{mm}, \mathrm{in})$ & D & 0.08 & 80 & 3.15 \\
\hline Axial Length $(\mathrm{m}, \mathrm{mm}, \mathrm{in})$ & $L$ & 0.08 & 80 & 3.15 \\
\hline $\begin{array}{l}\text { Number of Pockets } \\
\text { Nominal Gap }(m, \mu \mathrm{m}, \mathrm{in})\end{array}$ & $\begin{array}{r}N \\
\text { ho }\end{array}$ & $\begin{array}{r}6 \\
0.000015\end{array}$ & 15 & 0.00059 \\
\hline Compensator Land Width (m,mm,in) & Llc & 0.003 & 3 & 0.118 \\
\hline Max Compensator Circumf. Length (m, $\mathrm{mm}$, in) & $\max$ Lcc & 0.0419 & 41.9 & 1.649 \\
\hline Compensator Circumf. Length $(\mathrm{m}, \mathrm{mm}, \mathrm{in})$ & Lcc & 0.0188 & 18.8 & 0.742 \\
\hline r Collector Groove Width (m, mm, in) & Lcg & 0.002 & 2 & 0.079 \\
\hline Compensator Corner Radii $(\mathrm{m}, \mathrm{mm}$, in) & Radc & 0.0007 & 0.7 & 0.026 \\
\hline Supply Groove Width $(\mathrm{m}, \mathrm{mm}$, in) & Las & 0.002 & 2 & 0.079 \\
\hline Leakage Land Length $(\mathrm{m}, \mathrm{mm}, \mathrm{in})$ & Lleak & 0.01 & 10 & 0.394 \\
\hline Pocket Middle Axial Land Width ( $m, \mathrm{~mm}$,in) & LImp & 0.003 & 3 & 0.118 \\
\hline Pocket End Axial Land Width $(\mathrm{m}, \mathrm{mm}$, in) & Llep & 0.003 & 3 & 0.118 \\
\hline $\begin{array}{l}\text { Ratio } \\
\text { Pocket Circumferential Land Width (m, mm, in) }\end{array}$ & & $\begin{array}{r}1.5 \\
0.0251\end{array}$ & & \\
\hline $\begin{array}{l}\text { Pocket Circumferential Land Width }(\mathrm{m}, \mathrm{mm}, \mathrm{in}) \\
\text { Pocket Circumferential Length }(\mathrm{m}, \mathrm{mm}, \mathrm{in})\end{array}$ & $\begin{array}{l}\text { Lclp } \\
\text { Lco }\end{array}$ & $\begin{array}{l}0.0251 \\
0.0168\end{array}$ & $\begin{array}{r}25.1 \\
16.8\end{array}$ & $\begin{array}{l}0.989 \\
0.660\end{array}$ \\
\hline $\begin{array}{l}\text { frcumferential Length }(\mathrm{m}, \mathrm{mm} \text {, } \\
\text { f Drain Grooves }(\mathrm{m}, \mathrm{mm}, \mathrm{in})\end{array}$ & Ld & 0.00200 & 2 & 0.079 \\
\hline Compensator Axial Width (m,mm,in) & Lac & 0.008 & 8.0 & 0.31 \\
\hline $\begin{array}{l}\text { Pocket Axial Length }(\mathrm{m}, \mathrm{mm}, \mathrm{in}) \\
\text { Nominal Resistance Ratio }\end{array}$ & $\begin{array}{l}\text { Lap } \\
\text { gamma }\end{array}$ & $\begin{array}{r}0.058 \\
1.02\end{array}$ & 58.0 & \\
\hline \multicolumn{5}{|l|}{ Bearing Load Capacity } \\
\hline Location of Effective Bearing Force & Lcen $L$ & 0.339 & & \\
\hline Pressure Difference / Pressure @ ec,ep above & DPP & 0.027 & & \\
\hline Load Capacity (N,kN,Ibf)@ ec,ep given above & Fy & 344 & 0.34 & \\
\hline 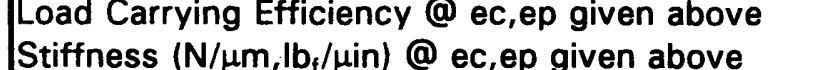 & $\begin{array}{l}\text { Feff } \\
\text { Ky }\end{array}$ & $\begin{array}{r}0.013 \\
2296\end{array}$ & 13.11 & \\
\hline Specific Stiffness @ ec,ep given above & Kspec & 1.292 & & \\
\hline $\begin{array}{l}\text { Initial Specific Stiffness } \\
\text { Load Carrying Efficiency @ 75\% of gap closure }\end{array}$ & $\begin{array}{l}\text { Kspec,o } \\
\text { Feff, } 0.75\end{array}$ & $\begin{array}{l}1.292 \\
0.540\end{array}$ & & \\
\hline \multicolumn{5}{|l|}{ Flow Rate and Pumping Power } \\
\hline Leakage Flow Rate $\left(\mathrm{m}^{\circ} 3 / \mathrm{s}, \mathrm{pm}, \mathrm{gpm}\right)$ & & 2.27E-05 & 1.36 & \\
\hline Total Flow Rate $\left(\mathrm{m}^{\wedge} 3 / \mathrm{s}, 1 \mathrm{pm}, \mathrm{gpm}\right)$ & & $5.58 \mathrm{E}-05$ & & \\
\hline $\begin{array}{l}\text { Pumping Power (W,kW,hp) } \\
\text { Specific Flow Rate }\end{array}$ & Ppump & 233 & 0.233 & 0.312 \\
\hline Specific Flc & Ospec & 27. & & \\
\hline $\begin{array}{l}\text { Ratio of Specific Initial Stiffness to Specific Flow } \\
\text { Rate }\end{array}$ & K/Ospec & 0.0466 & & \\
\hline
\end{tabular}




\section{E. Self-Compensated Bearing \#3}

The pad layout of Self-Compensated Bearing \#3 is shown in Figure 3.14. This bearing is nearly identical to SC Bearing \#1 except that it does not have drainage grooves between its supply pressure region and its pockets. Fluid enters the fluid supply area, denoted in Figure 3.14 with $\mathrm{P}_{\mathrm{s}}$, through a hole located somewhere in that region. After entering the supply pressure region, some of the fluid leaks across the leakage land to the pockets, and some of the fluid flows over the lands of the small compensation pads to a fluid collector groove in the center of the compensators. The fluid is then routed to the opposite side of the bearing to the bearing pockets. The fluid flows throughout the pockets until it crosses the lands and out of the bearing to atmospheric pressure. The axial drainage grooves that run partially between the pockets serve to isolate the pocket pressures and enable a pressure difference to exist between them. An axial land with a width of $\mathrm{L}_{\text {clp }}$ runs the remainder of the pad length. It also serves to isolate the pockets and enable a pressure difference to exist between them. 


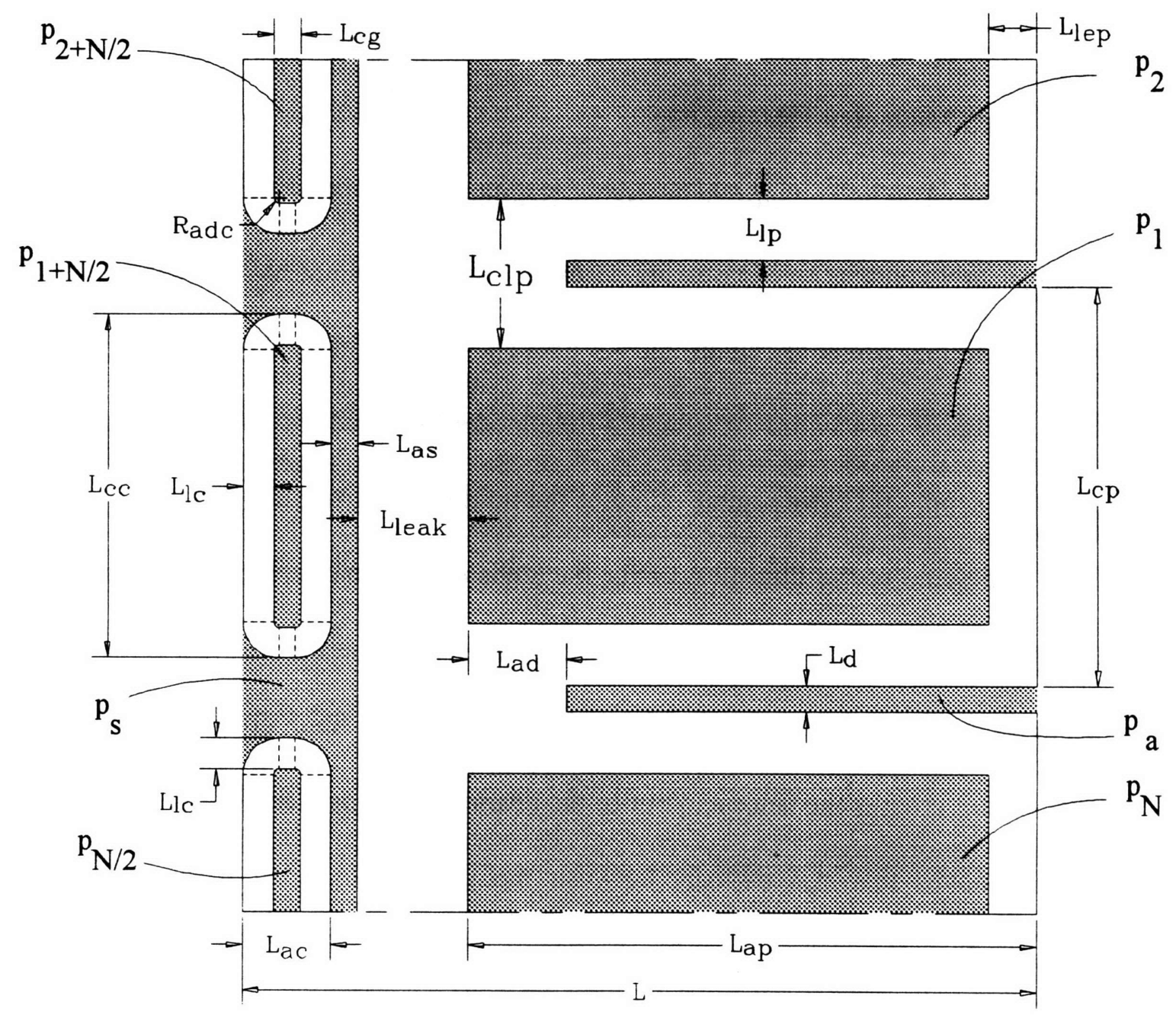

Figure 3.14. Schematic diagram of the pad layout of a self-compensated hydrostatic bearing with drainage grooves partially between its pockets and without a drainage groove between its supply area and its pockets. The shaded regions represent depressed regions.

The procedure for calculating the load-carrying capacity of SC Bearing \#3 is nearly identical to that of the previous bearings except that the leakage flow between the supply pressure area and the pockets must be accounted for. The resistance to leakage flow to the ith pocket is given by: 


$$
R_{\text {leak }, i}=R_{\text {circum }}\left(\mu, h_{o}, L_{\text {leak }}, L_{c p}-2 L_{l p}+L^{*}, \theta_{i}, e_{\text {circum }}\left(e_{1}, e_{2}, \theta_{i}\right)\right)
$$

where the eccentricities $e_{1}$ and $e_{2}$ are given by:

$$
\begin{aligned}
& e_{1}=e_{c}+\left(e_{p}-e_{c}\right) \frac{L-L_{a p}}{L} \\
& e_{2}=e_{c}+\left(e_{p}-e_{c}\right) \frac{L-L_{a p}-L_{\text {leak }}}{L}
\end{aligned}
$$

and $\mathrm{L}^{*}$ is given approximately by:

$$
L^{*} \approx 0.8 L_{l p}
$$

The leakage resistance of Equation (3.96) is combined in parallel with the compensation resistance to the ith pocket, $\mathbf{R}_{\mathrm{c}, \mathrm{i}}$, to yield an overall equivalent compensation resistance:

$$
R_{c e q, i}=\frac{1}{\frac{1}{R_{c, i}}+\frac{1}{R_{\text {leak }, i}}}
$$

This equivalent compensation resistance is used in place of $R_{c, i}$ to solve Fluid Circuit $\# 2$ of Appendix B.

Table 3.6 shows the results of representative calculations for a $80 \mathrm{~mm}$ diameter, 80 $\mathrm{mm}$ long bearing. The pocket land widths are $3 \mathrm{~mm}$. The compensator land widths are 3 $\mathrm{mm}$ and the collector groove width is $2 \mathrm{~mm}$. The compensator length is 0.95 times $\pi \mathrm{D} / \mathrm{N}$ in order to make the resistance ratio approximately equal to 1 . A leakage land width of 14 $\mathrm{mm}$ was found to approximately maximize the initial specific stiffness of the bearing. The performance shown is representative of this type of bearing. The initial specific stiffness is 1.16 , the load-carrying efficiency at $75 \%$ of gap closure is 0.487 , and the specific flow rate is 26.6. 


\section{Table 3.6}

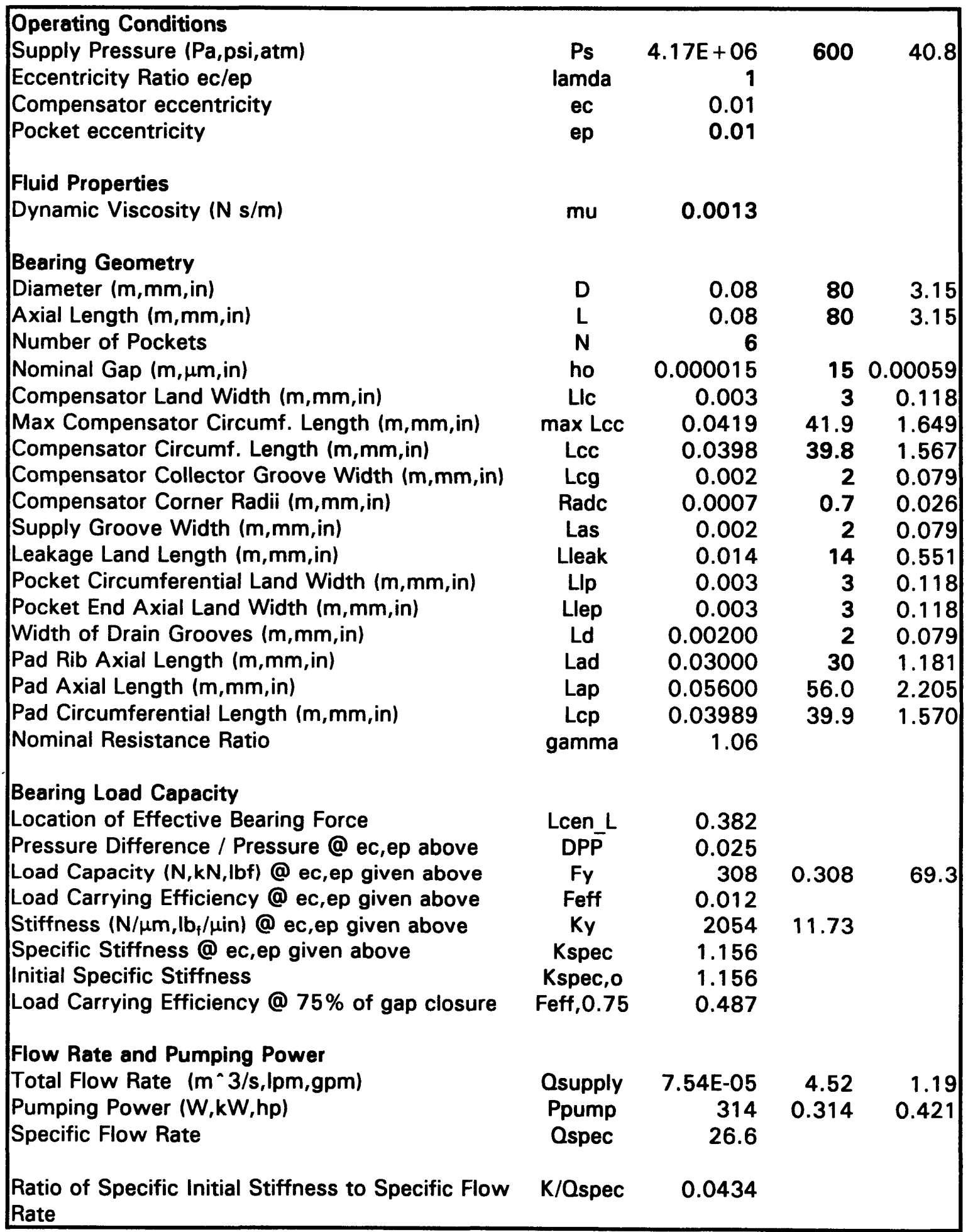


The leakage land width in Table 3.6 was set at $14 \mathrm{~mm}$. This was found to be the near-optimal width of this land that maximized the bearing stiffness, as shown in Figure 3.15. As the width of the leakage land is increased, the effect of the leakage flow between the supply pressure to the pockets is reduced; however, increasing the leakage land width reduces the area of the bearing that is utilized for load-carrying capacity. An optimal leakage land width therefore exists that maximizes the stiffness of the bearing. Increasing the leakage land width reduces the flow rate somewhat, and so a leakage land width somewhat greater than optimal is desired.

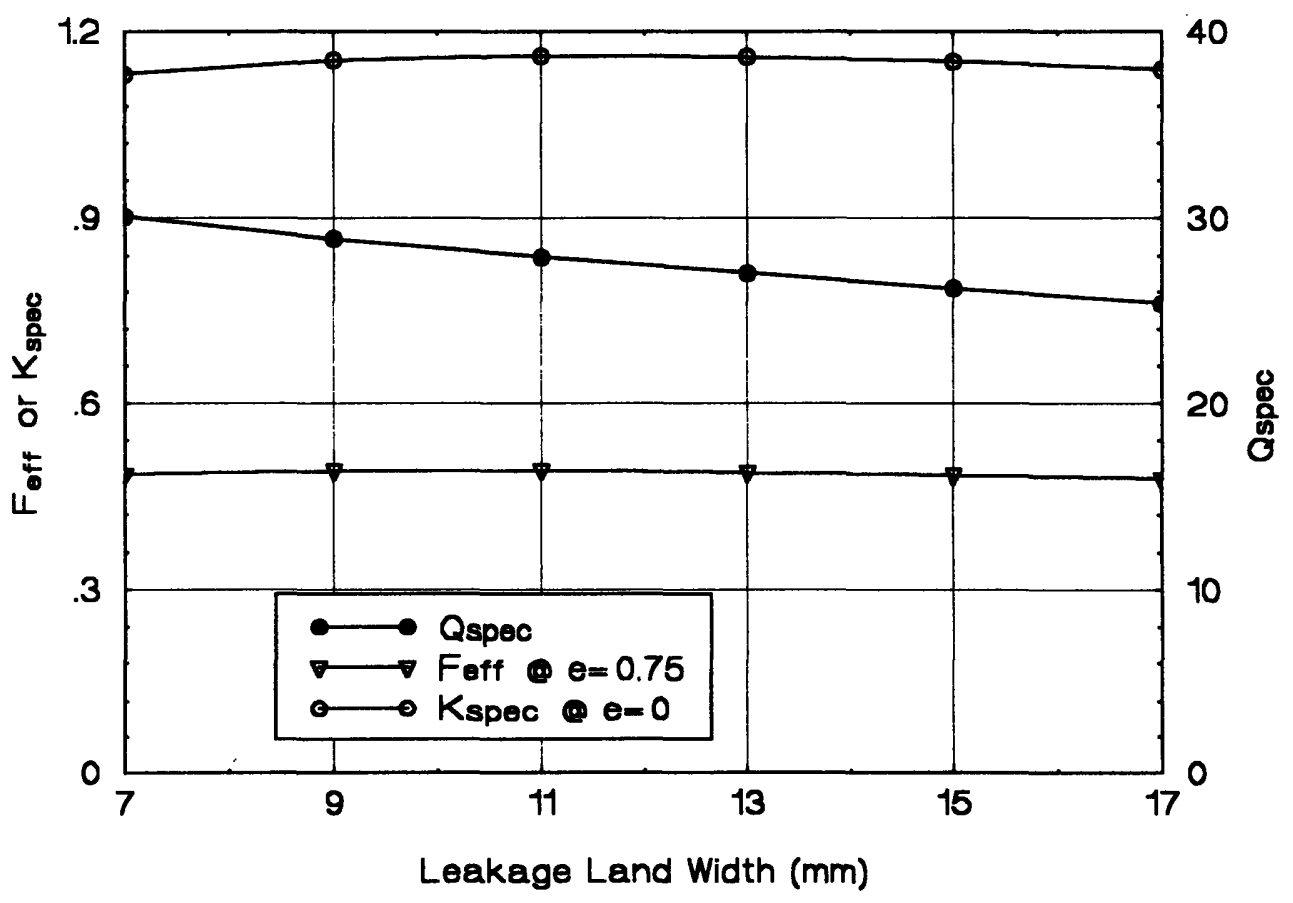

Figure 3.15. Effect of the leakage land width on the 6-pocket, $80 \mathrm{~mm}$ diameter SC Bearing \#3 of Table 3.6. 


\section{F. Self-Compensated Bearing \#4}

The pad layout of Self-Compensated Bearing \#4 is shown in Figure 3.16. This bearing is nearly identical to SC Bearing \#3 except that it does not have drainage grooves between its pockets.

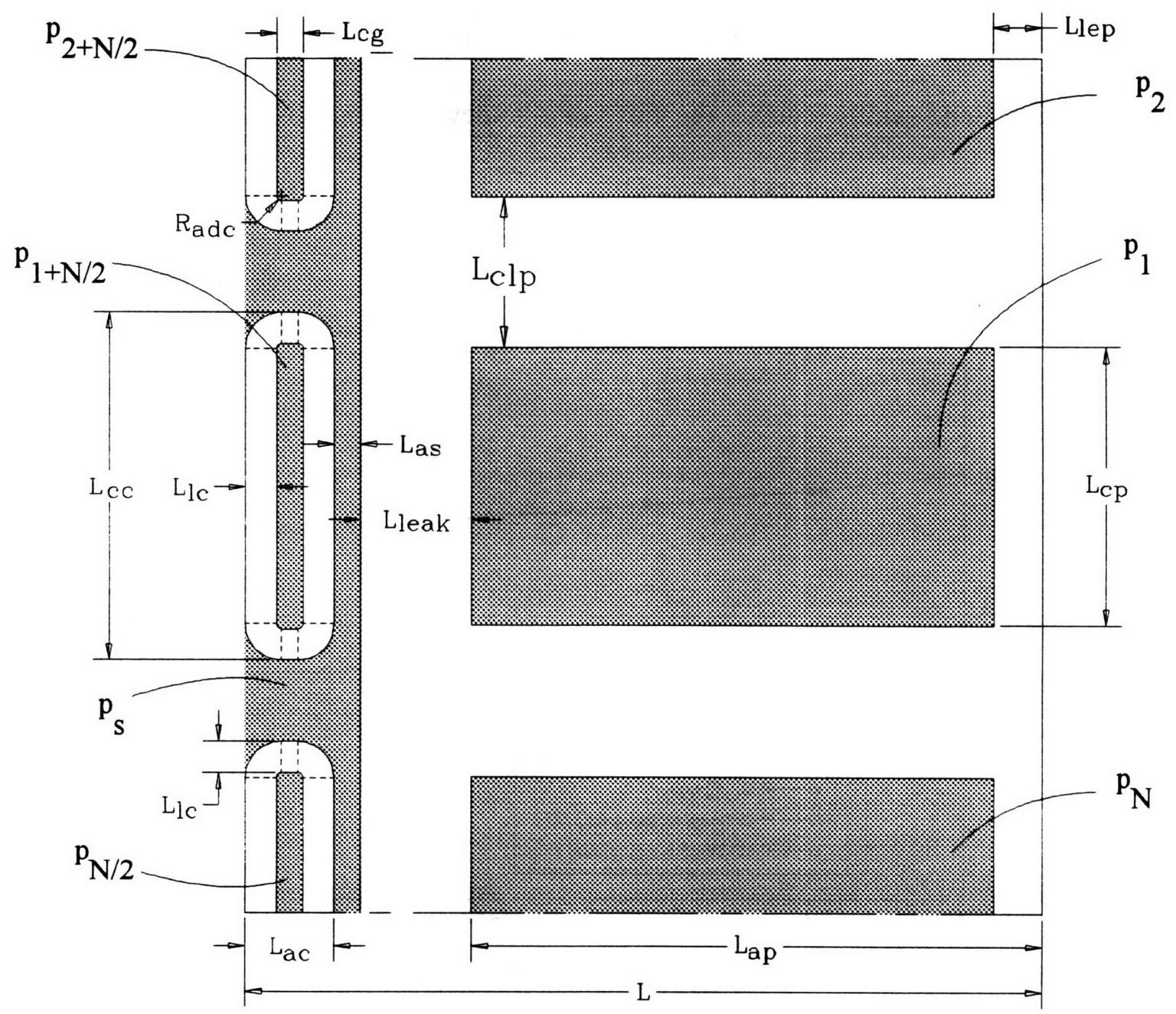

Figure 3.16. Schematic diagram of the pad layout of a self-compensated hydrostatic bearing without drainage grooves between its pockets and without a drainage groove between its supply area and its pockets. The shaded regions represent depressed regions. 
The procedure for calculating the load-carrying capacity of SC Bearing \#4 is nearly identical to that of the previous bearings, and will not be repeated here.

Table 3.7 shows input and output values for a representative $80 \mathrm{~mm}$ diameter, 80 $\mathrm{mm}$ long bearing. The pocket land widths are $3 \mathrm{~mm}$. The compensator land widths are 3 $\mathrm{mm}$ and the collector groove width is $2 \mathrm{~mm}$. The compensator length is 0.3 times $\pi \mathrm{D} / \mathrm{N}$ in order to make the resistance ratio approximately equal to 0.8 ; this value was found to approximately optimize the initial specific stiffness of the bearing. The width of the axial lands between the pockets was also optimized; its value is approximately $25 \mathrm{~mm}$. The performance shown in Table 3.7 is representative of the performance of this type of bearing. The initial specific stiffness is 1.01 , the load-carrying efficiency at $75 \%$ of gap closure is 0.425 , and the specific flow rate is 7.3 .

The effect of leakage from the supply pressure region to the pockets can be studied by comparing SC Bearing \#4 to SC Bearing \#2. The load-supporting pads of the two bearings are nearly identical. However, the specific stiffness of SCB \#2 is 1.29 , while that of SCB \#4 is 1.01 . The leakage flow between the supply pressure region and the pockets therefore reduces the bearing stiffness by about $22 \%$. However, the specific flow rate of SCB \#2 is 27.7 , which is approximately 4 times higher than that of SCB \#4. Therefore, in most applications SCB \#4 is preferred over SCB \#2 because it achieves nearly-equal stiffness and much lower flow rate. 
Table 3.7

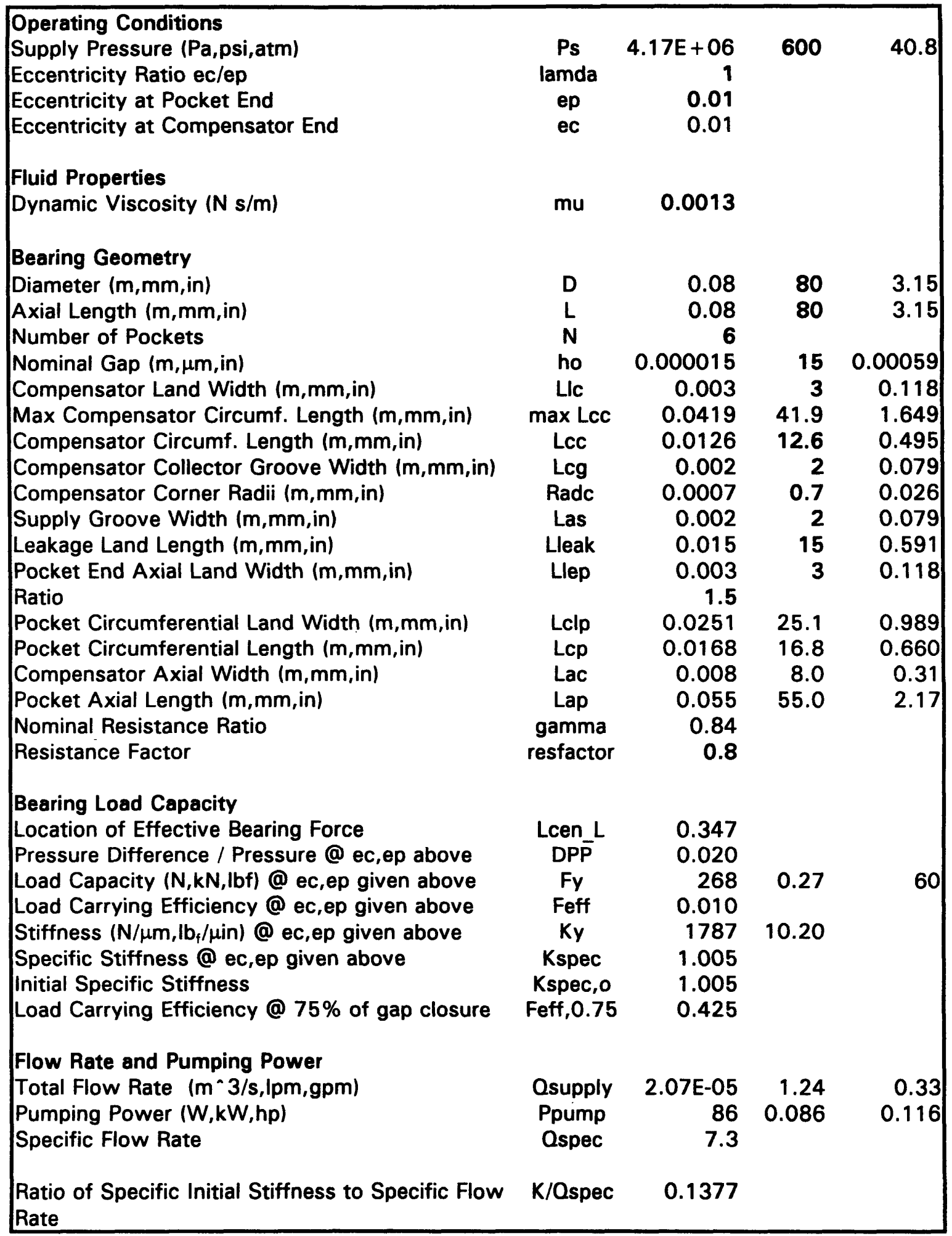




\section{G. Self-Compensated Bearing \#5}

The pad layout of Self-Compensated Bearing \#5 is shown in Figure 3.17. This bearing differs from the previous self-compensated bearings in that the compensator is no longer completely isolated from the pockets and drains. Fluid enters the bearing at supply pressure, $\mathrm{P}_{\mathrm{s}}$, at the left edge of the bearing. It then crosses the compensation lands to the fluid collection grooves. Some of the fluid then leaks to the drain groove, which is at atmospheric pressure. The rest of the fluid is routed to a pocket on the opposite side of the bearing where it crosses the pocket lands to atmospheric pressure.

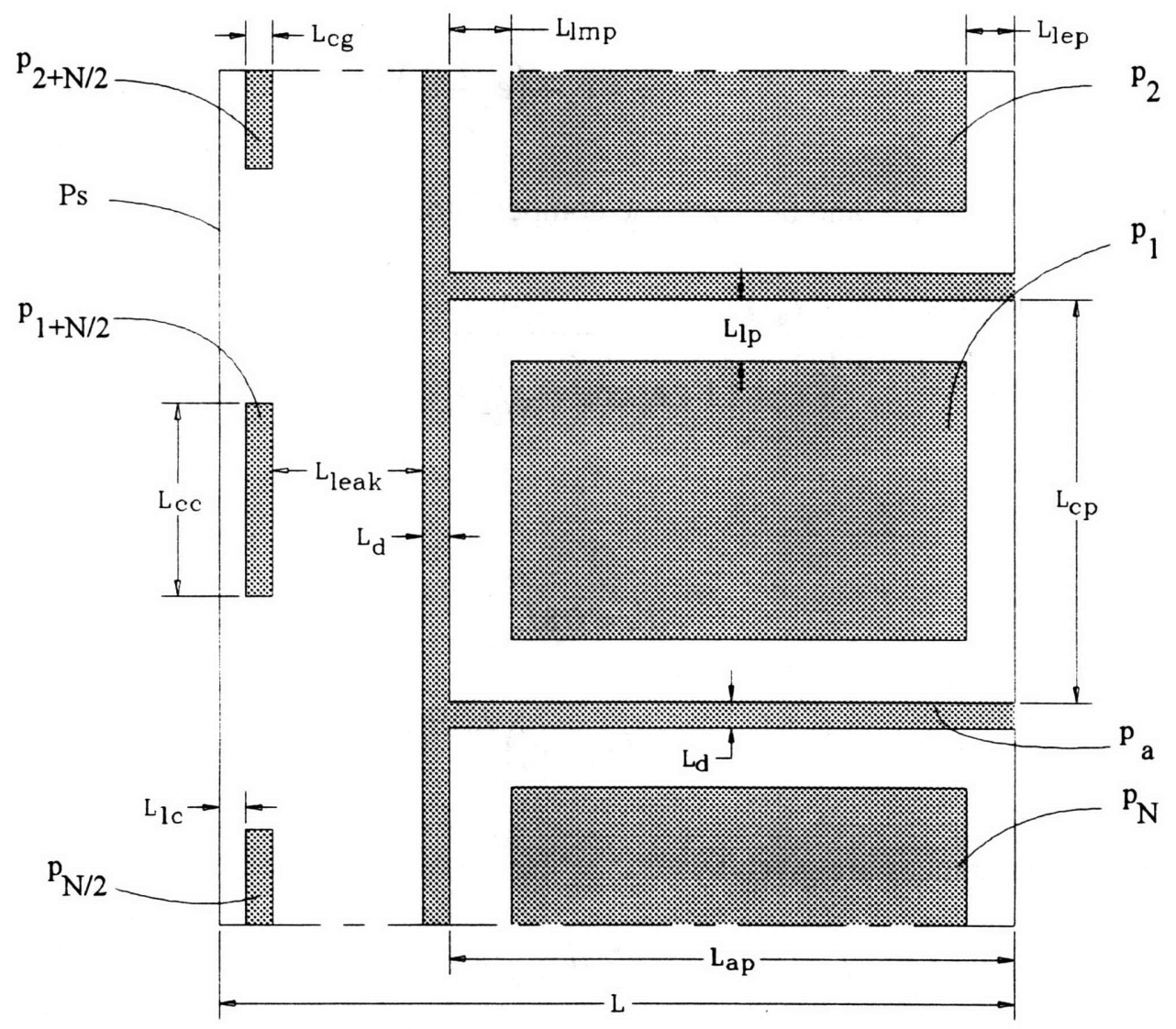

Figure 3.17. Schematic diagram of the pad layout of a self-compensated hydrostatic bearing with compensators that are not completely surrounded by supply pressure. The shaded regions represent depressed regions. 
The procedure for calculating the load-carrying capacity of SC Bearing \#5 is nearly identical to that for the previous bearings, except the leakage that occurs between the compensators and from the compensators to the drain groove must be accounted for. The resistance of the ith compensators is given by:

$$
R_{c, l}=R_{\text {crcum }}\left(\mu, h_{o}, L_{l c}, L_{c c}+L_{1}^{*}, \theta_{i+N / 2}, e_{\text {circum }}\left(e_{c}, e_{3}, \theta_{i+N / 2}\right)\right)
$$

where:

$$
\begin{aligned}
& L_{1}^{*} \approx 0.8 L_{l c} \\
& e_{3}=e_{c}+\left(e_{p}-e_{c}\right) \frac{L_{l c}}{L}
\end{aligned}
$$

The resistance of the ith land between the compensators is given by:

$$
R_{b, i}=\frac{12 \mu\left(\pi D / N-L_{c c}\right)}{h_{o}^{3}\left(L_{c g}+L_{2}^{*}\right)} \frac{1}{\left(1-e_{4} \cos \left(\theta_{i+N / 2}+\frac{\pi D / N}{D}\right)\right)^{3}}
$$

where:

$$
\begin{gathered}
L_{2}^{*} \approx 0.8 L_{l c} \\
e_{4}=e_{c}+\left(e_{p}-e_{c}\right) \frac{L_{l c}+L_{c g} / 2}{L}
\end{gathered}
$$

The leakage resistances are given by:

$$
R_{\text {leak }, i}=R_{\text {circum }}\left(\mu, h_{o}, L_{\text {leak }}, L_{c c}+L_{3}^{*}, \theta_{i+N / 2}, e_{\text {circum }}\left(e_{4}, e_{5}, \theta_{i+N / 2}\right)\right)
$$

where:

$$
L_{1}^{*} \approx 0.8\left\{\min \left(L_{\text {leak }}, \pi D / N-L_{c c}\right)\right\}
$$




$$
\begin{aligned}
& e_{4}=e_{c}+\left(e_{p}-e_{c}\right) \frac{L_{l c}+L_{c g}}{L} \\
& e_{5}=e_{c}+\left(e_{p}-e_{c}\right) \frac{L_{l c}+L_{c g}+L_{l e a k}}{L}
\end{aligned}
$$

The leakage resistance of Equation (3.107) is combined in parallel with the resistance to the ith pocket, $\mathbf{R}_{\mathrm{a}, \mathrm{i}}$, to yield an overall equivalent outlet resistance:

$$
R_{a e q, i}=\frac{1}{\frac{1}{R_{a, i}}+\frac{1}{R_{\text {leak }, i}}}
$$

This equivalent resistance is used in place of $R_{a, i}$ to solve Fluid Circuit \#2 of Appendix B.

Table 3.8 shows the results of calculations for a representative $80 \mathrm{~mm}$ diameter, 80 $\mathrm{mm}$ long bearing. The compensator land widths are $3 \mathrm{~mm}$ and the collector groove width is $2 \mathrm{~mm}$. The compensator length is 0.95 times $\pi \mathrm{D} / \mathrm{N}$ in order to make the resistance ratio as small as possible; the realized resistance ratio was 3.05 . The pocket land widths are 5 $\mathrm{mm}$; although reducing them to $3 \mathrm{~mm}$ increases the available load-supporting area, it also increases the resistance ratio and the stiffness is reduced. The performance shown is representative of this type of bearing. The initial specific stiffness is 0.729 , the loadcarrying efficiency at $75 \%$ of gap closure is 0.323 , and the specific flow rate is 20.2 .

The primary problem with SC Bearing \#5 is that it is difficult to design the bearing with an adequately low resistance ratio. The width of the pocket lands must inevitably be increased to increase the resistance of the pads. However, as the width of the pocket lands are increased, a lesser percentage of the available load-supporting area is utilized. This bearing has low stiffness and high flow rate and is not recommended in any applications. 
Table 3.8

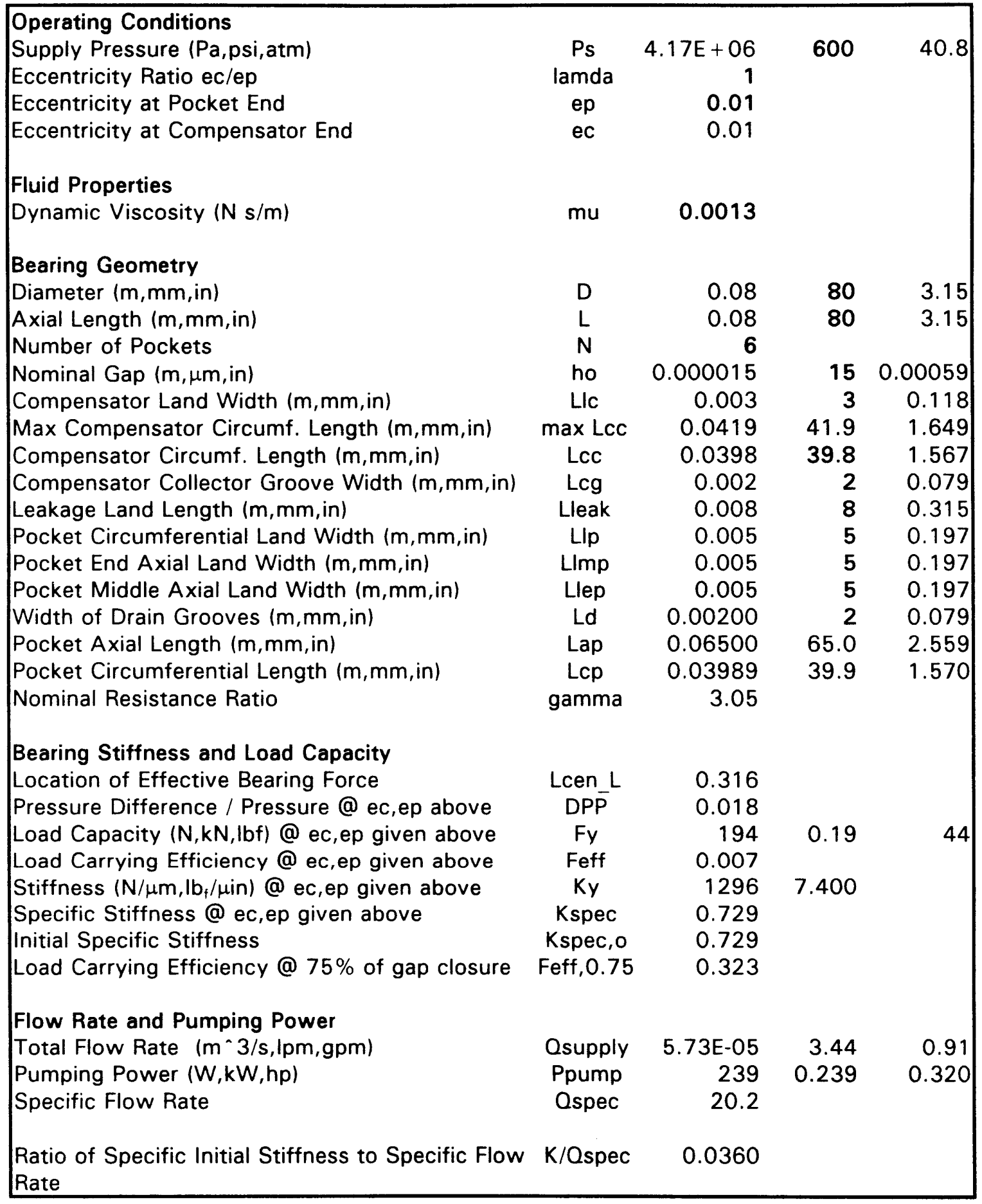




\section{H. Self-Compensated Bearing \#6}

The pad layout of Self-Compensated Bearing \#6 is shown in Figure 3.18. This bearing differs from SC Bearing \#5 in that there are no drain grooves between its pockets.

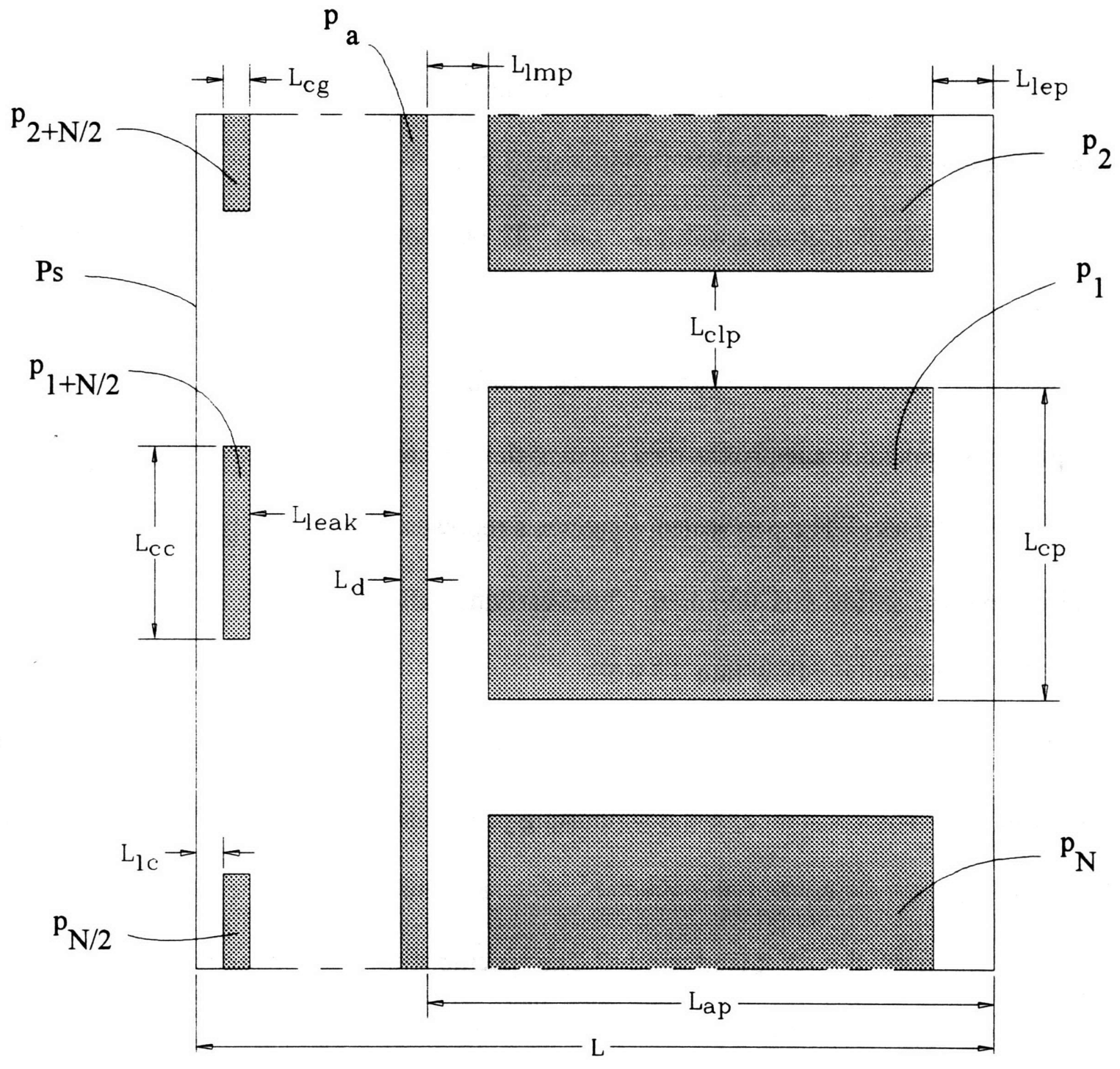

Figure 3.18. Schematic diagram of the pad layout of a self-compensated hydrostatic bearing with compensators that are not completely surrounded by supply pressure. The shaded regions represent depressed regions. 
The procedure for calculating the load-carrying capacity of SC Bearing \#6 is nearly identical to that for the SC Bearing \#5, except the leakage that occurs between the pockets must be accounted for. This was illustrated for FC Bearing \#2 and will not be repeated here.

Table 3.9 shows the results of calculations for a representative $80 \mathrm{~mm}$ diameter, 80 $\mathrm{mm}$ long bearing. The pocket land widths are $3 \mathrm{~mm}$. The compensator land widths are 3 $\mathrm{mm}$ and the collector groove width is $2 \mathrm{~mm}$. The compensator length is 0.75 times $\pi \mathrm{D} / \mathrm{N}$ in order to make the resistance ratio approximately equal to 1.6 ; this configuration was found to approximately maximize the bearing stiffness. The width of the axial lands that separate the pockets is approximately $22 \mathrm{~mm}$; this was found to approximately achieve the maximum stiffness. The performance shown is representative of this type of bearing. The initial specific stiffness is 0.934 , the load-carrying efficiency at $75 \%$ of gap closure is 0.392 , and the specific flow rate is 13.6 .

The load-carrying performance of SC Bearing \#6 is much better than that of SC Bearing \#5. The resistance of the supporting pads has been increased by eliminating the drainage grooves between the pockets. A respectable resistance ratio can now be achieved with small pocket land widths. The specific stiffness is now competetive with the self-compensated bearings that have supply pressure completely surrounding their compensators. 
Table 3.9

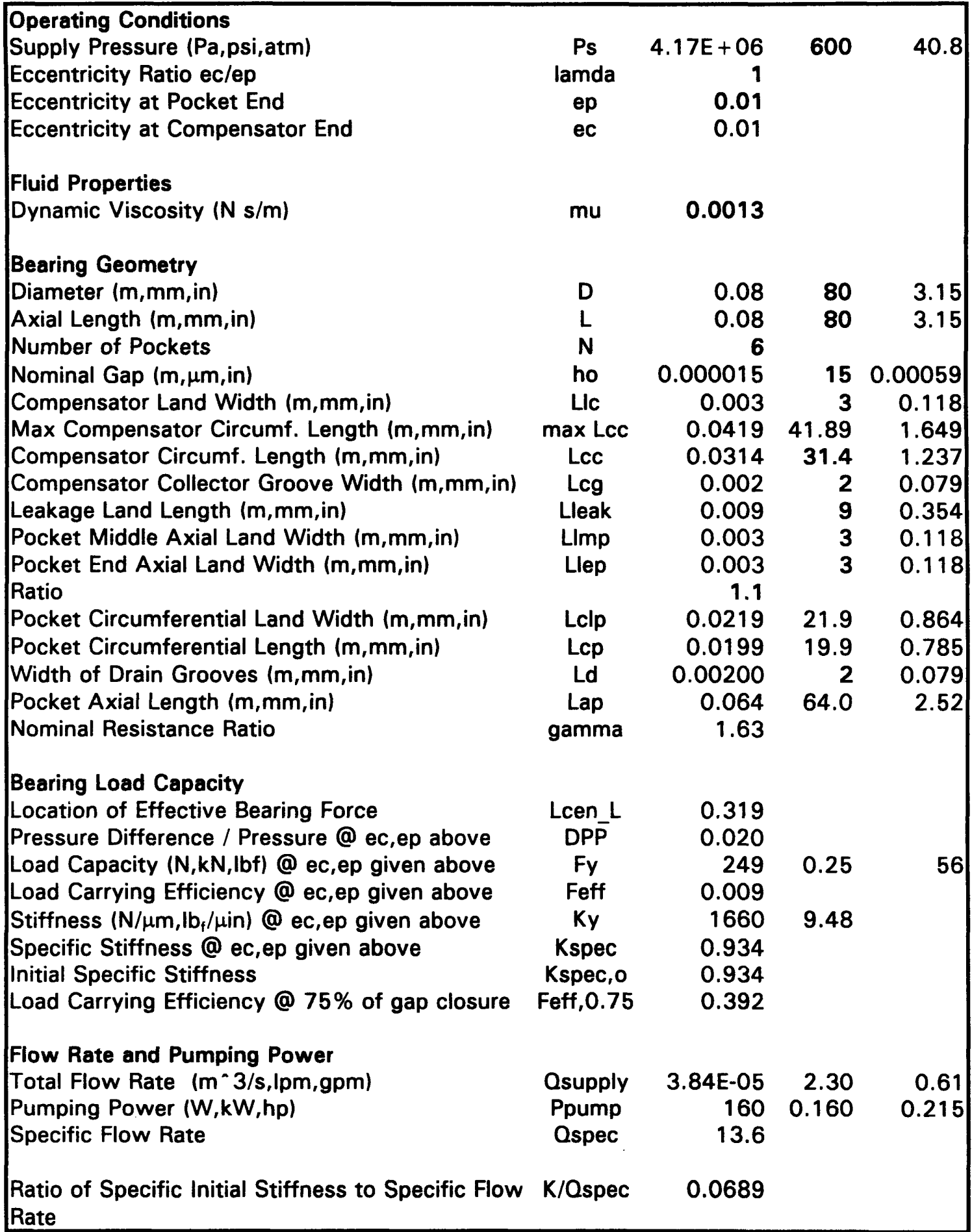




\section{I. Self-Compensated Bearing \#7}

The pad layout of Self-Compensated Bearing \#7 is shown in Figure 3.19. This bearing differs from SC Bearing \#5 only in that there is no drain grooves between its compensators and its pockets.

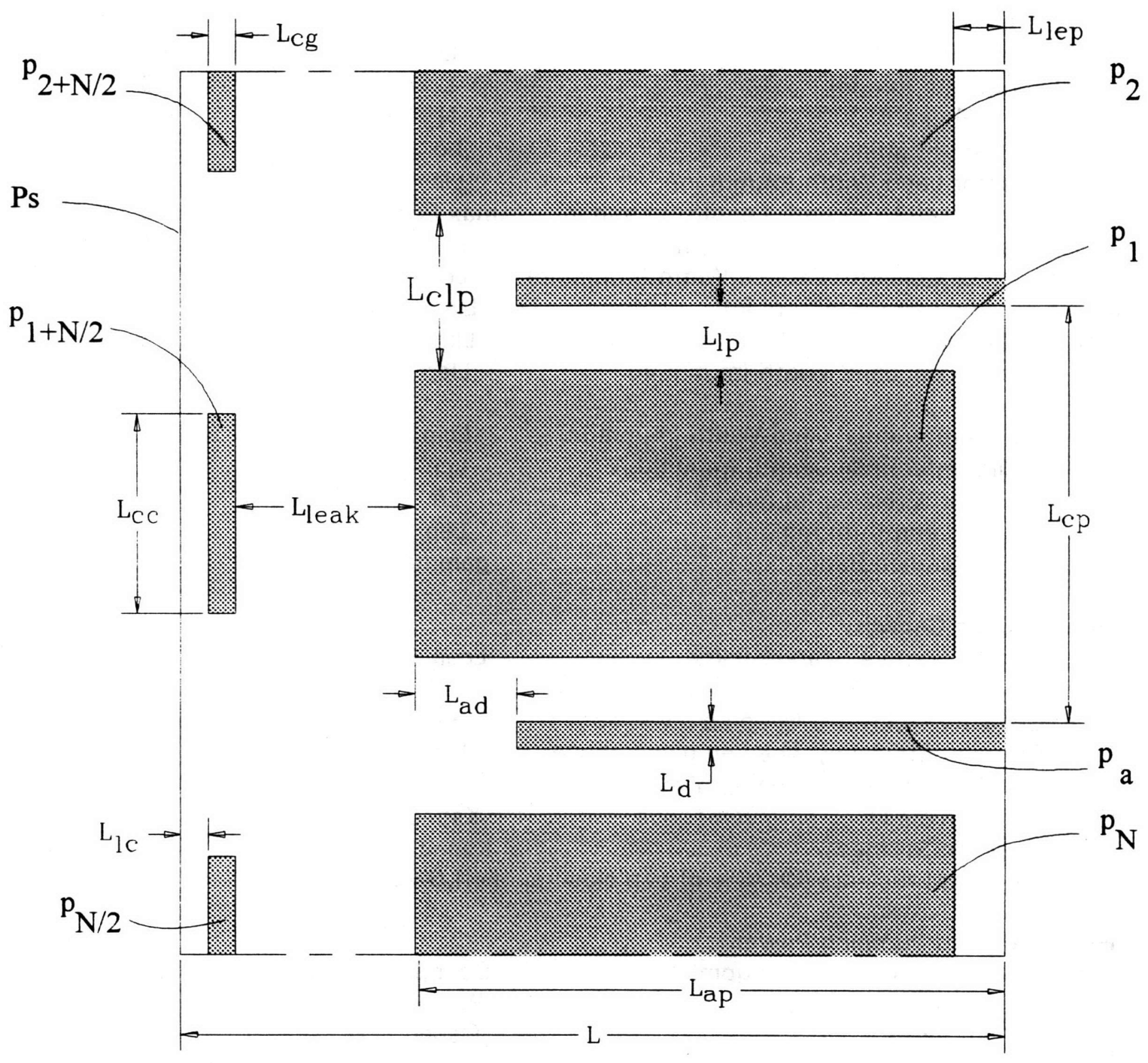

Figure 3.19. Schematic diagram of the pad layout of a self-compensated hydrostatic bearing with compensators that are not completely surrounded by supply pressure. The shaded regions represent depressed regions. 
The procedure for calculating the load-carrying capacity of SC Bearing \#7 is nearly identical to that for the SC Bearing \#6, except the leakage that occurs between the compensators and the pockets must be accounted for. The resistance of the ith compensator is given by:

$$
R_{c, i}=R_{\text {circum }}\left(\mu, h_{o}, L_{l c}, L_{c c}+L_{1}^{*}, \theta_{i+N / 2}, e_{\text {circum }}\left(e_{c}, e_{4}, \theta_{i+N / 2}\right)\right)
$$

where:

$$
\begin{aligned}
& L_{1}^{*} \approx 0.8 L_{l c} \\
& e_{4}=e_{c}+\left(e_{p}-e_{c}\right) \frac{L_{l c}}{L}
\end{aligned}
$$

The resistance of the ith land between the compensators is given by:

$$
R_{c c, i}=\frac{12 \mu\left(\pi D / N-L_{c c}\right)}{h_{o}^{3}\left(L_{c g}+L_{2}^{*}\right)} \frac{1}{\left(1-e_{5} \cos \left(\theta_{1+N / 2}+\frac{\pi D / N}{D}\right)\right)^{3}}
$$

where:

$$
\begin{gathered}
L_{2}^{*} \approx 0.8 L_{l c} \\
e_{5}=e_{c}+\left(e_{p}-e_{c}\right) \frac{L_{l c}+L_{c g} / 2}{L}
\end{gathered}
$$

The resistance of the ith land between the pockets is given by:

$$
R_{p p, i}=R_{\text {axial }}\left(\mu, h_{o}, L_{c l p}, L_{a d}-L_{d}, \theta_{i}+\frac{L_{c p}-2 L_{l p}+L_{c l p}}{D}, e_{\text {axid }}\left(e_{2}, e_{3}, \theta_{i}+\frac{L_{c p}-2 L_{l p}+L_{c l p}}{D}\right)\right)
$$

where:

$$
e_{2}=e_{p}+\left(e_{c}-e_{p}\right) \frac{L_{a p}-L_{a d}}{L}
$$




$$
e_{3}=e_{p}+\left(e_{c}-e_{p}\right) \frac{L_{l e p}}{L}
$$

The resistances $R_{c c, i}$ and $R_{p p, i}$ act in parallel. An equivalent resistance to be used as $R_{b, i}$ in Circuit \#3 of Appendix B is given by:

$$
R_{b e q, i}=\frac{1}{\frac{1}{R_{c c, i}}+\frac{1}{R_{p p, i}}}
$$

The resistance of the land between the compensator and the ith pocket is used for $R_{d, i}$ in Circuit \#3 of Appendix B, and is given by:

$$
R_{d, 1}=R_{\text {crcum }}\left(\mu, h_{o}, L_{\text {leak }}, L_{z z}, \theta_{i}, e_{\text {circum }}\left(e_{3}, e_{4}, \theta_{i}\right)\right)
$$

where:

$$
\begin{aligned}
& L_{z z} \approx \min \left(L_{c c}, L_{c p}-2 L_{l p}\right) \\
& e_{4}=e_{c}+\left(e_{p}-e_{c}\right) \frac{L_{l c}+L_{c g}}{L}
\end{aligned}
$$

The last resistances needed for Circuit \#3 of Appendix $B$ is $R_{a, i}$. These are found by combining the three pocket land resistances in parallel, as described in previous sections. Once Circuit \#3 has been solved for the flow rates and pressures, the net bearing force is calculated as described in previous sections.

Table 3.10 shows the results of calculations for a representative $80 \mathrm{~mm}$ diameter, $80 \mathrm{~mm}$ long bearing. The pocket land widths are $3 \mathrm{~mm}$. The compensator land widths are $3 \mathrm{~mm}$ and the collector groove width is $2 \mathrm{~mm}$. The compensator length is 0.95 times $\pi \mathrm{D} / \mathrm{N}$ in order to make the resistance ratio approximately equal to 1.3 . The performance 
shown is representative of this type of bearing. The initial specific stiffness is 1.03 , the load-carrying efficiency at $75 \%$ of gap closure is 0.453 , and the specific flow rate is 15.6 .

Table 3.10

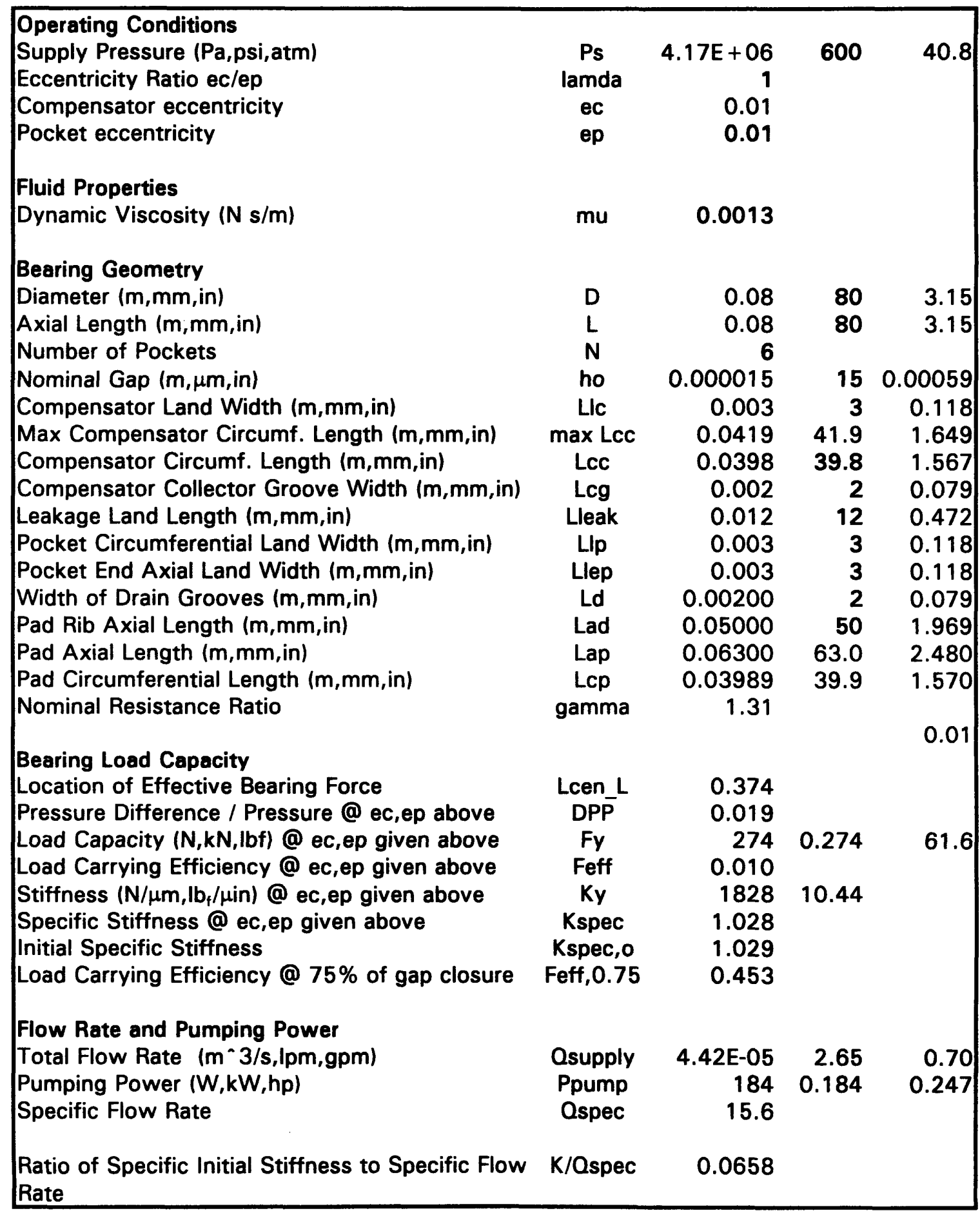




\section{J. Self-Compensated Bearing \#8}

The pad layout of Self-Compensated Bearing \#8 is shown in Figure 3.20. This bearing differs from SC Bearing \#7 only in that there is no drain grooves between its pockets.

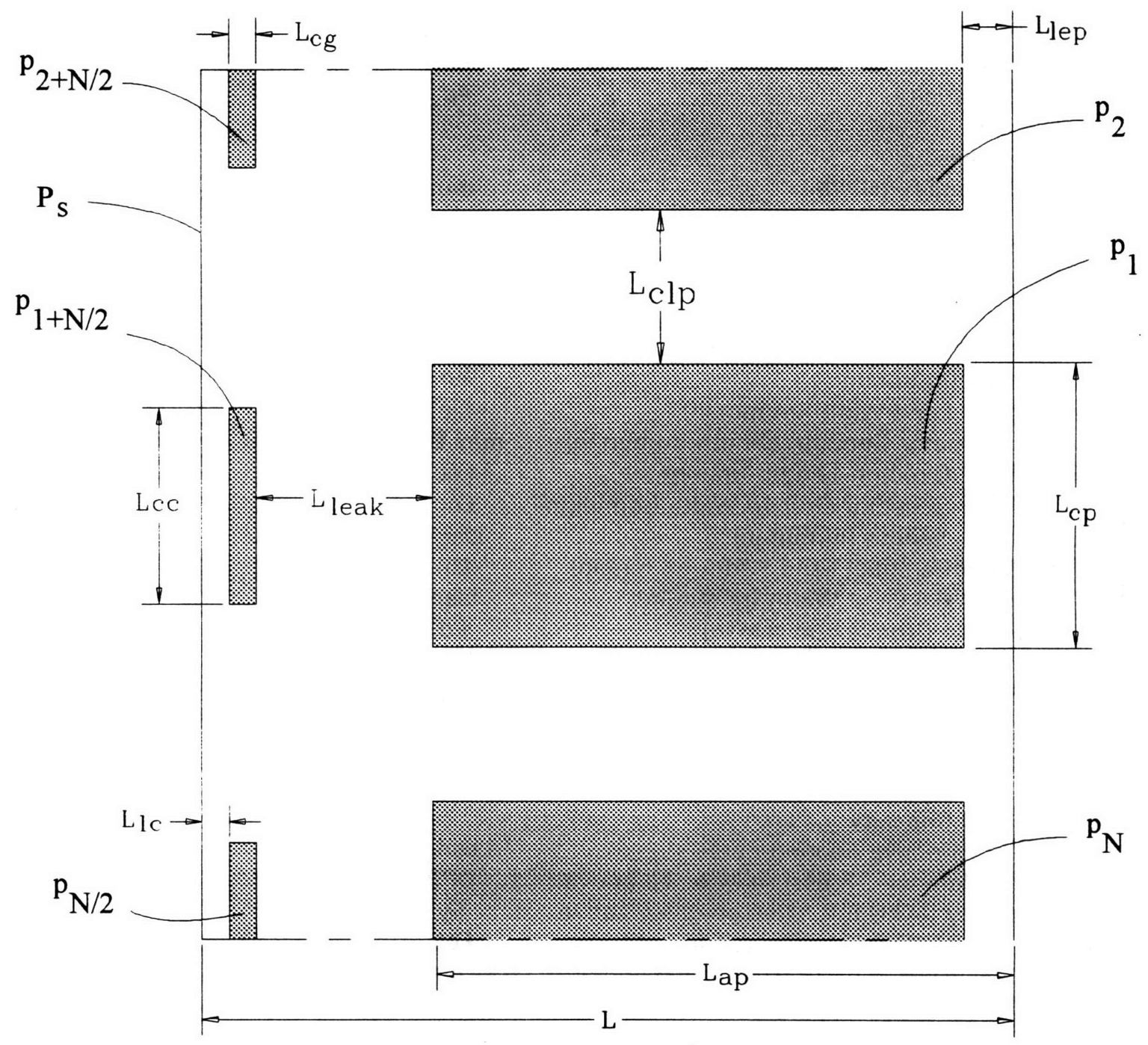

Figure 3.20. Schematic diagram of the pad layout of a self-compensated hydrostatic bearing with compensators that are not completely surrounded by supply pressure. The shaded regions represent depressed regions. 
The procedure for calculating the load-carrying capacity of SC Bearing \#8 is nearly identical to that for the SC Bearing \#7, and will not be described here.

Table 3.11 shows the results of calculations for a representative $80 \mathrm{~mm}$ diameter, $80 \mathrm{~mm}$ long bearing. The pocket land width is $3 \mathrm{~mm}$. The compensator land width is also $3 \mathrm{~mm}$ and the collector groove width is $2 \mathrm{~mm}$. The compensator length is 0.65 times $\pi \mathrm{D} / \mathrm{N}$ in order to make the resistance ratio approximately equal to 0.75 . This configuration was found to approximately maximize the initial stiffness of the bearing. The performance shown is representative of this type of bearing. The initial specific stiffness is 1.03 , the load-carrying efficiency at $75 \%$ of gap closure is 0.450 , and the specific flow rate is 8.1 .

Self-Compensated Bearing \#8 is an excellent bearing. It has exceptional performance and is easier to manufacture than any of the self-compensated bearings previously considered. Its combined qualities of high stiffness, low flow rate, and ease of construction make it attractive in a wide variety of spindle applications. 
Table 3.11

\begin{tabular}{|c|c|c|c|c|}
\hline $\begin{array}{l}\text { Operating Conditions } \\
\text { Supply Pressure (Pa,psi,atm) } \\
\text { Eccentricity Ratio ec/ep } \\
\text { Eccentricity at Pocket End } \\
\text { Eccentricity at Compensator End }\end{array}$ & $\begin{array}{c}\text { Ps } \\
\text { lamda } \\
\text { ep } \\
\text { ec }\end{array}$ & $\begin{array}{r}4.17 E+06 \\
1 \\
0.01 \\
0.01\end{array}$ & 600 & 40.8 \\
\hline \multicolumn{4}{|l|}{ Fluid Properties } & \\
\hline \multicolumn{5}{|l|}{ Bearing Geometry } \\
\hline Axial Length $(m, m m, i n)$ & $\mathrm{L}$ & 0.08 & 80 & 3.15 \\
\hline Number of Pockets & $\mathbf{N}$ & 6 & & \\
\hline Nominal Gap $(m, \mu m$,in) & ho & 0.000015 & 15 & 0.00059 \\
\hline Compensator Land Width ( $\mathrm{m}, \mathrm{mm}$,in) & LIc & 0.003 & 3 & 0.118 \\
\hline Max Compensator Circumf. Length $(\mathrm{m}, \mathrm{mm}, \mathrm{in})$ & $\max \operatorname{Lcc}$ & 0.0419 & 41.89 & 1.649 \\
\hline Compensator Circumf. Length (m, $\mathrm{mm}$, in) & Lcc & 0.0272 & 27.2 & 1.072 \\
\hline Compensator Collector Groove Width (m, $\mathrm{mm}$,in) & Lcg & 0.002 & 2 & 0.079 \\
\hline Leakage Land Length $(\mathrm{m}, \mathrm{mm}$, in) & Lleak & 0.012 & 12 & 0.472 \\
\hline Pocket End Axial Land Width $(\mathrm{m}, \mathrm{mm}, \mathrm{in})$ & Llep & 0.003 & 3 & 0.118 \\
\hline Ratio & & 1.1 & & \\
\hline Pocket Circumferential Land Width (m, $\mathrm{mm}$, in) & Lclp & 0.0219 & 21.9 & 0.864 \\
\hline Pocket Circumferential Length $(\mathrm{m}, \mathrm{mm}, \mathrm{in})$ & Lcp & 0.0199 & 19.9 & 0.785 \\
\hline $\begin{array}{l}\text { Pocket Axial Length ( } \mathrm{m}, \mathrm{mm} \text {,in) } \\
\text { Nominal Resistance Ratio }\end{array}$ & $\begin{array}{l}\text { Lap } \\
\text { gamma }\end{array}$ & $\begin{array}{r}0.063 \\
0.75\end{array}$ & 63.0 & 2.48 \\
\hline \multicolumn{5}{|l|}{ Bearing Load Capacity } \\
\hline Location of Effective Bearing Force & Lcen_L & 0.368 & & \\
\hline Pressure Difference / Pressure @ ec,ep above & DPP & 0.019 & & \\
\hline Load Capacity (N,kN,lbf) @ ec,ep given above & Fy & 275 & 0.28 & 62 \\
\hline Load Carrying Efficiency @ ec,ep given above & Feff & 0.010 & & \\
\hline Stiffness $\left(N / \mu \mathrm{m}, \mathrm{l}_{\mathrm{f}} / \mu \mathrm{in}\right) @$ ec,ep given above & Ky & 1837 & 10.49 & \\
\hline Specific Stiffness@ec,ep given above & Kspec & 1.033 & & \\
\hline Initial Specific Stiffness & Kspec,o & 1.034 & & \\
\hline Load Carrying Efficiency @ 75\% of gap closure & Feff, 0.75 & 0.450 & & \\
\hline Flow Rate and Pumping Power & & & & \\
\hline $\begin{array}{l}\text { Total Flow Rate (m`3/s,lpm,gpm) } \\
\text { Pumpina Power (W, kW hp) }\end{array}$ & $\begin{array}{l}\text { Osupply } \\
\text { Ppump }\end{array}$ & $\begin{array}{r}2.30 E-05 \\
96\end{array}$ & $\begin{array}{r}1.38 \\
0.096\end{array}$ & $\begin{array}{r}0.36 \\
0.128\end{array}$ \\
\hline Specific Flow Rate & Qspec & 8.1 & & \\
\hline $\begin{array}{l}\text { Ratio of Specific Initial Stiffness to Specific Flow } \\
\text { Rate }\end{array}$ & K/Qspec & 0.1275 & & \\
\hline
\end{tabular}




\section{K. Self-Compensated Bearing \#9}

The pad layout of Self-Compensated Bearing \#9 is shown in Figure 3.21. This bearing differs significantly from the previously described self-compensating bearings. Fluid enters the bearing at the left side, which is at supply pressure, $P_{s}$, as indicated in Figure 3.21. The fluid then flows axially down grooves which are at a depth $d_{c}$. The depth of the grooves is typically 2 to 3 times the bearing clearance, $h_{0}$. As the fluid flows axially down these grooves, some of it leaks circumferentially across the axial lands which separate them. These lands help to reduce circumferential leakage of fluid around the bearing and increase the load-carrying capacity of the bearing. At the end of the grooves, the fluid transitions back to the bearing clearance, $h_{0}$, and across the outlet lands to atmospheric pressure.

It will be shown in this section this bearing exhibits very poor performance compared to the other bearings considered in this chapter. Also, its load capacity is sensitive to the ratio of two tight clearances, $d_{d} / h_{0}$, which makes its it difficult to manufacture. However, this bearing is used in many commercial aerostatic spindles because it does not experience pneumatic hammer instability when a compressible fluid such as air is used [5]. Also, it will be shown in Chapter 4 that, although the bearing itself has low stiffness, it can be used to build spindles with relatively high stiffness. 


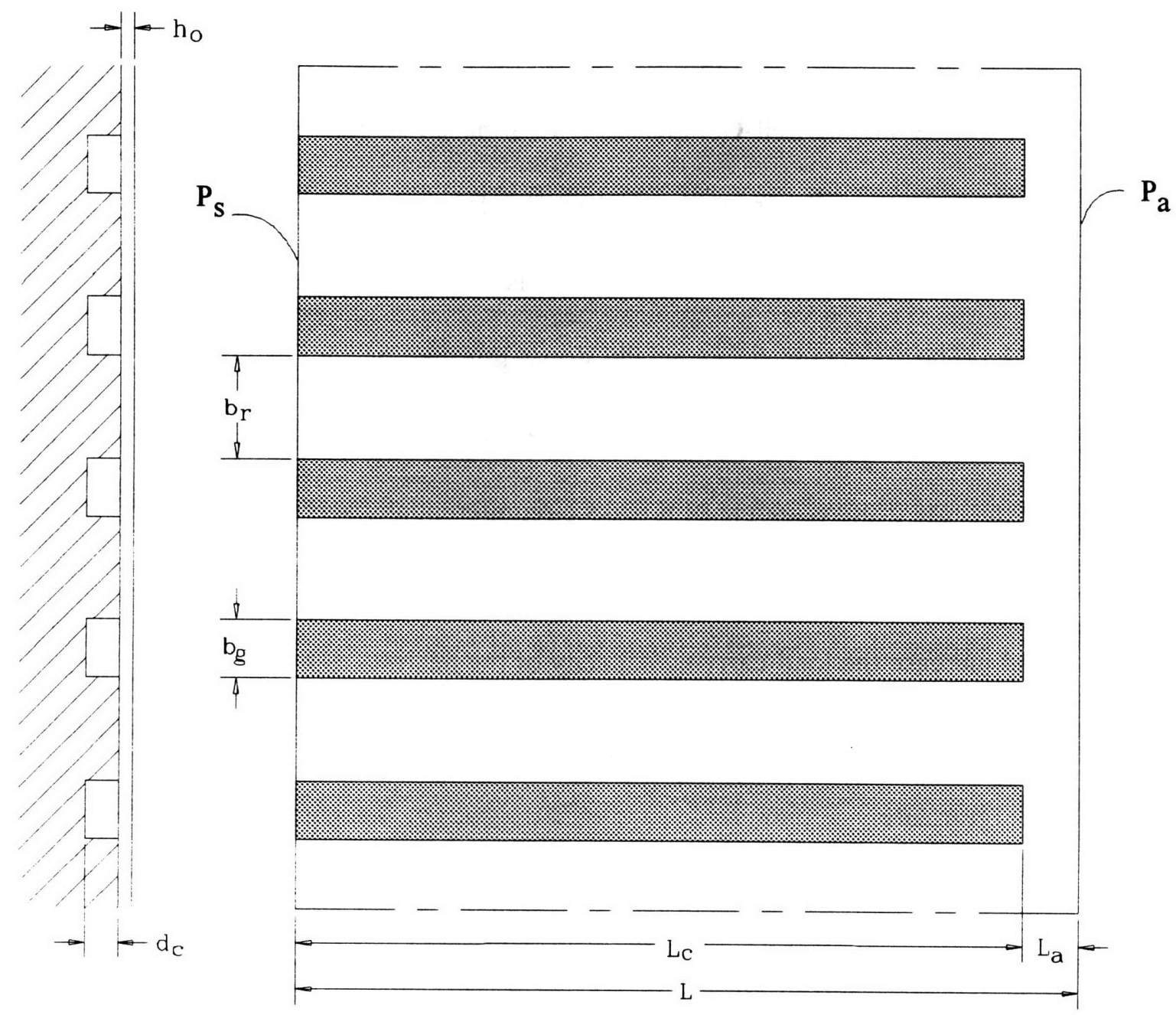

Figure 3.21. Schematic diagram of the pad layout of a step-compensated hydrostatic bearing with axial lands between its compensating pockets. The shaded regions represent regions that are at a depth of $d_{c}$. The magnitude of both clearances are exxagerated for clarity.

The procedure for calculating the load-carrying capacity of SC Bearing \#9 is significantly different than that of the other bearings considered in this chapter. The method is more complicated because the pressure in the grooves varies along the length of the compensation grooves. To account for this effect, the groove region of the bearing is 
broken up into $\mathrm{M}$ sections, as shown in Figure 3.22. The incremental groove resistances and axial land resistances will be considered as the fluid leaks circumferentially between successive grooves and flows axially down the length of the grooves. The fluid circuit used to model this fluid flow representation is Fluid Circuit \#4 of Appendix B. The resistances used to set up this circuit will be developed in this section.

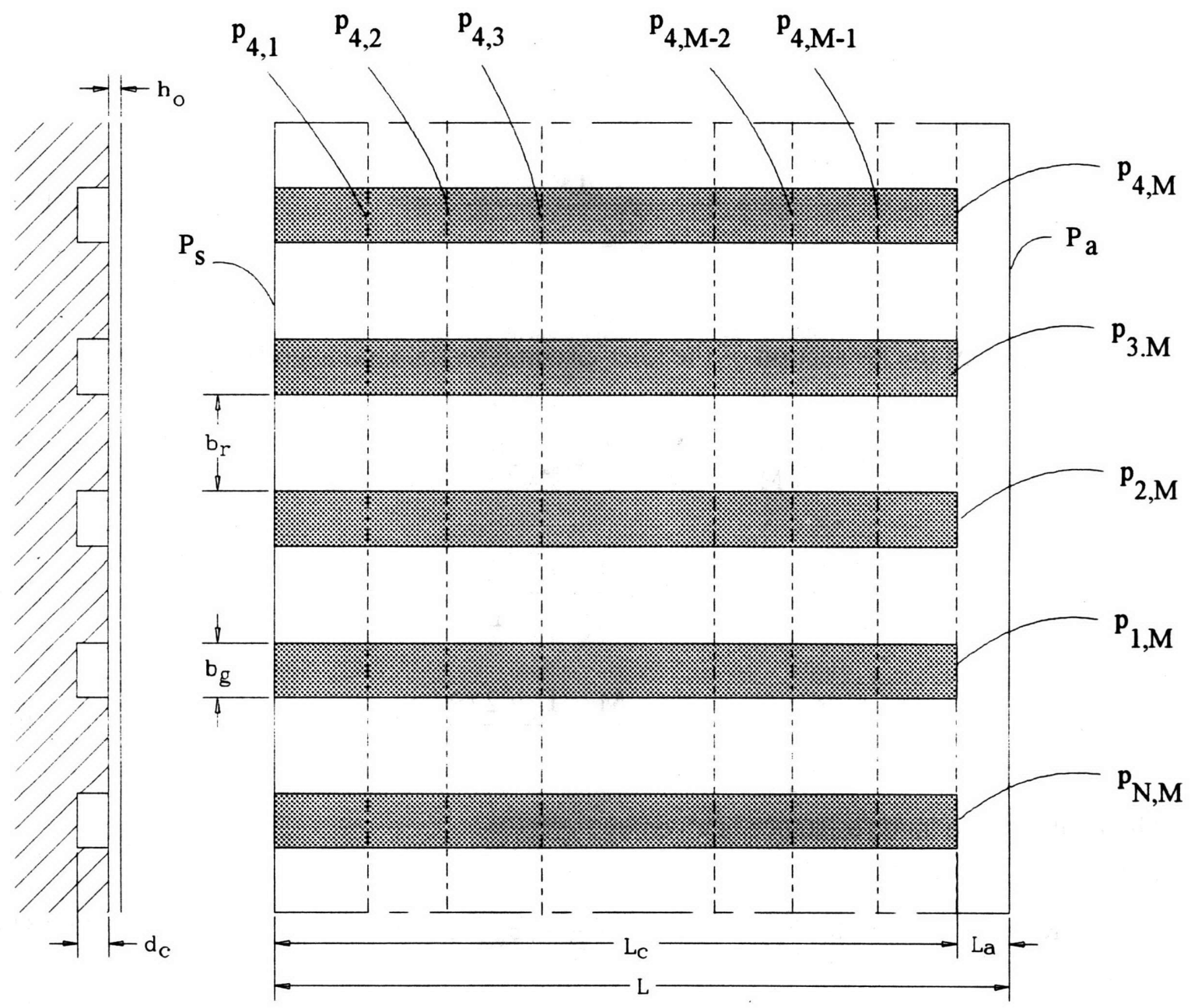

Figure 3.22. Schematic diagram of SC Bearing \#9. The shaded regions represent regions that are at a depth of $d_{c}$. The magnitude of both clearances are exagerated for clarity. 
The resistances for Fluid Circuit \#4 of Appendix B will now be developed. The jth groove section resistance of the ith groove is given by:

$$
\mathbf{R}_{c, i, j}=\mathbf{R}_{\text {circum }}\left(\mu, h_{o}+d_{c}, \frac{L_{c}}{M}, b_{g}, \theta_{i}, e_{\text {circum }}\left(e_{i, j}^{1-}, e_{i, j}^{1+}, \theta_{i}\right)\right)
$$

where:

$$
\begin{array}{r}
e_{i, j}^{1-}=\frac{e_{c}+\left(e_{p}-e_{c}\right)\left[(j-1) \frac{L_{c} / M}{L}\right]}{1+\frac{d_{c}}{h_{o}}} \\
e_{i, j}^{l+}=\frac{e_{c}+\left(e_{p}-e_{c}\right)\left[(j-1) \frac{L_{c} / M}{L}+\frac{L_{c} / M}{L}\right]}{1+\frac{d_{c}}{h_{o}}}
\end{array}
$$

The resistance of the jth section of the ither circumferential land is given by:

$$
\begin{gathered}
R_{c b, i, j}=R_{a x i a l}\left(\mu, h_{c}, b_{r}, \frac{L_{c}}{M}, \theta_{i}+\frac{b_{r}+b_{g}}{D}, e_{\text {axial }}\left(e_{i, j}^{2-}, e_{i, j}^{2+}, \theta_{i}+\frac{b_{r}+b_{g}}{D}\right)\right) \\
e_{i, j}^{2-}=e_{c}+\left(e_{p}-e_{c}\right)\left[j \frac{L_{c} / M}{L}-\frac{L_{c} / 2 / M}{L}\right] \\
e_{1, j}^{2+}=e_{c}+\left(e_{p}-e_{c}\right)\left[j \frac{L_{c} / M}{L}+\frac{L_{c} / 2 / M}{L}\right]
\end{gathered}
$$

The final leakage land resistance is given by:

$$
R_{b, i}=R_{a x i a l}\left(\mu, h_{0}, b_{r}, \frac{L_{c} / 2}{M}+L_{1}^{*}, \theta_{i}+\frac{b_{r}+b_{g}}{D}, e_{\text {axial }}\left(e_{3}, e_{4}, \theta_{i}+\frac{b_{r}+b_{g}}{D}\right)\right)
$$

where:

$$
\mathrm{L}_{1}^{*} \approx 0.4 \mathrm{~L}_{\mathrm{a}}
$$




$$
\begin{gathered}
e_{3}=e_{c}+\left(e_{p}-e_{c}\right)\left[\frac{L_{c}-L_{c} / 2 / M}{L}\right] \\
e_{4}=e_{c}+\left(e_{p}-e_{c}\right)\left[\frac{L_{c}}{L}\right]
\end{gathered}
$$

The outlet land resistances are given by:

$$
\mathbf{R}_{\mathrm{a}, \mathrm{i}}=\mathbf{R}_{\text {circum }}\left(\mu, \mathrm{h}_{\mathrm{o}}, \mathrm{L}_{\mathrm{a}}, \mathrm{b}_{\mathrm{g}}+\mathrm{L}_{2}^{*}, \theta_{\mathrm{i}}, \mathbf{e}_{\text {circum }}\left(\mathrm{e}_{4}, \mathrm{e}_{\mathrm{p}}, \theta_{\mathrm{i}}\right)\right)
$$

where:

$$
\mathrm{L}_{2}^{*} \approx 0.8\left\{\min \left(\mathrm{L}_{\mathrm{a}}, \mathrm{br}\right)\right\}
$$

Now that all of the resistances of Circuit \#4 of Appendix B have been developed, the circuit may be solved for the flow rates and the pressures.

The next step is to calculate the net bearing force generated by those pressures as described in previous sections. First the groove and land areas nearest the inlet are considered:

$$
\begin{aligned}
& \mathrm{F}_{\mathrm{y}, \mathrm{i}, \mathrm{l}}^{\mathrm{gr}}=\mathrm{D} \frac{\mathrm{L}_{\mathrm{c}}}{\mathrm{M}} \sin \left(\frac{\mathrm{b}_{\mathrm{g}}}{\mathrm{D}}\right)\left(\frac{\mathrm{P}_{\mathrm{s}}+\mathrm{p}_{\mathrm{i}, 1}}{2}\right) \cos \left(\theta_{\mathrm{i}}\right)
\end{aligned}
$$

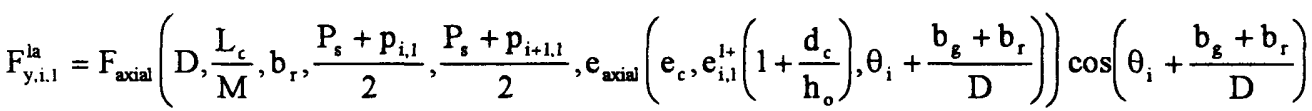

The forces generated by the jth section of the bearing are given by:

$$
\begin{aligned}
& F_{y, i, j}^{g r}=D \frac{L_{c}}{M} \sin \left(\frac{b_{g}}{D}\right)\left(\frac{p_{i, j-1}+p_{i, j}}{2}\right) \cos \left(\theta_{i}\right)
\end{aligned}
$$

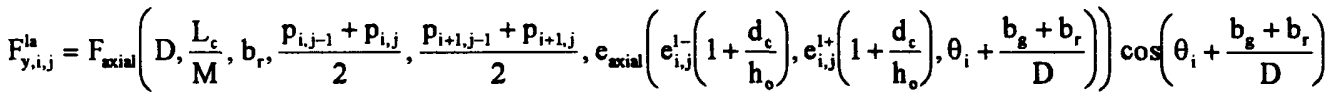

where $j=2,3 \ldots M$. The forces generated by the final sections of the bearing are given by: 


$$
\begin{gathered}
\mathrm{F}_{\mathrm{y}, \mathrm{i}, \mathrm{M}+1}^{\mathrm{r}}=\mathrm{D} \frac{\mathrm{L}_{\mathrm{a}}}{\mathrm{M}} \sin \left(\frac{\mathrm{b}_{\mathrm{g}}}{\mathrm{D}}\right)\left(\frac{\mathrm{p}_{\mathrm{i}, \mathrm{M}}}{2}\right) \cos \left(\theta_{\mathrm{i}}\right) \\
\mathrm{F}_{\mathrm{y}, \mathrm{i}, \mathrm{M}+1}^{\mathrm{la}}=\mathrm{D} \frac{\mathrm{L}_{\mathrm{a}}}{\mathrm{M}} \sin \left(\frac{\mathrm{b}_{\mathrm{r}}}{\mathrm{D}}\right)\left(\frac{\mathrm{p}_{\mathrm{i}, \mathrm{M}}+\mathrm{p}_{\mathrm{i}+\mathrm{l}, \mathrm{M}}}{2}\right) \cos \left(\theta_{\mathrm{i}}+\frac{\mathrm{b}_{\mathrm{r}}+\mathrm{b}_{\mathrm{g}}}{\mathrm{D}}\right)
\end{gathered}
$$

The total net bearing force in the y-direction is found by summing all of these forces for all $\mathrm{N}$ grooves and $\mathrm{M}$ sections.

Table 3.12 shows the results of calculations for a representative $80 \mathrm{~mm}$ diameter, $80 \mathrm{~mm}$ long bearing. The number of compensator sections used in the calculations was $\mathrm{M}$ $=5$; this was found to be adequate since the calculated stiffness changed by less than $1 \%$ going from $M=4$ to $M=5$. The outlet land width is $3 \mathrm{~mm}$. A rib ratio, $b_{r} / b_{g}$, of 0.85 was found to maximize the initial bearing stiffness. A clearance ratio, $d_{d} / h_{0}$, of 2.5 was found to maximize the inital bearing stiffness. The performance shown is representative of this type of bearing. The initial specific stiffness is 0.27 , the load-carrying efficiency at $75 \%$ of gap closure is 0.18 , and the specific flow rate is 9.6 .

The performance of SC Bearing \#9 exhibits very good performance compared to the other bearings considered in this chapter. However, this bearing is used in many commercial aerostatic spindles because it does not experience pneumatic hammer instability when a compressible fluid such as air is used. 
Table 3.12

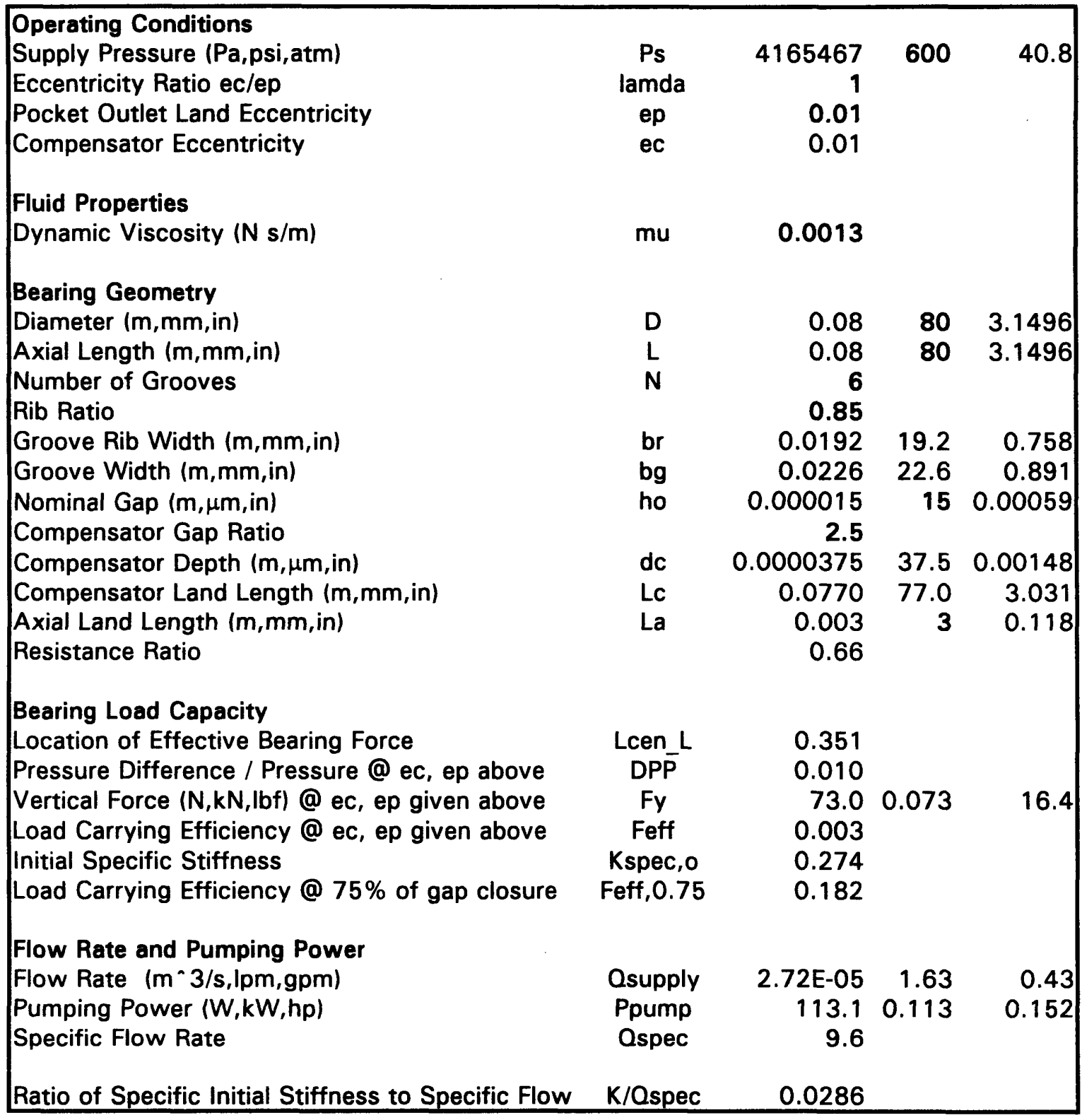

There is an optimum ratio, $d / h_{0}$, that maximizes the bearing stiffness. As shown in Figure 3.23, the six-pocket bearing of Table 3.12 has an optimal clearance ratio of approximately 2.5 that maximizes the initial stiffness of the bearing. Although the optimal clearance ratio for maximum bearing load capacity is approximately 2.0 , the bearing stiffness is nearly optimal for a clearance ratio of 2.5 . 


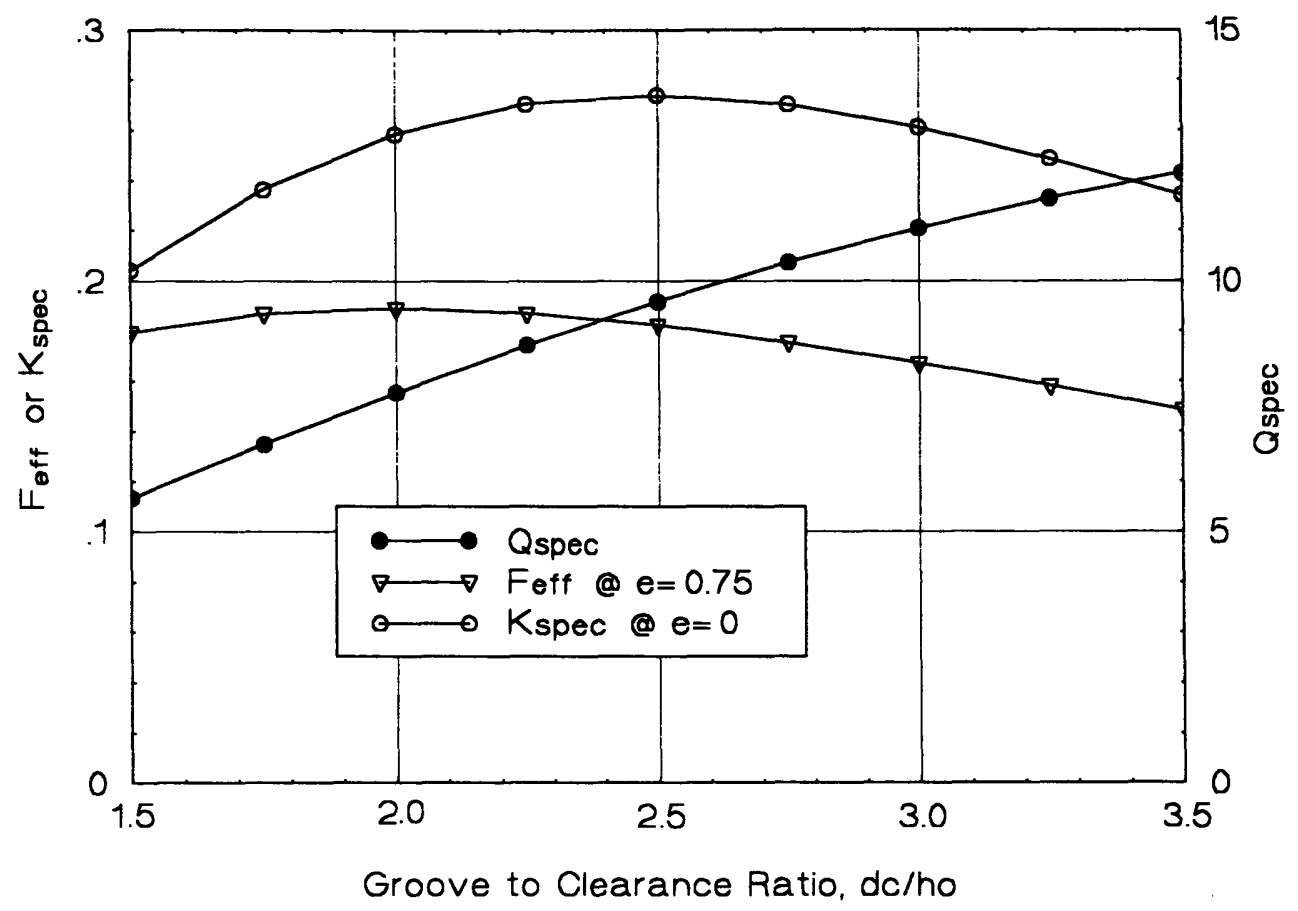

Figure 3.23. Effect of the groove clearance ratio on the 6-pocket, $80 \mathrm{~mm}$ diameter SC Bearing \#9 of Table 3.12 .

\section{L. Self-Compensated Bearing \#10}

The pad layout of Self-Compensated Bearing \#10 is shown in Figure 3.24. This bearing differs significantly from the previously described self-compensating bearings. Fluid enters the bearing at the left side, which is at supply pressure, $P_{s}$, as indicated in Figure 3.24. The fluid then flows across compensation lands and down grooves which twist circumferentially to the load-supporting pockets. The fluid then flows across the pocket lands to atmospheric pressure. The lands that separate the grooves and the pockets serve to enable a pressure difference to exist between them. This bearing has the advantage that all of the fluid routing is done on the bearing surface [6], thereby simplifying the spindle manufacture. 


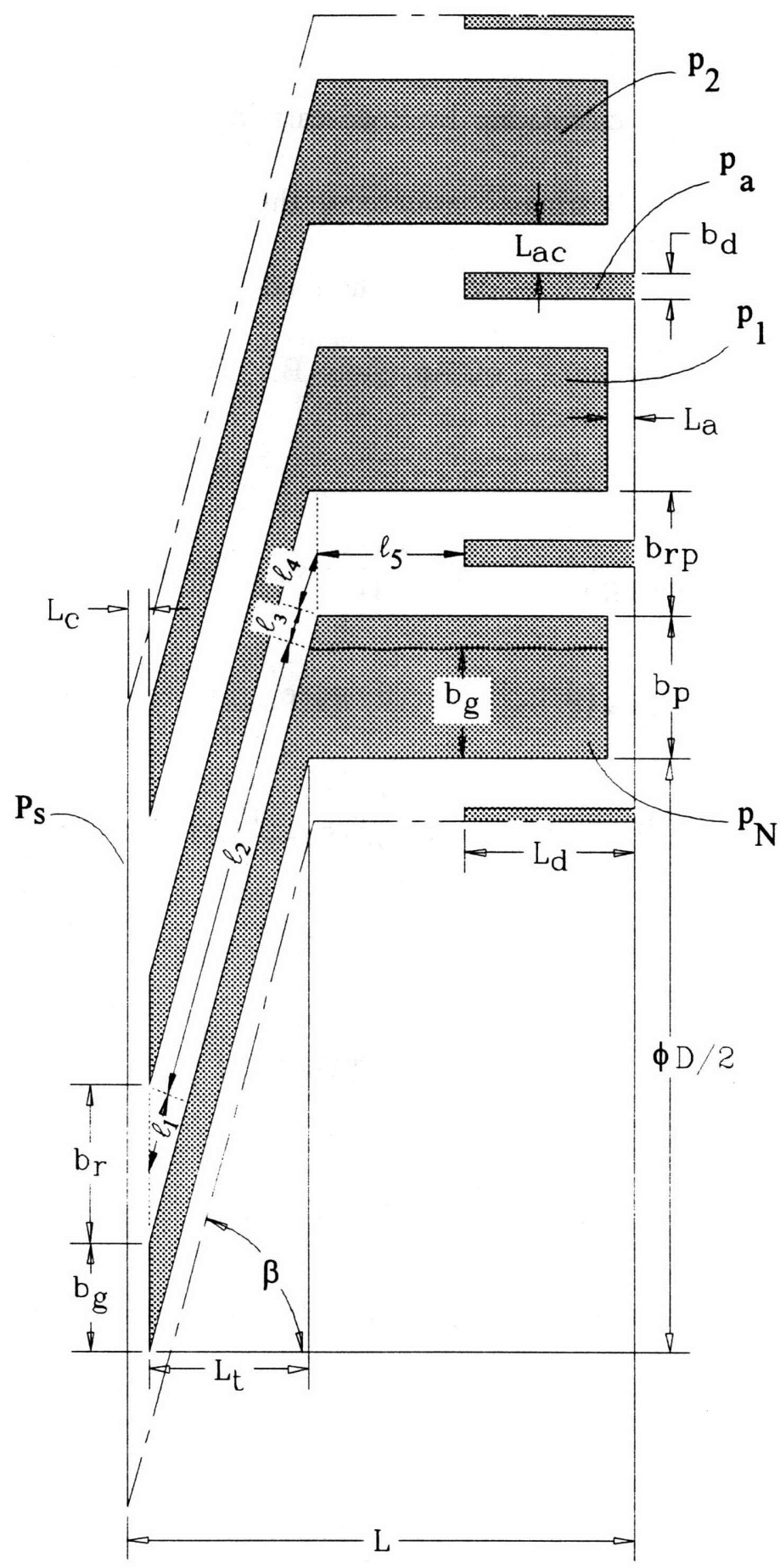

Figure 3.24. Schematic diagram of the pad layout of a self-compensated hydrostatic bearing in which the fluid is routed from the compensation resistances to the supporting pockets via grooves machined into the surface of the bearing itself. The shaded regions represent regions that are at a depth of $d_{c}$. 
The procedure for calculating the load-carrying capacity of SC Bearing \#10 is similar to that of the other bearings considered in this chapter, except that the slanting of the lands and grooves must be considered. The fluid circuit used to model this fluid flow representation is Fluid Circuit \#2 of Appendix B. The compensation resistances are broken up into three parts:

$$
\begin{aligned}
& \mathbf{R}_{\mathrm{cl}, \mathrm{i}}=\mathbf{R}_{\text {croum }}\left(\mu, \mathrm{h}_{\mathrm{o}}, \mathrm{L}_{\mathrm{c}}+\frac{\mathbf{b}_{\mathrm{r}}}{2 \tan (\beta)}, \mathbf{b}_{\mathrm{r}}, \theta_{\mathrm{i}}+\phi-\frac{\mathbf{b}_{\mathrm{g}}+\mathrm{b}_{\mathrm{r}}}{\mathrm{D}}, \mathbf{e}_{\text {ciroum }}\left(\mathrm{e}_{\mathrm{c}}, \mathrm{e}_{1}, \theta_{\mathrm{i}}+\phi-\frac{\mathrm{b}_{\mathrm{g}}+\mathbf{b}_{\mathrm{r}}}{\mathrm{D}}\right)\right) \\
& \mathbf{R}_{\mathrm{c} 2, \mathrm{i}}=\mathbf{R}_{\text {circum }}\left(\mu, \mathrm{h}_{\mathrm{o}}, \mathrm{L}_{\mathrm{c}}, \mathrm{b}_{\mathrm{g}}, \theta_{\mathrm{i}}+\phi, \mathbf{e}_{\text {circum }}\left(\mathrm{e}_{\mathrm{c}}, \mathrm{e}_{1}, \theta_{\mathrm{i}}+\phi\right)\right) \\
& \mathbf{R}_{\mathrm{c} 3, \mathrm{i}}=\mathbf{R}_{\text {circum }}\left(\mu, \mathrm{h}_{\mathrm{o}}, \mathrm{L}_{\mathrm{c}}, \mathrm{L}_{1}^{*}, \theta_{\mathrm{i}}+\phi+\frac{L_{1}^{*}}{\mathrm{D}}, \mathbf{e}_{\text {circum }}\left(\mathbf{e}_{\mathrm{c}}, \mathbf{e}_{1}, \theta_{\mathrm{i}}+\phi+\frac{\mathrm{L}_{1}^{*}}{\mathrm{D}}\right)\right)
\end{aligned}
$$

where:

$$
\begin{aligned}
& e_{1}=e_{c}+\left(e_{p}-e_{c}\right) \frac{L_{c}}{L} \\
& L_{1}^{*} \approx 0.4 b_{r}\left(1-\beta \frac{2}{\pi}\right)
\end{aligned}
$$

The three compensation resistances act in parallel, so the overall compensation resistance to use in Circuit \#2 of Appendix B is:

$$
\mathbf{R}_{\mathrm{c}, \mathrm{i}}=\frac{1}{\frac{1}{\mathbf{R}_{\mathrm{c} 1, \mathrm{i}}}+\frac{1}{\mathbf{R}_{\mathrm{c} 2, \mathrm{i}}}+\frac{1}{\mathbf{R}_{\mathrm{c}, 3, \mathrm{i}}}}
$$

Next consider the lands over which fluid leaks from groove to groove and pocket to pocket. The slating lands are broken up into four parts, as shown in Figure 3.24. The first region may be neglected, but its resistance is given conservatively by: 


$$
\mathbf{R}_{\mathrm{bl}, \mathrm{i}}=\frac{12 \mu}{\mathrm{h}_{\mathrm{o}}^{3}} \frac{\mathrm{b}_{\mathrm{r}} \cos (\beta)}{l_{1}^{*}} \frac{1}{\left(1-\mathrm{e}_{2} \cos \left(\theta_{\mathrm{i}}+\phi+\frac{\mathrm{b}_{\mathrm{g}}+\mathrm{b}_{\mathrm{r}}}{\mathrm{D}}\right)\right)^{3}}
$$

where:

$$
\begin{array}{r}
l_{1} \approx 0.8 \mathrm{~b}_{\mathrm{r}} \sin (\beta) \\
l_{1} \approx \frac{\mathrm{b}_{\mathrm{r}}}{2} \sin (\beta) \\
\mathrm{e}_{2}=\mathrm{e}_{\mathrm{c}}+\left(\mathrm{e}_{\mathrm{p}}-\mathrm{e}_{\mathrm{c}}\right) \frac{\mathrm{L}_{\mathrm{c}}+l_{1} \cos (\beta)}{\mathrm{L}}
\end{array}
$$

The resistance of the second slanted region is given approximately by:

$$
\begin{gathered}
\mathrm{R}_{\mathrm{b} 2 \mathrm{i}}=\frac{12 \mu}{\mathrm{h}_{\mathrm{o}}^{3}} \frac{\mathrm{b}_{\mathrm{r}} \cos (\beta)}{l_{2}} \frac{1}{\left(1-\mathrm{e}_{3} \cos \left(\theta_{\mathrm{i}}+\frac{\phi}{2}+\frac{\mathrm{b}_{\mathrm{g}}+\mathrm{b}_{\mathrm{r}}}{\mathrm{D}}\right)\right)^{3}} \\
\mathrm{l}_{2}=\left[\mathrm{L}_{\mathrm{t}}^{2}+\left(\frac{\phi \mathrm{D}}{2}\right)^{2}\right]^{1 / 2}-2\left(\frac{\mathrm{b}_{\mathrm{r}}}{2}\right) \sin (\beta) \\
\mathrm{e}_{3}=\mathrm{e}_{\mathrm{c}}+\left(\mathrm{e}_{\mathrm{p}}-\mathrm{e}_{\mathrm{c}}\right) \frac{\mathrm{L}_{\mathrm{c}}+\frac{\mathrm{L}_{\mathrm{t}}}{2}}{\mathrm{~L}}
\end{gathered}
$$

The resistance of the third slanted region is given approximately by:

$$
\begin{gathered}
R_{b 3, i}=\frac{12 \mu}{h_{o}^{3}} \frac{b_{r} \cos (\beta)}{l_{3}} \frac{1}{\left(1-e_{4} \cos \left(\theta_{i+1}-\frac{b_{g}}{D}-\frac{b_{p}-b_{g}}{D}-\frac{b_{r} \sin (\beta) \cos (\beta)}{D}\right)\right)^{3}} \\
e_{4}=e_{c}+\left(e_{p}-e_{c}\right) \frac{L_{c}+L_{t}-\frac{b_{p}-b_{g}}{2 \tan (\beta)}}{L}
\end{gathered}
$$




$$
l_{3}=\frac{\mathrm{b}_{\mathrm{p}}-\mathrm{b}_{\mathrm{g}}}{\sin (\beta)}
$$

The fourth region may be omitted but its resistance is given conservatively by:

$$
\mathbf{R}_{\mathrm{b} 4, \mathrm{i}}=\frac{12 \mu}{\mathrm{h}_{\mathrm{o}}^{3}} \frac{\mathrm{b}_{\mathrm{r}} \cos (\beta)}{l_{4}} \frac{1}{\left(1-\mathrm{e}_{4} \cos \left(\theta_{\mathrm{i}}+\frac{\mathrm{b}_{\mathrm{g}}+\mathrm{b}_{\mathrm{rp}}}{\mathrm{D}}\right)\right)^{3}}
$$

where:

$$
l_{4} \approx \frac{b_{p}-b_{g}}{2 \tan (\beta)}+\frac{b_{r}}{2} \sin (\beta)-\frac{l_{3}}{2}
$$

The resistance of the land between the pockets is given by:

$$
\begin{aligned}
& \mathbf{R}_{\mathrm{bs}, \mathrm{i}}=\mathbf{R}_{\text {axial }}\left(\mu, \mathbf{h}_{\mathrm{o}}, \mathrm{b}_{\mathrm{p}}, l_{\mathrm{s}}-l_{2}^{\prime}, \theta_{\mathrm{i}}+\frac{\mathbf{b}_{\mathrm{g}}+\mathbf{b}_{\mathrm{pp}}}{\mathrm{D}}, \mathbf{e}_{\text {axial }}\left(\mathbf{e}_{4}, \mathbf{e}_{5}, \theta_{\mathrm{i}}+\frac{\mathbf{b}_{\mathrm{g}}+\mathbf{b}_{\mathrm{p}}}{\mathrm{D}}\right)\right) \\
& \ell_{s}=\mathrm{L}-\mathrm{L}_{\mathrm{c}}-\mathrm{L}_{\mathrm{t}}-\mathrm{L}_{\mathrm{d}}-\frac{\mathrm{b}_{\mathrm{p}}-\mathrm{b}_{\mathrm{g}}}{\tan (\beta)} \\
& l_{2}^{\prime} \approx b_{d} \\
& e_{5}=e_{p}+\left(e_{c}-e_{p}\right) \frac{L_{d}}{L}
\end{aligned}
$$

The leakage resistances are combined in parallel to form the overall leakage resistance to use in Circuit \#2 of Appendix B:

$$
\mathbf{R}_{\mathrm{b}, \mathrm{i}}=\frac{1}{\frac{1}{\mathbf{R}_{\mathrm{b} 1, \mathrm{i}}}+\frac{1}{\mathbf{R}_{\mathrm{b} 2, \mathrm{i}}}+\frac{1}{\mathbf{R}_{\mathrm{b} 3, \mathrm{i}}}+\frac{1}{\mathbf{R}_{\mathrm{b} 4, \mathrm{i}}}+\frac{1}{\mathbf{R}_{\mathrm{b} 5, \mathrm{i}}}}
$$

There are four outlet land resistances. The two circumferential land resistances are given by:

$$
\mathbf{R}_{\mathrm{al}, \mathrm{i}}=\mathbf{R}_{\text {cram }}\left(\mu, \mathrm{h}_{\mathrm{o}}, \mathrm{L}_{\mathrm{a}}, \mathrm{b}_{\mathrm{g}}+\mathrm{l}_{2}^{*}, \theta_{\mathrm{i}}, \mathrm{e}_{\text {craum }}\left(\mathrm{e}_{6}, \mathrm{e}_{\mathrm{p}}, \theta_{\mathrm{i}}\right)\right)
$$




$$
R_{a 2, i}=R_{\text {cram }}\left(\mu, h_{0}, L_{a}, b_{p}-b_{g}+l_{3}^{*}, \theta_{i}-\frac{b_{p}}{D}, e_{\text {ciram }}\left(e_{6}, e_{p}, \theta_{i}-\frac{b_{p}}{D}\right)\right)
$$

where:

$$
\begin{aligned}
& e_{6}=e_{p}+\left(e_{c}-e_{p}\right) \frac{L_{a}}{L} \\
& l_{3}^{\prime} \approx 0.4 \min \left\{L_{a}, L_{a c}\right\}
\end{aligned}
$$

The axial land resistances are given by:

$$
\begin{gathered}
R_{a 3, i}=R_{a x i a l}\left(\mu, h_{0}, L_{a c}, L_{d}-L_{a}+l_{4}^{*}, \theta_{i}+\frac{b_{g}+L_{a c}}{D}, e_{a x i a l}\left(e_{5}, e_{6}, \theta_{i}+\frac{b_{g}+L_{a c}}{D}\right)\right) \\
R_{a 4, i}=R_{a x i a l}\left(\mu, h_{0}, L_{a c}, L_{d}-L_{2}+l_{3}^{*}, \theta_{i}-\frac{b_{g}+2\left(b_{p}-b_{g}\right)+L_{a c}}{D}, e_{a x i l a}\left(e_{s}, e_{6}, \theta_{i}-\frac{b_{g}+2\left(b_{p}-b_{g}\right)+L_{a c}}{D}\right)\right) \\
l_{4}^{*} \approx 0.8 L_{a c}
\end{gathered}
$$

The outlet resistances are combined in parallel to form the overall outlet resistance to use in Circuit \#2 of Appendix B:

$$
\mathbf{R}_{\mathrm{a}, \mathrm{i}}=\frac{1}{\frac{1}{\mathbf{R}_{\mathrm{a} 1, \mathrm{i}}}+\frac{1}{\mathbf{R}_{\mathrm{a} 2, \mathrm{i}}}+\frac{1}{\mathbf{R}_{\mathrm{a} 3, \mathrm{i}}}+\frac{1}{\mathrm{R}_{\mathrm{a} 4, \mathrm{i}}}}
$$

Now that all of the resistances of Circuit \#2 of Appendix B have been developed, the circuit may be solved for the flow rates and the pressures.

The next step is to calculate the net bearing force generated by those pressures as described in previous sections. The net force in the $y$-direction of each portion of the bearing are given by:

$$
\mathrm{F}_{\mathrm{y}, \mathrm{i}}^{1}=\mathrm{L}_{\mathrm{a}} \mathrm{D} \sin \left(\frac{\mathrm{b}_{\mathrm{g}}}{\mathrm{D}}\right)\left(\frac{\mathrm{p}_{\mathrm{i}}}{2}\right) \cos \left(\theta_{\mathrm{i}}\right)
$$




$$
\begin{aligned}
& F_{y, i}^{2}=L_{a} D \sin \left(\frac{b_{p}-b_{g}}{D}\right)\left(\frac{p_{i}}{2}\right) \cos \left(\theta_{i}-\frac{b_{p}}{D}\right) \\
& \mathrm{F}_{\mathrm{y}, \mathrm{i}}^{3}=\mathrm{L}_{\mathrm{a}} \mathrm{D} \sin \left(\frac{\mathrm{L}_{\mathrm{ac}}}{\mathrm{D}}\right)\left(\frac{\mathrm{p}_{\mathrm{i}}}{4}\right) \cos \left(\theta_{\mathrm{i}}+\frac{\mathrm{b}_{\mathrm{g}}+\mathrm{L}_{\mathrm{ac}}}{\mathrm{D}}\right) \\
& \mathrm{F}_{\mathrm{y}, \mathrm{i}}^{4}=\mathrm{L}_{\mathrm{a}} \mathrm{D} \sin \left(\frac{\mathrm{L}_{\mathrm{ac}}}{\mathrm{D}}\right)\left(\frac{\mathrm{p}_{\mathrm{i}}}{4}\right) \cos \left(\theta_{\mathrm{i}}-\frac{\mathrm{b}_{\mathrm{g}}}{\mathrm{D}}-\frac{2\left(\mathrm{~b}_{\mathrm{p}}-\mathrm{b}_{\mathrm{g}}\right)}{\mathrm{D}}-\frac{\mathrm{L}_{\mathrm{ac}}}{\mathrm{D}}\right) \\
& F_{y, 1}^{5}=\left(L_{d}-L_{a}\right) D \sin \left(\frac{L_{a c}}{D}\right)\left(\frac{p_{i}}{2}\right) \cos \left(\theta_{i}+\frac{b_{g}+L_{a c}}{D}\right) \\
& F_{y, 1}^{6}=\left(L_{d}-L_{a}\right) D \sin \left(\frac{L_{a c}}{D}\right)\left(\frac{p_{i}}{2}\right) \cos \left(\theta_{i}-\frac{b_{g}}{D}-\frac{2\left(b_{p}-b_{g}\right)}{D}-\frac{L_{a c}}{D}\right) \\
& F_{y, 1}^{7}=\left(L-L_{c}-L_{t}-L_{a}\right) D \sin \left(\frac{b_{g}}{D}\right)\left(p_{i}\right) \cos \left(\theta_{i}\right) \\
& F_{y, i}^{8}=\left(L-L_{c}-L_{t}-L_{a}-\frac{b_{p}-b_{g}}{\tan (\beta)}\right) D \sin \left(\frac{b_{p}-b_{g}}{D}\right)\left(p_{i}\right) \cos \left(\theta_{i}-\frac{b_{p}}{D}\right) \\
& F_{y, 1}^{8}=\left(\frac{b_{p}-b_{g}}{2 \tan (\beta)}\right) D \sin \left(\frac{b_{p}-b_{g}}{D}\right)\left(p_{i}\right) \cos \left(\theta_{i}-\frac{b_{g}}{D}-\frac{2\left(b_{p}-b_{g}\right)}{3 D}\right) \\
& F_{y, 1}^{9}=F_{\text {axial }}\left(D, l_{5}, b_{\text {rp }}, p_{i}, p_{i+1}, e_{\text {axial }}\left(e_{4}, e_{5}, \theta_{i}+\frac{b_{g}}{D}+\frac{b_{r p}}{D}\right)\right) \cos \left(\theta_{i}+\frac{b_{g}}{D}+\frac{b_{r p}}{D}\right) \\
& F_{y, i}^{10}=\left(\frac{b_{p}-b_{g}}{\tan (\beta)}\right) D \sin \left(\frac{b_{r}+b_{r p}}{2 D}\right)\left(\frac{p_{i}+p_{i+1}}{2}\right) \cos \left(\theta_{i}+\frac{b_{g}}{D}+\frac{b_{r p}}{D}\right) \\
& F_{y, i}^{\prime \prime}=\left(L_{t}\right) D \sin \left(\frac{b_{g}}{D}\right) \frac{\sin \left(\frac{\phi}{2}\right)}{\left(\frac{\phi}{2}\right)}\left(p_{i}\right) \cos \left(\theta_{i}+\frac{\phi}{2}\right)
\end{aligned}
$$




$$
\begin{gathered}
F_{y, i}^{12}=\left(L_{t}\right) D \sin \left(\frac{b_{r}}{D}\right) \frac{\sin \left(\frac{\phi}{2}\right)}{\left(\frac{\phi}{2}\right)}\left(\frac{p_{i}+p_{i+1}}{2}\right) \cos \left(\theta_{i}+\frac{\phi}{2}+\frac{b_{g}}{D}+\frac{b_{r}}{D}\right) \\
F_{y, i}^{13}=\left(L_{c}\right) D \sin \left(\frac{b_{g}}{D}\right)\left(\frac{P_{s}+p_{i}}{2}\right) \cos \left(\theta_{i}+\phi\right) \\
F_{y, i}^{14}=\left(L_{c}\right) D \sin \left(\frac{b_{r}}{D}\right)\left(\frac{2 P_{s}+p_{i}+p_{i+1}}{4}\right) \cos \left(\theta_{i}+\phi+\frac{b_{g}}{D}+\frac{b_{r}}{D}\right)
\end{gathered}
$$

The net force in the $y$-direction is found by summing all components of force, $\mathrm{i}=1,2 \ldots \mathrm{N}$. Unlike the bearings previously considered, an $\mathrm{x}$-direction force is also created by the twisted grooves. The $\mathrm{x}$-direction restoring force is computed exactly as the y-direction force, except the cosine term is replace by a sine term. For example:

$$
\mathrm{F}_{\mathrm{x}, \mathrm{i}}^{1}=-\mathrm{L}_{\mathrm{a}} \mathrm{D} \sin \left(\frac{\mathrm{b}_{\mathrm{g}}}{\mathrm{D}}\right)\left(\frac{\mathrm{p}_{\mathrm{i}}}{2}\right) \sin \left(\theta_{\mathrm{i}}\right)
$$

The negative sign is used so that the direction corresponds to that of Figure 3.2. The other $\mathrm{x}$-direction force components are developed in the same way from the $\mathrm{y}$-direction force components, and will not be given here. The resultant bearing force is then given by:

$$
F_{\text {res }}=\sqrt{F_{x}^{2}+F_{y}^{2}}
$$

The angle between the direction of displacement and the direction of the resultant bearing force, called the angle of incidence, is given by:

$$
\theta_{\text {res }}=\tan ^{-1}\left(-\frac{F_{x}}{F_{y}}\right)
$$


If the angle of incidence is equal to zero then the bearing moves in a direction coincident with the direction of applied radial force, and the bearing is stable at all rotational speeds. However, if the angle of incidence is not equal to zero, then a critical speed will exist at which bearing instability causes a failure. For this reason, the bearing should be designed with an angle of incidence less than approximately $45^{\circ}$. This will be discussed further later in this thesis.

Table 3.13 shows the results of calculations for a representative $80 \mathrm{~mm}$ diameter, $80 \mathrm{~mm}$ long bearing. The bearing was designed with maximum initial stiffness while keeping the angle of incidence less than $45^{\circ}$. The compensator land width is $3 \mathrm{~mm}$. The outlet land width is $3 \mathrm{~mm}$. The drain groove is $2 \mathrm{~mm}$ wide and $10 \mathrm{~mm}$ long. A groove rib ratio, $b_{r} / b_{g}$, of 1.4 and a pocket rib ratio, $b_{\pi \mathrm{T}} / b_{\mathrm{p}}$, of 0.85 was found to provide excellent bearing performance for this configuration. The The performance shown is representative of this type of bearing. The initial specific stiffness is 1.02 , the load-carrying efficiency at $75 \%$ of gap closure is 0.337 , and the specific flow rate is 9.8 . 
Table 3.13

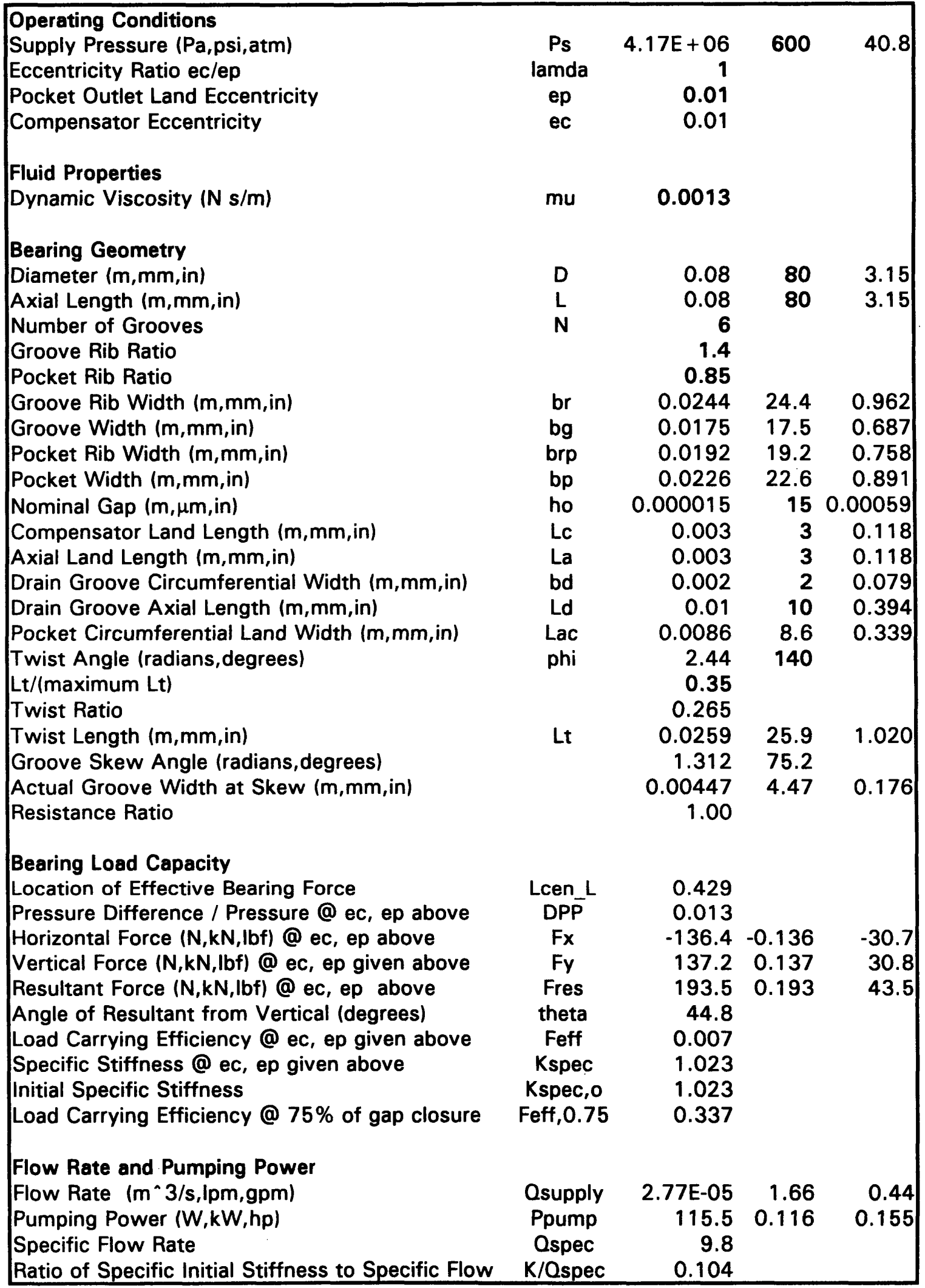




\section{L. Comparison of Twelve Radial Hydrostatic Bearings}

Twelve radial hydrostatic bearing configurations were considered in this chapter. For each case, a representative bearing with a diameter of $80 \mathrm{~mm}$ and a length of $80 \mathrm{~mm}$ was designed with approximately optimal initial stiffness while adhering to certain practical constraints. These constraints included a minimum groove width of $2 \mathrm{~mm}$ and a minimum land with of $3 \mathrm{~mm}$. The performance of each bearing is summarized in Table 3.14. As shown, some bearings provide high stiffness and load capacity, but also require high flow rates. Other bearings provide low flow rates but only low or moderate stiffness and load capacity. Other bearings, such as Bearings \#4, \#8, and \#10, provide both high stiffness and low flow rates. The proper selection of the radial bearing depends strongly on the application. In low speed applications, a bearing with a low flow rate is desirable; however, in high speed applications it is often desirable to have a higher bearing flow rate in order to keep the bearing temperature rise to an acceptable level.

It should be clearly emphasized that other characteristics of radial bearings besides their stiffness have an impact on the stiffness of the spindle. First, the effective load center of the bearing is important; how close the effective load center of the front radial bearing is to the spindle nose often has a dramatic impact on how stiff the spindle is. The effective load centers shown in Table 3.14 were not considered when the bearing geometry was designed. Second, how sensitive the front bearing is to shaft displacement at the front of the spindle is very important. The front radial bearing can be designed to have greater sensitivity to shaft deflection near the front of the spindle so that higher spindle stiffness is achieved. This 
The bearing performances illustrated in Table 3.14 were developed by considering the shaft to move parallel to the bearing housing. However, this is often not a very good approximation to a bearing of a machine tool spindle; when a radial force is applied to the front of the spindle, the shaft tilts within the bearing housing and often a substantially different bearing performance is realized. Therefore, the performance parameters shown in Table 3.14 do not adequately reflect the relative performance of each bearing when implemented into a machine tool spindle; although one bearing may have higher stiffness than another, it may not necessarily yield a spindle with a higher stiffness. In order to evaluate the true merits of the different bearings, they will be implemented into a representative spindle in the next chapter and the stiffness of the different spindles will be compared. 
Table 3.14

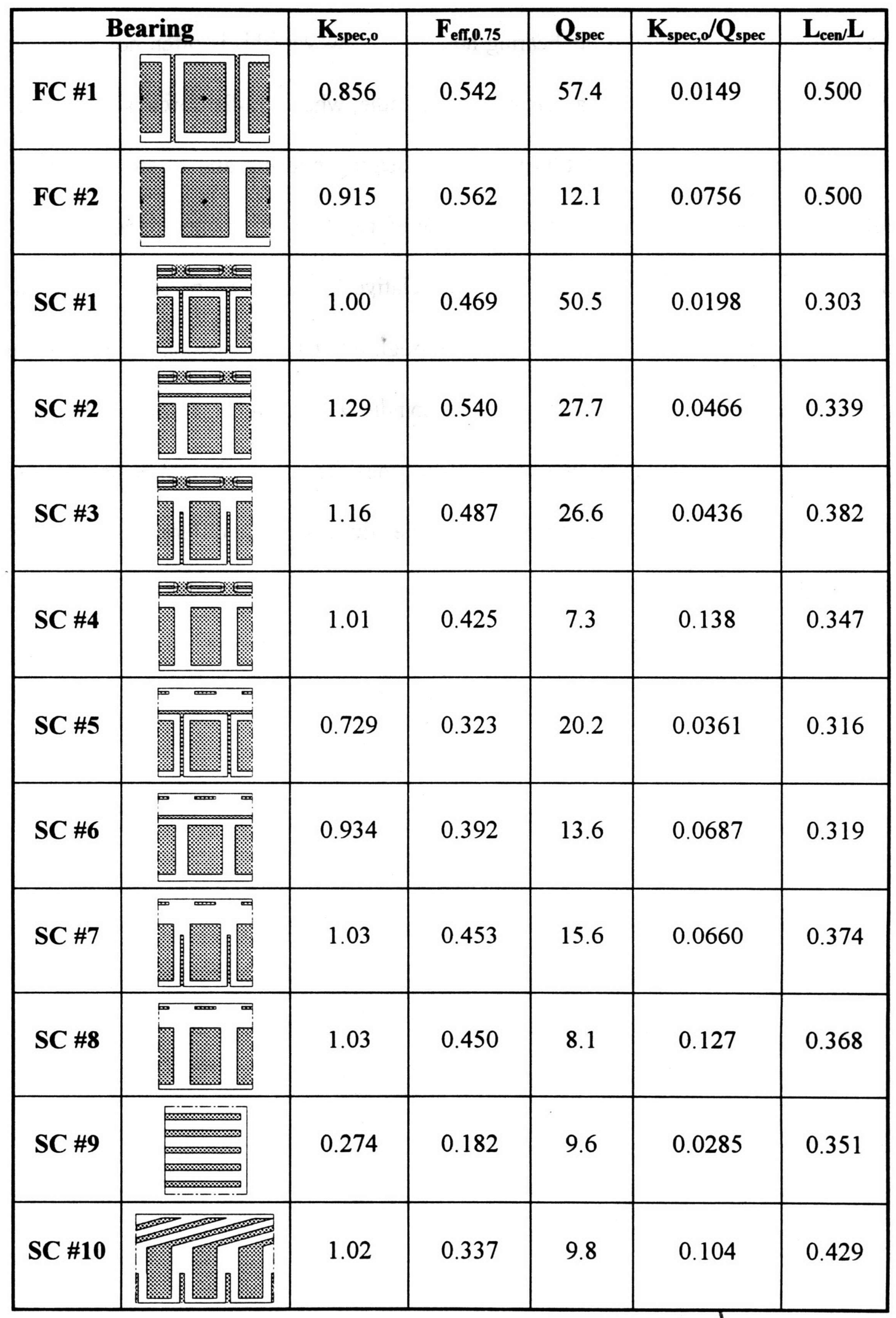




\section{STATIC SPINDLE RADIAL STIFFNESS}

For most applications, the most important characteristic of a machine tool spindle is its static radial stiffness. A spindle with high stiffness can be used to machine with high accuracy even when significant machining forces are placed on the spindle. The focus of this chapter is to develop methods and relations to accurately predict the radial stiffness of hydrostatic spindles.

The bearings of commercial machine tool spindles are usually so stiff that the flexibility of the spindle is dominated by the bending of the shaft. In nearly all applications, the flexibility of the shaft must be considered when computing the spindle stiffness. Adequate modeling of shaft bending in hydrostatic spindle systems is complicated by several factors. First, the shaft of a hydrostatic spindle may be complicated in geometry; the cross-section may change several times over the length of the shaft. Second, the length of the shaft is often not very long compared to its diameter, making shear deformations non-negligible. Finally, the tilting of the shaft often has a significant effect on the load-carrying characteristics of the front radial hydrostatic bearing; this complicates the solution procedure because the system must be solved iteratively while updating the bearing reaction. All of these effects are considered in this chapter.

Some simple relations are developed in the first section of this chapter using classical beam theory. A simple model of a constant cross-section spindle held by two bearings is considered. The relations developed for this simple model are often adequate for quick designs, but more accurate methods are needed for detailed design and 
optimization. For this purpose, finite element methods are developed to model shafts of varying cross-section. The four-node isoparametric beam elements include the effect of shear deformations, yet they are much more efficient than three-dimensional finite elements. In all cases, the effect of shaft bending on the bearing performance is incorporated into the analysis.

\section{A. Simple Model of a Constant Cross-Section Spindle Using Classical Beam Theory}

Figure 4.1 shows a simple model of a constant cross-section spindle with a force placed on its end while held by two bearings. The shaft has an elastic modulus $E$, and a cross-sectional moment of inertia, I. The front bearing has a stiffness of $\mathrm{K}_{\mathrm{f}}$, and the rear bearing has a stiffness of $\mathrm{K}_{\mathrm{r}}$. For this simplified analysis, the shaft is long and slender such that shear deformations are negligible and classical beam theory is applicable. When this assumption is adequate will be discussed in a future section of this chapter.

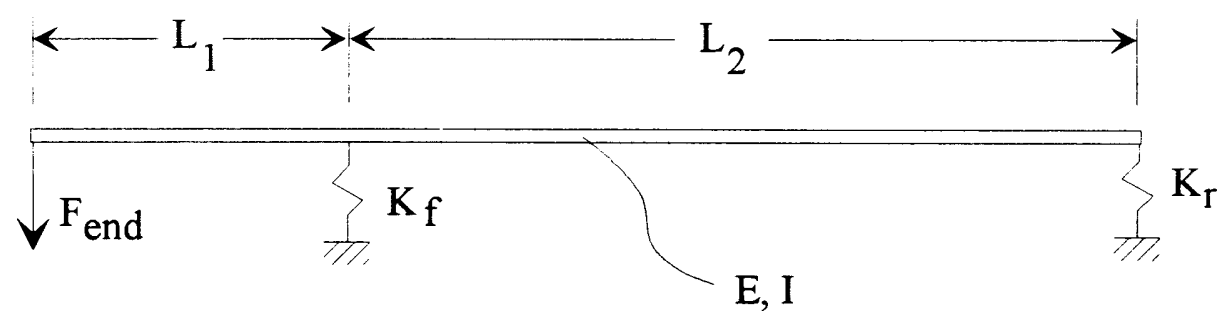

Figure 4.1. Simple model of a constant cross-section spindle with a force placed on its end while held by two bearings. 


\section{A.i. Calculated Spindle Stiffness}

The stiffness of the spindle is found by dividing the applied force by the deflection of the end of the spindle. This simple derivation can be found in numerous publications [7], and will not be repeated here. The stiffness of the spindle is given by:

$$
\mathrm{K}_{\mathrm{s}}=\frac{\frac{\mathrm{EI}}{\mathrm{L}_{1}^{3}}}{\frac{1}{3}+\frac{1}{3}\left(\frac{\mathrm{L}_{2}}{\mathrm{~L}_{1}}\right)+\frac{\mathrm{EI}}{\mathrm{K}_{\mathrm{r}} \mathrm{L}_{1}^{3}}\left(\frac{\mathrm{L}_{1}}{\mathrm{~L}_{2}}\right)^{2}+\frac{\mathrm{EI}}{\mathrm{K}_{\mathrm{f}} \mathrm{L}_{1}^{3}}\left[\left(\frac{\mathrm{L}_{1}}{\mathrm{~L}_{2}}\right)^{2}+2\left(\frac{\mathrm{L}_{1}}{\mathrm{~L}_{2}}\right)+1\right]}
$$

The spindle stiffness decreases with the cube of the distance between the front of the spindle and the front bearing; this distance should minimized as much as possible. The bearing spacing must also be considered. Increasing the distance between the bearings increases the bearing moment capability, thereby reducing the forces applied to the bearings (thereby reducing the bearing deflections); however, spacing them further apart causes the shaft to bend more. For any spindle, there is an optimum spacing ratio, $L_{1} / L_{2}$, that maximizes the spindle stiffness. The optimal ratio is found by taking the derivative of the stiffness given by Equation 4.1 with respect to $L_{1} / L_{2}$ and setting it equal to zero. This results in the following relation:

$$
\left(\frac{E I}{\mathrm{~K}_{\mathrm{r}} \mathrm{L}_{1}^{3}}\right)\left(\frac{\mathrm{L}_{1}}{\mathrm{~L}_{2}}\right)+\left(\frac{\mathrm{EI}}{\mathrm{K}_{\mathrm{f}} \mathrm{L}_{1}^{3}}\right)\left[\left(\frac{\mathrm{L}_{1}}{\mathrm{~L}_{2}}\right)+1\right]-\frac{1}{6\left(\frac{\mathrm{L}_{1}}{\mathrm{~L}_{2}}\right)^{2}}=0
$$

This relation can be easily solved numerically for the optimal spacing ratio, given the two non-dimensional bearing stiffness parameters.

Figure 4.2 shows the effect of bearing stiffnesses on an $80 \mathrm{~mm}$ diameter steel spindle with $L_{1}=120 \mathrm{~mm}$. The bearing spacing, $L_{2}$, was optimized for each point on the 
plots. As shown, the spindle stiffness increases sharply as the front bearing stiffness is increased but then levels off to an asymptotic limit that is a function of the rear bearing stiffness. The plots clearly indicate that the front bearing stiffness has a more dramatic impact on the spindle stiffness than the rear bearing stiffness. It is for this reason that spindles are often designed with rear bearings that are less stiff than the front bearings.

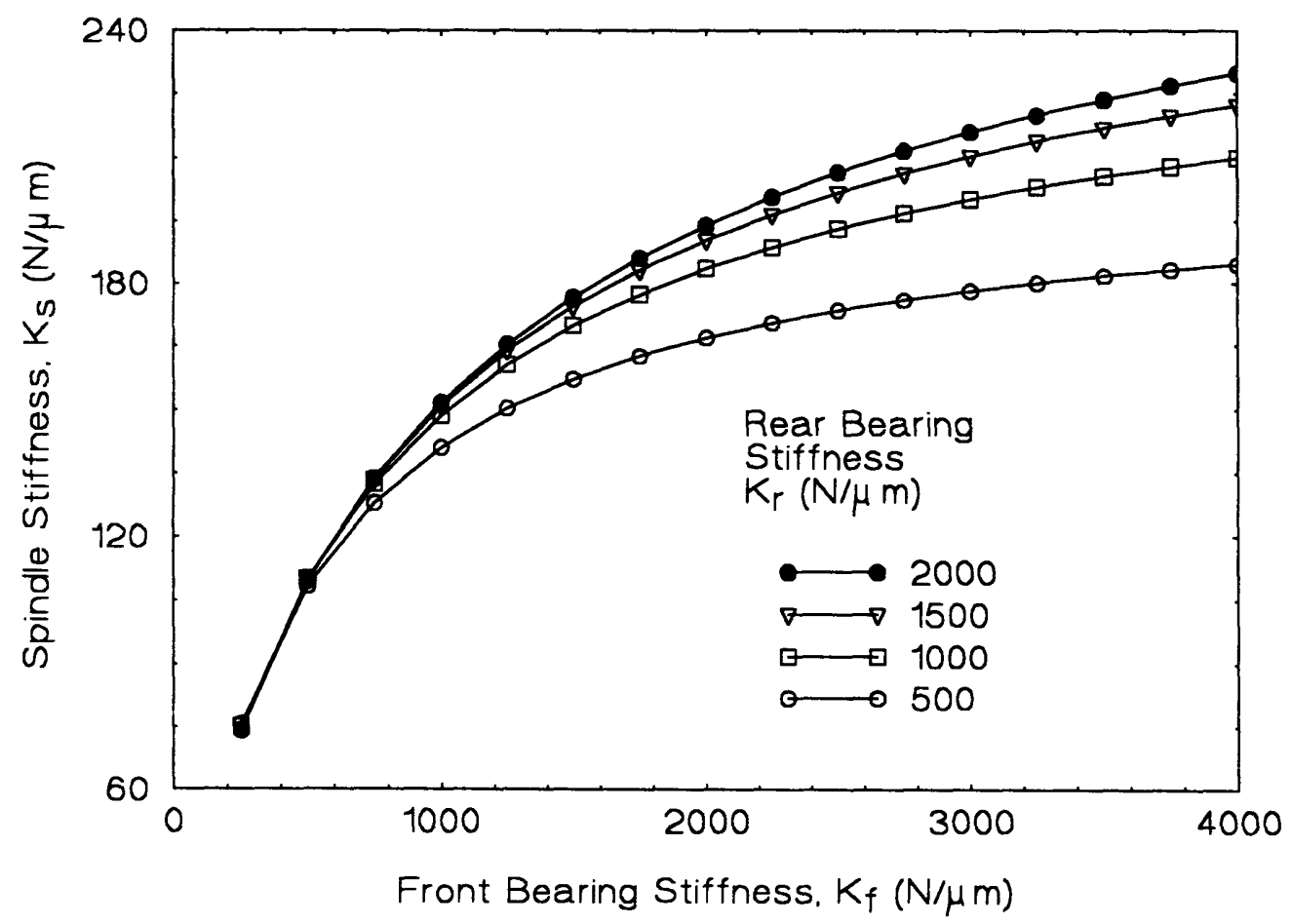

Figure 4.2. Effect of bearing stiffnesses on the stiffness of a $80 \mathrm{~mm}$ diameter steel spindle with $L_{1}=120 \mathrm{~mm}$. The bearing spacing, $\mathrm{L}_{2}$, was optimized for each point on the plots.

The theoretical maximum spindle stiffness that could be achieved if the bearings were infinitely stiff can be obtained by setting the bearing stiffnesses of Equation 4.1 equal to infininity to yield: 


$$
\mathrm{K}_{\mathrm{s}, \max }=\frac{\mathrm{EI} / \mathrm{L}_{1}^{3}}{\frac{1}{3}+\frac{1}{3}\left(\frac{\mathrm{L}_{2}}{\mathrm{~L}_{1}}\right)}
$$

The optimal bearing spacing for this case is clearly $L_{2} \ll L_{1}$. In this case, the maximum spindle stiffness is given by:

$$
\mathrm{K}_{\mathrm{s}, \max }=\frac{3 \mathrm{EI}}{\mathrm{L}_{1}^{3}}
$$

This is the same relation as that for the stiffness of a cantilevered beam. It is often useful to compare the stiffness of a spindle to the theoretical maximum stiffness given by Equation 4.4.

\section{A.ii. Incorporation of the Effect of Shaft Tilting into the Calculated Spindle Stiffness}

The bearing stiffnesses that are used in Equation 4.1 will now be considered. In Chapter 3, the stiffness of a radial bearing was calculated by moving the shaft eccentric to the bearing housing and then calculating the restoring force. Although the representative calculations were performed for a uniform eccentricity, the relations were developed such that the shaft could be tilted out of parallel with the bearing housing such that the eccentricity was a linear function of the axial position within the bearing. The bearing restoring force was then a function of the two eccentricities at the ends of the bearing. This extension of the model enables the bearing characterstics to be integrated into the design of the spindle. Figure 4.3 illustrates a shaft that is supported by two hydrostatic bearings. As a force is placed on the front end of the spindle, the shaft bends within the bearings. The eccentricity of the shaft in the front bearing is a strong function of the axial 
location within the housing. Most hydrostatic bearings are sufficiently short that the shaft eccentricity can be approximately modeled as a linear function of axial position; this approximation is usually adequate if $\mathrm{L} / \mathrm{D}$ is less than approximately 1.5 . A procedure will developed in this section to integrate the effect of shaft tilting into the spindle stiffness calculation.

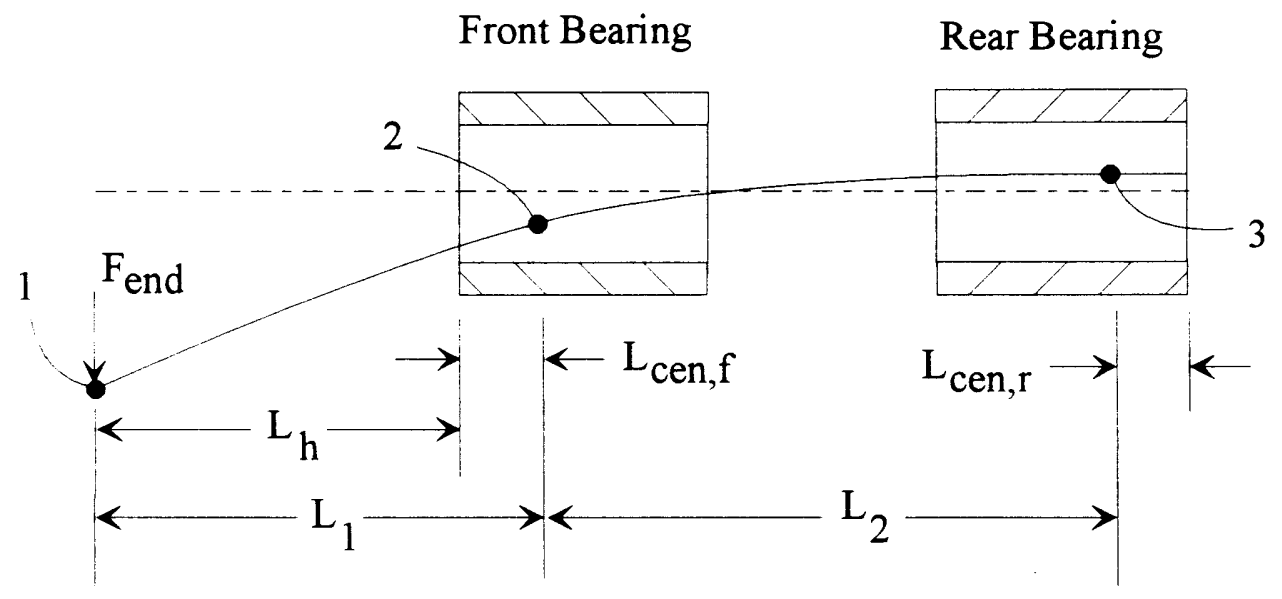

Figure 4.3. Schematic diagram of a shaft held by two hydrostatic bearings while a force is placed on its end.

In order to incorporate the effect of shaft tilting on the bearing stiffness, the eccentricity at each end of the bearing must be calculated as the force is applied to the end of the shaft. The classical beam theory used to derive Equation 4.1 can be used to calculate the shaft deflection at any location $\mathrm{x}$ from the front end of the spindle. If $\mathrm{x}<\mathrm{L}_{1}$, then the deflection is given by:

$u(x)=u_{1}+\theta_{1} L_{1} \frac{x}{L_{1}}+\left[3\left(u_{2}-u_{1}\right)-\left(\theta_{2} L_{1}-\theta_{1} L_{1}\right)-3 \theta_{1} L_{1}\right]\left(\frac{x}{L_{1}}\right)^{2}+$ 


$$
\frac{1}{3}\left[3\left(\theta_{2} L_{1}-\theta_{1} L_{1}\right)-6\left(u_{2}-u_{1}\right)+6 \theta_{1} L_{1}\right]\left(\frac{x}{L_{1}}\right)^{3}
$$

If $x>L_{1}$, then the deflection is given by:

$$
\begin{array}{r}
u(x)=u_{2}+\theta_{2} L_{2}\left(\frac{x-L_{1}}{L_{1}}\right)+\left[3\left(u_{3}-u_{2}\right)-\left(\theta_{3} L_{2}-\theta_{2} L_{2}\right)-3 \theta_{2} L_{2}\right]\left(\frac{x-L_{1}}{L_{2}}\right)^{2}+ \\
\frac{1}{3}\left[3\left(\theta_{3} L_{2}-\theta_{2} L_{2}\right)-6\left(u_{3}-u_{2}\right)+6 \theta_{2} L_{2}\right]\left(\frac{x-L_{1}}{L_{2}}\right)^{3}
\end{array}
$$

where the three nodal deflections and rotations are given by:

$$
\begin{gathered}
u_{1}=\frac{F_{\text {end }} L_{1}^{3}}{E I}\left[\frac{1}{3}+\frac{1}{3}\left(\frac{L_{2}}{L_{1}}\right)+\left(\frac{E I}{K_{r} L_{1}^{3}}\right)\left(\frac{L_{1}}{L_{2}}\right)^{2}+\left(\frac{E I}{K_{f} L_{1}^{3}}\right)\left\{\left(\frac{L_{1}}{L_{2}}\right)^{2}+2\left(\frac{L_{1}}{L_{2}}\right)+1\right\}\right] \\
u_{2}=\frac{F_{\text {end }}}{K_{f}}\left(\frac{L_{1}+L_{2}}{L_{2}}\right) \\
u_{3}=-\frac{F_{\text {end }}}{K_{r}}\left(\frac{L_{1}}{L_{2}}\right) \\
\theta_{1}=-F_{\text {end }}\left[\frac{L_{1} L_{2}}{3 E I}+\frac{L_{1}}{L_{2}^{2} K_{f}}+\frac{1}{K_{r} L_{2}}+\frac{L_{1}^{2}}{2 E I}\right] \\
\theta_{2}=-F_{\text {end }}\left[\frac{L_{1} L_{2}}{3 E I}+\frac{L_{1}}{L_{2}^{2} K_{f}}+\frac{1}{K_{f} L_{2}}+\frac{L_{1}}{L_{2}^{2} K_{r}}\right] \\
\theta_{3}=-F_{\text {end }}\left[-\frac{L_{1} L_{2}}{6 E I}+\frac{L_{1}}{L_{2}^{2} K_{f}}+\frac{1}{K_{f} L_{2}}+\frac{L_{1}}{L_{2}^{2} K_{r}}\right]
\end{gathered}
$$

A procedure will now be outlined to calculate the stiffness of the spindle: 
1. Calculate $K_{f}, K_{r}$ from the bearing characteristics with uniform, small bearing eccentricities of approximately 0.001 .

2. Calculate the optimal bearing spacing, $\mathrm{L}_{2}$, using Equation 4.2 .

3. Calculate the spindle stiffness using Equation 4.1.

4. Calculate the shaft deflections at both ends of the bearings, using Equations 4.5 and 4.6 and the current bearing stiffness values.

5. Calculate updated bearing stiffnesses based on the eccentricities from Step \#4.

6. Return to Step \#2 and repeat until the calculated spindle stiffness converges. Convergence can be judged by noting when the calculated spindle stiffness changes by less than $1 \%$ from one iteration to the next.

\section{A.iii. Representative Calculations}

A representative spindle will now be considered to illustrate the methods developed. A solid steel $\left(E=2 \times 10^{11} \mathrm{~Pa}\right)$ spindle with a diameter of $80 \mathrm{~mm}$ and an overhang length, $L_{h}$, of $100 \mathrm{~mm}$ will be considered. Self-Compensated Bearing \#1 will be used to support the spindle. For simplicity, the front and rear bearings used will be identical. A bearing with the parameters given in Table 3.3 will be used. Recall that the geometry was optimized for maximum initial specific stiffness, with the shaft displaced parallel to the bearing housing. A supply pressure of 600 psig is used to support the spindle. Table 4.1 shows the calculated spindle stiffness for four configurations corresponding to different locations of the bearing compensators. As shown, the location of the rear bearing compensator has little effect on the spindle stiffness; this is because the shaft is nearly parallel with the rear bearing housing for an optimized bearing spacing 
configuration. However, the location of the front bearing compensator has a substantial effect on the spindle stiffness; placing the compensators in the rear location places the effective bearing center nearer to the front of the spindle, thereby increasing the spindle stiffness.

Table 4.1

\begin{tabular}{|c|c|c|}
\hline $\begin{array}{c}\text { Front Bearing } \\
\text { Compensator } \\
\text { Location }\end{array}$ & $\begin{array}{c}\text { Rear Bearing } \\
\text { Compensator } \\
\text { Location }\end{array}$ & $\begin{array}{c}\text { Spindle Stiffness } \\
(\mathbf{N} / \boldsymbol{\mu m})\end{array}$ \\
\hline Front & Front & 139 \\
\hline Front & Rear & 141 \\
\hline Rear & Front & 150 \\
\hline Rear & Rear & 151 \\
\hline
\end{tabular}

For illustrative purposes, the stiffness of the spindle described above was computed without accounting for the effect of shaft tilting on the bearing stiffnesses. The compensator configuration used was "Rear/Rear." The computed spindle stiffness was $174 \mathrm{~N} / \mu \mathrm{m}$, which is approximately $15 \%$ higher than the $151 \mathrm{~N} / \mu \mathrm{m}$ predicted when accounting for the effect of shaft bending on the bearing stiffnesses. This is a typical error incurred when calculating the stiffness of a spindle that uses self-compensated bearings without accounting for the effect of shaft tilting on the bearing characteristics.

\section{B. Modeling Spindle Stiffness using Finite Element Methods}

The relations presented in the previous section were developed using classical beam theory that neglects shear deformations. Also, they were developed for a shaft of uniform cross-section. However, machine tool spindles are often designed with a shaft that is short compared to its diameter such that shear deformations are not negligible, and 
with many changes in cross-section. Methods will be developed in this section to accomodate both of these effects.

Three-dimensional finite element models are complicated to implement and computationally-intensive to execute. More efficient methods are therefore developed in this section to predict shaft bending and tool-point stiffness in spindle systems using more simple finite element methods. Four-node isoparametric beam elements (elements with cubic displacement interpolations) are used to model shaft bending and predict static spindle deflections. Shown in Figure 4.4 is a representative spindle modeled with an assembly of seven beam elements. The use of beam elements is a very simple and powerful way to model complex spindle deflections.

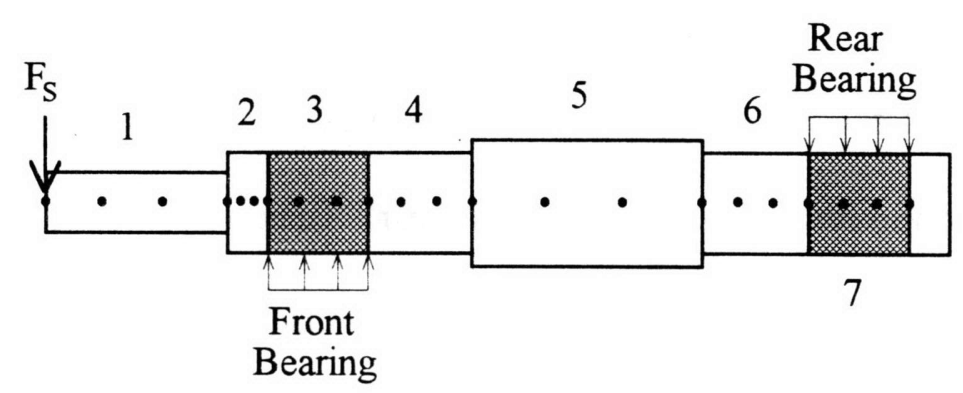

Figure 4.4. A shaft held by two radial hydrostatic bearings and subjected to a radial force at the spindle face.

The stiffness matrix for a four-node isoparametric beam element is developed in Appendix A. The development is complete except for the equivalent shear strain of the beam cross-section. In the development, it was assumed that the cross-section remained straight during shaft bending. The shear strain in an actual shaft will vary across its crosssection, being largest in magnitude along the neutral axis and smallest in magnitude near the upper and lower edges. To accommodate the assumption of a straight cross-section, 
an equivalent constant shear strain was defined such that, acting over an equivalent shear area $A_{6}$, the resulting shear strain energy is the same as that in the actual shaft:

$$
\gamma=\frac{\tau}{\mathrm{G}}=\frac{\mathrm{V}}{\mathrm{GA}_{\mathrm{s}}}
$$

where $\tau$ is the shear stress, $G$ is the shear modulus, and $V$ is the shear force. As a matter of convenience, the equivalent shear area is replaced with a shear correction factor,

$$
k=\frac{A_{s}}{A}
$$

The value of the shear correction factor has been computed for hollow and solid shafts and is available in the literature [8]. However, a general relation for $\mathrm{k}$ is not available for a hollow shaft with an inner diameter $D_{i}$, and an outer diameter $D_{0}$. In order to include the effect of shear deformations, a general relation for the effective shear area of a hollow shaft is developed in this chapter using three-dimensional finite-element computations. Simple benchmark cases are considered first, and the elements are found to agree closely with classical beam theory for cases where shear deformations are negligible. The elements are then combined to predict more complex spindle deflections.

\section{B.i. Benchmark Validation of the Finite Element Model for a "Long" Cylinder}

The finite element equations developed in the appendices will be tested in this section against classical beam theory for the simple cantilevered cylinder shown in Figure 4.5 in the limit that its length is much greater than its diameter.

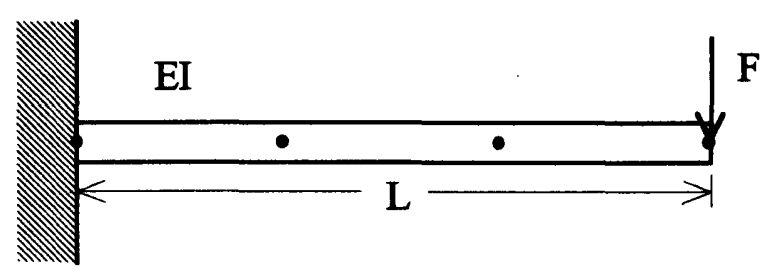


Figure 4.5. Schematic diagram of a cantilevered shaft. The shaft is modeled with a single four-node beam element.

Classical beam theory can be used to approximate the shaft deflection if the shaft is long and slender and deflections caused by shear forces are negligible. The resulting deflection is given by [8]:

$$
\delta(x)=-\frac{F}{6 E I}(3 L-x) x^{2}
$$

Figure 4.6 below shows a comparison of the deflection predicted using a single 4-node beam element and that by beam theory, Equation (4.15). In the examples used, the ratio of $\mathrm{D} / \mathrm{L}$ was equal to $1 / 100$, and so shear deformations were negligible. The finite element results agree with the analytical solution within $0.005 \%$.

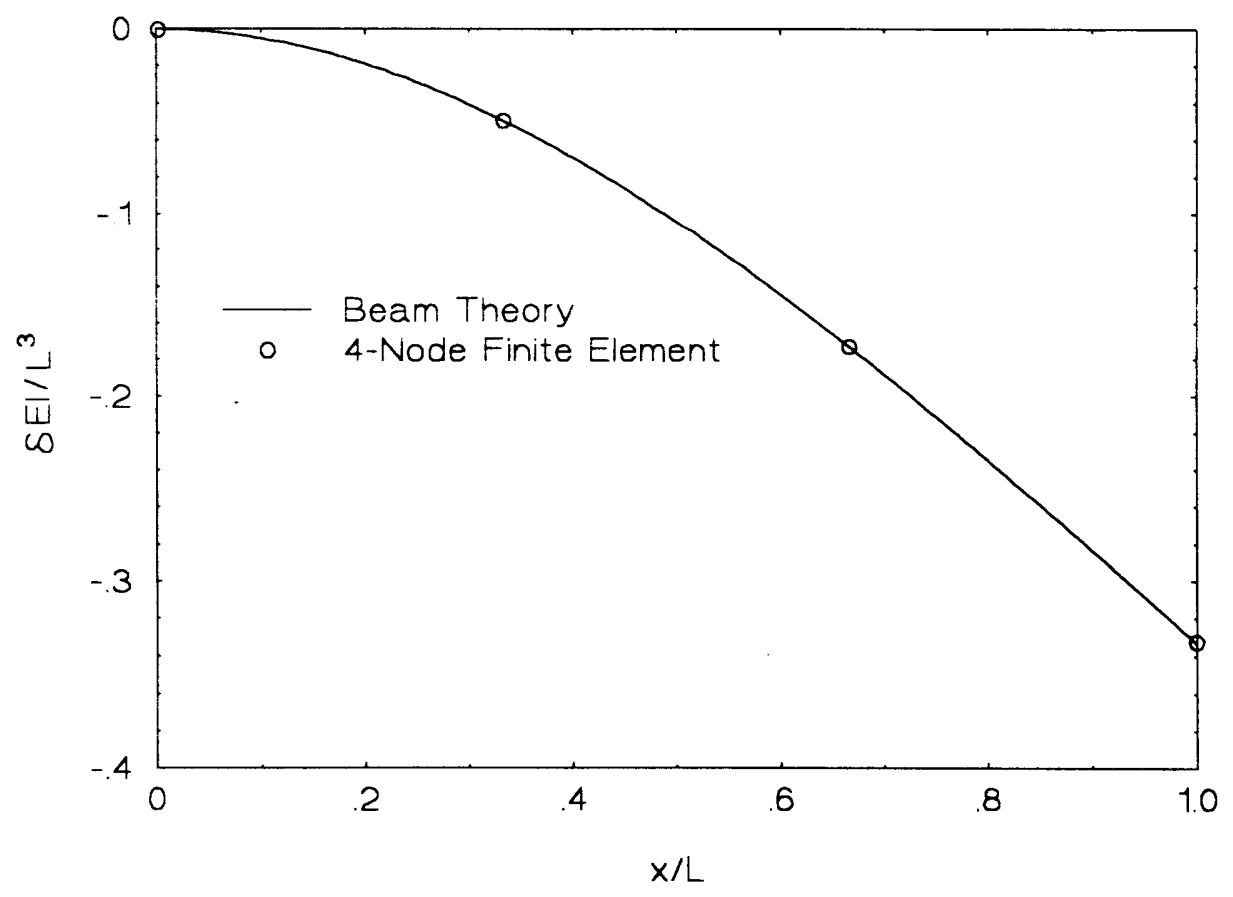


Figure 4.6. Static deflection of a cantilevered solid shaft with a uniform cross-section and $D / L=1 / 100$. The deflections predicted using a single four-node beam element agree with classical beam theory within $0.005 \%$.

As illustrated by Equation 4.15, the deflection obtained from classical beam theory is cubic. Since the interpolation functions used in the finite element solution were cubic interpolations, the finite element solution is exact. Thus, the finite elements used in this work provide adequate accuracy in modeling bending even when few elements are used. In addition, they provide ample functionality in that they allow the effects of shear forces to be accounted for in a straightforward manner, and elements can be easily combined to model beams with changes in cross-section and/or material properties.

\section{B.ii. Shaft Bending with Non-Negligible Shear Deformations}

The cantilivered beam shown in Figure 4.5 is again considered, but it is now allowed to be short and have non-negligible shear deformations. The stiffness matrices of the 4-node beam element, Equations A.32 and A.33, are used to determine the deflection of the beam at its free end. Eliminating the first two degrees of freedom, the deflection and rotation at the base, and applying a force at the free end yields the following system of equations: 


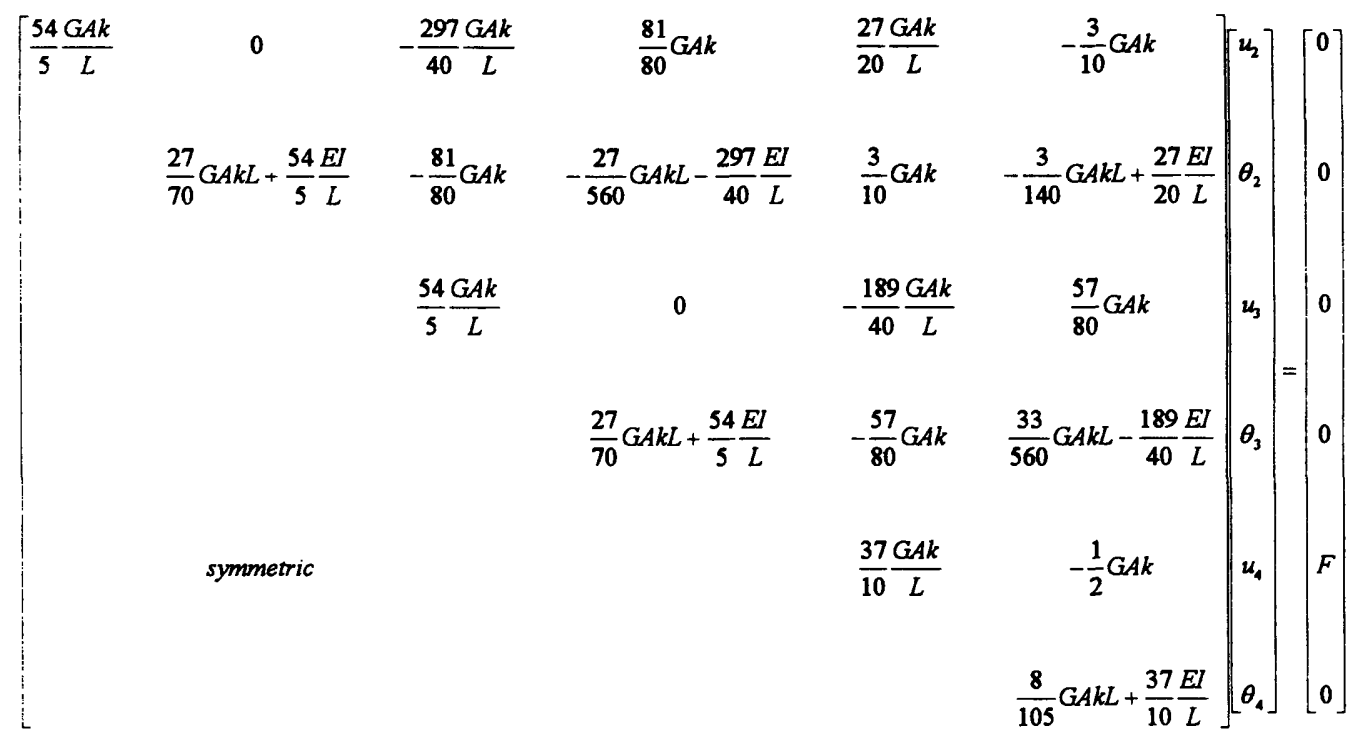

This system of equations is solved to obtain $u_{4}=\delta$, the deflection at the free end:

$$
\frac{\delta E I}{F L^{3}}=\frac{1}{3}+\frac{E I}{G A k L^{2}}
$$

Replacing the shear modulus with Young's modulus and Poisson's ratio,

$$
\frac{\delta E I}{F L^{3}}=\frac{1}{3}+\frac{2(1+v) I}{A k L^{2}}
$$

The added term of Equation 4.18 is the deflection caused by shear forces. In the next section, three-dimensional finite element computations will be used to investigate the effect of shear forces on short cylindrical shafts and to develop a general relation for the shear correction factor, $k$.

\section{B.iii. Development of the Shear Correction Factor for a General Hollow Shaft using Three-Dimensional Finite Element Computations}


Three-dimensional finite-element computations were made in order to determine an approximate relation for $k=f\left(D_{i} / D_{o}\right)$. Half of a shaft was meshed as shown in Figure 4.7. To approximate a simple cantilevered shaft, all nodes were held rigidly at one end, and a uniform traction was applied to the face of the other end. A symmetry boundary condition was used along the centerline.

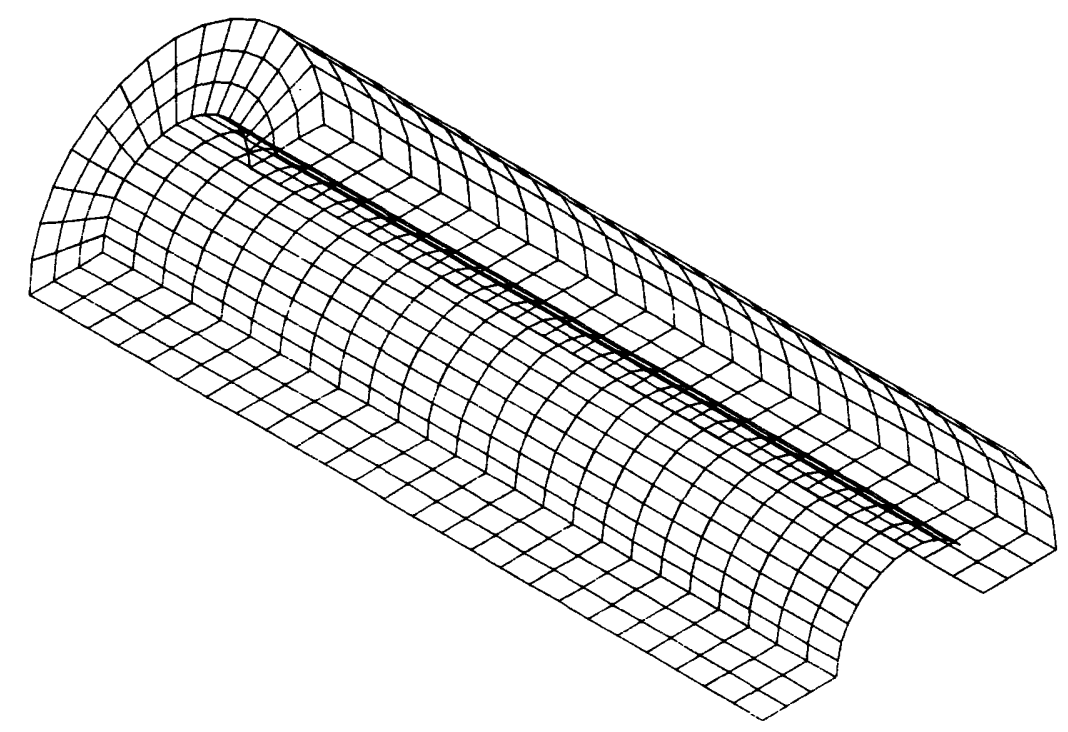

Figure 4.7. A typical mesh used in the three-dimensional finite element computations. All nodes are held rigidly at one end, and a uniform traction is applied to the face of the other end. A symmetry boundary condition was used along the centerline.

Computations were first made for a shaft with $L=0.25 \mathrm{~m}, D_{0}=0.1 \mathrm{~m}, E=200$ $\mathrm{MPa}, v=0.3$, and various $\mathrm{D}_{\mathrm{i}}$. The resulting center-line deflections at the free end are shown in Figure 4.8. These deflections were used to compute the shear correction factor for each case using Equation 4.18 and are plotted along with an approximate curve fit,

$$
k=0.74-0.16 \tan ^{-1}\left[6.3\left(\frac{D_{i}}{D_{o}}\right)-2.6\right]
$$


in Figure 4.8.

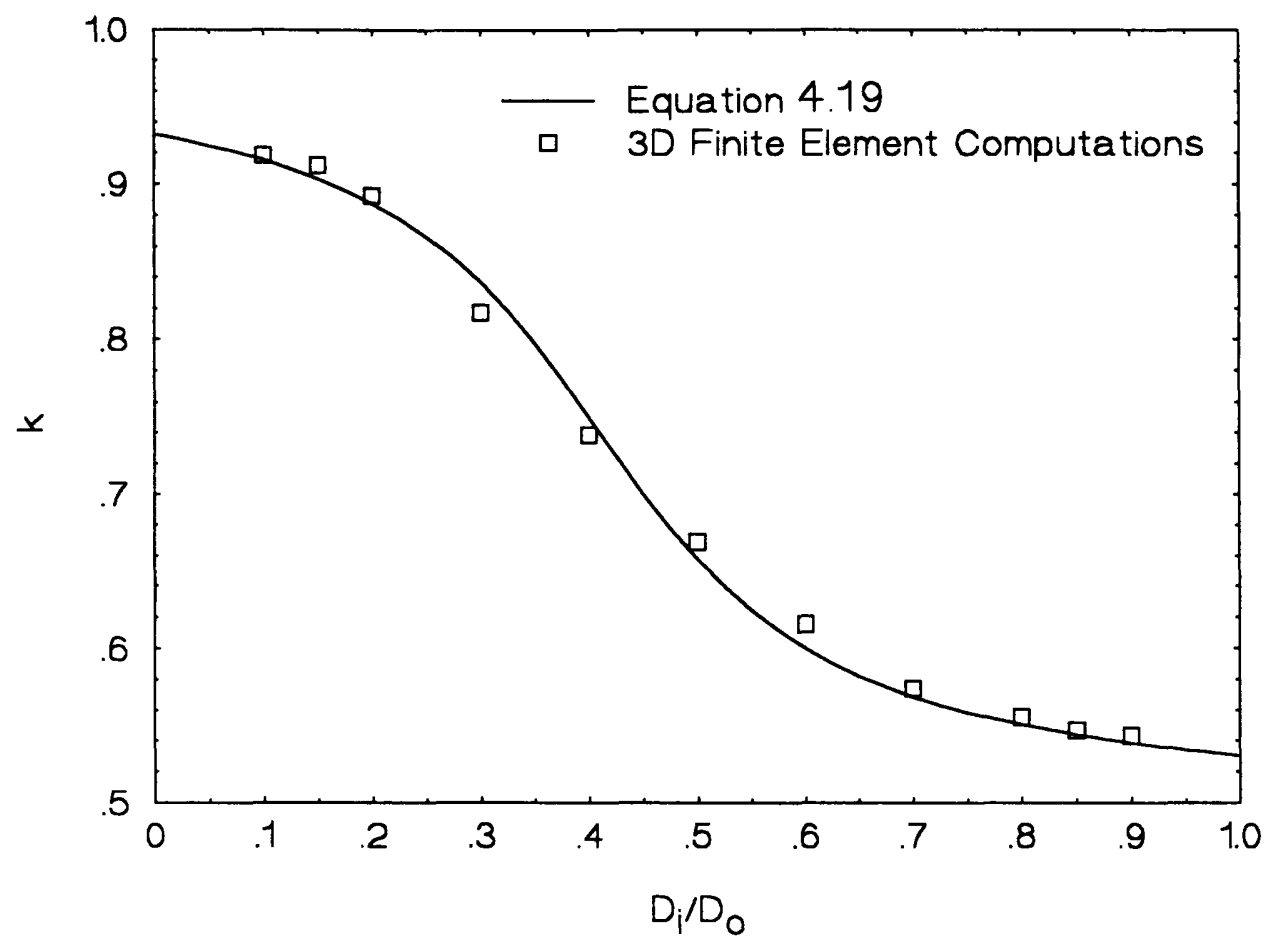

Figure 4.8. Shear correction factor, $k$, as a function of $D_{i} / D_{0}$. Three-dimensional finite element simulations were made of a cantilevered shaft with $\mathrm{L}=0.25 \mathrm{~m}, \mathrm{D}_{0}=0.1 \mathrm{~m}, \mathrm{E}=$ $200 \mathrm{MPa}, v=0.3$, and various $\mathrm{D}_{\mathrm{i}}$.

The end-point deflections predicted using Equations 4.18 and 4.19 are compared with the finite-element results in Figure 4.9. As shown, the agreement is excellent. 


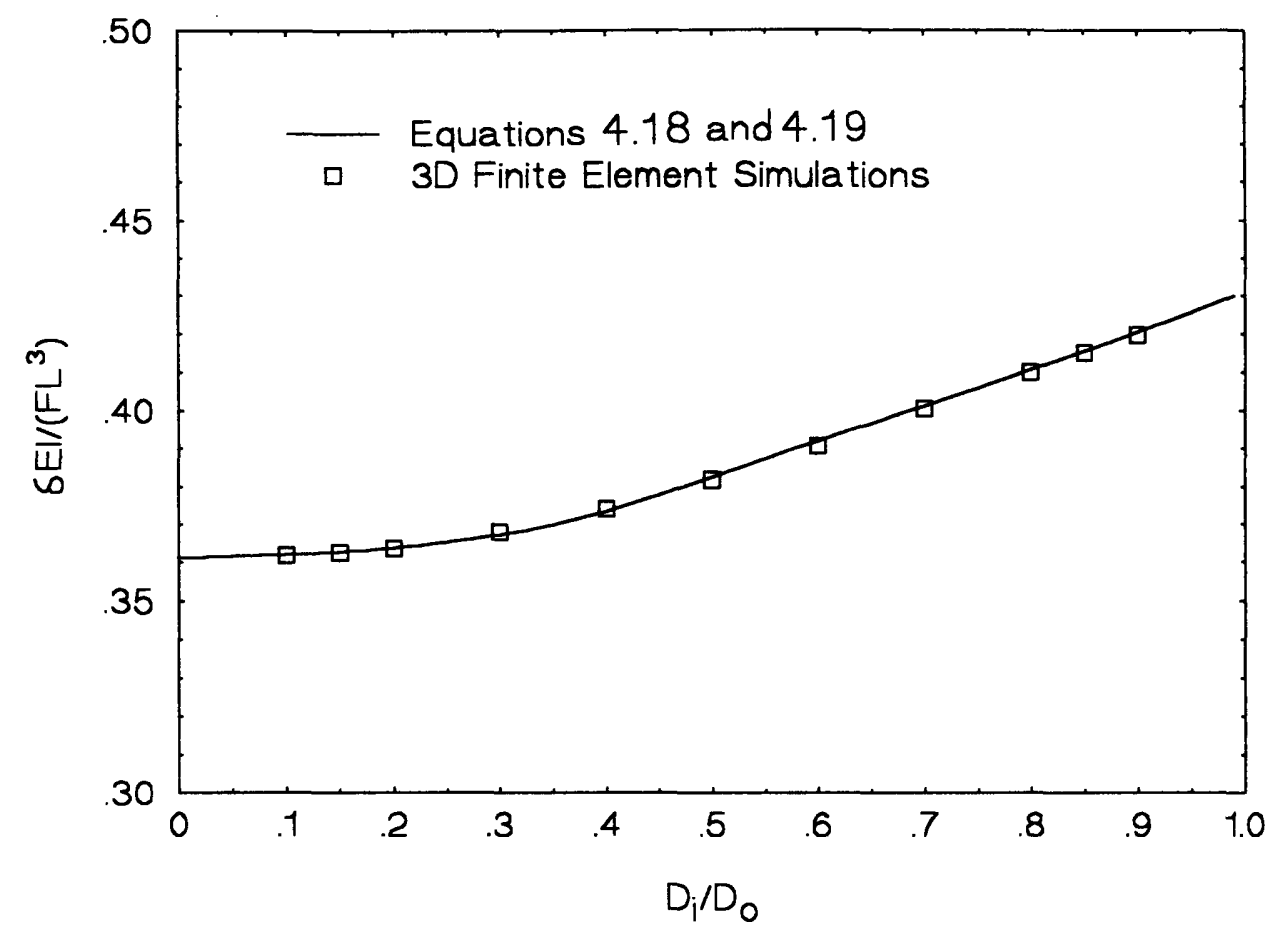

Figure 4.9. Deflection of the free end of a cantilevered shaft of varying cross-section. Three-dimensional finite element simulations were made with $\mathrm{L}=0.25 \mathrm{~m}, \mathrm{D}_{\mathrm{o}}=0.1 \mathrm{~m}, \mathrm{E}=$ $200 \mathrm{MPa}, v=0.3$, and various $\mathrm{D}_{\mathrm{i}}$.

All of the data used to curve fit the functional relation for $\mathrm{k}$ were for a fixed shaft aspect ratio, $L / D_{0}=2.5$. More simulations were made, this time for $D_{i} / D_{o}=0.5$, and various $L / D_{0}$. The resulting end-point deflections, shown in Figure 4.10, are compared with those predicted using Equations 4.18 and 4.19. Note that a cantilevered circular shaft with $L / D_{0}=1$ has an end-point deflection about twice that predicted using classical beam theory which neglects shear deformations. For $L / D_{0}=0.5$, the deflection is almost 5 times higher. 


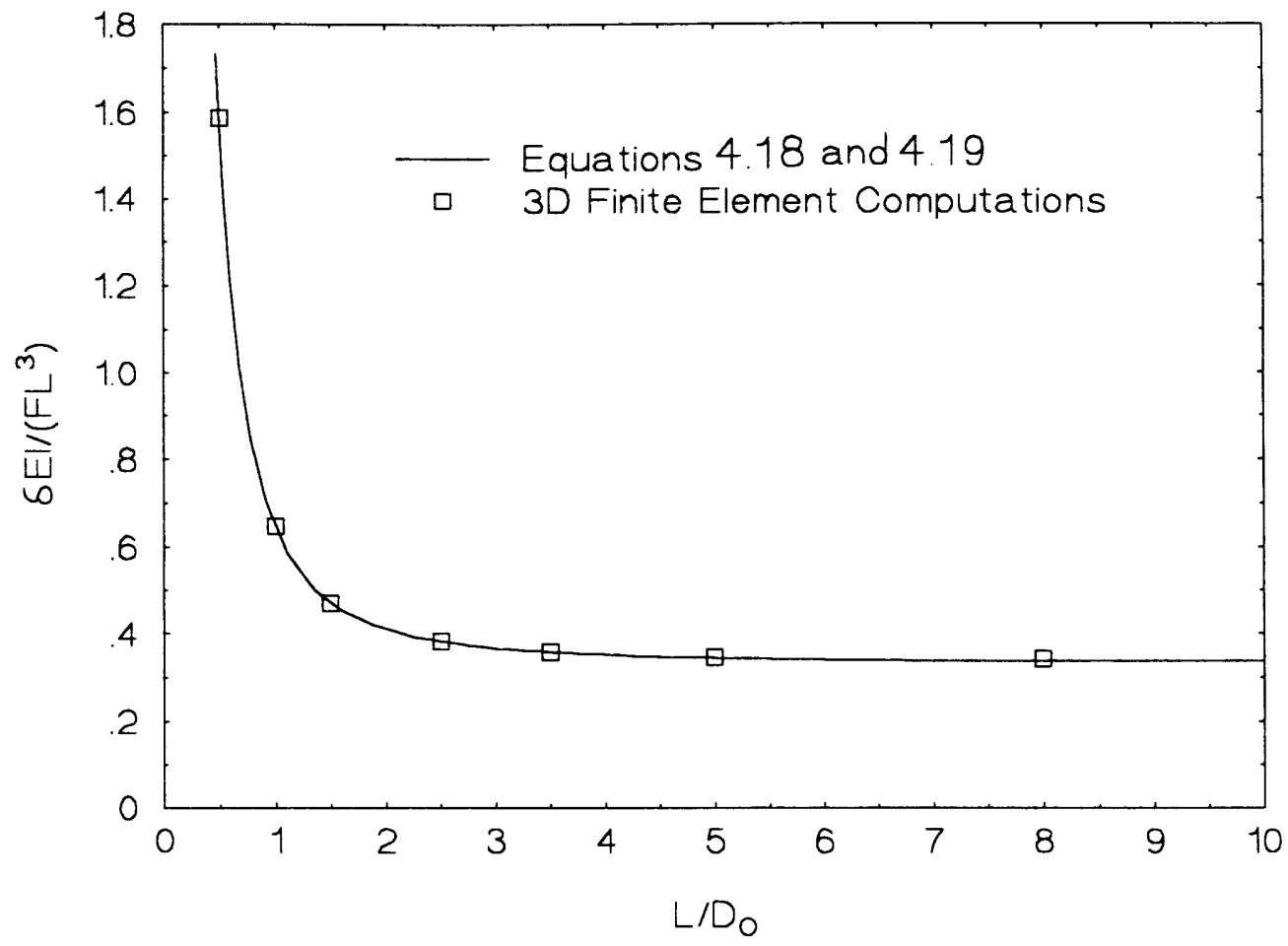

Figure 4.10. Deflection of the free end of a cantilevered shaft of constant cross-section and varying length. Three-dimensional finite element simulations were made with $D_{0}=0.1$ $\mathrm{m}, \mathrm{D}_{\mathrm{i}}=0.05 \mathrm{~m}, \mathrm{E}=200 \mathrm{MPa}, v=0.3$, and various $\mathrm{L}$.

\section{B.iv. Formation of the Global Stiffness Matrix}

As shown in Figure 4.4, a hydrostatic spindle is composed of several regions. Two of these regions are constrained by hydrostatic bearings. Each region may have a different cross-section. To appropriately model the shaft, seven beam elements will be combined and solved together to obtain the overall shaft deformation. In Appendix A, the stiffness matrix of a general 4-node beam element was derived. In this section, methods will be developed to combine seven stiffness matrices will be combined into one global stiffness matrix. 
A complete shaft is constructed by attaching seven beams together in series. Since the beams are 4-node elements, a total of 28 nodal displacements and 28 nodal rotations are solved for. However, beams attached to each other share common displacements and rotations at their end points. Figure 4.11 illustrates the interconnectivity of the beam elements. The displacement and rotation of the preceding and following beams are equal. Thus, the number degrees of freedom is equal to $2\left(3 \mathrm{~N}_{e}+1\right)$ where $\mathrm{N}_{e}$ is the number of elements. In the present work 7 elements are combined, and so there are 44 degrees of freedom.

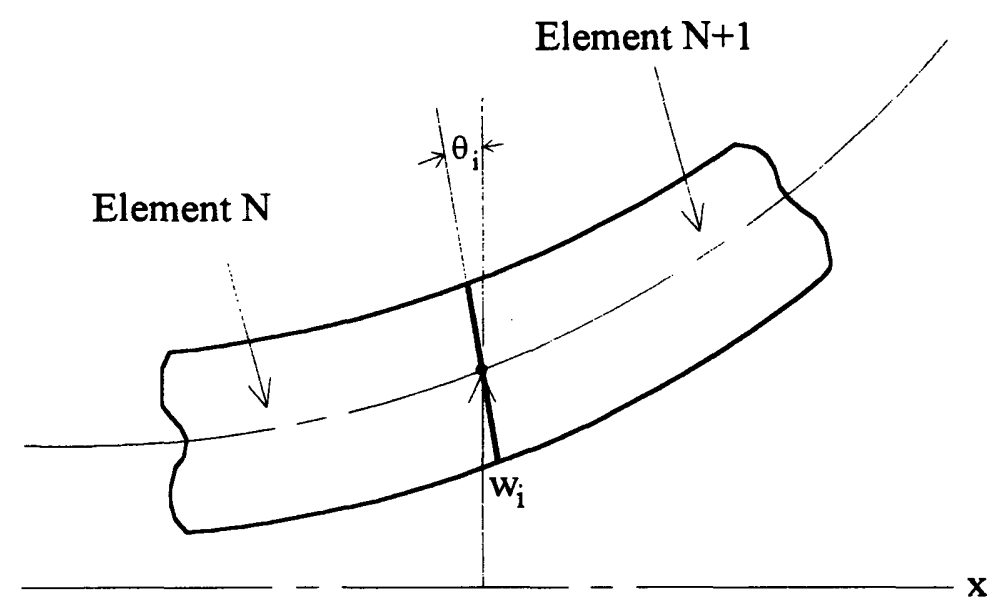

Figure 4.11. Diagram showing the interconnectivity of the beam elements. Nodal Displacements and nodal rotations are equated at element connections.

The global stiffness matrix is constructed by eliminating one pair of displacements which are shared by connected beams. Equation below shows the global stiffness matrix of two beams connected in series. The stiffness matrix corresponding to the first beam is denoted by $\underline{K}^{1}$, and that of the second by $\underline{K}^{2}$. The places in the matrix which are not 
filled contain zeroes. By examining equation, one can easily see how the global stiffness matrix for seven beams connected in series would be constructed.

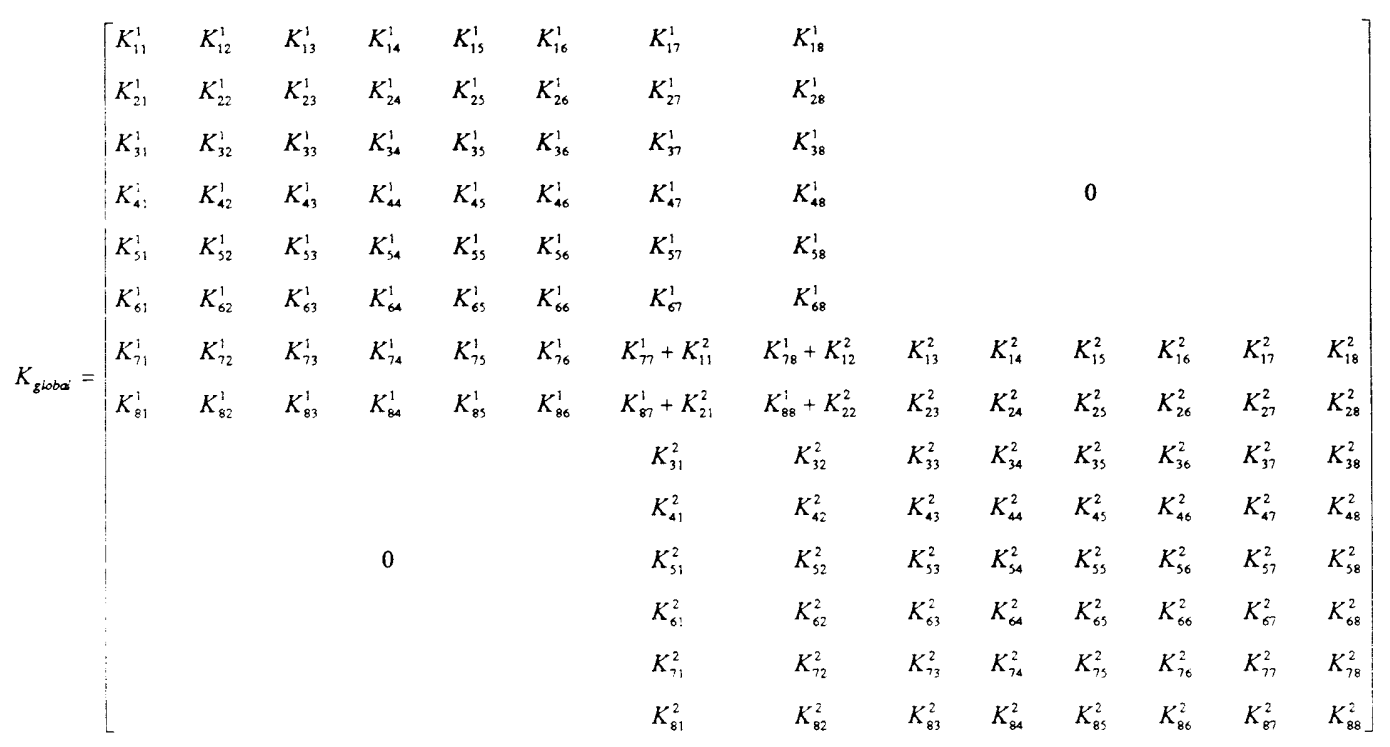

\section{B.v. Boundary Conditions}

Consider a general situation in which a shaft is held by front and rear radial hydrostatic bearings. As a radial force is applied to the spindle face, restoring pressures are established in the bearings, shown schematically in Figure 4.12. The distances $L_{a}$ and $\mathrm{L}_{\mathrm{b}}$ are the distances from the applied force at the spindle face to the centers of pressure of the front and rear bearings, respectively. The magnitudes of the bearing pressures can be calculated by summing the forces and moments and setting them equal to zero:

$$
\begin{aligned}
\sum F & =0: \quad f_{a} \ell_{a}=F_{s}+f_{b} \ell_{b} \\
\sum M_{\text {tool-point }} & =0: \quad f_{a} \ell_{a} L_{a}=f_{b} \ell_{b} L_{b}
\end{aligned}
$$


where $f$ is the force per unit length in the axial direction developed by the bearings. Solving these two equations for the two unknown bearing pressures:

$$
\begin{gathered}
f_{a}=\frac{F_{s}}{\ell_{a}} \frac{1}{1-\frac{L_{a}}{L_{b}}} \\
f_{b}=\frac{F_{s}}{\ell_{b}} \frac{1}{\frac{L_{b}}{L_{a}}-1}
\end{gathered}
$$

These pressures (forces per unit length), applied to their respective bearing areas, ensure that the shaft is in a state of static equilibrium.

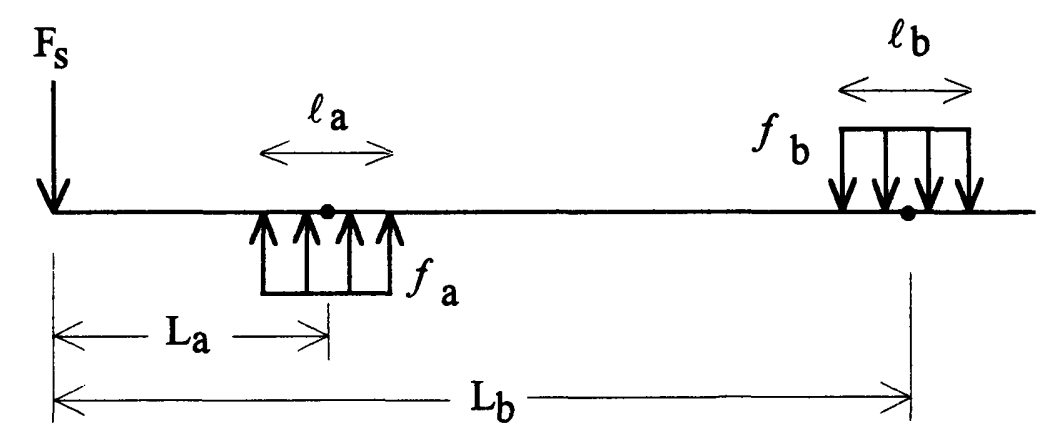

Figure 4.12. Schematic diagram of a shaft held by two radial hydrostatic bearings and subjected to a radial force at the spindle face. The hydrostatic bearings are modeled as areas of uniform pressure.

Once the pressures developed by the two hydrostatic bearings are known, the deflections of the shaft's neutral axis at the two bearing locations are also known from bearing stiffness calculations. The shaft deflections at the effective centers of the front and rear bearings are given by: 


$$
\begin{aligned}
& \delta_{a}=\frac{f_{a} \ell_{a}}{K_{a}} \\
& \delta_{b}=\frac{f_{b} \ell_{b}}{K_{b}}
\end{aligned}
$$

where $\mathrm{K}_{\mathrm{a}}$ and $\mathrm{K}_{\mathrm{b}}$ are the bearing stiffnesses which may be calculated using the methods developed in Chapter 3. In general, the bearing stiffnesses are a function of the shaft bending and must be solved for iteratively, as described in section 4.A.

\section{B.vi. Formation of the Global Load Vector}

In this work, a shaft is loaded with pressures over two bearing areas, and with a singular force at the end of the shaft. A schematic diagram of the arrangement is shown in Figure 13. Seven elements are combined in series to model the shaft. Note that the last section of shaft behind the rear bearing is not modeled since this is not necessary.

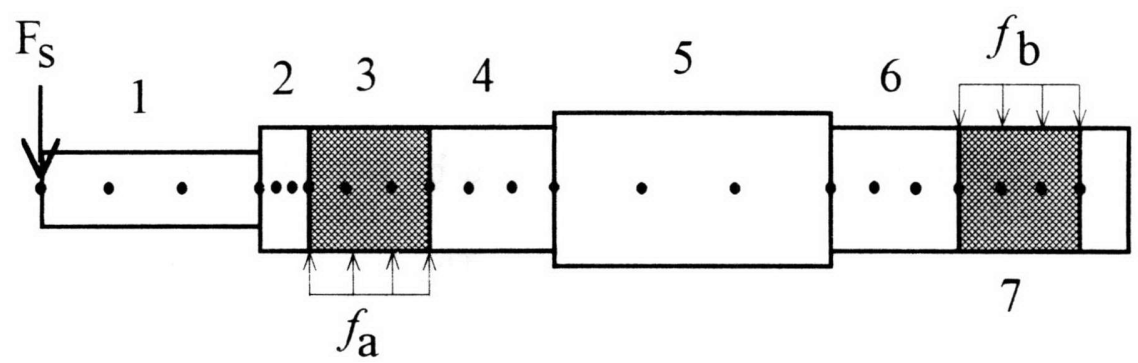

Figure 4.13. A shaft held by two radial hydrostatic bearings and subjected to a radial force at the spindle face. The shaft is modeled with seven elements connected in series.

The global load vector for the configuration of Figure 4.13 is found by summing the forces on the appropriate nodes into a total vector. The pressures exerted on the two bearing areas are implemented using Equation A.34. The force on the shaft tool-point is 
simply added to the first nodal position in the matrix. Sign convention is positive forces up, negative forces down. The global load vector for this arrangement is thus given by:

$$
\underline{R}=\left[\begin{array}{c}
-F_{s} \\
0 \\
0 \\
0 \\
0 \\
0 \\
\frac{1}{8} f_{a} L_{a} \\
\frac{3}{8} f_{a} L_{a} \\
\frac{3}{8} f_{a} L_{a} \\
0 \\
. \\
. \\
. \\
0 \\
-\frac{1}{8} f_{b} L_{b} \\
-\frac{3}{8} f_{b} L_{b} \\
-\frac{3}{8} f_{b} L_{b} \\
-\frac{1}{8} f_{b} L_{b}
\end{array}\right]
$$

\section{B.vii. Imposition of Boundary Conditions}

Two boundary conditions must be imposed on the shaft: the displacement at the front bearing, and the displacement at the rear bearing. The two boundary conditions are easily applied using the "big number" approach. The approach is to simply add a very large positive number (much larger than the other terms in the matrix) to the global stiffness matrix in the diagonal location corresponding to the node to be constrained. The 
same big number, multiplied by the nodal deflection, is added to the load vector in the location corresponding to the same node. This effectively acts to make the other terms in the corresponding equation negligible, forcing the nodal displacement to the desired value.

An example of the big number approach will be applied to a three-equation system:

$$
\left[\begin{array}{ccc}
5 & 9 & -11 \\
3 & 0.5 & 3 \\
-2 & 6.5 & 2.3
\end{array}\right]\left[\begin{array}{l}
x_{1} \\
x_{2} \\
x_{3}
\end{array}\right]=\left[\begin{array}{c}
1 \\
5 \\
-10
\end{array}\right]
$$

Suppose that a boundary condition of $\mathrm{x}_{2}=3.2$ was to be imposed on this system of equations. This could be done using the big number approach as follows:

$$
\left[\begin{array}{ccc}
5 & 9 & -11 \\
3 & 0.5+1 \times 10^{10} & 3 \\
-2 & 6.5 & 2.3
\end{array}\right]\left[\begin{array}{l}
x_{1} \\
x_{2} \\
x_{3}
\end{array}\right]=\left[\begin{array}{c}
1 \\
5+(3.2)\left(1 \times 10^{10}\right) \\
-10
\end{array}\right]
$$

The other terms of the second equation become negligible, and $x_{2}$ is forced to a value of 3.2 .

\section{B.vii. Representative Calculations}

The same representative spindle considered in section 4.A.iii will now be considered to illustrate the methods developed in this section. The shaft is solid steel $(\mathrm{E}=$ $2 \times 10^{11} \mathrm{~Pa}$ ) with a diameter of $80 \mathrm{~mm}$ and an overhang length, $\mathrm{L}_{\mathrm{h}}$, of $100 \mathrm{~mm}$. SelfCompensated Bearing \#1 will be used to support the spindle. A supply pressure of 600 psig will again be used. For simplicity, the front and rear bearings used will be identical. A bearing with the parameters given in Table 3.3 will again be used. Recall that the geometry was optimized for maximum initial specific stiffness, with the shaft displaced 
parallel to the bearing housing. A supply pressure of $600 \mathrm{psig}$ is used to support the spindle. Both compensators were oriented toward the rear of the spindle. The resulting spindle stiffness was calculated to be $129 \mathrm{~N} / \mu \mathrm{m}$. This is approximately $17 \%$ lower than the $151 \mathrm{~N} / \mu \mathrm{m}$ spindle stiffness calculated with methods that neglect the effect of shear deformations. This is a typicall error incurred for spindles with $L_{1} / D$ of 1 to 1.5 For shorter spindles, the error can be significantly larger.

\section{C. Comparison of Spindle Stiffness Using the Twelve Radial Hydrostatic Bearings of Chapter 3}

The twelve radial hydrostatic bearings of Chapter 3 will now each be integrated into a simple two-bearing spindle and their stiffnesses will be evaluated. For simplicity, bearings of identical geometry will be used for both the front and rear radial bearings; although in practice the two bearings may be different, the rear bearing generally has a small impact on the stiffness of the spindle and so using identical bearings is warranted for this comparison study. In each case, the solid steel $\left(E=2 \times 10^{11} \mathrm{~Pa}\right)$ shaft will have a diameter of $80 \mathrm{~mm}$ and an overhang length, $\mathrm{L}_{\mathrm{h}}$, of $120 \mathrm{~mm}$. The spindle stiffness will be calculated using the finite element methods developed in section 4.B. The effect of shaft tilting on the bearing performance will be included. The finite element model also includes the effect of shear formations on the shaft bending. Although this model will be used to calculate the spindle stiffness, the bearing spacing distance, $\mathrm{L}_{2}$, will be optimized in each case according to the more simple analysis developed in section 4.A; Equation (4.2) will be used to calculate the optimal bearing spacing. This is approach is adequate to provide approximately maximum spindle stiffness. 


\section{C.i. Fixed-Compensated Bearing \#1}

First, the Fixed-Compensated Bearing \#1 with a geometry of Table 3.1 was integrated into a complete two-bearing spindle. The resulting spindle stiffness was 99.6 $\mathrm{N} / \mu \mathrm{m}$. Next, the geometry of the bearing was optimized for maximum spindle stiffness. First, the length of the bearing was increased to $90 \mathrm{~mm}$; doing so increases the bearing stiffness, however, increasing the length of the bearing also places the effective load center of the front bearing further away to the front of the spindle. There is therefore an optimal bearing length that provides maximum spindle stiffness. In this case, the optimal bearing length was $90 \mathrm{~mm}$. Second, the width of the bearing circumferential land furthest from the front of the spindle, $L_{1 m p}$, was increased from $3 \mathrm{~mm}$ to $41 \mathrm{~mm}$; although doing so reduces the bearing stiffness, it makes the spindle more sensitive the fluid flowing over the other circumferential land, $\mathrm{L}_{\text {lep}}$, which is closer to the front of the spindle and increases the spindle stiffness. Increasing the width of the axial lands, $\mathrm{L}_{\mathrm{lp}}$, was found to decrease the load-supporting area too much and the spindle stiffness was reduced, so its width was left at $3 \mathrm{~mm}$. A comparison of the new and old spindle stiffness, bearing stiffness, and bearing flow rate is shown in Table 4.1. Although the bearing stiffness was considerably reduced, the spindle stiffness was increased by approximately $8 \%$. As a bonus, the bearing flow rate was reduced considerably. The parameters of the bearing optimized for maximum spindle stiffness are shown in Table 4.2.

\section{Table 4.1}

\begin{tabular}{|c|c|c|c|}
\hline & $\begin{array}{c}\text { Bearing } \\
\text { Stiffness } \\
(\mathbf{N} / \mu \mathrm{m})\end{array}$ & $\begin{array}{c}\text { Bearing } \\
\text { Flow Rate } \\
(\mathrm{gpm})\end{array}$ & $\begin{array}{c}\text { Spindle } \\
\text { Stiffness } \\
(\mathbf{N} / \mu \mathrm{m})\end{array}$ \\
\hline Bearing Optimized & 1520 & 2.58 & 99.6 \\
\hline Spindle Optimized & 1330 & 1.92 & 108 \\
\hline
\end{tabular}


Table 4.2.

\begin{tabular}{|c|c|c|c|c|}
\hline $\begin{array}{l}\text { Operating Conditions } \\
\text { Supply Pressure ( } \mathrm{Pa} \text {,psi,atm) } \\
\text { Eccentricity Ratio em/ee } \\
\text { Eccentricity at End } \\
\text { Eccentricity at Middle End }\end{array}$ & $\begin{array}{l}\text { Ps } \\
\text { lamda } \\
\text { ee } \\
\text { em }\end{array}$ & $\begin{array}{r}4.17 \mathrm{E}+06 \\
1 \\
0.01 \\
0.01\end{array}$ & 600 & 40.8 \\
\hline \multicolumn{5}{|l|}{$\begin{array}{l}\text { Fluid Properties } \\
\text { Dynamic Viscosity (N s/m) }\end{array}$} \\
\hline \multicolumn{5}{|l|}{ Bearing Geometry } \\
\hline Diameter $(m, m m, i n)$ & D & 0.08 & 80 & 3.15 \\
\hline Axial Length $(\mathrm{m}, \mathrm{mm}, \mathrm{in})$ & $\mathrm{L}$ & 0.09 & 90 & 3.54 \\
\hline Number of Pockets & $\mathbf{N}$ & 6 & & \\
\hline Nominal Gap (m, $\mu \mathrm{m}$, in) & ho & 0.000015 & 15 & 0.00059 \\
\hline Nominal Resistance Ratio & gamma & 1.5 & & \\
\hline Pocket Circumferential Land Width (m, mm, in) & LIp & 0.003 & 3 & 0.118 \\
\hline Pocket End Axial Land Width (m,mm,in) & LImp & 0.041 & 41 & 1.614 \\
\hline Pocket Middle Axial Land Width (m, $\mathrm{mm}$, in) & Llep & 0.003 & 3 & 0.118 \\
\hline Width of Drain Grooves $(\mathrm{m}, \mathrm{mm}, \mathrm{in})$ & Ld & 0.00200 & 2 & 0.079 \\
\hline Pocket Circumferential Length (m,mm,in) & Lcp & 0.03989 & 39.9 & 1.570 \\
\hline \multicolumn{5}{|l|}{ Bearing Stiffness and Load Capacity } \\
\hline $\begin{array}{l}\text { Location of Effective Bearing Force } \\
\text { Pressure Difference / Pressure @ ec,ep above }\end{array}$ & $\begin{array}{l}\text { Lcen_L } \\
\text { DPP }\end{array}$ & $\begin{array}{l}0.429 \\
0.013\end{array}$ & & \\
\hline Load Capacity ( $N, k N$, lbf) @ ec,ep given above & Fy & 200 & 0.20 & 45 \\
\hline Load Carrying Efficiency @ ec,ep given above & Feff & 0.007 & & \\
\hline Stiffness $(N / \mu \mathrm{m}, \mathrm{lb} / \mu \mathrm{in}) @$ ec,ep given above & Ky & 1332 & 7.608 & \\
\hline Specific Stiffness @ ec,ep given above & Kspec & 0.666 & & \\
\hline Initial Specific Stiffness & Kspec,o & 0.666 & & \\
\hline Load Carrying Efficiency @ 75\% of gap closure & Feff, 0.75 & 0.421 & & \\
\hline $\begin{array}{l}\text { Flow Rate and Pumping Power } \\
\left.\text { Total Flow Rate ( }{ }^{`} 3 / \mathrm{s}, \mathrm{lpm}, \mathrm{gpm}\right) \\
\text { Pumping Power }(\mathrm{W}, \mathrm{kW}, \mathrm{hp}) \\
\text { Specific Flow Rate }\end{array}$ & $\begin{array}{l}\text { Qsupply } \\
\text { Ppump } \\
\text { Qspec }\end{array}$ & $\begin{array}{r}1.21 E-04 \\
506 \\
48.2\end{array}$ & $\begin{array}{r}7.28 \\
0.506\end{array}$ & $\begin{array}{r}1.92 \\
0.679\end{array}$ \\
\hline $\begin{array}{l}\text { Ratio of Specific Initial Stiffness to Specific } \\
\text { Flow Rate }\end{array}$ & K/Qspec & 0.0138 & & \\
\hline
\end{tabular}




\section{C.ii. Fixed-Compensated Bearing \#2}

First, the Fixed-Compensated Bearing \#2 with a geometry of Table 3.2 was integrated into a complete two-bearing spindle. The resulting spindle stiffness was 102 $\mathrm{N} / \mu \mathrm{m}$. Next, the geometry of the bearing optimized for maximum spindle stiffness. First, the length of the bearing was increased to $84 \mathrm{~mm}$. Second, the width of the bearing circumferential land furthest from the front of the spindle, $L_{l m p}$, was increased from $3 \mathrm{~mm}$ to $28 \mathrm{~mm}$; although doing so reduces the bearing stiffness, it makes the spindle more sensitive the fluid flowing over the other circumferential land, $\mathrm{L}_{\text {lep }}$, which is closer to the front of the spindle. A comparison of the new and old spindle stiffness, bearing stiffness, and bearing flow rate is shown in Table 4.3. Although the bearing stiffness was considerably reduced, the spindle stiffness was increased by approximately $14 \%$. As a bonus, the bearing flow rate was reduced considerably. The parameter of the bearing optimized for maximum spindle stiffness are shown in Table 4.4.

Table 4.3

\begin{tabular}{|c|c|c|c|}
\hline & $\begin{array}{c}\text { Bearing } \\
\text { Stiffness } \\
(\mathbf{N} / \mu \mathrm{m})\end{array}$ & $\begin{array}{c}\text { Bearing } \\
\text { Flow Rate } \\
(\mathrm{gpm})\end{array}$ & $\begin{array}{c}\text { Spindle } \\
\text { Stiffness } \\
(\mathbf{N} / \mu \mathrm{m})\end{array}$ \\
\hline Bearing Optimized & 1630 & 0.54 & 102 \\
\hline Spindle Optimized & 1400 & 0.46 & 116 \\
\hline
\end{tabular}


Table 4.4.

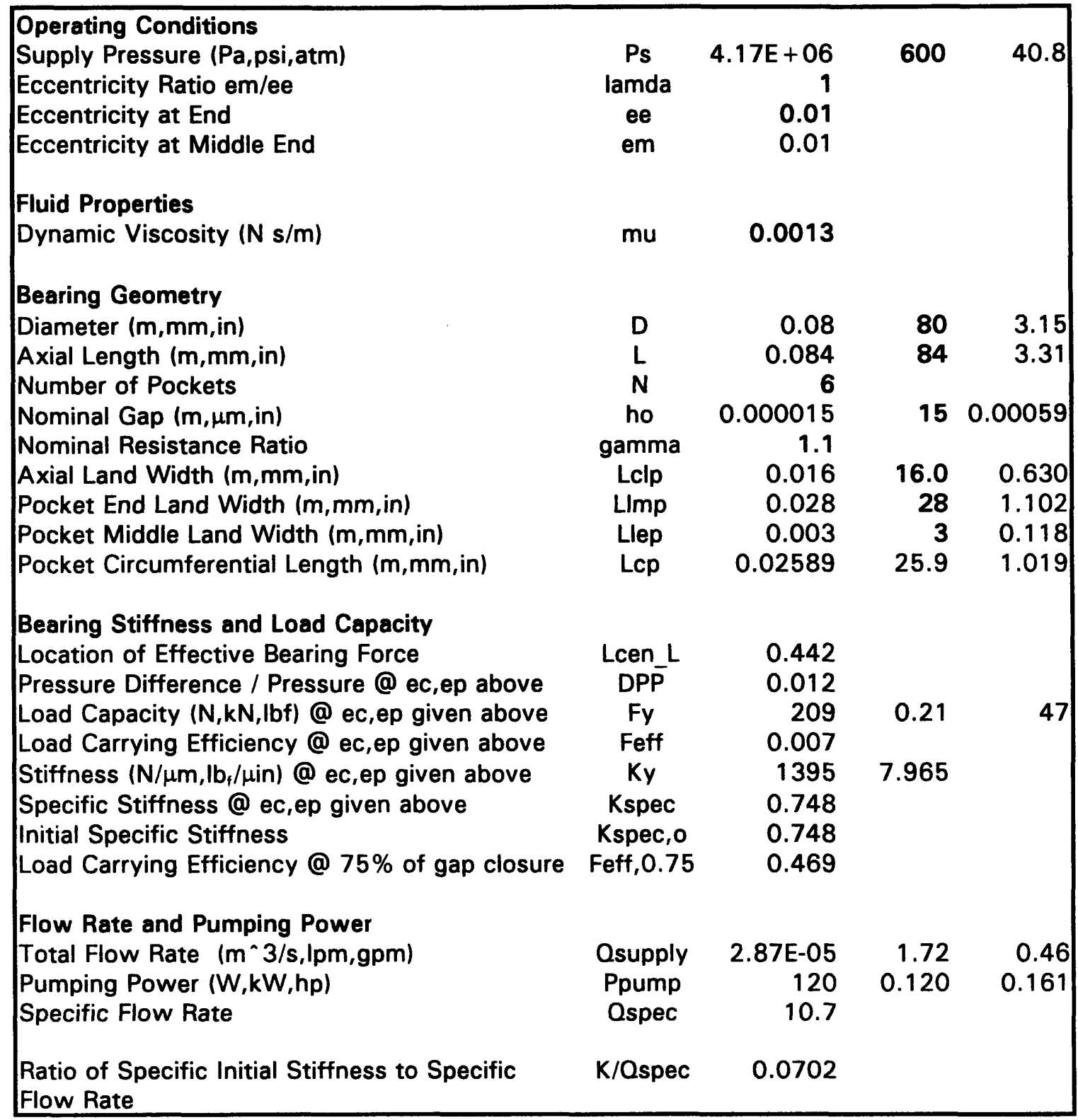




\section{C.iii. Self-Compensated Bearing \#1}

First, the Self-Compensated Bearing \#2 with a geometry of Table 3.3 was integrated into a complete two-bearing spindle. The configuration used was the front compensator oriented toward the rear and the rear compensator oriented toward the front. The resulting spindle stiffness was $102 \mathrm{~N} / \mu \mathrm{m}$. Next, the geometry of the bearing optimized for maximum spindle stiffness. The length of the bearing was unchanged since a bearing length of $80 \mathrm{~mm}$ was found to be approximately optimal. The width of the bearing pad circumferential land furthest from the front of the spindle, $\mathrm{L}_{\text {lmp }}$, was increased from $3 \mathrm{~mm}$ to $18 \mathrm{~mm}$; although doing so reduces the bearing stiffness, it makes the spindle more sensitive the fluid flowing over the other circumferential land, $\mathrm{L}_{\text {lep }}$, which is closer to the front of the spindle and increases the spindle stiffness. Increasing the width of the axial lands, $\mathrm{L}_{\mathrm{lp}}$, from $3 \mathrm{~mm}$ to $4.5 \mathrm{~mm}$ was also found to increase the spindle stiffness for the same reason. A comparison of the new and old spindle stiffness, bearing stiffness, and bearing flow rate is shown in Table 4.5. Although the bearing stiffness was considerably reduced, the spindle stiffness was increased by approximately $6 \%$. As a bonus, the bearing flow rate was reduced somewhat. The parameter of the bearing optimized for maximum spindle stiffness are shown in Table 4.6.

Table 4.5

\begin{tabular}{|c|c|c|c|}
\hline & $\begin{array}{c}\text { Bearing } \\
\text { Stiffness } \\
(\mathbf{N} / \mu \mathrm{m})\end{array}$ & $\begin{array}{c}\text { Bearing } \\
\text { Flow Rate } \\
(\mathrm{gpm})\end{array}$ & $\begin{array}{c}\text { Spindle } \\
\text { Stiffness } \\
(\mathbf{N} / \mu \mathrm{m})\end{array}$ \\
\hline Bearing Optimized & 1880 & 1.91 & 102 \\
\hline Spindle Optimized & 1760 & 1.62 & 108 \\
\hline
\end{tabular}


Table 4.6.

\begin{tabular}{|c|c|c|c|c|}
\hline $\begin{array}{l}\text { Operating Conditions } \\
\text { Supply Pressure (Pa,psi,atm) } \\
\text { Eccentricity Ratio ec/ep } \\
\text { Eccentricity at Pocket End } \\
\text { Eccentricity at Compensator End }\end{array}$ & $\begin{array}{c}\text { Ps } \\
\text { lamda } \\
\text { ep } \\
\text { ec }\end{array}$ & $\begin{array}{r}4.17 E+06 \\
1 \\
0.01 \\
0.01\end{array}$ & 600 & 40.8 \\
\hline $\begin{array}{l}\text { Fluid Properties } \\
\text { Dynamic Viscosity (N s/m) }\end{array}$ & mu & 0.0013 & & \\
\hline \multicolumn{5}{|l|}{ Bearing Geometry } \\
\hline Diameter $(\mathrm{m}, \mathrm{mm}, \mathrm{in})$ & D & 0.08 & 80 & 3.15 \\
\hline Axial Length $(\mathrm{m}, \mathrm{mm}$, in) & $\mathbf{L}$ & 0.08 & 80 & 3.15 \\
\hline Number of Pockets & $\mathbf{N}$ & 6 & & \\
\hline Nominal Gap $(m, \mu \mathrm{m}$, in) & ho & 0.000015 & 15 & 0.00059 \\
\hline Compensator Land Width (m, mm,in) & LIC & 0.003 & 3 & 0.118 \\
\hline Max Compensator Circumf. Length ( $\mathrm{m}, \mathrm{mm}$, in) & $\max$ Lcc & 0.0419 & 41.9 & 1.649 \\
\hline Compensator Circumf. Length (m, mm, in) & Lcc & 0.0398 & 39.8 & 1.567 \\
\hline Compensator Collector Groove Width $(\mathrm{m}, \mathrm{mm}, \mathrm{in})$ & Lcg & 0.002 & 2 & 0.079 \\
\hline Compensator Corner Radii (m,mm,in) & Radc & 0.0007 & 0.7 & 0.026 \\
\hline Supply Groove Width (m,mm,in) & Las & 0.002 & 2 & 0.079 \\
\hline Leakage Land Length $(\mathrm{m}, \mathrm{mm}, \mathrm{in})$ & Lleak & 0.009 & 9 & 0.354 \\
\hline Pocket Circumferential Land Width ( $\mathrm{m}, \mathrm{mm}$,in) & LIp & 0.0045 & 4.5 & 0.177 \\
\hline Pocket End Axial Land Width (m, mm, in) & LImp & 0.018 & 18 & 0.709 \\
\hline Pocket Middle Axial Land Width (m, $\mathrm{mm}$,in) & Llep & 0.003 & 3 & 0.118 \\
\hline Width of Drain Grooves $(\mathrm{m}, \mathrm{mm}$, in) & Ld & 0.00200 & 2 & 0.079 \\
\hline Pocket Axial Length (m,mm,in) & Lap & 0.05900 & 59.0 & 2.323 \\
\hline Pocket Circumferential Length $(\mathrm{m}, \mathrm{mm}$,in) & Lcp & 0.03989 & 39.9 & 1.570 \\
\hline Nominal Resistance Ratio & gamma & 1.16 & & \\
\hline \multicolumn{5}{|l|}{ Bearing Stiffness and Load Capacity } \\
\hline Location of Effective Bearing Force & Lcen_L & 0.257 & & \\
\hline Pressure Difference / Pressure @ ec,ep above & DPP & 0.028 & & \\
\hline Load Capacity (N,kN,lbf) @ ec,ep given above & Fy & 263 & 0.26 & 59 \\
\hline Load Carrying Efficiency @ ec,ep given above & Feff & 0.010 & & \\
\hline 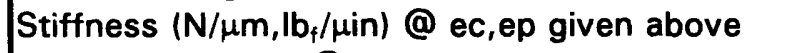 & Ky & 1755 & 10.020 & \\
\hline Specific Stiffness @ ec,ep given above & Kspec & 0.987 & & \\
\hline Initial Specific Stiffness & Kspec,o & 0.988 & & \\
\hline Load Carrying Efficiency @ 75\% of gap closure & Feff, 0.75 & 0.408 & & \\
\hline \multicolumn{5}{|l|}{ Flow Rate and Pumping Power } \\
\hline Leakage Flow Rate $\left(\mathrm{m}^{\wedge} 3 / \mathrm{s}, \mathrm{lpm}, \mathrm{gpm}\right)$ & Qleak & $2.52 \mathrm{E}-05$ & 1.51 & $\begin{array}{l}0.40 \\
1.62\end{array}$ \\
\hline Total Flow Rate $\left(\mathrm{m}^{\wedge} 3 / \mathrm{s}, \mathrm{lpm}, \mathrm{gpm}\right)$ & Qsupply & $1.02 E-04$ & 6.12 & $\begin{array}{r}1.62 \\
0.570\end{array}$ \\
\hline $\begin{array}{l}\text { Pumping Power (W,kW,hp) } \\
\text { Specific Flow Rate }\end{array}$ & $\begin{array}{l}\text { Ppump } \\
\text { Ospec }\end{array}$ & $\begin{array}{r}425 \\
44.9\end{array}$ & 0.425 & 0.570 \\
\hline $\begin{array}{l}\text { Ratio of Specific Initial Stiffness to Specific } \\
\text { Flow Rate }\end{array}$ & K/Ospec & 0.0220 & & \\
\hline
\end{tabular}




\section{C.iv. Self-Compensated Bearing \#2}

First, the Self-Compensated Bearing \#2 with a geometry of Table 3.4 was integrated into a complete two-bearing spindle. The resulting spindle stiffness was 109 $\mathrm{N} / \mu \mathrm{m}$. Next, the geometry of the bearing optimized for maximum spindle stiffness. First, the length of the bearing was reduced slightly to $77 \mathrm{~mm}$; although doing so reduces the bearing stiffness, it also places the effective load center of the front bearing closer to the front of the spindle and increases the spindle stiffness. Second, the width of the bearing pad circumferential land furthest from the front of the spindle, $\mathrm{L}_{\mathrm{lmp}}$, was increased from 3 $\mathrm{mm}$ to $11 \mathrm{~mm}$; although doing so reduces the bearing stiffness, it makes the spindle more sensitive to the fluid flowing over the other circumferential land, $\mathrm{L}_{\mathrm{lep}}$, which is closer to the front of the spindle, thereby increasing the spindle stiffness. A comparison of the new and old spindle stiffness, bearing stiffness, and bearing flow rate is shown in Table 4.7. Although the bearing stiffness was reduced, the spindle stiffness was increased by approximately $3 \%$. As a bonus, the bearing flow rate was reduced somewhat. The parameters of the bearing optimized for maximum spindle stiffness are shown in Table 4.8.

Table 4.7

\begin{tabular}{|c|c|c|c|}
\hline & $\begin{array}{c}\text { Bearing } \\
\text { Stiffness } \\
(\mathbf{N} / \mu \mathbf{m})\end{array}$ & $\begin{array}{c}\text { Bearing } \\
\text { Flow Rate } \\
(\mathbf{g p m})\end{array}$ & $\begin{array}{c}\text { Spindle } \\
\text { Stiffness } \\
(\mathbf{N} / \mu \mathbf{m})\end{array}$ \\
\hline Bearing Optimized & 2300 & 0.88 & 109 \\
\hline Spindle Optimized & 1980 & 0.79 & 112 \\
\hline
\end{tabular}


Table 4.8.

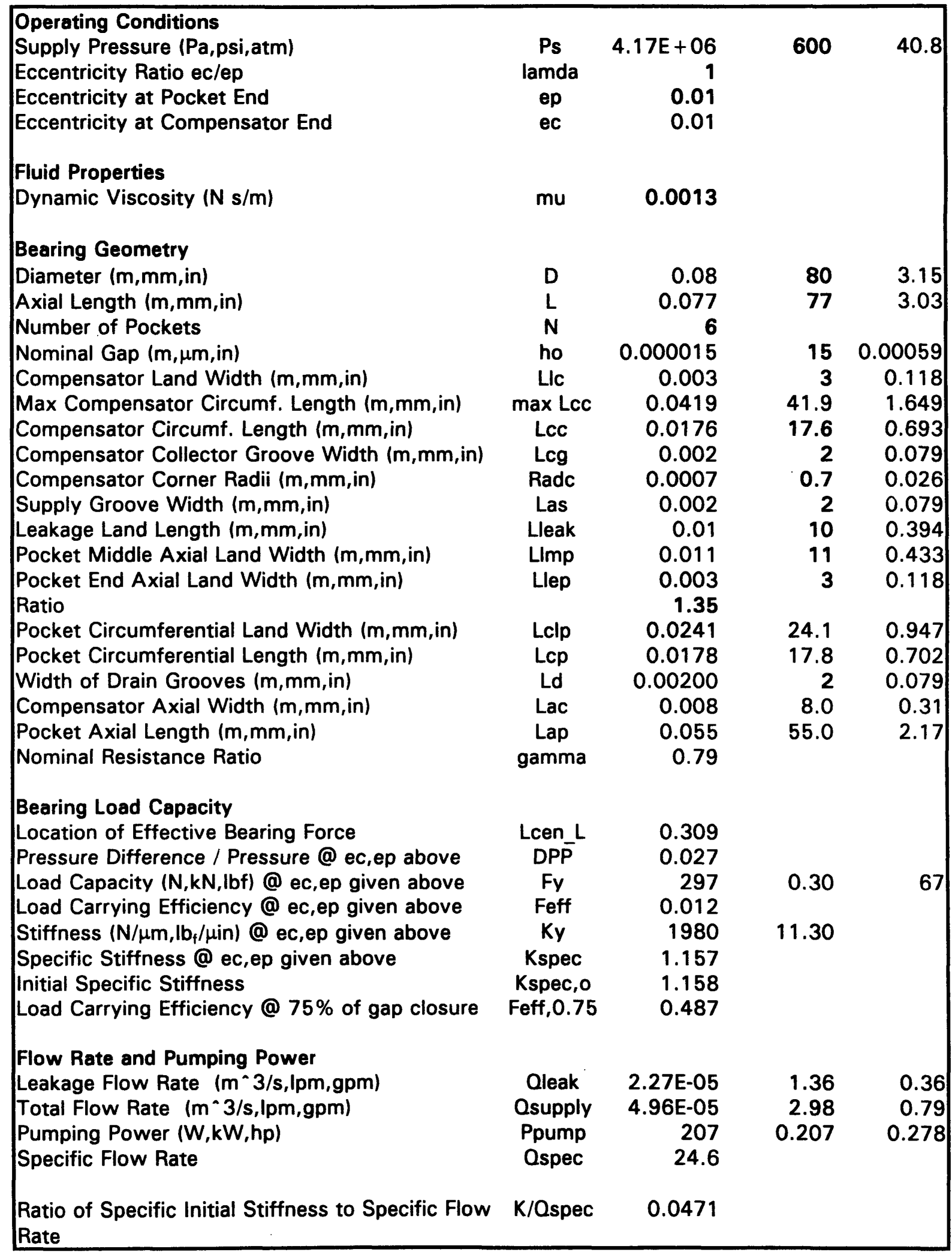




\section{C.v. Self-Compensated Bearing \#3}

First, the Self-Compensated Bearing \#3 with a geometry of Table 3.6 was integrated into a complete two-bearing spindle. The resulting spindle stiffness was 114 $\mathrm{N} / \mu \mathrm{m}$. Next, the geometry of the bearing optimized for maximum spindle stiffness. For this case, the optimized bearing geometry also provided the optimum spindle stiffness. A comparison of the new and old spindle stiffness, bearing stiffness, and bearing flow rate is shown in Table 4.9. The parameters of the bearing are shown in Table 4.10.

Table 4.9

\begin{tabular}{|c|c|c|c|}
\hline & $\begin{array}{c}\text { Bearing } \\
\text { Stiffness } \\
(\mathbf{N} / \mu \mathrm{m})\end{array}$ & $\begin{array}{c}\text { Bearing } \\
\text { Flow Rate } \\
(\mathrm{gpm})\end{array}$ & $\begin{array}{c}\text { Spindle } \\
\text { Stiffness } \\
(\mathbf{N} / \mu \mathrm{m})\end{array}$ \\
\hline Bearing Optimized & 2050 & 1.19 & 114 \\
\hline Spindle Optimized & 2050 & 1.19 & 114 \\
\hline
\end{tabular}


Table 4.10.

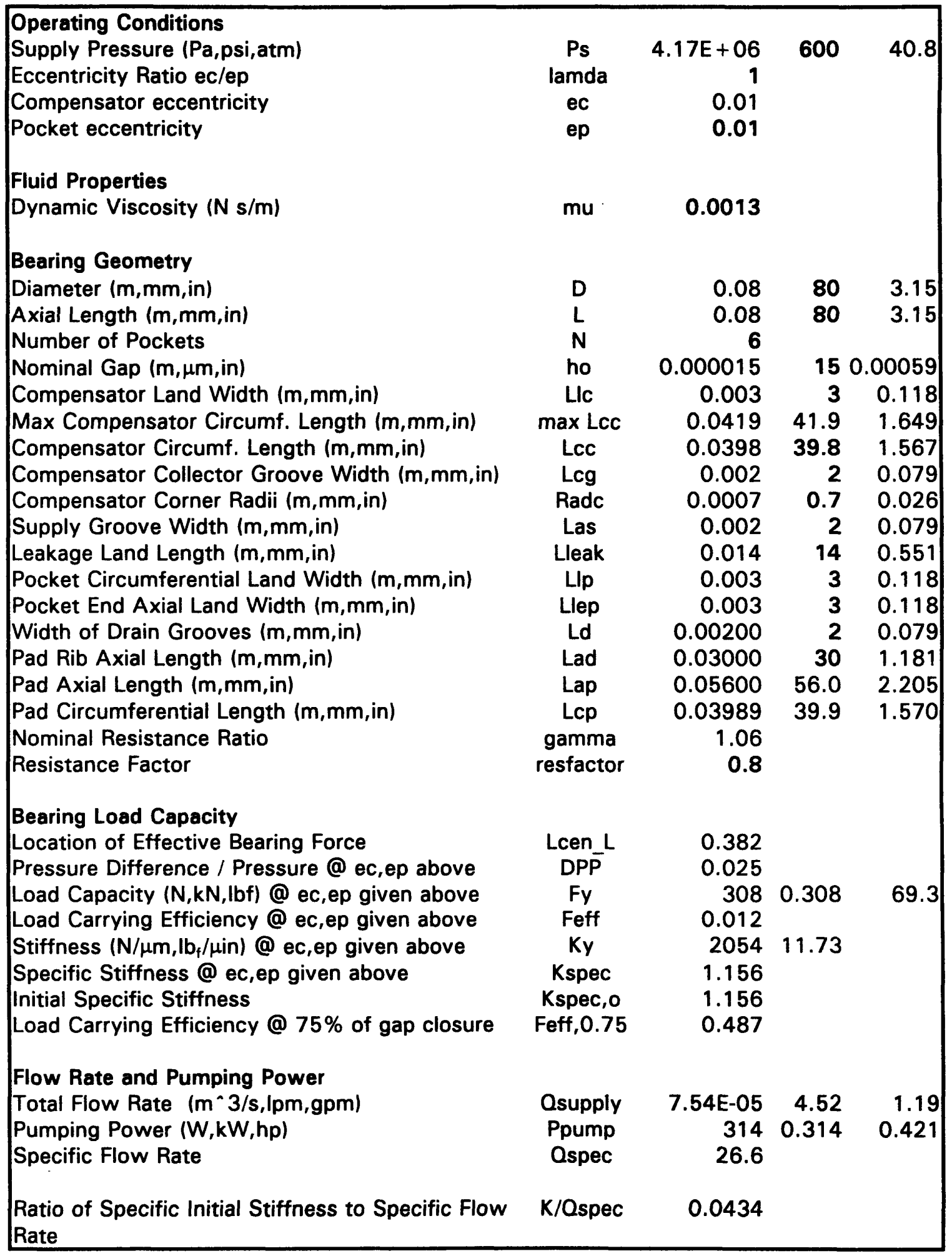




\section{C.vi. Self-Compensated Bearing \#4}

First, the Self-Compensated Bearing \#4 with a geometry of Table 3.7 was integrated into a complete two-bearing spindle. The optimal configuration was found to be with both compensators towards the rear of the spindle. The resulting spindle stiffness was $117 \mathrm{~N} / \mu \mathrm{m}$. Next, the geometry of the bearing was optimized for maximum spindle stiffness. The length of the bearing was increased to $97 \mathrm{~mm}$. A comparison of the new and old spindle stiffness, bearing stiffness, and bearing flow rate is shown in Table 4.11. As shown, the configuration that was optimized for bearing stiffness is nearly equivalent to that optimized for spindle stiffness for this case. The parameters of the bearing optimized for maximum spindle stiffness are shown in Table 4.12.

\section{Table 4.11}

\begin{tabular}{|c|c|c|c|}
\hline & $\begin{array}{c}\text { Bearing } \\
\text { Stiffness } \\
(\mathbf{N} / \mu \mathbf{m})\end{array}$ & $\begin{array}{c}\text { Bearing } \\
\text { Flow Rate } \\
(\mathrm{gpm})\end{array}$ & $\begin{array}{c}\text { Spindle } \\
\text { Stiffness } \\
(\mathbf{N} / \mu \mathbf{m})\end{array}$ \\
\hline Bearing Optimized & 1790 & 0.33 & 117 \\
\hline Spindle Optimized & 2200 & 0.34 & 120 \\
\hline
\end{tabular}


Table 4.12.

\begin{tabular}{|c|c|c|c|c|}
\hline $\begin{array}{l}\text { Operating Conditions } \\
\text { Supply Pressure (Pa,psi,atm) } \\
\text { Eccentricity Ratio ec/ep } \\
\text { Eccentricity at Pocket End } \\
\text { Eccentricity at Compensator End }\end{array}$ & $\begin{array}{l}\text { Ps } \\
\text { lamda } \\
\text { ep } \\
\text { ec }\end{array}$ & $\begin{array}{r}4.17 E+06 \\
1 \\
0.01 \\
0.01\end{array}$ & 600 & 40.8 \\
\hline $\begin{array}{l}\text { Fluid Properties } \\
\text { Dynamic Viscosity (N s/m) }\end{array}$ & $\mathrm{mu}$ & 0.0013 & & \\
\hline 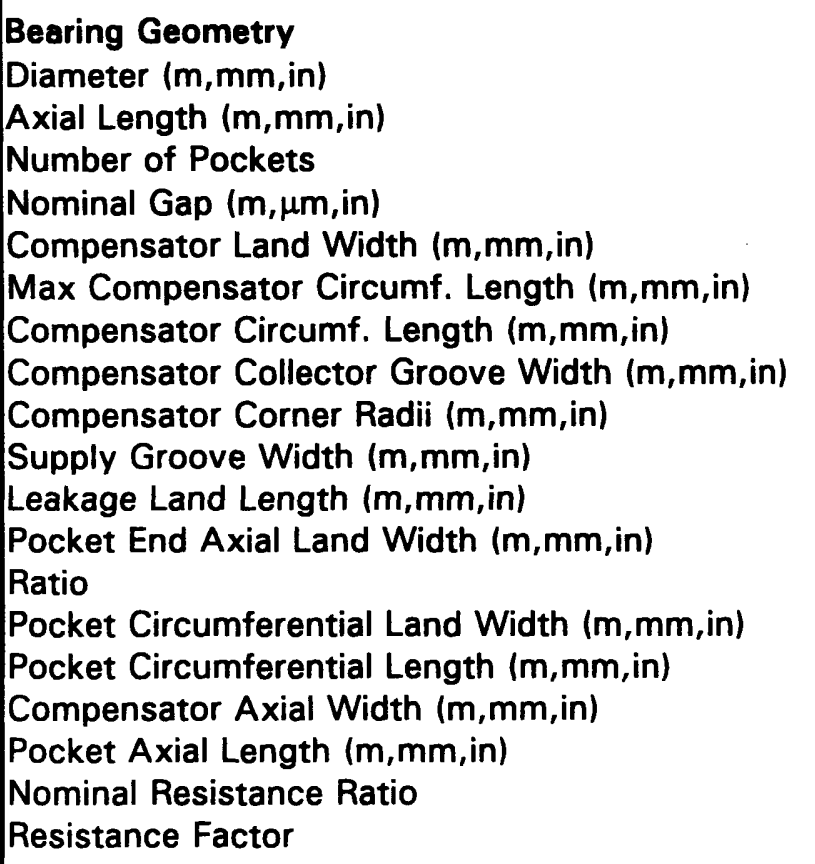 & $\begin{array}{c}D \\
L \\
N \\
\text { ho } \\
\text { Llc } \\
\text { max Lcc } \\
\text { Lcc } \\
\text { Lcg } \\
\text { Radc } \\
\text { Las } \\
\text { Lleak } \\
\text { Llep } \\
\text { Lclp } \\
\text { Lcp } \\
\text { Lac } \\
\text { Lap } \\
\text { gamma } \\
\text { resfactor }\end{array}$ & $\begin{array}{r}0.08 \\
0.097 \\
6 \\
0.000015 \\
0.003 \\
0.0419 \\
0.0126 \\
0.002 \\
0.0007 \\
0.002 \\
0.01 \\
0.003 \\
1.5 \\
0.0251 \\
0.0168 \\
0.008 \\
0.077 \\
0.84 \\
0.8\end{array}$ & $\begin{array}{r}80 \\
97 \\
\\
15 \\
3 \\
41.9 \\
12.6 \\
2 \\
0.7 \\
2 \\
10 \\
3\end{array}$ & $\begin{array}{r}3.15 \\
3.82 \\
\\
0.00059 \\
0.118 \\
1.649 \\
0.495 \\
0.079 \\
0.026 \\
0.079 \\
0.394 \\
0.118\end{array}$ \\
\hline 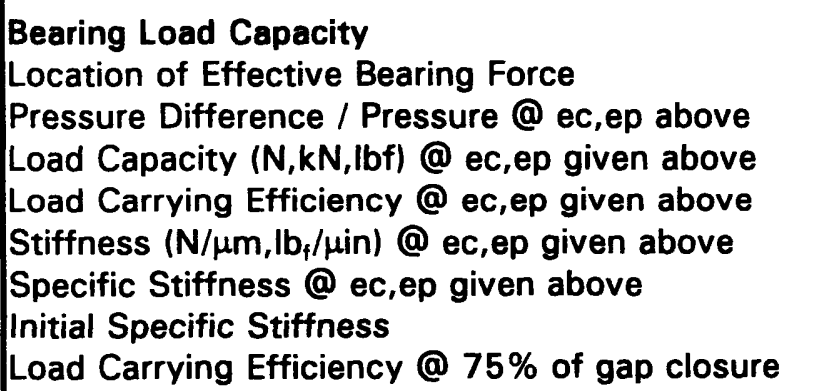 & $\begin{array}{c}\text { Lcen_L } \\
\text { DPP } \\
\text { Fy } \\
\text { Feff } \\
\text { Ky } \\
\text { Kspec } \\
\text { Kspec,o } \\
\text { Feff,0.75 }\end{array}$ & $\begin{array}{r}0.393 \\
0.018 \\
330 \\
0.010 \\
2201 \\
1.021 \\
1.021 \\
0.440\end{array}$ & $\begin{array}{r}0.33 \\
12.57\end{array}$ & 74 \\
\hline $\begin{array}{l}\text { Flow Rate and Pumping Power } \\
\text { Total Flow Rate (m^3/s,lpm,gpm) } \\
\text { Pumping Power }(W, k W, h p) \\
\text { Specific Flow Rate }\end{array}$ & $\begin{array}{l}\text { Qsupply } \\
\text { Ppump } \\
\text { Qspec }\end{array}$ & $\begin{array}{r}2.11 \mathrm{E}-05 \\
88 \\
9.1\end{array}$ & $\begin{array}{r}1.27 \\
0.088\end{array}$ & $\begin{array}{r}0.34 \\
0.118\end{array}$ \\
\hline $\begin{array}{l}\text { Ratio of Specific Initial Stiffness to Specific Flow } \\
\text { Rate }\end{array}$ & K/Ospec & 0.1128 & & \\
\hline
\end{tabular}




\section{C.vii. Self-Compensated Bearing \#5}

First, the Self-Compensated Bearing \#5 with a geometry of Table 3.8 was integrated into a complete two-bearing spindle. The optimal configuration was found to be with both compensators towards the rear of the spindle. The resulting spindle stiffness was $87.6 \mathrm{~N} / \mu \mathrm{m}$. Next, the geometry of the bearing was optimized for maximum spindle stiffness. Second, the width of the bearing pad circumferential land furthest from the front of the spindle, $\mathrm{L}_{\mathrm{lmp}}$, was increased from $5 \mathrm{~mm}$ to $12 \mathrm{~mm}$; although doing so reduces the bearing stiffness, it makes the spindle more sensitive the fluid flowing over the other circumferential land, $\mathrm{L}_{\mathrm{lep}}$, which is closer to the front of the spindle. The width of the axial lands, $\mathrm{L}_{\text {lep }}$, was reduced to $3 \mathrm{~mm}$ for the same reason. A comparison of the new and old spindle stiffness, bearing stiffness, and bearing flow rate is shown in Table 4.13. The parameters of the bearing optimized for maximum spindle stiffness are shown in Table 4.14.

Table 4.13

\begin{tabular}{|c|c|c|c|}
\hline & $\begin{array}{c}\text { Bearing } \\
\text { Stiffness } \\
(\mathbf{N} / \mu \mathbf{m})\end{array}$ & $\begin{array}{c}\text { Bearing } \\
\text { Flow Rate } \\
(\mathbf{g p m})\end{array}$ & $\begin{array}{c}\text { Spindle } \\
\text { Stiffness } \\
(\mathbf{N} / \mu \mathbf{m})\end{array}$ \\
\hline Bearing Optimized & 1300 & 0.91 & 87.6 \\
\hline Spindle Optimized & 1210 & 0.88 & 90.4 \\
\hline
\end{tabular}


Table 4.14.

\begin{tabular}{|c|c|c|c|c|}
\hline $\begin{array}{l}\text { Operating Conditions } \\
\text { Supply Pressure (Pa,psi,atm) } \\
\text { Eccentricity Ratio ec/ep } \\
\text { Eccentricity at Pocket End } \\
\text { Eccentricity at Compensator End }\end{array}$ & $\begin{array}{l}\text { Ps } \\
\text { lamda } \\
\text { ep } \\
\text { ec }\end{array}$ & $\begin{array}{r}4.17 E+06 \\
1 \\
0.01 \\
0.01\end{array}$ & 600 & 40.8 \\
\hline \multicolumn{5}{|l|}{$\begin{array}{l}\text { Fluid Properties } \\
\text { Dynamic Viscosity (N s }\end{array}$} \\
\hline \multicolumn{5}{|l|}{ Bearing Geometry } \\
\hline $\begin{array}{l}\text { Diameter }(\mathrm{m}, \mathrm{mm}, \mathrm{in}) \\
\text { Axial Length }(\mathrm{m}, \mathrm{mm}, \mathrm{in})\end{array}$ & $\begin{array}{l}D \\
L\end{array}$ & $\begin{array}{l}0.08 \\
0.08\end{array}$ & $\begin{array}{l}80 \\
80\end{array}$ & $\begin{array}{l}3.15 \\
3.15\end{array}$ \\
\hline Number of Pockets & $\mathbf{N}$ & 6 & & \\
\hline Nominal Gap $(m, \mu m, i n)$ & ho & 0.000015 & 15 & 0.00059 \\
\hline Compensator Land Width (m, mm,in) & Llc & 0.003 & 3 & 0.118 \\
\hline Max Compensator Circumf. Length $(\mathrm{m}, \mathrm{mm}$, in) & $\max$ Lcc & 0.0419 & 41.9 & 1.649 \\
\hline Compensator Circumf. Length $(\mathrm{m}, \mathrm{mm}$,in) & Lcc & 0.0398 & 39.8 & 1.567 \\
\hline Compensator Collector Groove Width $(\mathrm{m}, \mathrm{mm}$, in) & Lcg & 0.002 & 2 & 0.079 \\
\hline Leakage Land Length (m,mm,in) & Lleak & 0.01 & 10 & 0.394 \\
\hline Pocket Circumferential Land Width ( $\mathrm{m}, \mathrm{mm}$, in) & LIp & 0.0055 & 5.5 & 0.217 \\
\hline Pocket End Axial Land Width ( $\mathrm{m}, \mathrm{mm}$,in) & LImp & 0.012 & 12 & 0.472 \\
\hline Pocket Middle Axial Land Width (m, mm, in) & Llep & 0.003 & 3 & 0.118 \\
\hline Width of Drain Grooves (m,mm,in) & Ld & 0.00200 & 2 & 0.079 \\
\hline Pocket Axial Length (m,mm,in) & Lap & 0.06300 & 63.0 & 2.480 \\
\hline Pocket Circumferential Length $(\mathrm{m}, \mathrm{mm}$, in) & Lcp & 0.03989 & 39.9 & 1.570 \\
\hline Nominal Resistance Ratio & gamma & 2.72 & & \\
\hline \multicolumn{5}{|l|}{ Bearing Stiffness and Load Capacity } \\
\hline Location of Effective Bearing Force & Lcen_L & 0.247 & & \\
\hline Pressure Difference / Pressure @ ec,ep above & DPP & 0.019 & & \\
\hline Load Capacity (N,kN,Ibf) @ ec,ep given above & Fy & 182 & 0.18 & 41 \\
\hline Load Carrying Efficiency @ ec,ep given above & Feff & 0.007 & & \\
\hline Stiffness $\left(N / \mu m, l b_{f} / \mu i n\right) @$ ec,ep given above & Ky & 1211 & 6.914 & \\
\hline Specific Stiffness @ ec,ep given above & Kspec & 0.681 & & \\
\hline Initial Specific Stiffness & Kspec,o & 0.681 & & \\
\hline Load Carrying Efficiency @ 75\% of gap closure & Feff,0.75 & 0.299 & & \\
\hline $\begin{array}{l}\text { Flow Rate and Pumping Power } \\
\text { Total Flow Rate (m^3/s,lpm,gpm) } \\
\text { Pumping Power }(\mathrm{W}, \mathrm{kW}, \mathrm{hp}) \\
\text { Specific Flow Rate }\end{array}$ & $\begin{array}{l}\text { Qsupply } \\
\text { Ppump } \\
\text { Qspec }\end{array}$ & $\begin{array}{r}5.56 \mathrm{E}-05 \\
232 \\
19.6\end{array}$ & $\begin{array}{r}3.34 \\
0.232\end{array}$ & $\begin{array}{r}0.88 \\
0.311\end{array}$ \\
\hline $\begin{array}{l}\text { Ratio of Specific Initial Stiffness to Specific Flow } \\
\text { Rate }\end{array}$ & K/Qspec & 0.0347 & & \\
\hline
\end{tabular}




\section{C.viii. Self-Compensated Bearing \#6}

First, the Self-Compensated Bearing \#6 with a geometry of Table 3.9 was integrated into a complete two-bearing spindle. The optimal configuration was found to be with both compensators towards the rear of the spindle. The resulting spindle stiffness was $88.1 \mathrm{~N} / \mu \mathrm{m}$. Next, the geometry of the bearing was optimized for maximum spindle stiffness. The width of the bearing pad circumferential land furthest from the front of the spindle, $\mathrm{L}_{\mathrm{lmp}}$, was increased from $3 \mathrm{~mm}$ to $8 \mathrm{~mm}$; although doing so reduces the bearing stiffness, it makes the spindle more sensitive the fluid flowing over the other circumferential land, $\mathbf{L}_{\text {lep }}$, which is closer to the front of the spindle. A comparison of the new and old spindle stiffness, bearing stiffness, and bearing flow rate is shown in Table 4.15. The parameters of the bearing optimized for maximum spindle stiffness are shown in Table 4.16

Table 4.15

\begin{tabular}{|c|c|c|c|}
\hline & $\begin{array}{c}\text { Bearing } \\
\text { Stiffness } \\
(\mathbf{N} / \mu \mathbf{m})\end{array}$ & $\begin{array}{c}\text { Bearing } \\
\text { Flow Rate } \\
(\mathrm{gpm})\end{array}$ & $\begin{array}{c}\text { Spindle } \\
\text { Stiffness } \\
(\mathbf{N} / \mu \mathbf{m})\end{array}$ \\
\hline Bearing Optimized & 1660 & 0.61 & $\mathbf{8 8 . 1}$ \\
\hline Spindle Optimized & 1600 & 0.54 & 93.0 \\
\hline
\end{tabular}


Table 4.16

\begin{tabular}{|c|c|c|c|c|}
\hline $\begin{array}{l}\text { Operating Conditions } \\
\text { Supply Pressure (Pa,psi,atm) } \\
\text { Eccentricity Ratio ec/ep } \\
\text { Eccentricity at Pocket End } \\
\text { Eccentricity at Compensator End }\end{array}$ & $\begin{array}{c}\text { Ps } \\
\text { lamda } \\
\text { ep } \\
\text { ec }\end{array}$ & $\begin{array}{r}4.17 E+06 \\
1 \\
0.01 \\
0.01\end{array}$ & 600 & 40.8 \\
\hline $\begin{array}{l}\text { Fluid Properties } \\
\text { Dynamic Viscosity (N s/m) }\end{array}$ & mu & 0.0013 & & \\
\hline \multicolumn{5}{|l|}{ Bearing Geometry } \\
\hline Diameter (m, mm,in) & D & 0.08 & 80 & 3.15 \\
\hline$m$, in) & $\mathrm{L}$ & 0.084 & 84 & 3.31 \\
\hline Number of Pockets & $\mathbf{N}$ & 6 & & \\
\hline Nominal Gap ( & ho & 0.000015 & 15 & 0.00059 \\
\hline Width $(\mathrm{m}, \mathrm{mm}, \mathrm{in})$ & Llc & 0.003 & & 0.118 \\
\hline (m, mm,in) & $\max$ Lcc & 0.0419 & 41.89 & 1.649 \\
\hline Co & Lc & 0.0314 & 31.4 & 1.237 \\
\hline Width (m,mm,in) & Lc & 0.002 & 2 & 0.079 \\
\hline Lea & Llea & 0.012 & 12 & 0.472 \\
\hline dth $(m, m m, i n)$ & LImp & 0.008 & 8 & 0.315 \\
\hline et End Axial Land Width (m,mm,in) & Llep & 0.003 & 3 & 0.118 \\
\hline $\begin{array}{l}\text { Ratio } \\
\text { Pocket Circumferential Land Width (m, mm, in) }\end{array}$ & Lclp & 0.0219 & 21.9 & 0.864 \\
\hline Pocket Circun & Lcp & 0.0199 & 19.9 & 0.785 \\
\hline Width of Drain Grooves (m, mm,in) & Ld & 0.00200 & & 0.079 \\
\hline$(m, m m, i n)$ & Lap & 0.065 & 65.0 & 2.56 \\
\hline Nominal Resistance Ratio & gamma & 1.20 & & \\
\hline \multicolumn{5}{|l|}{ Bearing Load Capacity } \\
\hline $\begin{array}{l}\text { Location of Effective Bearing Force } \\
\text { Pressure Difference / Pressure @ ec,ep above }\end{array}$ & $\begin{array}{l}\text { Lcen_L } \\
\text { DPP }\end{array}$ & $\begin{array}{l}0.269 \\
0.020\end{array}$ & & \\
\hline Load Capacity (N,kN,lbf) @ ec,ep given above & Fy & 239 & 0.24 & 54 \\
\hline Load Carrying Efficiency @ ec,ep given above & Feff & 0.009 & & \\
\hline Stiffness $\left(N / \mu m, l b_{f} / \mu i n\right) @$ ec,ep given above & Ky & 1594 & 9.10 & \\
\hline Specific Stiffness @ ec,ep given above & Kspec & 0.854 & & \\
\hline Initial Specific Stiffness & Kspec,o & 0.854 & & \\
\hline Load Carrying Efficiency @ 75\% of gap closure & Feff, 0.75 & 0.367 & & \\
\hline Flow Rate and Pumping Power & & & & \\
\hline $\begin{array}{l}\text { Total Flow Rate }(\mathrm{m} ` 3 / \mathrm{s}, \mathrm{lpm}, \mathrm{gpm}) \\
\text { Pumping Power }(\mathrm{W}, \mathrm{kW}, \mathrm{hp})\end{array}$ & Qsupply & $\begin{array}{r}3.39 E-05 \\
141\end{array}$ & $\begin{array}{r}2.04 \\
0.141\end{array}$ & $\begin{array}{r}0.54 \\
0.190\end{array}$ \\
\hline & & 12.6 & & \\
\hline $\begin{array}{l}\text { Ratio of Specific Initial Stiffness to Specific Flow } \\
\text { Rate }\end{array}$ & K/Qspec & 0.0679 & & \\
\hline
\end{tabular}




\section{C.ix. Self-Compensated Bearing \#7}

First, the Self-Compensated Bearing \#7 with a geometry of Table 3.10 was integrated into a complete two-bearing spindle. The optimal configuration was found to be with both compensators towards the rear of the spindle. The resulting spindle stiffness was $109 \mathrm{~N} / \mu \mathrm{m}$. Next, the geometry of the bearing was optimized for maximum spindle stiffness. First, the length of the bearing was reduced to $77 \mathrm{~mm}$; although doing so reduces the bearing stiffness, it also places the effective load center of the front bearing closer to the front of the spindle and increases the spindle stiffness. Next, the leakage land width was increased to $15.5 \mathrm{~mm}$. A comparison of the new and old spindle stiffness, bearing stiffness, and bearing flow rate is shown in Table 4.16. As shown, the configuration that was optimized for bearing stiffness is nearly equivalent to that optimized for spindle stiffness for this case. The parameters of the bearing optimized for maximum spindle stiffness are shown in Table 4.17.

\section{Table 4.16}

\begin{tabular}{|c|c|c|c|}
\hline & $\begin{array}{c}\text { Bearing } \\
\text { Stiffness } \\
(\mathbf{N} / \mu \mathrm{m})\end{array}$ & $\begin{array}{c}\text { Bearing } \\
\text { Flow Rate } \\
(\mathrm{gpm})\end{array}$ & $\begin{array}{c}\text { Spindle } \\
\text { Stiffness } \\
(\mathbf{N} / \mu \mathrm{m})\end{array}$ \\
\hline Bearing Optimized & 1830 & 0.70 & 109 \\
\hline Spindle Optimized & 1730 & 0.60 & 110 \\
\hline
\end{tabular}


Table 4.17

\begin{tabular}{|c|c|c|c|c|}
\hline $\begin{array}{l}\text { Operating Conditions } \\
\text { Supply Pressure (Pa,psi,atm) } \\
\text { Eccentricity Ratio ec/ep } \\
\text { Compensator eccentricity } \\
\text { Pocket eccentricity }\end{array}$ & $\begin{array}{l}\text { Ps } \\
\text { lamda } \\
\text { ec } \\
\text { ep }\end{array}$ & $\begin{array}{r}4.17 E+06 \\
1 \\
0.01 \\
0.01\end{array}$ & 600 & 40.8 \\
\hline \multicolumn{5}{|l|}{$\begin{array}{l}\text { Fluid Properties } \\
\text { Dynamic Viscosity ( } \mathrm{N} / \mathrm{m} \text { ) }\end{array}$} \\
\hline \multicolumn{5}{|l|}{ Bearing Geometry } \\
\hline Diameter $(\mathrm{m}, \mathrm{mm}, \mathrm{in})$ & $D$ & 0.08 & 80 & 3.15 \\
\hline , mm,in) & $\mathbf{L}$ & 0.077 & 77 & 3.03 \\
\hline of Pockets & $\mathbf{N}$ & 6 & & \\
\hline$n, \mu m$, in) & ho & 0.000015 & 15 & 0.00059 \\
\hline and Width $(\mathrm{m}, \mathrm{mm}$, in) & Llc & 0.003 & 3 & 0.118 \\
\hline Max Compensator Circumf. Length (m, mm, in) & $\max$ Lcc & 0.0419 & 41.9 & 1.649 \\
\hline Compensator Circumf. Length $(\mathrm{m}, \mathrm{mm}, \mathrm{in})$ & Lcc & 0.0398 & 39.8 & 1.567 \\
\hline $\begin{array}{l}\text { Compensator Collector Groove Width } \\
(\mathrm{m}, \mathrm{mm}, \mathrm{in})\end{array}$ & Lcg & 0.002 & 2 & 0.079 \\
\hline Leakage Land Length ( $\mathrm{m}, \mathrm{mm}$,in) & Lleak & 0.0155 & 15.5 & 0.610 \\
\hline Pocket Circumferential Land Width ( $m, \mathrm{~mm}$, in) & Llp & 0.00375 & 3.75 & 0.148 \\
\hline Pocket End Axial Land Width (m,mm,in) & Llep & 0.003 & 3 & 0.118 \\
\hline Width of Drain Grooves (m, mm,in) & Ld & 0.00200 & 2 & 0.079 \\
\hline Pad Rib Axial Length (m, mm,in) & Lad & 0.05000 & 50 & 1.969 \\
\hline Pad Axial Length ( $m, m m$,in) & Lap & 0.05650 & 56.5 & 2.224 \\
\hline $\begin{array}{l}\text { Pad Circumferential Length (m,mm,in) } \\
\text { Nominal Resistance Ratio }\end{array}$ & $\begin{array}{l}\text { Lcp } \\
\text { gamma }\end{array}$ & $\begin{array}{r}0.03989 \\
0.95\end{array}$ & 39.9 & 1.570 \\
\hline & & & & 0.0035 \\
\hline \multicolumn{5}{|l|}{ Béaring Load Capacity } \\
\hline $\begin{array}{l}\text { Location of Effective Bearing Force } \\
\text { Pressure Difference / Pressure @ ec,ep above }\end{array}$ & $\underset{\text { DPP }}{\text { Lcen } L}$ & $\begin{array}{l}0.339 \\
0.020\end{array}$ & & \\
\hline $\begin{array}{l}\text { Load Capacity (N,kN,lbf) @ ec,ep given above } \\
\text { Load Carrying Efficiency @ ec,ep given above }\end{array}$ & $\begin{array}{l}\text { Fy } \\
\text { Feff }\end{array}$ & $\begin{array}{r}259 \\
0.010\end{array}$ & 0.259 & 58.3 \\
\hline Stiffness $\left(N / \mu m, l b_{f} / \mu i n\right) @$ ec,ep given above & Ky & 1729 & 9.87 & \\
\hline Specific Stiffness @ ec,ep given above & Kspec & 1.011 & & \\
\hline Initial Specific Stiffness & Kspec,o & 1.011 & & \\
\hline Load Carrying Efficiency @ 75\% of gap closure & Feff, 0.75 & 0.437 & & \\
\hline $\begin{array}{l}\text { Flow Rate and Pumping Power } \\
\text { Total Flow Rate (m^3/s,lpm,gpm) } \\
\text { Pumping Power (W,kW,hp) } \\
\text { Specific Flow Rate }\end{array}$ & $\begin{array}{c}\text { Osupply } \\
\text { Ppump } \\
\text { Ospec }\end{array}$ & $\begin{array}{r}3.81 \mathrm{E}-05 \\
159 \\
12.9\end{array}$ & $\begin{array}{r}2.28 \\
0.159\end{array}$ & $\begin{array}{r}0.60 \\
0.213\end{array}$ \\
\hline $\begin{array}{l}\text { Ratio of Specific Initial Stiffness to Specific } \\
\text { Flow Rate }\end{array}$ & K/Ospec & 0.0781 & & \\
\hline
\end{tabular}




\section{C.x. Self-Compensated Bearing \#8}

First, the Self-Compensated Bearing \#8 with a geometry of Table 3.11 was integrated into a complete two-bearing spindle. The optimal configuration was found to be with both compensators towards the rear of the spindle. The resulting spindle stiffness was $110 \mathrm{~N} / \mu \mathrm{m}$. Next, the geometry of the bearing was optimized for maximum spindle stiffness. First, the length of the bearing was increased to $84 \mathrm{~mm}$. Next, the width of the leakage land, Lleak, was increased to $15 \mathrm{~mm}$; although doing so decreases the effective supporting area of the bearing, it also pushes the effective bearing center closer to the front of the spindle and reduces leakage between the compensators and the pockets. A comparison of the new and old spindle stiffness, bearing stiffness, and bearing flow rate is shown in Table 4.18. The parameters of the bearing optimized for maximum spindle stiffness are shown in Table 4.19.

\section{Table 4.18}

\begin{tabular}{|c|c|c|c|}
\hline & $\begin{array}{c}\text { Bearing } \\
\text { Stiffness } \\
(\mathbf{N} / \mu \mathrm{m})\end{array}$ & $\begin{array}{c}\text { Bearing } \\
\text { Flow Rate } \\
(\mathrm{gpm})\end{array}$ & $\begin{array}{c}\text { Spindle } \\
\text { Stiffness } \\
(\mathbf{N} / \mu \mathrm{m})\end{array}$ \\
\hline Bearing Optimized & 1840 & 0.36 & 110 \\
\hline Spindle Optimized & 1950 & 0.36 & 111 \\
\hline
\end{tabular}


Table 4.19

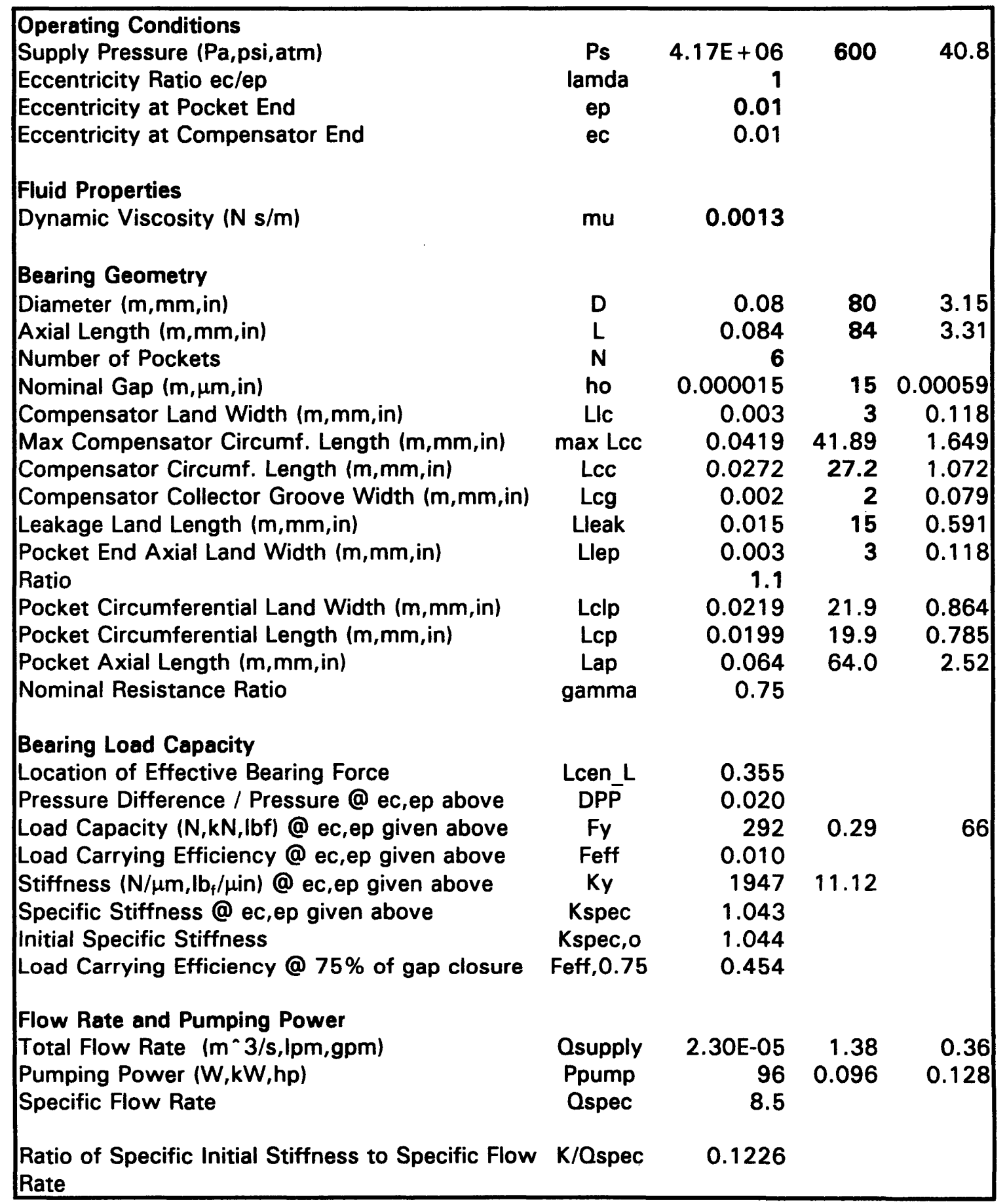




\section{C.xi. Self-Compensated Bearing \#9}

First, the Self-Compensated Bearing \#9 with a geometry of Table 3.12 was integrated into a complete two-bearing spindle. The optimal configuration was found to be with both compensators towards the rear of the spindle. The resulting spindle stiffness was $104 \mathrm{~N} / \mu \mathrm{m}$. Although SC Bearing \#9 has considerably lower stiffness than the other bearings considered in this section, it provides comparable spindle stiffness. This is understood by realizing that as the shaft bends at the front bearing, the compensating region remains at relatively small eccentricity while the outlet land is displaced to a large eccentricity. The front bearing therefore behaves similar to a fixed-compensated bearing when the shaft bends.

Next, the geometry of the bearing was optimized for maximum spindle stiffness. First, the length of the bearing was increased to $90 \mathrm{~mm}$; although doing so reduces the distance between the effective load center of the front bearing and the front of the spindle, it enhances the effect described above. A comparison of the new and old spindle stiffness, bearing stiffness, and bearing flow rate is shown in Table 4.20. The parameters of the bearing optimized for maximum spindle stiffness are shown in Table 4.21 .

\section{Table 4.20}

\begin{tabular}{|c|c|c|c|}
\hline & $\begin{array}{c}\text { Bearing } \\
\text { Stiffness } \\
(\mathbf{N} / \mu \mathrm{m})\end{array}$ & $\begin{array}{c}\text { Bearing } \\
\text { Flow Rate } \\
(\mathrm{gpm})\end{array}$ & $\begin{array}{c}\text { Spindle } \\
\text { Stiffness } \\
(\mathbf{N} / \mu \mathrm{m})\end{array}$ \\
\hline Bearing Optimized & 487 & 0.43 & 104 \\
\hline Spindle Optimized & 550 & 0.41 & 108 \\
\hline
\end{tabular}


Table 4.21

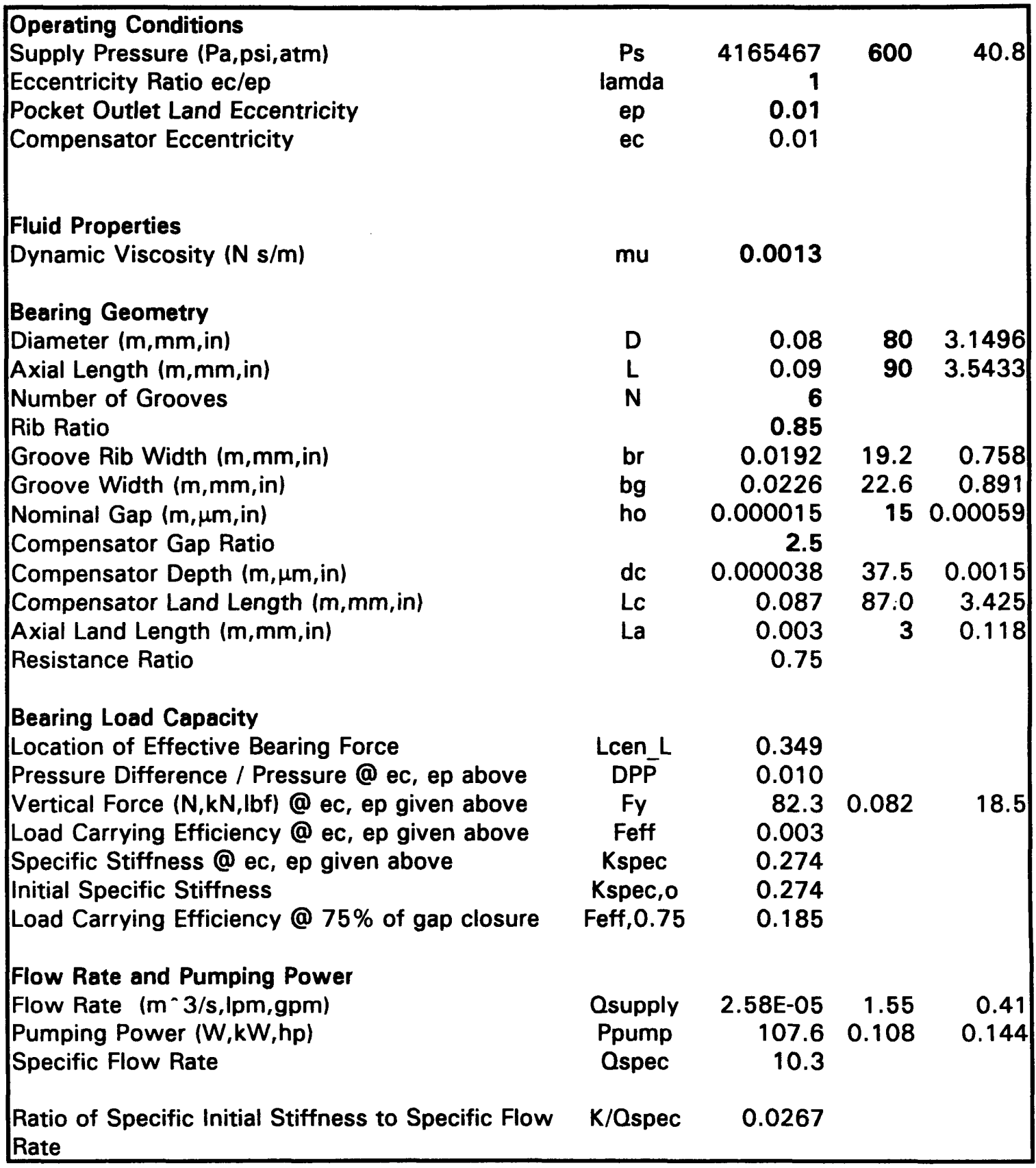




\section{C.xii. Self-Compensated Bearing \#10}

First, the Self-Compensated Bearing \#10 with a geometry of Table 3.13 was integrated into a complete two-bearing spindle. Note that this bearing was designed with a length of $80 \mathrm{~mm}$; radial hydrostatic bearings are commonly designed with their length equal to their diameter. The spindle stiffness using this bearing geometry was $99.9 \mathrm{~N} / \mu \mathrm{m}$. Next, the bearing geometry was optimized for maximum spindle stiffness. The optimal bearing length that produced the maximum spindle stiffness was $94 \mathrm{~mm}$. A comparison of the new and old spindle stiffness, bearing stiffness, and bearing flow rate is shown in Table 4.21. The parameters of the bearing optimized for maximum spindle stiffness are shown in Table 4.23.

Table 4.22

\begin{tabular}{|c|c|c|c|}
\hline & $\begin{array}{c}\text { Bearing } \\
\text { Stiffness } \\
(\mathbf{N} / \mu \mathrm{m})\end{array}$ & $\begin{array}{c}\text { Bearing } \\
\text { Flow Rate } \\
(\mathrm{gpm})\end{array}$ & $\begin{array}{c}\text { Spindle } \\
\text { Stiffness } \\
(\mathbf{N} / \mu \mathrm{m})\end{array}$ \\
\hline Bearing Optimized & 1930 & 0.44 & 99.9 \\
\hline Spindle Optimized & 2520 & 0.40 & 108 \\
\hline
\end{tabular}




\section{Table 4.23}

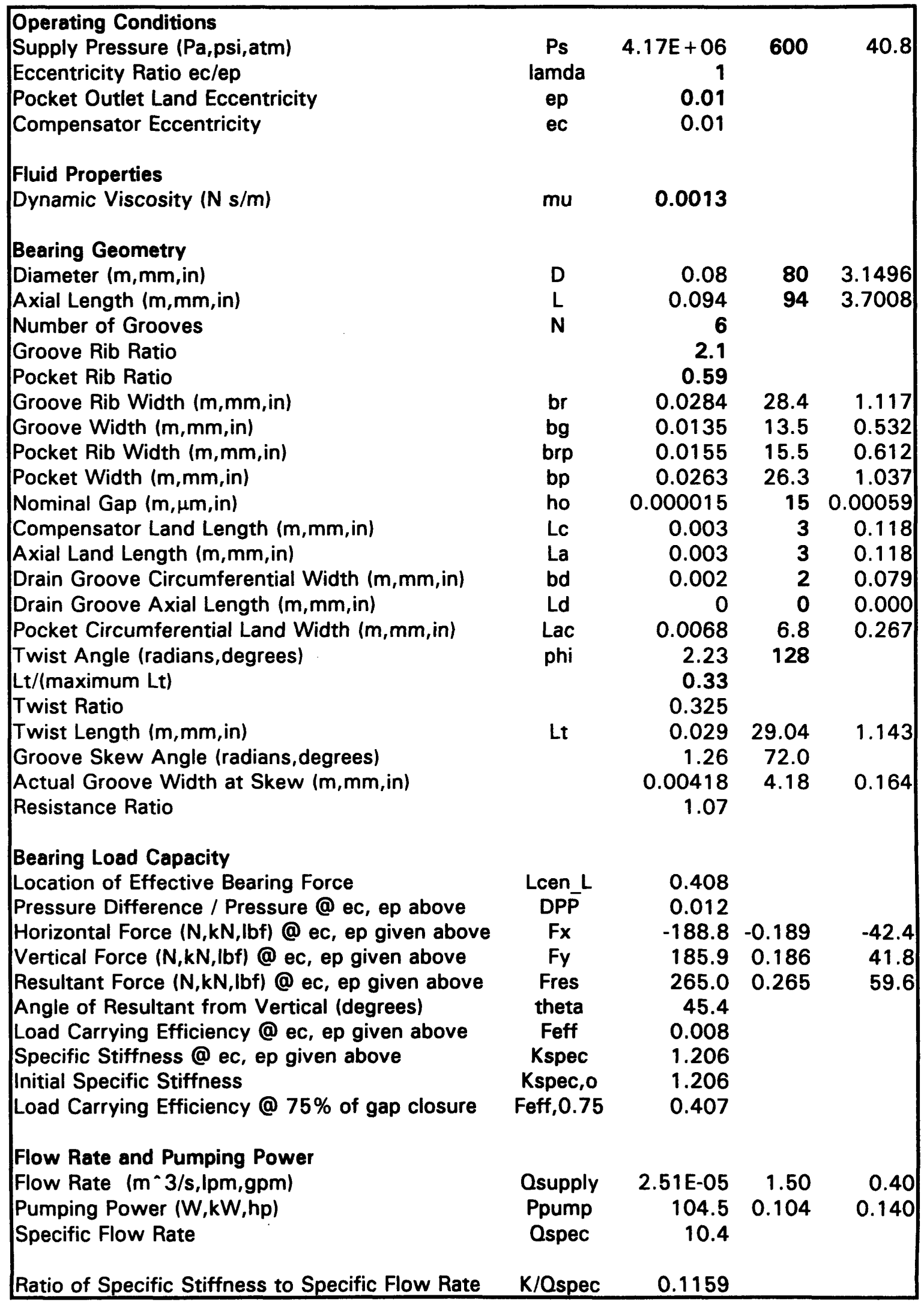




\section{C.xiii. Summary of Spindle Stiffness for Twelve Radial Bearings}

In the preceding sections, the twelve radial hydrostatic bearings of Chapter 3 were

each integrated into a simple two-bearing spindle and their stiffnesses were evaluated. A summary of their performances is shown in Table 4.24. 


\section{.}




\section{THRUST BEARING DESIGN: STIFFNESS, LOAD CAPACITY,}

AND FLOW RATE

The stiffness and load capacity of a machine tool spindle in the axial direction is usually just as important as its stiffness and load capacity in the radial direction. The primary purpose of the thrust bearings are to hold the shaft rigidly in the axial direction while allowing for free angular rotation. This should be accomplished with reasonably low flow rate and pumping power. Often the goals of high stiffness and low pumping power are competing and the most adequate design will depend upon the overall project objective. In some cases, achieving high spindle stiffness is the most important goal and high pumping power is an acceptable cost if it yields higher spindle stiffness. In other cases, the size and cost of the pump must be kept to a minimum, even if that means realizing lower axial stiffness. In the design of most machine tool spindles, both goals of high stiffness and low pumping power are important and a well-balanced bearing design is sought. Other important bearing attributes include the bearing damping, shearing power consumption, and temperature rise. These factors will be considered in future chapters. The focus of this chapter will be load-carrying capacity and pumping power.

The purpose of this chapter is to appropriately model hydrostatic thrust bearings so that their stiffness can be calculated and their design can be integrated into the overall design of the spindle. This is accomplished by calculating the restoring force that the bearing generates when the shaft is displaced from its equilibrium position. Fortunately, in nearly all cases the axial compliance of the shaft is much less than that of the thrust bearing and so the axial stiffness of the spindle is simply equal to the stiffness of its thrust bearing. 
Some hydrostatic thrust bearings used in machine tool spindles contain more than one pocket per side. These bearings are located at the front of the spindle so that they provide restoring moments to the shaft when a radial load is placed onto its nose, thereby increasing the radial stiffness of the spindle. However, the size of the thrust bearing must often be made large in order to contribute significantly to radial spindle stiffness. In this chapter, only bearings with a single pocket on each side are considered. It should be clearly emphasized that these bearings provide only axial support for the shaft.

The relations developed in this chapter can be readily implemented into computer programs to quickly design and optimize hydrostatic thrust bearings for machine tool spindles. In the derivations that follow, several important assumptions are made about the bearing characteristics and the operating conditions. It is assumed that:

- The depressed areas of the bearings, including the pockets, supply grooves, and drainage grooves, are at a depth sufficiently great that the pressure drop throughout them is negligible compared to the pressure drop across the bearing land regions. This assumption allows the depressed regions to be modeled as regions of constant pressure and enables fluid circuit theory to be used to solve for the bearing pressures.

- The fluid is incompressible so that density changes caused by changes in the fluid pressure or temperature are negligible as it passes through the bearing.

- The fluid has a constant dynamic viscosity as it passes through the bearing. This is a good assumption if the temperature rise of the fluid from the bearing inlet to the bearing outlet causes a negligible change in the fluid viscosity. 
- The fluid flow on the land areas of the bearing are laminar and fully-developed, as discussed in Chapter 2. In this case, the flow rate across a land is linearly proportional to the pressure difference across it and so the resistances of the fluid circuit are constant and linear fluid circuit theory can be used to solve for the flow rates and pressures.

These assumptions must be considered before the methods and results generated in this chapter are used in hydrostatic bearing design.

Three hydrostatic thrust bearings are considered in this chapter. In order to compare their relative merits and characteristics, some non-dimensional performance parameters will now be defined. The most important attribute of a bearing is its load carrying efficiency, which is the bearing restoring force divided by the supply pressure times the projected bearing area:

$$
\mathrm{F}_{\mathrm{eff}}=\frac{\mathrm{F}}{\mathrm{P}_{\mathrm{s}} \pi\left(\mathrm{R}_{\mathrm{o}}^{2}-\mathrm{R}_{\mathrm{i}}^{2}\right)}
$$

where $F$ is the applied load, $P_{s}$ is the supply pressure, $R_{i}$ is the inner diameter of the thrust area, and $R_{0}$ is the outer diameter of the thrust area. The load-carrying efficiency is a strong function of the displacement; it is usually fairly linear for deflections up to $50 \%$ of gap closure and then its slope decreases as the shaft comes closer to touch-down. At touch-down, most hydrostatic bearings have load-carrying efficiencies between 0.4 and 0.6 . The load-carrying efficiency at $75 \%$ of gap closure is usually a good indication of the load-capacity of the bearing, and will be used as an indication of such in this chapter.

Perhaps more important than the load-carrying efficiency of a bearing is its stiffness. The specific stiffness quantifies the bearing stiffness: 


$$
\mathrm{K}_{\text {spec }}=\frac{\mathrm{F}_{\text {eff }}}{\varepsilon}
$$

where $\varepsilon=\delta / h_{0}, \delta$ is the displacement, and $h_{0}$ is the nominal axial clearance per side. Hydrostatic bearings behave non-linearly and so the specfic stiffness is a function of the displacement. For applications where the cutting loads are low relative to the bearing load capacity, the stiffness of the bearing at small displacements is usually the most important consideration. Fortunately, the bearing stiffness is usually highest at small displacements. The initial specific stiffness of a bearing generally corresponds to very low displacements $(\varepsilon<0.01)$, and usually ranges between 0.8 and 1.2 for most hydrostatic bearings, but can be even higher.

The above bearing parameters describe the load-carrying performance of the bearing. Another important consideration is the amount of flow rate that is needed to achieve the load-carrying performance. A parameter that will be used to compare the flow rates for different bearings is the specific flow rate, which is the supply flow rate divided by the flow rate that would occur if the entire length of the bearing were used as a hydraulic resistance:

$$
Q_{\text {spec }}=\frac{Q_{s}}{\left(\frac{P_{s} \pi h_{o}^{3}}{3 \mu \ln \left(R_{o} / R_{i}\right)}\right)}
$$

The specific flow rate of hydrostatic thrust bearings vary from as little as 2 to as much as 20. The specific flow rate of a bearing is an indication of the pumping power required to support it since the pumping power is the supply pressure times the flow rate. 
Relations are developed in the following sections to predict the load-carrying performance of three hydrostatic thrust bearings. A representative bearing with an outer diameter of $100 \mathrm{~mm}$ and an inner diameter of $80 \mathrm{~mm}$ is considered in each case and its characteristics are presented. Although the flow rate is considered when the dimensions of the bearing geometries are chosen, the design in each case is primarily optimized to generate the maximum initial specific stiffness. In addition, the dimensions of the bearing geometry are chosen while considering manufacturing limitations. For example, the minimum groove width used is $2 \mathrm{~mm}$; this groove size can be generated fairly easily using conventional machining methods. The minimum land width used is $3 \mathrm{~mm}$; reducing the land width further would make manufacturing variations become significant. Also, the land width should be kept to at least $3 \mathrm{~mm}$ to keep the fluid flow across the lands laminar and fully-developed when water is used at supply pressures over 500 psi. Using these limitations and considerations, a representative bearing is designed and optimized for each bearing type.

\section{A. Thrust Bearing \#1}

A schematic diagram of Thrust Bearing \#1 is shown in Figure 5.1. This bearing is a conventional, fixed-compensated thrust bearing. Fluid at supply pressure, $P_{s}$, is supplied across inlet compensation resistances to the pockets. It then flows radially across the outlet resistances, which are at a clearance $h_{0}$, and to drainage grooves at atmospheric pressure, $\mathrm{P}_{\mathrm{a}}$. Note that the pockets shown in Figure 5.1 have been machined into the surface of the shaft flange; they may be equivalently machined into the housing member. 


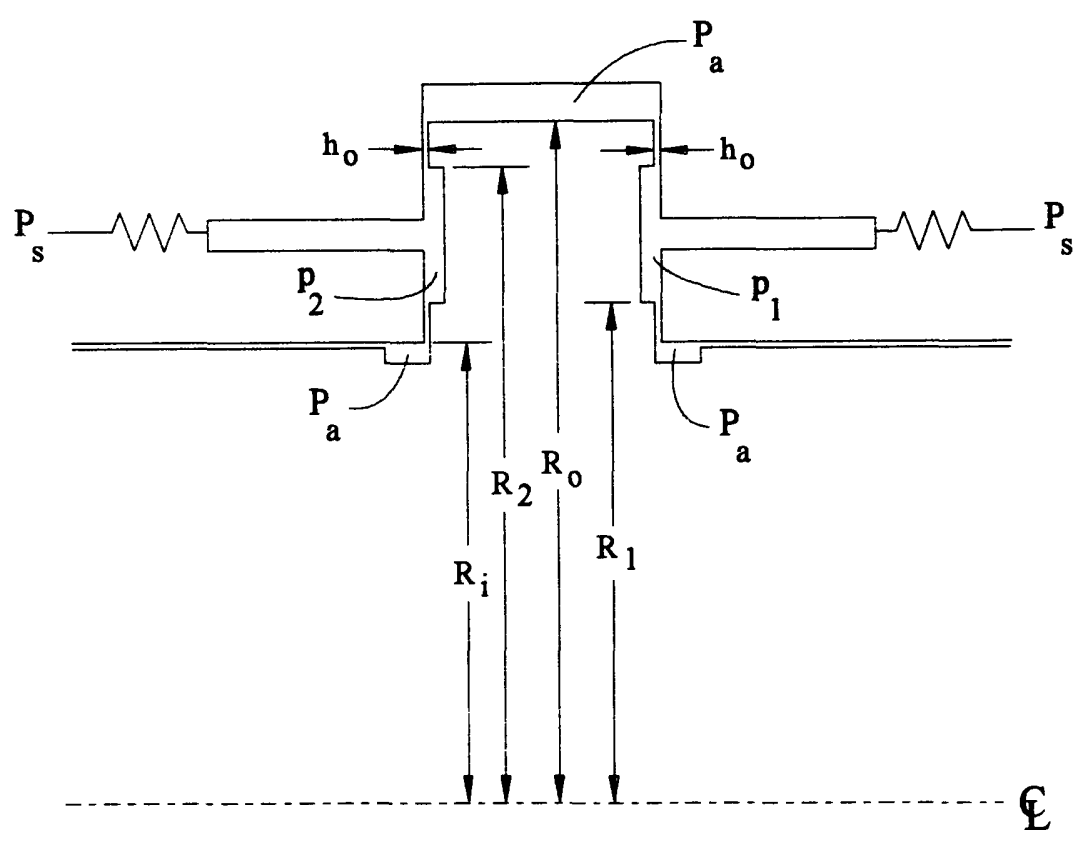

Figure 5.1. Schematic diagram of Thrust Bearing \#1. The two axial clearances, denoted with a magnitude $h_{0}$, are exagerated for clarity.

\section{A.i. Description of Calculations for Thrust Bearing \#1}

To calculate the characteristics of this thrust bearing, the resistance of each bearing land is needed. The resistance of the inner lands are given by:

$$
\begin{aligned}
& \mathbf{R}_{1}^{1}=\frac{6 \mu}{\pi \mathrm{h}_{\mathrm{o}}^{3}(1-\varepsilon)^{3}} \ln \left(\frac{\mathbf{R}_{1}}{\mathbf{R}_{\mathrm{i}}}\right) \\
& \mathbf{R}_{1}^{2}=\frac{6 \mu}{\pi \mathrm{h}_{\mathrm{o}}^{3}(1+\varepsilon)^{3}} \ln \left(\frac{\mathbf{R}_{1}}{\mathrm{R}_{\mathrm{i}}}\right)
\end{aligned}
$$

where the shaft is displaced such that the clearance at pocket 1 decreases. The resistance of the outer lands are given by:

$$
\mathrm{R}_{2}^{\prime}=\frac{6 \mu}{\pi \mathrm{h}^{3}(1-\varepsilon)^{3}} \ln \left(\frac{\mathrm{R}_{\mathrm{o}}}{\mathrm{R}_{2}}\right)
$$




$$
\mathrm{R}_{2}^{2}=\frac{6 \mu}{\pi \mathrm{h}_{\mathrm{o}}^{3}(1+\varepsilon)^{3}} \ln \left(\frac{\mathrm{R}_{\mathrm{o}}}{\mathrm{R}_{2}}\right)
$$

The overall pocket resistances are given by:

$$
\begin{aligned}
& \mathbf{R}_{\text {eq }}^{1}=\frac{1}{\frac{1}{\mathbf{R}_{1}^{1}}+\frac{1}{\mathbf{R}_{2}^{1}}} \\
& \mathbf{R}_{\text {eq }}^{2}=\frac{1}{\frac{1}{\mathbf{R}_{1}^{2}}+\frac{1}{\mathbf{R}_{2}^{2}}}
\end{aligned}
$$

The compenation resistances are chosen such the ratio of a compensation resistance to a nominal equivalent resistance is equal to some value, $\gamma$. The resistances of Circuit \#1 of Appendix B are now known. The solution for the pocket pressures is given by:

$$
\frac{p_{1}-p_{2}}{P_{s}}=\frac{\frac{1}{(1-\varepsilon)^{3}}}{\frac{1}{(1-\varepsilon)^{3}}+\gamma}-\frac{\frac{1}{(1+\varepsilon)^{3}}}{\frac{1}{(1+\varepsilon)^{3}}+\gamma}
$$

The bearing restoring force is this pressure difference times the "effective pocket area." The effective pocket area is that area which, when multiplied by the pocket pressure, results in the same force as if the detailed pressure profiles on the lands were considered. The effective area of a pocket is given by:

$$
A_{\text {eff }}=\frac{\pi}{2}\left(\frac{R_{0}^{2}-R_{2}^{2}}{\ln \left(R_{0} / R_{2}\right)}\right)-\frac{\pi}{2}\left(\frac{R_{1}^{2}-R_{i}^{2}}{\ln \left(R_{1} / R_{i}\right)}\right)
$$

The bearing restoring force is then given by:

$$
F=A_{\text {eff }}\left(p_{1}-p_{2}\right)
$$


In addition to the bearing load-carrying capability, the supply flow rate is needed. The supply flow rate is typically evaluated for small displacements, $\varepsilon=0$, since it varies only slightly with small displacements. The supply flow rate is simply the supply pressure divided by the overall resistance of both pockets. Nominally, both pocket resistances are equal, so the supply flow rate is given by:

$$
\mathrm{Q}_{\mathrm{s}}=\frac{2 \mathrm{P}_{\mathrm{s}}}{\mathrm{R}_{\mathrm{eq}}^{1}} \frac{1}{1+\gamma}=\frac{2 \mathrm{P}_{\mathrm{s}}}{\mathrm{R}_{\mathrm{eq}}^{2}} \frac{1}{1+\gamma}
$$

where the resistances are evaluated with $\varepsilon=0$.

The bearing performance is clearly a function of the resistance ratio, $\gamma$. The resistance ratio that provides maximum bearing load capacity can be found by taking the derivative of Equation (5.10) with respect to $\gamma$ and setting it equal to zero. This yields the following relation for the optimal resistance ratio:

$$
\gamma_{\mathrm{opt}}=\frac{\frac{\left(\frac{1-\varepsilon}{1+\varepsilon}\right)^{3 / 2}}{(1-\varepsilon)^{3}}-\frac{1}{(1-\varepsilon)^{3}}}{1-\left(\frac{1-\varepsilon}{1+\varepsilon}\right)^{3 / 2}}
$$

A plot of the optimal resistance ratio versus the displacement ratio is shown in Figure 5.2. As shown, a resistance ratio of 1 should be used to maximize the initial stiffness of the bearing; however, a value of approximately 3.5 should be used to maximize the bearing load capacity. 


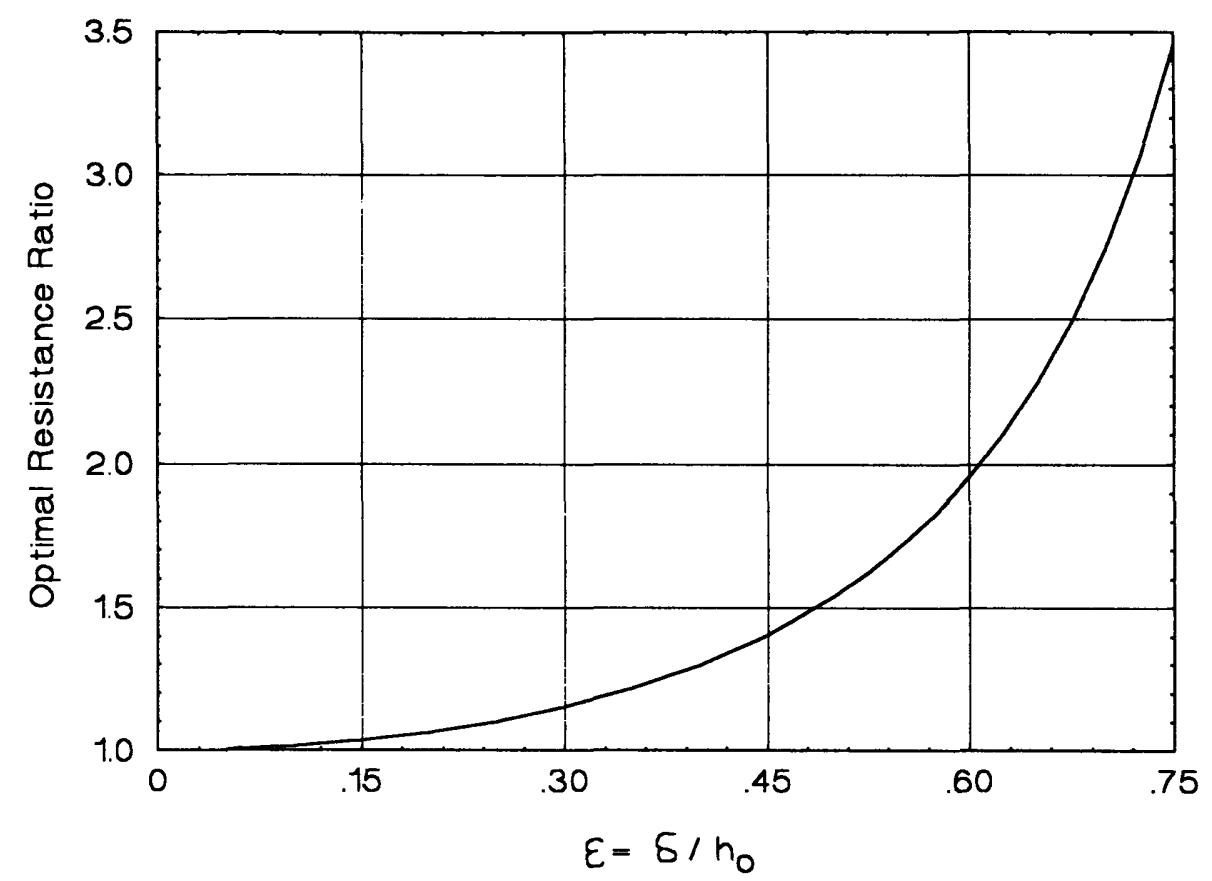

Figure 5.2. Optimal resistance ratio that provides maximum load-carrying efficiency as a function of the bearing displacement.

\section{A.ii. Representative Calculations for Thrust Bearing \#1}

Table 5.1 shows the input and output values for a representative bearing with an outer diameter of $100 \mathrm{~mm}$, an inner diameter of $80 \mathrm{~mm}$, and a nominal clearance of $15 \mu \mathrm{m}$ per side. The land widths are all $3 \mathrm{~mm}$. The resistance ratio is 1 , which provides maximum initial stiffness. As shown, the initial specific stiffness is 1.05 , the load-carrying efficiency at $75 \%$ of gap closure is 0.579 , and the specific flow rate is 3.35 . 


\section{Table 5.1}

\begin{tabular}{|c|c|c|c|c|}
\hline \multicolumn{5}{|l|}{ Operating Conditions } \\
\hline $\begin{array}{l}\text { Supply Pressure (Pa,psi,atm) } \\
\text { Percentage of Gap Closure }\end{array}$ & $\begin{array}{l}\text { Ps } \\
\text { pct }\end{array}$ & $\begin{array}{r}4.17 E+06 \\
0.1\end{array}$ & 600 & 40.8 \\
\hline \multicolumn{5}{|l|}{ Fluid Properties } \\
\hline Dynamic Viscosity ( $\mathrm{N} \mathrm{s} / \mathrm{m}$ ) & mu & 0.0013 & & \\
\hline \multicolumn{5}{|l|}{ Bearing Geometry } \\
\hline Outer Thrust Diameter (m, mm, in) & Do & 0.1 & 100 & 3.937 \\
\hline Inner Thrust Diameter (m,mm,in) & $\mathrm{Di}$ & 0.08 & 80 & 3.150 \\
\hline Inner Land Width (m, mm,in) & bi & 0.003 & 3 & 0.118 \\
\hline Diameter to Inner Land (m, $\mathrm{mm}$, in) & D1 & 0.086 & 86 & 3.386 \\
\hline Outer Land Width $(\mathrm{m}, \mathrm{mm}$, in) & bo & 0.003 & 3 & 0.118 \\
\hline Diameter to Outer Land $(\mathrm{m}, \mathrm{mm}$, in) & D2 & 0.094 & 94 & 3.701 \\
\hline Nominal Clearance per Side $(m, \mu \mathrm{m}$,in) & ho & 0.000015 & 15 & 0.00059 \\
\hline Nominal Resistance Ratio & gamma & 1 & & \\
\hline Effective Load-Supporting Area $\left(\mathrm{m}^{2}, \mathrm{~mm}^{2}, \mathrm{in}^{2}\right)$ & Aeff & 0.00198 & 1979 & 3.07 \\
\hline \multicolumn{5}{|l|}{ Bearing Load Capacity } \\
\hline Pressure Difference / Pressure & DPP & 0.149 & & \\
\hline Load Capacity (N,kN,lbf) & $F$ & 1231 & & \\
\hline Load Carrying Efficiency @ pct above & Feff & 0.105 & & \\
\hline Specific Stiffness @ pct above & Kspec & 1.046 & & \\
\hline Initial Specific Stiffness & Kspec,o & 1.050 & & \\
\hline Load Carrying Efficiency @ pct = 0.75 & Feff, 0.75 & 0.579 & & \\
\hline \multicolumn{5}{|l|}{ Flow Rate and Pumping Power } \\
\hline Equivalent Pad Resistance & Req & $2.45 E+10$ & & \\
\hline Supply Flow Rate $\left(m^{\wedge} 3 / \mathrm{s}, 1 \mathrm{pm}, \mathrm{gpm}\right)$ & Qsupply & $1.70 \mathrm{E}-04$ & 10.19 & 2.69 \\
\hline Specific Flow Rate & Ospec & 3.35 & & \\
\hline Pumping Power (W,kW,hp) & Ppump & 707 & 0.71 & 0.95 \\
\hline
\end{tabular}

\section{B. Thrust Bearing \#2}

A schematic diagram of Thrust Bearing $\# 2$ is shown in Figure 5.3. This bearing differs from Thrust Bearing \#1 in that no external compensation resistances are needed [9]. The Fluid is supplied at supply pressure, $P_{s}$, into the supply region. It then flows axially across the compensating lands, which are at a clearance $h_{c}$, to the pockets. It then flows radially across the outlet lands, which are at a clearance $h_{0}$, to drainage grooves at 
atmospheric pressure, $P_{\mathbf{a}}$. Note that the pockets shown in Figure 5.3 have been machined into the surface of the shaft flange; they may be equivalently machined into the housing member. Also note that the compensation lands have been placed at the outer diameter of the thrust bearing; they may also be placed at the inner diameter by placing the bearing lands at the bearing outer diameter.

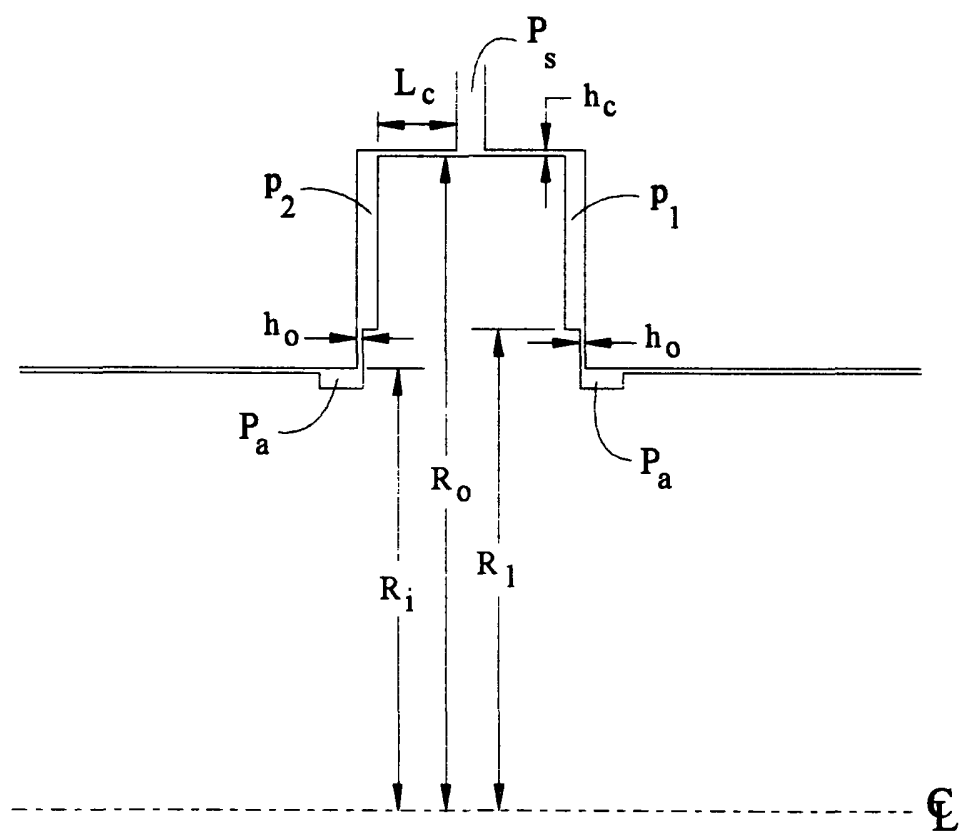

Figure 5.3. Schematic diagram of Thrust Bearing \#2. The two axial clearances, denoted by $h_{0}$, and the two radial clearances, denoted by $h_{c}$, are exagerated for clarity.

\section{B.i. Description of Calculations for Thrust Bearing \#2}

To calculate the characteristics of this thrust bearing, the resistance of each bearing land is needed. The resistance of the inner lands are given by:

$$
\mathrm{R}_{\mathrm{p}}^{1}=\frac{6 \mu}{\pi \mathrm{h}_{\mathrm{o}}^{3}(1-\varepsilon)^{3}} \ln \left(\frac{\mathbf{R}_{1}}{\mathrm{R}_{\mathrm{i}}}\right)
$$




$$
R_{p}^{2}=\frac{6 \mu}{\pi h_{o}^{3}(1+\varepsilon)^{3}} \ln \left(\frac{R_{1}}{R_{i}}\right)
$$

The resistance of the compensation lands are given by:

$$
R_{c}^{1}=R_{c}^{2}=\frac{12 \mu L_{c}}{\pi D_{0} h_{0}^{3}}
$$

Note that it has been assumed that the radial eccentricity of the shaft is equal to zero. The compenation resistances are chosen such the ratio of a compensation resistance to a nominal equivalent resistance is equal to some value, $\gamma$. The resistances of Circuit \#1 of Appendix B are now known. The solution for the pocket pressures is given by:

$$
\frac{p_{1}-p_{2}}{P_{s}}=\frac{\frac{1}{(1-\varepsilon)^{3}}}{\frac{1}{(1-\varepsilon)^{3}}+\gamma}-\frac{\frac{1}{(1+\varepsilon)^{3}}}{\frac{1}{(1+\varepsilon)^{3}}+\gamma}
$$

Note that this equation is the same as Equation (5.10), the pressure difference for Thrust Bearing \#1. The bearing restoring force is this pressure difference times the effective pocket area. The effective area of a pocket is given by:

$$
A_{\text {eff }}=\pi R_{o}^{2}-\frac{\pi}{2}\left(\frac{R_{1}^{2}-R_{i}^{2}}{\ln \left(R_{1} / R_{i}\right)}\right)
$$

The bearing restoring force is then given by:

$$
F=A_{\text {eff }}\left(p_{1}-p_{2}\right)
$$

In addition to the bearing load-carrying capability, the supply flow rate is needed. The supply flow rate is typically evaluated for small displacements, $\varepsilon=0$, since it varies only slightly with small displacements. The supply flow rate is simply the supply pressure 
divided by the overall resistance of both pockets. Nominally, both pocket resistances are equal, so the supply flow rate is given by:

$$
\mathrm{Q}_{\mathrm{s}}=\frac{2 \mathrm{P}_{\mathrm{s}}}{\mathrm{R}_{\mathrm{p}}^{1}} \frac{1}{1+\gamma}=\frac{2 \mathrm{P}_{\mathrm{s}}}{\mathrm{R}_{\mathrm{p}}^{2}} \frac{1}{1+\gamma}
$$

where the resistances are evaluated with $\varepsilon=0$.

The optimal resistance ratio that can be used to maximize the bearing load capacity is the same as that for Thrust Bearing \#1; Equation (5.14) is applicable for this bearing as well.

With the use of Equation (5.17), it was assumed that the radial eccentricity of the the shaft is zero. Consider the shaft to have an eccentricity e, such that the actual clearance is a function of the angular coordinate, $\theta$ :

$$
h_{c}(\theta)=h_{c, o}[1-e \cos (\theta)]
$$

The actual resistance of a compensation land is given by:

$$
R_{c}^{\prime}=\frac{12 \mu L_{c}}{\int_{0}^{2 \pi} h_{c}(\theta)^{3}\left(\frac{D_{0}}{2}\right) d \theta}
$$

Inserting Equation (5.22) and integrating,

$$
R_{c}^{1}=\frac{12 \mu L_{c}}{\left(\frac{D_{o}}{2}\right) h_{c, o}^{3} \int_{0}^{2 \pi}(1-e \cos (\theta))^{3} d \theta}=\frac{12 \mu L_{c}}{D_{o} h_{c, o}^{3}} \frac{1}{1+\frac{3}{2} e^{2}}
$$

Dividing Equation (5.24) by Equation (5.17), the actual resistance ratio of the bearing becomes:

$$
\gamma_{\text {actual }}=\frac{\gamma}{1+\frac{3}{2} \mathrm{e}^{2}}
$$


Figure 5.4 shows the effect of radial eccentricity on the initial stiffness of bearings with various designed resistance ratios. As shown, the bearing should be designed with a resistance ratio somewhat greater than 1 to ensure near-optimal performance.

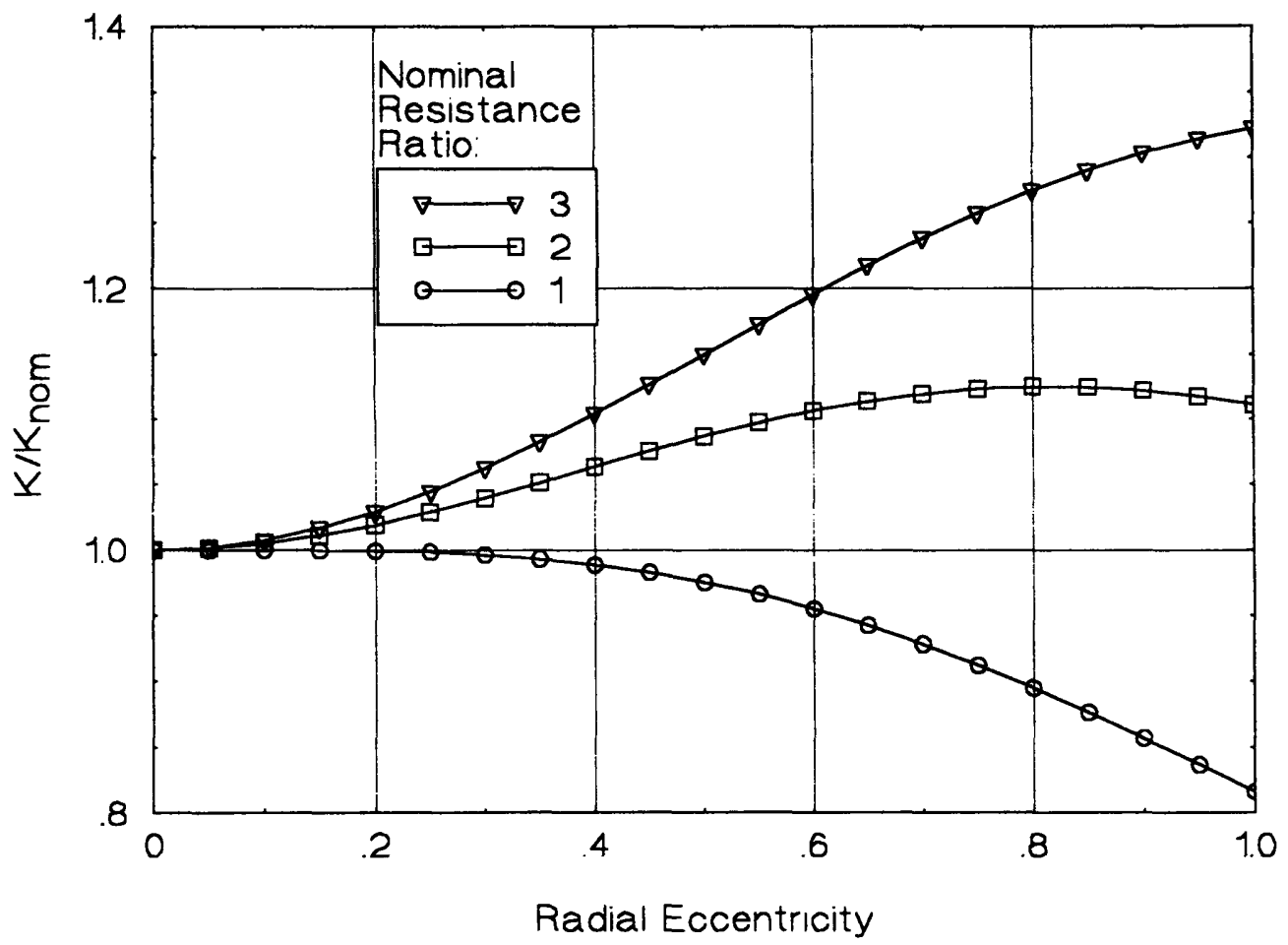

Figure 5.4. Effect of radial eccentricity on the initial stiffness of the Thrust Bearing \#2, for several different nominal resistance ratios.

A drawback of Thrust Bearing $\# 2$ is that its performance depends upon the ratio of two tight clearances, $h_{d} / h_{0}$. Figure 5.5 shows the effect of variations in the compensaiton land clearance on the initial stiffness of the bearing. The plot is for a constant axial clearance, $h_{0}$. As shown, the nominal resistance ratio should be chosen to be 
approximately 1 to 1.25 to minimize the effect of manufacturing variations of the clearances.

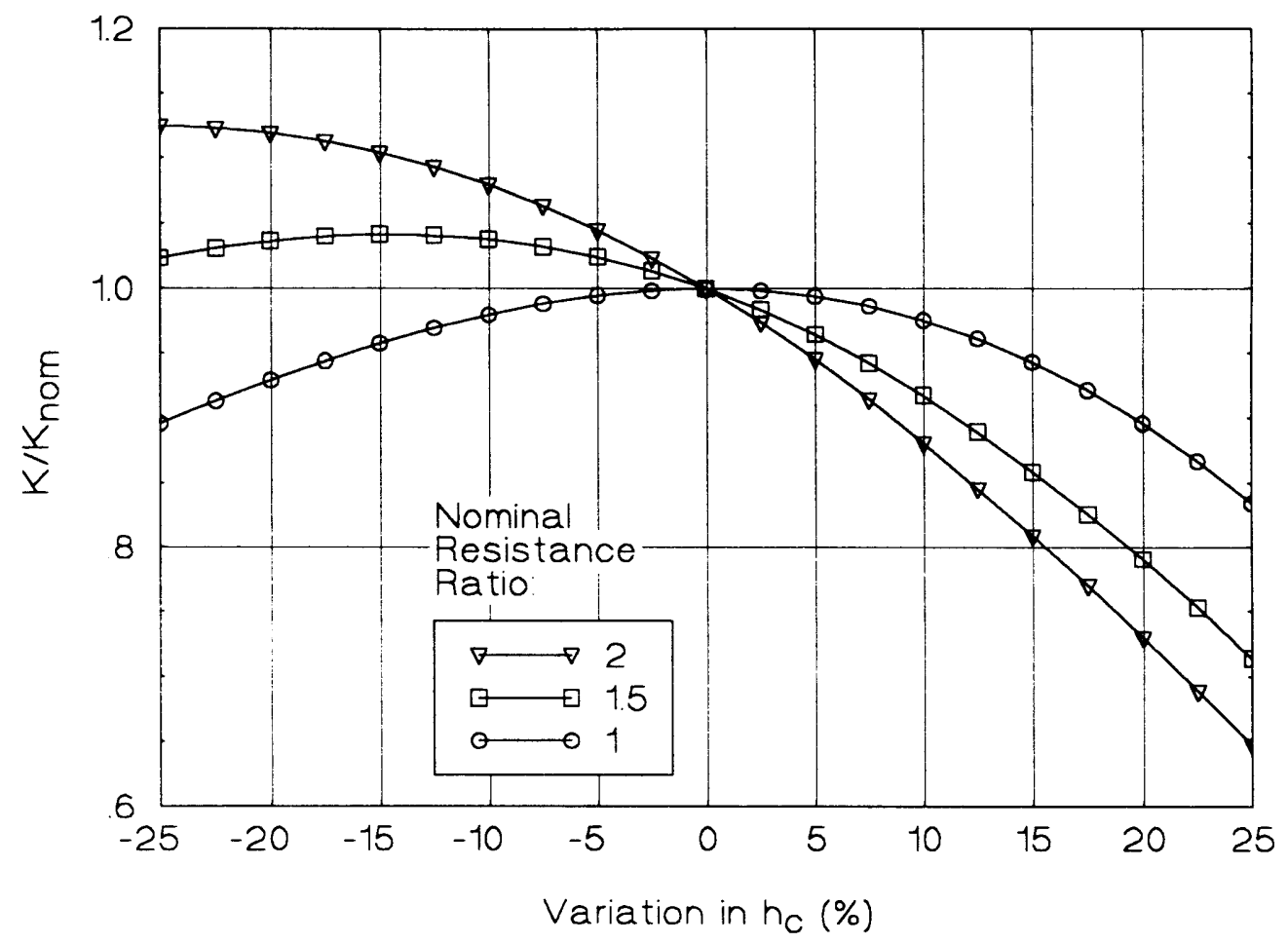

Figure 5.5. Effect of variation in the compensating land clearance on the stiffness of Thrust Bearing \#2.

\section{B.ii. Representative Calculations for Thrust Bearing \#2}

Table 5.2 shows the input and output values for a representative bearing with an outer diameter of $100 \mathrm{~mm}$, an inner diameter of $80 \mathrm{~mm}$, and a nominal clearance of $15 \mu \mathrm{m}$ per side. The bearing land widths are $3 \mathrm{~mm}$. The resistance ratio is 1.25 , which provides nearly maximum initial bearing stiffness. As shown, the initial specific stiffness is 1.28 , the load-carrying efficiency at $75 \%$ of gap closure is 0.736 , and the specific flow rate is 1.37 . 


\section{Table 5.2}

\begin{tabular}{|c|c|c|c|c|}
\hline \multicolumn{5}{|l|}{ Operating Conditions } \\
\hline Supply Pressure (Pa,psi,atm) & Ps & 4.17E+06 & 600 & 40.8 \\
\hline Percentage of Gap Closure & pct & 0.1 & & \\
\hline \multicolumn{5}{|l|}{ Fluid Properties } \\
\hline Dynamic Viscosity ( $\mathrm{N} \mathrm{s/m}$ ) & mu & 0.0013 & & \\
\hline \multicolumn{5}{|l|}{ Bearing Geometry } \\
\hline Outer Thrust Diameter $(\mathrm{m}, \mathrm{mm}, \mathrm{in})$ & Do & 0.1 & 100 & 3.937 \\
\hline Inner Thrust Diameter (m,mm,in) & $\mathrm{Di}$ & 0.08 & 80 & 3.150 \\
\hline Inner Land Width (m,mm,in) & bi & 0.003 & 3 & 0.118 \\
\hline Diameter to Inner Land (m, mm,in) & D1 & 0.086 & 86 & 3.386 \\
\hline Nominal Clearance per Side $(m, \mu m, i n)$ & ho & 0.000015 & 15 & 0.00059 \\
\hline Nominal Resistance Ratio & gamma & 1.25 & & \\
\hline Radial Clearance of Compenation Land ( $m, \mathrm{~mm}$,in) & hc & 0.000015 & 15 & 0.00059 \\
\hline Compenation Land Width $(\mathrm{m}, \mathrm{mm}$, in) & Lc & 0.00452 & 4.52 & 0.178 \\
\hline Effective Load-Supporting Area $\left(\mathrm{m}^{2}, \mathrm{~mm}^{2}, \mathrm{in}^{2}\right)$ & Aeff & 0.00245 & 2446 & 3.79 \\
\hline \multicolumn{5}{|l|}{ Bearing Load Capacity } \\
\hline Pressure Difference / Pressure & DPP & 0.148 & & \\
\hline Load Capacity (N,kN,lbf) & $\mathbf{F}$ & 1506 & & \\
\hline Load Carrying Efficiency @ pct above & Feff & 0.128 & & \\
\hline Specific Stiffness @ pct above & Kspec & 1.279 & & \\
\hline Initial Specific Stiffness & Kspec,o & 1.281 & & \\
\hline Load Carrying Efficiency @ pct = 0.75 & Feff, 0.75 & 0.736 & & \\
\hline \multicolumn{5}{|l|}{ Flow Rate and Pumping Power } \\
\hline Equivalent Pad Resistance & $\mathrm{Rp}$ & $5.32 E+10$ & & \\
\hline Supply Flow Rate $\left(m^{\wedge} 3 / \mathrm{s}, 1 \mathrm{pm}, \mathrm{gpm}\right)$ & Qsupply & $6.96 \mathrm{E}-05$ & 4.18 & 1.10 \\
\hline Specific Flow Rate & Ospec & 1.37 & & \\
\hline Pumping Power $(\mathrm{W}, \mathrm{kW}, \mathrm{hp})$ & Ppump & 290 & 0.29 & 0.39 \\
\hline
\end{tabular}




\section{C. Thrust Bearing \#3}

A schematic diagram of Thrust Bearing \#3 is shown in Figure 5.6. This bearing differs from the previous thrust bearings in that one side of the thrust bearing is larger than the other. This configuration enables the thrust bearing to have preferential load capacity in a one direction, a characteristic which is especially desirable in drilling applications [10]. The fluid is supplied at supply pressure, $P_{s}$, into the supply region. It then flows radially across the secondary thrust face land, which is at a clearance $h_{o s}$, to the pocket. Note that the groove that channels the fluid axially to the pocket has a clearance much greater than the bearing clearances such that the pressure drop across it is negligible. One the fluid has reached the pocket, it then flows radially across the outlet land, which is at a clearance $h_{o p}$, to drainage grooves at atmospheric pressure, $\mathrm{P}_{\mathrm{a}}$. Note that the pockets shown in Figure 5.6 have been machined into the surface of the shaft flange; they may be equivalently machined into the housing member.

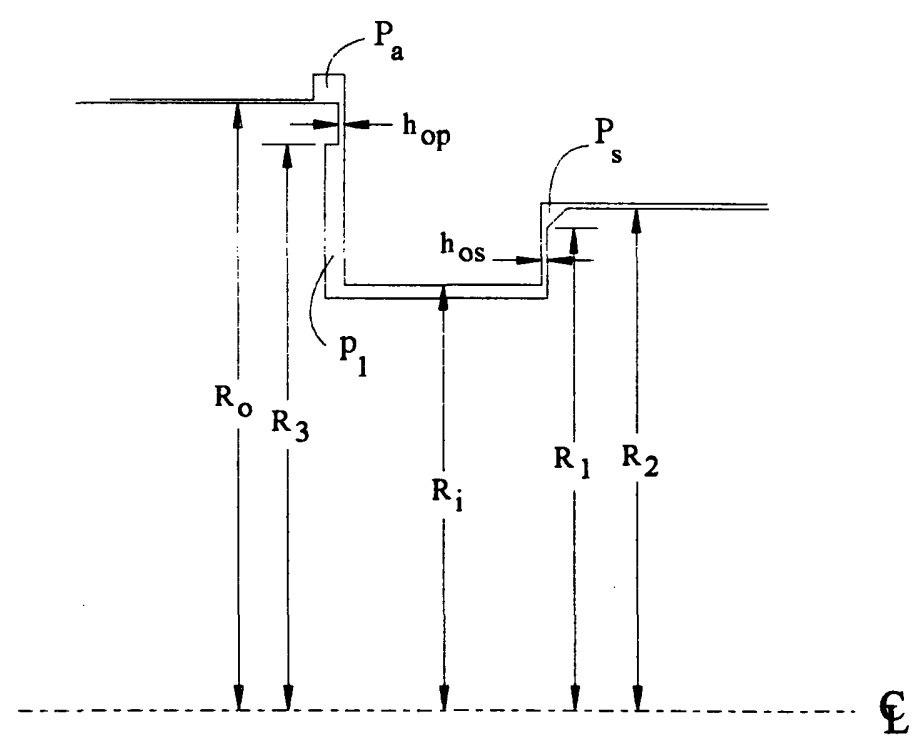

Figure 5.6. Schematic diagram of Thrust Bearing \#3. The two axial clearances denoted by $h_{\mathrm{os}}$ and $h_{\mathrm{op}}$ are exaggerated for clarity. 


\section{C.i. Description of Calculations for Thrust Bearing \#3}

To calculate the characteristics of this thrust bearing, the resistance of each bearing land is needed. The resistance of the secondary thrust face land is given by:

$$
\mathbf{R}_{\mathrm{s}}=\frac{6 \mu}{\pi\left(\mathrm{h}_{\mathrm{os}}+\delta\right)^{3}} \ln \left(\frac{\mathrm{R}_{1}}{\mathrm{R}_{\mathrm{i}}}\right)
$$

The resistance of the pocket land is given by:

$$
\mathbf{R}_{\mathrm{p}}=\frac{6 \mu}{\pi\left(\mathrm{h}_{\mathrm{op}}-\delta\right)^{3}} \ln \left(\frac{\mathrm{R}_{\mathrm{o}}}{\mathrm{R}_{3}}\right)
$$

The resistances of Circuit \#1 of Appendix B are now known. The solution for the pocket pressure is given by:

$$
\frac{\mathrm{p}_{1}}{\mathrm{P}_{\mathrm{s}}}=\frac{\mathrm{R}_{\mathrm{p}}}{\mathrm{R}_{\mathrm{p}}+\mathrm{R}_{\mathrm{s}}}=\frac{1}{1+\frac{\ln \left(\mathrm{R}_{1} / \mathrm{R}_{\mathrm{i}}\right)}{\ln \left(\mathrm{R}_{\mathrm{o}} / \mathrm{R}_{3}\right)}\left(\frac{\mathrm{h}_{\text {op }}-\delta}{\mathrm{h}_{\text {os }}+\delta}\right)^{3}}
$$

where the equilibrium clearances, $h_{o p}$ and $h_{o s}$, will be determined from a force balance. The force exerted by the primary thrust face is given by:

$$
\mathrm{F}_{\mathrm{p}}=\left[\frac{\pi}{2}\left(\frac{\mathrm{R}_{\mathrm{o}}^{2}-\mathrm{R}_{3}^{2}}{\ln \left(\mathrm{R}_{\mathrm{o}} / \mathrm{R}_{3}\right)}\right)-\pi \mathrm{R}_{\mathrm{i}}^{2}\right] \mathrm{p}_{1}
$$

The force exerted by the secondary thrust face is given by:

$$
\mathrm{F}_{\mathrm{s}}=\pi \mathrm{R}_{2}^{2} \mathrm{P}_{\mathrm{s}}-\pi \mathrm{R}_{\mathrm{i}}^{2} \mathrm{p}_{1}-\frac{\pi}{2}\left(\frac{\mathrm{R}_{1}^{2}-\mathrm{R}_{\mathrm{i}}^{2}}{\ln \left(\mathrm{R}_{1} / \mathrm{R}_{\mathrm{i}}\right)}\right)\left(\mathrm{P}_{\mathrm{s}}-\mathrm{p}_{1}\right)
$$

At equilibrium, these two opposing forces must equal each other. Solving for the equilibrium pocket pressure, 


$$
\frac{\mathrm{p}_{1, \text { eq }}}{\mathrm{P}_{\mathrm{s}}}=\frac{\pi \mathrm{R}_{2}^{2}-\frac{\pi}{2}\left(\frac{\mathrm{R}_{1}^{2}-\mathbf{R}_{\mathrm{i}}^{2}}{\ln \left(\mathrm{R}_{1} / \mathrm{R}_{\mathrm{i}}\right)}\right)}{\frac{\pi}{2}\left(\frac{\mathrm{R}_{\mathrm{o}}^{2}-\mathrm{R}_{3}^{2}}{\ln \left(\mathrm{R}_{\mathrm{o}} / \mathrm{R}_{3}\right)}\right)-\frac{\pi}{2}\left(\frac{\mathrm{R}_{1}^{2}-\mathrm{R}_{\mathrm{i}}^{2}}{\ln \left(\mathrm{R}_{1} / \mathrm{R}_{\mathrm{i}}\right)}\right)}
$$

Once the equilibrium pocket pressure has been evaluated, the equilibrium clearance ratio can be determined by substuting $\delta=0$ into Equation (5.28):

$$
\left(\frac{h_{o p}}{h_{\text {os }}}\right)_{\text {eq }}=\left[\left\{\left(\frac{P_{s}}{p_{l, e q}}\right)-1\right\} \frac{\ln \left(R_{o} / R_{3}\right)}{\ln \left(R_{1} / R_{i}\right)}\right]^{1 / 3}
$$

Now that the equilibrium clearance ratio is known, the pocket pressure can be determined for any deflection, d, using Equation (5.28). The net bearing force is then found by substracting Equation (5.30) from Equation (5.29).

The nominal bearing flow rate is found by combining the resistances given by Equations (5.26) and (5.27):

$$
\mathrm{Q}_{\mathrm{s}}=\frac{\mathrm{P}_{\mathrm{s}}}{\frac{6 \mu}{\pi \mathrm{h}_{\mathrm{os}}^{3}} \ln \left(\frac{\mathrm{R}_{1}}{\mathrm{R}_{\mathrm{i}}}\right)+\frac{6 \mu}{\pi \mathrm{h}_{\mathrm{op}}^{3}} \ln \left(\frac{\mathrm{R}_{\mathrm{o}}}{\mathrm{R}_{3}}\right)}
$$

\section{C.ii. Representative Calculations for Thrust Bearing \#3}

Table 5.3 shows the input and output values for a representative bearing with an outer diameter of $100 \mathrm{~mm}$, an inner diameter of $80 \mathrm{~mm}$, and a secondary face outer diameter of $87 \mathrm{~mm}$. The tota nominal clearance, $h_{o p}+h_{o s}$, is $30 \mu \mathrm{m}$. The bearing land width is $3 \mathrm{~mm}$. The supply groove width is $2 \mathrm{~mm}$. As shown, the initial specific stiffness is 1.07 , the primary direction load-carrying efficiency at $75 \%$ of gap closure is 0.508 , the 
secondary direction load-carrying efficiency at $75 \%$ of gap closure is 0.256 , and the specific flow rate is 1.04 .

\section{Table 5.3}

\begin{tabular}{|c|c|c|c|c|}
\hline \multicolumn{5}{|l|}{ Operating Conditions } \\
\hline Supply Pressure (Pa,psi,atm) & Ps & $4.17 E+06$ & 600 & 40.8 \\
\hline Deflection from Equilibrium ( $m, \mu \mathrm{m}$,in) & d & 5.00E-06 & 5.00 & 0.00020 \\
\hline \multicolumn{5}{|l|}{ Fluid Properties } \\
\hline Dynamic Viscosity ( $\mathrm{N} / \mathrm{m}$ ) & mu & 0.0013 & & \\
\hline \multicolumn{5}{|l|}{ Bearing Geometry } \\
\hline Outer Thrust Diameter $(\mathrm{m}, \mathrm{mm}, \mathrm{in})$ & Do & 0.1 & 100 & 3.937 \\
\hline Inner Thrust Diameter (m,mm,in) & $\mathrm{Di}$ & 0.08 & 80 & 3.150 \\
\hline Secondary Face Outer Diameter $(\mathrm{m}, \mathrm{mm}, \mathrm{in})$ & D2 & 0.087 & 87 & 3.425 \\
\hline Primary Face Land Width $(\mathrm{m}, \mathrm{mm}, \mathrm{in})$ & bo & 0.003 & 3 & 0.118 \\
\hline Diameter to Primary Land $(m, m m, i n)$ & D3 & 0.094 & 94 & 3.701 \\
\hline Supply Groove Width $(m, m m, i n)$ & bs & 0.002 & 2 & 0.079 \\
\hline Outer Diameter of Secondary Land $(\mathrm{m}, \mathrm{mm}, \mathrm{in})$ & D1 & 0.083 & 83 & 3.268 \\
\hline Total Clearance (hos + hop) $(m, \mu m$, in) & htot & 0.00003 & 30 & 0.00118 \\
\hline Equilibrium Pocket Pressure Ratio & peq_Ps & 0.336 & & \\
\hline Equilibrium Clearance Ratio & hop_hos & 1.493 & & \\
\hline Nominal Primary Clearance $(m, \mu m, i n)$ & hop & $1.80 \mathrm{E}-05$ & 17.967 & 0.00071 \\
\hline Nominal Secondary Clearance $(m, \mu m, i n)$ & hos & $1.20 \mathrm{E}-05$ & 12.033 & 0.00047 \\
\hline \multicolumn{5}{|l|}{ Bearing Load Capacity } \\
\hline Pocket Pressure / Supply Pressure $@ \delta$ above & p1_Ps & 0.792 & & \\
\hline Load Capacity (N,kN,lbf) @ $\delta$ above & $\overline{\mathrm{F}}$ & 4129 & & \\
\hline Load Carrying Efficiency @ $\delta$ above & Feff & 0.351 & & \\
\hline Specific Stiffness @ $\delta$ above & Kspec & 1.052 & & \\
\hline Initial Specific Stiffness & Kspec,o & 1.071 & & \\
\hline Primary Load Carrying Efficiency @ pct = 0.75 & Feff, 0.75 & 0.508 & & \\
\hline Secondary Load Carrying Efficiency @ pct $=0.75$ & Feff, -0.75 & -0.256 & & \\
\hline \multicolumn{5}{|l|}{ Flow Rate and Pumping Power } \\
\hline Overall Bearing Resistance & Rbear & $7.89 E+10$ & & \\
\hline Supply Flow Rate $\left(\mathrm{m}^{\wedge} 3 / \mathrm{s}, 1 \mathrm{pm}, \mathrm{gpm}\right)$ & Osupply & $5.28 \mathrm{E}-05$ & 3.17 & 0.84 \\
\hline Specific Flow Rate & Qspec & 1.04 & & \\
\hline Pumping Power $(\mathrm{W}, \mathrm{kW}, \mathrm{hp})$ & Ppump & 220 & 0.22 & 0.29 \\
\hline
\end{tabular}




\section{FRICTIONAL AND THERMAL CHARACTERISTICS}

The power consumed by viscous shearing of the fluid within hydrostatic bearings should be minimized as much as possible for two reasons. First, reducing viscous shearing power leaves more of the available motor power to be used for machining. Adding a larger motor to the spindle to compensate for bearing drag increases the cost of the machine and the cost of its operation. Second, the viscous power consumed by the bearings is equal to the heat generated within the bearings. This heat generation causes a temperature rise of the shaft and the housing, resulting in unwanted thermal growth errors.

Excessive shearing power is the reason why hydrostatic bearings have been limited to low speed machine tool applications over the past few decades. The recent implementation of water-based coolants as the lubricant has substantially extended their speed capability, but shearing power consumption still remains a principal consideration. Even if water is used as the bearing lubricant, hydrostatic spindles usually have higher viscous shearing power than rolling element spindles at speeds greater than 1 million DN.

It is important for a spindle designer to be able to readily predict the power consumed by hydrostatic bearings of various geometries. The shearing power of a bearing can be approximately modeled by breaking the bearing up into pocket, groove, and land regions and summing the shearing powers generated by each region. Figure 6.1 shows a schematic diagram of the pockets of a hydrostatic bearing with axial lands separating them. The axial lands effectively act to create a pressure gradient inside the pocket as the shaft rotates, resulting in a recirculation of the fluid within the pockets. The depth of the pockets, $h_{p}$, is usually much greater than the bearing land clearance, $h$. In this case, the 
fluid flow within the pockets does not depend upon the fluid flow over the lands, and the two regions may be considered separately. The fluid flow within the pockets may be modeled as simple cavity flow for the purpose of shearing power calculations. Also, centrifugal effects caused by curvature of the pockets are usually negligible unless they extend for a significant distance around the circumference of the shaft and they are deep compared to the radius of the bearing. The cavity flow used to approximate the pocket shear power will be considered in the second section of this chapter.

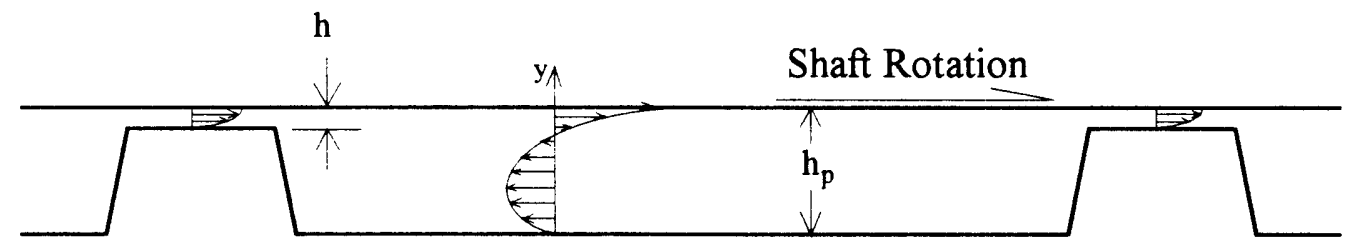

Figure 6.1. Velocity profiles developed in hydrostatic bearings. The lands tend to dam up the pocket flow and cause it to recirculate.

The other bearing regions which must be considered for shearing power calculations are the bearing lands. The flow over the axial and circumferential lands can usually be adequately modeled as uninterupted flow between concentric cylinders with zero pressure gradient. The assumption of uninterrupted flow is valid as long as the "entry length" of the flow is small compared to the circumferential length being considered. This is true if either (1) the flow is laminar and fully-developed on the land as described in Appendix C, or (2) the flow is turbulent. The assumption of zero pressure gradient circumferentially is valid as long as the pressure gradient within the fluid film times the radial clearance of the fluid film is much less than the shear stress on the wall. This is true 
for nearly all lands of hydrostatic bearings, as long as the assumptions given above are adequate.

The first thing that must be considered when the bearing friction in a particular region of the bearing is calculated is whether the flow is laminar or turbulent in that region. Although the flow between the shaft and lands of a hydrostatic bearing generally remains laminar for most operating speeds, the flow within the pockets and grooves usually becomes turbulent even at moderate operating speeds. For typical water bearings with deep pockets, transition in the pockets occurs at low operating speeds, as shown in Table 6.1. However, for applications in which radial space requirements are critical and the bearing pockets are made very shallow, transition in the pockets may not occur until the bearing is taken to moderate operating speeds. The flow on the lands of hydrostatic bearings typically remains laminar over most operating speeds.

Table 6.1.

\begin{tabular}{|c|c|}
\hline Bearing Region & DN \\
\hline Deep Pocket, $\mathrm{h}_{\mathrm{p}}=1 \mathrm{~mm}$ & 0.02 million \\
\hline Shallow Pocket, $\mathrm{h}_{\mathrm{p}}=100 \mu \mathrm{m}$ & 0.2 million \\
\hline Lands, $\mathrm{h}_{\mathrm{a}}=20 \mathrm{~mm}$ & 1.5 million \\
\hline
\end{tabular}

Approximate formulations for the bearing friction at laminar and turbulent speeds were developed by prior researchers and the relations will be summarized in this chapter. In each case, the relations will be presented in the form of a skin friction factor, which is a common way to express the shear stress non-dimensionally:

$$
C_{f}=\frac{\tau}{\frac{1}{2} \rho V^{2}}
$$


where $\tau$ is the shear stress, $\rho$ is the fluid density, and $V$ is the surface velocity of the rotating shaft. This definition will be used throughout this chapter as the frictional relations for each bearing region are presented. The friction factor is a function of the Reynolds number as well as another flow parameter such as a non-dimensional spacing ratio. Once the friction factor is computed for a given bearing region at a given flow condition, the viscous shearing power of that region may be computed by the following relation:

$$
\rho_{s}=C_{f} \frac{1}{2} \rho V^{3} A_{s}
$$

where $A_{s}$ is the shear area of the region.

\section{A. Bearing Land and Circumferential Groove Flow}

The flow over the lands that results from shaft rotation will be discussed in this section. The analysis is also applicable to circumferential drainage grooves which run completely around the circumference of the bearing. This flow is modeled as uninterupted flow between two concentric cylinders with no pressure gradient. Since the cylinders are considered concentric, the relations presented are only valid when the eccentricity of the shaft is small (less than about 0.1 ); however, this is the case in all spindles except those which are loaded with heavy forces.

The fluid flow on the lands of hydrostatic bearings usually remains laminar up to fairly high operating speeds. However, the circumferential drainage grooves of typical hydrostatic bearings can become turbulent at moderate operating speeds, and at very high 
operating speeds, even the lands can become turbulent. Both laminar and turbulent regimes will therefore be considered in this section.

Consider two concentric cylinders rotating with fluid contained between them. The inner cylinder has a radius $R_{i}$ and rotates with a speed $\omega$. The radial clearance between the two cylinders is $h$. The fluid has a density $\rho$ and a dynamic viscosity $\mu$. Correlations have been made to experimental data that can be used to approximately calculate the friction factor for this case [11]:

$$
\begin{array}{rr}
0<\mathrm{N}_{\mathrm{Ta}}<41 & \mathrm{C}_{\mathrm{f}}=\frac{2}{\operatorname{Re}} \frac{\left(1+\mathrm{h} / \mathrm{R}_{\mathrm{i}}\right)^{2}}{1+0.5\left(\mathrm{~h} / \mathrm{R}_{\mathrm{i}}\right)} \\
41<\mathrm{N}_{\mathrm{Ta}}<63 & \mathrm{C}_{\mathrm{f}}=\frac{0.11\left(\mathrm{~N}_{\mathrm{Ta}}\right)^{0.854}}{\mathrm{Re}} \\
63<\mathrm{N}_{\mathrm{Ta}}<\left(\mathrm{N}_{\mathrm{Ta}}\right)_{\mathrm{V}-\mathrm{T}} & \mathrm{C}_{\mathrm{f}}=\frac{0.476\left(\mathrm{~N}_{\mathrm{Ta}}\right)^{0.5}}{\mathrm{Re}} \\
\mathrm{N}_{\mathrm{Ta}}>\left(\mathrm{N}_{\mathrm{Ta}}\right)_{\mathrm{V}-\mathrm{T}} & \frac{\left(1+\mathrm{h} / \mathrm{R}_{\mathrm{i}}\right)}{12 \sqrt{2 \mathrm{C}_{\mathrm{f}}}\left(1+0.5\left(\mathrm{~h} / \mathrm{R}_{\mathrm{i}}\right)\right\}}-\ln \left(\frac{\mathrm{Re} \sqrt{\mathrm{C}_{\mathrm{f}} / 2}}{2\left(1+\mathrm{h} / \mathrm{R}_{\mathrm{i}}\right)}\right)-8.58=0
\end{array}
$$

where $\operatorname{Re}=\rho h \omega R_{i} / \mu$ is the characteristic Reynolds number, and $N_{T a}=\operatorname{Re}\left(h / R_{i}\right)^{0.5}$ is the Taylor number. $\left(\mathrm{N}_{\mathrm{T}_{2}}\right)_{\mathrm{V}-\mathrm{T}}$ is the value of the Taylor number at transition from vortexdominant flow to turbulence-dominant flow. Its value depends on the clearance ratio $h / R_{i}$. Which equation to use can be determined by (1) calculating the value of $\mathrm{C}_{\mathrm{f}}$ using Equation (6.5), (2) calculating the value of $C_{f}$ using Equation (6.6), and (3) using the value of $C_{f}$ which is highest.

The value of $C_{f}$ calculated using Equation (6.3) through (6.6) is plotted versus the Taylor number for several different clearance ratios in Figure 6.2. For low Taylor numbers, $\mathrm{N}_{\mathrm{Ta}}<41$, the flow is laminar. For very low clearance ratios, the value of $\mathrm{C}_{\mathrm{f}} \mathrm{Re}$ is equal to a constant. However, for significant clearance ratios, the value of $C_{f} R e$ increases 
as the clearance ratio is increased because the vortices generated by the fluid centrifugal forces cause higher shear stress on the cylinder than that if the vortices did not occur. For $41<\mathrm{N}_{\mathrm{Ta}}<63$, the flow is transitional. For $63<\mathrm{N}_{\mathrm{Ta}}<\left(\mathrm{N}_{\mathrm{Ta}}\right)_{\mathrm{V}-\mathrm{T}}$, the flow is vortexdominated flow and the value of $\mathrm{C}_{\mathrm{f}} \mathrm{Re}$ is independent of the clearance ratio for a given Taylor number. Once the speed of the cylinder is increased to a Taylor number corresponding to $\left(\mathrm{N}_{T_{\mathrm{a}}}\right)_{\mathrm{V}-\mathrm{T}}$, the flow becomes turbulence-dominated and the vortices generated by the fluid centrifugal forces have a negligible effect on the wall friction. The transitions between flow regimes shown in Figure 6.2 are distinct; however, in reality the transitions are gradual and the relations given by Equation (6.3) through (6.6) are only approximate in these regions.

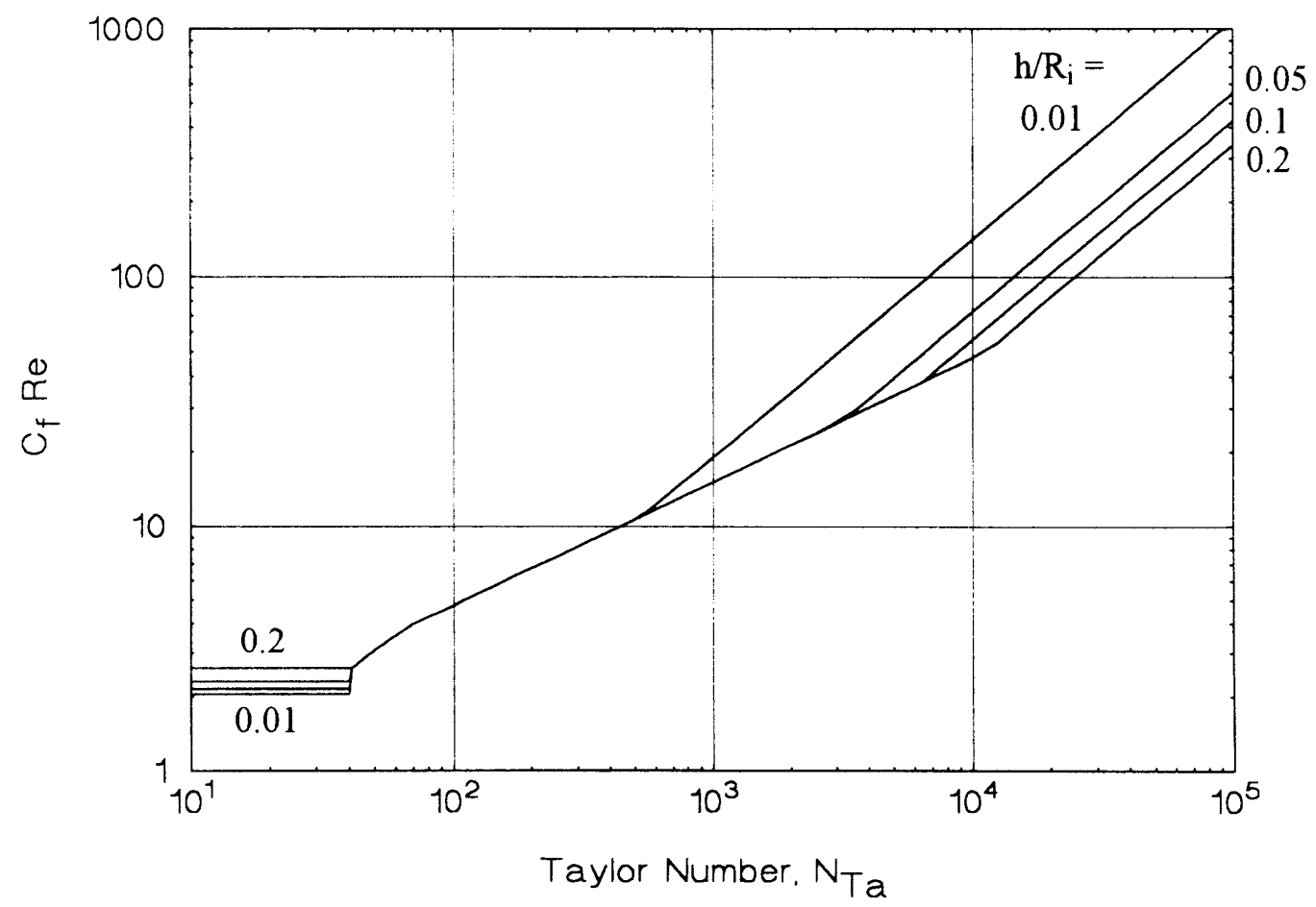

Figure 6.2. The skin friction factor for flow between two concentric cylinders for several different clearance ratios. 
Sample calculations are now performed on a $90 \mathrm{~mm}$ diameter inner cylinder, using a water-based coolant with $\rho=1000 \mathrm{~kg} / \mathrm{m}^{3}$ and $\mu=0.0013 \mathrm{Nm} / \mathrm{s}$ as the fluid. Figure 6.3 shows the calculated shearing power for a $10 \mathrm{~mm}$ long cylinder for several different clearances. As expected, the shearing power increases as the clearance is decreased. However, there is one exception; for speeds greater than about 13,000 rpm, the friction developed using a clearance of $20 \mu \mathrm{m}$ is less than that developed using a clearance of 50 $\mu \mathrm{m}$. Using a smaller clearance causes less shear power at these speeds because the flow has not made the transition to turbulence-dominated flow. This transition will occur at $41,000 \mathrm{rpm}$, at which point using a $50 \mu \mathrm{m}$ clearance generates less friction. Also note in Figure 6.3 that increasing the clearance from $1 \mathrm{~mm}$ to $10 \mathrm{~mm}$ decreases the shearing power by only a small amount. Increasing the clearance past $10 \mathrm{~mm}$ has no effect.

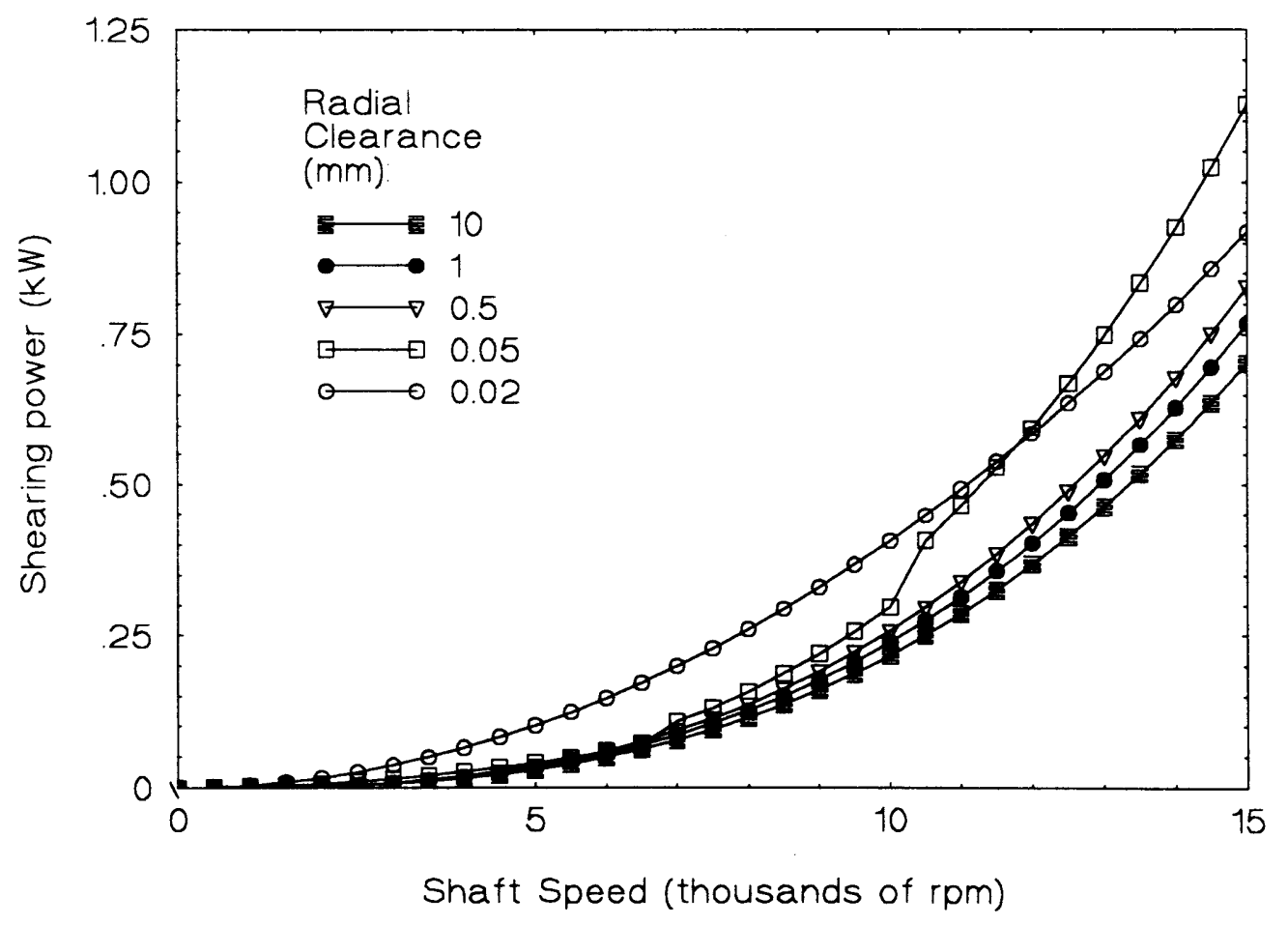

Figure 6.3. Shearing power produce by a $10 \mathrm{~mm}$ long circumferential groove which contains a water-based coolant with $\rho=1000 \mathrm{~kg} / \mathrm{m}^{3}$ and $\mu=0.0013 \mathrm{Nm} / \mathrm{s}$. The shaft diameter is $90 \mathrm{~mm}$. 
The clearance between the lands of hydrostatic bearings are very small compared to the bearing diameter, such that the clearance ratio $h / R_{i}$ is nearly always very small. In these cases, an adequate model of the fluid flow is that of Couette flow, and the following relations may be used [12]:

$$
\begin{array}{rc}
0<\operatorname{Re}<1600 & \mathrm{C}_{\mathrm{f}}=\frac{2}{\operatorname{Re}} \\
1600<\operatorname{Re}<2400 & \mathrm{C}_{\mathrm{f}}=0.00125 \\
\operatorname{Re}>2400 & \sqrt{2 \mathrm{C}_{\mathrm{f}}}=\frac{0.182}{\log _{10}(\operatorname{Re} / 4)}
\end{array}
$$

where again $R e=\rho h \omega R_{i} / \mu$ is the characteristic Reynolds number. The simplified relations given by Equations (6.7) through (6.9) give nearly the same results as those of Equations (6.3) through (6.6) for small clearance ratios.

\section{A. Bearing Pocket and Axial Groove Flow}

As previously described, the fluid flow within the pockets and axial grooves of hydrostatic bearings can be modeled as simple cavity flow if the pocket depth or groove depth is at least approximately ten times greater than the bearing clearance. A schematic representation of this fluid flow is shown in Figure 6.4. The upper wall moves with a surface speed, V, and causes the fluid to continuously recirculate within the pocket. Although the flow on the lands is usually laminar over the entire range of bearing operating speeds, the flow in the pockets of hydrostatic bearings usually becomes turbulent at even low operating speeds. Both laminar and turbulent regimes will therefore be considered in this section. 


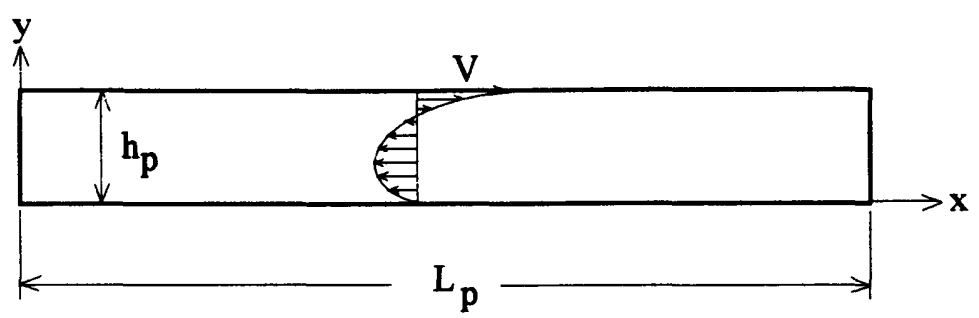

Figure 6.4. Model used to approximate the flow within the pockets and axial grooves of hydrostatic bearing with deep pockets and grooves.

Research previously done [13] illustrates that turbulence in the pockets of hydrostatic bearings begins at a Reynolds number of about 1,000 based on the pocket depth. This transition Reynolds number was shown to be approximately true for pocket aspect ratios of $15<\mathrm{L}_{\mathrm{p}} / \mathrm{h}_{\mathrm{p}}<240$. For lack of other data, this transitional Reynolds number can be approximately used for other aspect ratios. Relations for the friction factor for laminar and turbulent cavity flow are given by [14]:

$$
\begin{gathered}
0<\operatorname{Re}_{p}<1000 \quad C_{f}=\frac{8}{\operatorname{Re}_{p}}\left\{1+2.76\left(\frac{h_{p}}{L_{p}}\right)\left[1+0.00135 \operatorname{Re}^{1.09}\left(\frac{h_{p}}{L_{p}}\right)^{-0.21}\right]\right. \\
1000<\operatorname{Re}_{p}<2000 \quad C_{f}=0.0088\left\{1+\ln \left[1+2.71 \operatorname{Re}_{p}^{-0.134}\left(\frac{h_{p}}{L_{p}}\right)^{3.51 R^{-0.131}}\right]\right\} \\
\operatorname{Re}_{p}>2000 \quad C_{f}=0.047 \operatorname{Re}_{p}^{-0.226}\left\{1+\ln \left[1+2.71 \operatorname{Re}_{p}^{-0.134}\left(\frac{h_{p}}{L_{p}}\right)^{3.51 R e^{-0.131}}\right]\right\}
\end{gathered}
$$

where $\operatorname{Re}_{\mathrm{p}}=\rho V h_{\mathrm{p}} / \mu$ is the pocket Reynolds number, $h_{\mathrm{p}} / L_{\mathrm{p}}$ is the pocket aspect ratio, and $V$ is the surface speed of the shaft. 
The value of $C_{f}$ calculated using Equations (6.10) through (6.12) is plotted versus the Reynolds number for several different pocket aspect ratios in Figure 6.5. As shown, the friction of a pocket increases as the pocket aspect ratio decreases for both laminar and turbulent speeds. This is true because the end regions of the pocket where the flow turns around contributes more and more as the pocket aspect ratio is decreased. This effect is more dramatic for laminar speeds than for turbulent speeds because the wall friction is determined primarily by the boundary layer on the wall for turbulent fluid flow. The transitions between flow regimes shown in Figure 6.5 are distinct; however, in reality the transitions are gradual and the relations given by Equation (6.10) through (6.12) are only approximate in these regions.

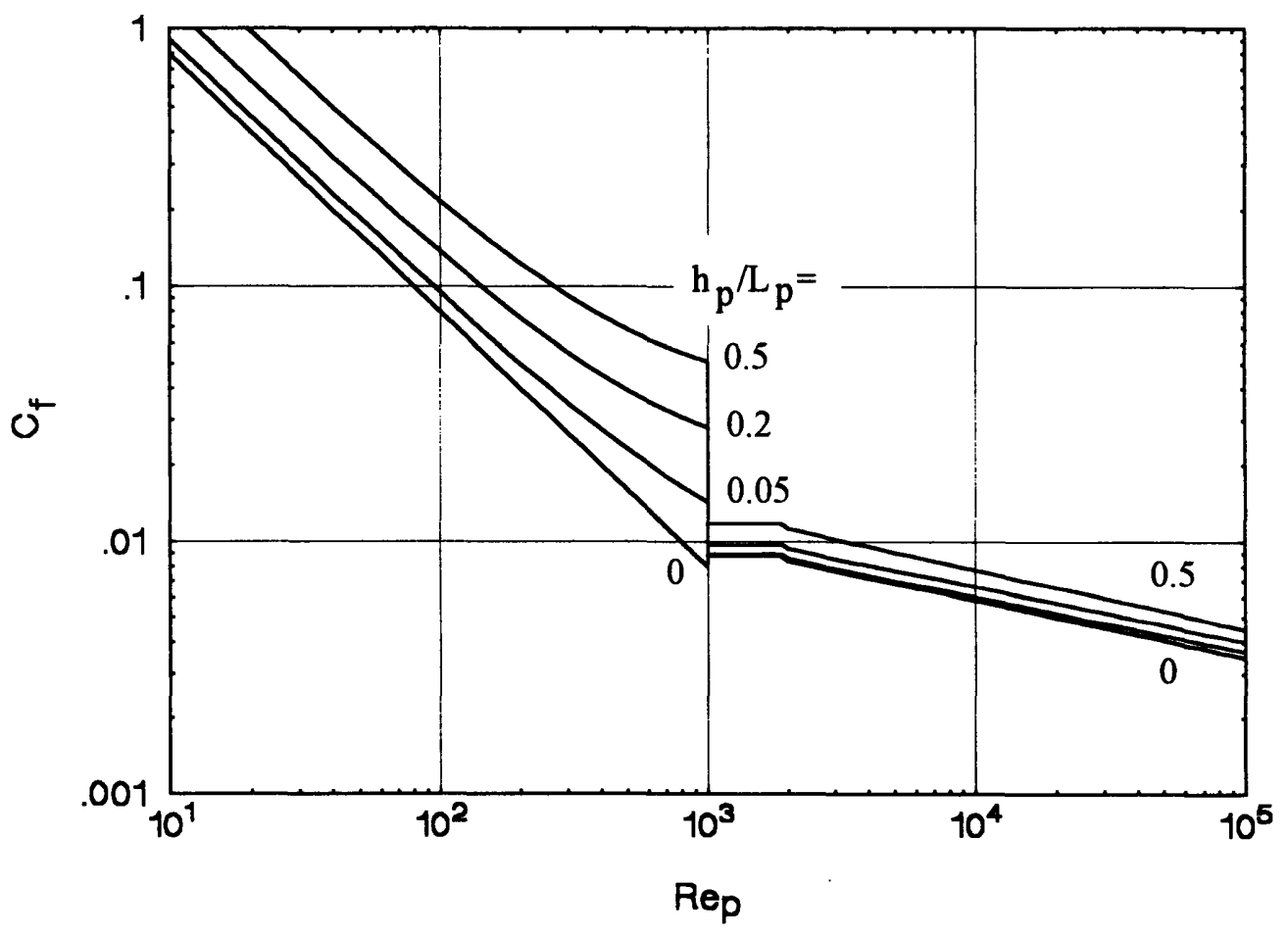

Figure 6.5. The skin friction factor for flow in a hydrostatic pocket for several different pocket aspect ratios. 
Sample calculations are now performed on a pocket with a circumferential length of $42 \mathrm{~mm}$ and an axial length of $10 \mathrm{~mm}$. The pocket contains a water-based coolant with $\rho=1000 \mathrm{~kg} / \mathrm{m}^{3}$ and $\mu=0.0013 \mathrm{Nm} / \mathrm{s}$. The shaft diameter is $90 \mathrm{~mm}$. Figure 6.6 shows the calculated shearing power for several different pocket depths. As expected, the shearing power decreases as the pocket depth is increased. However, increasing the pocket depth greater than approximately $10 \mathrm{~mm}$ decreases the frictional power only slightly.

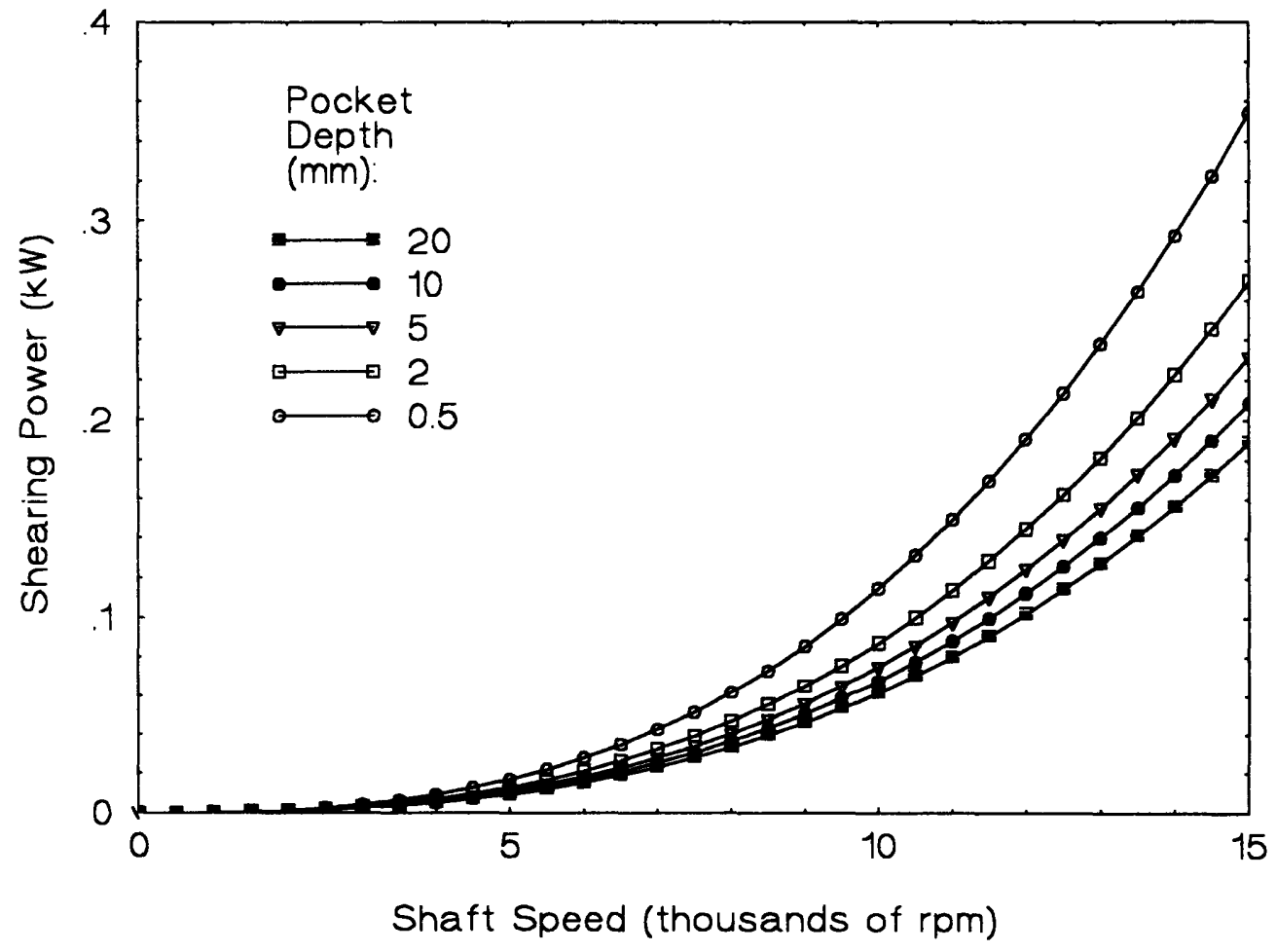

Figure 6.6. Shearing power produce by a pocket with a circumferential length of $42 \mathrm{~mm}$ and an axial length of $10 \mathrm{~mm}$. The pocket contains a water-based coolant with $\rho=1000$ $\mathrm{kg} / \mathrm{m}^{3}$ and $\mu=0.0013 \mathrm{Nm} / \mathrm{s}$. The shaft diameter is $90 \mathrm{~mm}$. 


\section{C. Temperature Rise}

Integrally related to the shearing power consumption of a hydrostatic bearing is its fluid temperature rise. The thermal behavior of a bearing is often equally important as its frictional behavior. In fact, the two are often coupled by a temperature-dependent viscosity. This coupling can be neglected if the temperature rise of the bearing is small, but often for high speeds the coupling is important. Both cases are considered in this chapter.

In addition to changes in viscosity, there can also be a significant change in the bearing clearance if the temperature rise of the bearing is high. The thermal expansion of the rotor can become a problem if the shaft diameter grows much faster than the inner diameter of the bearing housing. In practice, the speed of the shaft should be increased slow enough to allow the housing temperature to rise with the temperature of the shaft. Also, schemes to actively cool the bearings by passing coolant through the housing should be carefully considered because the growth of the housing bore will be inhibited while the shaft continues to grow and the bearing clearance can become dangerously tight. A better scheme to cool the bearings is to simply pre-chill the bearing lubricant before it enters the bearing such that the average fluid temperature within the bearing is approximately room temperature. Another method that is often used in conjunction with through-the-tool cooling is to pass coolant through the center of the shaft. 


\section{C.i. Constant-Viscosity Model}

At steady state, the shaft of a hydrostatic bearing is approximately adiabatic. This is true because the conduction of heat axially to a cooler part of the shaft is nearly always small compared to the overall heat being generated in the bearings. If the shaft is adiabatic, the power generated by viscous shearing within the bearing must either leave with the leaking fluid or be conducted radially through the housing. For a nearly uniform lubricant temperature $T_{\text {bulk, }}$ a one-dimensional heat balance on the bearing results in:

$$
\rho_{s}=U A_{i}\left(T_{\text {bulk }}-T_{\text {outer }}\right)+\rho c_{p} Q_{\text {supply }}\left(T_{\text {bulk }}-T_{\text {supply }}\right)
$$

where $\mathcal{P}_{\mathrm{s}}$ is the total shearing power generated, $\mathrm{Q}_{\text {supply }}$ is the volume flow rate of lubricant through the bearing, $\rho c_{p}$ is the lubricant heat capacity per unit volume, $T_{\text {supply }}$ is the lubricant supply temperature, $\mathrm{T}_{\text {outer }}$ is the sleeve outer temperature, $\mathrm{U}$ is the overall heat transfer coefficient to $T_{\text {outer, }}$ and $A_{i}$ is the projected area of the inner sleeve $\approx 2 \pi r_{i} L_{i}$. Note that, since the flow within the pockets is well-mixed, $\mathrm{T}_{\text {bulk }} \approx \mathrm{T}_{\text {shaft }} . \mathrm{A}$ one-dimensional approximation for $\mathrm{U}$ is:

$$
\mathrm{U}=\left[\frac{1}{\overline{\mathrm{h}}}+\frac{\overline{\mathrm{r}_{\mathrm{i}}}}{\mathrm{k}_{\text {sleeve }}} \ln \left(\frac{\mathrm{r}_{\mathrm{o}}}{\overline{\mathrm{r}_{\mathrm{i}}}}\right)\right]^{-1}
$$

where $\bar{r}_{\mathrm{i}}$ is the mean inner sleeve radius, $r_{0}$ is the outer sleeve radius, and $k_{\text {sleeve }}$ is the sleeve thermal conductivity. The heat transfer coefficient in this expression is a function of bearing geometry, shaft rotation rate, and lubricant properties. The heat transfer coefficient can be evaluated numerically usually commercial computational fluid dynamics software packages. However, doing so is almost never necessary, as discussed next. 
Consider the outer housing temperature, $\mathrm{T}_{\text {outer }}$. In some machine tool applications, the spindle is an integral part of a machine. Thus, the machine would act as a heat sink to which the heat generated by the spindle would be conducted. In other applications, the outer edge of the bearing sleeve might be cooled convectively by a coolant. Still another boundary condition occurs when the bearing housing is exposed to ambient air. In this case, the heat rate from the bearing housing by natural convection is usually small compared with that removed by the leaking fluid, and may possibly be neglected.

Consider first the situation in which convection from the sleeve is negligible. In this case $T_{\text {outer }} \approx T_{\text {shaft }}$. Equation (6.13) therefore reduces to:

$$
\left(\mathrm{T}_{\text {shaft }}-\mathrm{T}_{\text {supply }}\right)=\frac{\rho_{\mathrm{s}}}{\rho \mathrm{c}_{\mathrm{p}} \mathrm{Q}_{\text {sup ply }}}
$$

Although this expression neglects any heat that may be convected or conducted from the bearing other than that carried away with the lubricant, it is usually an excellent approximation of the bearing temperature rise, especially in situations where the leakage rate is high.

Consider next a situation in which the bearing housing is actively cooled using the coolant supply to such a degree that $T_{\text {outer }} \approx T_{\text {supply }}$. For this case, Equation (6.13) reduces to:

$$
\left(\mathrm{T}_{\text {shaft }}-\mathrm{T}_{\text {supply }}\right)=\frac{\rho_{\mathrm{s}}}{\mathrm{UA}_{\text {slecve }}+\rho \mathrm{c}_{\mathrm{p}} \mathrm{Q}_{\text {sup ply }}}
$$

For cases in which the inner sleeve heat transfer coefficient is high, the thermal resistance of the sleeve dominates and the overall heat transfer coefficient given by Equation (6.14) can be approximated as: 


$$
\mathrm{U}=\left[\frac{\overline{\mathrm{r}_{\mathrm{i}}}}{\mathrm{k}_{\text {sleeve }}} \ln \left(\frac{\mathrm{r}_{\mathrm{o}}}{\overline{\mathrm{r}_{\mathrm{i}}}}\right)\right]^{-1}
$$

Use of this equation greatly simplifies bearing thermal predictions. It is usually valid for water bearings at moderately high rotation rates, low sleeve conductivities, or large sleeve thicknesses. Neglecting curvature of the sleeve, a suitable criterion for which Equation (6.17) is valid is:

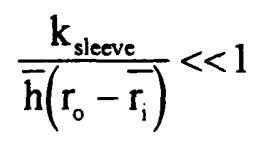

The use of Equation (6.15) is recommended for nearly all cases. Unless the bearing is active cooled, the heat conducted through the bearing housing is very small compared the heat leaving with the leaking fluid. For the case of an actively cooled bearing housing, Equation (6.17) is recommended for almost all cases since the criterion given by Equation (6.18) is usually valid.

Next consider the temperature rise of the fluid that results from the pressure driven flow. As the fluid is passed through the bearing, it is sheared by the restrictions of the bearing lands. The temperature rise caused by the fluid pumping is simply the pumping power, $\rho_{\mathrm{p}}=\left(\mathrm{P}_{\text {supply }}\right)\left(\mathrm{Q}_{\text {supply }}\right)$, divided by the flow rates times the heat capacity:

$$
\Delta T_{p}=\frac{P_{\text {supply }}}{\rho c_{p}}
$$

This temperature rise is often small compared to the temperature rise caused by the heat generated from shaft rotation. For example, the temperature rise of water caused by pumping it from $600 \mathrm{psig}$ is approximately $1^{\circ} \mathrm{C}$. 


\section{C.ii. A Simple Model to Account for Temperature-Dependent Viscosity}

The relations developed in Chapter 3 to predict the bearing load-carrying characteristics and the bearing flow rate, as well as those developed in the previous section to predict the bearing shearing power, were all developed assuming that the fluid properties were constant within the bearing. This approximation is good if the temperature rise through the bearing is low enough that the change in viscosity from the bearing inlet to the bearing outlet is negligible. However, when the bearings are used at high speeds, the isothermal model is often not adequate.

Consider a fluid with a temperature-dependent viscosity that obeys the following empirical relation:

$$
\mu=\mu_{\mathrm{i}}\left(\frac{\mathrm{T}}{\mathrm{T}_{\mathrm{i}}}\right)^{\mathrm{n}}
$$

where $T$ is the absolute temperature of the fluid, $T_{i}$ is the absolute temperature of the fluid at the bearing inlet, $\mu_{\mathrm{i}}$ is the viscosity of the fluid at the bearing inlet, and $\mathrm{n}$ is an exponent that is characteristic of a fluid. The relation given by Equation (6.19) can be used as an excellent curve fit to the viscosity curve of most lubricants, such as water and oil, over a wide range of temperatures. For example, the viscosity of pure water is $8.665 \times 10^{-4} \mathrm{Nm} / \mathrm{s}$ at $300 \mathrm{~K}$, and an exponent of -6.72 provides agreement within about $1 \%$ for a temperature range of $290 \mathrm{~K}$ to $320 \mathrm{~K}$.

In order to develop a simplifed model that accounts for the temperature-dependent viscosity, a simplifed relation for the shearing power is needed. For many bearings, most of the heat generated is caused by the bearing land flow. For nearly all operating speeds, 
this flow can be approximately modeled as laminar Couette flow. If the entire bearing area is modeled as such, the shearing power per unit length is given by:

$$
\frac{\rho_{s}}{L}=\frac{\mu V^{2} \pi D}{h}
$$

where $\mathrm{V}$ is the surface velocity of the shaft, and $\mathrm{h}$ is the clearance between the shaft and housing.

Consider the flow of fluid from one side of a bearing to the other, as shown in Figure 6.7. The fluid enters at a temperature $T_{i}$, and is heated to a temperature of $T_{0}$ as it leaves the bearing. As the fluid is heated, its viscosity changes according to Equation (6.20). The local shearing power per unit length changes as the the fluid passes through the bearing because its viscosity changes.

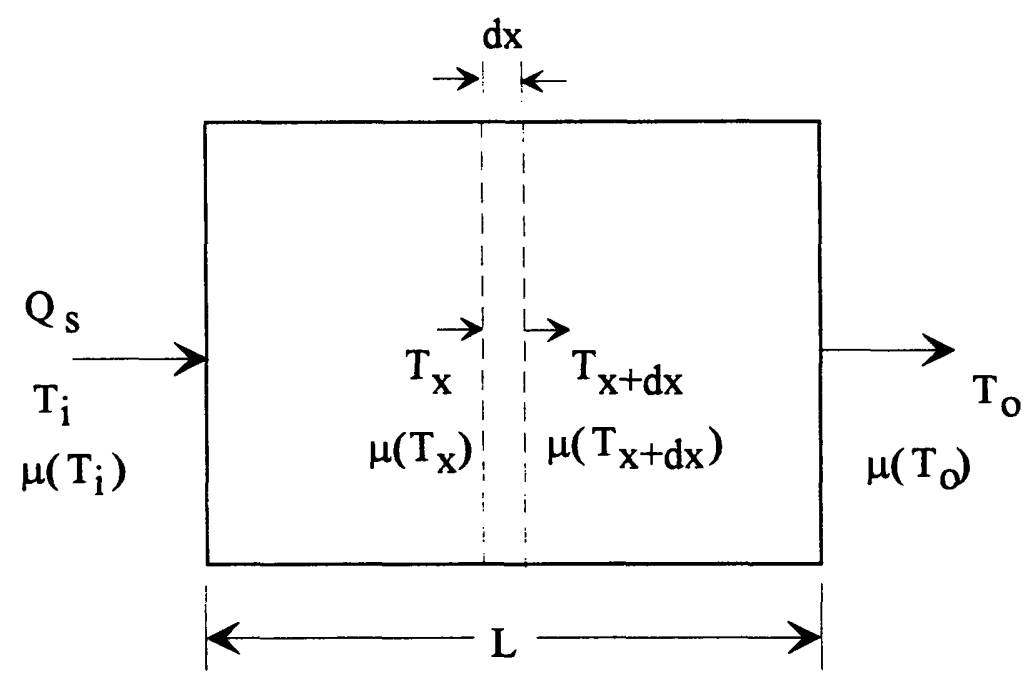

Figure 6.7. Schematic diagram of a hydrostatic bearing with the fluid heated as it passes through it. 
A local energy balance can be made on the small fluid element, $\mathrm{dx}$, by considering all of the heat generated as removed with the leaking fluid:

$$
T_{x+d x}-T_{x}=\frac{\left(\frac{\rho}{L}\right)_{x} d x}{Q_{s} \rho c_{p}}
$$

As the size of the fluid element becomes very small,

$$
\frac{d T}{d x}=\frac{\left(\frac{\rho}{L}\right)_{x}}{Q_{s} \rho c_{p}}
$$

Inserting Equation (6.21) for the power generation:

$$
\frac{d T}{d x}=\mu(T) \frac{V^{2} \pi D}{Q_{s} \rho c_{p} h}
$$

Rearranging this relation,

$$
\frac{d T}{\mu(T)}=\frac{V^{2} \pi D}{Q_{s} \rho c_{p} h} d x
$$

Inserting the viscosity relation given by Equation (6.19),

$$
\frac{d T}{\mu_{i}\left(\frac{T}{T_{i}}\right)^{n}}=\frac{V^{2} \pi D}{Q_{s} \rho c_{p} h} d x
$$

It will be assumed at this point that the bearing clearance, $h$, is a constant. By integrating this relation and rearranging its terms, the following formula is obtained:

$$
T=\frac{n}{n+1}\left[T_{i}+(n+1) \frac{\mu_{i} V^{2} \pi D L}{Q_{s} \rho c_{p} h}\left(\frac{x}{L}\right)\right]^{\frac{1}{n+1}}
$$


The supply flow rate, $Q_{s}$, also depends on the temperature change that occurs within the bearing. For most bearings, the supply flow rate is a function of the inlet and outlet resistance only. The resistance of the inlet land depends upon the inlet temperature, $T_{i}$, and the outlet resistance depends upon the outlet temperature, $T_{0}$. If the bearing has a ratio of inlet to outlet resistance, $\gamma$, it can be shown that its actual flow rate will be given by:

$$
Q_{s}=Q_{s}^{*}\left(\frac{1+\gamma\left(\frac{T_{o}}{T_{i}}\right)^{n}}{1+\gamma}\right)
$$

where $Q_{s}^{*}$ is the flow rate computed using a constant viscosity of $\mu_{\mathrm{i}}$. Its value can be computed using the methods developed in Chapter 3. Inserting Equation (6.28) into Equation (6.27):

$$
T=\frac{n}{T_{i}^{n+1}}\left[T_{i}+(n+1) \frac{\mu_{i} V^{2} \pi D L}{h} \frac{1}{Q_{s}^{*} \rho c_{p}} \frac{1+\gamma}{1+\gamma\left(\frac{T_{0}}{T_{i}}\right)^{n}}\left(\frac{x}{L}\right)\right]^{\frac{1}{n+1}}
$$

The term that represents the total bearing friction evaluated with the inlet fluid viscosity, $\mu_{\mathrm{i}}$, will be replaced with the actual power calculated with the more complete methods developed in the previous sections of this chapter, also evaluated with the inlet fluid viscosity: 


$$
T=T_{i}^{\frac{n}{n+1}}\left[T_{i}+(n+1) \frac{\rho^{*}}{Q_{s}^{*} \rho c_{p}} \frac{1+\gamma}{1+\gamma\left(\frac{T_{0}}{T_{i}}\right)^{n}}\left(\frac{x}{L}\right)\right]^{\frac{1}{n+1}}
$$

The outlet temperature is therefore given by:

$$
T_{o}=T_{1}^{\frac{n}{n+1}}\left[T_{1}+(n+1) \frac{\rho^{*}}{Q_{s}^{*} \rho c_{p}} \frac{1+\gamma}{1+\gamma\left(\frac{T_{0}}{T_{i}}\right)^{n}}\right]^{\frac{1}{n+1}}
$$

This implicit relation can be used to solve iteratively for the actual outlet bearing temperature, $T_{0}$. The temperature rise of the fluid caused by the fluid pumping can be approximately accounted for by simply calculating this component using Equation (6.19) and adding it to the outlet temperature found using Equation (6.31). Once the outlet temperature is known, the actual outlet flow rate can be calculated using Equation (6.28). The actual shearing power can be approximately evaluated by considering the average viscosity within the bearing:

$$
\begin{array}{r}
\frac{\rho}{\rho^{*}}=\frac{\int_{T_{i}}^{T_{0}}\left(\frac{T}{T_{i}}\right)^{-n} d T}{T_{0}-T_{i}} \\
\frac{\rho}{\rho^{*}}=\frac{T_{i}^{(1-n)}-T_{o}^{(1-n)}}{\left(T_{0}-T_{i}\right) T_{i}^{-n}(n-1)}
\end{array}
$$

The simple model developed in this section can be used to approximately account for temperature-dependent fluid viscosity for bearings whose shearing power is predominantly determined by the laminar bearing land flow. It is also approximately valid for other 
bearings, since the actual power used in the equations is calculated with the more detailed methods of this chapter.

\section{D. Thermal and Frictional Design of a Hydrostatic Bearing}

The frictional relations presented in the previous sections can be used to study the relative shear stress of the different bearing areas in order to optimize the bearing design for minimal shearing power consumption. In sections $6 . \mathrm{A}$ and $6 . \mathrm{B}$, a $90 \mathrm{~mm}$ diameter bearing was considered and the shearing power developed by the lands and the pockets of the bearing were calculated. Figure 6.8 shows the friction developed on a land with a clearance of $20 \mu \mathrm{m}$ to that in a pocket with a depth of $2 \mathrm{~mm}$ using fluids of various dynamic viscosities. A viscosity of $0.0013 \mathrm{Nm} /$ scorresponds to that of a water-based coolant at room temperature. A viscosity of $0.0008 \mathrm{Nm} / \mathrm{s}$ corresponds to that of a waterbased coolant at approximately $40^{\circ} \mathrm{C}$; this temperature might exist in a bearing operating at high speeds, greater than $12,000 \mathrm{rpm}$. As shown, the frictional shear stress on the lands is lower than that in the pockets for speeds greater than 5,000 to $8,000 \mathrm{rpm}$. This can be understood by recognizing that the flow in the pockets is very turbulent while the the flow on the lands remains laminar for all speeds shown. Also shown in Figure 6.8, a viscosity of $0.005 \mathrm{Nm} / \mathrm{s}$ corresponds to that of a very light-weight spindle oil at $40^{\circ} \mathrm{C}$. As shown, the shear stress on the lands remains greater than that in the pockets for speeds up to $15,000 \mathrm{rpm}$. Although the flow within the pocket is turbulent, the shear stress on the lands is very high because the viscosity is high. 


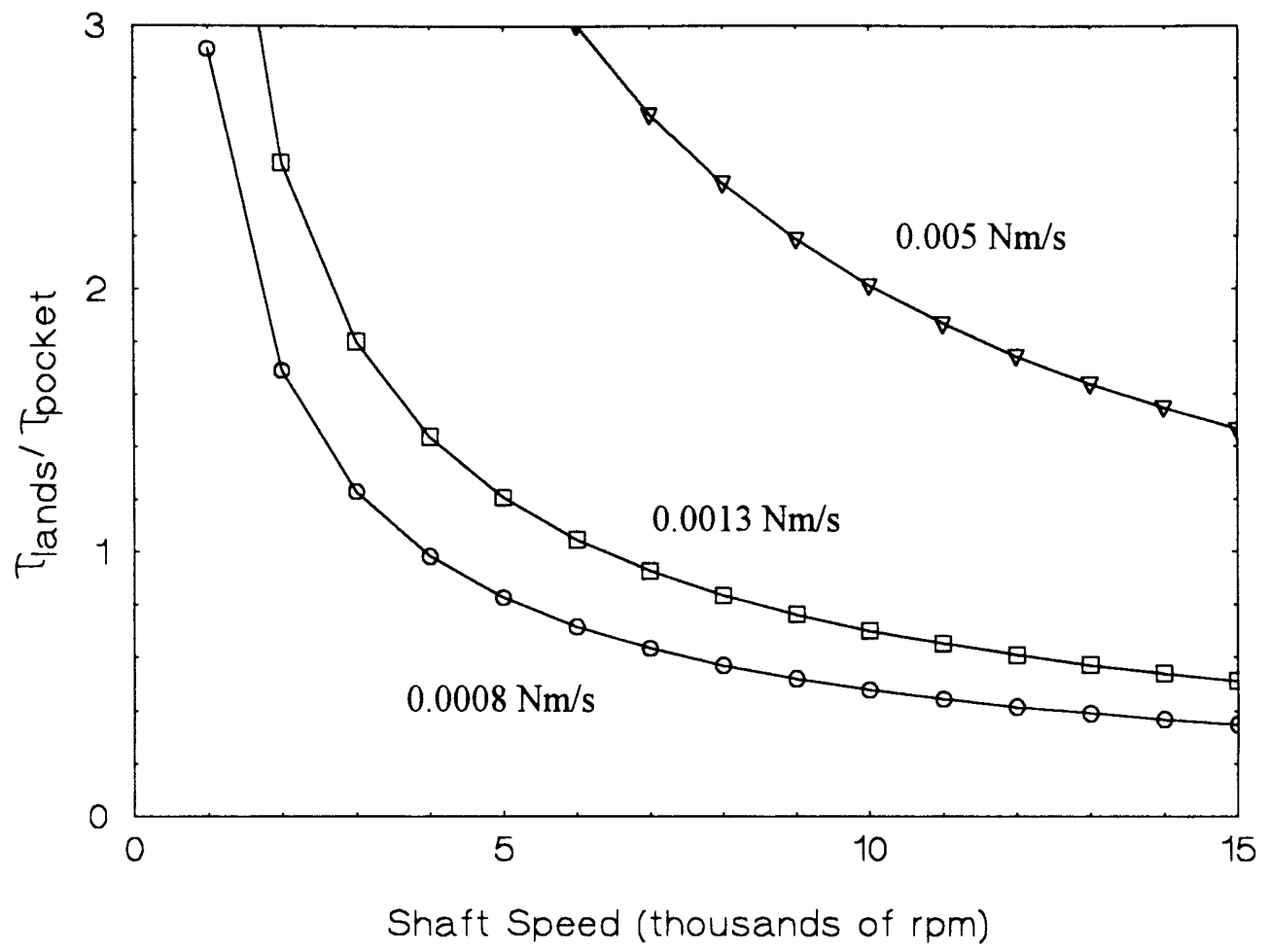

Figure 6.8. Comparison of the friction developed on a land with a clearance of $20 \mu \mathrm{m}$ to that in a pocket with a depth of $2 \mathrm{~mm}$ using fluids of various dynamic viscosities. The bearing diameter is $90 \mathrm{~mm}$.

Figure 6.8 clearly shows that, when using a water-based coolant as the bearing lubricant, the friction is higher in the pockets than on the lands when the bearing is operated at high speeds. Since friction is usually only a concern at high speeds, it appears that as much of the bearing area should be land area as possible. Increasing the land area is also desirable to achieve higher bearing damping. Figure 6.9 illustrates how the land area of Self-Compensated Bearing \#10 can be increased. Figure 6.9(a) shows the theoretical configuration used in the bearing model of Chapter 3, and Figure 6.9(b) shows 
the actual manufactured configuration. The raised areas in the center of the pockets serve to increase the bearing damping and reduce the bearing friction.
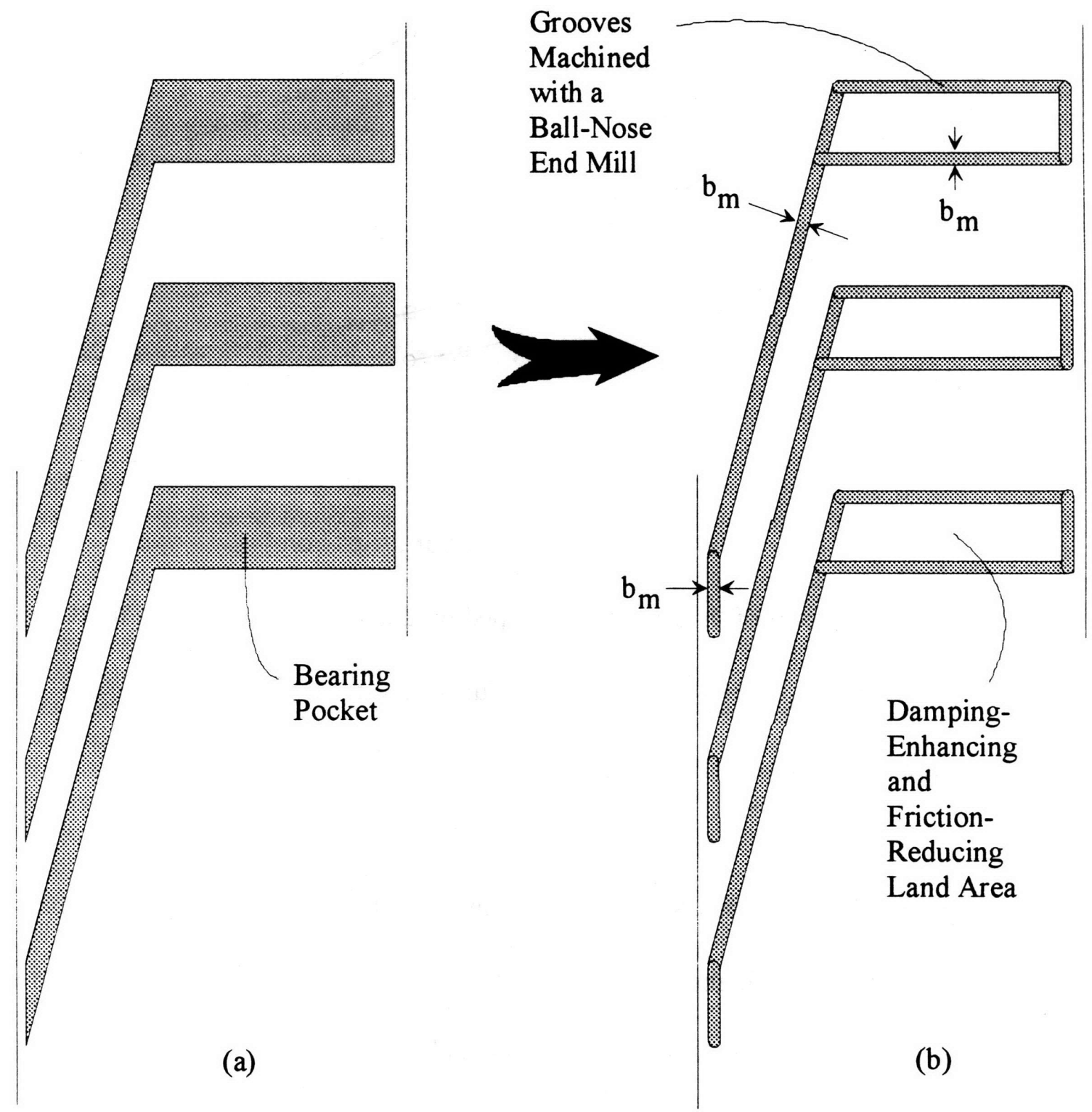

Figure 6.9. Illustratation of the pad lay-out of SC Bearing \#10, including (a) the theoretical configuration used in the bearing model, and (b) the actual manufactured configuration. The shaded areas represent depressed regions. The raised areas in the center of the pockets serve to increase the bearing damping and reduce the bearing friction. 
The frictional and thermal characteristics of a radial bearing will now be considered. The bearing is a SC Bearing \#10 with a diameter of $80 \mathrm{~mm}$ and a length of 80 $\mathrm{mm}$. A water-based coolant is used that a viscosity approximately $30 \%$ higher than that of water at all temperatures. The bearing is manufactured with an end mill, as shown in Figure 6.9. The width of the grooves, $b_{m}$, is $3 \mathrm{~mm}$. Their average depth is $0.75 \mathrm{~mm}$. Table 6.2 shows the resulting shearing power consumption of the bearing is approximately $2.7 \mathrm{~kW}$ at $10,000 \mathrm{rpm}$. In this analysis, a constant clearance of $20 \mu \mathrm{m}$ was used. This is approximately valid if the housing bore has sufficient time to grow as the shaft grows due to thermal expansion. The change in clearance due to centrifugal forces for a steel shaft at this speed is approximately $0.48 \mu \mathrm{m}$, and so this effect is not considered. At $15,000 \mathrm{rpm}$, the shaft will grow radially by approximately $1.08 \mu \mathrm{m}$, and this effect starts to become more important.

Table 6.2 also shows that the total temperature rise of the fluid passing through the bearing is approximately $9.6^{\circ} \mathrm{C}$ at $10,000 \mathrm{rpm}$. The flow rate has increased from $1.08 \mathrm{gpm}$ to $1.20 \mathrm{gpm}$ due to the decrease in fluid viscosity with the temperature temperature. Note that the effect of fluid pumping by the grooves has been neglected in the analysis; the actual flow rate may be somewhat higher or lower, depending on which way the grooves are oriented. 
Table 6.2

\begin{tabular}{|c|c|c|c|c|}
\hline \multicolumn{5}{|l|}{ Operating Conditions } \\
\hline Shaft Speed (rad/s,rpm) & $\mathbf{w}$ & 1047 & 10,000 & \\
\hline Supply Pressure (Pa,psi,atm) & Ps & 4.17E + 06 & 600 & 40.8 \\
\hline \multicolumn{5}{|l|}{ Fluid Properties } \\
\hline Dynamic Viscosity ( $\mathrm{s} / \mathrm{m}$ ) & mu & 0.00126 & & \\
\hline Bearing Inlet Temperature (K,oC,oF) & Tmu & 295 & & \\
\hline Viscosity-Temperature Exponent & nmu & 6.69 & & \\
\hline Density $(\mathrm{kg} / \mathrm{m} ` 3)$ & rho & 1000 & & \\
\hline Specific Heat Capacity $(\mathrm{J} / \mathrm{kg} / \mathrm{oC})$ & $\mathrm{Cp}$ & 4180 & & \\
\hline \multicolumn{5}{|l|}{ Bearing Geometry } \\
\hline Diameter (m,mm,in) & D & 0.08 & 80 & 3.150 \\
\hline Axial Length $(\mathrm{m}, \mathrm{mm}, \mathrm{in})$ & $L$ & 0.08 & 80 & 3.150 \\
\hline Number of Grooves & $\mathbf{N}$ & 6 & & \\
\hline Groove Rib Ratio & & 1.4 & & \\
\hline Pocket Rib Ratio & & 0.85 & & \\
\hline Groove Rib Width (m, mm, in) & br & 0.0244 & 24.4 & 0.962 \\
\hline Groove Width (m,mm,in) & bg & 0.0175 & 17.5 & 0.687 \\
\hline Pocket Rib Width (m, mm, in) & brp & 0.0192 & 19.2 & 0.758 \\
\hline Pocket Width $(\mathrm{m}, \mathrm{mm}, \mathrm{in})$ & bp & 0.0226 & 22.6 & 0.891 \\
\hline Nominal Gap $(m, \mu m$, in) & ho & 0.00002 & \multicolumn{2}{|c|}{200.00079} \\
\hline Compensator Land Length ( $\mathrm{m}, \mathrm{mm}$, in) & Lc & 0.003 & 3 & 0.118 \\
\hline Axial Land Length (m,mm,in) & La & 0.003 & 3 & 0.118 \\
\hline Drain Groove Circumferential Width ( $\mathrm{m}, \mathrm{mm}$, in) & bd & 0.003 & 3 & 0.118 \\
\hline Drain Groove Axial Length (m,mm,in) & Ld & 0.01 & 10 & 0.394 \\
\hline Pocket Circumferential Land Width $(\mathrm{m}, \mathrm{mm}$, in) & Lac & 0.0081 & 8.1 & 0.320 \\
\hline Twist Angle (radians, degrees) & phi & 2.44 & 140 & \\
\hline Twist Ratio & & 0.2650 & & \\
\hline Twist Length (m,mm,in) & $\mathrm{Lt}$ & 0.0259 & 25.9 & 1.020 \\
\hline Groove Skew Angle (radians, degrees) & Beta & 1.31 & 75.2 & \\
\hline Theoretical Groove Width at Skew $(\mathrm{m}, \mathrm{mm}$, in) & & 0.0045 & 4.47 & 0.176 \\
\hline End Mill Width $(\mathrm{m}, \mathrm{mm}, \mathrm{in})$ & bm & 0.003 & 3 & 0.118 \\
\hline End Mill Average Depth (m,mm,in) & bmd & 0.00075 & 0.75 & 0.030 \\
\hline Resistance Ratio & gamma & 1.0 & & \\
\hline \multicolumn{5}{|l|}{ Supply Flow Rate and Pumping Power } \\
\hline Specific Flow Rate & Qspec & 9.8 & & \\
\hline Isothermal Supply Flow Rate (m`3/s,lpm,gpm) & $\mathrm{Q}^{*}$ & $6.79 \mathrm{E}-05$ & 4.07 & 1.08 \\
\hline Actual Supply Flow Rate (m -3/s,lpm,gpm) & Qsupply & 7.6E-05 & 4.56 & 1.20 \\
\hline Pumping Power (W,kW, hp) & Ppump & 316.6 & 0.317 & 0.425 \\
\hline \multicolumn{5}{|l|}{ Power Consumption and Temperature Rise } \\
\hline Isothermal Bearing Power Consumption (W,kW,hp) & & 3029 & 3.03 & 4.06 \\
\hline Actual Bearing Power Consumption (W,kW,hp) & & 2724 & 2.72 & 3.65 \\
\hline Pumping Temperature Rise (oC,oF) & & 1.0 & 1.8 & \\
\hline Total Temperature Rise (oC, oF) & & 9.6 & 17.3 & \\
\hline
\end{tabular}


Figure 6.10 shows the bearing shearing power consumption as a function of shaft speed. The power increases approximately with the cube of the shaft speed until the temperature-dependence of the fluid viscosity becomes important, and then levels off somewhat.

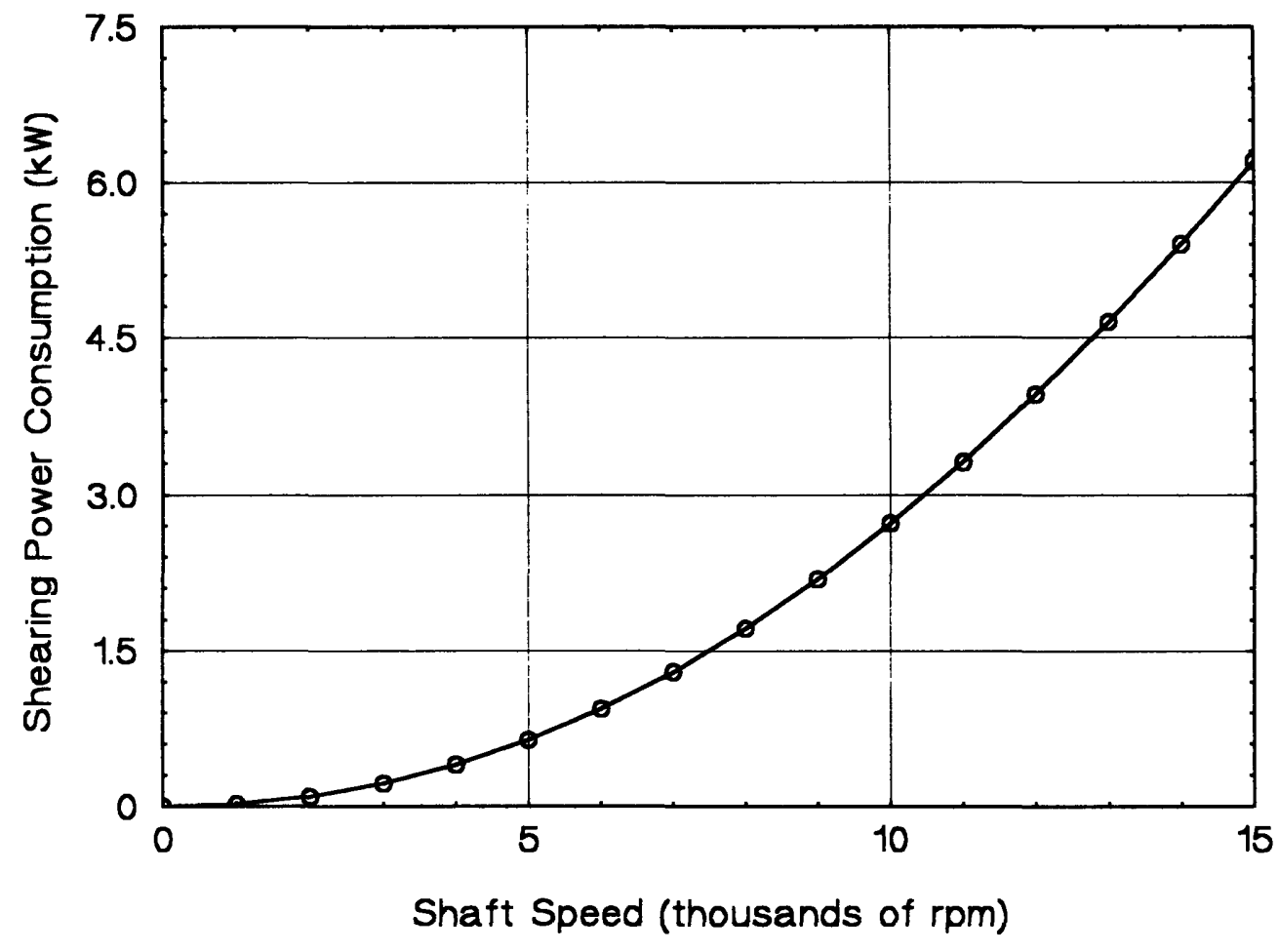

Figure 6.10. Shearing power consumption of the bearing with the parameters shown in Table 6.2. The analysis includes the effect of a temperature-dependent fluid viscosity. 
Figure 6.11 shows the temperature rise of the fluid through the bearing as a function of shaft speed. The $1^{\circ} \mathrm{C}$ temperature rise at $0 \mathrm{rpm}$ corresponds to the temperature rise from the pumping of the fluid from a pressure of 600 psig to a pressure of 0 psig. As the shaft speed is increased, the temperature rise of the fluid increases as it absorbs more shearing power generated within the bearing. The temperature rise increases approximately with the cube of the shaft speed until the temperature-dependence of the fluid viscosity becomes important; at speeds greater than approximately 12,000 rpm, it increases almost linearly with the shaft speed.

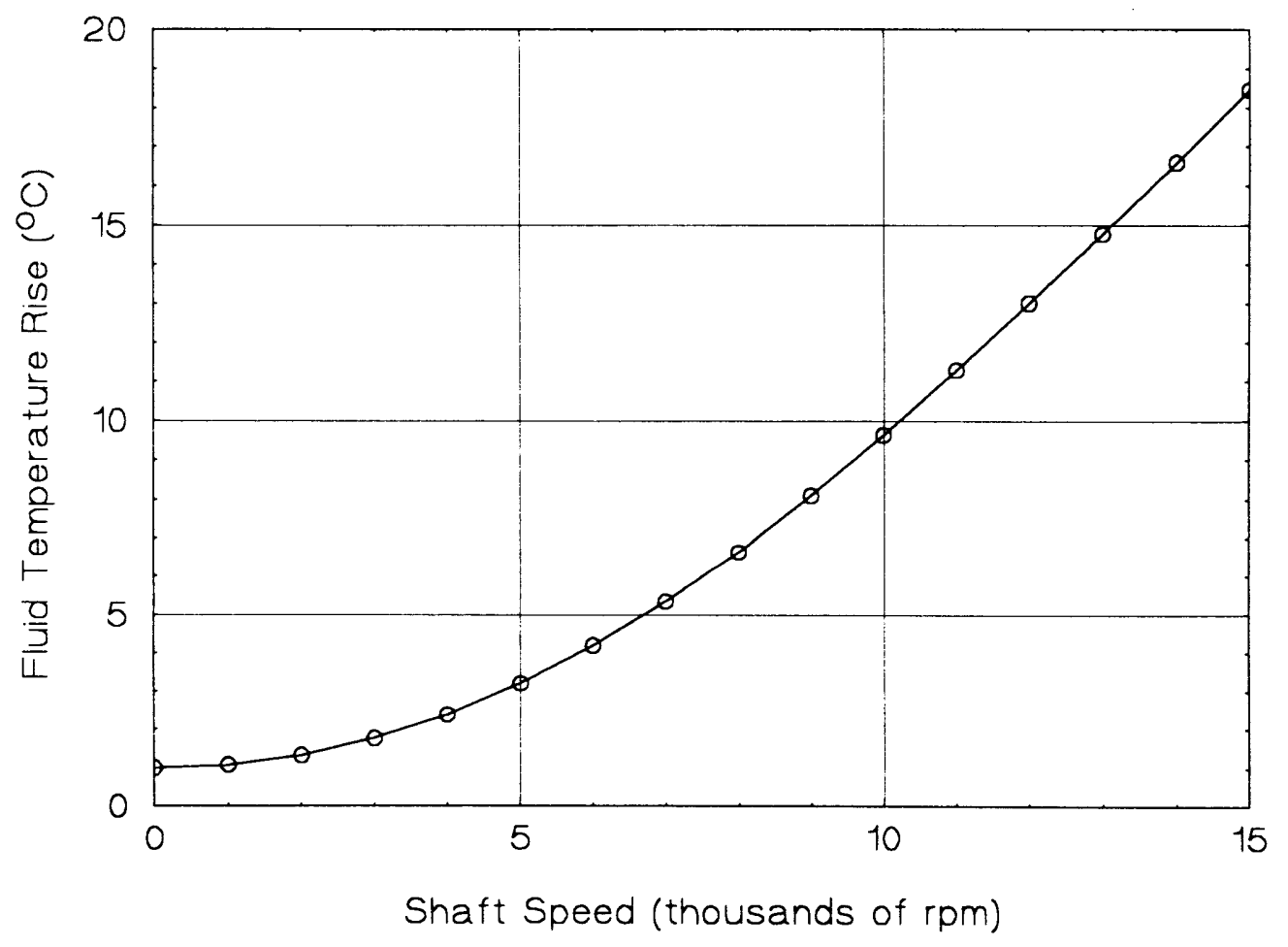

Figure 6.11. Temperature rise of the fluid through the bearing with the parameters shown in Table 6.2. The analysis includes the effect of a temperature-dependent fluid viscosity. 
Figure 6.12 shows the volumetric flow rate of fluid through the bearing as a function of shaft speed. The increased flow rate is due solely to the decrease in fluid viscosity with increase temperature. The effect of fluid pumping by the grooves has been neglected in the analysis; the actual flow rate may be somewhat higher or lower, depending on which way the grooves are oriented.

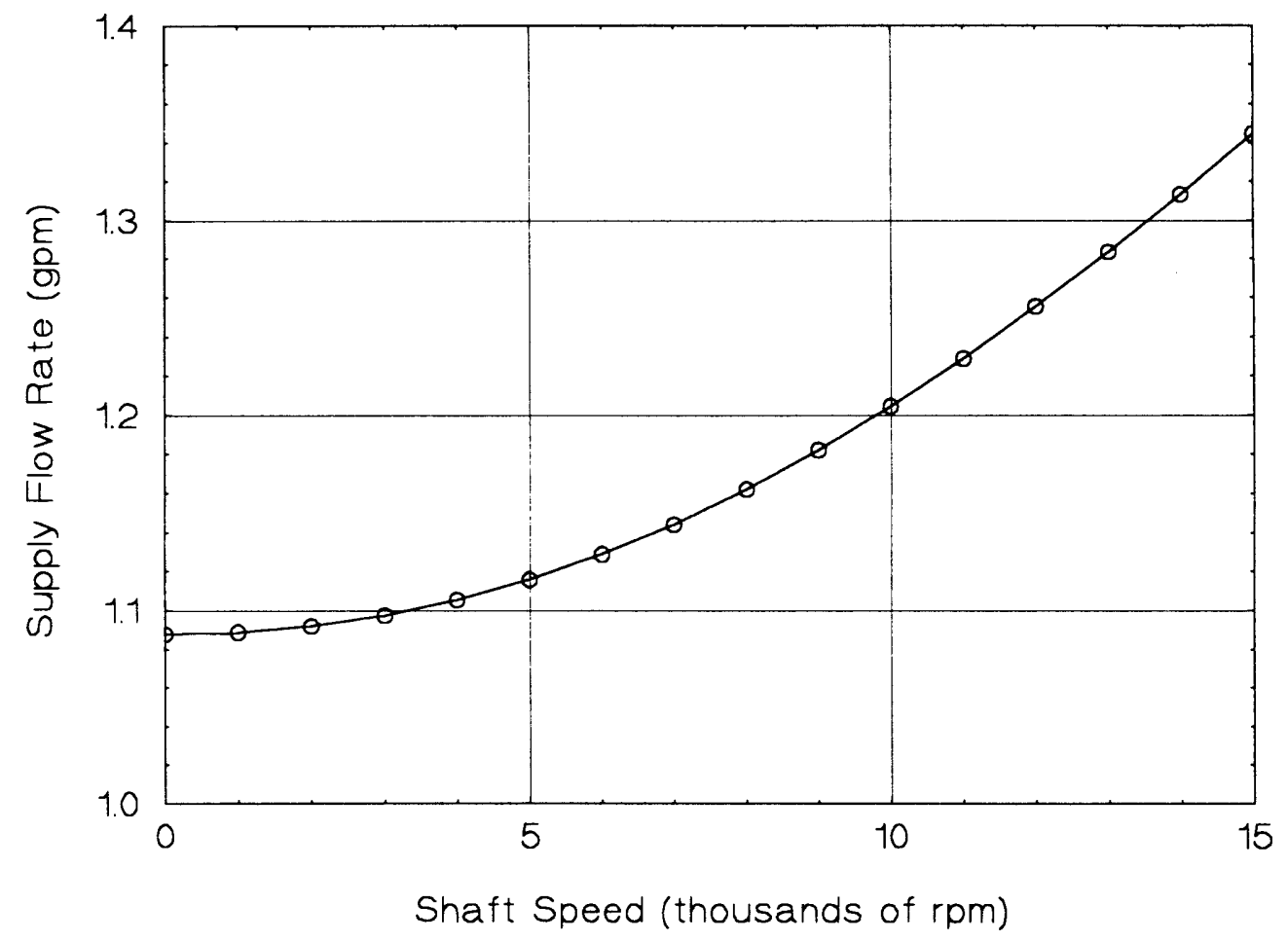

Figure 6.12. Supply flow rate of fluid through the bearing with the parameters shown in Table 6.2. The analysis includes the effect of a temperature-dependent fluid viscosity. 


\section{EXPERIMENTAL TESTING: THREE PROTOTYPE SPINDLES}

This chapter describes the tests performed at Setco Whitnon on the three fluid film test spindles. The primary test spindle was built with SC Bearing \#1, and the two secondary test spindles were built with SC Bearing \#10. The three spindles were each evaluated for use in a machine that can be used to drill clusters of holes in steel components. The design of a 4-cluster machine is described in Chapter 8.

The tests performed on the primary test spindle were successful. The radial stiffness of the spindle was measured to be over 130,000 pounds per inch at a shaft location 4" from the front of the air seal. The load capacity of the thrust bearing was tested and found to about 1,100 pounds. The spindle was driven to $18,000 \mathrm{rpm}$ and subjected to impact hits on the end of the shaft exceeding 150 pounds while rotating at $10,000 \mathrm{rpm}$ without failure. Modal plots show that the spindle is very well damped with a dynamic stiffness of about 73,000 pounds per inch at a resonant frequency of $550 \mathrm{~Hz}$. In addition, the temperature rise of the water flowing through the pockets was measured to be $4.2^{\circ} \mathrm{C}$ when the shaft is rotating at $10,000 \mathrm{rpm}, 12.5^{\circ} \mathrm{C}$ when rotating at $18,000 \mathrm{rpm}$. All of these results were predicted within a reasonably close margin, and the behavior of the spindle is believed to be well understood. The test results indicate that the prototype spindle has exceptional qualities that meet or exceed the intended project goals.

The tests performed on the secondary test spindles were also very successful. The new spindle configuration has grooves on the surface of the shaft to route the fluid from the compensating lands to the load-supporting pockets. With this scheme the need to cross-drill through the shaft is eliminated and the spindle fabrication is greatly simplified. 
In addition, these spindles require substantially less flow rate and pumping power to achieve the same stiffness. The goals of the tests were to verify the static stiffnesses of the spindles and to verify their stability at high speeds. The two spindles had excellent static stiffnesses that were comparable to that achieved with the primary test spindle. Also, the four-pocket spindle was sucessfully driven to $20,000 \mathrm{rpm}$ and its stability was verified. These tests verify the basis of the design.

\section{A. Primary Self-Compensated Test Spindle}

The primary fluid film test spindle is shown in Figure 7.1. Four-pocket selfcompensated hydrostatic bearings are used for the front and rear radial bearings. A smaller bearing on the pulley drive shaft is used to provide substantial belt load capability and effectively isolate the noise of the pulley drive system from the rest of the spindle. A striking characterstic of the spindle of Figure 7.1 is that all of the bearing detail is machined into the surface of the shaft itself, grooves machined into the shaft with a ball end-mill are used to create the bearing "pockets" and integral compensators. This method of hydrostatic spindle construction, which greatly simplifies spindle fabrication, is enabled by the use of self-compensation. With this technique the compensation resistances can be placed on the surface of the shaft and made to rotate at the same rate as the pockets. 


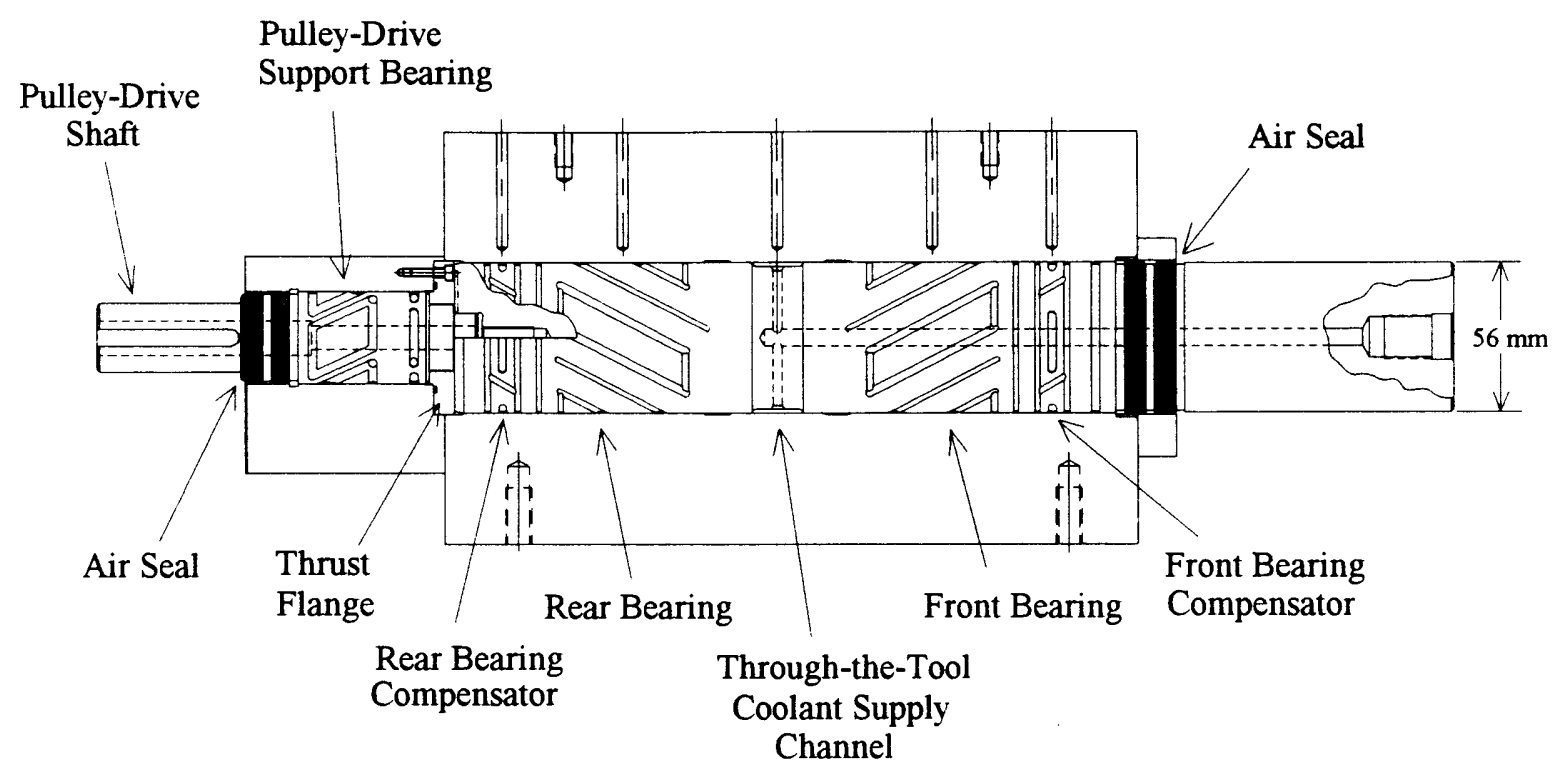

Figure 7.1. Layout of the primary test spindle. The radial bearings are self-compensated bearings with feed holes cross-drilled through the shaft.

Also shown in the test spindle of Figure 7.1 are air seals on the front and rear of the spindle. These seals are non-contact seals that are pressurized with normal shop air to prevent any leakage of the coolant used to pressurize the bearings. The seals were added to the spindle for ergonomics and to minimize the routine maintenance of refilling the coolant reservoir. Also shown in Figure 7.1 is a unique method to supply through-thetool coolant. Coolant is supplied to the groove in the center of the spindle and then routed through holes drilled radially to the center of the shaft and then to the front face of the spindle. The thrust bearing, another unique characteristic of this spindle, is designed with one thrust face larger than the other to provide preferential load capacity in the pushing direction for this drilling application. 
As mentioned previously, the bearings of the primary prototype spindle are selfcompensated bearings that have features on the surface of the shaft itself to provide compensation to the bearings. In the primary test spindle, the fluid is routed from the compensators to the pockets on the opposite side of the shaft through holes that are crossdrilled through the shaft, as illustrated in Figure 7.2 and shown in Figure 7.3. The holes reduce the stiffness of the shaft only negligibly and provide a convenient means to route the fluid. Since the fluid is nearly incompressible and the holes begin and end at the same radius, fluid inertia forces induced by shaft rotation do not affect the bearing performance. This method of cross-drilling through the shaft is an effective manufacturing method. However, a new method in which the fluid is routed on the surface of the shaft itself simplifies fabrication and reduces the flow rate of fluid required to pressurize the bearings. Two test spindles were also fabricated with this method, and tests of these spindles will be described later in this chapter. Tests of the cross-drilled spindle will be described first.

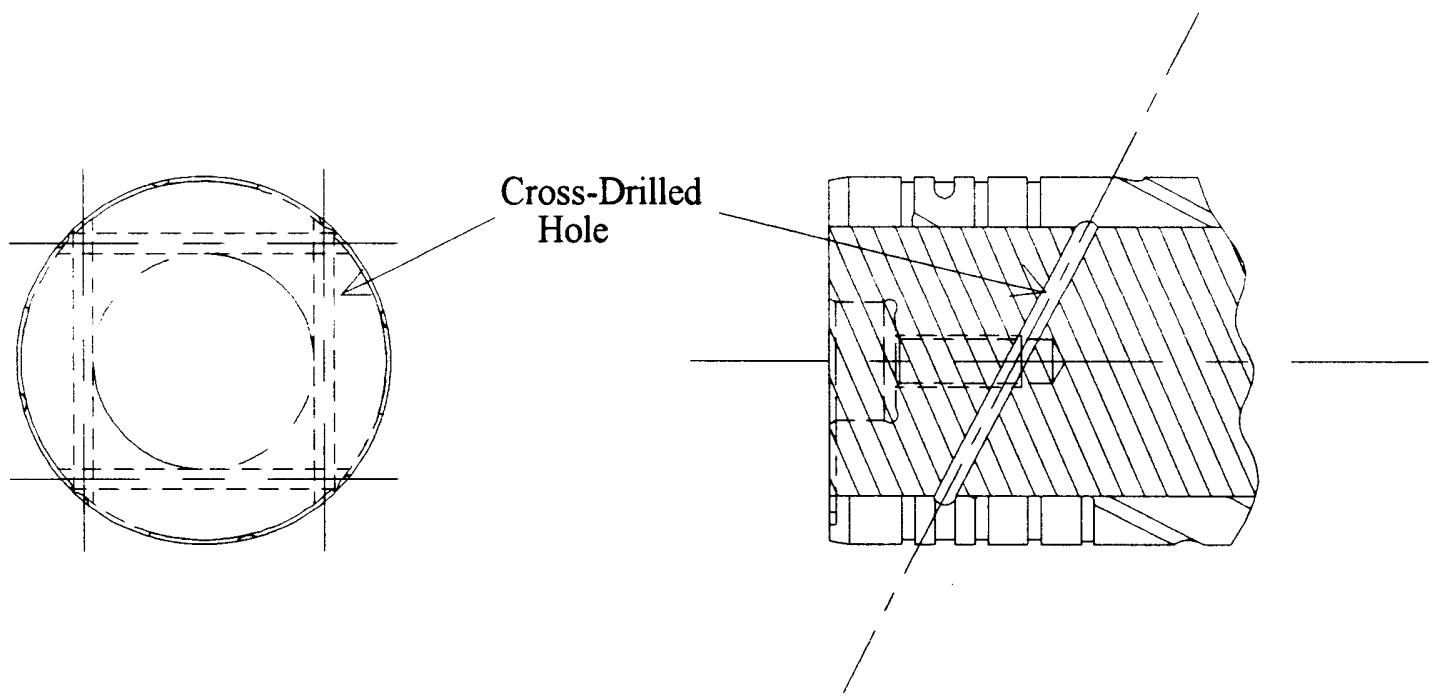

Figure 7.2. Illustration of the holes cross-drilled through the shaft from the compensators to the pockets on the opposite side of the shaft. 


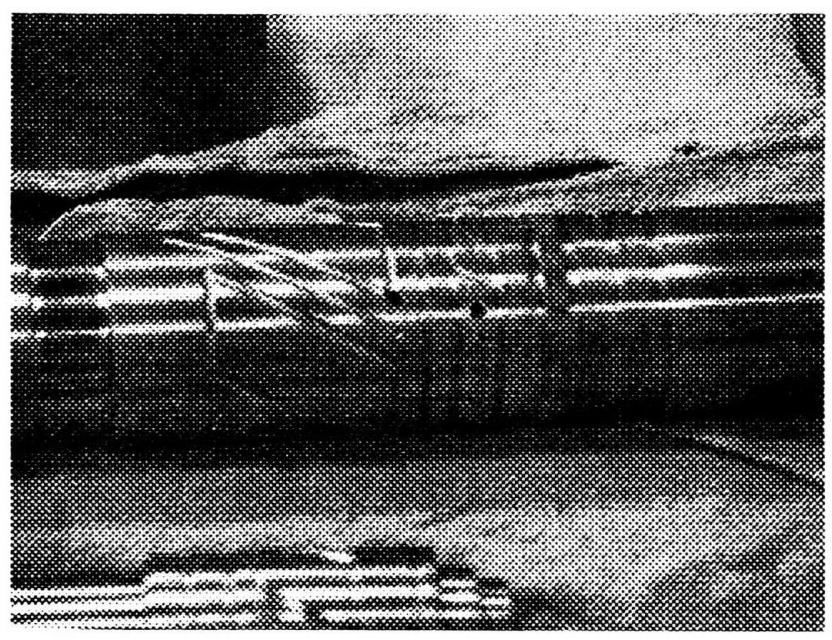

Figure 7.3. Picture of the shaft of the Phase I test spindle showing the holes that are cross-drilled through the shaft from the compensators to the load-supporting pockets.

\section{A.i. Static Radial Stiffness}

The static stiffness of the spindle was measured by hanging a dead weight of 25 pounds from its end and measuring the deflection with a Starrett indicator. The resolution of the indicator was 0.00001 " and its accuracy was verified by testing it against the positioning lead of a capacitance probe. The positions on the spindle where the weight was suspended and the displacement was measured are shown in Figure 7.4. With a supply pressure of 590 psi and a back pressure of 11 psi (about 580 psi pressure difference), the deflection of the spindle loaded with 25 pounds was 0.000190 inches. This yields a static stiffness of 131,500 pounds per inch. Since the shaft material in this test was steel, it is apparent that we can meet our goal stiffness of 250,000 pounds per 
inch using a carbide shaft with an elastic modulus of $60 \times 10^{6} \mathrm{psi}$. This is discussed further below.

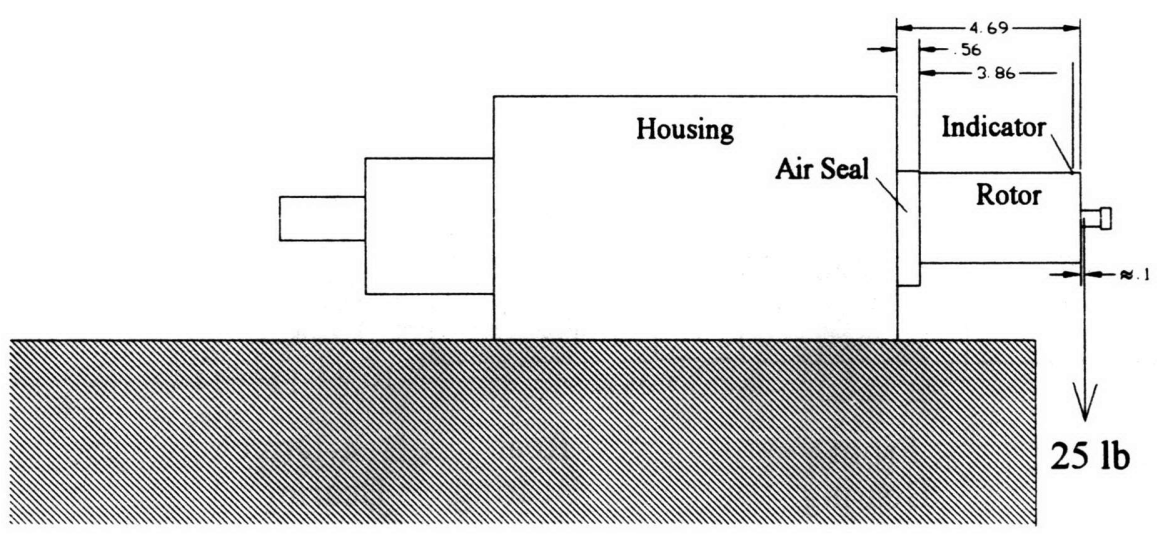

Figure 7.4. Schematic diagram showing the axial locations of the indicator and the applied weight that were used in the static stiffness tests.

A goal of the spindle testing was to verify the theory of the spreadsheets so that the next generation spindle could be optimally designed with confidence. A comparison will therefore be made between the measured stiffness and the predicted stiffness for this configuration. Figure 7.5 shows a schematic diagram of the rotor illustrating the finite elements and boundary conditions used to model the shaft bending. Pressures were applied at the appropriate bearing locations with magnitudes corresponding to the predicted bearing stiffnesses. Table 7.1 shows the values of the parameters used in the analysis. In addition, the clearances used on the front and rear radial bearings were 15.5 $\mu \mathrm{m}$ and $15.4 \mu \mathrm{m}$, respectively. Figure 7.6 shows the predicted centerline deflection of the spindle subjected to a force of 25 pounds on its front end and no belt pressure on its rear 
end. The predicted stiffness is $131,700 \mathrm{lb} /$ in . Although an accuracy of about $10 \%$ is expected, the exceptional agreement between the predicted stiffness of $131,700 \mathrm{lb} / \mathrm{in}$ with the measured stifness of $131,500 \mathrm{lb} /$ in is most likely coincidental.

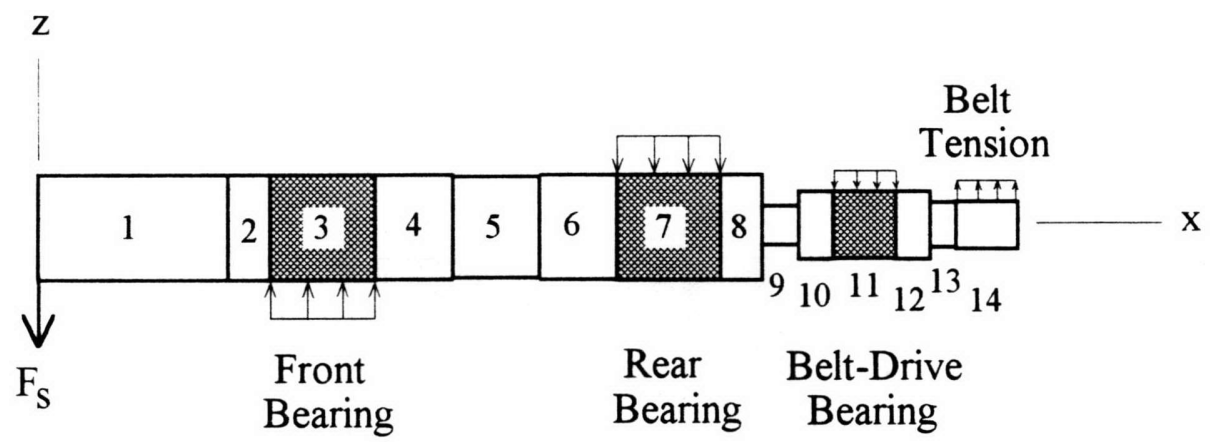

Figure 7.5. Schematic diagram of the rotor illustrating elements and boundary conditions used to model the shaft bending.

Table 7.1.

\begin{tabular}{|c|c|c|c|c|c|}
\hline Section & $\begin{array}{c}\text { Elastic } \\
\text { Modulus } \\
(\mathbf{P a})\end{array}$ & $\begin{array}{c}\text { Length } \\
(\mathbf{m})\end{array}$ & $\begin{array}{c}\text { Outer } \\
\text { Diameter } \\
(\mathbf{m})\end{array}$ & $\begin{array}{c}\text { Inner } \\
\text { Diameter } \\
(\mathbf{m})\end{array}$ & $\begin{array}{c}\text { Poisson } \\
\text { Ratio }\end{array}$ \\
\hline 1 & $2 \times 10^{11}$ & 0.1271 & 0.056 & 0.01 & 0.3 \\
\hline 2 & $2 \times 10^{11}$ & 0.038 & 0.056 & 0.006 & 0.3 \\
\hline 3 & $2 \times 10^{11}$ & 0.06 & 0.056 & 0.006 & 0.3 \\
\hline 4 & $2 \times 10^{11}$ & 0.0175 & 0.056 & 0.006 & 0.3 \\
\hline 5 & $2 \times 10^{11}$ & 0.019 & 0.053 & 0.006 & 0.3 \\
\hline 6 & $2 \times 10^{11}$ & 0.0175 & 0.056 & 0 & 0.3 \\
\hline 7 & $2 \times 10^{11}$ & 0.06 & 0.056 & 0 & 0.3 \\
\hline 8 & $2 \times 10^{11}$ & 0.032 & 0.056 & 0 & 0.3 \\
\hline 9 & $2 \times 10^{11}$ & 0.01 & 0.024 & 0.009 & 0.3 \\
\hline 10 & $2 \times 10^{11}$ & 0.019 & 0.034 & 0.009 & 0.3 \\
\hline 11 & $2 \times 10^{11}$ & 0.029 & 0.034 & 0.009 & 0.3 \\
\hline 12 & $2 \times 10^{11}$ & 0.022 & 0.032 & 0.013 & 0.3 \\
\hline 13 & $2 \times 10^{11}$ & 0.0254 & 0.025 & 0.013 & 0.3 \\
\hline 14 & $2 \times 10^{11}$ & 0.0254 & 0.025 & 0.013 & 0.3 \\
\hline
\end{tabular}




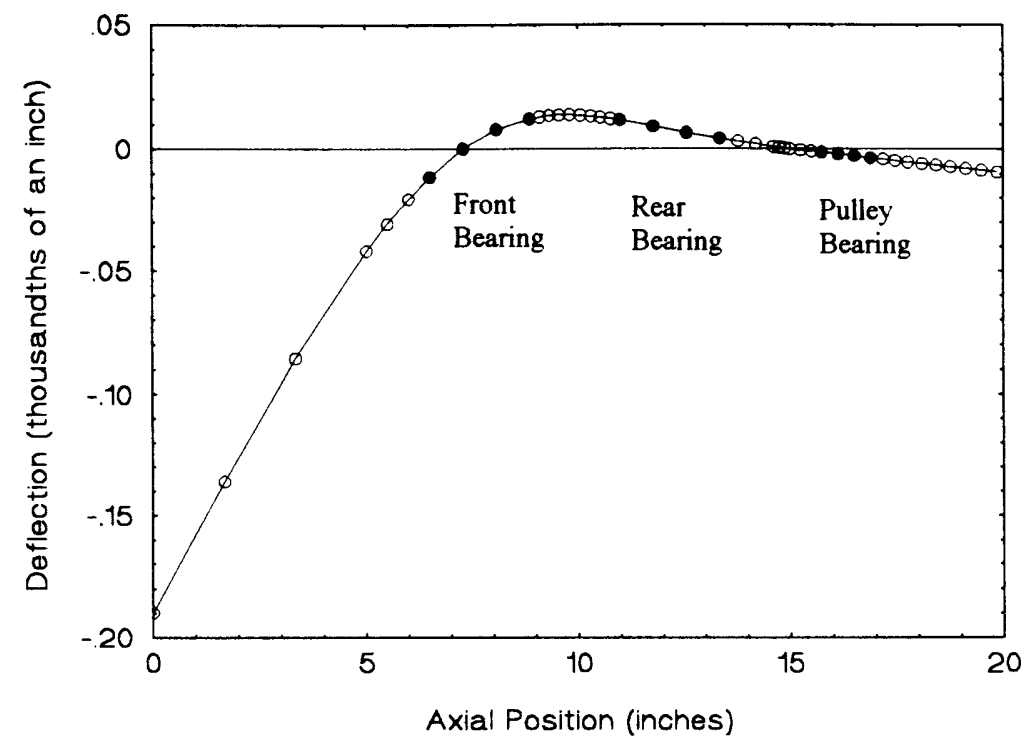

Figure 7.6. Predicted deflection of the spindle subjected to a force of 25 pounds on the nose of the spindle. The dark circles indicate the positions of the bearing pads.

The original goal stiffness of the project was $250,000 \mathrm{lb} /$ in at a location 4 " from the front of the housing using a carbide shaft. The calculations previously described were repeated using a carbide shaft with an elastic modulus of $60 \times 10^{6} \mathrm{psi}$, and the predicted stiffness of this configuration is $236,000 \mathrm{lb} / \mathrm{in}$. This calculation indicates that, with a few modifications of the spindle design based on knowledge gained from the spindle testing (e.g., the orientation of the bearing pads), we can meet our project goal stiffness of $250,000 \mathrm{lb} /$ in using a carbide shaft if it is deemed necessary.

In addition to the primary stiffness test, a second spindle configuration was also tested for static stiffness. In this test the fluid drains which lead from the pocket isolation channels were closed off. The function of the pocket isolation channels are to eliminate leakage from the fluid supply regions of the bearing to the bearing pockets. With the drains from the isolation channels closed, both the stiffness of the bearings and the overall 
flow rate of the spindle decrease. With the drains leading from the isolation channels closed off and a supply pressure of $590 \mathrm{psi}$, a back pressure of $0 \mathrm{psi}$, the deflection of the spindle loaded with 25 pounds was 0.000210 inches. This yields a static stiffness of about 119,000 pounds per inch. Although the stiffness of this configuration is about $10 \%$ lower than that with the drains open, the flow rate through the spindle and the required pumping power are reduced $30 \%$ from about $4.8 \mathrm{gpm}$ to about $3.7 \mathrm{gpm}$ as discussed in the section of this report on flow rate.

In addition to the front spindle stiffness tests, the rear belt drive extension of the spindle was also tested for static stiffness. The stiffness was measured by hanging a dead weight of 25 pounds from the rear end of the belt drive extension and measuring the deflection with a Starrett indicator with a resolution of 0.00001 ". The position on the spindle where the weight was suspended and where the displacement was measured are shown in Figure 7.7. With a supply pressure of 590 psi and no back pressure, the deflection of the spindle loaded with 25 pounds was 0.0005 inches. This yields a static stiffness of about 50,000 pounds per inch.

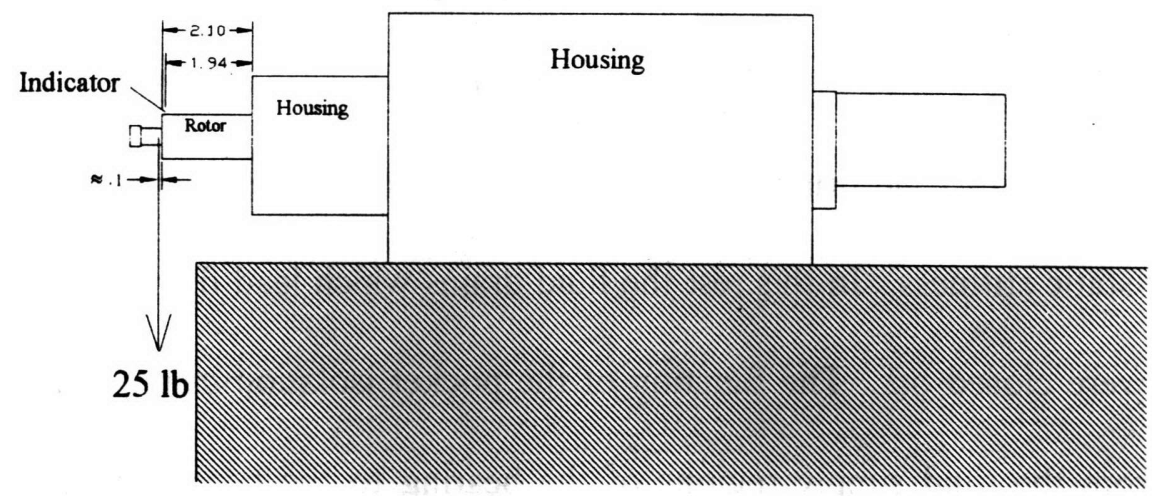

Figure 7.7. Schematic diagram showing the axial locations of the indicator and the applied weight that were used in the static stiffness tests on the rear of the spindle. 
A comparison will again be made between the measured stiffness and the predicted stiffness of the spindle for this configuration. In this case a point force is applied to the rear end of the spindle and no force is applied to the front end. The parameters used in the calculations are again those of Table 7.1. The clearance used on the rear pulley bearing was $14 \mu \mathrm{m}$. Figure 7.8 shows the predicted centerline deflection of the spindle subjected to a force of 25 pounds on its rear end. The predicted stiffness is $60,000 \mathrm{lb} / \mathrm{in}$, which agrees with the measured stiffness of $50,000 \mathrm{lb} /$ in within about $20 \%$. The reason why the rear stiffness is slightly lower than expected may be the compliance of the interface between the rear belt-drive extension and the main front shaft. The difference is small, however, and the rear extension will certainly serve its intended purpose of supporting the shaft under a belt load. Note also that in this test the stiffness was measured on the very end of the pulley drive extension. In operation the belt will be applied to the center of the pulley drive extension where the stiffness will be significantly higher. 


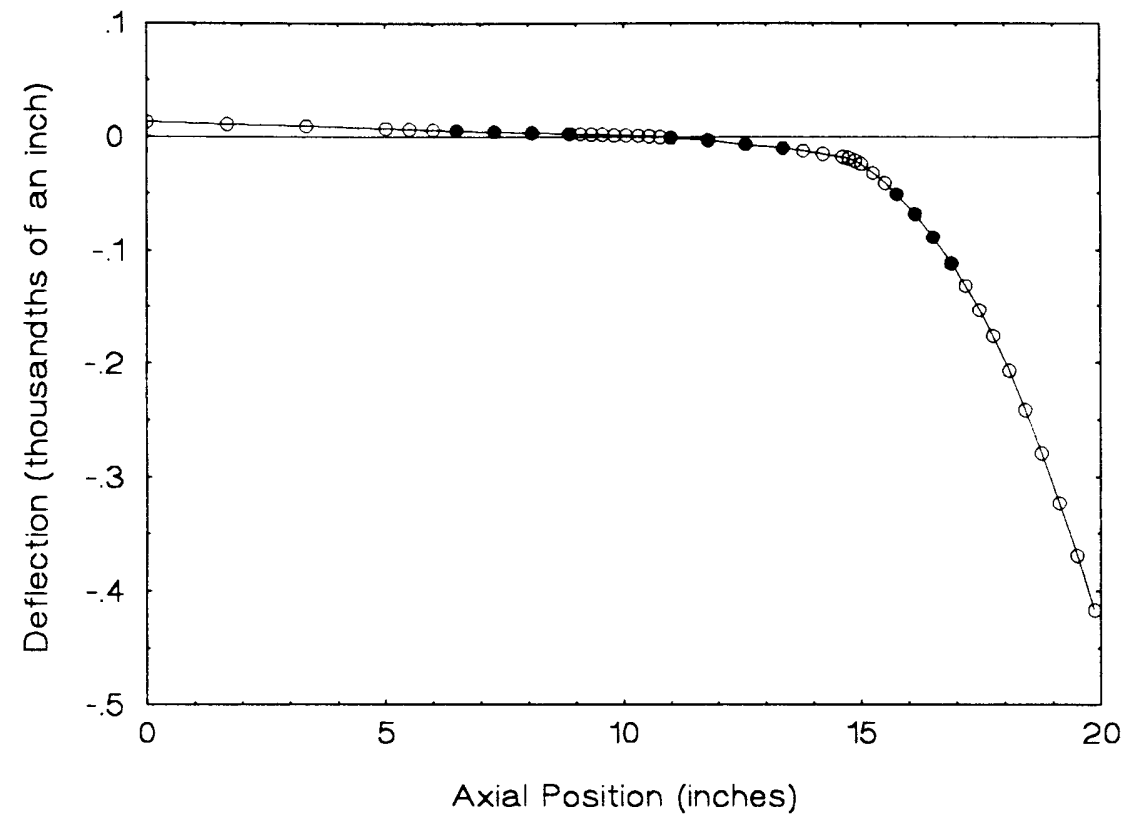

Figure 7.8. Predicted deflection of the spindle subjected to a force of 25 pounds on the rear end of the spindle. The dark circles indicate the positions of the bearing pads.

Note in Figure 7.8 that when 25 pounds of forces is applied to the rear belt drive extention that the predicted deflection of the front spindle face is only about 0.00002 inches. This observation indicates that the belt system used to drive the spindle is effectively isolated from the front of the spindle where the tool is held. This observation was confirmed with TIR measurements that illustrate that the runout at the front face of the spindle is only marginally affected by whether the spindle is driven with a belt drive or with an in-line drive.

\section{A.ii. Dynamic Radial Stiffness}

In addition to the static stiffness tests performed on the stationary shaft, dynamic radial stiffness tests were performed with and without the shaft rotating. For these tests the spindle was excited with a load cell hammer and its resulting displacment was 
measured with a capacitance probe. Both the force and displacement measurements were made at a location on the shaft approximately 4 inches from the front of the air seal. In all of the dynamic tests the spindle was connected on its rear belt drive extension to the torque sensor using a flexible coupling. Also, in all of the dynamic tests the drains leading from the pocket isolation grooves were closed. Note that this configuration had a lower static stiffness than that with the pocket isolation drains open.

The capacitance probe was mounted with the use of a magnetic holder onto an angle-iron that was bolted down to the same table to which the spindle housing was bolted. This configuration was found to be the best configuration possible given the limited fixturing available at the time of testing. A better configuration would be obtained if the capacitance probe were mounted directly to the spindle housing. This would eliminate the effects of vibrations of the angle iron, vibrations between the angle iron and the table, and vibrations between the spindle housing and the table. Although the tests performed with the limited fixturing are probably adequate, if more accurate results are needed then a more suitable mounting structure should be fabricated and the tests should be repeated with the capacitance probe mounted directly to the spindle housing.

Dynamic tests were performed first with the shaft not rotating. For these tests a light hammer with a sensitivity of $10 \mathrm{mV} / \mathrm{lb}$ was used with hits in the range of 10 to 30 pounds. A five-sample averaging scheme was used to reduce the effect of noise and obtain a cleaner plot. The resulting frequency response spectrum is shown in Figure 7.9. The natural frequency of the spindle is about $550 \mathrm{~Hz}$, with a dynamic stiffness at this peak 
of about 73,000 lb/in. This high magnitude of dynamic stiffness is expected of a welldamped hydrostatic spindle.

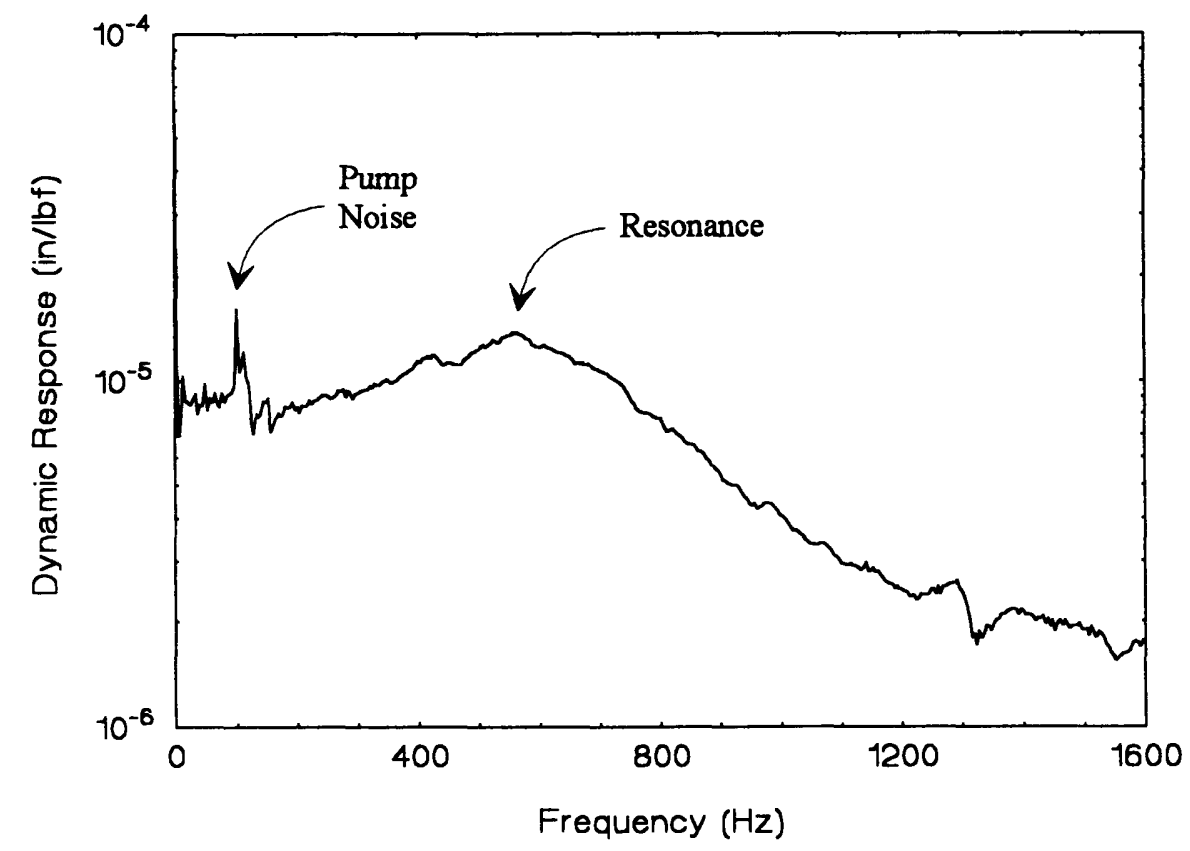

Figure 7.9. Measured dynamic response of the unrotating spindle measured at a point 4" front the front of the air seal. Note the noise spike less then $200 \mathrm{~Hz}$ due to the pump.

Note the noise spike of Figure 7.9 that occured at a frequency less then $200 \mathrm{~Hz}$. This noise was isolated by measuring the spectral power density of the capacitance probe output with and without the pump running. This test proved that the noise in Figure 7.9 less than about $200 \mathrm{~Hz}$ was attributable to the pump. Note also that the stiffness of the spindle as the frequency becomes small in Figure 7.9 limits to about 115,000 lb/in, which is close to the static stiffness of $119,000 \mathrm{lb} /$ in measured for this spindle configuration. This observation adds to our confidence that the dynamic measurements made are accurate. 
The dynamic tests were next repeated with the shaft rotating up to $10,000 \mathrm{rpm}$. In these tests, the electrical noise emitted by the drive motor tended to drown out the measurements made using the light hammer. This noise was confirmed to be electrical by noting that even with the shaft unrotating the noise was excessive with the motor turned on. A heavier hammer with a sensitivity of $0.9 \mathrm{mV} / \mathrm{lb}$ was therefore used with hits on the end of the shaft in the range of 50 to 150 pounds. These heavy hits tended to excite other components of the test set-up, including the angle-iron to which the capacitance probe was mounted. The response spectrum of the shaft rotating at $10,000 \mathrm{rpm}$ is shown in Figure 7.10. Note that the peak at about $100 \mathrm{~Hz}$ is thought to be attributable to the vibration of the angle iron. The peak at $550 \mathrm{~Hz}$ found in the previous measurements made with the shaft unrotating is not evident in Figure 7.10. This peak was likely drowned out by the excessive electrical noise as well as other structural noise resulting from the heavy hits. The data is therefore unreliable as a means to measure the dynamic stiffness of the rotating shaft, and it will be necessary to eliminate the electrical noise emitted by the drive motor before accurate data can be obtained. The data presented here is useful data, however, in that it illustrates that no excessive resonances were witnessed with the shaft rotating at high speed. Perhaps the most useful product of these tests is that the spindle absorbed hits on its end up to 150 pounds while rotating at $10,000 \mathrm{rpm}$ without failure. 


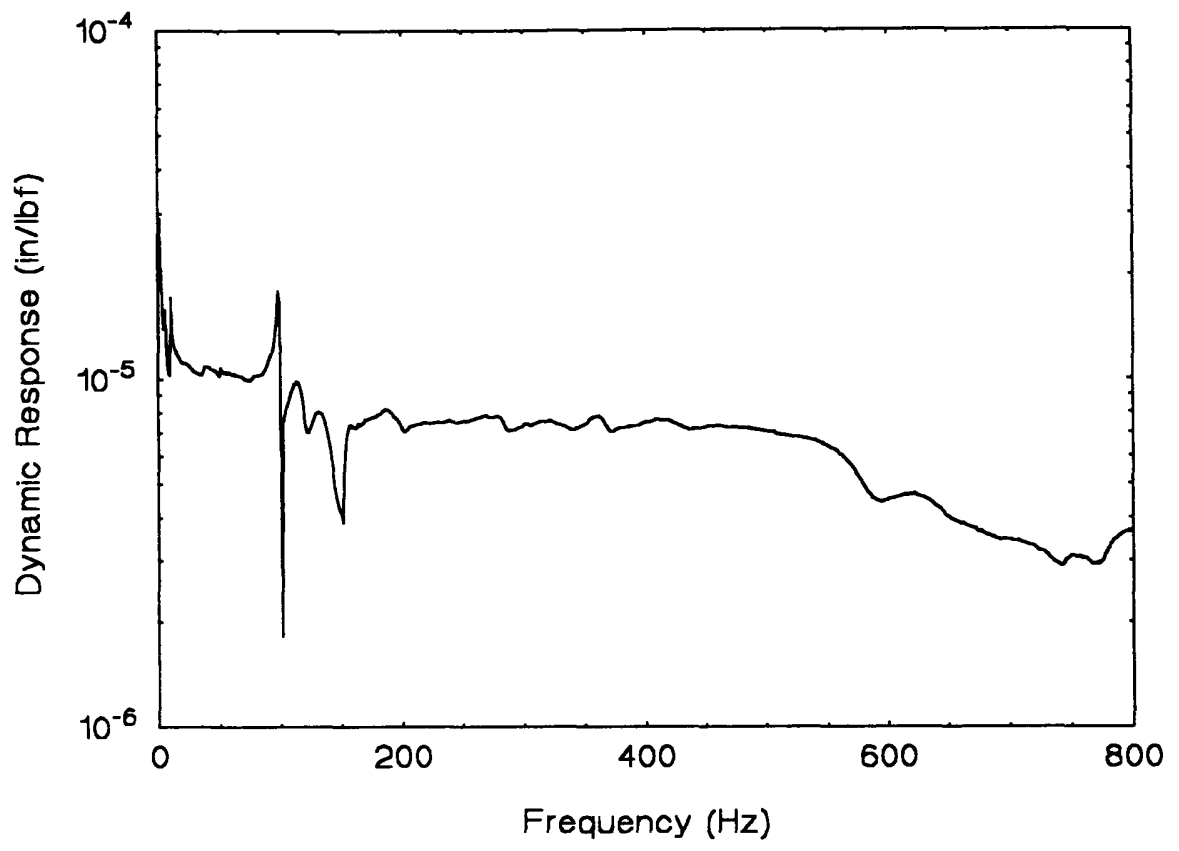

Figure 7.10. Measured dynamic response of the spindle measured at a point 4" front the front of the air seal. The speed of the spindle was $10,000 \mathrm{rpm}$.

\section{A.iii. Static Thrust Stiffness and Load Capacity}

In addition to the radial stiffness tests, tests were also performed to determine the static thrust stiffness and load capacity of the spindle. These tests indicate that the thrust bearing is not performing according to its expected behavior. Although the load capacity of the thrust bearing is close to what was expected, the stiffness of the thrust bearing is about half that predicted by the spreadsheets. This behavior was found to be the result of excessive housing compliance.

The total clearance of the two thrust gaps was measured first by moving the unpressurized spindle forward and rearward until contact was made with the housing. An overall displacement of about 0.0013 inches was measured during this test. Next the spindle was pushed all the way forward so that the primary thrust face was in contact with 
the housing. The spindle was pressurized and the displacement back to equilibrium was measured to be about 0.0007 inches. This is consistent with the equilibrium position of 0.00074 inches predicted by the spreadsheets (note that the two faces of the thrust bearing have unequal areas and so at no-load conditions the spindle rests about $15 \%$ off-center).

Once the clearances of the thrust bearing had been measured, the thrust tests were conducted by loading the front face of the spindle axially using a hydraulic cylinder and measuring the applied force with transducers placed between the cylinder and the shaft. The displacement was measured with two Starrett indicators; the first indicator had a resolution of 0.00001 inches and the second one 0.0001 inches. The indicator used for higher applied forces had a lower accuracy because the required travel for these tests was higher. The measured forces and displacements of the thrust test are tabulated in Table 7.2 and plotted in Figure 7.11. The shaft was found to bottom out at an applied load of approximately 1,100 pounds, which is about $10 \%$ less than the touch-down load capacity of 1,200 pounds predicted by the spreadsheets. Touchdown was determined by turning the rotor by hand at each applied load to feel for friction between the thrust face and the housing. Although the touch-down load capacity of the thrust bearing was close to expected, the overall deflection of the shaft at this load was about twice that expected. As shown in Figure 7.11, the displacement of the shaft from equilibrium was measured to be about 0.0011 inches at touch-down. This displacement is greater than the 0.0007 inches of measured clearance in this direction, indicating that there is excessive housing compliance that is causing the stiffness to be lower than expected. The stiffness of the thrust bearing with the current housing design is about $1,000,000 \mathrm{lb} / \mathrm{in}$. This stiffness 
could be doubled by designing a stiffer housing member to hold the shaft under thrust loads. However, this may not be necessary to meet the intended goals of the project.

Table 7.2.

\begin{tabular}{|c|c|c|}
\hline $\begin{array}{c}\text { Force } \\
\text { (pounds) }\end{array}$ & $\begin{array}{c}\text { Deflection } \\
\text { (inches) }\end{array}$ & $\begin{array}{c}\text { Indicator } \\
\text { Resolution } \\
\text { (inches) }\end{array}$ \\
\hline-75 & -0.00016 & 0.00001 \\
\hline-50 & -0.00011 & 0.00001 \\
\hline-25 & -0.00005 & 0.00001 \\
\hline 25 & 0.000025 & 0.00001 \\
\hline 50 & 0.00005 & 0.00001 \\
\hline 449.6 & 0.0005 & 0.0001 \\
\hline 562 & 0.0006 & 0.0001 \\
\hline 674.4 & 0.0008 & 0.0001 \\
\hline 899.2 & 0.0009 & 0.0001 \\
\hline 1011.6 & 0.00097 & 0.0001 \\
\hline 1079 & 0.0011 & 0.0001 \\
\hline
\end{tabular}

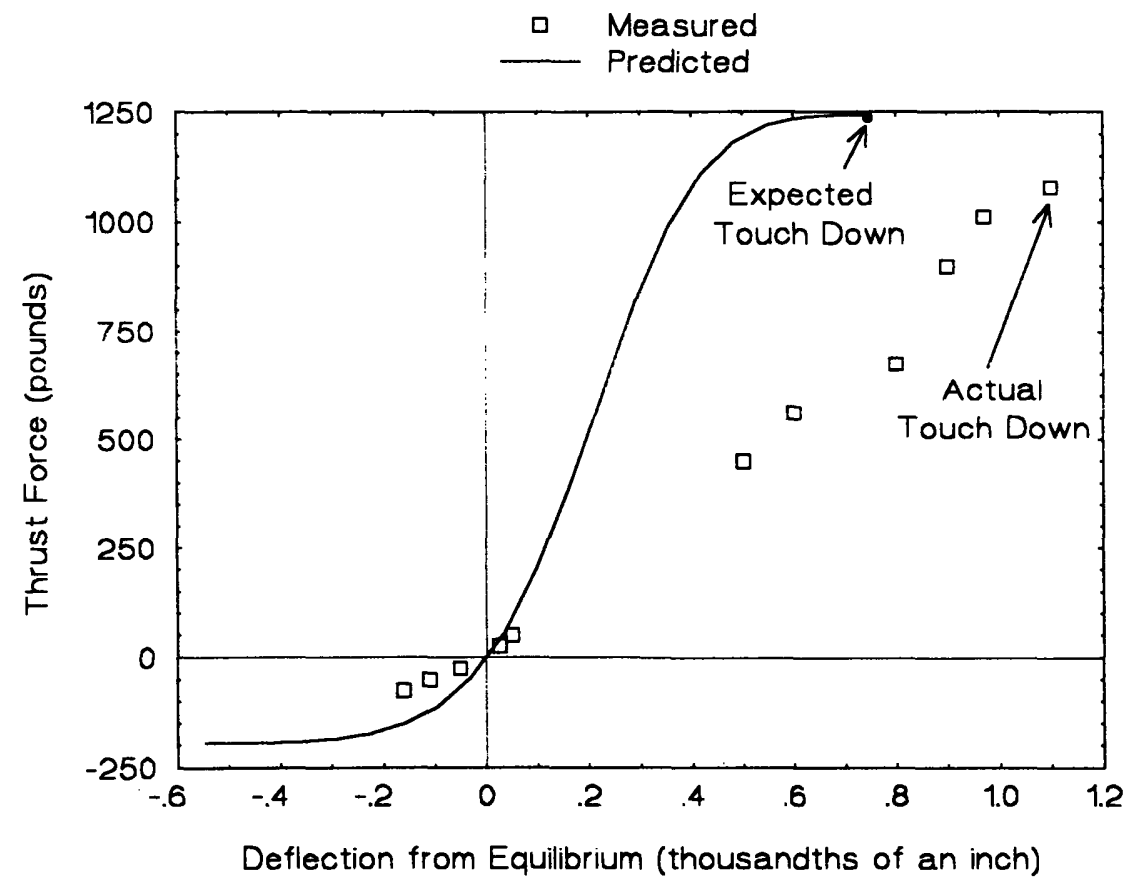

Figure 7.11. Measured and predicted load performances of the thrust bearing. The disagreement is thought to be the result of compliance in the spindle housing. 


\section{A.iv. Description of the Thrust Bearing "Failure"}

A goal of the test was to drive the prototype spindle to a speed of $20,000 \mathrm{rpm}$ while measuring the torque required to turn the shaft and the temperature rise of the coolant. Although a speed of $18,000 \mathrm{rpm}$ was successfully reached, the spindle seized when the shaft speed was increased from $18,000 \mathrm{rpm}$ to $19,000 \mathrm{rpm}$. Upon removing the shaft from the housing, there were pick-ups on the secondary face of the thrust bearing and on the front-most leakage land of the front radial bearing. There was no pick-up on the primary face of the thrust bearing. From this evidence it was concluded that centrifugal forces on the fluid in the primary thrust face recess caused the shaft to move forward to such an extent that the secondary thrust face touched down on the housing and caused the seizure. Although the thrust design used in this application has an upper speed limitation, it is clearly adequate for the $10,000 \mathrm{rpm}$ maximum spindle speed for which it was designed.

To understand the speed limitation of the thrust bearing, a brief description of the thrust bearing will be given here. The thrust bearing of the prototype spindle is illustrated in Figure 7.12. It is of the type Thrust Bearing \#3 illustrated in Chapter 5. Fluid at supply pressure is routed to the supply channel via a hole drilled through the housing. This hole is not shown in Figure 7.12. Upon entering the supply channel, it crosses the secondary thrust land which is at a tight axial clearance (about 0.0006 ") from the housing. Upon exiting the secondary thrust land, the fluid is at a pressure of about half the supply pressure. It then flows through the feed clearance to the thrust recess which is machined into the primary face of the thrust bearing. The fluid flows radially outward through the 
thrust recess to the primary thrust land which is at a tight axial clearance (about 0.0007 ") from the housing. The fluid crosses the primary thrust land and exits to the drainage channel, which is at a pressure of approximately atmospheric pressure. The fluid is routed out of the drainage channel through a hole that is not shown in Figure 7.12.

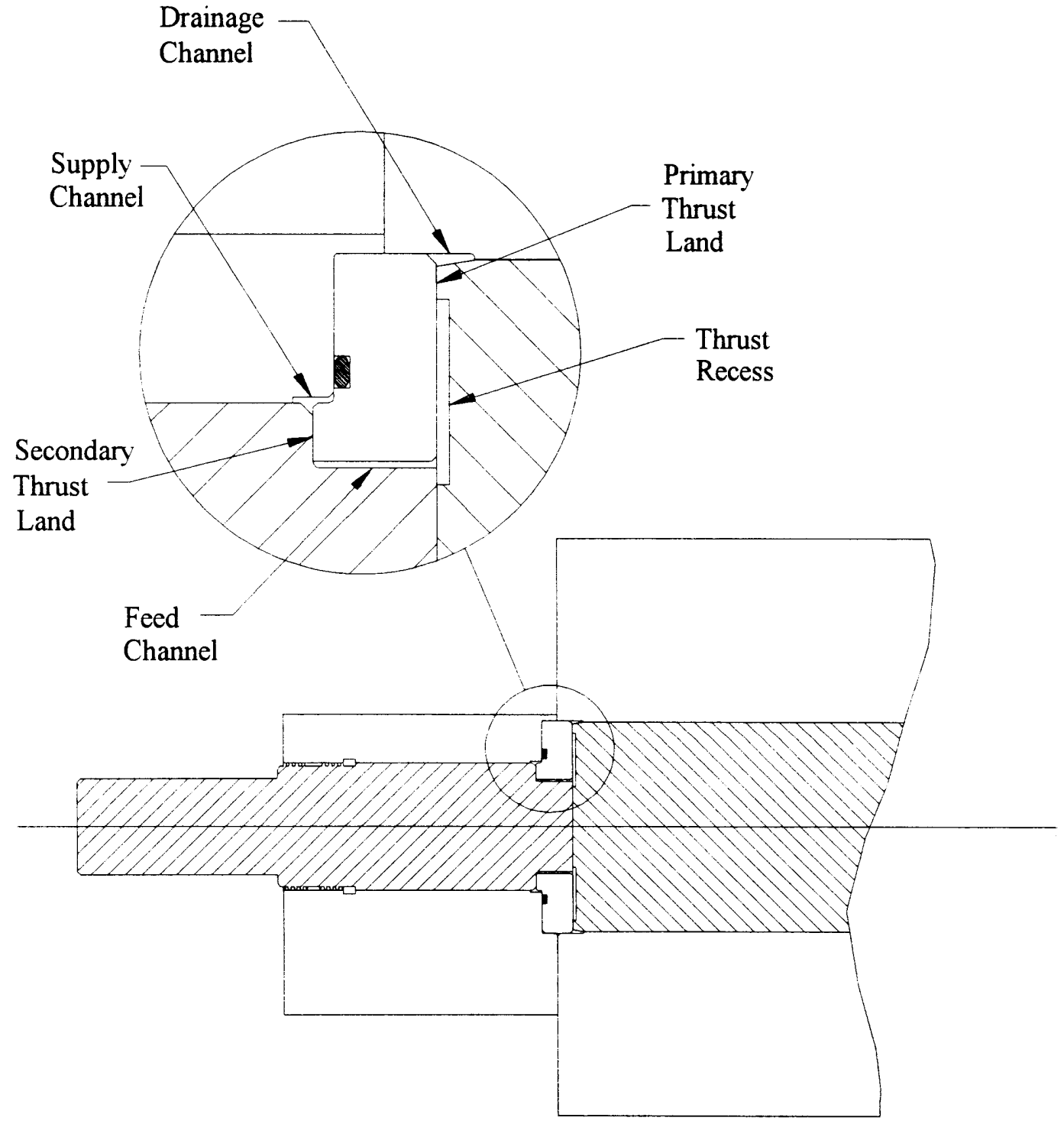

Figure 7.12. Illustration of the thrust bearing used in the prototype spindle. 
At low speeds, the two faces of the thrust bearing work together to keep the spindle centered axially such that the clearance of the primary thrust land is approximately equal to the clearance of the secondary thrust land. As the shaft is turned, centrifugal forces acting on the fluid in the primary thrust recess cause an increase in the average recess pressure. Centrifugal forces also act on the fluid in the land clearances, but to a lesser extent. There is therefore a net force imbalance resulting from the increase in primary thrust recess pressure that causes the shaft to move forward until a new bearing equilibrium is reached. As the speed is increased, the shaft moves further and further forward axially. At some critical speed, the shaft has moved forward 0.0006 " and the secondary thrust land touches down on the housing. This critical speed is the maximum speed at which the bearing can operate. For the Phase I fluid film test spindle, this speed was somewhere between $18,000 \mathrm{rpm}$ and $19,000 \mathrm{rpm}$. This speed capability is certainly adequate for the cluster spindle project, since the maximum speed with which the spindles will operate is $3,000 \mathrm{rpm}$.

\section{A.v. Flow Rate}

The flow rate of water through the spindle was found to depend only on the pressure difference across it and the temperature of the bearing lands, not on the shaft rotation speed. This important result was expected but verification was needed from the spindle tests.

The flow rate of water through the spindle was measured first with the drain lines leading from the pocket isolation channels closed. Three Omega rotameter water flow meters with accuracies of $0.07 \mathrm{gpm}$ were used to measure the flow rates through the three 
supply lines. The first one led to the front radial bearing, the second one led to the rear radial bearing, and the third one led to both the thrust bearing and the belt-supporting radial bearing. The three supply flow rates were each found to increase proportionately with the pressure difference across the spindle, the supply pressure minus the back pressure. As the shaft speed was increased to low speeds (less than about 5,000 rpm), the flow rate remained constant. As the shaft speed was increased further to $10,000 \mathrm{rpm}$, the flow rate was found to increase by about $5 \%$. However, this increased flow rate was found to be the result of the increased temperature of the bearing lands from $24^{\circ} \mathrm{C}$ to about $28^{\circ} \mathrm{C}$ and resulting decrease in the viscosity of the water flowing over them. This was verified by stopping the spindle from $10,000 \mathrm{rpm}$ and noting that the flow rate through the spindle remained constant until the shaft was cooled back to its original temperature where the flow rate was lower. The flow rates measured at $600 \mathrm{psi}$ supply pressure and no back pressure are shown in Table 7.3. A separate test performed indicates that approximately $0.2 \mathrm{gpm}$ of the flow rate to the rearest supply line flows to the rear pulley bearing, and the rest flows to the thrust bearing.

Table 7.3

\begin{tabular}{|c|c|c|c|c|c|c|}
\hline $\begin{array}{c}\text { Time } \\
(\text { min.) }\end{array}$ & $\begin{array}{c}\text { Speed } \\
(\mathbf{r p m})\end{array}$ & $\begin{array}{c}\text { Inlet } \\
\text { Temp. }\end{array}$ & $\begin{array}{c}\text { Drainage } \\
\text { Temp. }\end{array}$ & $\begin{array}{c}\text { Front } \\
\text { Radial } \\
\text { Bearing }\end{array}$ & $\begin{array}{c}\text { Rear } \\
\text { Radial } \\
\text { Bearing }\end{array}$ & $\begin{array}{c}\text { Thrust Bearing and } \\
\text { Belt-Supporting } \\
\text { Radial Bearing }\end{array}$ \\
\hline 0 & 0 & $23^{\circ} \mathrm{C}$ & $24^{\circ} \mathrm{C}$ & $1.40 \mathrm{gpm}$ & $1.38 \mathrm{gpm}$ & $0.98 \mathrm{gpm}$ \\
\hline 30 & 10,000 & $23^{\circ} \mathrm{C}$ & $28^{\circ} \mathrm{C}$ & $1.47 \mathrm{gpm}$ & $1.43 \mathrm{gpm}$ & $1.05 \mathrm{gpm}$ \\
\hline 30 & 0 & $23^{\circ} \mathrm{C}$ & $28^{\circ} \mathrm{C}$ & $1.47 \mathrm{gpm}$ & $1.43 \mathrm{gpm}$ & $1.05 \mathrm{gpm}$ \\
\hline 60 & 0 & $23^{\circ} \mathrm{C}$ & $24^{\circ} \mathrm{C}$ & $1.40 \mathrm{gpm}$ & $1.38 \mathrm{gpm}$ & $0.98 \mathrm{gpm}$ \\
\hline
\end{tabular}


The flow rate measurements were repeated with the drain lines leading from the pocket isolation channels open. The flow rates to the front and rear radial bearings were each found to increase by about $0.55 \mathrm{gpm}$. The total flow rate therefore increased from $3.7 \mathrm{gpm}$ to $4.8 \mathrm{gpm}$, an increase of about $30 \%$. As discussed in a previous section, the pocket isolation channels effectively act to eliminate leakage from the fluid supply regions of the bearing to the bearing pockets. With the drains from the isolation channels open, the stiffness of the bearings are increased although it is paid for with a higher flow rate (and therefore a higher required pumping power).

The flow rates predicted by the spreadsheets were found to agree with the measured flow rates only if the clearances between the shaft and housing were chosen to do so. A clearance of $15.5 \mu \mathrm{m}(0.000610 ")$ was used on the front bearing and a clearance of $15.4 \mu \mathrm{m}(0.000606 ")$ was used on the rear bearing. The clearance used on the rear pulley bearing was $14 \mu \mathrm{m}\left(0.00055^{\prime \prime}\right)$. The actual bearing clearances were measured by three different independent companies: Setco/Whitnon, Edmunds Gage, and Setco/Cincinatti. The shaft and housing diameters were measured in four places, as shown in Figure 7.13. The measured diameters are tabulated in Table 7.4. As shown, the measured clearances varied between the three sets of measurements. Since the flow rate varies with the cube of the bearing clearance, the measured flow rate probably yields the most accurate indication of the actual bearing clearance. 
(a)

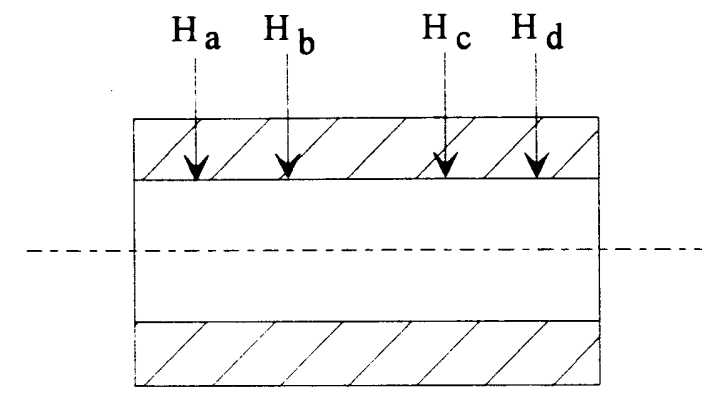

(b)

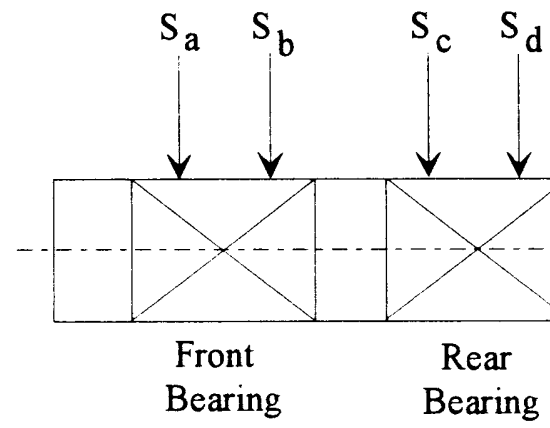

Figure 7.13. Schematic diagram illustrating the locations on the (a) housing and (b) shaft where the diameters were measured.

Table 7.4.

\begin{tabular}{|c|c|c|c|}
\hline Measured By & $\begin{array}{c}\text { Housing Diameter } \\
\text { (inches) }\end{array}$ & $\begin{array}{c}\text { Shaft Diameter } \\
\text { (inches) }\end{array}$ & $\begin{array}{c}\text { Clearance per Side } \\
\text { (inches) }\end{array}$ \\
\hline \multirow{4}{*}{ Setco/Whitnon } & $\mathrm{H}_{\mathrm{a}}=2.2055$ & $\mathrm{~S}_{\mathrm{a}}=2.2046$ & 0.00045 \\
\hline & $\mathrm{H}_{\mathrm{b}}=2.2055$ & $S_{b}=2.2046$ & 0.00045 \\
\hline & $\mathrm{H}_{\mathrm{c}}=2.2055$ & $S_{c}=2.2046$ & 0.00045 \\
\hline & $\mathrm{H}_{\mathrm{d}}=2.2055$ & $S_{d}=2.2046$ & 0.00045 \\
\hline \multirow{4}{*}{ Edmunds Gage } & $\mathrm{H}_{\mathrm{a}}=2.20559$ & $S_{a}=2.20446$ & 0.00056 \\
\hline & $\mathrm{H}_{\mathrm{b}}=2.20555$ & $\mathrm{~S}_{\mathrm{b}}=2.20448$ & 0.00053 \\
\hline & $\mathrm{H}_{\mathrm{c}}=2.20564$ & $S_{c}=2.20452$ & 0.00056 \\
\hline & $\mathrm{H}_{\mathrm{d}}=2.20555$ & $S_{d}=2.20453$ & 0.00051 \\
\hline \multirow{4}{*}{ Setco/Cincinatti } & $\mathrm{H}_{\mathrm{a}}=2.2057$ & $S_{a}=2.2045$ & 0.0006 \\
\hline & $\mathrm{H}_{\mathrm{b}}=2.2059$ & $S_{b}=2.2045$ & 0.0007 \\
\hline & $\mathrm{H}_{\mathrm{c}}=2.2056$ & $S_{c}=2.2045$ & 0.00055 \\
\hline & $\mathrm{H}_{\mathrm{d}}=2.2057$ & $S_{d}=2.2045$ & 0.0006 \\
\hline
\end{tabular}


Flow rates were also tested to determine the effect of feeding the fluid radially or tangentially at a $45^{\circ}$ angle to the pressure grooves on the bearing surface. The flow rate tests performed with the fluid fed to the bearing at a $45^{\circ}$ angle were repeated with the supply fluid fed perpendicular to the shaft. With the shaft unrotating, the measured flow rates were found to be equal to within the accuracy of the flow meters for the two configurations. The spindle was rotated up to $10,000 \mathrm{rpm}$ and again there was no noticable difference in the measured flow rates, the measured torque, or the measured temperature rise between the two configurations. It is therefore concluded that it is not necessary to feed the fluid into the bearing at an angle for this spindle. The reason why this technique is not important in this case is because the dynamic head of the fluid caused by the shaft rotating at $10,000 \mathrm{rpm}$ is about one-tenth of the supply pressure of $600 \mathrm{psi}$. In higher speed bearings where the dynamic head of the rotating fluid becomes a significant portion of the supply pressure, there is a chance that the supply flow rate to the bearing may be reduced somewhat. This is not the case in this application and the fluid may simply be fed radially to the bearing, perpendicular to the surface of the shaft.

The flow rate of coolant through the spindle was next measured at speeds up to $18,000 \mathrm{rpm}$. The flow rates measured using 600 psig supply pressure are shown in Figure 7.14. As the spindle speed was increased, the flow rate through each bearing also increased. The increased flow rate is the result of the increased temperature of the bearing lands and resulting decrease in the viscosity of the coolant flowing over them. 


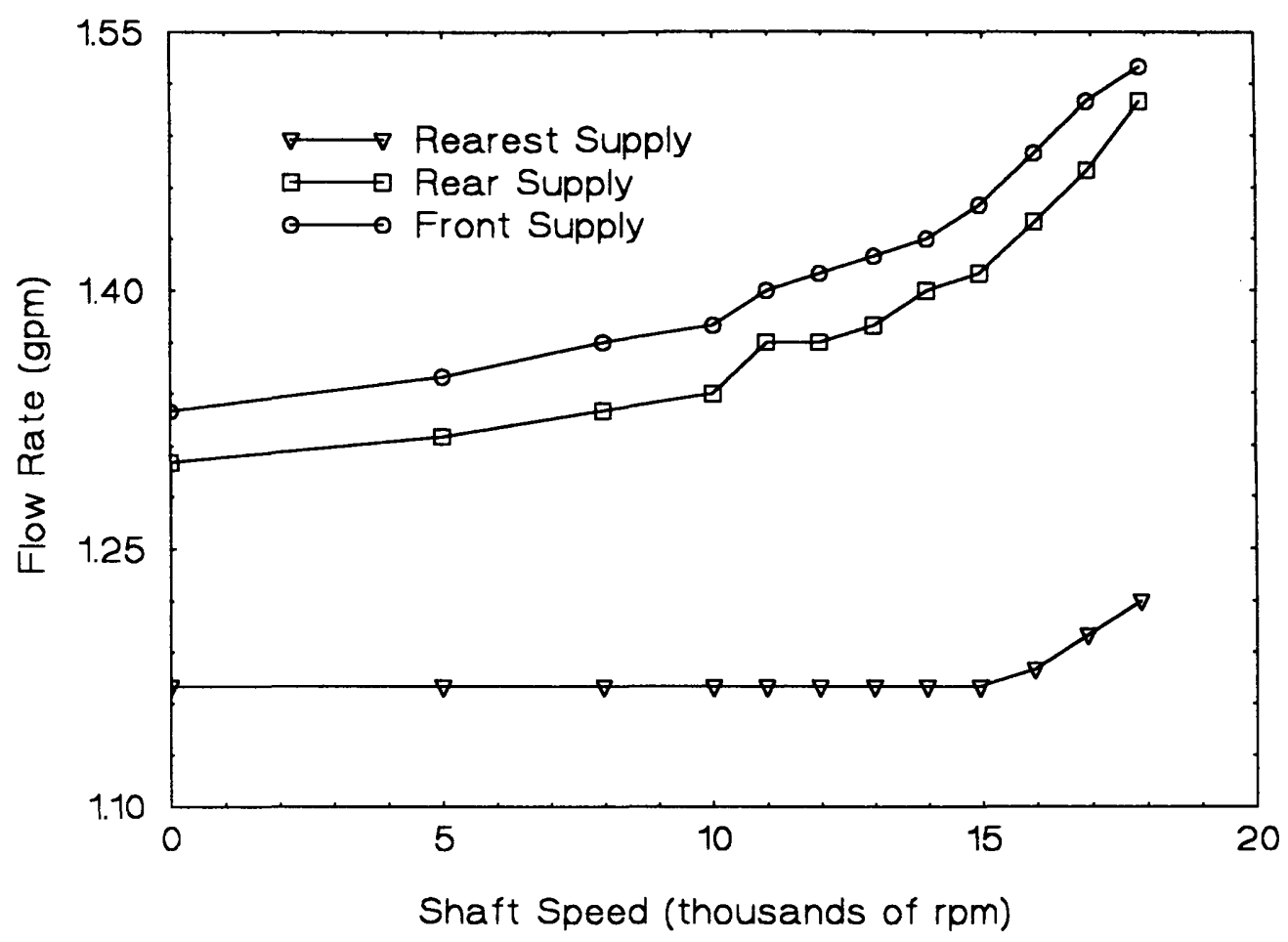

Figure 7.14. Measured flow rates of coolant supplied to the three supply ports of the spindle as a function of shaft speed, using a fixed supply pressure of 600 psig.

\section{A.vi. Coolant Supply Flow}

A method was provided in the prototype spindle to supply a flow of coolant to the center of the shaft to facilitate the use of a through-the-tool coolant system. As shown in Figure 7.15, the fluid is first routed to a fluid collection channel located on the outer edge of the shaft. Axial leakage lands with tight clearances are provided on both sides of the fluid collection channel to prevent excessive leakage flow. Although a small flow of the coolant leaks off over these lands, the rest of it flows through holes drilled radially inward to the center of the shaft. A hole drilled axially through the center of the shaft is used to route the coolant to the front of the spindle where it can be used to cool the tool. This 
method is an excellent way in which to route fluid to the tool without making contact with the rotating shaft.

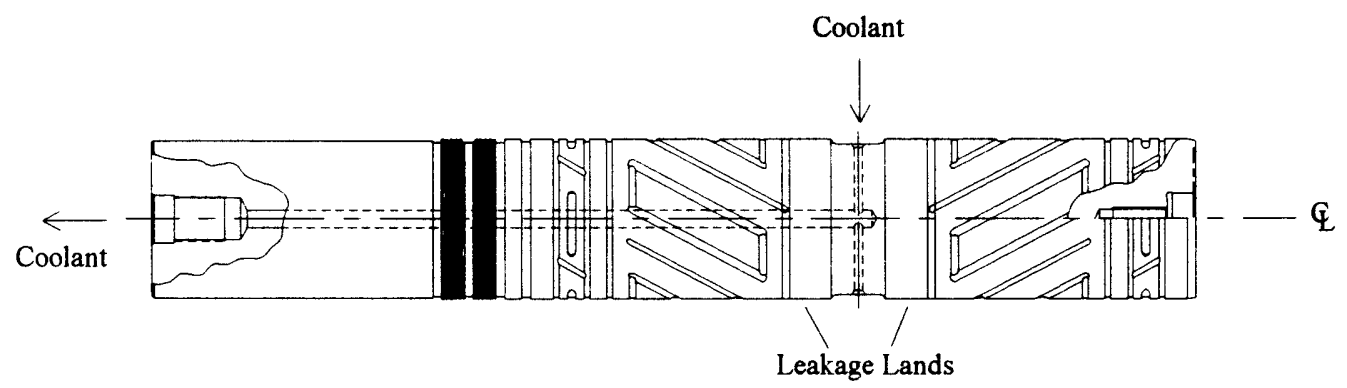

Figure 7.15. Drawing of the rotor illustrating the scheme used to route coolant to the center of the shaft so that it may be used for through-the-tool cooling.

Measurements were made to determine the added pressure required to overcome the centripetal forces exerted outward on the fluid caused by shaft rotation. This pressure can be calculated by setting the outward pressure gradient in the radial holes equal to the body force exerted on the fluid:

$$
\frac{d p}{d r}=\rho \omega^{2} r
$$

where $p$ is the pressure, $\rho$ is the fluid density, $\omega$ is the shaft rotational speed, and $r$ is the radial coordinate. Integrating with respect to $r$, the pressure difference from the outer edge to the shaft center is given by:

$$
p_{\text {outer }}-p_{\text {inner }}=\frac{1}{2} \rho \omega^{2}\left(R_{\text {outer }}^{2}-R_{\text {inner }}^{2}\right)
$$

where $R_{\text {outer }}$ is the outer radius where the fluid enters the radial holes and $R_{\text {inner }}$ is the inner radius where the fluid exits the radial holes. 
The pressure required to pump coolant to the center of the shaft was measured over a shaft speed range from 0 to $10,000 \mathrm{rpm}$. At $0 \mathrm{rpm}$, the supply pressure required to pump the fluid at $1.5 \mathrm{gpm}$ was $7.6 \mathrm{psi}$. This pressure drop was the frictional component required to pump the coolant through the radial holes, through the central supply hole, and through the hose back to the coolant supply tank. As the shaft speed was increased, the supply pressure required to pump the coolant increased as shown in Figure 7.16. Also shown in Figure 7.16 is the predicted supply pressure required to pump the coolant at 1.5 gpm. Note that $7.6 \mathrm{psi}$ was added to account for the friction pressure drop.

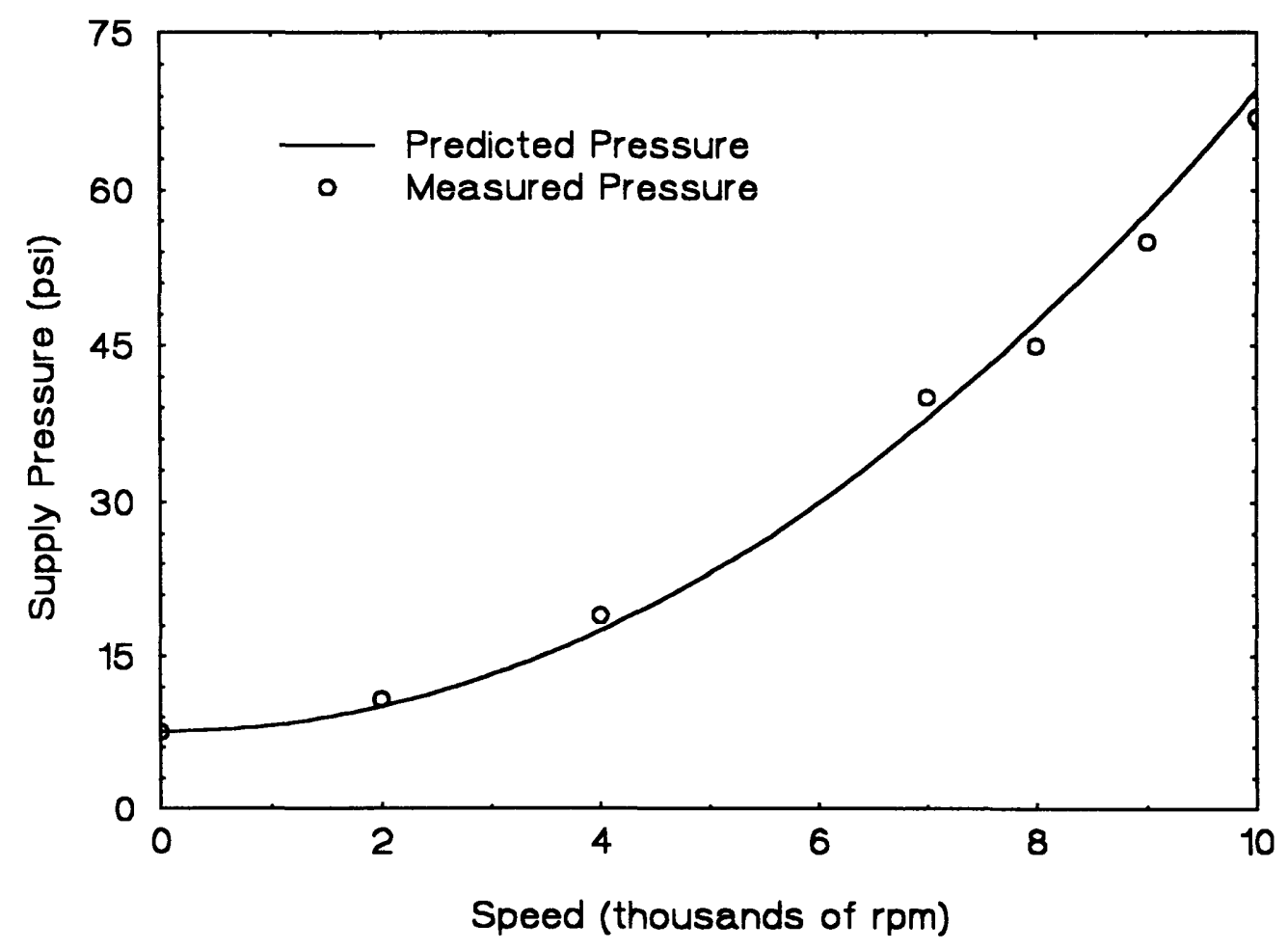

Figure 7.16. Supply pressures required to pump coolant to the center of the shaft, through a hose, and back to the coolant supply tank at $1.5 \mathrm{gpm}$. 
In addition to the primary coolant supply flow, the flow of coolant across the leakage lands which surround the fluid collection channel was also evaluated. The flow rate of coolant to the outer collection groove and that from the central rotor coolant channel were simultaneously measured and were found to be equal to within the accuracy of the flow meters $( \pm 0.07 \mathrm{gpm})$ at all shaft speeds. This is consistent with the $0.043 \mathrm{gpm}$ of leakage flow predicted by the spreadsheets for a coolant supply pressure of $70 \mathrm{psi}$.

\section{A.vii. Torque and Shearing Power}

The torque required to drive the spindle was measured using a dynamic torque sensor connected in-line between the drive motor and the spindle. The torque meter used had a measurable torque range of 0 to $500 \mathrm{in}-\mathrm{lb}$. Although the torque expected to turn the spindle was less than $20 \mathrm{in}-\mathrm{lb}$, the $500 \mathrm{in}-\mathrm{lb}$ meter was used because it was the only torque sensor available at the time of the testing. The measured torque required to turn the spindle up to $10,000 \mathrm{rpm}$ is shown in Figure 7.17. The torque shown in this plot is the measured torque minus the hysteresis of the torque sensor, which was found to be about 5 in-lb. This value was obtained by adjusting the curve of Figure 7.17 until the torque limited to a value of zero at zero $\mathrm{rpm}$. The torque predicted by the spreadsheets for a speed of $10,000 \mathrm{rpm}$ is about $19.0 \mathrm{in}-\mathrm{lb}$, which is about $25 \%$ higher than the $15.1 \mathrm{in}-\mathrm{lb}$ measured torque at this speed. However, the accuracy of the torque meter in this range is certainly greater than \pm 5 in-lb ( $1 \%$ of full-scale reading), and so the agreement is adequate. The measured torque data presented here is only ball-park and is not an accurate recording of the spindle torque. 


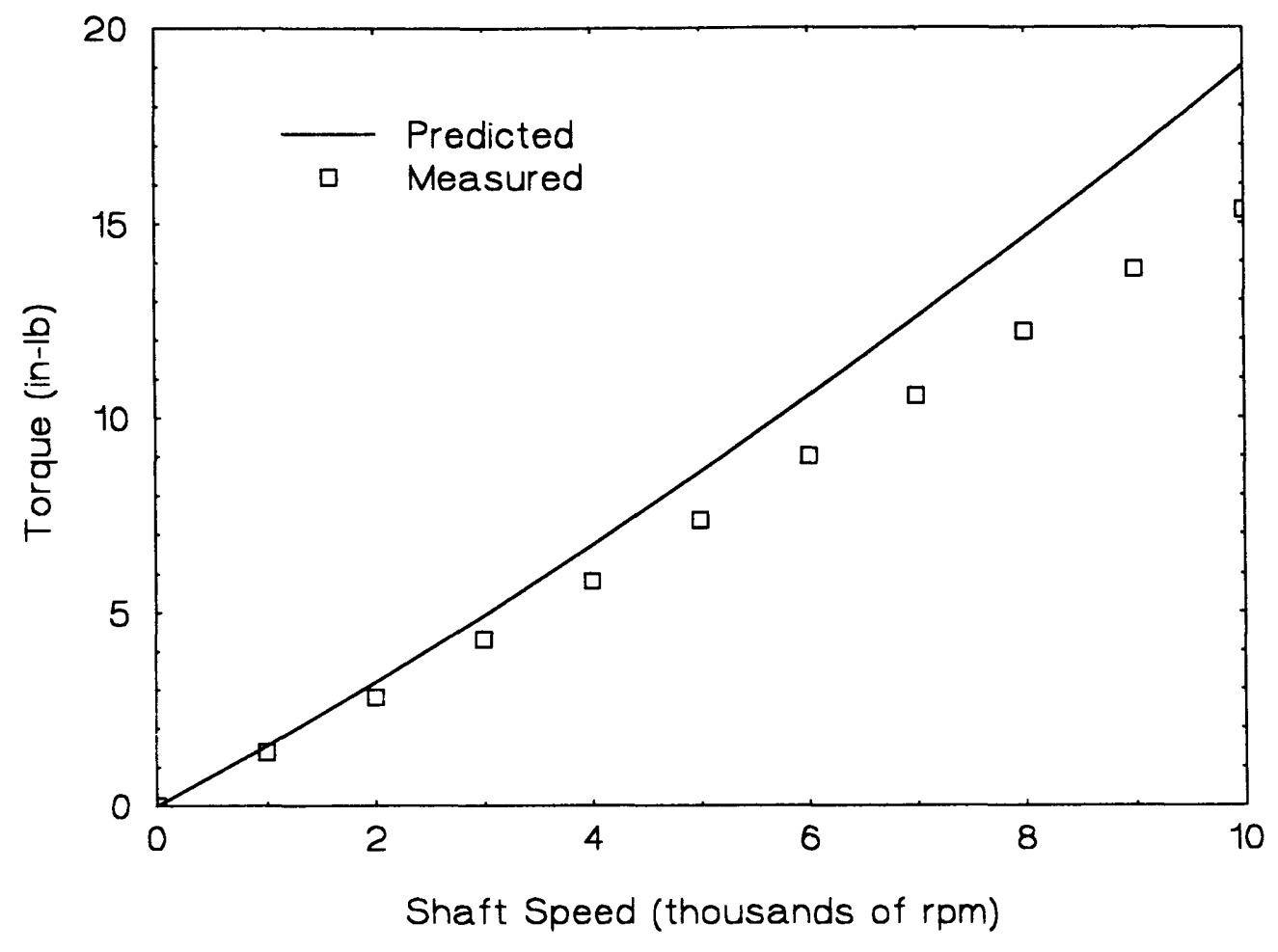

Figure 7.17. Measured torque required to drive the spindle up to $10,000 \mathrm{rpm}$.

The drive power consumed by the spindle is found by multiplying the measured torque of Figure 7.17 by the shaft speed. The measured shearing power curve of Figure 7.18 is again presented only as a ball-park indicator of the bearing power consumption and is not an accurate recording of this data. 


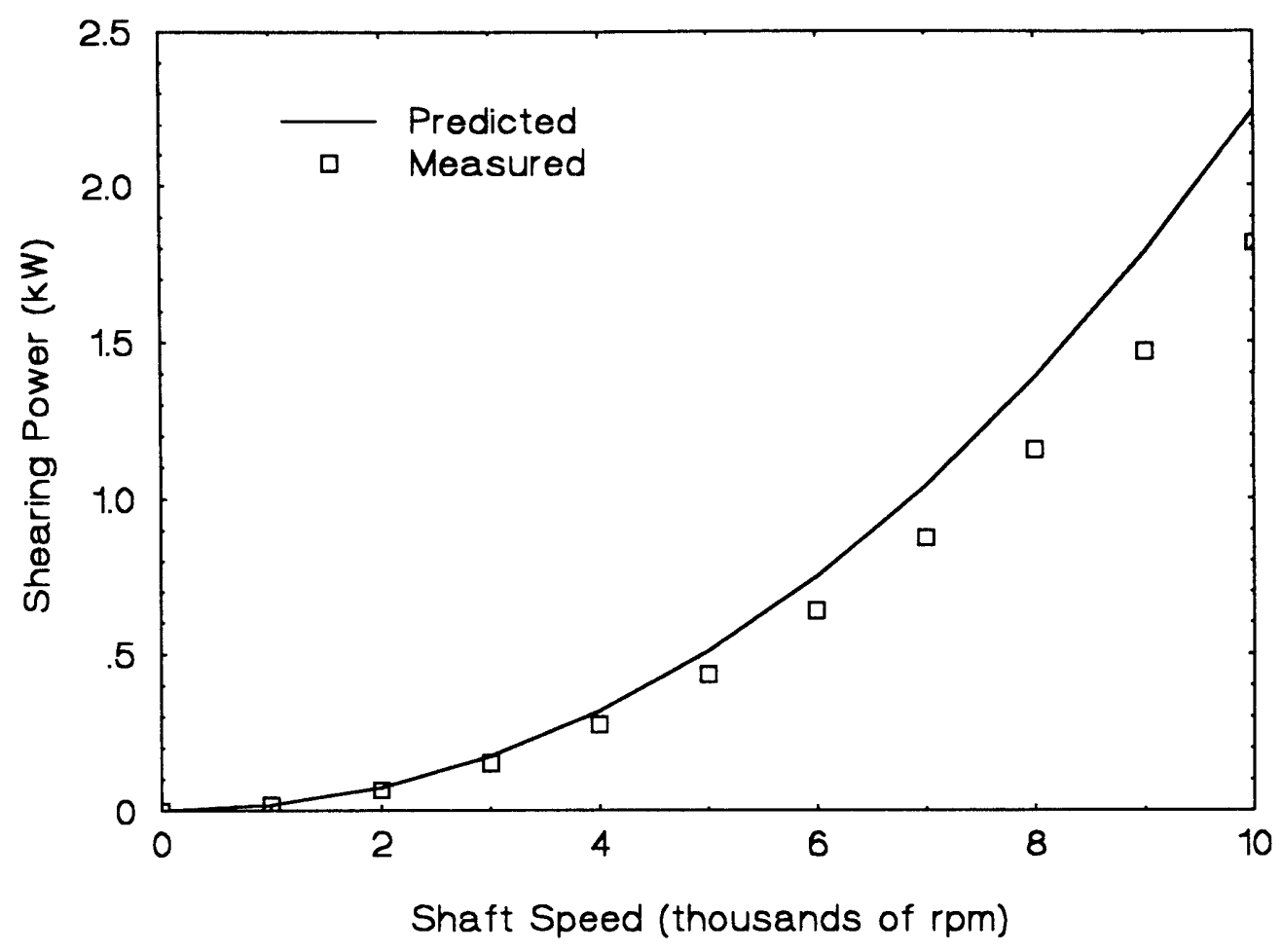

Figure 7.18. Measured shearing speed required to turn the spindle up to $10,000 \mathrm{rpm}$.

The measured torque required to turn the spindle up to $18,000 \mathrm{rpm}$ is shown in Figure 7.19. The torque predicted by the spreadsheets for a speed of $18,000 \mathrm{rpm}$ is about 38.4 in-lb, which is about $35 \%$ higher than the 28.2 in-lb measured torque at this speed. The measured torque data presented here is only ball-park and is not an accurate recording of the spindle torque. 


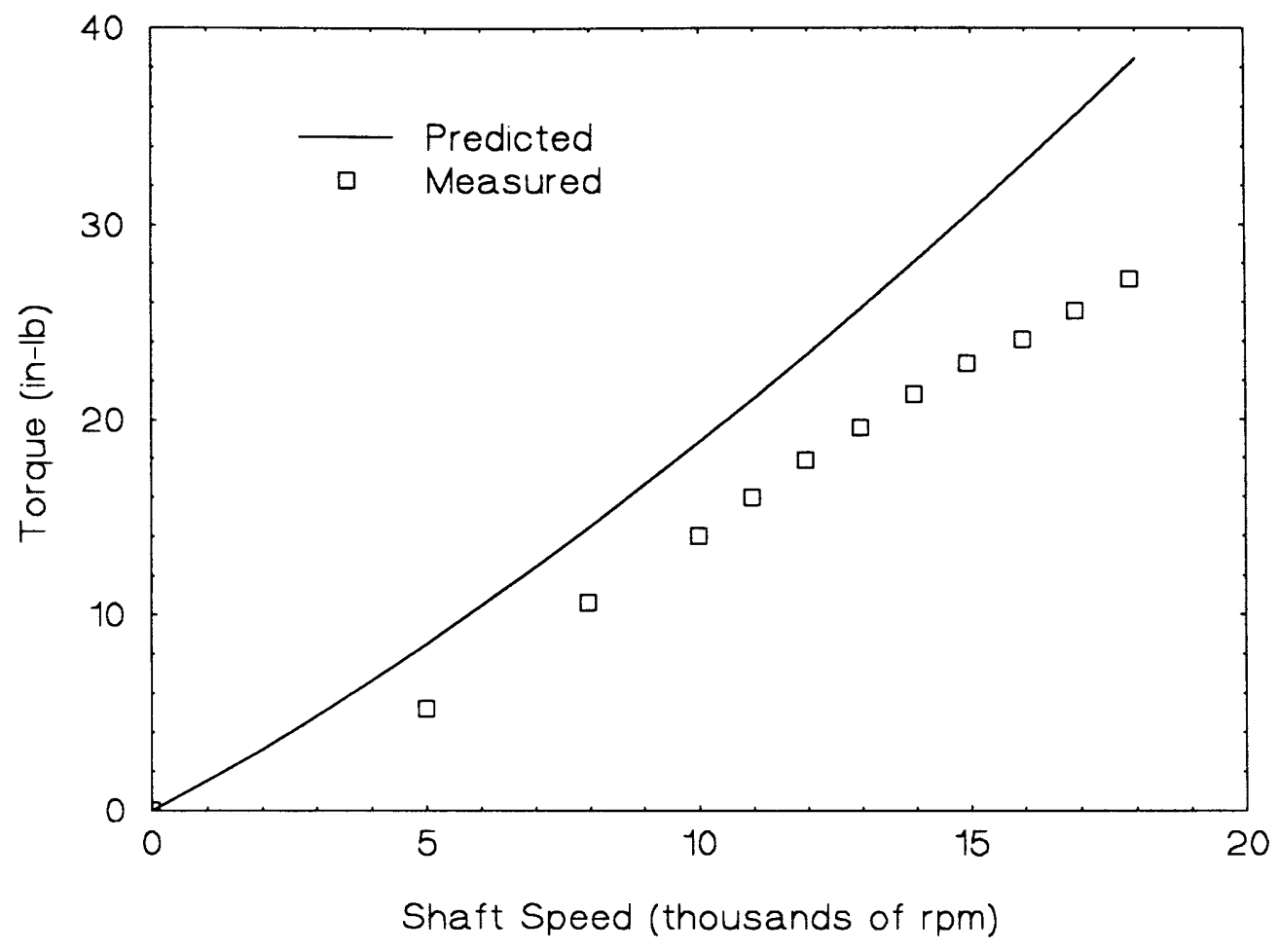

Figure 7.19. Measured torque required to drive the spindle up to $18,000 \mathrm{rpm}$.

The drive power consumed by the spindle is found by multiplying the measured torque of Figure 7.19 by the shaft speed. The measured shearing power curve of Figure 7.20 is again presented only as a ball-park indicator of the bearing power consumption and is not an accurate recording of this data. The predicted shear power is thought to be quite accurate because the temperature rise of the coolant calculated using this power agreed well with the measured temperature rise, as discussed in the next section. 


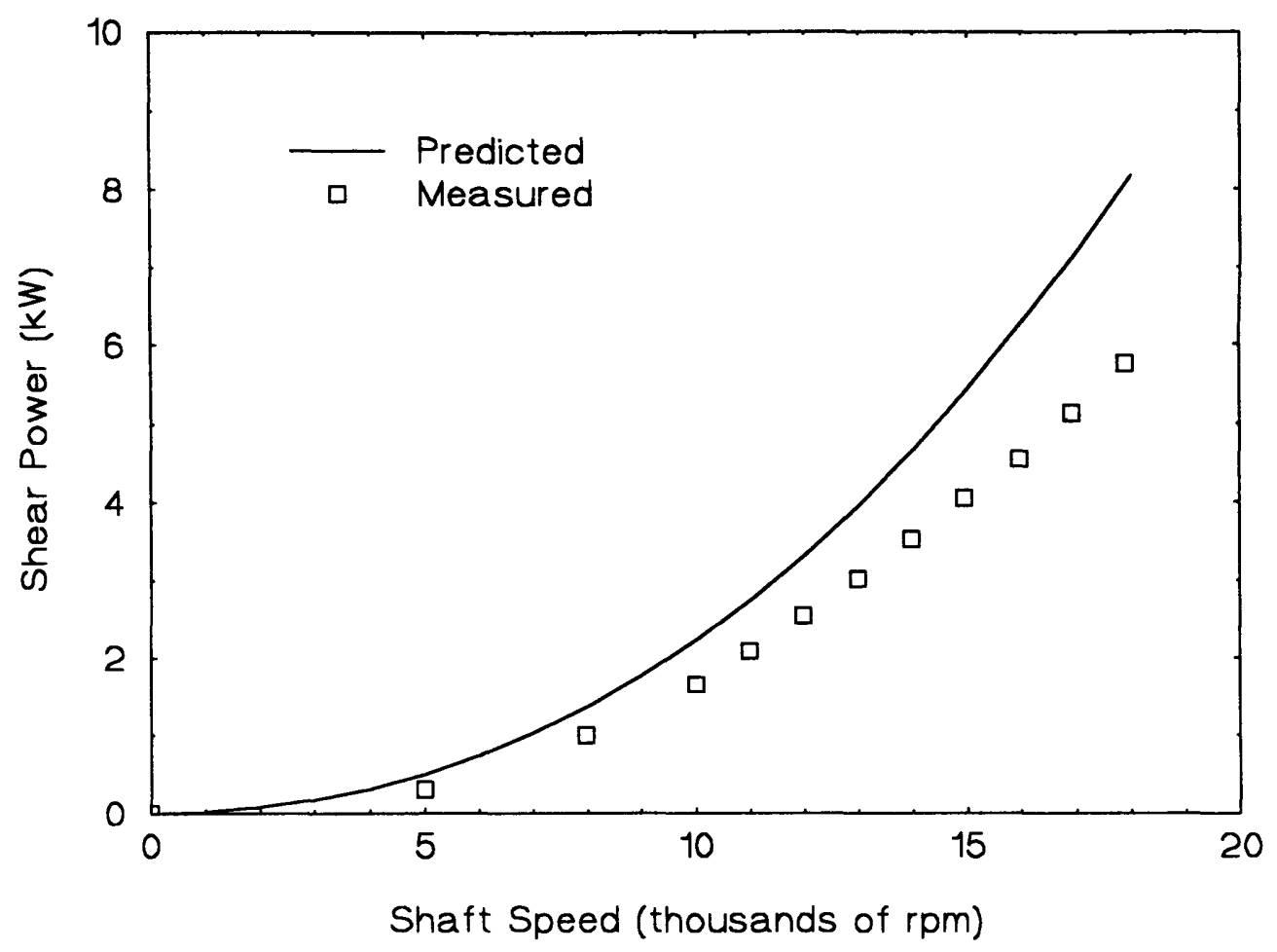

Figure 7.20. Measured shearing power required to turn the spindle up to $18,000 \mathrm{rpm}$.

\section{A.viii. Temperature Rise}

The temperature rise of the spindle was measured using thermistors which have an accuracy of less than $\pm 0.1^{\circ} \mathrm{C}$. A considerable amount of electrical noise was added to the system by the drive motor, however, and filtering of the data was needed after the testing. The thermistors used to measure the supply fluid and the drain fluids were positioned such that they were in direct contact with the fluid to obtain the most accurate reading. An additional thermistor was positioned down into a hole drilled radially through the housing to within about $1 / 4$ " of the bearing surface. The hole was positioned axially such that it was in the center of the spindle housing, mid-way between the two radial bearings. Figure 7.21 shows the measured temperatures of the supply fluid, the drain fluids, and the 
housing as the spindle speed was increased from 0 to $9,000 \mathrm{rpm}$. A periodic cycle of the temperature is visible because the period of the chiller was about 5 minutes. Note that although the system was not quite at equilibrium during the 12 minute test period, the temperature differences (the temperatures minus the supply temperature) remained constant after only a few minutes. This was typical of all of the tests at all of the shaft speeds.

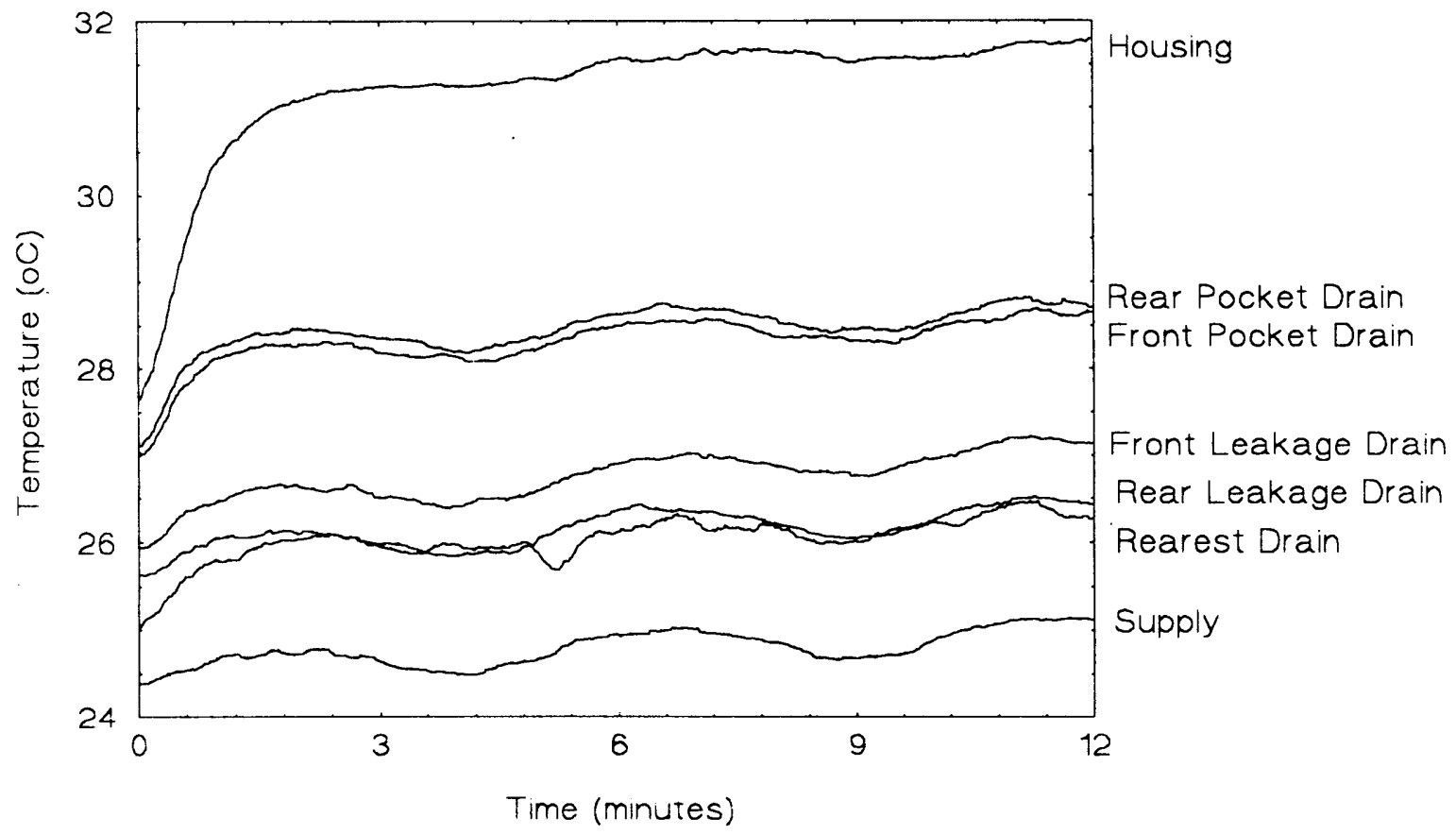

Figure 7.21. Measured temperatures of the spindle supply fluid, housing, and drains as it was warmed up at $9,000 \mathrm{rpm}$.

The temperature differences measured for each shaft speed are plotted in Figure 7.22. At zero shaft speed, the temperature rise of the fluid through the pocket leakage drains is about $0.95^{\circ} \mathrm{C}$. This is very close to the $1{ }^{\circ} \mathrm{C}$ temperature rise that is expected for 
water crossing a resistance with a pressure drop of $600 \mathrm{psi}$. This component of temperature rise remains fixed as the shaft speed is increased. The remainder of the temperature rises of Figure 7.22 are caused by the viscous shearing of the fluid in the bearings due to the rotational motion of the shaft.

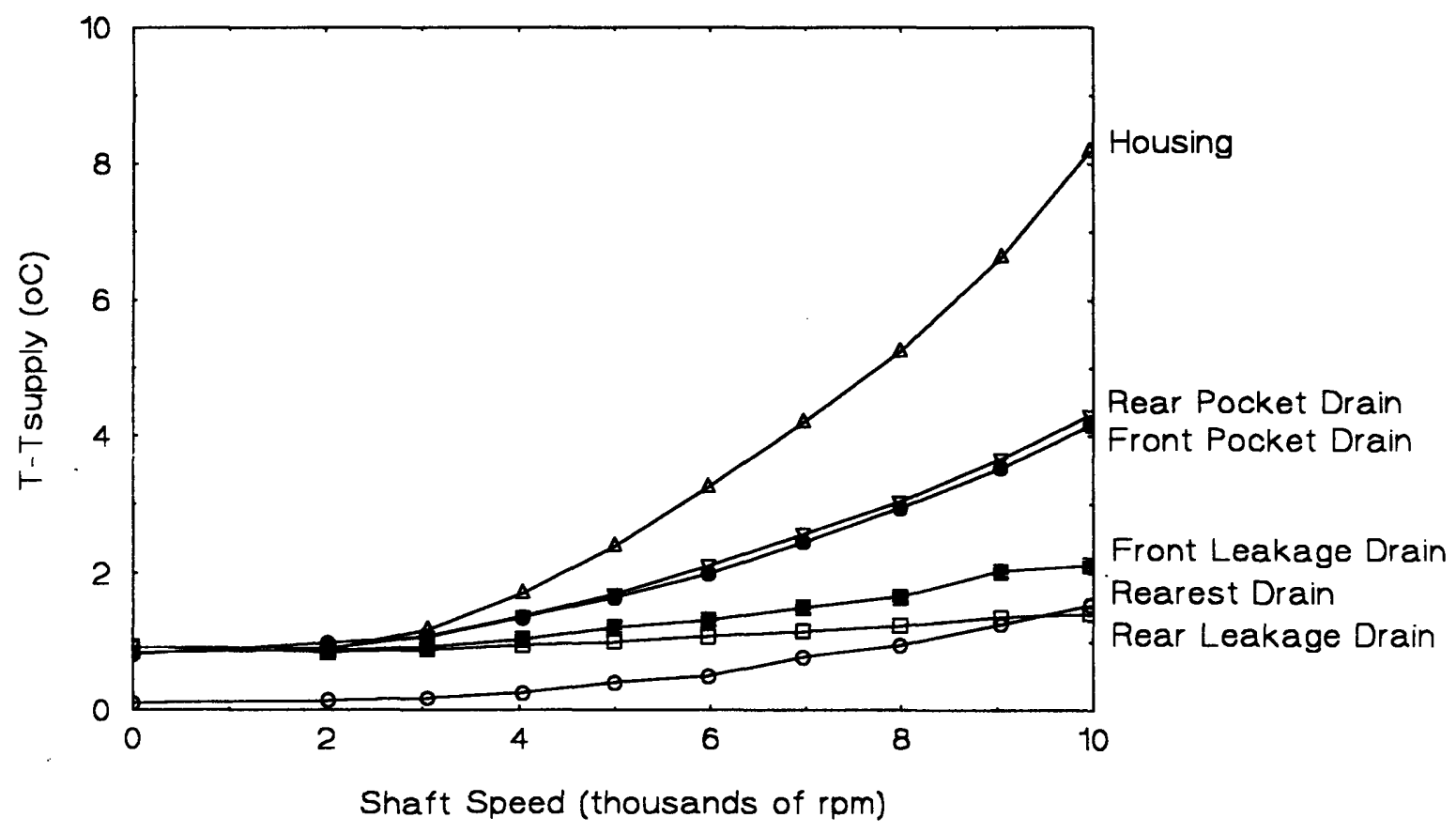

Figure 7.22. Measured temperature differences across the spindle at speeds up to 10,000 rpm.

Note in Figure 7.22 that the the drains from the front and rear radial pockets are nearly the same temperature at all speeds; although the shearing power was nearly the same for the two bearings the rear drain temperature was slightly higher because the flow rate was lower due to the slightly tighter gap on this end of the spindle. Also note that the rear leakage drain is significantly cooler than the front leakage drain. The flow added to 
this drain from the thrust bearing acts to cool this fluid and cause a lower overall temperature rise. The most evident attribute of Figure 7.22 is that the housing temperature was significantly warmer than the fluid leaving the bearing drains. The housing temperature was higher than the other temperatures because there was no fluid being fed to the coolant supply channel near the housing temperature reading during the testing. Under normal operating conditions, coolant will be fed to this channel in order to route it to the center of the shaft. Therefore, under normal operating conditions this central housing temperature will be significantly cooler than the drain temperatures and the rest of the bearing. The $4.2^{\circ} \mathrm{C}$ temperature rise of the fluid flowing through the compensators and pockets of the two main radial bearings therefore represents the maximum temperature rise that will occur in the spindle in its normal mode of operation at a speed of $10,000 \mathrm{rpm}$.

A comparison will now be made between the temperature rises of Figure 7.22 and that predicted by the spreadsheets. The temperature of the fluid leaving the front pocket drain provides a characteristic test of whether the spreadsheets are adequately predicting the heat generated in the bearings. This flow absorbs shearing power generated in the lands and pockets of the compensators as well as the lands and pockets of the radial bearing pads. Figure 7.23 shows a comparison of the predicted and measured temperature rise of this fluid with shaft speeds ranging from 0 to $10,000 \mathrm{rpm}$. The temperature rise at low speeds is simply that generated from pumping the fluid from 600 psi over the lands and out to atmospheric pressure. As the speed is increased, the flow begins to absorb more and more heat generated in the bearings due to the viscous shearing of the fluid 
caused by the shaft rotation. Although the flow over the lands is still laminar at a speed of $10,000 \mathrm{rpm}$, the flow through the pockets and drainage grooves are turbulent with a Reynolds number of about 18,000. As shown, the agreement between the predicted and measured temperature rise is very good.

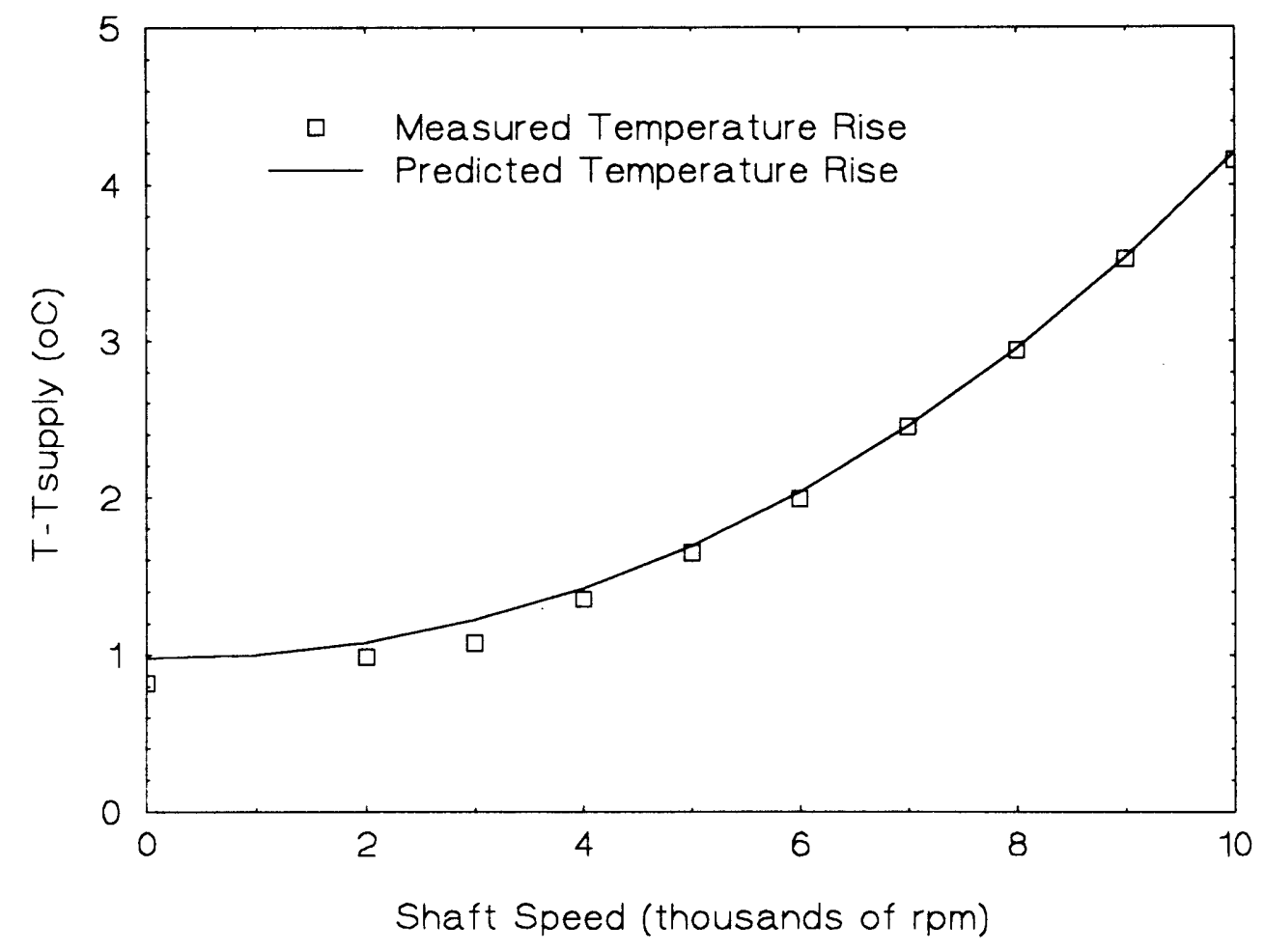

Figure 7.23. Comparison of the measured and predicted temperature rise of the fluid flowing through the compensators and pockets of the front radial bearing.

In a separate test, the primary prototype spindle was taken up to higher speed. The temperature rise of the spindle was measured in this test using thermocouples which have an accuracy of about $\pm 0.5^{\circ} \mathrm{C}$. The thermocouples were again positioned such that they were in direct contact with the fluid to obtain the most accurate reading, one in the 
coolant entering the spindle and one in the coolant leaving the spindle from the rear radial bearing drain port. The difference between these two temperatures is plotted for shaft speeds up to $18,000 \mathrm{rpm}$ in Figure 7.24. At zero shaft speed, the temperature rise of the fluid through the pocket leakage drain is about $0.7^{\circ} \mathrm{C}$. This is very close to the $1^{\circ} \mathrm{C}$ temperature rise that is expected for water crossing a resistance with a pressure drop of $600 \mathrm{psi}$. The maximum temperature rise of the coolant was $12.5^{\circ} \mathrm{C}$ at $18,000 \mathrm{rpm}$. This value is very close to the $12^{\circ} \mathrm{C}$ temperature rise computed using the shear power predicted by the spreadsheets and the measured flow rates of Figure 7.14.

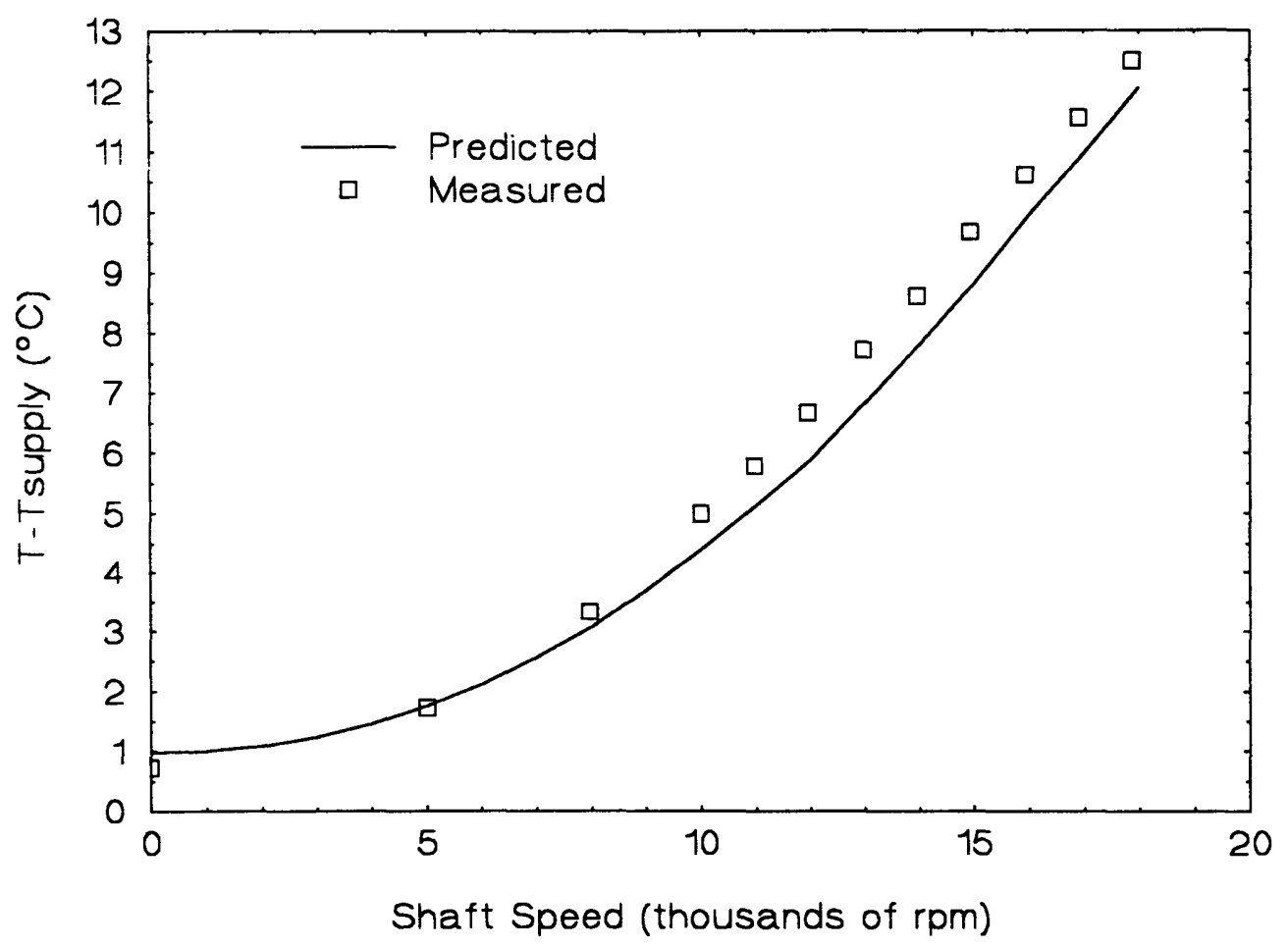

Figure 7.24. Comparison of the measured and predicted temperature rise of the fluid flowing through the compensators and pockets of the rear radial bearing. 


\section{A.ix. Spindle Error Motion}

The error motion of the spindle was measured by a representative of Lyon Inc., a manufacturer of error motion instrumentation. The set-up that was used for this testing is shown in Figure 7.25. Capacitance probes were mounted off of a small steel ball which was mounted to the front nose of the spindle [15]. The ball was displaced from the centerline of the spindle by a radial distance of $50 \mu$ in, enabling the computer to track the rotational speed of the spindle. Error motions were measured first with the spindle drive off (no rotation) and the pump supplying the bearings with a full pressure of 600 psig. The radial and axial displacement were both found to be equal to $30.0 \mu \mathrm{in}$. This contribution to the spindle error motion, which remains approximately constant as the spindle speed is increased, is the result of pressure fluctuations in the bearing supply fluid caused by the noisy axial piston pump used in these tests. With the use of a more quiet pump such as a screw pump, this displacement could be substantially reduced. Accumulators can also be used to reduce the effect of pressure fluctuations on the error motion of the spindle.

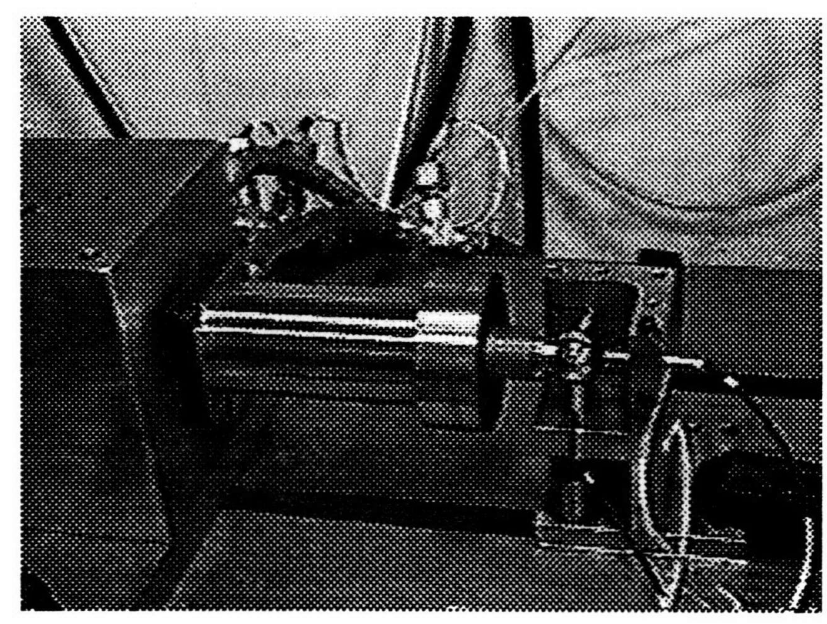

Figure 7.25. Picture of the test set-up used to measure the error motion of the spindle. 
The radial error motion of the spindle was next measured at various spindle rotation rates. The spindle was first driven with a ball bearing drive spindle connected inline to the test spindle with flexible couplings. Table 7.5 shows that a maximum total radial runout of $76.5 \mu$ in was experienced at a rotational speed of $7,000 \mathrm{rpm}$. The total runout was $67.0 \mu$ in at $10,000 \mathrm{rpm}$. A radial runout this low would be extremely difficult to achieve with a comparably sized rolling element spindle. The shaft of a hydrostatic spindle makes no mechanical contact with the housing, and the fluid film tends to average variations in the fluid gap. Any minor perturbations that are induced on the shaft are substantially dampened by squeeze-film effects in the bearings. These qualities make fluid film bearings unparalleled in terms of spindle accuracy. With proper attention to the fluid system and bearing geometry, the error motion could be reduced to less than $10 \mu \mathrm{in}$. Although this level of accuracy is not necessary in this drilling application, it makes hydrostatic spindles extremely attractive for grinding applications.

Table 7.5.

\begin{tabular}{|c|c|c|c|}
\hline $\begin{array}{c}\text { Speed } \\
(\mathbf{r p m})\end{array}$ & $\begin{array}{c}\text { Asynchronous } \\
\text { Runout } \\
(\mu \mathrm{in})\end{array}$ & $\begin{array}{c}\text { Average } \\
\text { Runout } \\
(\mu \mathrm{in})\end{array}$ & $\begin{array}{c}\text { Total } \\
\text { Runout } \\
(\mu \mathrm{in})\end{array}$ \\
\hline 1000 & 18.3 & 14.9 & 29.0 \\
\hline 1010 & 22.9 & 13.7 & 25.6 \\
\hline 1010 & 19.4 & 14.5 & 25.7 \\
\hline 2000 & 22.5 & 17.6 & 31.2 \\
\hline 2000 & 28.2 & 14.1 & 34.4 \\
\hline 5000 & 27.5 & 23.0 & 36.2 \\
\hline 7000 & 61.2 & 47.7 & 76.5 \\
\hline 10000 & 54.0 & 40.6 & 67.0 \\
\hline
\end{tabular}


The spindle was next driven with a belt drive system as shown in Figure 7.26. Two tests were done with different belt tensions. The measured radial runout of the spindle using a belt tension of $50 \mathrm{~N}\left(11.2 \mathrm{lb}_{\mathrm{f}}\right)$ is shown in Table 7.6. A maximum total radial runout of $44.2 \mu$ in was experienced at a rotational speed of $5,000 \mathrm{rpm}$. This is only marginally higher than the $44.2 \mu$ in of run-out measured at this speed with the in-line drive system. This fact illustrates the effectiveness of the rear pulley shaft in isolating the noisy belt drive from the rest of the spindle. The measured radial runout of the spindle using a belt tension of $69 \mathrm{~N}\left(15.5 \mathrm{lb}_{\mathrm{f}}\right)$ is shown in Table 7.7. A maximum total radial runout of $39.3 \mu$ in was experienced at a rotational speed of $5,000 \mathrm{rpm}$.

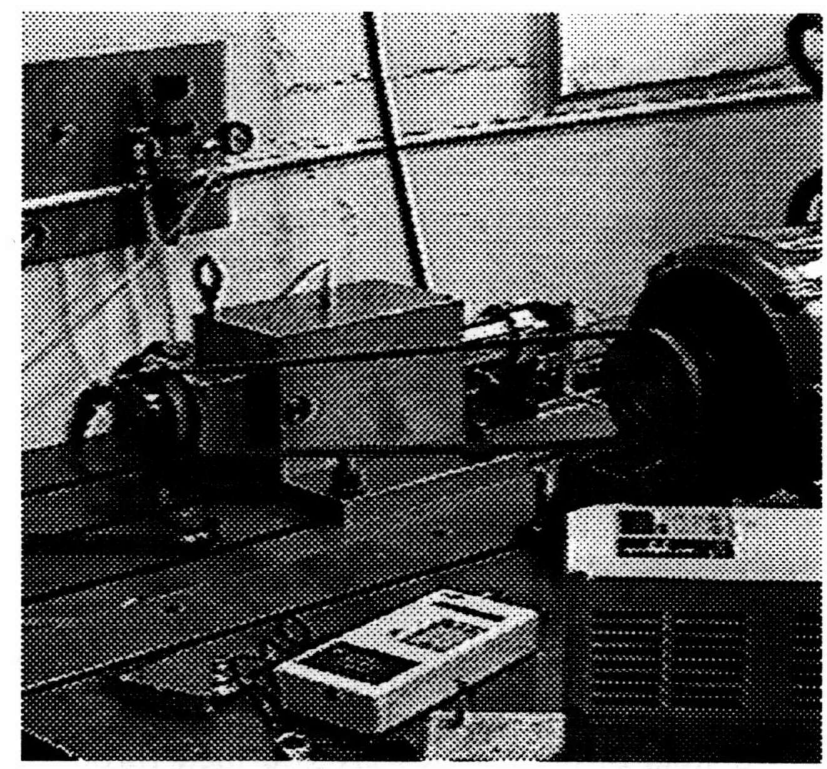

Figure 7.26. Picture of belt-drive system used to drive the spindle during the error motion tests. 
Table 7.6.

\begin{tabular}{|c|c|c|c|}
\hline $\begin{array}{c}\text { Speed } \\
(\mathbf{r p m})\end{array}$ & $\begin{array}{c}\text { Asynchronous } \\
\text { Runout } \\
(\boldsymbol{\mu} \text { in) }\end{array}$ & $\begin{array}{c}\text { Average } \\
\text { Runout } \\
(\boldsymbol{\mu} \text { in) }\end{array}$ & $\begin{array}{c}\text { Total } \\
\text { Runout } \\
(\boldsymbol{\mu} \text { in) }\end{array}$ \\
\hline 1000 & 26.2 & 13.0 & 31.6 \\
\hline 1000 & 25.3 & 13.7 & 29.9 \\
\hline 2000 & 27.1 & 14.6 & 30.9 \\
\hline 2800 & 24.0 & 16.0 & 31.2 \\
\hline 4050 & 29.3 & 24.1 & 35.7 \\
\hline 4625 & 24.8 & 24.2 & 38.3 \\
\hline 4990 & 23.7 & 20.9 & 44.2 \\
\hline
\end{tabular}

Table 7.7.

\begin{tabular}{|c|c|c|c|}
\hline $\begin{array}{c}\text { Speed } \\
(\mathbf{r p m})\end{array}$ & $\begin{array}{c}\text { Asynchronous } \\
\text { Runout } \\
(\mu \mathrm{in})\end{array}$ & $\begin{array}{c}\text { Average } \\
\text { Runout } \\
(\mu \mathrm{in})\end{array}$ & $\begin{array}{c}\text { Total } \\
\text { Runout } \\
(\mu \mathrm{in})\end{array}$ \\
\hline 920 & 27.8 & 16.5 & 32.7 \\
\hline 1052 & 24.8 & 14.4 & 32.6 \\
\hline 2729 & 25.5 & 22.8 & 38.6 \\
\hline 2729 & 24.6 & 23.4 & 37.5 \\
\hline 2844 & 35.2 & 22.8 & 39.2 \\
\hline 4995 & 24.9 & 22.3 & 39.3 \\
\hline
\end{tabular}

\section{B. Twisted-Groove Spindle \#1: Eight-Pocket Spindle}

To this point, this chapter has presented measurements made on the primary Phase I fluid film test spindle, which is a self-compensated spindle with the fluid routed from the compensators to the pockets through holes cross-drilled through the shaft. The results clearly shown that this method can produce an excellent machine tool spindle with superior qualities. However, a new method in which the fluid is routed on the surface of the shaft itself simplifies fabrication and reduces the flow rate of fluid required to pressurize the bearings. Two test spindles were fabricated with this method, one with 
eight-pocket bearings and one with four-pocket bearings, and tests of these spindles will be presented next.

The eight-pocket secondary test spindle is shown in Figure 7.27. The shaft diameter is $56 \mathrm{~mm}$. A picture of the shaft pulled part-way out of the housing is shown in Figure 7.28. In this spindle, coolant is supplied to the central supply channel at about 600 psig. It then flows axially across compensating lands and enters grooves which route it part-way around the shaft to the pockets. The pockets serve as the primary loadsupporting area of the bearing. The fluid then flows axially across outlet lands to a drain groove which in this spindle is exterior to the housing. No provision was made to seal the fluid at the ends of the spindle and keep it from spraying outward since this spindle was intended only for simplified testing purposes. Note that this spindle has no thrust bearing and so the shaft is free to float axially in the housing. This spindle was only intended for simplified testing and so no thrust capacity was provided.

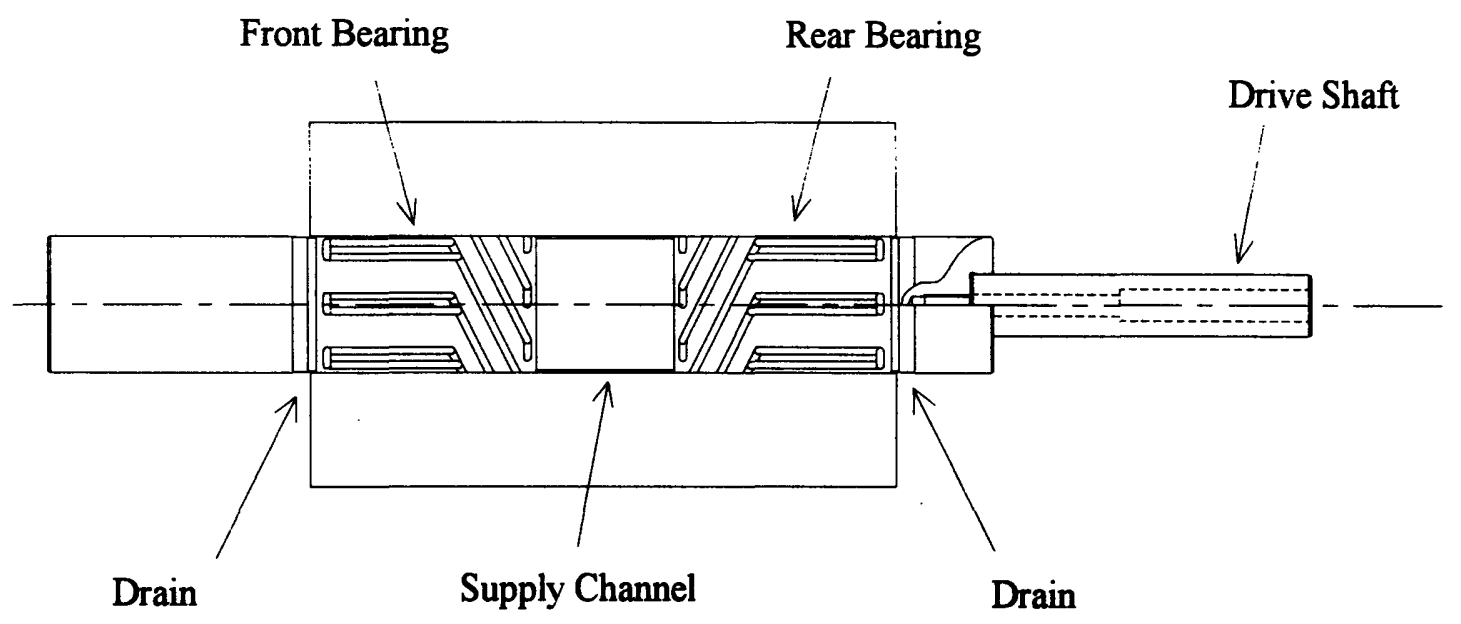

Figure 7.27. Layout of the eight-pocket secondary Phase I test spindle. 


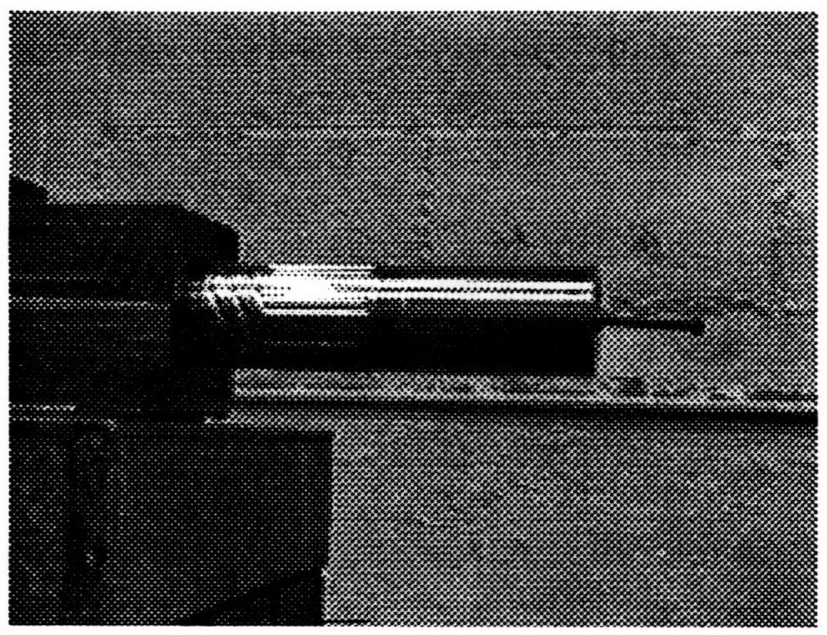

Figure 7.28. Picture of the eight-pocket secondary Phase I test spindle. The shaft is pulled part-way out of the housing to show it off.

The experimental spindle shown in Figures 7.27 and 7.28 was fabricated for testing. After fabrication, the inner diameter of the housing and the outer diameter of the shaft were measured. The shaft had a relatively uniform diameter of 2.2045 "; its diameter was constant to within the measuring accuracy of the indicator, which was approximately $0.00005 "$. The inner diameter of the bore, however, varied significantly. There was found to be a large amount of "bell mouth" at both ends of the bore. In the inner regions of the bore, it had a relatively uniform diameter of 2.2056 ". At 0.5 " from its end, however, its diameter was measured to be 2.2059 ". This makes the clearance $0.00055^{\prime \prime}(14.0 \mu \mathrm{m})$ in the inner regions of the bearings, and $0.00065 "(16.5 \mu \mathrm{m})$ at a location 0.5 " from the end of the housing. The outlet lands of the bearings extended to within approximately 0.085 " from the end of the housing. The bell-mouth was thought to be much higher at this location, although it was not measured. Although this amount of clearance variation is not acceptable for a production spindle, the experimental spindle was tested nevertheless. The 
performance of the spindle was found to be adequate in every respect except for its flow rate. The flow rate through both bearings was found to be approximately $1.76 \mathrm{gpm}$ at a supply pressure of $600 \mathrm{psig}$, using a water-based coolant with a viscosity approximately $30 \%$ greater than that of water. This corresponds to approximately $0.88 \mathrm{gpm}$ per bearing. This is much more than expected. For example, for a uniform clearance of $16.5 \mathrm{~mm}$, the flow rate through one bearing should only be about $0.4 \mathrm{gpm}$. The high flow rate through the bearing indicates that the housing bore had substantial bell-mouth.

The static stiffness of the eight-pocket test spindle was measured by hanging a 25 pound weight from the front of the spindle and measuring the displacement with a Starrett indicator. The positions on the spindle where the weight was suspended and the displacement was measured are shown in Figure 7.29. With a supply pressure of 600 psig, the deflection of the spindle loaded with 25 pounds was 0.000105 inches. This yields a static stiffness of 238,100 pounds per inch. This is much higher than the static stiffness of the cross-drilled spindle presented previously. However, the front pad of the eight-pocket surface routing spindle is closer to nose of the spindle by 1.5 inches.

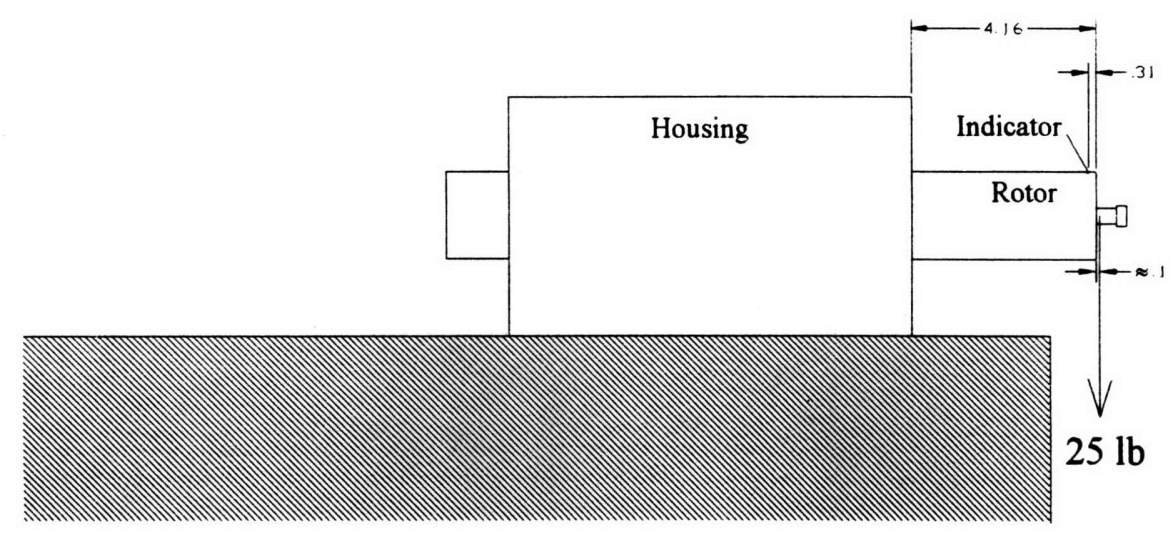

Figure 7.29. Schematic diagram showing the axial locations of the indicator and the applied weight that were used in the static stiffness tests. Dimensions are in inches. 
The static stiffness of the spindle was next simulated with the methods developed in Chapter 4. The bearing characteristics are tabulated in Table 7.8. The bearing clearance used in the calculations was $16.5 \mu \mathrm{m}$. Although the clearance was thought to be much greater at the bearing exit lands as described above, the calculation with a uniform clearance of $16.5 \mu \mathrm{m}$ is given here for comparison purposes. The calculated stiffness of the spindle is $246,000 \mathrm{lb}_{\mathrm{f}} / \mathrm{in}$. Given the uncertainity in the bearing clearance, the agreement with the $238,000 \mathrm{lb}_{\mathrm{f}} /$ in measured stiffness is adequate. 
Table 7.8

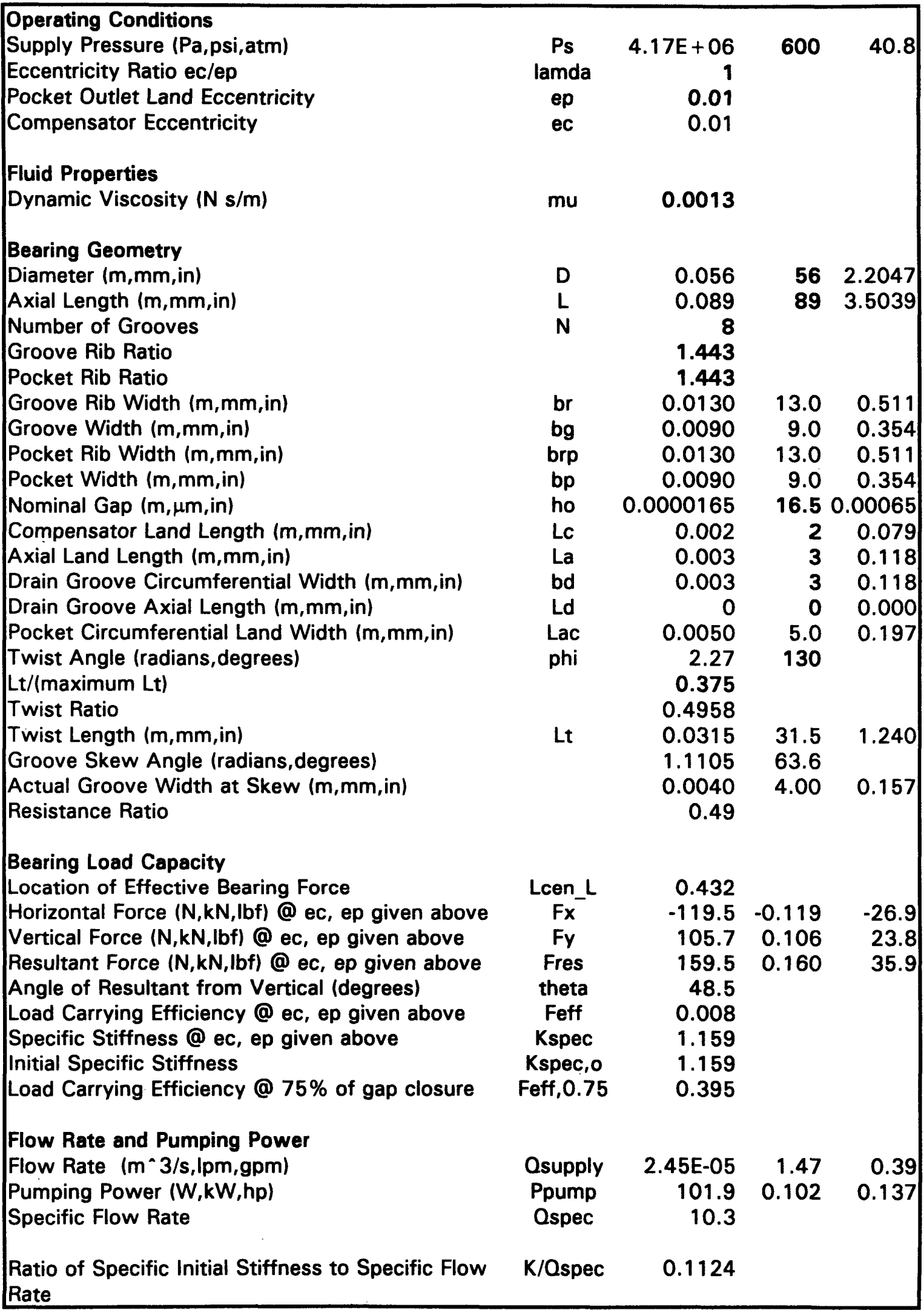


The eight-pocket test spindle was next rotated at high speed to test its stability and thermal characteristics. During the test, the chiller used to cool the bearing supply fluid did not operate properly. Nevertheless, the spindle was driven to $13,000 \mathrm{rpm}$ with an inline drive using a flexible coupling. The inlet and outlet temperatures of the fluid were measured with thermocouples that had accuracies of approximately $0.25^{\circ} \mathrm{F}$. Shown in Table 7.9 are the measured steady-state inlet and outlet fluid temperatures. Also shown is the bearing supply flow rate, which decreased with increasing shaft speed. The flow rate decreased because the bearing grooves were oriented such that the fluid was pumped from the pockets back toward the compensators as the shaft was rotated. The supply flow rate is also plotted in Figure 7.30. Note that, if the grooves were oriented in the opposite direction, the flow rate through the spindle would increase with increasing shaft speed.

Table 7.9

\begin{tabular}{|c|c|c|c|}
\hline $\begin{array}{c}\text { Speed } \\
\text { (rpm) }\end{array}$ & $\begin{array}{c}\text { Inlet Temp. } \\
\left({ }^{\circ} \mathbf{F}\right)\end{array}$ & $\begin{array}{c}\text { Outlet Temp. } \\
\text { ( } \mathbf{F} \text { ) }\end{array}$ & $\begin{array}{c}\text { Flow Rate } \\
(\mathbf{g p m})\end{array}$ \\
\hline 0 & 77.2 & 77.7 & 2.05 \\
\hline 1,000 & 78.6 & 79.9 & 2.03 \\
\hline 2,000 & 82.2 & 83.5 & 1.98 \\
\hline 3,000 & 85.3 & 86.9 & 2 \\
\hline 4,000 & 84.4 & 86.7 & 1.97 \\
\hline 5,000 & 87.7 & 90.7 & 1.95 \\
\hline 6,000 & 82.8 & 86.7 & 1.93 \\
\hline 7,000 & 85.1 & 89.5 & 1.88 \\
\hline 8,000 & 90 & 95.3 & 1.83 \\
\hline 9,000 & 94.9 & 101.5 & 1.79 \\
\hline 10,000 & 91.1 & 100.6 & 1.77 \\
\hline 11,000 & 91.3 & 102.5 & 1.72 \\
\hline 12,000 & 90.3 & 103.5 & 1.63 \\
\hline 13,000 & 100 & 115.5 & 1.5 \\
\hline
\end{tabular}




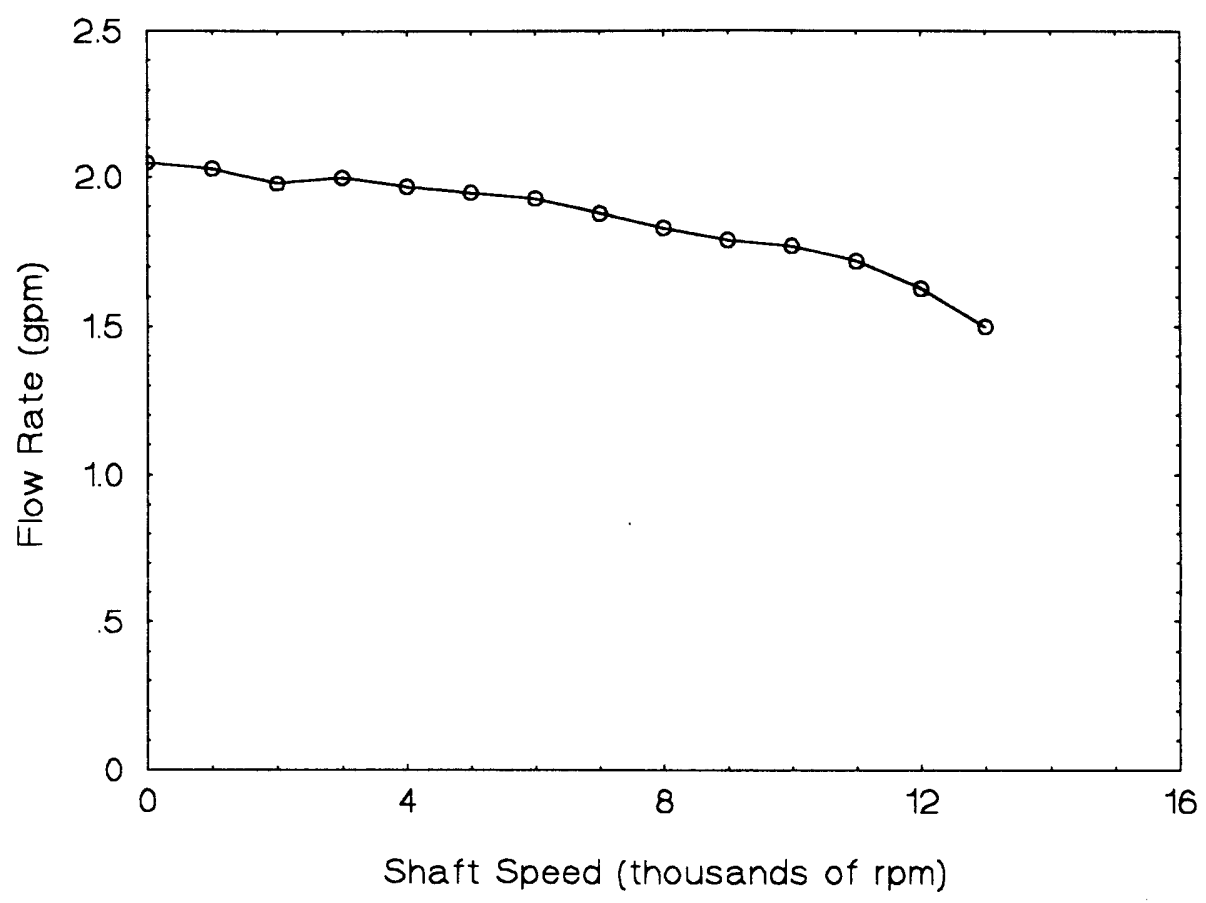

Figure 7.30. Measured flow rate of coolant supplied to the eight-pocket test spindle versus operating speed.

As shown in Figure 7.30, the pumping effect of the grooves of SC Bearing \#10 plays a large role in determining the flow rate through the bearing. For this reason, a separate test was done to isolate the change in flow rate caused pumping effect from the change in flow rate caused by the temperature-dependent viscosity. An attempt was made to measure the flow rate of the bearing at different speeds but the same fluid inlet temperature. The data collected is shown in Table 7.10. As shown, the inlet temperature was approximately $87^{\circ} \mathrm{F}$ for each case. The supply pressure was also approximately 665 psig for each case. Neglecting the change in viscosity caused by the temperature rise across the bearing, $\Delta \mathrm{Q} / \mathrm{Q}_{\mathrm{o}}$ in Table 7.10 represents the decrease in flow rate that was caused by the pumping effect of the grooves. 
Table 7.10

\begin{tabular}{|c|c|c|c|c|}
\hline Speed (rpm) & Inlet Temp. $\left({ }^{\circ} \mathrm{F}\right)$ & $\begin{array}{c}\text { Supply Pressure } \\
\text { (psig) }\end{array}$ & $\begin{array}{c}\text { Measured Flow } \\
\text { Rate (gpm) }\end{array}$ & $\Delta \mathrm{Q} / \mathrm{Q}_{\mathbf{o}}$ \\
\hline 0 & 87.1 & 660 & 2.0 & 0 \\
\hline 5,000 & 87.3 & 664 & 1.92 & 0.04 \\
\hline 7,000 & 85.1 & 676 & 1.88 & 0.06 \\
\hline 8,000 & 90.0 & 672 & 1.83 & 0.085 \\
\hline 10,000 & 87.3 & 672 & 1.78 & 0.11 \\
\hline
\end{tabular}

Next, the change in flow rate was calculated using the methods of Appendix D. The viscosity used was that of the coolant at $87^{\circ} \mathrm{F}$, which is $0.00105 \mathrm{Nm} / \mathrm{s}$, and the fluid density was $995 \mathrm{~kg} / \mathrm{m}^{3}$. The value of $L p=b_{e} / \cos (\beta)=(3 \mathrm{~mm}) / \cos \left(63.6^{\circ}\right)=6.75 \mathrm{~mm}$. The value of $\mathrm{L}_{\mathrm{p}}{ }^{*}$ used was $70 \mathrm{~mm}$, which is the circumferential distance of the groove extending from the compensating land to the pocket exit land. The value of the groove depth used was $0.4 \mathrm{~mm}$; this is the approximate average depth of the groove, as shown in Figure 7.31. The calculated change in flow rate is compared with the measured change in flow rate in Figure 7.32. As shown, the agreement is surprisingly good.

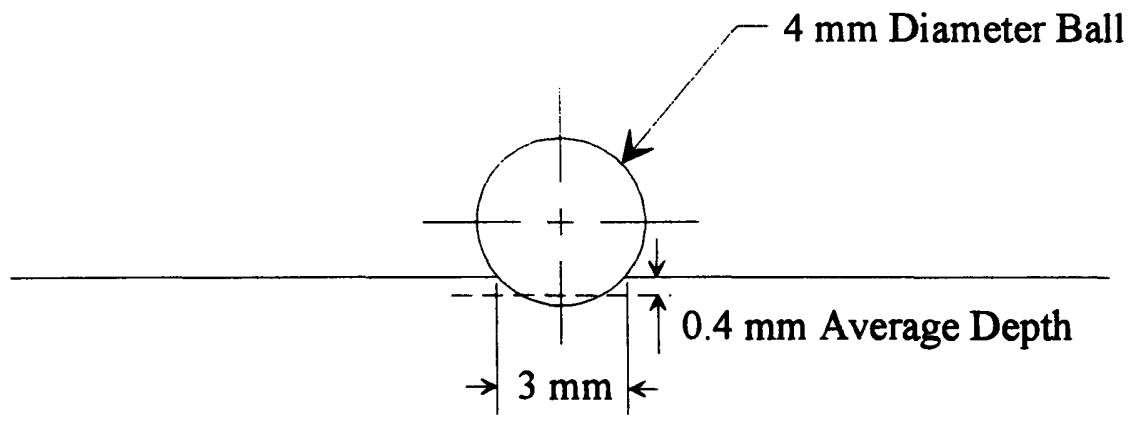

Figure 7.31. Schematic diagram illustrating the ball of the end mill used to machine the grooves in the experimental test spindle. 


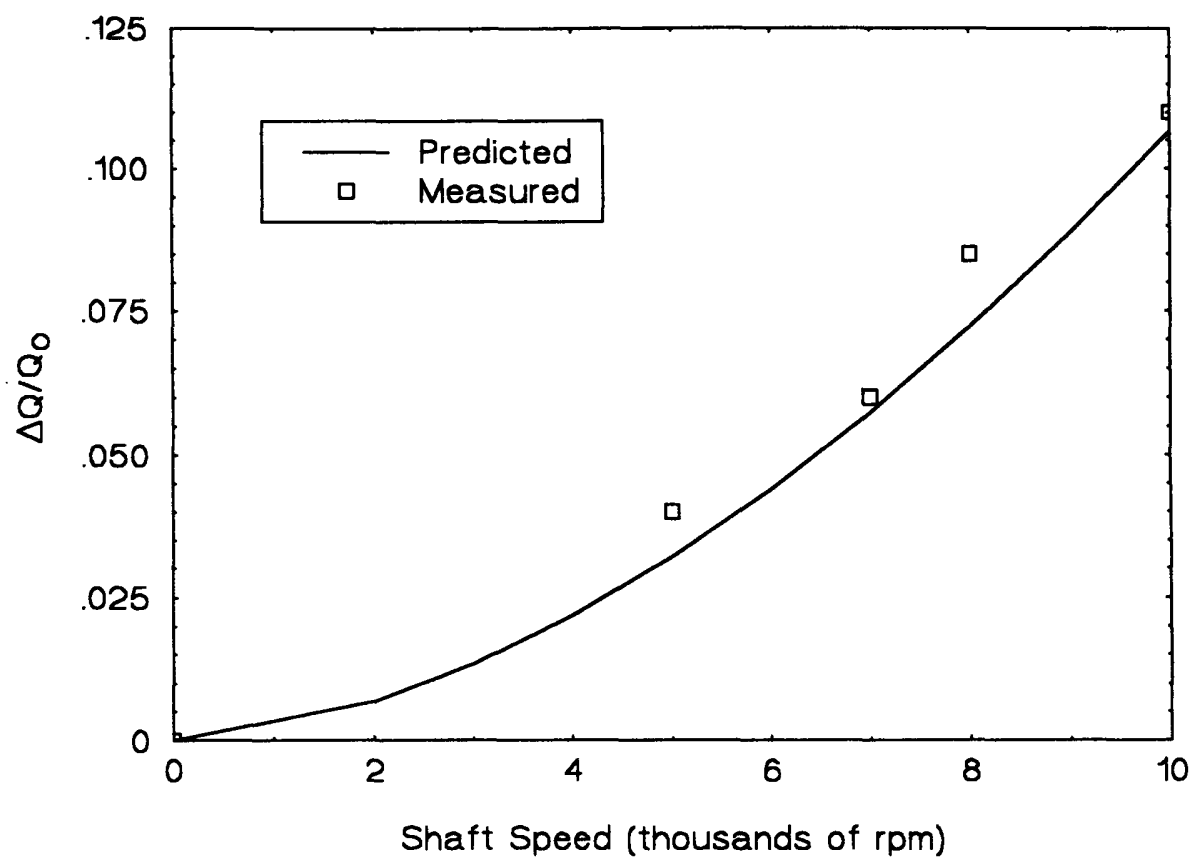

Figure 7.32. Comparison of the predicted and measured reduction in flow rate caused by the fluid pumping within the grooves of the experimental test spindle.

\section{C. Twisted-Groove Spindle \#2}

Tests performed on the four-pocket test spindle with SC Bearing \#10 will be presented next. This spindle is illustrated Figure 7.33. A picture of the grooves on the surface of the shaft is shown in Figure 7.34. The lay-out of this spindle is identical to that of the spindle of Figure 7.27. Coolant is supplied to the central supply channel at about 600 psig. It then flows axially across compensating lands and enters grooves which route it part-way around the shaft to the pockets. The fluid then flows axially across outlet lands to a drain groove which in this spindle is exterior to the housing. The housing used for the 4-pocket spindle was the same as that used for the 8-pocket spindle described in 
the previous section. Although the housing had substantial bell-mouth at its ends, it was nevertheless used for testing purposes.

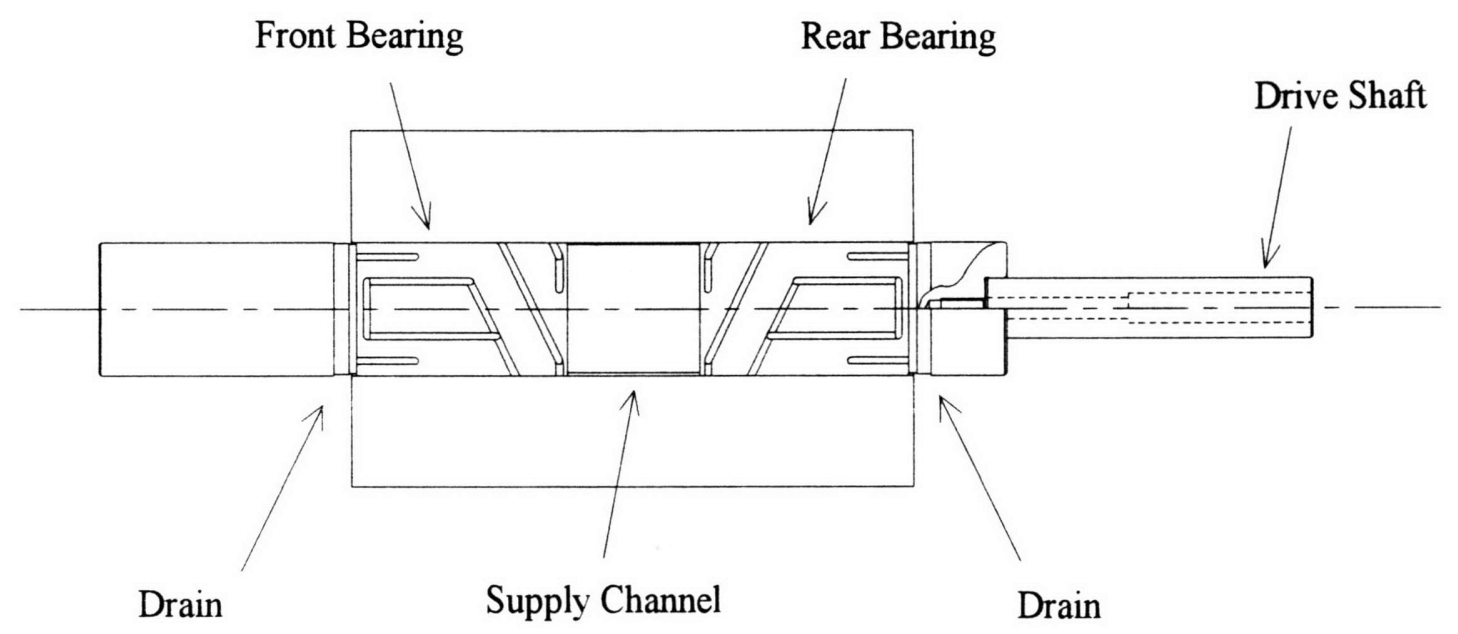

Figure 7.33. Layout of the four-pocket secondary Phase I test spindle.

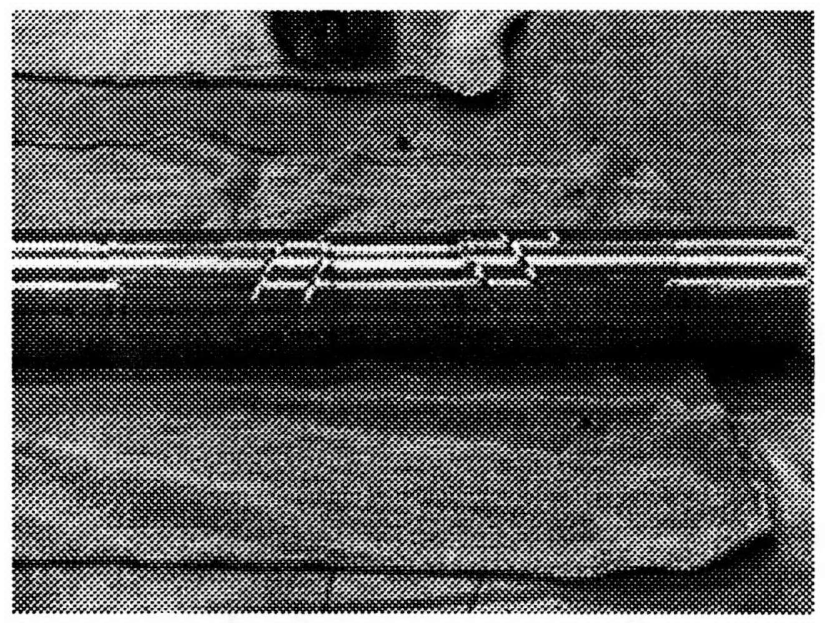

Figure 7.34. Picture of the four-pocket secondary Phase I test shaft.

The static stiffness of the eight-pocket test spindle was measured first. This was again found by hanging a 25 pound weight from the front of the spindle and measuring the 
displacement with a Starrett indicator. The positions on the spindle where the weight was suspended and the displacement was measured are shown in Figure 7.35. With a supply pressure of $600 \mathrm{psig}$, the deflection of the spindle loaded with 25 pounds was 0.00011 inches. This yields a static stiffness of 227,300 pounds per inch. This is slightly lower than that of the eight-pocket spindle.

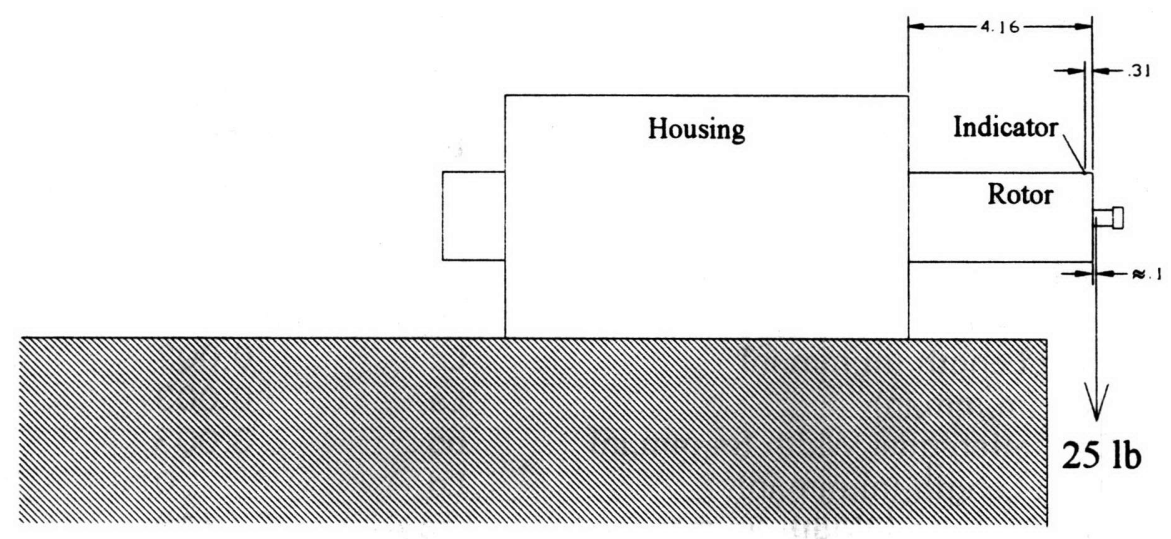

Figure 7.35. Schematic diagram showing the axial locations of the indicator and the applied weight that were used in the static stiffness tests. Dimensions are in inches.

The static stiffness of the spindle was next simulated with the methods developed in Chapter 4. The bearing characteristics are tabulated in Table 7.11. The bearing clearance used in the calculations was $16.5 \mu \mathrm{m}$. Although the clearance was thought to be much greater at the bearing exit lands as described above, the calculation with a uniform clearance of $16.5 \mu \mathrm{m}$ is given here for comparison purposes. The calculated stiffness of the spindle is $229,000 \mathrm{lb}_{\mathrm{f}}$ in. Given the uncertainity in the bearing clearance, the agreement with the $227,000 \mathrm{lb}_{\mathrm{f}}$ /in measured stiffness is certainly adequate. 
Table 7.11

\begin{tabular}{|c|c|c|c|c|}
\hline $\begin{array}{l}\text { Operating Conditions } \\
\text { Supply Pressure (Pa,psi,atm) } \\
\text { Eccentricity Ratio ec/ep } \\
\text { Pocket Outlet Land Eccentricity } \\
\text { Compensator Eccentricity }\end{array}$ & $\begin{array}{c}\text { Ps } \\
\text { lamda } \\
\text { ep } \\
\text { ec }\end{array}$ & $\begin{array}{r}4.17 E+06 \\
1 \\
0.01 \\
0.01\end{array}$ & 600 & 40.8 \\
\hline $\begin{array}{l}\text { Fluid Properties } \\
\text { Dynamic Viscosity }(\mathrm{N} \mathrm{s} / \mathrm{m})\end{array}$ & $\mathrm{mu}$ & 0.0013 & & \\
\hline \multicolumn{5}{|l|}{ Bearing Geometry } \\
\hline Diameter $(m, m m, i n)$ & $\mathrm{D}$ & 0.056 & 56 & 2.2047 \\
\hline Axial Length $(\mathrm{m}, \mathrm{mm}, \mathrm{in})$ & $\mathbf{L}$ & 0.089 & 89 & 3.5039 \\
\hline Number of Grooves & $N$ & 4 & & \\
\hline $\begin{array}{l}\text { Groove Rib Ratio } \\
\text { Pocket Rib Ratio }\end{array}$ & & $\begin{array}{l}1.5706 \\
1.5706\end{array}$ & & \\
\hline Groove Rib Width (m, mm, in) & br & 0.0269 & 26.9 & 1.058 \\
\hline Groove Width $(\mathrm{m}, \mathrm{mm}, \mathrm{in})$ & bg & 0.0171 & 17.1 & 0.674 \\
\hline Pocket Rib Width $(\mathrm{m}, \mathrm{mm}$, in) & brp & 0.0269 & 26.9 & 1.058 \\
\hline Pocket Width $(\mathrm{m}, \mathrm{mm}$,in) & bp & 0.0171 & 17.1 & 0.674 \\
\hline Nominal Gap $(m, \mu m$, in $)$ & ho & 0.0000165 & 16.5 & 0.000650 \\
\hline Compensator Land Length ( $\mathrm{m}, \mathrm{mm}$,in) & LC & 0.002 & 2 & 0.079 \\
\hline Axial Land Length $(\mathrm{m}, \mathrm{mm}$, in) & La & 0.003 & 3 & 0.118 \\
\hline Drain Groove Circumferential Width ( $\mathrm{m}, \mathrm{mm}$, in) & bd & 0.003 & 3 & 0.118 \\
\hline Drain Groove Axial Length $(\mathrm{m}, \mathrm{mm}$,in) & Ld & 0 & 0 & 0.000 \\
\hline Pocket Circumferential Land Width $(\mathrm{m}, \mathrm{mm}, \mathrm{in})$ & Lac & 0.0119 & 11.9 & 0.470 \\
\hline Twist Angle (radians, degrees) & phi & 2.443461 & 140 & \\
\hline$L t /($ maximum $L t)$ & & 0.3971 & & \\
\hline Twist Ratio & & 0.4875462 & & \\
\hline Twist Length $(\mathrm{m}, \mathrm{mm}, \mathrm{in})$ & Lt & 0.0334 & 33.4 & 1.313 \\
\hline Groove Skew Angle (radians, degrees) & & 1.12 & 64.0 & \\
\hline Actual Groove Width at Skew $(\mathrm{m}, \mathrm{mm}, \mathrm{in})$ & & 0.0075 & 7.50 & 0.295 \\
\hline Resistance Ratio & & 0.49 & & \\
\hline \multicolumn{5}{|l|}{ Bearing Load Capacity } \\
\hline Location of Effective Bearing Force & Lcen_L & 0.422 & & \\
\hline Horizontal Force ( $N, k N, l b f) @ e c$, ep given above & $F x^{-}$ & -95.8 & -0.096 & -21.5 \\
\hline Vertical Force (N,kN,lbf) @ ec, ep given above & Fy & 90.6 & 0.091 & 20.4 \\
\hline Resultant Force $(\mathrm{N}, \mathrm{kN}, \mathrm{lbf}) @$ ec, ep given above & Fres & 131.9 & 0.132 & 29.7 \\
\hline Angle of Resultant from Vertical (degrees) & theta & 46.6 & & \\
\hline Load Carrying Efficiency @ ec, ep given above & Feff & 0.006 & & \\
\hline Specific Stiffness @ ec, ep given above & Kspec & 0.925 & & \\
\hline Initial Specific Stiffness & Kspec,o & 0.925 & & \\
\hline Load Carrying Efficiency @ 75\% of gap closure & Feff, 0.75 & 0.329 & & \\
\hline Flow Rate and Pumping Power & & & & \\
\hline Flow Rate $(\mathrm{m} \wedge 3 / \mathrm{s}, \mathrm{lpm}, \mathrm{gpm})$ & Qsupply & 2.09E-05 & 1.26 & 0.33 \\
\hline $\begin{array}{l}\text { Pumping Power (W,kW,hp) } \\
\text { Specific Flow Rate }\end{array}$ & $\begin{array}{l}\text { Ppump } \\
\text { Qspec }\end{array}$ & $\begin{array}{r}87.2 \\
8.8\end{array}$ & 0.087 & 0.117 \\
\hline $\begin{array}{l}\text { Ratio of Specific Initial Stiffness to Specific Flow } \\
\text { Rate }\end{array}$ & K/Ospec & 0.1048 & & \\
\hline
\end{tabular}


The four-pocket cross-routed spindle of Figures 7.33 and 7.34 was sucessfully driven to $20,000 \mathrm{rpm}$. However, the thermocouple used to measure the inlet temperature of the coolant was corrupted by noise from the drive spindle. Also, the flow rate through the spindle was off of the scale of the only flow meter available at the time of the tests. The torque and outlet temperature were therefore the only measurements sucessfully recorded during the high-speed test.

The torque required to drive the spindle up to $20,000 \mathrm{rpm}$ was measured using a dynamic torque sensor connected in-line between the drive motor and the spindle with the use of flexible couplings. The torque meter used had a measurable torque range of 0 to $500 \mathrm{in}-\mathrm{lb}$. Although the torque expected to turn the spindle was less than $40 \mathrm{in}-\mathrm{lb}$, the 500 in-lb meter was used because it was the only torque sensor available at the time of the testing. The measured torque required to turn the spindle up to $20,000 \mathrm{rpm}$ is shown in Figure 7.36. The torque predicted by the spreadsheets is in good agreement with the measured torque at all speeds. The measured torque data presented here is only ball-park and is not an accurate recording of the spindle torque. 


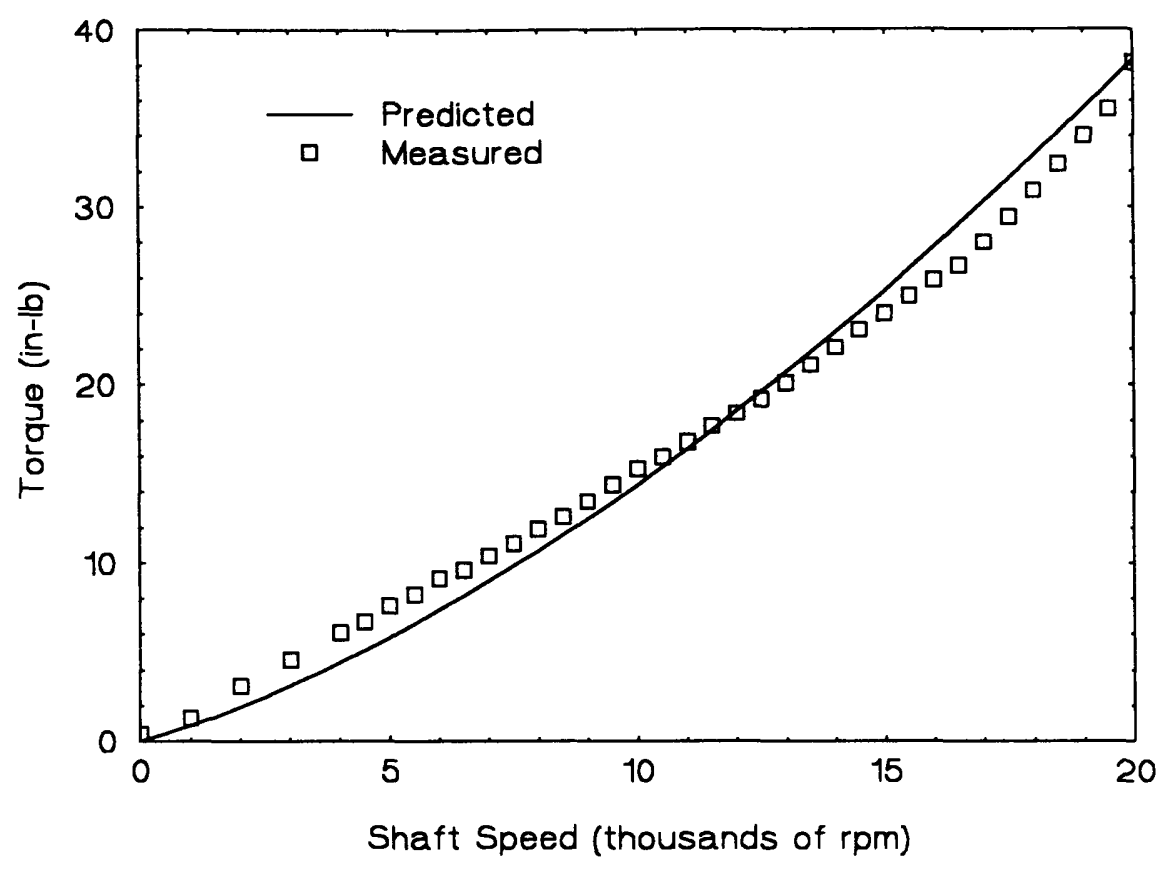

Figure 7.36. Torque required to turn the four-pocket surface routed spindle up to 20,000 rpm.

Note that at $20,000 \mathrm{rpm}$, the Reynolds number of the flow on the tight-clearance lands is approximately 800 , and so the flow is laminar even at this speed of 1.1 million DN. The Reynolds number of the flow in the grooves at $20,000 \mathrm{rpm}$ is approximately 24,000 , and so the flow is turbulent even at this speed. As the speed is increased from 0 to $20,000 \mathrm{rpm}$, the power consumed by the turbulent flow in the grooves becomes a greater portion of the total shear power. The slope of the torque curve of Figure 7.36 therefore increases as the speed is increased. If the speed were increased further, this trend would continue up to about 2.5 million DN. At a speed of 3 million DN the flow on the lands would be fully turbulent, and the slope of the torque curve would increase somewhat sharply. 
The drive power consumed by the spindle is found by multiplying the measured torque of Figure 7.36 by the shaft speed. The measured shearing power curve of Figure 7.37 is again presented only as a ball-park indicator of the bearing power consumption and is not an accurate recording of this data.

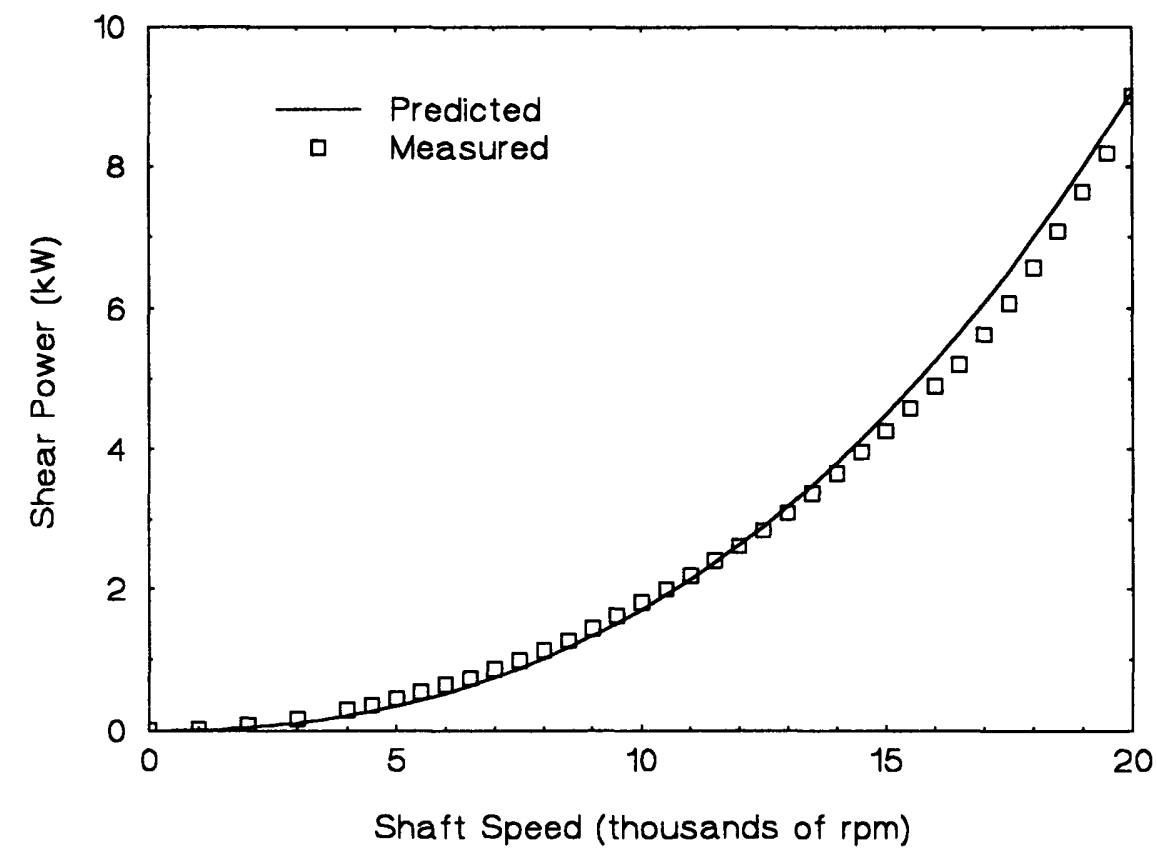

Figure 7.37. Measured shearing power required to turn the four-pocket surface routed spindle up to $20,000 \mathrm{rpm}$. 


\section{CLUSTER DRILLING MACHINE}

Although the prototype spindles tested in the previous chapters provided valuable information about hydrostatic spindles in general, they were designed with a specific application in mind. The spindles will be integrated into an assembly that is capable of drilling a cluster of four tightly spaced holes into steel components. Spindles that are currently used in this application are built with rolling element bearings. The stiffness of these spindles are very low because the bearings take up a substantial amount of the available radial space between the centerlines, resulting in spindles with small shaft diameters. Because the spindles have low radial stiffness, the holes must be drilled with multiple passes using tools of increasing diameter in order to limit the loads placed on the spindles. Having to make multiple passes and to make tool changes substantially increases the cost of the parts being manufactured. An improved machine with higher radial stiffness is very desirable in this application.

\section{A. The Cluster Spindle Assembly}

Figure 8.1 shows the cluster spindle assembly developed in this project. The four spindles are supported by self-compensating hydrostatic bearings with the bearing geometries machined into the surface of the shafts. This configuration greatly simplifies the spindle manufacture and enables the shaft diameters to be increased. The centerline distance between the spindle centers is $61.02 \mathrm{~mm}$. The shafts each have a diameter of 56 $\mathrm{mm}$. This configuration has substantially increased radial stiffness compared to the rolling element spindle assembly it is designed to replace. 


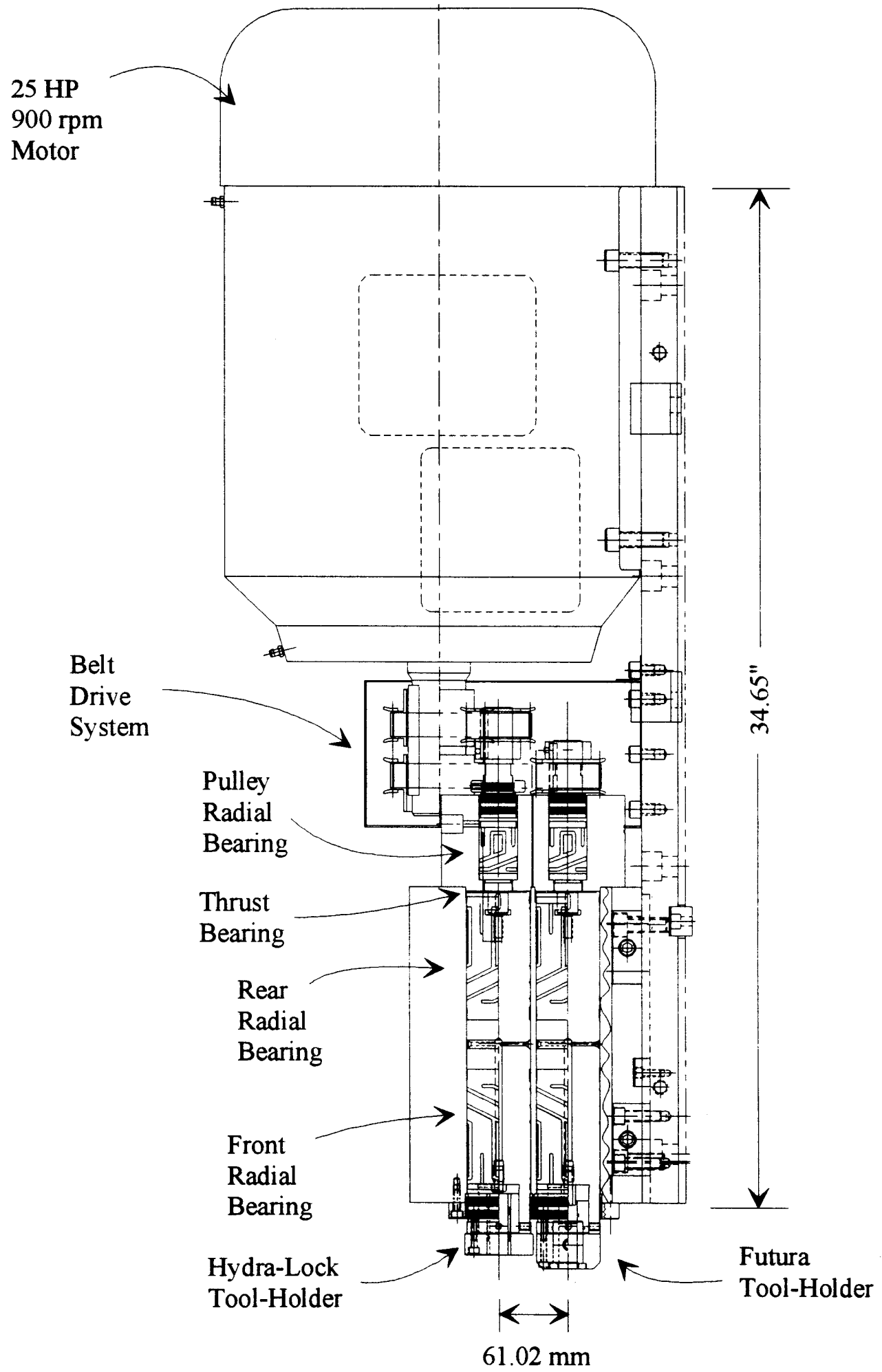

Figure 8.1. Four-spindle assembly that drills clusters of holes in a single pass. The novel hydrostatic bearings developed in this work support the spindles. 
Other features of the spindle assembly shown in Figure 8.1 include:

- The radial bearings are each of the Self-Compensating Bearing \#10 type described in Chapter 3. The grooves are oriented in a direction to pump more fluid through the bearings with increasing shaft speed, thereby reducing the temperature rise of the spindles.

- The thrust bearing is of the Thrust Bearing \#3 type described in Chapter 5. This thrust bearing is designed to handle over 1,000 pounds of force in the pushing direction and 200 pounds of force in the pulling direction when supplied with $600 \mathrm{psig}$.

- A small bearing in the rear of each spindle helps to provide substantial belt load capability and to reduce the effect of the vibrations from the belt drive system on the error motion of the spindles.

- Through-the-tool cooling is provided with the non-contact coupling described in Chapter 7.

- The spindles are pressurized with TECH $\mathrm{COOL}^{1}$, a water-based coolant.

- The spindle can use both Futura and Hydra-Lock tool holders.

- The motor is $10-\mathrm{hp}, 900-\mathrm{rpm}$. With the belt drive system, the spindle speed can be varied between 1,000 and $3,000 \mathrm{rpm}$.

- Non-contact air seals are used to seal the front and rear of the spindles.

The geometry of the bearings and their performance will be described next.

\footnotetext{
${ }^{1}$ Chemical Technologies Incorporated, Jackson, MI 49203.
} 
The front radial bearing is shown in Figure 8.2. Note that the rear bearing is identical in geometry to the first. The shaft diameter is $56 \mathrm{~mm}$, the bearing pad length is $89.5 \mathrm{~mm}$. The bearing is a Self-Compensated Bearing \#10, as described in Chapter 3, with 6 pockets. The performance of the bearing shown in Figure 8.2 is illustrated in Table 8.1. As shown, the bearing has an initial specific stiffness of 1.22 , and a specific flow rate of 14.1 .

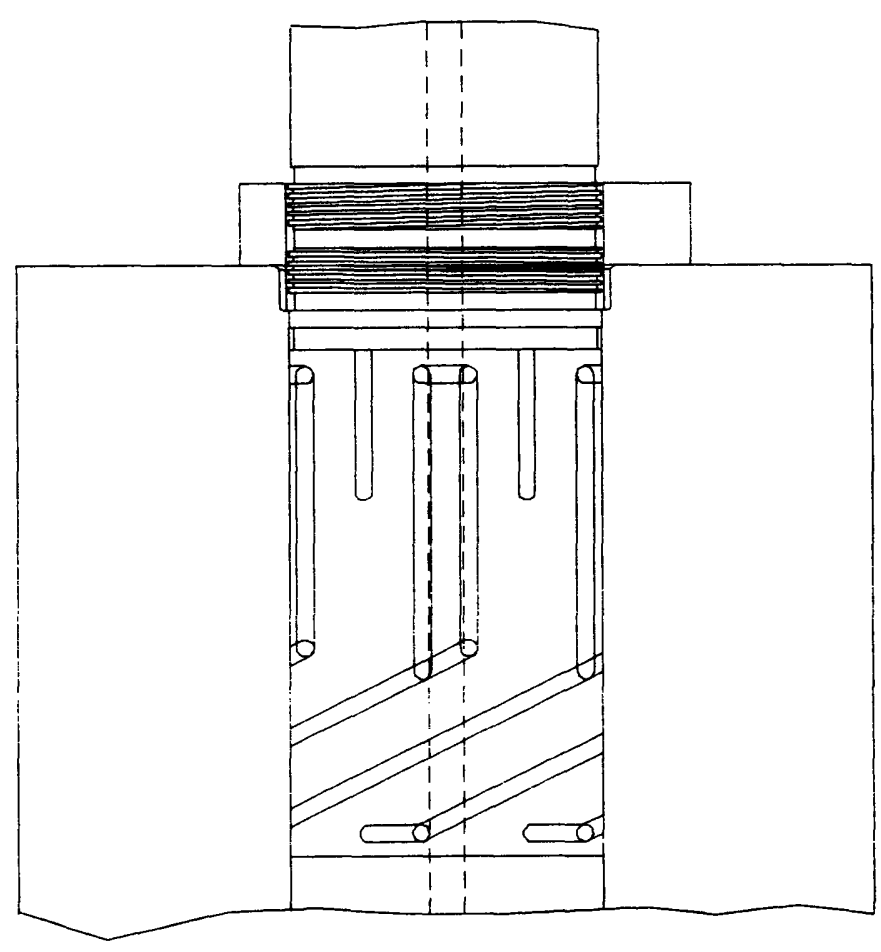

Figure 8.2. Pad layout of the front and rear radial bearings of the cluster spindles. 
Table 8.1

\begin{tabular}{|c|c|c|c|c|}
\hline $\begin{array}{l}\text { Operating Conditions } \\
\text { Supply Pressure (Pa,psi,atm) } \\
\text { Eccentricity Ratio ec/ep } \\
\text { Pocket Outlet Land Eccentricity } \\
\text { Compensator Eccentricity }\end{array}$ & $\begin{array}{l}\text { Ps } \\
\text { lamda } \\
\text { ep } \\
\text { ec }\end{array}$ & $\begin{array}{r}4.17 E+06 \\
1 \\
0.01 \\
0.01\end{array}$ & 600 & 40.8 \\
\hline \multicolumn{5}{|l|}{ Fluid Properties } \\
\hline $\begin{array}{l}\text { Bearing Geometry } \\
\text { Diameter }(\mathrm{m}, \mathrm{mm}, \mathrm{in}) \\
\text { Axial Length }(\mathrm{m}, \mathrm{mm}, \mathrm{in}) \\
\text { Number of Grooves } \\
\text { Groove Rib Ratio } \\
\text { Pocket Rib Ratio }\end{array}$ & $\begin{array}{l}D \\
L \\
N\end{array}$ & $\begin{array}{r}0.056 \\
0.08953 \\
6 \\
1.57 \\
1.57\end{array}$ & $\begin{array}{r}56 \\
89.53\end{array}$ & $\begin{array}{l}2.2047 \\
3.5248\end{array}$ \\
\hline Groove Rib Width $(\mathrm{m}, \mathrm{mm}, \mathrm{in})$ & br & 0.0179 & 17.9 & 0.705 \\
\hline Groove Width $(\mathrm{m}, \mathrm{mm}$, in $)$ & bg & 0.0114 & 11.4 & 0.449 \\
\hline Pocket Rib Width (m, mm,in) & brp & 0.0179 & 17.9 & 0.705 \\
\hline Pocket Width $(\mathrm{m}, \mathrm{mm}, \mathrm{in})$ & bp & 0.0114 & 11.4 & 0.449 \\
\hline Nominal Gap $(m, \mu m, i n)$ & ho & 0.0000127 & 12.7 & 0.00050 \\
\hline Compensator Land Length $(m, m m$, in) & Lc & 0.0025 & 2.5 & 0.098 \\
\hline Axial Land Length $(\mathrm{m}, \mathrm{mm}$, in) & La & 0.003 & 3 & 0.118 \\
\hline Drain Groove Circumferential Width $(\mathrm{m}, \mathrm{mm}$, in) & bd & 0.003 & 3 & 0.118 \\
\hline Drain Groove Axial Length ( $\mathrm{m}, \mathrm{mm}$,in) & Ld & 0.025 & 25 & 0.984 \\
\hline Pocket Circumferential Land Width (m, $\mathrm{mm}$,in) & Lac & 0.0075 & 7.5 & 0.294 \\
\hline Twist Angle (radians, degrees) & phi & 2.444 & 140 & \\
\hline$L t /(\operatorname{maximum} \mathrm{Lt})$ & & 0.394 & & \\
\hline Twist Ratio & & 0.484 & & \\
\hline Twist Length $(\mathrm{m}, \mathrm{mm}, \mathrm{in})$ & Lt & 0.03317 & 33.1 & 1.303 \\
\hline Groove Skew Angle (radians, degrees) & & 1.1201011 & 64.2 & \\
\hline Actual Groove Width at Skew $(\mathrm{m}, \mathrm{mm}, \mathrm{in})$ & & 0.00497 & 4.97 & 0.196 \\
\hline Resistance Ratio & & 1.35 & & \\
\hline \multicolumn{5}{|l|}{ Bearing Load Capacity } \\
\hline Location of Effective Bearing Force & Lcen_L & 0.472 & & \\
\hline Pressure Difference / Pressure @ ec, ep above & $\mathrm{DPP}$ & 0.016 & & \\
\hline Horizontal Force ( $N, k N, l b f) @ e c$, ep given above & $\mathrm{Fx}$ & -125.2 & -0.125 & -28.1 \\
\hline Vertical Force (N,kN,Ibf) @ ec, ep given above & Fy & 102.2 & 0.102 & 23.0 \\
\hline Resultant Force ( $\mathrm{N}, \mathrm{kN}, \mathrm{lbf}) @$ ec, ep given above & Fres & 161.6 & 0.162 & 36.3 \\
\hline Angle of Resultant from Vertical (degrees) & theta & 50.8 & & \\
\hline Load Carrying Efficiency @ ec, ep given above & Feff & 0.008 & & \\
\hline Specific Stiffness @ ec, ep given above & Kspec & 1.223 & & \\
\hline Initial Specific Stiffness & Kspec, 0 & 1.224 & & \\
\hline Load Carrying Efficiency @ 75\% of gap closure & Feff, 0.75 & 0.353 & & \\
\hline $\begin{array}{l}\text { Flow Rate and Pumping Power } \\
\text { Flow Rate (m^3/s,lpm,gpm) } \\
\text { Pumping Power }(W, k W, h p) \\
\text { Specific Flow Rate }\end{array}$ & $\begin{array}{l}\text { Qsupply } \\
\text { Ppump } \\
\text { Ospec }\end{array}$ & $\begin{array}{r}1.51 \mathrm{E}-05 \\
62.9 \\
14.1 \\
\end{array}$ & $\begin{array}{r}0.91 \\
0.063\end{array}$ & $\begin{array}{r}0.24 \\
0.084\end{array}$ \\
\hline
\end{tabular}


The small pulley-support bearing is shown in Figure 8.3. The dimater of the shaft is $34 \mathrm{~mm}$, the length of the bearing pad is $46.5 \mathrm{~mm}$. The bearing is a Self-Compensated Bearing \#10, as described in Chapter 3 , with 4 pockets. The performance of the bearing shown in Figure 8.2 is illustrated in Table 8.2. As shown, the bearing has a load-carrying efficiency of 0.277 , and a specific flow rate of 8.1.

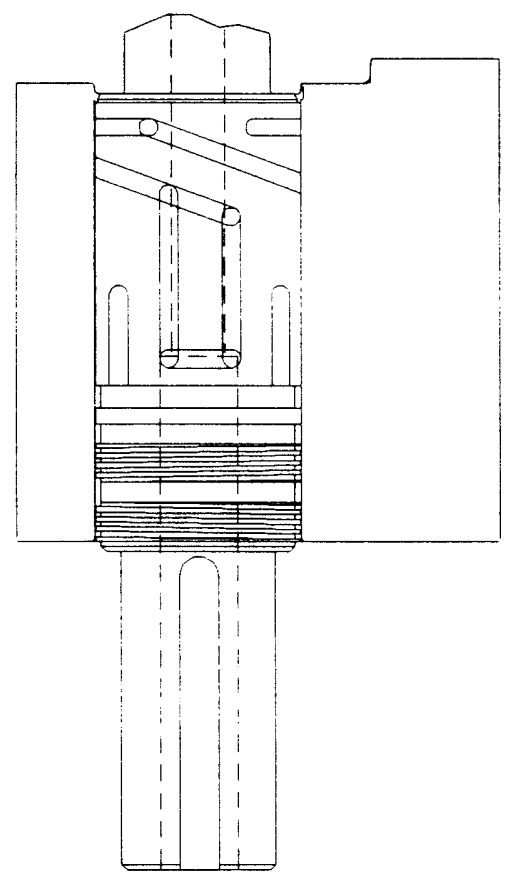

Figure 8.3. Pad layout of the pulley-support radial bearing of the cluster spindles. 
Table 8.2.

\begin{tabular}{|c|c|c|c|c|}
\hline $\begin{array}{l}\text { Operating Conditions } \\
\text { Supply Pressure (Pa,psi,atm) } \\
\text { Eccentricity Ratio ec/ep } \\
\text { Pocket Outlet Land Eccentricity } \\
\text { Compensator Eccentricity }\end{array}$ & $\begin{array}{l}\text { Ps } \\
\text { lamda } \\
\text { ep } \\
\text { ec }\end{array}$ & $\begin{array}{r}4.17 E+06 \\
1 \\
0.01 \\
0.01\end{array}$ & 600 & 40.8 \\
\hline $\begin{array}{l}\text { Fluid Properties } \\
\text { Dynamic Viscosity ( } \mathrm{N} / \mathrm{m})\end{array}$ & mu & 0.0013 & & \\
\hline $\begin{array}{l}\text { Bearing Geometry } \\
\text { Diameter (m,mm,in) } \\
\text { Axial Length }(\mathrm{m}, \mathrm{mm}, \mathrm{in}) \\
\text { Number of Grooves } \\
\text { Groove Rib Ratio } \\
\text { Pocket Rib Ratio }\end{array}$ & $\begin{array}{l}D \\
L \\
N\end{array}$ & $\begin{array}{r}0.034 \\
0.0465 \\
4 \\
1 \\
1\end{array}$ & $\begin{array}{r}34 \\
46.48\end{array}$ & $\begin{array}{l}1.3386 \\
1.8299\end{array}$ \\
\hline $\begin{array}{l}\text { Groove Rib Width }(\mathrm{m}, \mathrm{mm}, \text { in) } \\
\text { Groove Width }(\mathrm{m}, \mathrm{mm}, \mathrm{in}) \\
\text { Pocket Rib Width }(\mathrm{m}, \mathrm{mm}, \mathrm{in}) \\
\text { Pocket Width }(\mathrm{m}, \mathrm{mm}, \mathrm{in})\end{array}$ & $\begin{array}{l}\text { br } \\
\text { bg } \\
\text { brp } \\
\text { bp }\end{array}$ & $\begin{array}{l}0.0134 \\
0.0134 \\
0.0134 \\
0.0134\end{array}$ & $\begin{array}{l}13.4 \\
13.4 \\
13.4 \\
13.4\end{array}$ & $\begin{array}{l}0.526 \\
0.526 \\
0.526 \\
0.526\end{array}$ \\
\hline Nominal Gap $(\mathrm{m}, \mu \mathrm{m}, \mathrm{in})$ & ho & $1.40 \mathrm{E}-05$ & 13.97 & 0.00055 \\
\hline Compensator Land Length ( $\mathrm{m}, \mathrm{mm}$, in) & Lc & 0.0025 & 2.5 & 0.098 \\
\hline Axial Land Length $(\mathrm{m}, \mathrm{mm}$, in) & La & 0.003 & 3 & 0.118 \\
\hline Drain Groove Circumferential Width ( $\mathrm{m}, \mathrm{mm}$,in) & bd & 0.003 & 3 & 0.118 \\
\hline Drain Groove Axial Length (m,mm,in) & Ld & 0.015 & 15 & 0.591 \\
\hline Pocket Circumferential Land Width (m, mm,in) & Lac & 0.0052 & 5.2 & 0.204 \\
\hline $\begin{array}{l}\text { Twist Angle (radians, degrees) } \\
L t / \text { maximum } L t \text { ) }\end{array}$ & phi & $\begin{array}{r}2.443 \\
0.37\end{array}$ & 140 & \\
\hline $\begin{array}{l}\text { Lt/(maximum Lt) } \\
\text { Twist Ratio }\end{array}$ & & $\begin{array}{r}0.37 \\
0.365\end{array}$ & & \\
\hline $\begin{array}{l}\text { Twist Length (m,mm,in) } \\
\text { Groove Skew Angle (radians, degrees) }\end{array}$ & Lt & $\begin{array}{l}0.015 \\
1.221\end{array}$ & $\begin{array}{r}15.163 \\
69.9\end{array}$ & 0.597 \\
\hline Actual Groove Width at Skew $(\mathrm{m}, \mathrm{mm}$, in) & & 0.005 & 4.58 & 0.180 \\
\hline Resistance Ratio & & 1.21 & & \\
\hline $\begin{array}{l}\text { Bearing Load Capacity } \\
\text { Location of Effective Bearing Force }\end{array}$ & Lcen_L & 0.451 & & \\
\hline Horizontal Force ( $N, k N, l b f) @ ~ e c$, ep given above & $\mathrm{Fx}^{-}$ & -27.3 & -0.027 & -6.1 \\
\hline Vertical Force (N,kN, Ibf) @ ec, ep given above & Fy & 21.6 & 0.022 & 4.9 \\
\hline $\begin{array}{l}\text { Resultant Force ( } \mathrm{N}, \mathrm{kN}, \mathrm{lbf}) @ \text { @ ec, ep given above } \\
\text { Angle of Resultant from Vertical (degrees) }\end{array}$ & $\begin{array}{l}\text { Fres } \\
\text { theta }\end{array}$ & $\begin{array}{l}34.9 \\
51.6\end{array}$ & 0.035 & 7.8 \\
\hline Load Carrying Efficiency @ ec, ep given above & Feff & 0.005 & & \\
\hline Specific Stiffness @ ec, ep given above & Kspec & 0.853 & & \\
\hline $\begin{array}{l}\text { Initial Specific Stiffness } \\
\text { Load Carrying Efficiency @ 75\% of gap closure }\end{array}$ & $\begin{array}{l}\text { Kspec,0 } \\
\text { Feff,0.75 }\end{array}$ & $\begin{array}{l}0.853 \\
0.277\end{array}$ & & \\
\hline $\begin{array}{l}\text { Flow Rate and Pumping Power } \\
\text { Flow Rate (m^ } 3 / \mathrm{s}, \mid \mathrm{pm}, \mathrm{gpm}) \\
\text { Pumping Power }(\mathrm{W}, \mathrm{kW}, \mathrm{hp}) \\
\text { Specific Flow Rate }\end{array}$ & $\begin{array}{l}\text { Osupply } \\
\text { Ppump } \\
\text { Ospec }\end{array}$ & $\begin{array}{r}1.36 \mathrm{E}-05 \\
56.7 \\
8.1\end{array}$ & $\begin{array}{r}0.82 \\
0.057\end{array}$ & $\begin{array}{r}0.22 \\
0.076\end{array}$ \\
\hline
\end{tabular}


The thrust bearings of the spindles are developed using the pulley-support shafts, as shown in Figure 8.4. The thrust bearing is of the type Thrust Bearing \#3 described in Chapter 5. The dimensional detail and the performance of the bearing are shown in Table 8.3. The thrust bearing is capable of supporting approximately 1150 pounds in the pushing direciton and 240 pounds in the pulling direction when supplied with a pressure of 600 psig. The stiffness is $952,000 \mathrm{lb}_{\mathrm{f}} /$ in using $600 \mathrm{psig}$. The flow rated requied for the thrust bearing at this pressure is $0.28 \mathrm{gpm}$ when a total clearance of $33 \mu \mathrm{m}$ is used.

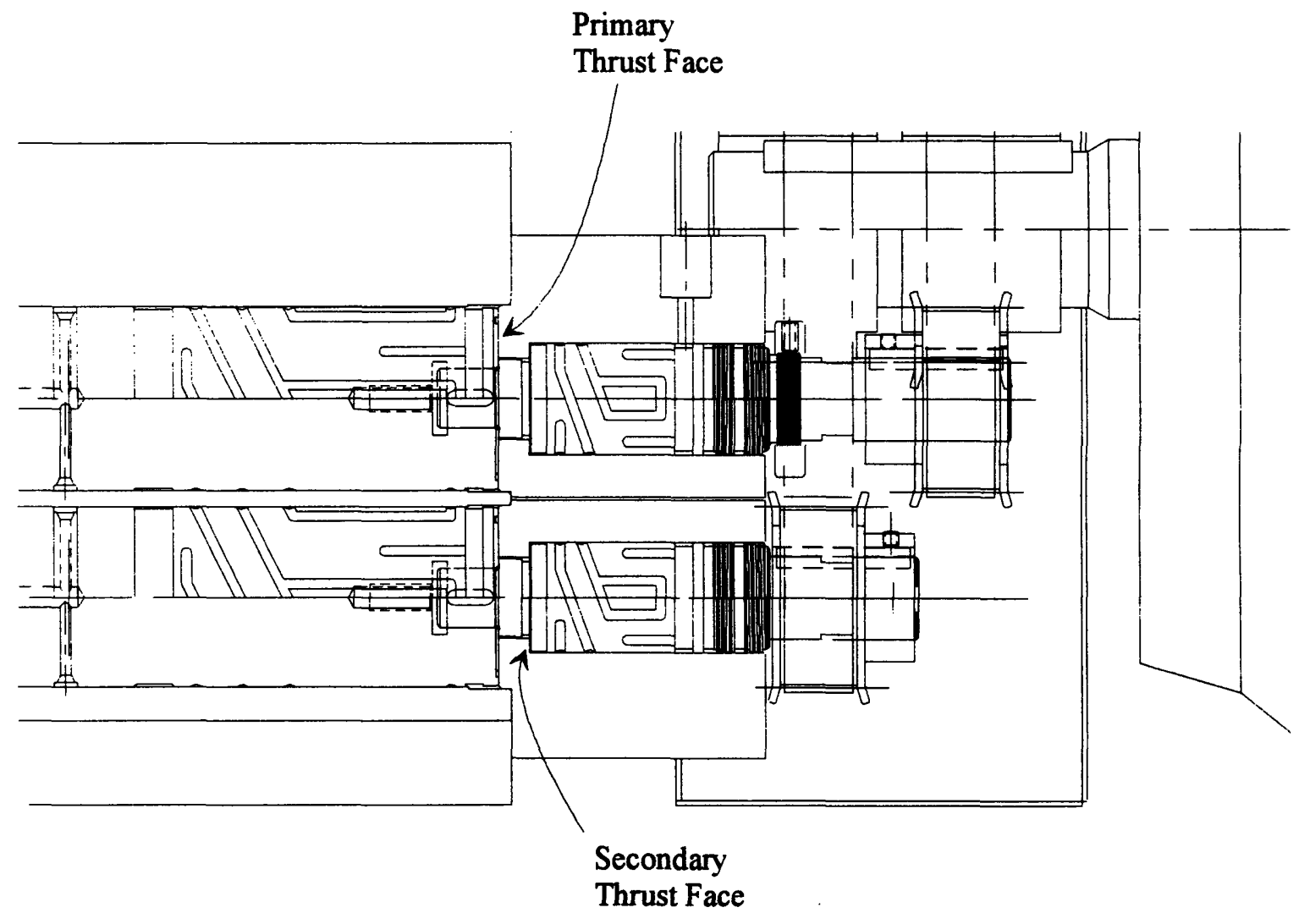

Figure 8.4. View of the rear of the cluster spindle assembly, illustrating the thrust bearing system developed using the rear pulley-support shafts. 
Table 8.3

\begin{tabular}{|c|c|c|c|c|}
\hline \multicolumn{5}{|l|}{ Operating Conditions } \\
\hline Supply Pressure (Pa,psi,atm) & Ps & 4.17E + 06 & 600 & 40.8 \\
\hline Deflection from Equilibrium $(m, \mu \mathrm{m}, \mathrm{in})$ & d & $1.00 \mathrm{E}-06$ & 1.00 & 0.00004 \\
\hline \multicolumn{5}{|l|}{ Fluid Properties } \\
\hline Dynamic Viscosity (N s/m) & mu & 0.0013 & & \\
\hline \multicolumn{5}{|l|}{ Bearing Geometry } \\
\hline Outer Thrust Diameter (m, $\mathrm{mm}$, in) & Do & 0.055 & 55 & 2.165 \\
\hline Inner Thrust Diameter (m, mm, in) & $\mathrm{Di}$ & 0.025 & 25 & 0.984 \\
\hline Secondary Face Outer Diameter $(\mathrm{m}, \mathrm{mm}, \mathrm{in})$ & D2 & 0.034 & 34 & 1.339 \\
\hline Primary Face Land Width $(\mathrm{m}, \mathrm{mm}$, in) & bo & 0.0025 & 2.5 & 0.098 \\
\hline Diameter to Primary Land $(\mathrm{m}, \mathrm{mm}$, in) & D3 & 0.05 & 50 & 1.969 \\
\hline Supply Groove Width (m, mm,in) & bs & 0.00076 & 0.76 & 0.030 \\
\hline Outer Diameter of Secondary Land $(\mathrm{m}, \mathrm{mm}, \mathrm{in})$ & D1 & 0.03248 & 32.48 & 1.279 \\
\hline Total Clearance (hos + hop) $(m, \mu \mathrm{m}$, in) & htot & 0.000033 & 33 & 0.00130 \\
\hline Equilibrium Pocket Pressure Ratio & peq_Ps & 0.173 & & \\
\hline Equilibrium Clearance Ratio & hop_hos & 1.202 & & \\
\hline Nominal Primary Clearance $(m, \mu m, i n)$ & hōp & $1.80 \mathrm{E}-05$ & 18.0 & 0.00071 \\
\hline Nominal Secondary Clearance $(m, \mu m, i n)$ & hos & $1.50 \mathrm{E}-05$ & 14.98 & 0.00059 \\
\hline \multicolumn{5}{|l|}{ Bearing Load Capacity } \\
\hline Pocket Pressure / Supply Pressure @ $\delta$ above & p1_Ps & 0.232 & & \\
\hline Load Capacity (N,kN,Ibf) @ $\delta$ above & $\bar{F}$ & 371 & & \\
\hline Load Carrying Efficiency @ $\delta$ above & Feff & 0.047 & & \\
\hline Specific Stiffness @ $\delta$ above & Kspec & 0.779 & & \\
\hline Initial Specific Stiffness & Kspec,o & 0.700 & & \\
\hline Primary Load Carrying Efficiency @ pct = 0.75 & Feff, 0.75 & 0.657 & & \\
\hline Secondary Load Carrying Efficiency @ pct = 0.75 & Feff, -0.75 & -0.139 & & \\
\hline \multicolumn{5}{|l|}{ Flow Rate and Pumping Power } \\
\hline Overall Bearing Resistance & Rbear & $2.337 E+11$ & & \\
\hline Supply Flow Rate $\left(m^{`} 3 / \mathrm{s}, 1 \mathrm{pm}, \mathrm{gpm}\right)$ & Qsupply & $1.78 \mathrm{E}-05$ & 1.07 & 0.28 \\
\hline Specific Flow Rate & Qspec & 0.93 & & \\
\hline Pumping Power (W,kW,hp) & Ppump & 74 & 0.07 & 0.10 \\
\hline
\end{tabular}

Consider the effect of supply pressure on the spindle performance. Table 8.4 lists the radial stiffness, thrust capacity, temperature rise, and flow rate as a function of supply pressure. All calculations were performed using the mean radial clearance of $0.00055^{\text {" }}$ (with tolerance, the clearance will be 0.0005 " to 0.0006 "). The shaft is steel. The first 
radial stiffness shown is that which would occur if the $56 \mathrm{~mm}$ diameter shaft were extended 4 inches from the front of the air seal. Although this configuration unrealistic, applying a force at this location applies the same moment to the spindle that would occur if a force were applied at the tool-point. The second spindle stiffness shown is that at the front face of the Hydra-Lock tool-holder, which is at a location 1.25" from the front of the air seal. As shown, increasing the supply pressure from 400 psig to 600 psig increases the radial spindle stiffness by only about $15 \%$. However, 1,000 pounds of thrust capacity is needed for this application, so a supply pressure of 600 psig is required. As shown, the temperature rise is quite low at $3,000 \mathrm{rpm}$, the maximum operating speed of the spindle. The total flow rate to the spindle assembly at this mean clearance is $4.76 \mathrm{gpm}$ at $600 \mathrm{psig}$; this represents only $1.25 \mathrm{~kW}$ of pumping power.

Table 8.4

\begin{tabular}{|c|c|c|c|c|c|c|}
\hline $\begin{array}{c}\text { Supply } \\
\text { Pressure } \\
\text { (psig) }\end{array}$ & $\begin{array}{c}\text { Spindle } \\
\text { Stiffness } \\
\text { @ 4" ext. } \\
\text { (lb/in) }\end{array}$ & $\begin{array}{c}\text { Spindle } \\
\text { Stiffness @ } \\
\text { tool-holder } \\
\text { (lbolin) }\end{array}$ & $\begin{array}{l}\text { Pushing } \\
\text { Load } \\
\text { Capacity } \\
\text { (lb }) \\
\end{array}$ & $\begin{array}{c}\text { Pulling } \\
\text { Load } \\
\text { Capacity } \\
\left(\mathbf{l b}_{\mathrm{f}}\right)\end{array}$ & $\begin{array}{c}\text { Temp. Rise } \\
\text { @ 3,000 } \\
\text { rpm } \\
\left({ }^{\circ} \mathrm{C}\right)\end{array}$ & $\begin{array}{c}\text { 4-Spindle } \\
\text { Flow Rate } \\
\text { (gpm) }\end{array}$ \\
\hline 300 & 138,000 & 399,000 & 580 & 120 & 2.9 & 2.44 \\
\hline 400 & 149,000 & 450,000 & 770 & 160 & 2.5 & 3.20 \\
\hline 500 & 158,000 & 491,000 & 960 & 200 & 2.3 & 3.96 \\
\hline 600 & 164,000 & 522,000 & 1150 & 240 & 2.3 & 4.76 \\
\hline
\end{tabular}




\section{B. Comparison with the Rolling Element Spindle}

A comparison will now be made between the radial stiffness of the existing rollingelement spindles used in this application with that of the hydrostatic spindles used in the new cluster machine. The shaft of the rolling element spindle is shown in Figure 8.5. For comparison purposes, the spindle was extended outward by 4 inches from the front face of its housing, which is a location corresponding to the tool-point. The diameter used in this extension was 1.97 inches, which is the diameter of the spindle at its tool-holder. The stiffness of the front bearing are each $145 \mathrm{~N} / \mu \mathrm{m}$. The stiffness of the rear bearings are each $102 \mathrm{~N} / \mu \mathrm{m}$. The simulated shaft bending is shown in Figure 8.6. The spindle stiffness is $68,400 \mathrm{lb}_{\mathrm{f}} /$ in. The stiffness of the hydrostatic spindle was shown in Table 8.4 to be $164,000 \mathrm{lb}_{\mathrm{f}}$ /in using the designed supply pressure of $600 \mathrm{psig}$. The hydrostatic spindle is therefore 2.4 times more stiff than the rolling element spindle it will replace.

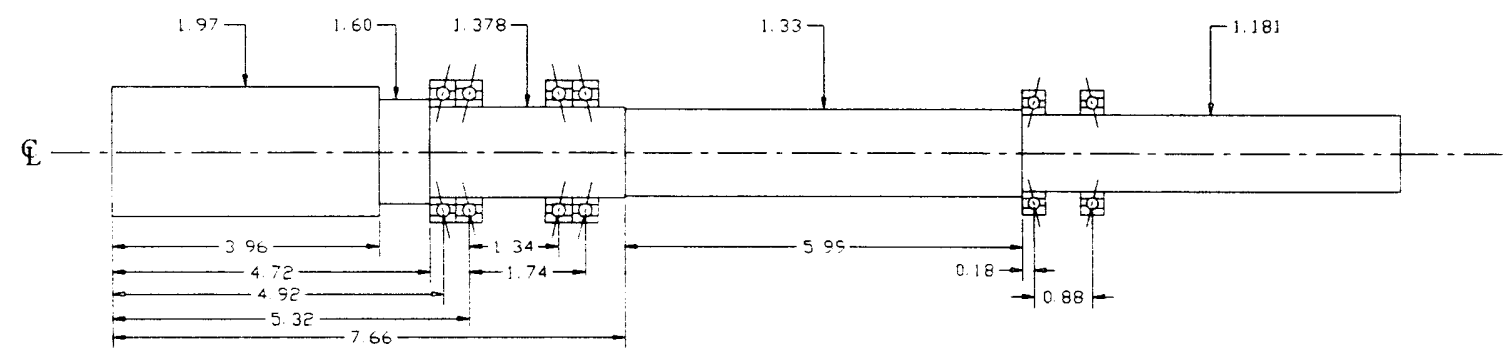

Figure 8.5. Configuration of one of the rolling element spindles of the prior cluster machine. The front diameter of the spindle is extended outward by 4 " from the front spindle face for comparison purposes. Dimensions are in inches. 


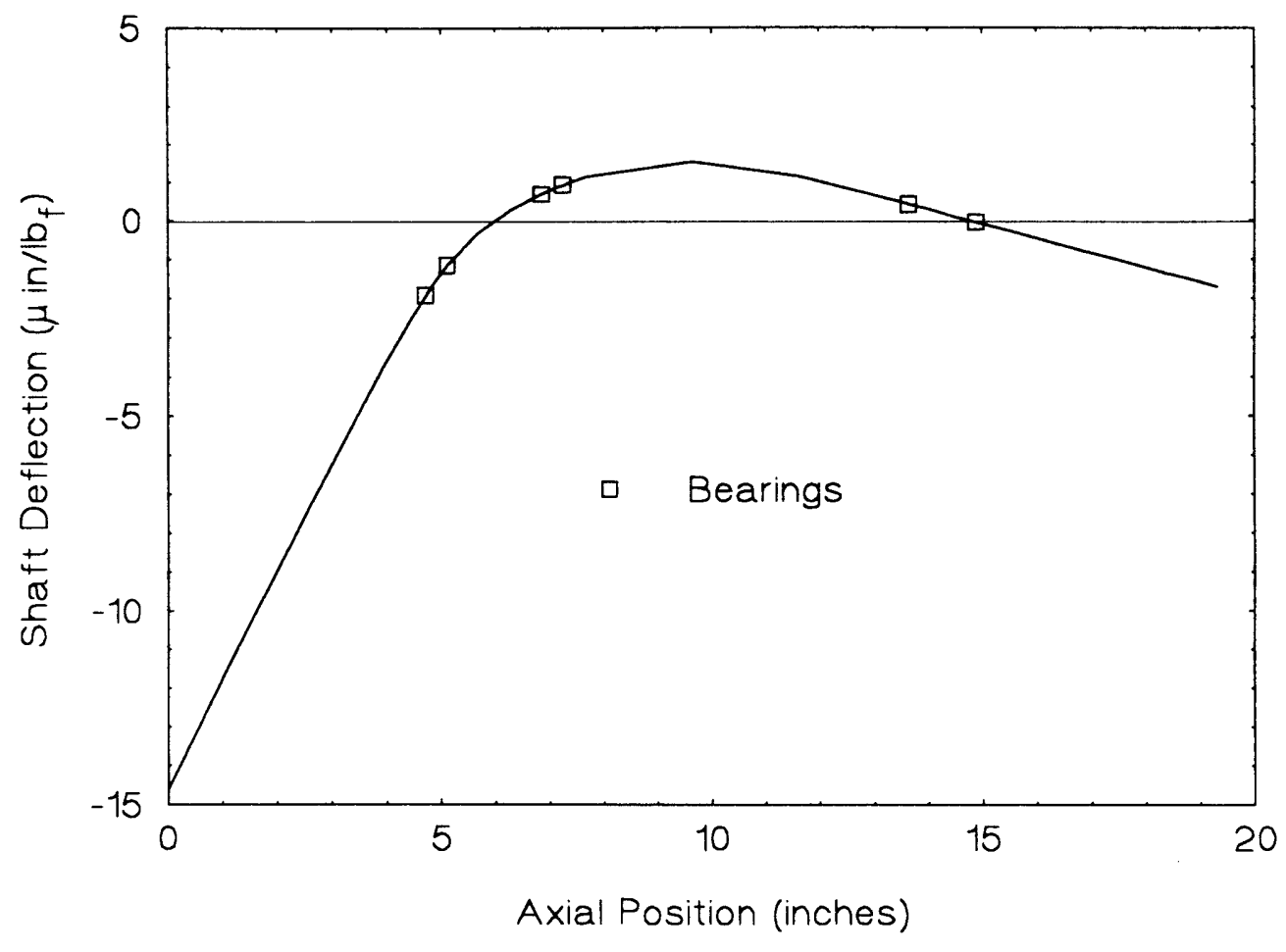

Figure 8.6. Computed deflection of the shaft of the rolling element spindle shown in Figure 8.5. 


\section{CONCLUSION}

This work investigated the application of hydrostatic bearings, particularly those of the self-compensating type, to precision machine tool spindles. First, relations were developed to calculate the hydraulic resistances of various bearing land flows in tilted and untilted orientations. These relations were then applied to predicting the load-carrying characteristics of twelve different radial hydrostatic bearings. The bearing calculations were then integrated with shaft bending calculations to predict static spindle stiffness. By integrating the bearing design with the spindle design, the geometry was optimized to provide maximum spindle stiffness. It was found that even small changes in the bearing geometry can often significantly increase the spindle stiffness. For example, decreasing the width of the lands nearest the front of the spindle can often significantly improve the spindle stiffness. These design improvements are missed when the bearing design is disconnected from the spindle design.

The design of three different hydrostatic thrust bearings were considered next. Self-compensating thrust bearings were found to provide higher stiffness and load capacity, as well as lower flow rates, than thrust bearings that use fixed-compensation. The self-compensating thrust bearings can also be readily supplied with aqueous fluids such as water-based coolants without potential clogging problems.

Methods were also developed to calculate the frictional and thermal characteristics of hydrostatic bearings. Relations were presented to calculate the viscous shearing power consumed by the land, pocket, and groove flow. It was determined that, when water or water-based coolants are used at moderate operating speeds, the friction developed by the 
laminar flow on the lands is often significantly lower than that developed by the turbulent flow within the pockets. Thus, as much of the bearing area should be made land area as possible in order to reduce drag as well as to increase bearing damping.

The effect of the temperature dependence of the fluid viscosity on the frictional and thermal characteristics of the bearings was considered next. A simple model was developed that can be used to account for the change in the viscosity of the fluid as its temperature increases while flowing through the bearing. It was found that, at moderate and high operating speeds, the change in viscosity caused by bearing temperature rise can have a significant effect on the bearing performance.

The relations developed in the thesis were compared to experimental data collected on three prototype test spindles. These spindles were found to have excellent qualities that represent a significant advance in machine tool spindle technology. The models developed in this work were found to agree with the experimental results within a reasonably close margin.

Finally, the design of a machine that drills clusters of holes in steel components was presented. Hydrostatic spindles were found to to be clearly superior to rolling element spindles this application. The use of hydrostatic bearings enabled the diameter of the shafts to be increased, thereby greatly increasing the radial stiffness of the spindles. It is expected that the new machine will be able to drill holes with fewer passes than the old machine, thereby reducing manufacturing costs. 


\section{REFERENCES}

1. Fuller, Dudley D. Theory and Practice of Lubrication for Engineers, Second Edition, John Wiley \& Sons, New York, 1984.

2. Stansfield, F.M., Hydrostatic Bearings for Machine Tools and Similar Applications, The Machinery Publishing Co., LTD., London, 1970.

3. Bassani, R. And B. Piccigallo, Hydrostatic Lubrication, Elsevier Science Publishers, Amsterdam, 1992.

4. Slocum, A.H., Self-Compensating Hydrostatic Bearings for Supporting Round Shaft for Rotary and/or Linear Motion, U.S. Patent \#5,281,032, February 20, 1994.

5. Arneson, H.E.G., Hydrostatic Bearing Structure, U.S. Patent \# 3,305,282, February 21, 1967.

6. Wasson, Kevin L. And Alexander H. Slocum, Self-Compensating Hydrostatic Bearing for Rotary Motion Applications, claim submitted June 1995, patent pending.

7. Wardle, F.P, S.J. Lacey and S.Y. Poon, "Dynamic and Static Characteristics of a Wide Speed Range Machine Tool Spindle," Precision Engineering, Volume 5, Number 4, October 1983, pp. 175-183.

8. Roark, R.J. and W.C. Young, Formulas for Stress and Strain, 5th Edition, McGrawHill Book Co., New York, 1975.

9. Slocum, Alexander H., and Kevin L. Wasson, Self-Compensating Hydrostatic Thrust Bearings, claim submitted January 1996, patent pending. 
10. Slocum, A.H., Low Profile Self Compensated Hydrostatic Thrust Bearing, claim submitted 1995, patent pending.

11. G.E. Fluid Flow Data Book, Genium Publishing, May 1971.

12. El Telbany, M. M. M., and A. J. Reynolds, "The Structure of Turbulent Plane Couette Flow," Journal of Fluids Engineering, Volume 104, 1982, pp. 367-372.

13. Shinkle, J.N., and K.G. Hornung, "Frictional Characteristic of Liquid Hydrostatic Journal Bearings," Transactions of the American Society of Mechanical Engineers, Journal of Basic Engineering, Volume 87, 1965, pp. 163-169.

14. Wasson, Kevin L., "Hydrostatic Radial Bearings for High Speed Precision Machine Tool Applications," M.S. Thesis, Department of Mechanical Engineering, Massachusetts Institute of Technology, Cambridge, MA, January 1994.

15. Axis of Rotation: Methods for Specifying and Testing, ANSI Standard B89.3.4M1985, American Society of Mechanical Engineers, United Engineering Center, 345 East 47th Street, New York, NY 10017.

16. Bathe, Klaus-Jurgen, Finite Element Procedures in Engineering Analysis, PrenticeHall, Inc.: Englewood Cliffs, NJ, 1982.

17. Hussain, A. K. M. F., and W. C. Reynolds, "Measurements in Fully Developed Turbulent Channel Flow," Transactions of the American Society of Mechanical Engineers, Journal of Fluids Engineering, Volume 97, 1975, pp. 568-578.

18. Shah, R. K., "A Correlation for Laminar Hydrodynamic Entry Length Solutions for Circular and Noncircular Ducts," Journal of Fluids Engineering, Volume 100, June 1978, pp. 177-179. 


\section{NOMENCLATURE}

A area

$\mathrm{C}_{\mathrm{f}} \quad$ skin friction factor

D shaft diameter; bearing diameter eccentricity of shaft with respect to bearing housing

E elastic modulus

F force

f force per unit length

G shear modulus

h land clearance; pocket depth

I cross-sectional moment of innertia

K stiffness

k shear correction factor; thermal conductivity

L land width; groove length

n fluid viscosity-temperature exponent

$\mathrm{N}_{\mathrm{Ta}} \quad$ Taylor number

$\mathrm{p}$ pressure

p power

Q volumetric flow rate

$\mathrm{R} \quad$ hydraulic resistance; bearing radius

$r$ radial coordinate

$\mathrm{Re} \quad$ Reynolds number

$\mathrm{u} \quad \mathrm{x}$-direction fluid velocity

$v \quad y$-direction fluid velocity

V shaft surface velocity

w $\quad$-direciton fluid velocity

$\mathrm{x} \quad$ principal direction

y principal direction

z principal direction

$\beta \quad$ land tilt angle; groove skew angle

$\delta$ deflection

$\gamma \quad$ resistance ratio; shear strain

$\mu \quad$ fluid dynamic viscosity

$\checkmark$ fluid kinematic viscosity; Poisson's ratio

$\theta \quad$ angular coordinate

$\rho \quad$ fluid density

$\tau \quad$ shear stress 


\section{Subscripts}

$\begin{array}{ll}\text { a } & \text { atmospheric } \\ \text { b } & \text { bottom } \\ \text { c } & \text { compensator } \\ \text { cen } & \text { effective center } \\ \text { d } & \text { drain } \\ \text { e } & \text { end } \\ \text { eff } & \text { efficiency } \\ \text { m } & \text { middle } \\ \text { o } & \text { nominal; equilibrium } \\ \text { p } & \text { pocket } \\ \text { s } & \text { supply; shear } \\ \text { spec } & \text { specific } \\ t & \text { top }\end{array}$




\section{APPENDIX A: BEAM THEORY AND DEVELOPMENT OF THE FINITE ELEMENT MATRICES}

In order to develop an adequate model which is readily usable in spindle design practice, certain simplifying assumptions about the bending of the shaft must be made. Beam theory that includes shear deformations will be employed to solve for the deflection of the neutral axis of the shaft as a function of axial position, $w(x)$. In the development of this analysis, the following assumptions will be made:

1. A normal to the neutral axis of the shaft remains straight during deformation.

2. The shaft material is isotropic and homogeneous.

3. The shaft material remains in the elastic region.

4. Shaft strains are small enough such that the response is linear (i.e., the deflection at any point on the shaft is linearly proportional to the applied load).

The first assumption is illustrated in Figure A.1. The dark line represents a crosssection of the shaft, initially perpendicular to the neutral axis, which remains straight during shaft deformation. If the shaft is long and slender and the effects of shear forces are negligible, then the cross-section will remain perpendicular to the neutral axis after deformation. However, the presence of a shear strain, $\gamma$, results in a total rotation of the cross-section given by:

$$
\theta=\frac{d w}{d x}-\gamma
$$




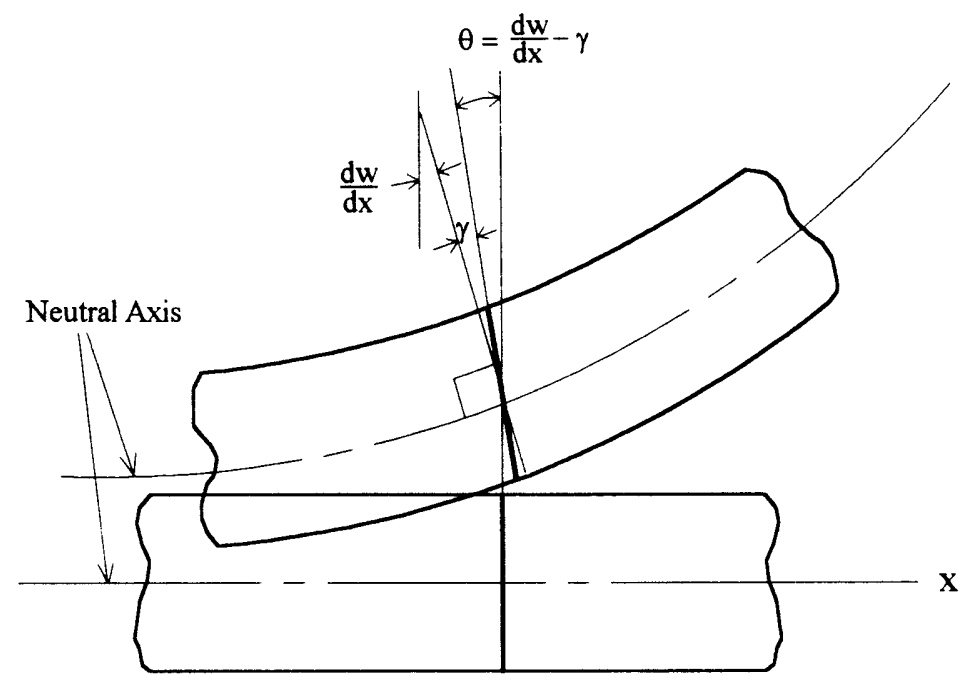

Figure A.1. Deformation of a shaft cross-section, illustrating the relationship between the shaft slope, $\mathrm{dw} / \mathrm{dx}$, the effective shear strain, $\gamma$, and the total rotation of the shaft crosssection, $\beta$.

Note that the shear strain used in Equation (A.1) and illustrated geometrically in Figure A. 1 is an equivalent shear strain of the cross-section. The shear strain in an actual shaft will vary across its cross-section, being largest in magnitude along the neutral axis and smallest in magnitude near the upper and lower edges. To accommodate the assumption of a straight cross-section, an equivalent constant shear strain is defined such that, acting over an equivalent shear area $A_{s}$, the resulting shear strain energy will be the same as that in the actual shaft:

$$
\gamma=\frac{\tau}{G}=\frac{V}{G A_{s}}
$$

where $\tau$ is the shear stress, $G$ is the shear modulus, and $V$ is the shear force. As a matter of convenience, the equivalent shear area is replaced with a shear correction factor, 


$$
k=\frac{A_{s}}{A}
$$

the value of which has been computed for hollow and solid shafts and is available in the literature [8]. A general relation for $k$ is developed in this work for a hollow shaft with an inner diameter $D_{i}$, and an outer diameter $D_{o}$ (see Chapter 4). Using the shear correction factor, the equivalent shear strain now becomes:

$$
\gamma=\frac{V}{G A k}
$$

and the total rotation of the shaft cross-section in Equation (A.1) can now be adequately computed

Consider a beam of length $L$, moment of inertia $I$, bulk modulus $E$, shear modulus $G$, and shear correction factor $k$. The beam is loaded with applied moments, $\mathrm{m}$, and forces per unit length, $f$. Summing up the potential energies caused by bending deformations and shear deformations, plus those of the external loads, the total potential energy of the beam is given by [16]:

$$
\Pi=\frac{E I}{2} \int_{0}^{L}\left(\frac{d \theta}{d x}\right)^{2} d x+\frac{G A k}{2} \int_{0}^{L}\left(\frac{d w}{d x}-\theta\right)^{2} d x-\int_{0}^{L} f w d x-\int_{0}^{L} m \theta d x
$$

The static equilibrium displacement of the shaft can be found by minimizing the total potential energy in the beam. This is accomplished by using the stationarity condition

$$
\begin{aligned}
& \delta \Pi=0 \\
& E I \int_{0}^{L}\left(\frac{d \theta}{d x}\right) \delta\left(\frac{d \theta}{d x}\right) d x+G A k \int_{0}^{L}\left(\frac{d w}{d x}-\theta\right) \delta\left(\frac{d w}{d x}-\theta\right) d x-\int_{0}^{L} f \delta w d x-\int_{0}^{L} m \delta \theta d x=0
\end{aligned}
$$

and solving this equation for the displacements. 
Finite element methods will now be used to implement the beam theory and solve Equation (A.6) for the shaft deflections. Four-node beam elements illustrated schematically in Figure A.2 will be used in this work. The continuous deflection and rotation of the beam is approximated with four nodal translations and four nodal rotations.

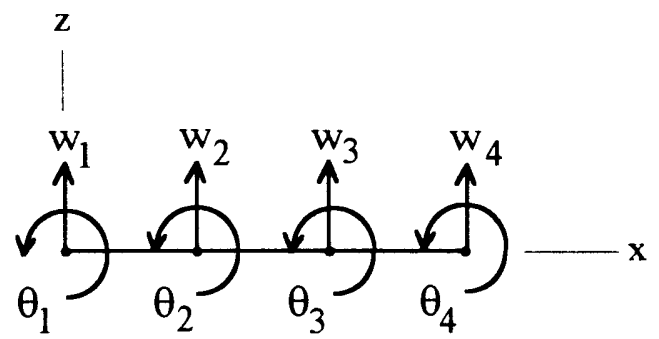

Figure A.2. Schematic diagram of a four-node beam element. Shown are the four nodal translations and the fur nodal rotations.

The displacements shown in Figure A.2 are discrete nodal displacements. Interpolation functions are needed in order to approximate the continuous deformations of the element when the total potential energy is minimized. The continuous and discrete deflections and rotations are related to each other with interpolation functions:

$$
\begin{gathered}
w=\sum_{i=1}^{4} h_{i} w_{i} \\
\theta=\sum_{i=1}^{4} h_{i} \theta_{i}
\end{gathered}
$$

where $h_{i}$ are the four interpolation functions, $w$ and $\theta$ are the continuous deflections and rotations of the element, and $w_{i}$ and $\theta_{i}$ are the discrete nodal deflections and rotations of the element. For the four-node element used in this analysis, the interpolation functions are cubic: 


$$
\begin{aligned}
& h_{1}=-\frac{9}{2}\left(\frac{x}{L}\right)^{3}+9\left(\frac{x}{L}\right)^{2}-\frac{11}{2}\left(\frac{x}{L}\right)+1 \\
& h_{2}=\frac{27}{2}\left(\frac{x}{L}\right)^{3}-\frac{45}{2}\left(\frac{x}{L}\right)^{2}+9\left(\frac{x}{L}\right) \\
& h_{3}=-\frac{27}{2}\left(\frac{x}{L}\right)^{3}+18\left(\frac{x}{L}\right)^{2}-\frac{9}{2}\left(\frac{x}{L}\right) \\
& h_{4}=\frac{9}{2}\left(\frac{x}{L}\right)^{3}-\frac{9}{2}\left(\frac{x}{L}\right)^{2}+\left(\frac{x}{L}\right)
\end{aligned}
$$

These functions were derived such that they equal 1 at their corresponding node and equal zero at all other nodes in the element. For example, $h_{l}$ is equal to 1 at $x / L=0$, which is the location of the first node, and equal to zero at $x / L=1 / 3,2 / 3,1$, which are the locations of nodes two, three, and four, respectively. The derivatives of the interpolation function will also be needed:

$$
\begin{aligned}
& h_{1}^{\prime}=\frac{\partial h_{1}}{\partial x}=-\frac{27}{2}\left(\frac{x}{L}\right)^{2} \frac{1}{L}+18\left(\frac{x}{L}\right) \frac{1}{L}-\frac{11}{2} \frac{1}{L} \\
& h_{2}^{\prime}=\frac{\partial h_{2}}{\partial x}=\frac{81}{2}\left(\frac{x}{L}\right)^{2} \frac{1}{L}-45\left(\frac{x}{L}\right) \frac{1}{L}+9 \frac{1}{L} \\
& h_{3}^{\prime}=\frac{\partial h_{3}}{\partial x}=-\frac{81}{2}\left(\frac{x}{L}\right)^{2} \frac{1}{L}+36\left(\frac{x}{L}\right) \frac{1}{L}-\frac{9}{2} \frac{1}{L} \\
& h_{4}^{\prime}=\frac{\partial h_{4}}{\partial x}=\frac{27}{2}\left(\frac{x}{L}\right)^{2} \frac{1}{L}-9\left(\frac{x}{L}\right) \frac{1}{L}+\frac{1}{L}
\end{aligned}
$$

In matrix form, the continuous and discrete deformations and their derivatives are represented by:

$$
\begin{aligned}
& w=\underline{H}_{w} \underline{\hat{u}} \\
& \frac{\partial w}{\partial x}=\underline{B}_{w} \underline{\hat{u}}
\end{aligned}
$$




$$
\begin{aligned}
& \theta=\underline{H}_{\theta} \underline{\hat{u}} \\
& \frac{\partial \theta}{\partial x}=\underline{B}_{\theta} \underline{\hat{u}}
\end{aligned}
$$

where:

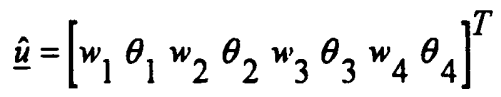

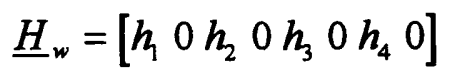

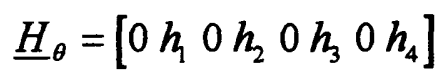

$$
\begin{aligned}
& \underline{B}_{w}=\left[\frac{\partial h_{1}}{\partial x} 0 \frac{\partial_{2}}{\partial x} 0 \frac{\partial h_{3}}{\partial x} 0 \frac{\partial h_{4}}{\partial x} 0\right] \\
& \underline{B}_{\theta}=\left[0 \frac{\partial_{1}}{\partial x} 0 \frac{\partial_{2}}{\partial x} 0 \frac{\partial_{3}}{\partial x} 0 \frac{\partial_{4}}{\partial x}\right]
\end{aligned}
$$

The beam deformations and their derivatives have now been properly interpolated from their nodal values. Substituting these relations into Equation (A.6), the condition for stationarity becomes:

$$
\left[E I \int_{0}^{L} \underline{B}_{\theta}^{T} \underline{B}_{\theta} d x\right] \underline{\hat{\hat{u}}}+\left[G A k \int_{0}^{L}\left(\underline{B}_{w}-\underline{H}_{\theta}\right)^{T}\left(\underline{B}_{w}-\underline{H}_{\theta}\right) d x\right] \hat{\underline{\hat{u}}}-\int_{0}^{L} \underline{H}_{w}^{T} p d x-\int_{0}^{L} \underline{H}_{\theta}^{T} m d x=0
$$

This equation is commonly represented by a stiffness maxtrix, $\underline{K}$, and a load vector, $\underline{R}$ :

$$
\underline{K \hat{u}}=\underline{R}
$$

where:

$$
\begin{gathered}
\underline{K}=\underline{K}_{1}+\underline{K}_{2} \\
\underline{K}_{1}=E I \int_{0}^{L} \underline{B}_{\theta}^{T} \underline{B}_{\theta} d x \\
\underline{K}_{2}=G A k \int_{0}^{L}\left(\underline{B}_{w}-\underline{H}_{\theta}\right)^{T}\left(\underline{B}_{w}-\underline{H}_{\theta}\right) d x
\end{gathered}
$$




$$
\underline{R}=\int_{0}^{L} \underline{H}_{w}^{T} f d x+\int_{0}^{L} \underline{H}_{\theta}^{T} m d x
$$

The stiffness matrix, $\underline{K}$, is assembled by substituting the interpolation matrices, Equations (A.22) through (A.25), into Equations (A.29) and (A.30) and integrating over the length of the element. This procedure results in the following matrices:

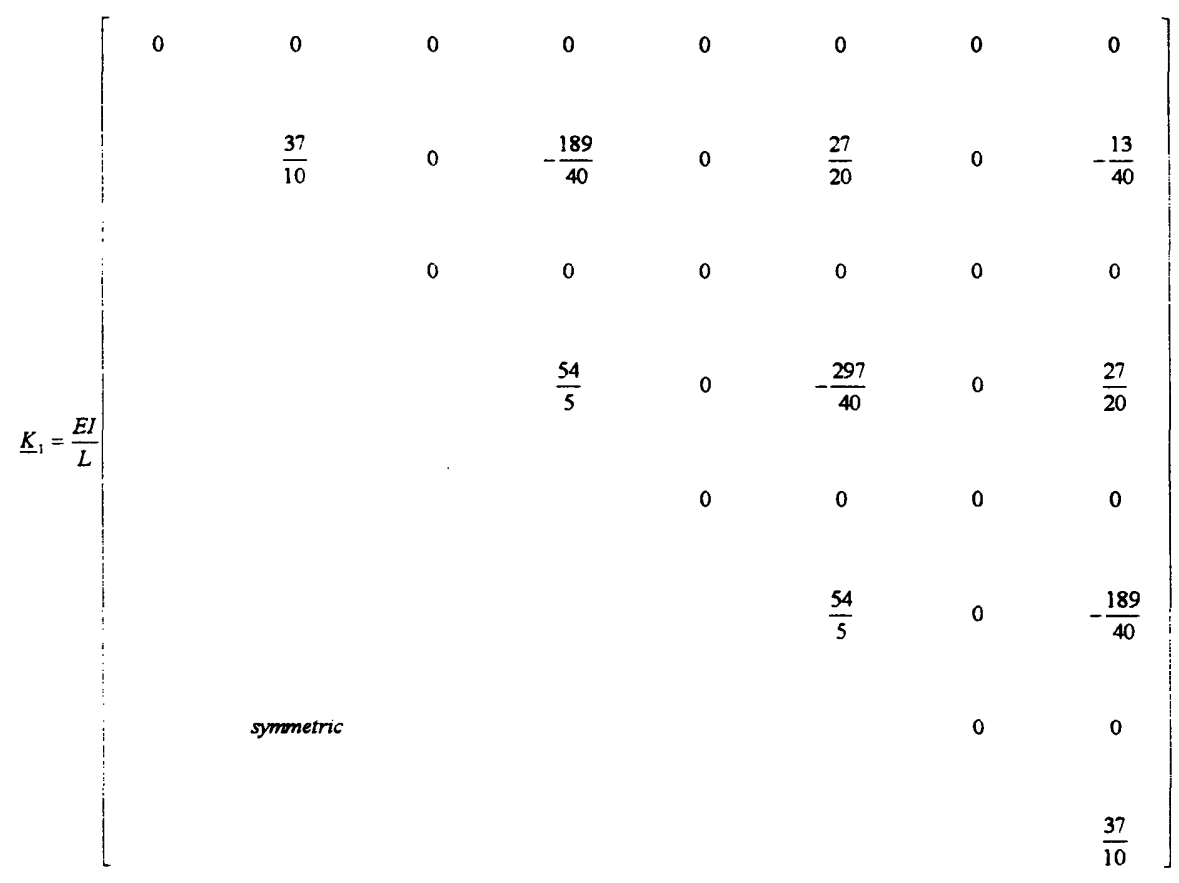

(A.32) 


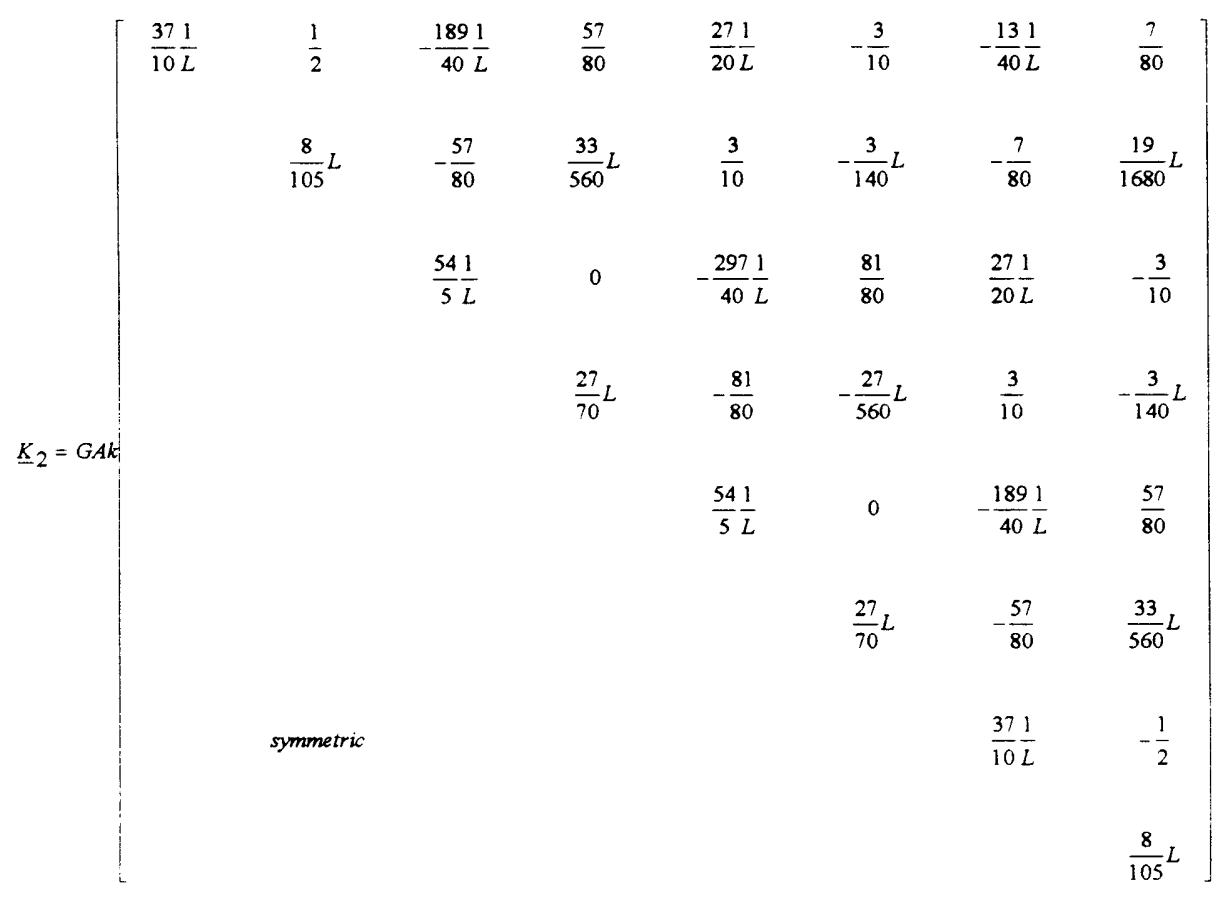

(A.33)

The right-hand-side load vector is similarly assembled by substituting Equations (A.22) and (A.23) into Equation (A.31) and integrating over the length of the element. In this work, no applied moments will be used. However, applied pressures will be used on the elements corresponding to the bearing areas. For an element with an applied force per unit length, $f$ :

$$
\underline{R}=f L\left[\begin{array}{c}
1 / 8 \\
0 \\
3 / 8 \\
0 \\
3 / 8 \\
0 \\
1 / 8 \\
0
\end{array}\right]
$$


The problem has now been reduced to solving a matrix system of equations, $\underline{K \hat{u}}=\underline{R}$, to obtain the discrete nodal point deflections and rotations, $\underline{\hat{u}}$. 


\section{APPENDIX B: SOLUTIONS TO FLUID CIRCUITS OF HYDROSTATIC BEARINGS}

The solutions to four fluid circuits commonly encountered in hydrostatic bearing design will be presented in this Appendix. The first circuit is a simple circuit used to model bearings with no leakage between their pockets. The second circuit is used to model bearings with leakage between their pockets and/or compensators. The third circuit is used to model bearings that have leakage between their pockets and/or compensators, as well as leakage between their pockets and compensators. The fourth circuit is used to model so-called step-compensated bearing that have leakage along the lengths of their compensating lands, as well as leakage between their pockets. In all of the derivations, it is assumed that the resistances are not dependent upon the flow rate of fluid through them.

\section{B.1. Fluid Circuit \#1}

The first fluid circuit is shown in Figure B.1. Fluid is pumped at supply pressure, Ps, across the compensating resistances, $\mathrm{Rc}(\mathrm{i})$, and across the outlet resistances $\mathrm{Ra}(\mathrm{i})$ to atmospheric pressure, $\mathrm{Pa}$. The flow rate through each compensator is equal to the flow rate through its corresponding pockets, and is given by:

$$
\mathrm{Q}(\mathrm{i})=\frac{\mathrm{Ps}-\mathrm{Pa}}{\mathrm{Rc}(\mathrm{i})+\mathrm{Ra}(\mathrm{i})} \quad i=1,2 \ldots N
$$

where $\mathrm{N}$ is the number of pockets. The supply flow rate is found by summing all of the pocket flow: 


$$
Q s=\sum_{i=1}^{N} Q(i)=(P s-P a)\left(\sum_{i=1}^{N} \frac{1}{R c(i)+R a(i)}\right)
$$

The corresponding pockets pressures are given by:

$$
\mathrm{P}(\mathrm{i})=\mathrm{Pa}+\left(\frac{\mathrm{Ra}(\mathrm{i})}{\operatorname{Rc}(\mathrm{i})+\mathrm{Ra}(\mathrm{i})}\right)(\mathrm{Ps}-\mathrm{Pa}) \quad i=1,2 \ldots N
$$

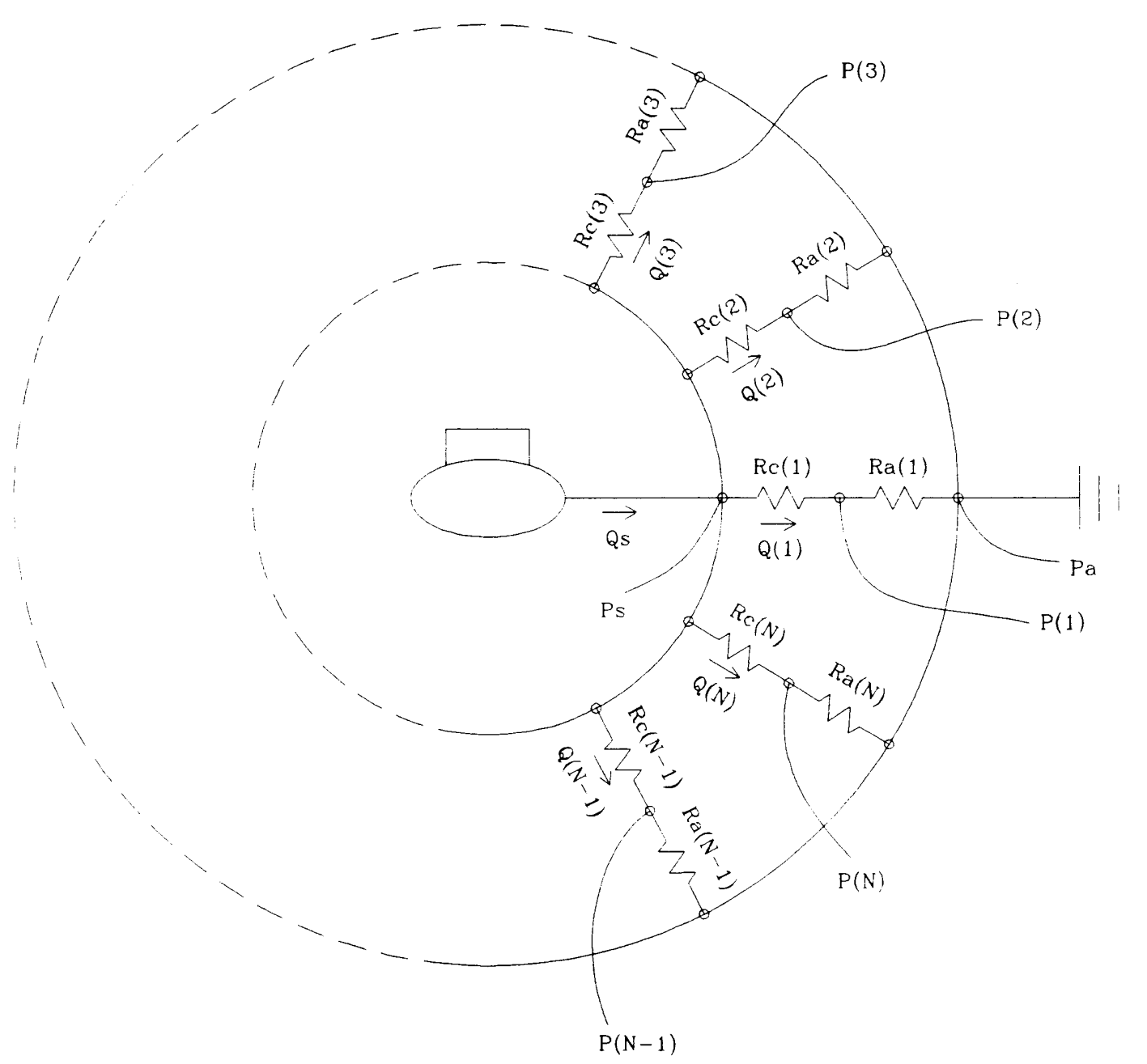

Figure B.1. Fluid circuit of a hydrostatic bearing with $\mathrm{N}$ pockets and no leakage between its compensators or its pockets. 


\section{B.2. Fluid Circuit \#2}

The second fluid circuit is shown in Figure B.2. Fluid is pumped at supply pressure, Ps, across the compensating resistances, $\operatorname{Rc}(\mathrm{i})$, to the pockets. Some of the fluid then leaks between the pockets across the leakage resistances $\mathrm{Rb}(\mathrm{i})$ before it passes across the outlet resistances $\mathrm{Ra}(\mathrm{i})$ to atmospheric pressure, $\mathrm{Pa}$. This circuit is more complicated than the first circuit because requires the solution of a systems of equations. These equations will be derived and then an algorithm to set up the matrices will be presented.

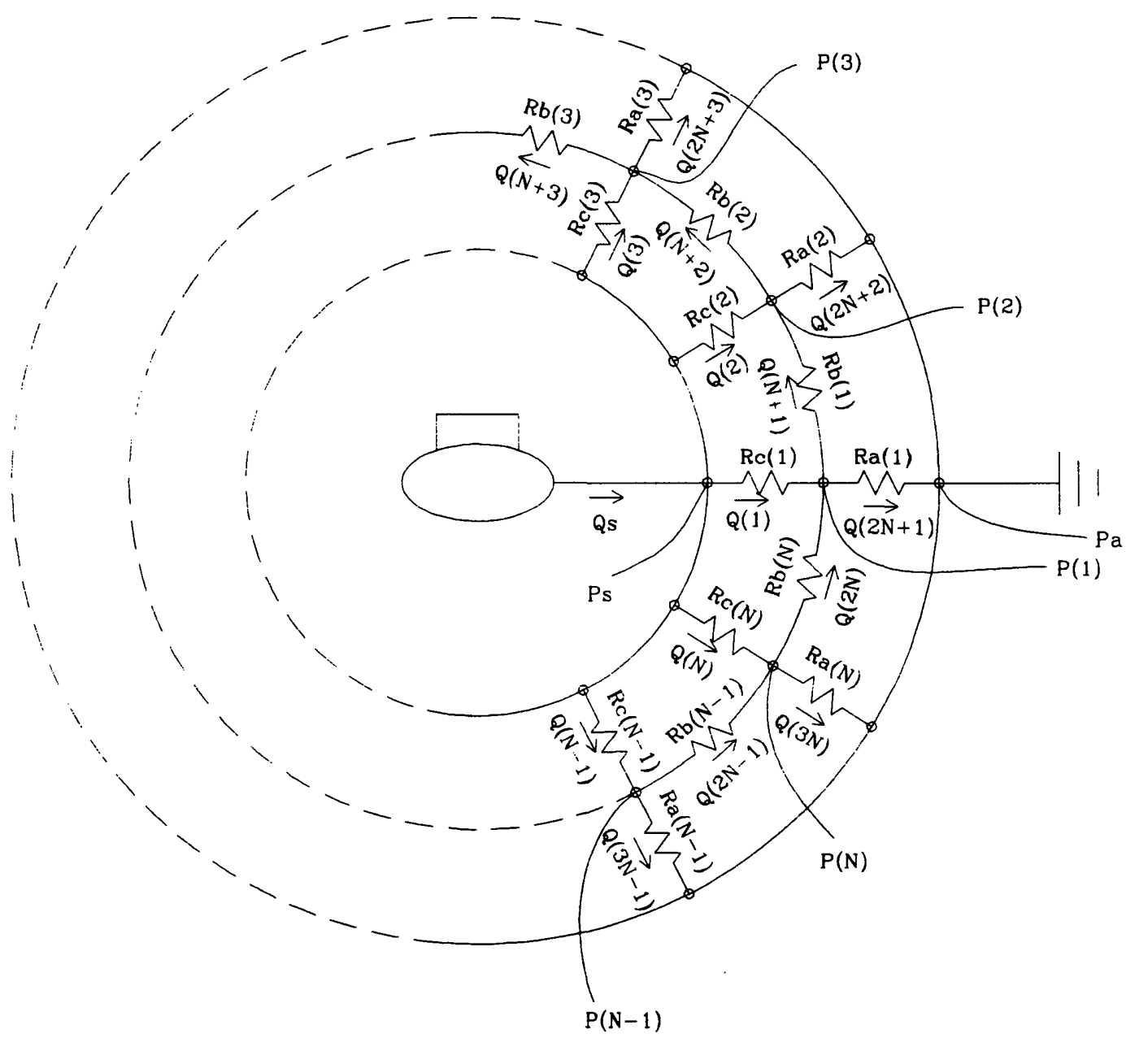

Figure B.2. Fluid circuit of a hydrostatic bearing with $\mathrm{N}$ pockets and leakage between its pockets and/or compensators. 
A brief derivation of the equations needed to solve the circuit will first be presented. A set of $3 \mathrm{~N}$ equations are required to solve for the $3 \mathrm{~N}$ unknown flow rates. The first $\mathrm{N}$ equations are determined by noting that the sum of the pressure drops around the innermost loops must equal zero:

$$
Q(i) \operatorname{Rc}(i)+Q(i+N) R b(i)-Q(i+1) R c(i+1)=0 \quad i=1,2 \ldots N
$$

The next set of $\mathrm{N}$ equations are determined by noting that the sum of the flow rates into each central node must equal zero:

$$
\mathrm{Q}(\mathrm{i})-\mathrm{Q}(2 \mathrm{~N}+\mathrm{i})-\mathrm{Q}(\mathrm{N}+\mathrm{i})+\mathrm{Q}(\mathrm{N}+\mathrm{i}-1)=0 \quad \mathrm{i}=1,2 \ldots \mathrm{N}
$$

The third set of $\mathrm{N}$ equations are determined by setting the pressure drop across the compensators and pockets equal to the bearing supply pressure difference:

$$
\mathrm{Q}(\mathrm{i}) \operatorname{Rc}(\mathrm{i})+\mathrm{Q}(2 \mathrm{~N}+\mathrm{i}) \operatorname{Ra}(\mathrm{i})=\mathrm{Ps}-\mathrm{Pa} \quad \mathrm{i}=1,2 \ldots \mathrm{N}
$$

These set of $3 \mathrm{~N}$ equations can then be solved for the $3 \mathrm{~N}$ unknown flow rates. Once the flow rates are known, the $\mathrm{N}$ pocket pressures can then be computed.

The following algorithm, written in BASIC, can be used to solve Equations B.4, B.5, and B. 6 for the unknown flow rates. First a $3 \mathrm{~N}$ by $3 \mathrm{~N}$ resistance matrix, $\mathrm{RR}(\mathrm{i}, \mathrm{j})$, will be set up:

$$
\begin{aligned}
& \text { For } i=1 \text { To } 3^{*} N \\
& \text { For } j=1 \text { To } 3^{*} N \\
& \operatorname{RR}(i, j)=0 \\
& \operatorname{Next} j \\
& \text { Next } i \\
& \text { For } i=1 \text { To } N \\
& \operatorname{RR}(i, i)=\operatorname{Rc}(i) \\
& \operatorname{RR}(i, i p(i, N))=-\operatorname{Rc}(i p(i, N)) \\
& \operatorname{RR}(i, i+N)=\operatorname{Rb}(i) \\
& \text { Next } i \\
& \text { For } i=1 \text { To } N \\
& \operatorname{RR}(i+N, i)=1 \\
& \operatorname{RR}(i+N, i+N)=-1
\end{aligned}
$$




$$
\begin{aligned}
& R R(i+N, \operatorname{im}(i, N)+N)=1 \\
& R R(i+N, i+2 * N)=-1
\end{aligned}
$$

\section{Next $i$}

$$
\begin{aligned}
& \text { For } i=1 \text { To } N \\
& \operatorname{RR}(i+2 * N, i)=\operatorname{Rc}(i) \\
& \operatorname{RR}(i+2 * N, i+2 * N)=\operatorname{Ra}(i)
\end{aligned}
$$

\section{Next I}

where $i m(i, N)$ and $i p(i, N)$ and are the following functions:

Function im(i, N)

$$
\begin{aligned}
& \text { If } \mathrm{i}=1 \text { Then } \\
& \text { im }=\mathrm{N} \\
& \text { Else } \\
& \text { im }=\mathrm{i}-1
\end{aligned}
$$

End If

End Function

Function ip(i, N)

If $i=N$ Then

$$
\text { ip }=1
$$

$$
\begin{aligned}
& \text { Else } \\
& \text { ip }=i+1
\end{aligned}
$$

End If

End Function

Next a 1 by $3 \mathrm{~N}$ pressure matrix, $\mathrm{PP}(\mathrm{i})$, is created:

$$
\begin{aligned}
& \text { For } \mathrm{i}=1 \text { To } 2 * \mathrm{~N} \\
& \text { PP(i) }=0 \\
& \text { Next } \mathrm{i} \\
& \text { For } \mathrm{i}=2 * \mathrm{~N}+1 \text { To } 3 * \mathrm{~N} \\
& \text { PP(i) }=\mathrm{Ps}-\mathrm{Pa} \\
& \text { Next } \mathrm{i}
\end{aligned}
$$

The systems of equations, $R R(i, j) Q(i)=P P(i)$, can be solved from the unknown flow rates, Q(i) using any standard matrix solution algorithm. The supply flow rate is calculated by:

$$
\mathrm{Qs}=\sum_{\mathrm{i}=1}^{\mathrm{N}} \mathrm{Q}(\mathrm{i})
$$

The pocket pressures are calculated by:

$$
P(i)=P a+Q(2 N+i) R a(i) \quad i=1,2 \ldots N
$$




\section{B.3. Fluid Circuit \#3}

The third fluid circuit is shown in Figure B.3. Fluid is pumped at supply pressure, Ps, across the compensating resistances, $\mathrm{Rc}(\mathrm{i})$, to the pockets. Some of the fluid then leaks across the leakage resistances $\mathrm{Rb}(\mathrm{i})$ and $\mathrm{Rd}(\mathrm{i})$ before it passes across the outlet resistances $\mathrm{Ra}(\mathrm{i})$ to atmospheric pressure, $\mathrm{Pa}$. The equations used to solve for the system of flow rates will be derived and then an algorithm to set up the matrices will be presented.

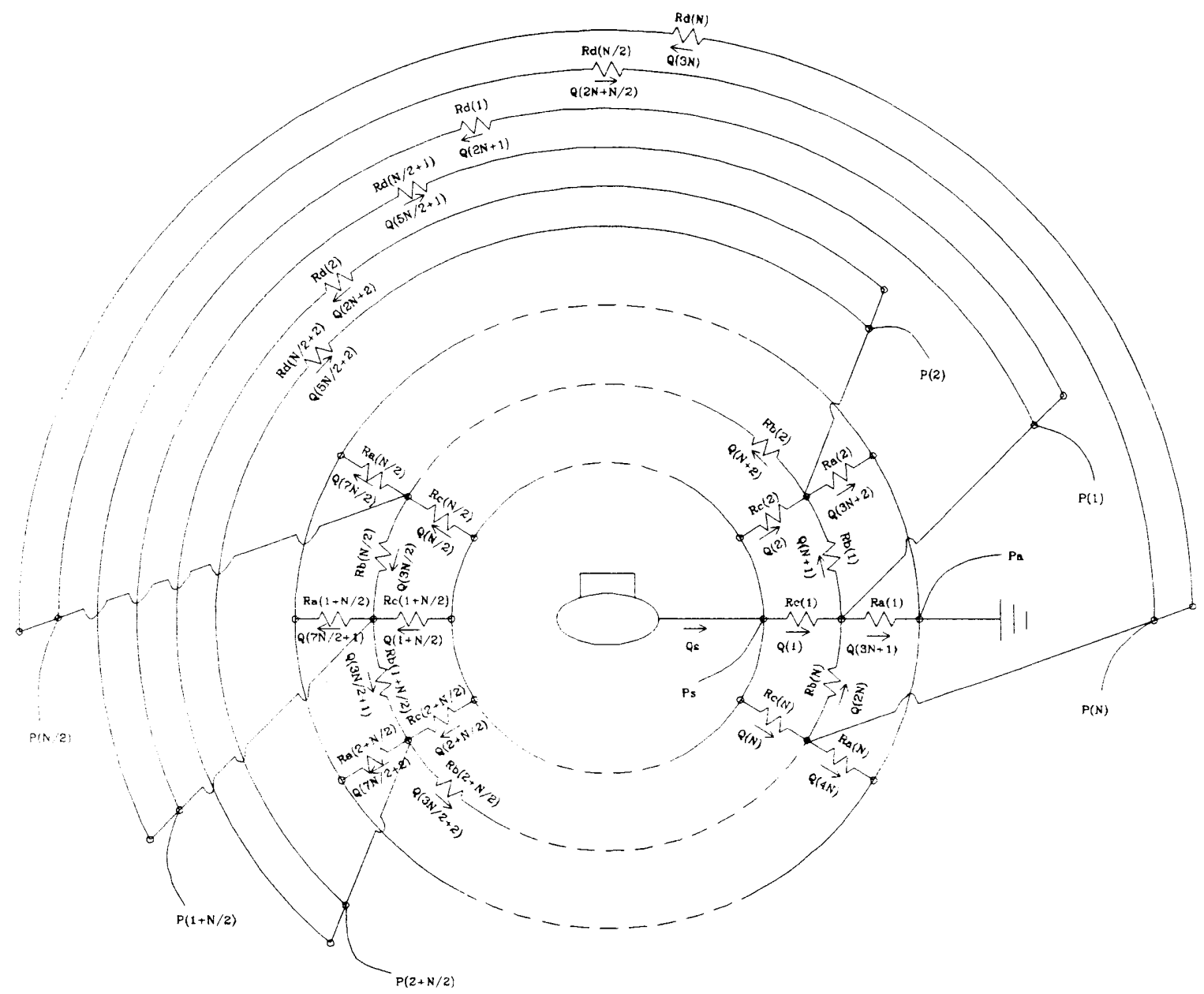

Figure B.3. Fluid circuit of a hydrostatic bearing with $\mathrm{N}$ pockets and leakage between its pockets and/or compensators, as well as leakage between its compensators and its pockets. 
A brief derivation of the equations needed to solve the circuit will first be presented. A set of $4 \mathrm{~N}$ equations are required to solve for the $4 \mathrm{~N}$ unknown flow rates. The first $\mathrm{N}$ equations are determined by noting that the sum of the pressure drops around the innermost loops must equal zero:

$$
Q(i) R c(i)+Q(i+N) R b(i)-Q(i+1) R c(i+1)=0 \quad i=1,2 \ldots N
$$

The next set of $\mathrm{N}$ equations are determined by setting the sum of the pressure drops around the outer loops equal to zero:

$$
Q(2 N+i) R d(i)-Q(N+i) R b(i) \ldots-Q(3 N / 2+i-1) R b(N / 2+i-1)=0 \quad i=1,2 \ldots N
$$

The next set of $\mathrm{N}$ equations are determined by noting that the sum of the flow rates into each central node must equal zero:

$$
Q(i)-Q(3 N+i)-Q(N+i)+Q(N+i-1)+Q(5 N / 2+i)-Q(2 N+i)=0 \quad i=1,2 \ldots N
$$

The fourth set of $\mathrm{N}$ equations are determined by setting the pressure drop across the compensators and pockets equal to the supply pressure and the outlet pressure:

$$
\mathrm{Q}(\mathrm{i}) \mathrm{Rc}(\mathrm{i})+\mathrm{Q}(3 \mathrm{~N}+\mathrm{i}) \mathrm{Ra}(\mathrm{i})=\mathrm{Ps}-\mathrm{Pa} \quad \mathrm{i}=1,2 \ldots \mathrm{N}
$$

These set of $4 \mathrm{~N}$ equations can then be solved for the $4 \mathrm{~N}$ unknown flow rates. Once the flow rates are known, the $\mathrm{N}$ pocket pressures can then be computed.

The following algorithm, written in BASIC, can be used to solve Equations B.9, B.10, B.11, and B. 12 for the unknown flow rates. First a $4 \mathrm{~N}$ by $4 \mathrm{~N}$ resistance matrix, RR(i,j), will be set up:

$$
\begin{aligned}
& \text { For } i=1 \text { To } 4 * N \\
& \text { For } j=1 \text { To } 4^{*} N \\
& R R(i, j)=0 \\
& \quad \text { Next } j \\
& \text { Next } i \\
& \text { For } i=1 \text { To N } \\
& R R(i, i)=\operatorname{Rc}(i)
\end{aligned}
$$




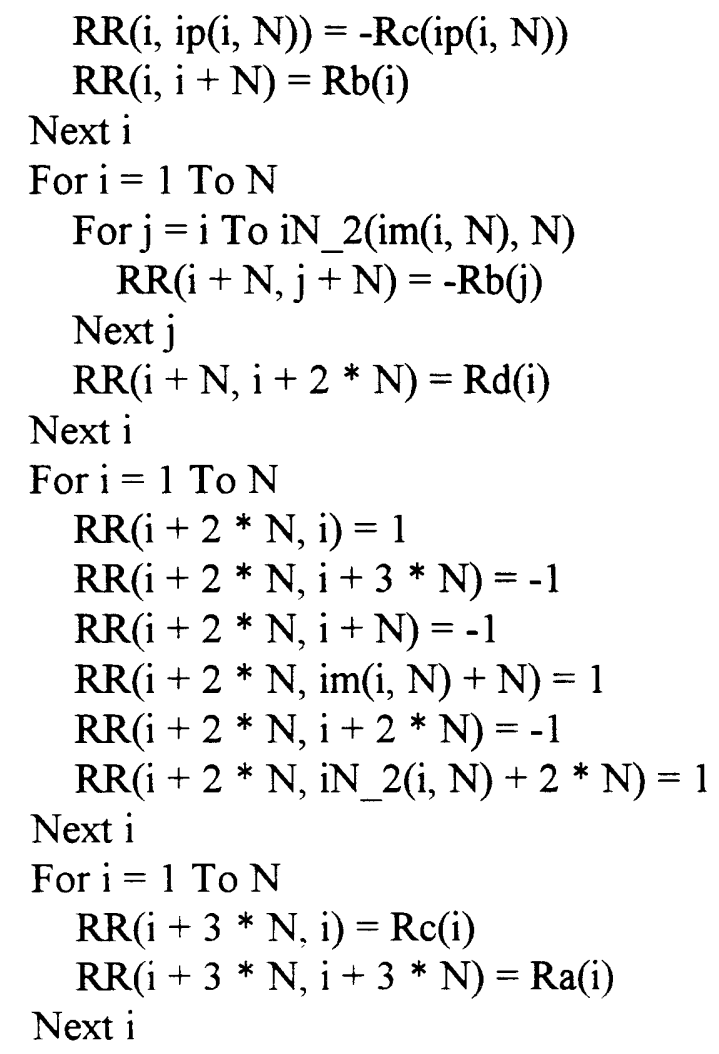

where im(i,N), ip $(i, N)$, and $\mathrm{N}_{\mathbf{N}} 2(\mathrm{i}, \mathrm{N})$ are functions that satisfy the following conditions:

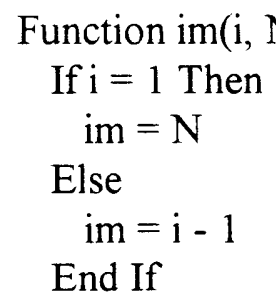

\section{End Function}

Function ip(i, N)

If $i=N$ Then ip $=1$

Else

ip $=i+1$

End If

End Function

Function iN_2(i, N)

If $\mathrm{i}<\mathrm{N} / \overline{2}$ Or $\mathrm{i}=\mathrm{N} / 2$ Then iN_2 $=\mathrm{i}+\mathrm{N} / 2$

Else

iN_2 $=\mathrm{i}-\mathrm{N} / 2$

End If

End Function 
Next a 1 by $4 \mathrm{~N}$ pressure matrix, $\mathrm{PP}(\mathrm{i})$, is created:

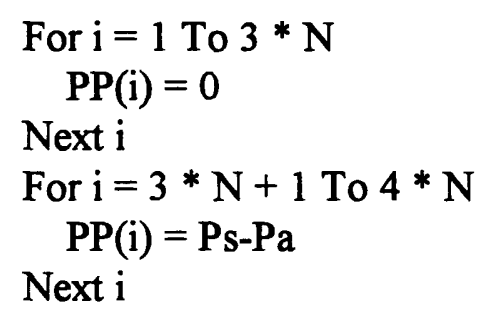

The systems of equations, $R R(i, j) Q(i)=P P(i)$, can be solved to obtain the unknown flow rates, Q(i) using any standard matrix solution algorithm. The supply flow rate is then calculated by:

$$
\mathrm{Q} s=\sum_{\mathrm{i}=1}^{\mathrm{N}} \mathrm{Q}(\mathrm{i})
$$

The pocket pressures are calculated by:

$$
P(i)=P a+Q(3 N+i) R a(i) \quad i=1,2 \ldots N
$$

\section{B.4. Fluid Circuit \#4}

The fourth fluid circuit is shown in Figure B.4. Fluid is pumped at supply pressure, Ps, across the compensating resistances, $\operatorname{Rc}(\mathrm{i}, \mathrm{j})$, to the pockets. The compensating lands are broken up into $\mathrm{M}$ divisions along their length. As the fluid crosses the compensating resistances, some of it leaks across the leakage resistances $R c b(i, j)$. After reaching the pockets, some of the fluid also leaks across the leakage resistances $\mathrm{Rb}$ (i) before it passes across the outlet resistances $\mathrm{Ra}(\mathrm{i})$ to atmospheric pressure, $\mathrm{Pa}$. The equations used to solve for the system of flow rates will be derived and then an algorithm to set up the matrices will be presented. 


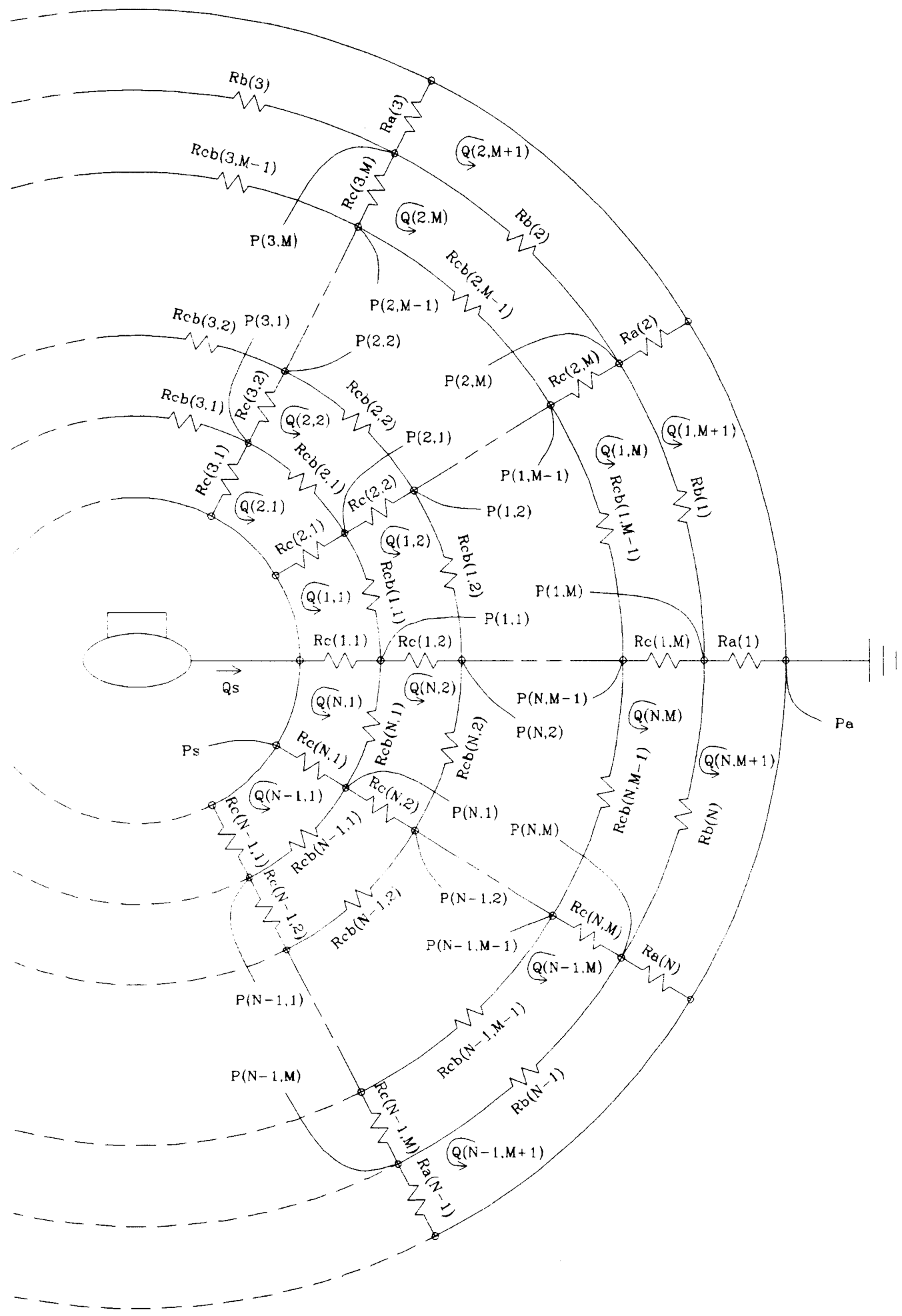

Figure B.4. Fluid circuit of a hydrostatic bearing with $\mathrm{N}$ pockets and leakage between its compensators along $\mathrm{M}$ divisions of their length, as well as leakage between the pockets. 
A brief derivation of the equations needed to solve the circuit will now be presented. A mesh-current method of circuit solution [ ] will be employed. This method is used because it is excellent for solving circuits with large numbers of degrees of freedom. Much fewer equations result using the mesh-current method and so the solution is much faster. Note that $(\mathrm{M}+2) \mathrm{N}$ mesh currents (or mesh flow rates) have been designated in Figure B.4. Since the circuit is essentially a three-dimensional circuit, N more mesh currents were needed to account for the supply loop. These currents, which will be designed as $Q(i, M+2)$ with $\mathrm{i}=1 \ldots \mathrm{N}$, are envisioned to begin at the nodes corresponding to atmospheric pressure, loop back to the pump, and connect to the nodes corresponding to supply pressure.

A set of $(M+2) N$ equations are required to solve for the $(M+2) N$ unknown flow rates. The first $\mathrm{N}$ equations are determined by noting that the sum of the pressure drops around the innermost loops must equal zero:

$$
\begin{gathered}
{[Q(i, 1)-Q(i-1,1)+Q(i, M+2)] \operatorname{Rc}(i, 1)+[Q(i, 1)-Q(i, 2)] \operatorname{Rcb}(i, 1)+} \\
{[Q(i, 1)-Q(i+1,1)-Q(i+1, M+2)] \operatorname{Rc}(i+1,1)=0 \quad i=1,2 \ldots N}
\end{gathered}
$$

The next set of equations are determined by setting the sum of the pressure drops around the inner loops equal to zero:

$$
\begin{gathered}
{[Q(i, j)-Q(i-1, j)+Q(i, M+2)] \operatorname{Rc}(i, j)+[Q(i, j)-Q(i, j+1)] \operatorname{Rcb}(i, j)+} \\
{[Q(i, j)-Q(i+1, j)-Q(i+1, M+2)] \operatorname{Rc}(i+1, j)+[Q(i, j)-Q(i, j-1)] \operatorname{Rcb}(i, j-1)=0} \\
i=1,2 \ldots N \quad j=2,3 \ldots M-1
\end{gathered}
$$

The next set of equations are determined by setting the sum of the pressure drops around the next inner loops equal to zero: 


$$
\begin{gathered}
{[Q(i, M)-Q(i-1, M)+Q(i, M+2)] R c(i, M)+[Q(i, M)-Q(i, M+1)] R b(i)+} \\
{[Q(i, M)-Q(i+1, M)-Q(i+1, M+2)] R c(i+1, M)+[Q(i, M)-Q(i, M-1)] R c b(i, M-1)=0} \\
i=1,2 \ldots N
\end{gathered}
$$

The next set of equations are determined by setting the sum of the pressure drops around the next outermost loops equal to zero:

$$
\begin{gathered}
{[Q(i, M+1)-Q(i-1, M+1)+Q(i, M+2)] R a(i)+} \\
{[Q(i, M+1)-Q(i+1, M+1)-Q(i+1, M+2)] R a(i+1)+[Q(i, M+1)-Q(i, M)] R b(i)=0} \\
i=1,2 \ldots N
\end{gathered}
$$

The last set of equations are determined by setting the sum of the pressure drops across each leg of the circuit (from supply pressure to atmospheric pressure) equal to the supply pressure difference:

$$
\begin{gathered}
{[Q(i, 1)-Q(i-1,1)+Q(i, M+2)] \operatorname{Rc}(i, 1)+\ldots+[Q(i, M)-Q(i-1, M)+Q(i, M+2)] \operatorname{Rc}(i, M)+} \\
{[Q(i, M+1)-Q(i-1, M+1)+Q(i, M+2)] \operatorname{Ra}(i)=P s-P a \quad i=1,2 \ldots N}
\end{gathered}
$$

This set of $(\mathrm{M}+2) \mathrm{N}$ equations are required to solve for the $(\mathrm{M}+2) \mathrm{N}$ unknown flow rates. Once the flow rates are known, the pocket pressures can then be computed.

The following algorithm, written in BASIC, can be used to solve Equations B.15, B.16, B.17, B.18, and B.19 for the unknown flow rates. First a $(\mathrm{M}+2) \mathrm{N}$ by $(\mathrm{M}+2) \mathrm{N}$ resistance matrix, $R R(i, j)$, will be set up:

$$
\begin{aligned}
& \text { For } i=1 \text { To }(M+2) * N \\
& \text { For } j=1 \text { To }(M+2) * N \\
& \quad \operatorname{RR}(i, j)=0 \\
& \text { Next } j \\
& \text { Next } i \\
& \text { For } i=1 \text { To N } \\
& \quad \operatorname{RR}(i, i)=\operatorname{Rc}(i, 1)+\operatorname{Rcb}(i, 1)+\operatorname{Rc}(i p(i, N), 1) \\
& \operatorname{RR}(i, i m(i, N))=-\operatorname{Rc}(i, 1)
\end{aligned}
$$




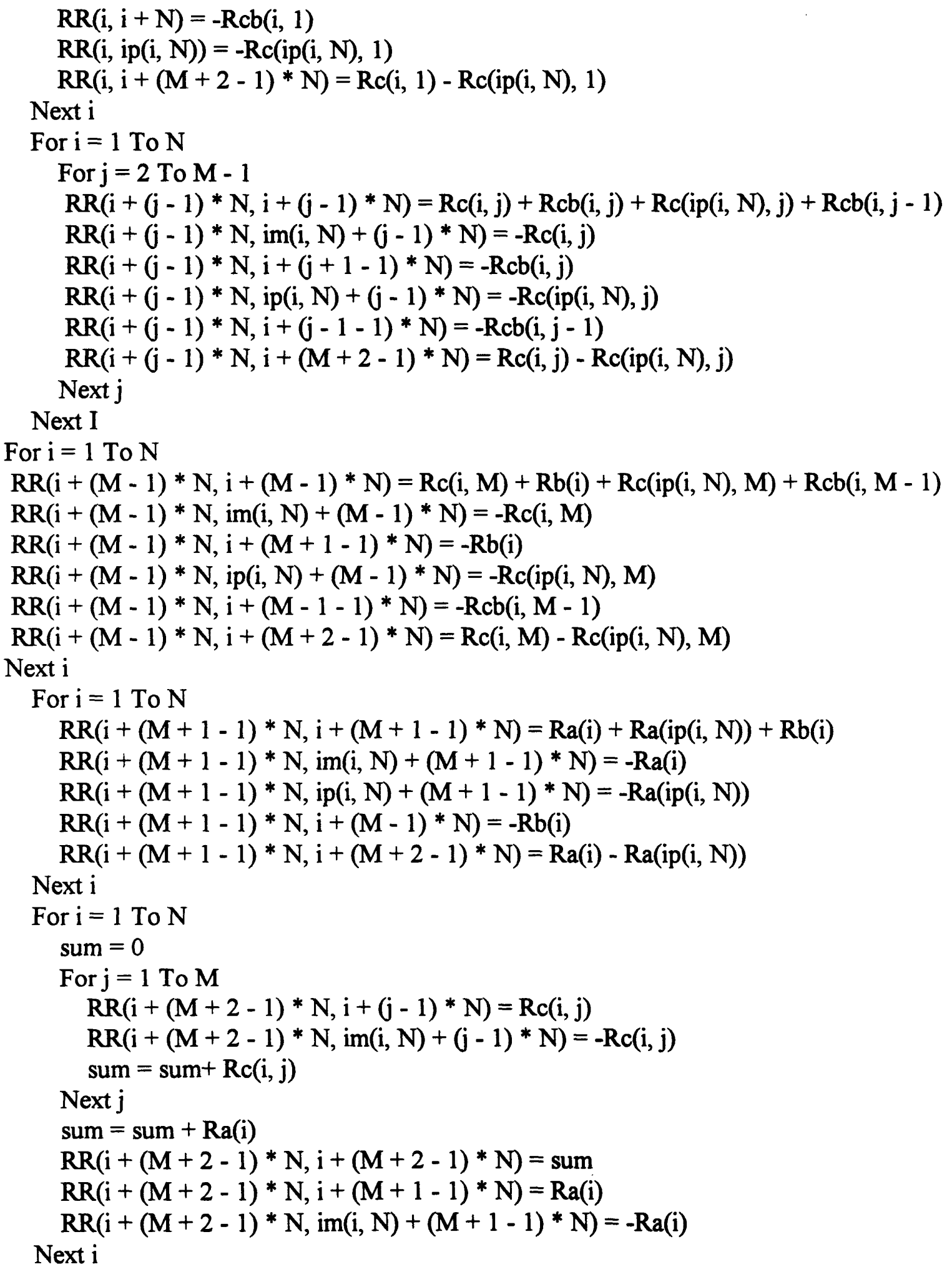

where $i m(i, N), i p(i, N)$, and $i N \_2(i, N)$ are functions that satisfy the following conditions:

Function im(i, N) 


$$
\begin{aligned}
& \text { If } i=1 \text { Then } \\
& \text { im }=N \\
& \text { Else } \\
& \text { im }=\mathrm{i}-1
\end{aligned}
$$

End If

End Function

Function ip(i, N)

If $i=N$ Then

ip $=1$

Else

ip $=i+1$

End If

End Function

Next a 1 by $(M+2) * N$ pressure matrix, $P P(i)$, is created:

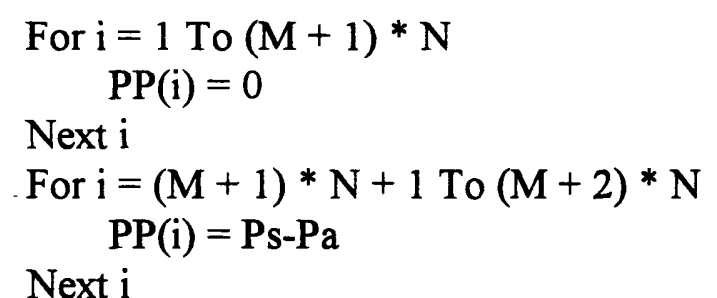

The systems of equations, $R R(i, j) Q(i)=P P(i)$, can be solved from the unknown flow rates, $\mathrm{Q}(\mathrm{i})$ using any standard matrix solution algorithm. The supply flow rate is then calculated by:

$$
Q s=\sum_{i=1}^{N}\{Q(i)-Q(i m(i, N))+Q(i+(M+1) * N)\}
$$

The first $\mathrm{N}$ pressures are calculated by:

$$
\mathrm{P}(\mathrm{i}, 1)=\mathrm{Ps}-(\mathrm{Q}(\mathrm{i})-\mathrm{Q}(\mathrm{im}(\mathrm{i}, \mathrm{N}))+\mathrm{Q}(\mathrm{i}+(\mathrm{M}+2-1) * \mathrm{~N})) * \mathrm{Rc}(\mathrm{i}, 1) \quad i=1,2 \ldots N
$$

The remaining pressures are calculated by:

$$
\begin{gathered}
P(i, j)=P(i, j-1)-\left(Q\left(i+(j-1)^{*} N\right)-Q(i m(i, N)+(j-1) * N)+Q(i+(M+2-1) * N)\right) * R c(i, j) \\
i=1,2 \ldots N \quad j=2,3 \ldots M
\end{gathered}
$$




\section{APPENDIX C: CRITERIA FOR LAMINAR AND FULLY- DEVELOPED LAND FLOW}

In the derivation of the hydraulic resistances of the bearing land flows given in Chapter 2, several important assumptions were made, including:

- The fluid flow resulting from the driving pressure difference across the land is laminar and fully-developed.

- The fluid flow resulting from shaft rotation is laminar and fully-developed.

If the bearing is operated at low speeds and low supply pressures, then both the flow induced by pressurization and the flow induced by shaft rotation are fully-developed fluid flows and the nonlinear terms of the governing equations are negligible. In this case, the combined fluid flow solution may be obtained by simply adding the two solutions considered separately. This is called the "principle of superposition." Therefore, the hydrostatic load-carrying characteristics of the bearing are not affected by the rotation of the shaft and may be determined by considering only the fluid flow resulting from pressurization. If either of the two combined fluid flows are not laminar and fullydeveloped, then convective effects must be considered and the analysis becomes far more complicated. This thesis has developed relations to predict the load-carrying characteristics of bearings that are operated at low speeds and low pressures such that the fluid flow across the lands remains laminar and fully-developed. Careful consideration must be given to the criteria developed in this appendix to ensure that the relations developed in Chapter 2 are applicable. 
Consider the developing laminar land flow shown in Figure C.1. The fluid is visualized as entering the clearance with a uniform velocity. As it proceeds to flow across the width of the land, it develops into a parabolic velocity profile that is characteristic of fully-developed viscous flow between parallel plates. The distance required for the flow to reach $99 \%$ of its fully-developed profile is called the "entrance length" of the flow. The relations given in Chapter 2 were developed assuming that the entrance length of the flow was much shorter than the width of the land. This is a good approximation if the fluid is very viscous, the land is very long with the respect to the clearance, or the flow rate is very low. However, for less viscous fluids, shorter land widths, or high flow rates, the assumption is not good and the entrance length can be a significant portion of the land width. Criteria will be developed in this appendix that must be satisfied for the pressureinduced flow to be laminar and fully-developed, so that the relations developed in Chapter 2 can be used to predict the bearing performance.

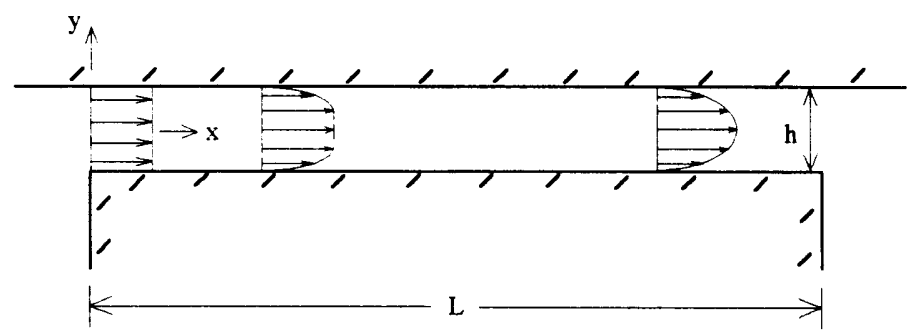

Figure C.1. Schematic diagram of developing laminar fluid flow over a flat land of uniform clearance.

A criterion will first be considered to determine whether the pressure-induced flow across a land is laminar. Generally, pressure-driven fluid flow through a channel remains 
laminar for Reynolds numbers based on the hydraulic diameter less than about 2300 [17]. This Reynolds number is defined as:

$$
\operatorname{Re}=\frac{\rho \bar{u} D_{h}}{\mu}
$$

where $D_{h}=2 h$ is the hydraulic diameter of the clearance and $\bar{u}$ is the average velocity given by:

$$
\overline{\mathrm{u}}=\frac{\mathrm{Q}}{\mathrm{L}_{\mathbf{z}} \mathrm{h}}
$$

where $\mathrm{Q}$ is the volumetric flow rate across the land and $\mathrm{L}_{\mathrm{z}}$ is the length of the land in the direction into the page. If the flow is fully-developed, then the flow rate can be determined from Equation (2.9). In this case, the critical pressure difference at which the flow is no longer laminar is then given by:

$$
\Delta \mathrm{p}_{\mathrm{cnt}} \approx 13800 \frac{\mu^{2}}{\rho} \frac{\mathrm{L}}{\mathrm{h}^{3}}
$$

The criterion of Equation (C.3) is conservative because the flow rate will be less if the flow is not fully-developed. Figure C.2 illustrates the critical pressure difference for a water-based coolant with a dynamic viscosity of $0.0013 \mathrm{~N} \mathrm{~s} / \mathrm{m}$ and a density of $995 \mathrm{~kg} / \mathrm{m}^{3}$, using various land widths and clearances. Note that for a $3 \mathrm{~mm}$ wide land with a clearance of $20 \mu \mathrm{m}$, the flow will no longer be laminar if the pressure difference is greater than approximately $1250 \mathrm{psid}$. Note that for most applications, the pressure drop across a land is approximately half of the supply pressure difference. This means that the flow over a 3 $\mathrm{mm}$ wide land with a clearance of $20 \mu \mathrm{m}$ will remain laminar up to a bearing supply pressure of approximately 2500 psig. 


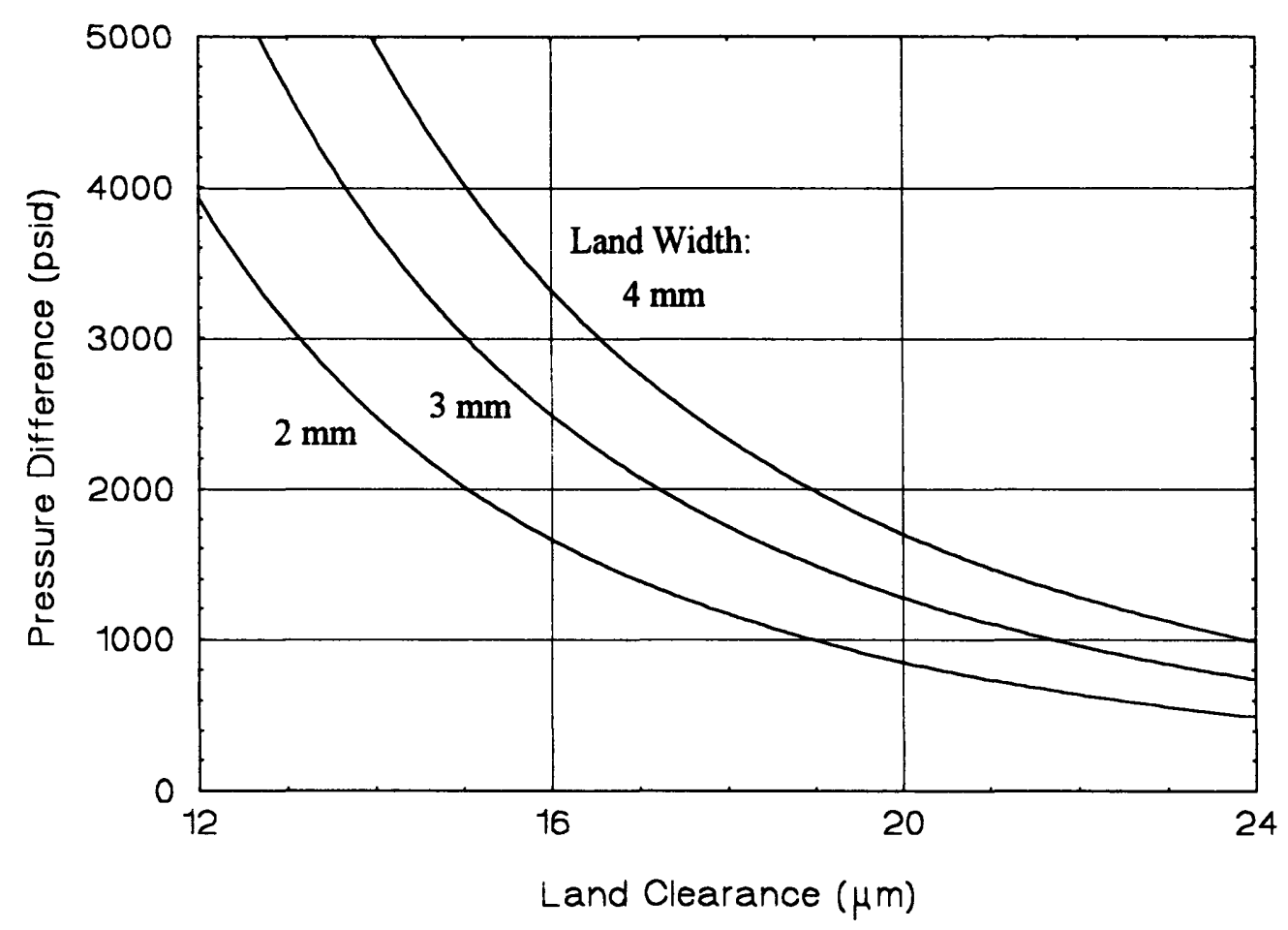

Figure C.2. Critical pressure difference for a rectangular land with a water-based coolant flowing over it. Below the critical pressure, the flow remains laminar.

The assumption of fully-developed pressure flow will be considered next. Note in the schematic diagram of Figure C.1 that the derivative of the velocity with respect to $y$ at the wall is greater while the flow is developing than it is when it is fully-developed. This means that, for a given flow rate, the pressure drop across the land will be greater than that predicted using the fully-developed relations of Chapter 2. An approximate correlation for the pressure drop in developing laminar channel flow is given by Shah [18]. The pressure difference required to drive the fluid at a mean velocity $\bar{u}$ is given by:

$$
\Delta \mathrm{p}=\frac{1}{2} \rho \overline{\mathrm{u}}^{2} \frac{4 \mathrm{~L}}{\mathrm{D}_{\mathrm{h}} \operatorname{Re}_{\overline{\mathrm{u}}}}\left(\mathrm{f}_{\mathrm{app}} \operatorname{Re}_{\overline{\mathrm{u}}}\right)
$$




$$
\begin{gathered}
f_{\text {app }} \operatorname{Re}_{\overline{\mathrm{u}}}=\frac{3.44}{\sqrt{\mathrm{L}^{+}}}+\frac{24+\frac{\mathrm{K}(\infty)}{4 \mathrm{~L}^{+}}-\frac{3.44}{\sqrt{\mathrm{L}^{+}}}}{1+0.000029\left(\mathrm{~L}^{+}\right)^{-2}} \\
\mathrm{~L}^{+}=\frac{\mathrm{L}}{\mathrm{D}_{\mathrm{h}} \operatorname{Re}_{\overline{\mathrm{u}}}}
\end{gathered}
$$

The value of $\mathrm{K}(\infty)$ has not yet been determined experimentally, but a value recommended by Shah is 0.66 ; he recommends this value based on theoretical work done by others. The entry length of the flow (the distance required for the flow to become $99 \%$ fully developed) is approximately $\mathrm{L}^{+}=0.011$.

Shown in Figure C. 3 is the ratio of flow rate calculated using Equations (C.4) through (C.6) divided by that predicted using the fully-developed relation, Equation (2.9), for several different clearances and a land width of $3 \mathrm{~mm}$. The fluid is a water-based coolant with a dynamic viscosity of $0.0013 \mathrm{~N} \mathrm{~s} / \mathrm{m}$ and a density of $995 \mathrm{~kg} / \mathrm{m}^{3}$. As shown, the predicted flow rate is approximately 0.96 times that for fully-developed flow for a clearance of $20 \mu \mathrm{m}$ and a pressure difference of $300 \mathrm{psid}$. Note that this corresponds to a bearing supply pressure of 600 psig. The curves of Figure C. 3 are all for a land width of 3 $\mathrm{mm}$; reducing the land width causes convective effects to become more important, and increasing the land width causes the convective effects to become less important. 


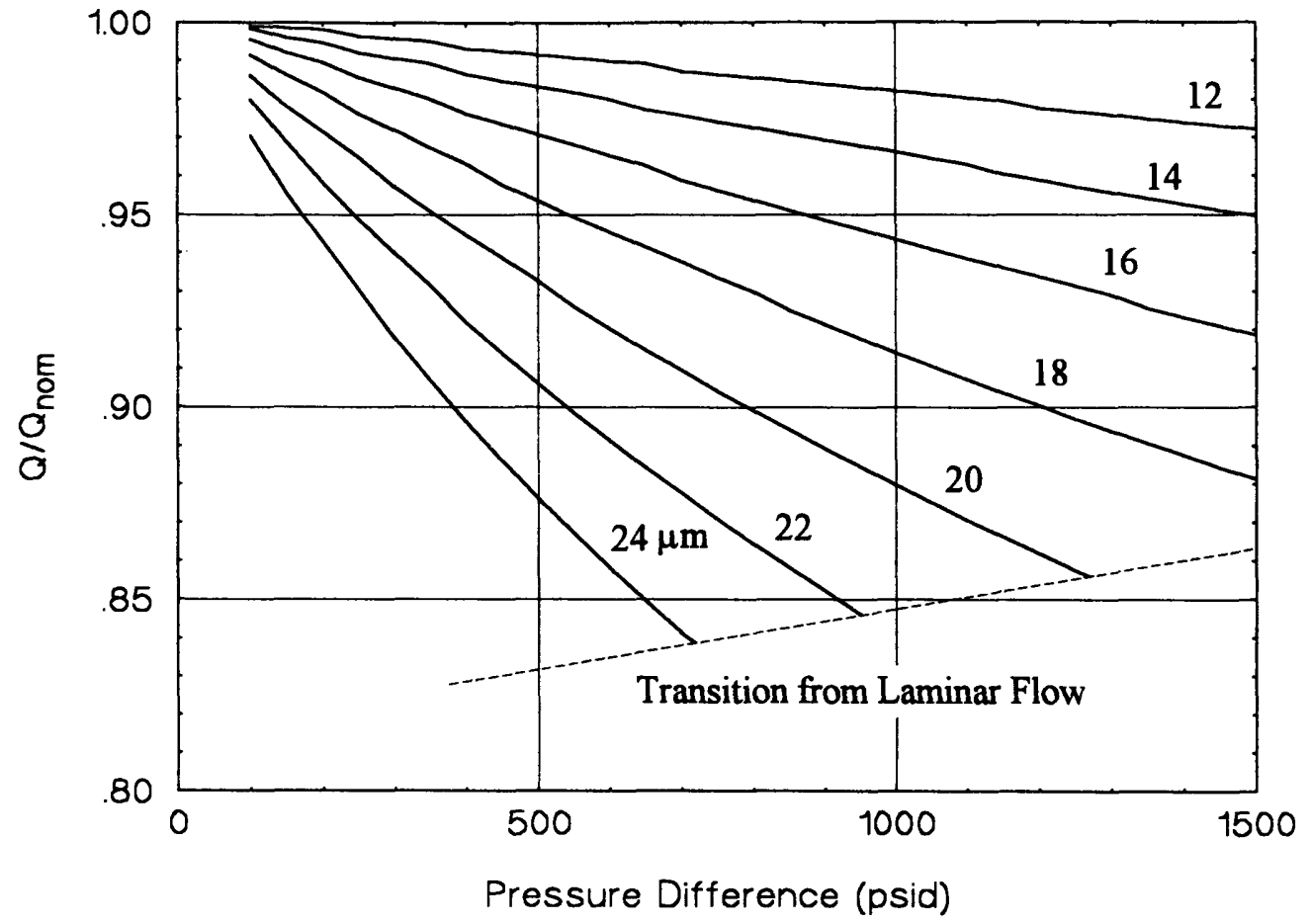

Figure C.3. Ratio of the flow rate across a land divided by that predicted using Equation (2.9), which was developed assuming fully-developed flow. The fluid is a water-based coolant. The different curves correspond to different clearances. The land width is $3 \mathrm{~mm}$.

The hydrodynamic entry length will be considered next. The distance over the land at which the flow reaches $99 \%$ of its fully developed velocity distribution is approximately $\mathrm{L}^{+}=0.011$. Shown in Figure C. 4 is the ratio of $\mathrm{L}^{+}$required to reach $99 \%$ of developed flow divided by $\mathrm{L}^{+}$corresponding to the entire $3 \mathrm{~mm}$ width of the land. The fluid is again a water-based coolant with a dynamic viscosity of $0.0013 \mathrm{~N} \mathrm{~s} / \mathrm{m}$ and a density of 995 $\mathrm{kg} / \mathrm{m}^{3}$. As shown, for a pressure difference of $300 \mathrm{psid}$ (corresponding to a bearing with a supply pressure of approximately $600 \mathrm{psig}$ ) and a land clearance of $20 \mu \mathrm{m}$, the flow reaches $99 \%$ of its fully-developed state at a location of approximately $7 \%$ of the width of 
the land. In this case the entry region is negligible. However, for higher pressures, larger clearances, or shorter land widths, the entry region can be significant.

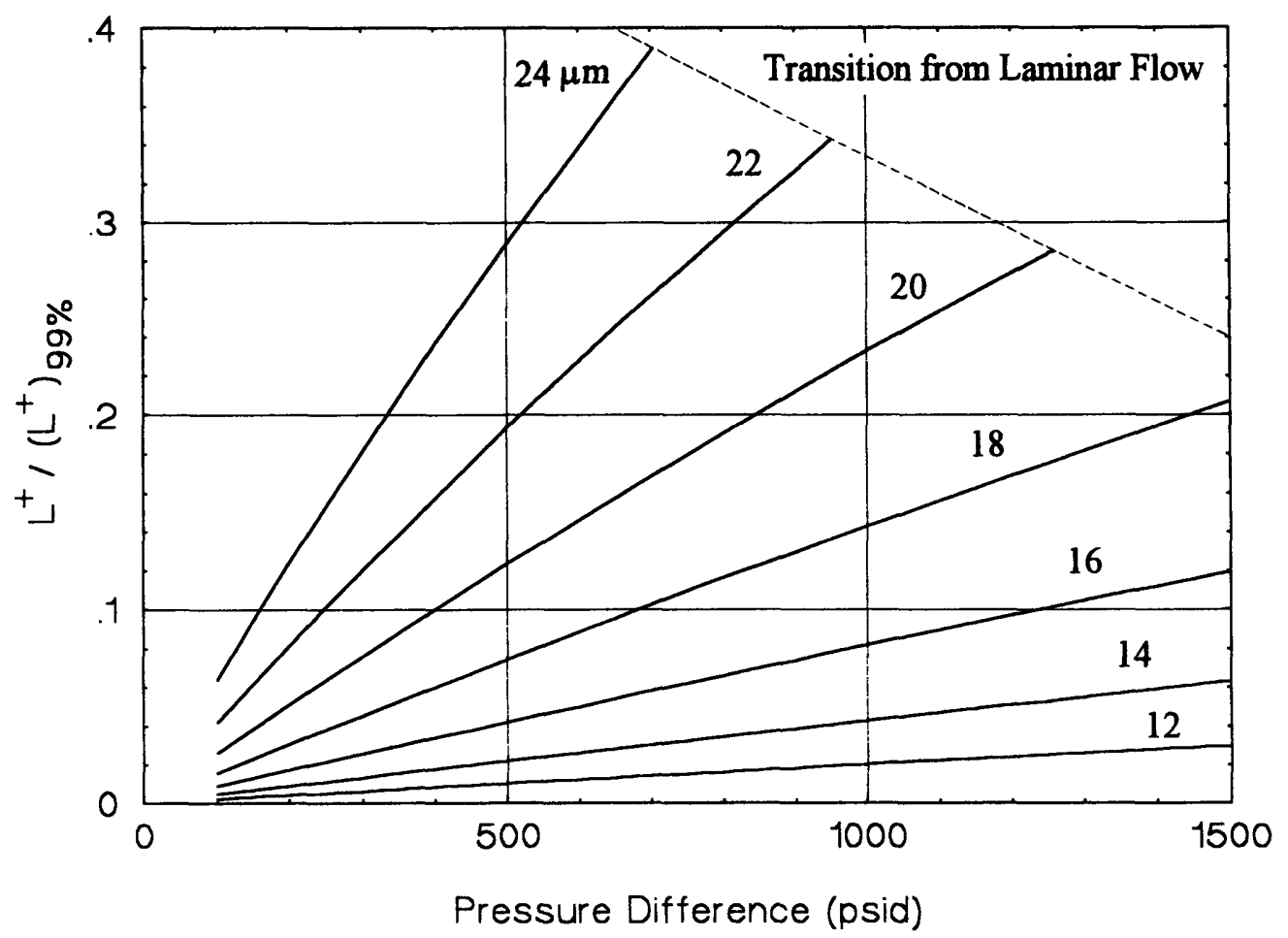

Figure C.4. Ratio of $\mathrm{L}^{+}$corresponding to the entire land width divided by $\mathrm{L}^{+}$required to reach $99 \%$ of fully-developed flow. The fluid is a water-based coolant. The different curves correspond to different clearances. The land width is $3 \mathrm{~mm}$.

Next consider the fluid flow over the lands that is caused by shaft rotation. Fluid flow that enters a land is shown schematically in figure C.5. The fluid is visualized as entering the land clearance with a uniform velocity. As the fluid is dragged across the width of the land, it develops into the linear velocity profile characteristic of Couette fluid flow. The relations given in Chapter 2 were developed assuming that the entrance length of the flow was much shorter than the width of the land. This is a good approximation if 
the fluid is very viscous, the land is very long with the respect to the clearance, or the shaft rotational speed is very low. However, for less viscous fluids, shorter land widths, or high speeds, the assumption is not good and the entrance length can be a significant portion of the land width. Criteria will be developed in this appendix that must be satisfied for the pressure-induced flow to be laminar and fully-developed, so that the relations developed in Chapter 2 can be used to predict the bearing performance. It is important to note that not all bearing lands have an entrance region caused by shaft rotation; some lands are uninterrupted circumferentially. Only those lands that are interuppted circumferentially by pockets or grooves must be considered.

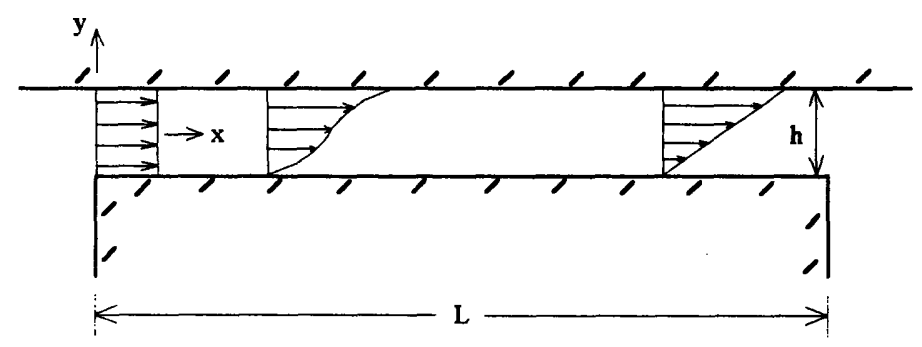

Figure C.5. Schematic diagram of developing laminar Couette flow over a flat land of uniform clearance.

A criterion will first be considered to determine whether the Couette flow across a land is laminar. Generally, the land fluid flow induced by shaft rotation remains laminar for Reynolds numbers based on the clearance dimension less than about 1600 [12]. This Reynolds number is defined as:

$$
\operatorname{Re}=\frac{\rho V h}{\mu}
$$


where $\mathrm{V}$ is the surface speed of the shaft and $\mathrm{h}$ is the clearance between the land and the housing. Rearranging, the critical shaft speed at which the fluid flow induced by shaft rotation is no longer laminar is given by:

$$
\omega_{\text {crit }}=3200 \frac{\mu}{\rho} \frac{1}{h D}
$$

where $\mathrm{D}$ is the shaft diameter. Consider a water-based coolant with a dynamic viscosity of $0.0013 \mathrm{~N} \mathrm{~s} / \mathrm{m}$ and a density of $995 \mathrm{~kg} / \mathrm{m}^{3}$. The critical speed at which the flow is no longer laminar is shown in Figure C. 5 for several different land clearances and shaft diameters.

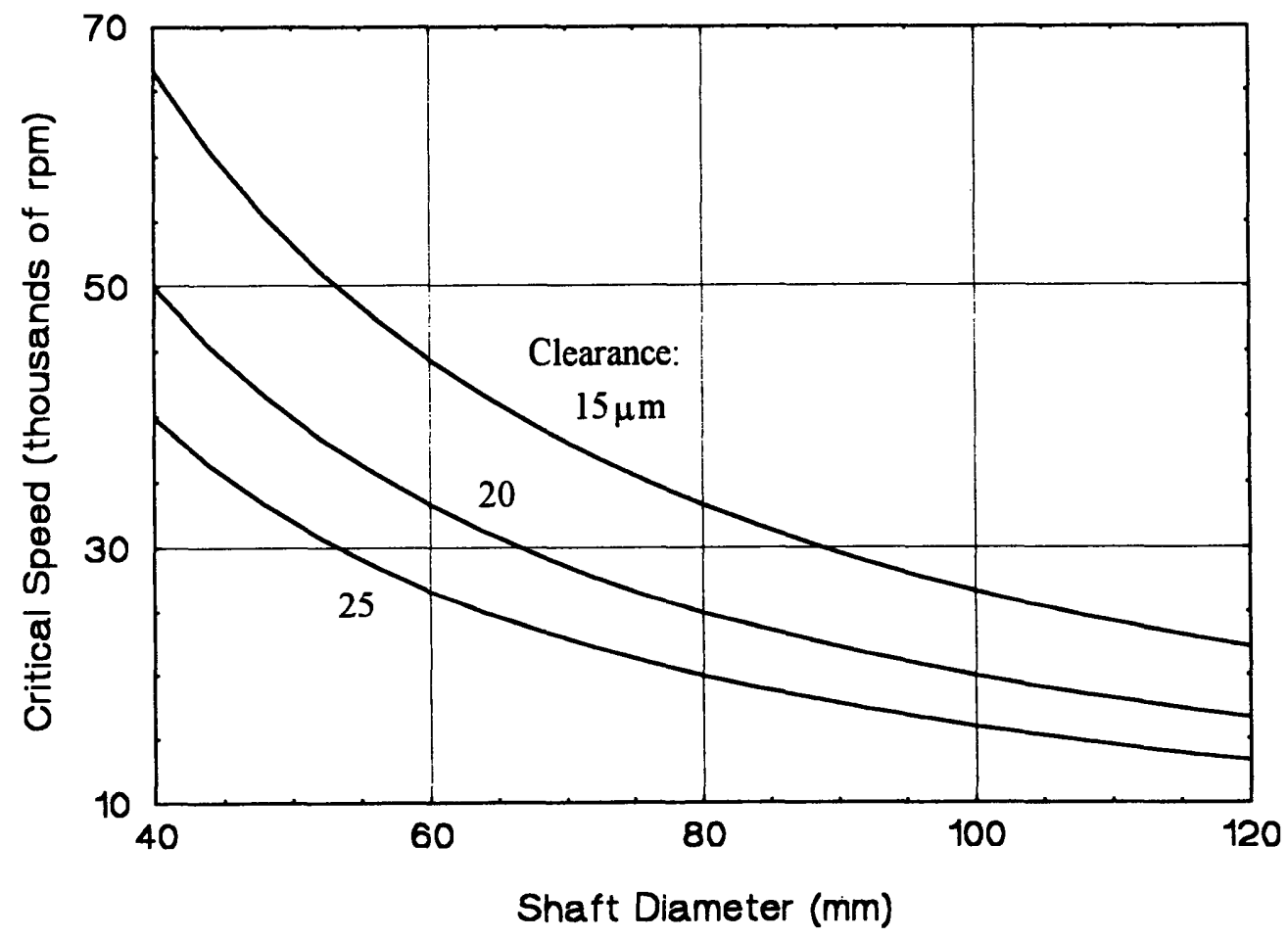

Figure C.5. Critical shaft speed above which the flow over the lands induced by shaft rotation is no longer laminar. The fluid is a water-based coolant. 
The speed at which the fluid flow induced by shaft rotation is no longer fullydeveloped will be considered next. An entry-region solution for Couette flow could not be found in the literature. However, an order-of-magnitude consideration will be given here. Consider the governing equation of motion, Equation (2.1). For the convective terms to be negligible,

$$
u \frac{\partial u}{\partial x}+v \frac{\partial u}{\partial y} \ll<\frac{\mu}{\rho} \frac{\partial^{2} u}{\partial y^{2}}
$$

An order-of-magnitude analysis can be performed by recognizing that, for the land flow at laminar speeds, $\mathrm{u} \sim \mathrm{V}, \mathrm{x} \sim \mathrm{L}$, and $\mathrm{y} \sim \mathrm{h}$. By substituting into Equation (2.2), continuity yields a characteristic y-velocity scale:

$$
\mathrm{v} \sim \frac{\mathrm{h}}{\mathrm{L}} \mathrm{V}
$$

Substituting into Equation (),

$$
\begin{gathered}
\mathrm{V} \frac{\mathrm{V}}{\mathrm{L}}+\mathrm{V} \frac{(\mathrm{Vh} / \mathrm{L})}{\mathrm{h}} \ll \frac{\mu}{\rho} \frac{\mathrm{V}}{\mathrm{h}^{2}} \\
\frac{\mathrm{V}^{2}}{\mathrm{~L}} \ll v \frac{\mathrm{V}}{\mathrm{h}^{2}} \\
\frac{\mathrm{Vh} \frac{\mathrm{h}}{\mathrm{L}}}{\mathrm{L}} \ll 1 \\
\operatorname{Re} \frac{\mathrm{h}}{\mathrm{L}} \ll 1
\end{gathered}
$$

Although this criterion accurately represents the scaling required for the flow to be fullydeveloped, it is only an order-of-magnitude estimate of the condition required for the land flow induced by shaft rotation to be fully-developed. 


\section{APPENDIX D: MODEL FOR THE POCKET PRESSURE GRADIENT}

Relations are presented in this appendix that can be used to approximately calculate the pressure gradient within the pockets or grooves of hydrostatic bearings at speeds sufficiently high such that the flow is highly turbulent $\left(\operatorname{Re}_{\mathrm{p}}=\rho \mathrm{Vh}_{\mathrm{p}} / \mu>1 \times 10^{4}\right)$. These relations were developed by Wasson [14]. Once the pressure gradient is known, it can be used to calculate the change in flow rate caused by fluid pumping within the grooves of Self-Compensated Bearing \#10, presented in Chapter 3. First, a "fullydeveloped" pressure gradient is calculated. Next, a correction factor is added to approximately account for the effect of turning the fluid around within the pocket. Note that the "walls" inside hydrostatic bearings are typically smooth and surface roughness was neglected.

A relation between the pressure gradient and the wall friction within the pocket was derived by Wasson by considering a small circumferential fluid element $\mathrm{dx}$, as shown in Figure D.1. The fluid is considered "fully-developed," meaning that the velocity profile is unchaning along the length of the pocket. Equating the forces on this fluid element,

$$
\begin{array}{r}
\tau_{t} d x+\tau_{b} d x=[(p+d p)-p] h_{p} \\
\frac{d p}{d x}=\frac{\tau_{t}+\tau_{b}}{h_{p}}=\rho \frac{u_{t}^{2}+u_{b}^{2}}{h_{p}}
\end{array}
$$

Letting $n=u_{b} / u_{t}$,

$$
\frac{d p}{d x}=\rho \frac{u_{t}^{2}}{h_{p}}\left(n^{2}+1\right)
$$


where $u_{t}=\sqrt{\tau_{t} / \rho}, u_{b}=\sqrt{\tau_{b} / \rho}, \tau_{t}$ is the top (shaft) wall shear stress, $\tau_{b}$ is the bottom (sleeve) wall shear stress, and $\rho$ is the fluid density.

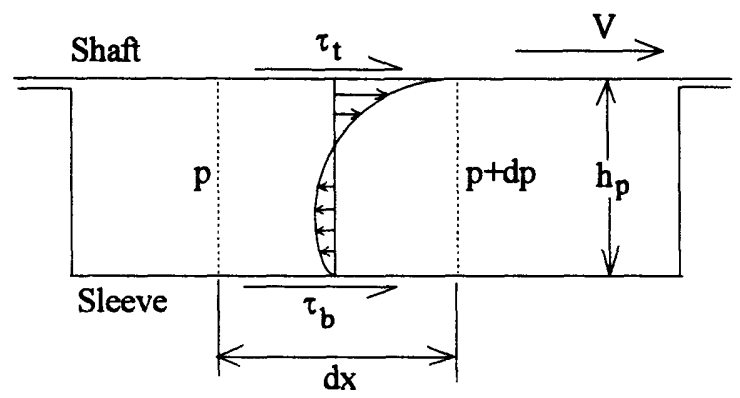

Figure D.1. Small circumferential fluid element within the pocket.

Using an eddy-viscosity model to account for the turbulence, the following relations were derived to relate the top wall friction factor to the shear stress ratio [14]:

$$
\begin{gathered}
\ln \left(\operatorname{Re}_{\mathrm{p}} \sqrt{\frac{C_{f}}{2}}\right)=\frac{-n^{3}+3 n^{2}+n+1}{2 n^{2}}+\frac{\kappa\left(1-2 n^{2}\right)}{6 n C_{c}}-\kappa C-\ln (n) \\
\sqrt{\frac{2}{C_{f}}}=C(1-n)+\frac{1-n}{\kappa} \ln \left(\operatorname{Re}_{p} \sqrt{\frac{C_{f}}{2}}\right)-\frac{n}{\kappa} \ln (n)+\frac{\left(n^{2}+1\right)(1-n)}{n \kappa}+\frac{1-n^{2}}{2 C_{c}}
\end{gathered}
$$

where $C_{f}=\tau_{t} /\left(\rho V^{2} / 2\right)$ and $R e=V h_{p} / \nu, \kappa$ is von Karmann's constant $(=0.42), C$ is the log-law constant $(=5.43)$, and $\mathrm{C}_{\mathrm{c}}$ is a constant that was determined empirically to be equal to -0.17 . These two relations can be solved simultaneously for the friction factor, $\mathrm{C}_{\mathrm{f}}$, and the shear stress ratio, $\mathrm{n}$. Once the values of $\mathrm{n}$ and $\mathrm{C}_{\mathrm{f}}$ are found, they can be substituted into Equation (D.3) to calculate the fully-developed pressure gradient. 
The relation presented above was derived assuming that the fluid flow profile within the pocket is fully-developed. To account for effect of the fluid turning at the leading and trailing edge of the pocket, the following relation may be used [14]:

$$
\frac{\mathrm{dp}}{\mathrm{dx}}=\left(\frac{\mathrm{dp}}{\mathrm{dx}}\right)_{\text {fully-developed }}\left\{1+\ln \left[1+2.71 \mathrm{Re}_{\mathrm{p}}^{-0.134}\left(\frac{\mathrm{h}_{\mathrm{p}}}{\mathrm{L}_{\mathrm{p}}}\right)^{3.51 \mathrm{Re}_{\mathrm{p}}^{-0.131}}\right]\right\}
$$

Note that the length, $L_{p}$, to use in Equation (D.6) is the approximate length in which the fluid completely turns around. In a conventional pocket, this would simply be the pocket circumferential length. However, in a slanted groove, the approximate distance is shown in Figure D.2. The pressure difference from one end of the groove to the other is given by:

$$
\Delta p=\left(\frac{d p}{d x}\right) L_{p}^{*}
$$

where $L_{p}{ }^{*}$ is the circumferential length from the beginning to the end of the groove, as shown in Figure D.2.

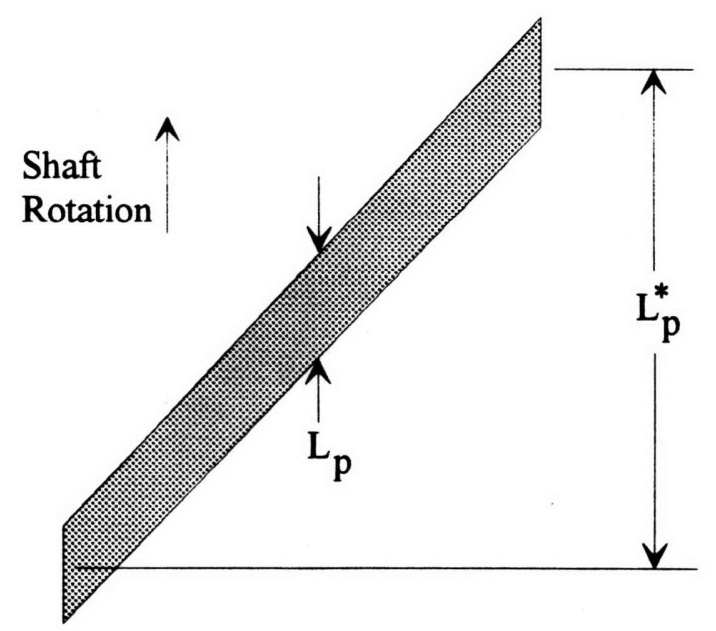

Figure D.2. Schematic diagram of a slanted groove. The top lid of the groove is moved at high speed in the direction shown, while the fluid recirculates within the length $L_{p}$. 